\title{
FREEZE-THAW SLUDGE DEWATERING AND STABILISATION USING FERRATE(VI)
}

\author{
by \\ James Diak, B.Eng, M.A.Sc.
}

A thesis submitted to the Faculty of Graduate and Postdoctoral Affairs in partial fulfilment of the requirements for the degree of

Doctor of Philosophy

in

\section{Environmental Engineering*}

\author{
Department of Civil and Environmental Engineering \\ Carleton University \\ Ottawa, Ontario \\ Canada
}

*The Doctor of Philosophy in Environmental Engineering program is a joint program with the University of Ottawa administered by the Ottawa-Carleton Institute for Environmental Engineering

(C)2015

James Diak 


\section{ABSTRACT}

The study examined the individual and combined effects of potassium ferrate(VI) additions and freeze-thaw conditioning for the stabilisation and dewatering of wastewater sludges. The purpose of the research was to develop a simple and effective sludge management approach for remote communities in cold climates.

Freeze-thaw sludge dewatering with gravity meltwater drainage reduced the sludge volume by up to $88 \%$, and resulted in approximately $2.4-\log$ inactivation of fecal coliform. For many of the test samples, increasing the time frozen from 1 to 15 days increased the level of inactivation of fecal coliform. However, some samples demonstrated >3-log inactivation of fecal coliform after only 1 day frozen, which suggests that fecal coliform inactivation occurs primarily as a result of the freezing process. Similarly, the freezing temperature did not have a significant effect on the level of inactivation, and increasing the duration of time spent frozen from 1 to 15 days did not improve sludge drainability during thaw, which indicates that particle consolidation, leading to improved sludge dewaterability, occurs during the freezing process.

Potassium ferrate( $\mathrm{VI})$ additions followed by a 15 -minute reaction period oxidised sludge constituents, which inactivated fecal coliform, reduced the concentrations of odour causing compounds (ammonia and sulphide), and solubilised sludge solids, resulting in an increase in soluble chemical oxygen demand (sCOD), soluble proteins and soluble carbohydrates. Potassium ferrate(VI) additions as low as $1.0 \mathrm{~g} / \mathrm{L}$ also reduced the concentrations of hormones in sludge. 
Co-treatment of anaerobically digested sludge using $5.0 \mathrm{~g} / \mathrm{L}$ of potassium ferrate(VI) followed by freeze-thaw with gravity meltwater drainage reduced fecal coliform to $<100$ colony forming units (CFU)/g dry solids (DS), and dewatered the sludge to $>12 \%$ total solids (TS), representing an $85 \%$ reduction in sludge mass following a 12 -hour thawing period. The sludge cake remaining was suitable for land application in terms of fecal coliform under the level 1 criteria for pathogens (CP1) (Ontario Ministry of Agriculture, Food and Rural Affairs (OMAFRA), 2013). Additionally, raw primary sludge was treated and dewatered to below CP2 limits using $5.1 \mathrm{~g} / \mathrm{L}$ potassium ferrate( $\mathrm{VI})$ followed by freeze-thaw with gravity meltwater drainage, and to below CP1 limits using $<15 \mathrm{~g} / \mathrm{L}$ pretreatment followed by freeze-thaw. 


\section{ACKNOWLEDGEMENTS}

First I would like to thank my supervisor, Dr. Banu Örmeci, for providing me this opportunity, and for her continued dedication, support and encouragement throughout this lengthy process. Next I would like to thank all the faculty and staff in the Department of Civil and Environmental Engineering at Carleton University, especially my previous professors, Dr. Marie-Josée Tudoret Chow, and all my close friends in the Örmeci lab group. To all the committee members who examined this research: Thank you for your time and contributions. I would also like to thank Corinne Proux and Lysane Bolduc for their contributions, and all of the great people at IMS.

Finally, I'd like to thank my family, close friends, and particularly Kelly, for their tolerance and unconditional support. You've all helped make this possible. I could not have done it without you.

This is dedicated to the ones I love, and all the ones I've thanked more than ten times. 


\section{TABLE OF CONTENTS}

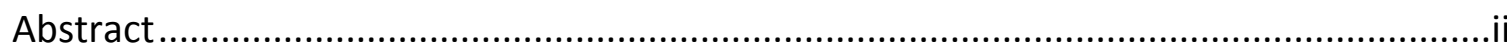

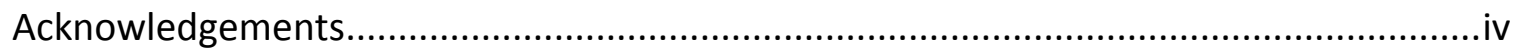

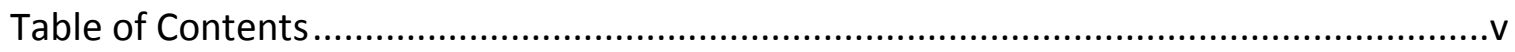

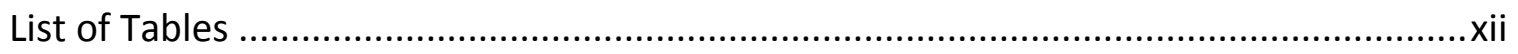

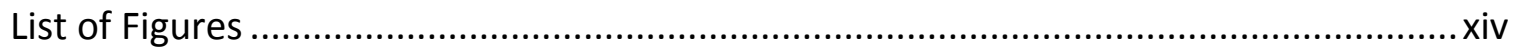

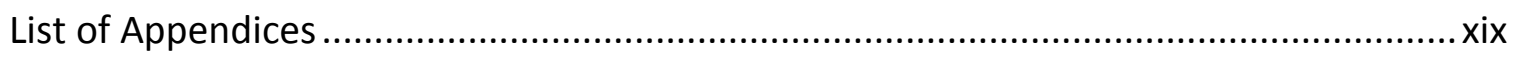

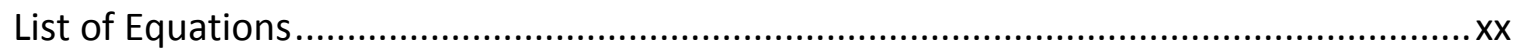

List of Acronyms and Nomenclature ................................................................

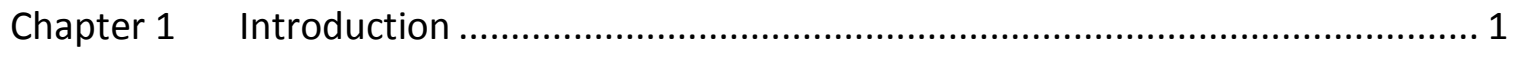

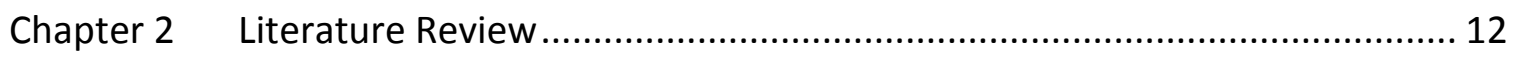

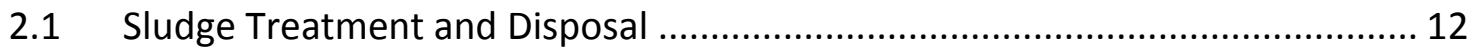

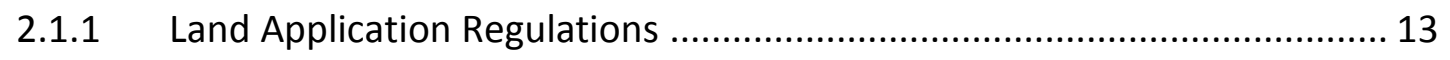

2.1.2 Emerging Organic Compounds (EOCs) ................................................. 19

2.1.3 Discussion on the Land Application of Treated Biosolids .......................... 24

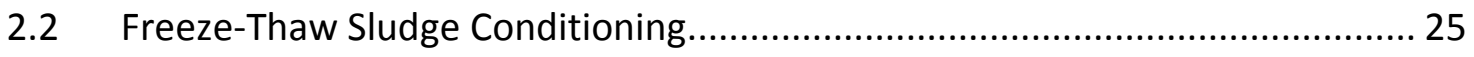

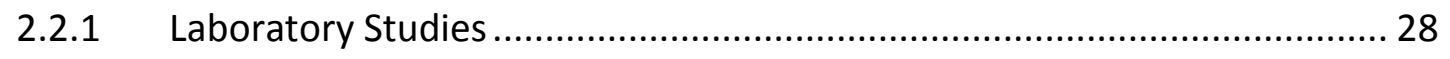

2.2.2 Pilot and Field-Scale Freezing Beds................................................. 42

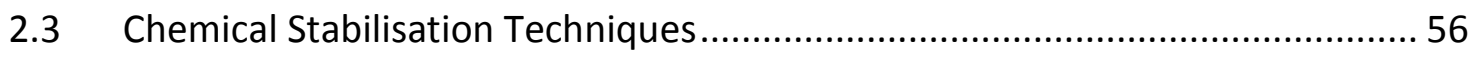

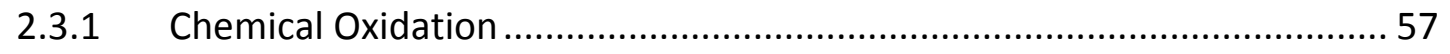

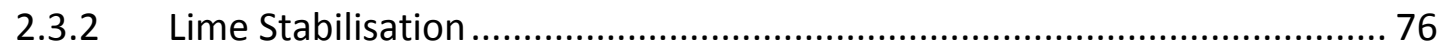

2.3.3 Discussion on the Use of Ferrate(VI) as a Chemical Oxidant for Wastewater

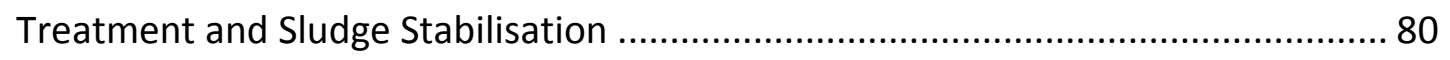

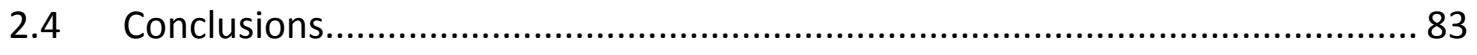

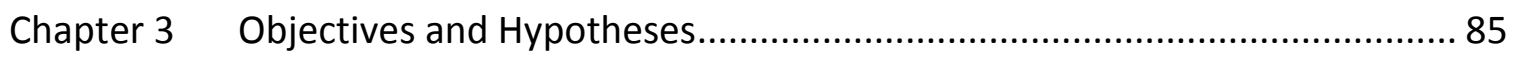

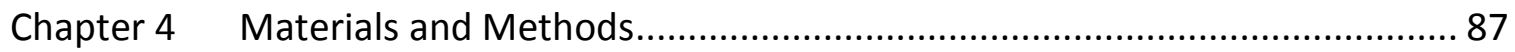


4.1 Freeze-Thaw Treatment of RBC Sludge from a Remote Mining Exploration Facility in Subarctic Canada (Chapter 5) ............................................................... 88

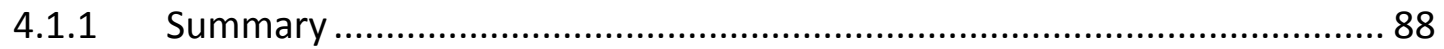

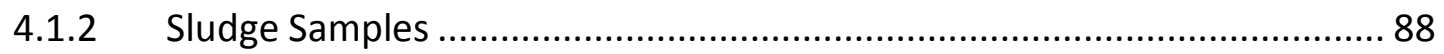

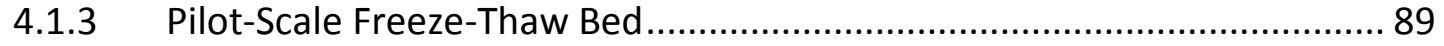

4.1.4 Sample Analysis: Initial Sludge and Sludge Cake Following Freeze-Thaw .. 91

4.1.5 Sample Analysis: Meltwater Collected During Sludge Thaw .................... 103

4.2 Combined Freeze-Thaw and Ferrate(VI) Oxidation Treatment on E. coli in a PBS

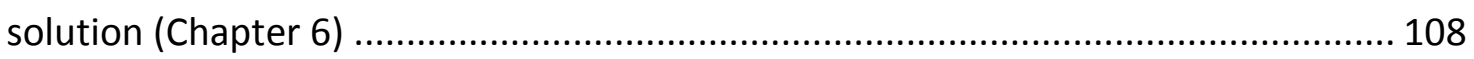

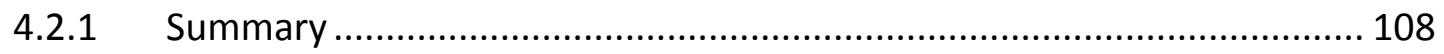

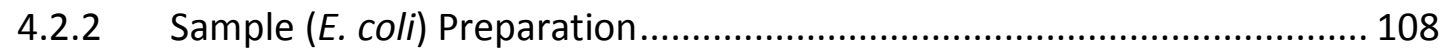

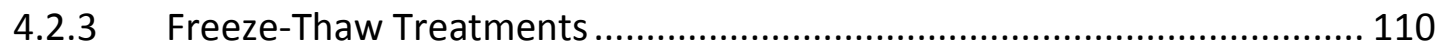

4.2.4 Pre- and Post-Treatment with Potassium ferrate(VI) ........................... 112

4.2.5 Sample Analysis: E. coli Enumeration ................................................ 113

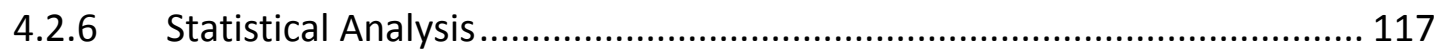

4.3 Individual and Combined Effects of Freeze-Thaw and Ferrate(VI) Oxidation for the Stabilisation and Dewatering of Wastewater Sludges (Chapter 7) ..................... 118

4.3.1 Summary ……….............................................................................. 118

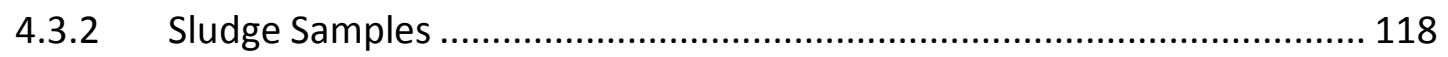

4.3.3 Part I: Freeze-Thaw Treatment in Bottles............................................... 119

4.3.4 Part II: Freeze-Thaw Treatment in Simulated Drainage Beds.................. 126

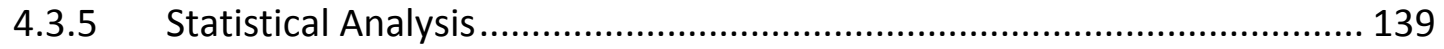

4.4 Stabilisation and Dewatering of Primary Sludge USING Ferrate(VI) PreTreatment Followed By Freeze-Thaw in Simulated Drainage Beds (Chapter 8)......... 139

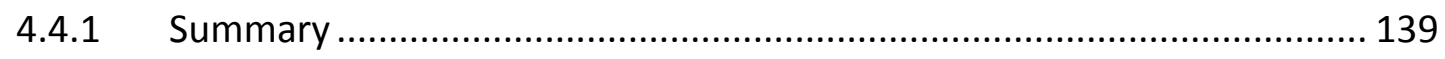

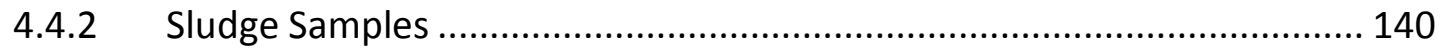

4.4.3 Pre-Treatment with Potassium Ferrate(VI) ........................................ 140

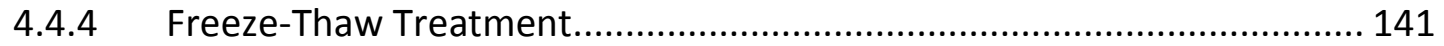

4.4.5 Sample Analysis: Initial Sludge and Ferrate(VI)-Treated Sludge.............. 142

4.4.6 Sample Analysis: Sludge Cake Following Freeze-Thaw .......................... 142 
4.4.7 Sample Analysis: Meltwater Collected During Sludge Thaw .....

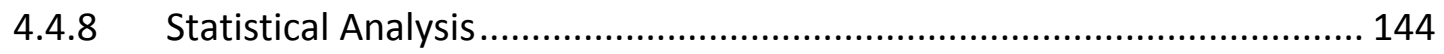

4.5 Oxidation of Selected Hormones in Anaerobically Digested (AD) Sludge

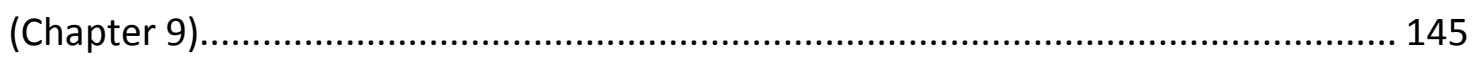

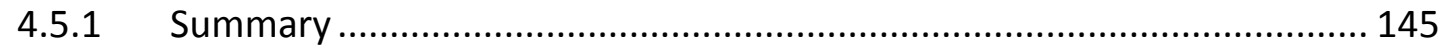

4.5.2 Hormone Stock Solutions............................................................... 145

4.5.3 Sludge Sample and Hormone Spiking ................................................. 146

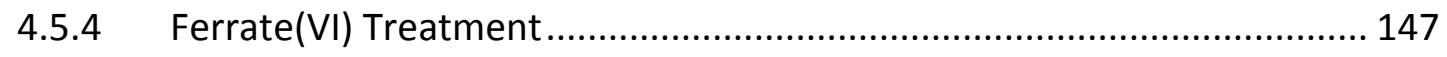

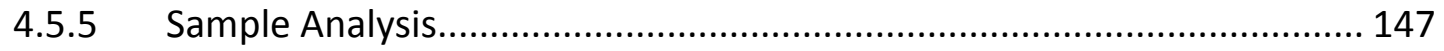

Chapter 5 Freeze-Thaw Treatment of Rotating Biological Contactor (RBC) Sludge from a Remote Mining Exploration Facility in Subarctic Canada .......................................... 151

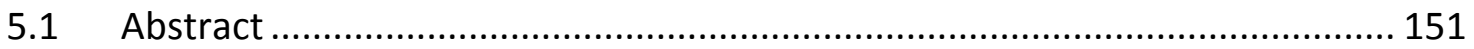

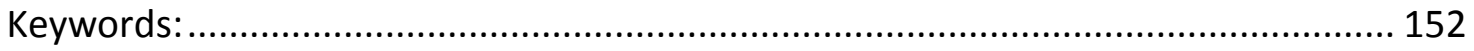

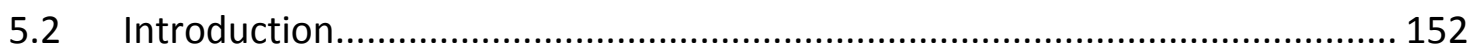

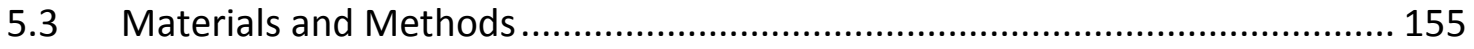

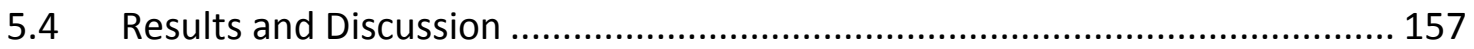

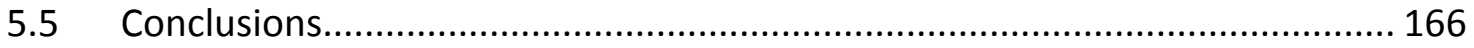

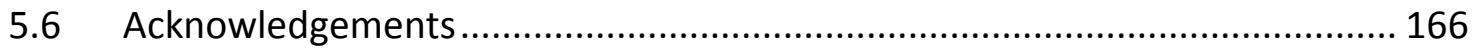

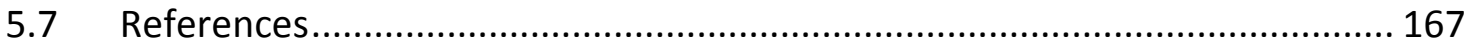

Chapter 6 Combined Freeze-Thaw and Ferrate(VI) Oxidation Treatment on E. coli in a

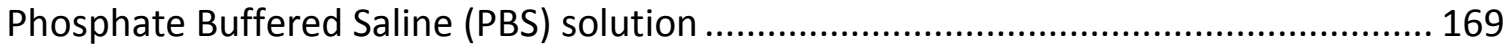

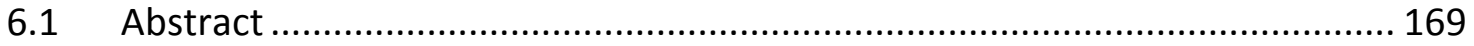

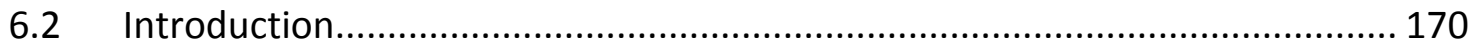

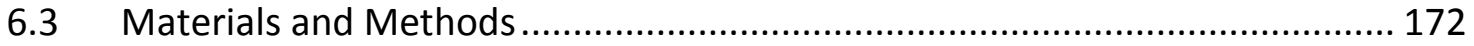

6.3.1 Sample (E. Coli) Preparation ............................................................. 172

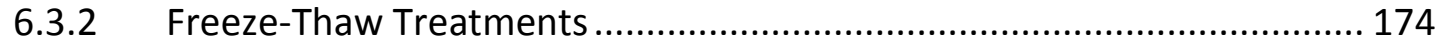

6.3.3 Pre-and Post-Treatments with Potassium Ferrate(VI) ........................... 176

6.3.4 E. Coli Enumeration....................................................................... 178

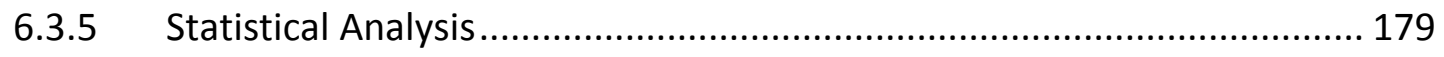

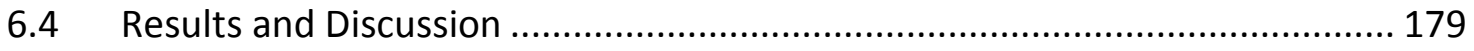


6.4.1 Effect of Ferrate(VI) Dose and Contact Time

6.4.2 Comparison of MTF and MF Enumeration Techniques, and 7-day FreezeThaw Reproducibility Test

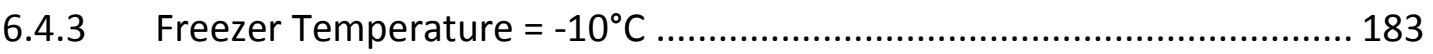

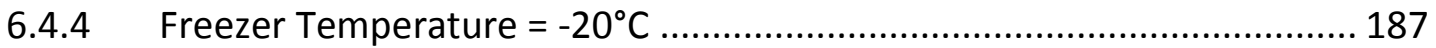

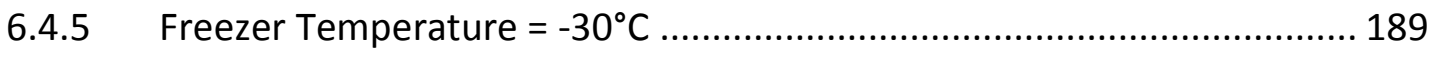

6.4.6 Effect of Freezing Time and Freezing Temperature (Freezing Rate) ........ 192

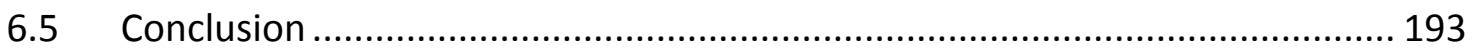

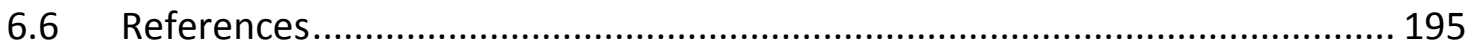

Chapter 7 Individual and Combined Effects of Freeze-Thaw and Ferrate(VI) Oxidation for the Stabilisation and Dewatering of Wastewater Sludges....................................... 199

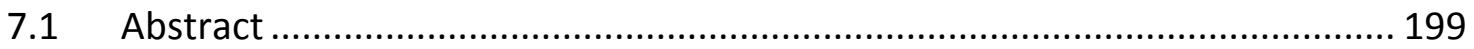

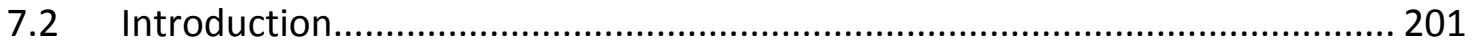

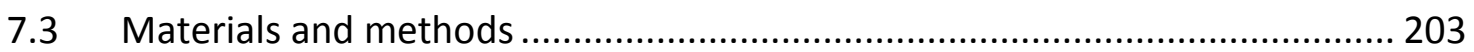

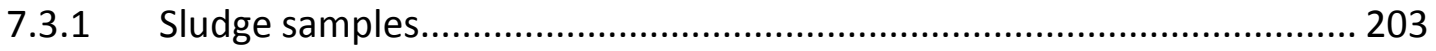

7.3.2 Part I: Freeze-thaw treatment in bottles .............................................. 204

7.3.3 Part II: Freeze-thaw treatment in simulated drainage beds.................... 206

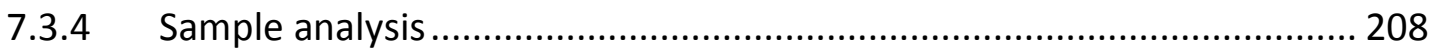

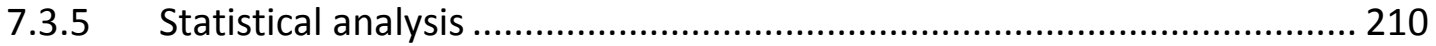

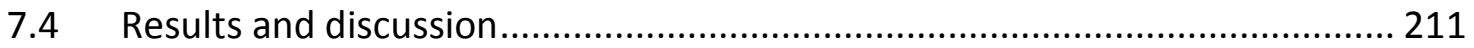

7.4.1 Part I: Freeze-thaw treatment in bottles ............................................. 211

7.4.2 Part II: Freeze-thaw treatment in simulated drainage beds..................... 221

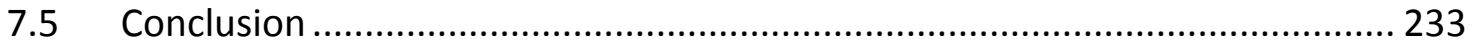

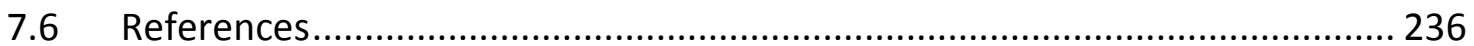

Chapter 8 Stabilisation and Dewatering of Primary Sludge USING Ferrate(VI) PreTreatment Followed By Freeze-Thaw in Simulated Drainage Beds............................... 243

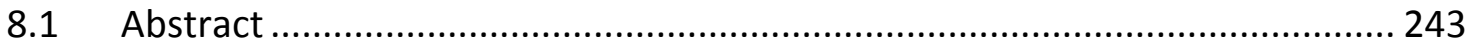

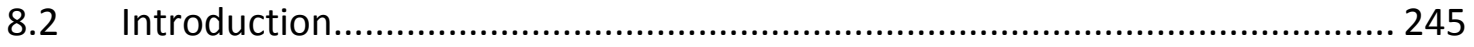

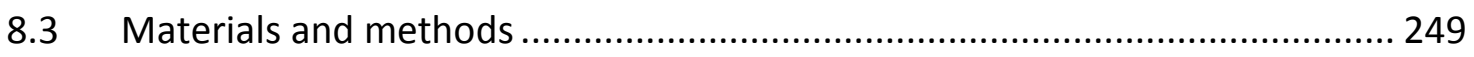

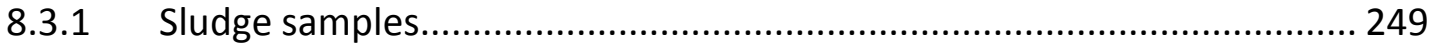




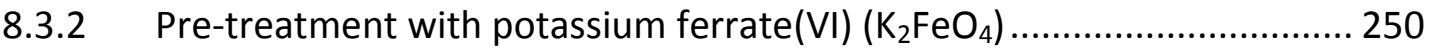

8.3.3 Freeze-thaw stabilisation and dewatering ....................................... 250

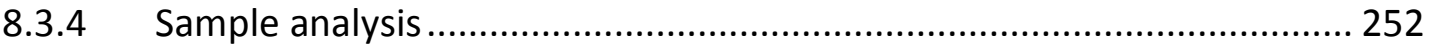

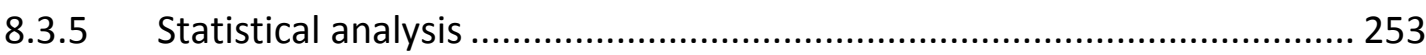

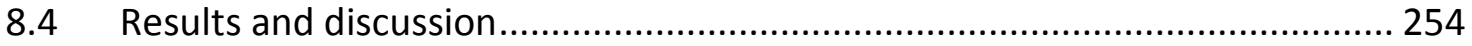

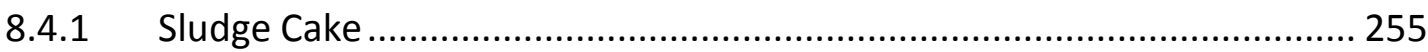

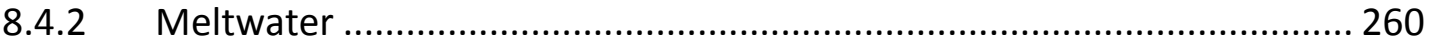

8.5 Follow-Up Experiments with Higher Doses of Ferrate(VI) ............................. 269

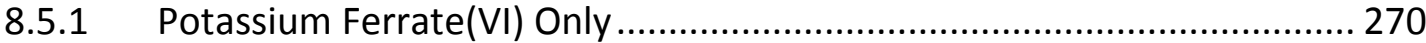

8.5.2 Freeze-thaw with potassium ferrate(VI) pre-treatment ........................ 273

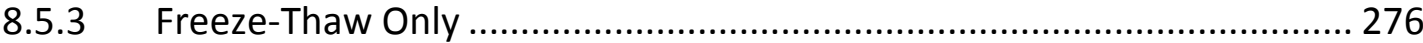

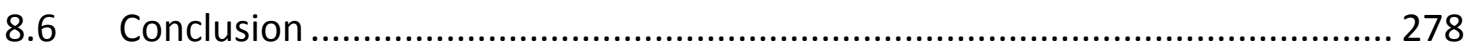

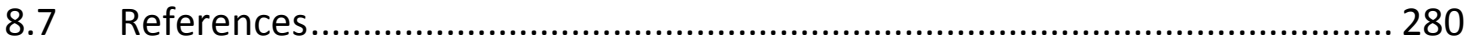

Chapter 9 Oxidation of Selected Hormones in Anaerobically Digested Sludge ........ 287

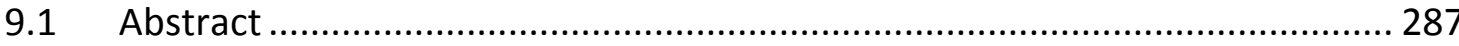

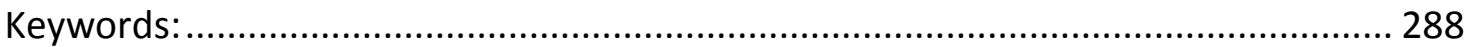

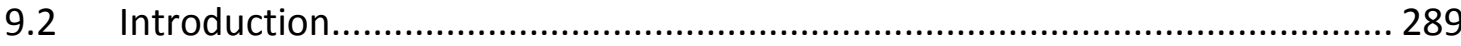

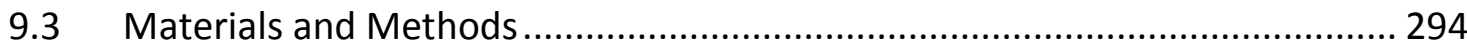

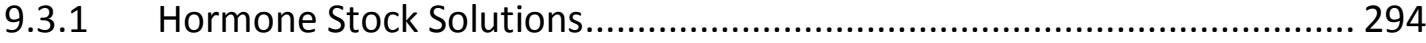

9.3.2 Sludge Sample and Hormone Spiking ................................................. 295

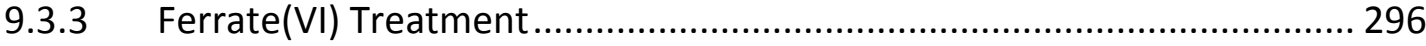

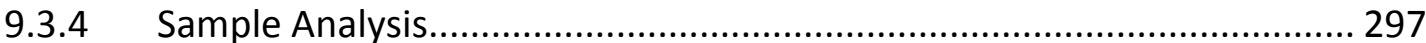

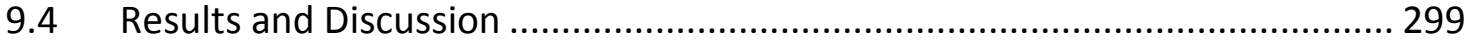

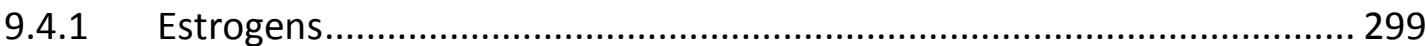

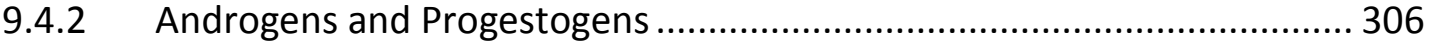

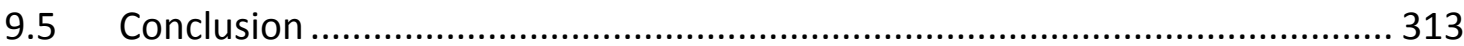

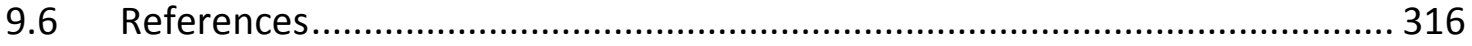

Chapter 10 Application of Ferrate(VI) Oxidation and Freeze-Thaw Sludge Dewatering at a Remote Facility in Northern Canada.... 


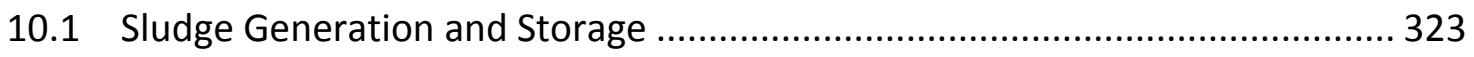

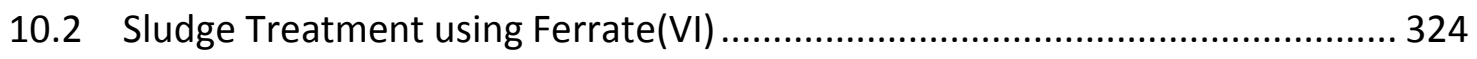

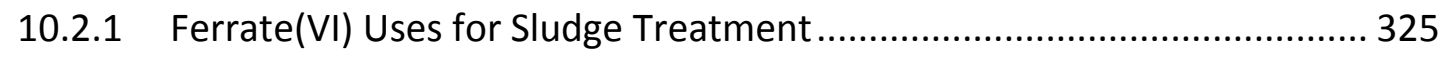

10.2.2 Sludge Pre- and Post-treatment with Ferrate(VI) ................................ 325

10.2.3 Pre-Treatment of RBC Sludge using Ferrate(VI) ................................. 326

10.3 Design and Operation of a Freeze-Thaw Sludge Dewatering Bed .................. 329

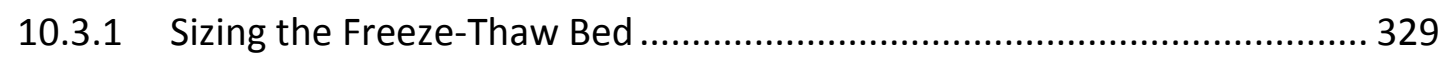

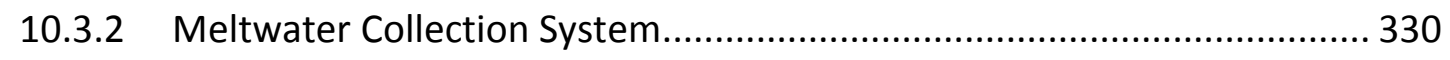

10.3.3 Preparation of the Freeze-Thaw Bed................................................. 330

10.3.4 Application of Sludge Layers to the Freeze-Thaw Bed ........................... 331

10.3.5 Meltwater Collection and Management .............................................. 333

10.3.6 Collection and Disposal of the Final Sludge Cake ................................. 336

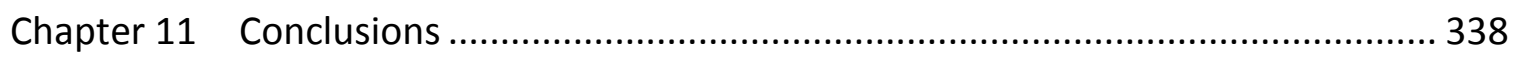

11.1 Freeze-Thaw Sludge Stabilisation and Dewatering ..................................... 338

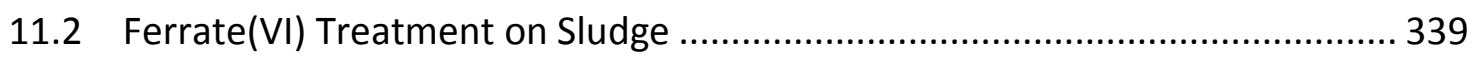

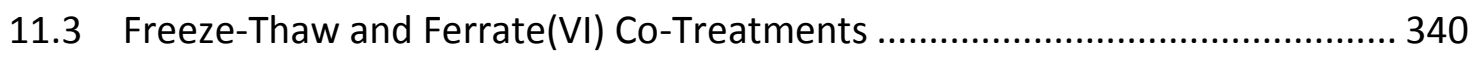

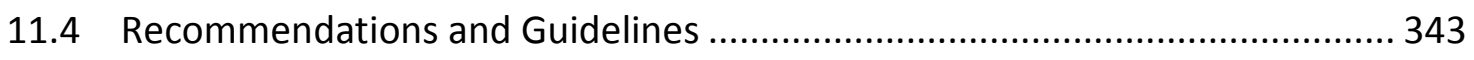

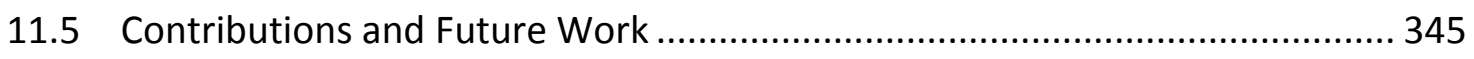

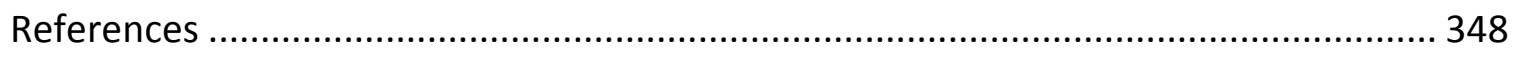

Appendix A1 Criteria for Selecting the Significant Dilutions Used to Determine the MPN Index and 95\% Confidence Limits (U.S. EPA, 2005a) ......................................... 375

Appendix A2 Fecal Coliform MPN Index and 95\% Confidence Limits for Various Combinations of Positive Results When Fives Tubes are used per Dilution (U.S. EPA,

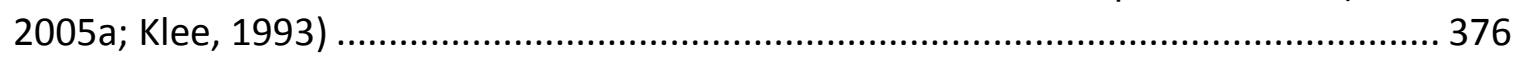

Appendix A3 Salmonella MPN Index and 95\% Confidence Limits for Various Combinations of Positive Results When Fives Tubes are used per 20.0, 10.0, and $1.0 \mathrm{~mL}$ Homogenised Sample Inoculation Volumes (U.S. EPA, 2005b; Klee, 1993) .................. 378

Appendix B1 Temperature Logs for Freeze-Thaw Experiments with E. coli in PBS, using Freezer Temperatures of $-10,-20$ and $-30^{\circ} \mathrm{C}$ 380 
Appendix B2 Temperature Logs for Freeze-Thaw Experiments with Primary Sludge,

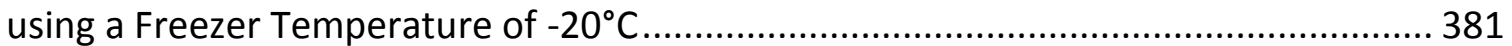

Appendix B3 Temperature Logs for Freeze-Thaw Experiments with Anaerobically Digested Sludge, using a Freezer Temperature of $-20^{\circ} \mathrm{C}$ 382

Appendix B4 Temperature Logs for Freeze-Thaw Experiments with Primary Sludge,

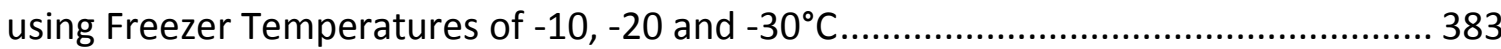

Appendix B5 Summary of Freezing Geometries, Temperature Log Data, Freezing Rates and Calculated Proportionality Coefficients 384

Appendix C1 Sludge Generation in a CLEMENTINE ${ }^{\circledR}$ Wastewater Treatment Unit with Combined Primary Settling and Biological Treatment using a RBC 385

Appendix C2 Annual Ferrate(VI) Production Costs for Electrochemically-Generated Ferrate(VI) Solutions and Ferrate(VI) Salts for Sludge Treatment 386

Appendix C3 Operation of a Freeze-Thaw Sludge Dewatering Bed: Estimated Sludge Freezing Time as a Function of Sludge Layer Thickness and Average Daily Freezing Temperature 387

Appendix C4 Meltwater Generation, Collection and Management 388 


\section{LIST OF TABLES}

Table 2-1: Ontario criteria pollutants for land application of biosolids (MOE, 2014)..... 15

Table 2-2: Concentrations of TSS, COD and BOD in the meltwater collected during

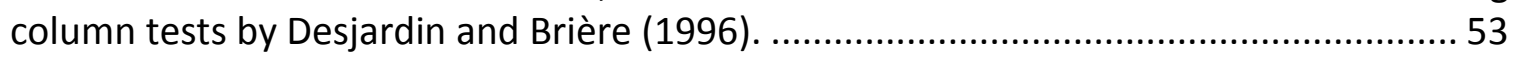

Table 2-3: Oxidation potential of common oxidants used in wastewater and sludge

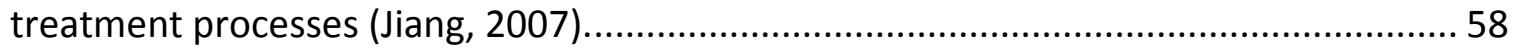

Table 2-4: Typical lime doses for pre-treatment sludge stabilisation (WEF, 1995)........ 79

Table 4-1: Characteristics of the RBC sludge used in the freeze-thaw pilot study........... 88

Table 4-2: Characteristics of the primary and anaerobically digested sludge samples used for part I and part II respectively of the combined freeze-thaw and ferrate(VI) oxidation experiments.

Table 4-3: Initial characteristics of the primary sludge samples. 140

Table 4-4: Spiked concentrations of the selected hormones in the low concentration and high concentration sludge samples used for subsequent tests with potassium ferrate(VI).

Table 6-1: Description of the treatments applied to the test samples and their representative abbreviations used in subsequent figures. 178

Table 7-1: Characteristics of the primary and anaerobically digested sludge samples used for part I and part II respectively. 204

Table 7-2: Description of the treatments applied to the primary sludge samples and their representative abbreviation. 206

Table 7-3: Volume of meltwater collected via gravity drainage during the thawing process and the corresponding mass of sludge cake remaining. 222

Table 8-1: Initial characteristics of the primary sludge samples. 250

Table 8-2: Description of the treatments applied to the primary sludge samples and their representative abbreviation. 254

Table 8-3: Volume of meltwater collected via gravity drainage during the thawing process and the corresponding mass of sludge cake remaining. . 255

Table 8-4: Percent reduction in sludge volume following freeze-thaw with gravity meltwater drainage. 255 
Table 9-1: Spiked concentrations of the selected hormones in the low concentration and high concentration sludge samples used for subsequent tests with potassium ferrate(VI). 296

Table 9-2: Percent change in the concentrations of the measured estrogens in the low concentration $(15-75 \mathrm{ng} / \mathrm{mL})$ and the high concentration $(60-300 \mathrm{ng} / \mathrm{mL})$ sludge samples, following the addition of $0.5 \mathrm{~g} / \mathrm{L}$ (LD) and $1.0 \mathrm{~g} / \mathrm{L}$ (HD) of potassium ferrate(VI). 306

Table 9-3: Percent change in the concentrations of the measured androgens and progestogens in the low concentration $(3-15 \mathrm{ng} / \mathrm{mL})$, and the high concentration (12-60 $\mathrm{ng} / \mathrm{mL}$ ) sludge samples, following the addition of $0.5 \mathrm{~g} / \mathrm{L}$ (LD) and $1.0 \mathrm{~g} / \mathrm{L}$ (HD) of potassium ferrate(VI).... 313 


\section{LIST OF FIGURES}

Figure 2-1: Postulated distribution of water in sludge by (a) Vesilind and Martel (1990), and (b) Vesilind (1994)...... 26

Figure 2-2: Sludge freezing bed used in full-scale tests by Hellström and Kvarnström (1997).

Figure 2-3: Conceptual drawing of a sludge freezing bed (Martel, 2001). 45

Figure 2-4: Potential depth of sludge $(\mathrm{cm})$ that could be frozen in the United States, if applied in sequential layers, $8 \mathrm{~cm}$ thick (Reed et al., 1986). 50

Figure 2-5: Potential depth of sludge $(\mathrm{m})$ that could be frozen in Canada and Alaska, if applied in sequential layers, $8 \mathrm{~cm}$ thick (Kinsley et al., 2012). 51

Figure 2-6: Speciation of ferrate(VI) for various pH (Delaude and Laszlo, 1996)..... 62

Figure 2-7: Percent reduction in Salmonella, 15 minute exposure time (Godfree et al., 1984). 79

Figure 4-1: Schematic of the pilot freezing bed used in preliminary freeze-thaw experiments with RBC sludge (Seprotech Systems Inc.). 90

Figure 4-2: Fecal coliform enumeration using the MTF method, demonstrating a positive result (left), and a negative result (right). 96

Figure 4-3: Summary of the incubation procedures used for the detection and enumeration of Salmonella using EPA method 1682 (U.S. EPA 2005b). 102

Figure 4-4: CST apparatus used to quantify the rate of release of water from sludge (APHA et al., 2005). 123

Figure 4-5: Schematic of (a) the freezing vessel and (b) the simulated drainage bed with meltwater collection, used for the freeze-thaw experiments with anaerobically digested sludge.

Figure 4-6: Protein calibration curve. 131

Figure 4-7: Carbohydrate calibration curve. 134

Figure 5-1: (a) Remote mining exploration facility in Matoush, Quebec (Strateco Resources Inc.), (b) Pilot-scale freezing bed used for freeze-thaw conditioning of RBC sludge.

Figure 5-2: Volume of meltwater collected during thaw at ambient temperatures...... 159 
Figure 5-3: Total solids and volatile solids content of the sludge cake during thaw..... 160

Figure 5-4: TSS and VSS concentrations of the meltwater during thaw. 161

Figure 5-5: Total COD concentrations of the meltwater during thaw..... 162

Figure 5-6: TP, TN, ammonia and nitrate concentrations of the meltwater during thaw. 163

Figure 5-7: Turbidity and $\mathrm{pH}$ of the meltwater during thaw. 164

Figure 6-1: E. coli concentration following (a) various contact times using a $0.5 \mathrm{mg} / \mathrm{L}$ of $\mathrm{K}_{2} \mathrm{FeO}_{4}$, (b) various potassium ferrate(VI) doses using a 15-minute contact time. 181

Figure 6-2: A comparison of the Multiple Tube Fermentation (MTF) and the Membrane Filtration (MF) methods for the enumeration of $E$. coli, and a reproducibility test on freeze-thaw treatments for the reduction of $E$. coli concentrations. 183

Figure 6-3: Effect of low ferrate( $\mathrm{VI})$ dose ( $\left.\mathrm{LD}=0.5 \mathrm{mg} / \mathrm{L} \mathrm{K}_{2} \mathrm{FeO}_{4}\right)$ when used alone (15-min contact time), and as a pre- and post-treatment to freeze-thaw with $\mathrm{T}_{\text {Freeze }}=-10^{\circ} \mathrm{C} \ldots . .185$

Figure 6-4: Effect of high ferrate( $\mathrm{VI})$ dose $\left(\mathrm{HD}=1.0 \mathrm{mg} / \mathrm{L} \mathrm{K}_{2} \mathrm{FeO}_{4}\right)$ when used alone (15min contact time), and as a pre- and post-treatment to freeze-thaw with $T_{\text {Freeze }}=-10^{\circ} \mathrm{C}$.

Figure 6-5: Effect of low ferrate(VI) dose ( $\left.\mathrm{LD}=0.5 \mathrm{mg} / \mathrm{L} \mathrm{K}_{2} \mathrm{FeO}_{4}\right)$ when used alone (15-min contact time), and as a pre- and post-treatment to freeze-thaw with $\mathrm{T}_{\text {Freeze }}=-20^{\circ} \mathrm{C} \ldots . .188$

Figure 6-6: Effect of high ferrate(VI) dose ( $\left.\mathrm{HD}=1.0 \mathrm{mg} / \mathrm{L} \mathrm{K}_{2} \mathrm{FeO}_{4}\right)$ when used alone (15min contact time), and as a pre- and post-treatment to freeze-thaw with $T_{\text {Freeze }}=-20^{\circ} \mathrm{C}$. 189

Figure 6-7: Effect of low ferrate( $\mathrm{VI})$ dose ( $\left.\mathrm{LD}=0.5 \mathrm{mg} / \mathrm{L} \mathrm{K}_{2} \mathrm{FeO}_{4}\right)$ when used alone (15-min contact time), and as a pre- and post-treatment to freeze-thaw with $\mathrm{T}_{\text {Freeze }}=-30^{\circ} \mathrm{C} \ldots . .191$

Figure 6-8: Effect of high ferrate( $\mathrm{VI})$ dose $\left(\mathrm{HD}=1.0 \mathrm{mg} / \mathrm{L} \mathrm{K}_{2} \mathrm{FeO}_{4}\right)$ when used alone (15min contact time), and as a pre- and post-treatment to freeze-thaw with $\mathrm{T}_{\text {Freeze }}=-30^{\circ} \mathrm{C}$. 192

Figure 7-1: Schematic of (a) the freezing vessel and (b) the simulated drainage bed with meltwater collection, used for the freeze-thaw experiments with anaerobically digested sludge. 208

Figure 7-2: Effect of LD and HD on the (a) CST and (b) ammonia and sulphide concentrations in the primary sludge, following a 15-minute contact time. 214

Figure 7-3: Effect of LD on the concentration of fecal coliform in primary sludge when used alone, and as a pre- and post-treatment with freeze-thaw. 216 
Figure 7-4: Effect of LD on the TS and VS when used alone, and as a pre- and posttreatment with freeze-thaw.

Figure 7-5: Effect of HD on the concentration of fecal coliform in primary sludge when used alone, and as a pre- and post-treatment with freeze-thaw.

Figure 7-6: Effect of HD on the TS and VS when used alone, and as a pre- and posttreatment with freeze-thaw.

Figure 7-7: Simulated drainage beds with meltwater collection used for the freeze-thaw experiments with anaerobically digested sludge samples.

Figure 7-8: Effect of LD and HD on the concentration of fecal coliform in the meltwater collected during the thawing stage. 224

Figure 7-9: Effect of LD and HD on the concentrations of soluble proteins, soluble carbohydrates and SCOD in the meltwater collected during the thawing stage. 226

Figure 7-10: Initial sludge and meltwater samples collected following freeze-thaw (1 day frozen). The visible text in the background was used to demonstrate the clarity of the samples following freeze-thaw with ferrate(VI) pre-treatment. 228

Figure 7-11: Effect of LD and HD on the turbidity and $\mathrm{pH}$ of the meltwater collected during the thawing stage. 229

Figure 7-12: Effect of LD and HD on the concentration of fecal coliform in the sludge cake when used alone, and as a pre-treatment to freeze-thaw with meltwater separation. 231

Figure 7-13: Effect of LD and HD on the TS and VS of the sludge cake when used alone and as a pre-treatment to freeze-thaw with meltwater separation. 233

Figure 8-1: Schematic of the (a) freezing vessel and (b) thawing/meltwater collection apparatus used in the freeze-thaw sludge dewatering experiments with primary sludge. 252

Figure 8-2: Effect of LD and HD pre-treatment with freeze-thaw on the TS and VS of the sludge cake following meltwater drainage. 258

Figure 8-3: Effect of LD and HD pre-treatment with freeze-thaw on the concentration of fecal coliform in the sludge cake following meltwater drainage. 260

Figure 8-4: Effect of LD and HD pre-treatment with freeze-thaw on the concentration of fecal coliform in meltwater. 262

Figure 8-5: Effect of LD and HD pre-treatment with freeze-thaw on the SCOD of the meltwater. 264 
Figure 8-6: Effect of LD and HD pre-treatment with freeze-thaw on the concentration of soluble proteins in the meltwater. 265

Figure 8-7: Effect of LD and HD pre-treatment with freeze-thaw on the concentration of soluble carbohydrates in the meltwater. 266

Figure 8-8: Effect of LD and HD pre-treatment with freeze-thaw on the turbidity of the meltwater. 268

Figure 8-9: Meltwater samples collected from sludge batch A, after being frozen for 1 day. 268

Figure 8-10: Effect of LD and HD pre-treatment with freeze-thaw on the $\mathrm{pH}$ of the meltwater. 269

Figure 8-11: Effect of potassium ferrate(VI) dose on the concentration of fecal coliform, TS and VS in the primary sludge. 272

Figure 8-12: Concentration of fecal coliform in the sludge cake remaining following pretreatment with potassium ferrate(VI), and freeze-thaw using a freezer temperature of $20^{\circ} \mathrm{C}$, and a 12 hour thaw period at room temperature, in a simulated drainage bed setup $\left(0^{*}\right.$ represents samples following a 15-minute reaction period with the potassium ferrate $(\mathrm{VI})$, without any freeze-thaw or cake-meltwater separation, and the downward arrows are to indicate that the measured concentration was at the lower method detection limit) 275

Figure 8-13: Concentration of fecal coliform in the meltwater samples collected following pre-treatment with potassium ferrate( $\mathrm{VI})$, and freeze-thaw using a freezer temperature of $-20^{\circ} \mathrm{C}$, and a 12 hour thaw period at room temperature, in a simulated drainage bed setup ( $0 *$ represents samples following a 15 -minute reaction period with the potassium ferrate $(\mathrm{VI})$, without any freeze-thaw or cake-meltwater separation, and the downward arrows are to indicate that the measured concentration was at the lower method detection limit). 276

Figure 8-14: Effect of stand-alone freeze-thaw treatment on the concentration of TS, VS and fecal coliform in the sludge cake, and the concentration of fecal coliform in the meltwater (Initial* represents the raw primary sludge without any meltwater-cake separation, the units are quoted on a wet and dry basis). 277

Figure 9-1: Concentrations of estrogens in the samples that were spiked with (a) the low concentration (15-75 ng/mL), and (b) the high concentration $(60-300 \mathrm{ng} / \mathrm{mL})$ of selected estrogens, and following the subsequent addition of $0.5 \mathrm{~g} / \mathrm{L}$ (LD) and $1.0 \mathrm{~g} / \mathrm{L}$ (HD) of potassium ferrate(VI). 305

Figure 9-2: Concentrations of androgens and progestogens in the sludge samples that were spiked with (a) the low concentration (3-12 ng/mL), and (b) the high concentration 
$(12-60 \mathrm{ng} / \mathrm{mL})$ of androgens and progestins, and following the subsequent addition of $0.5 \mathrm{~g} / \mathrm{L}(\mathrm{LD})$ and $1.0 \mathrm{~g} / \mathrm{L}(\mathrm{HD})$ of potassium ferrate(VI) ................................................ 312 


\section{LIST OF APPENDICES}

Appendix A1 Criteria for Selecting the Significant Dilutions Used to Determine the MPN Index and 95\% Confidence Limits (U.S. EPA, 2005a) 375

Appendix A2 Fecal Coliform MPN Index and 95\% Confidence Limits for Various Combinations of Positive Results When Fives Tubes are used per Dilution (U.S. EPA, 2005a; Klee, 1993) 376

Appendix A3 Salmonella MPN Index and 95\% Confidence Limits for Various Combinations of Positive Results When Fives Tubes are used per 20.0, 10.0, and $1.0 \mathrm{~mL}$ Homogenised Sample Inoculation Volumes (U.S. EPA, 2005b; Klee, 1993) .... 378

Appendix C1 Sludge Generation in a CLEMENTINE ${ }^{\circledR}$ Wastewater Treatment Unit with Combined Primary Settling and Biological Treatment using a RBC 385

Appendix C2 Annual Ferrate(VI) Production Costs for Electrochemically-Generated Ferrate(VI) Solutions and Ferrate(VI) Salts for Sludge Treatment. 386

Appendix C3 Operation of a Freeze-Thaw Sludge Dewatering Bed: Estimated Sludge Freezing Time as a Function of Sludge Layer Thickness and Average Daily Freezing Temperature 387

Appendix C4 Meltwater Generation, Collection and Management 388 


\section{LIST OF EQUATIONS}

(2)

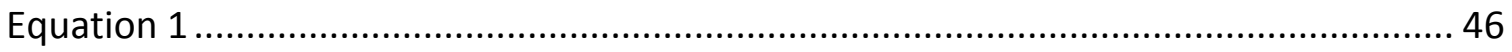

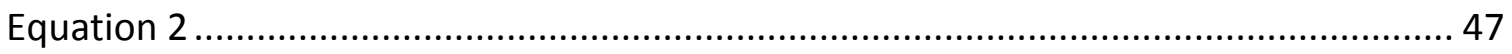

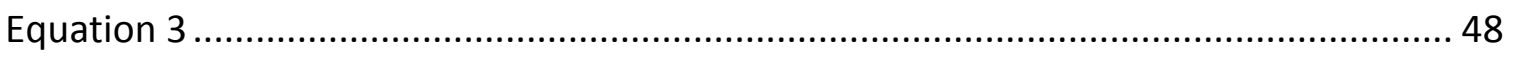

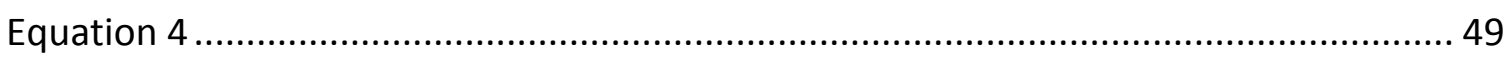

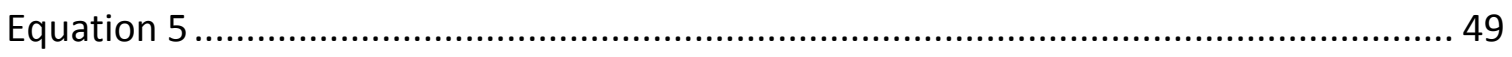

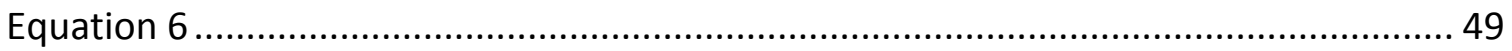

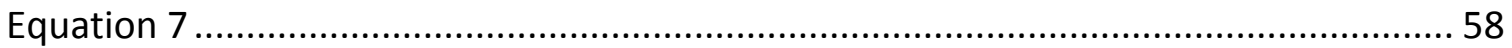

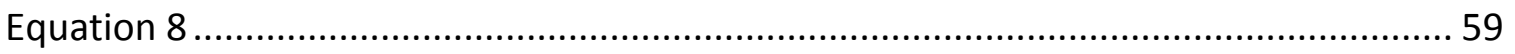

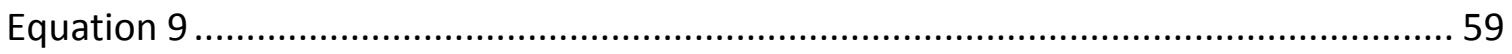

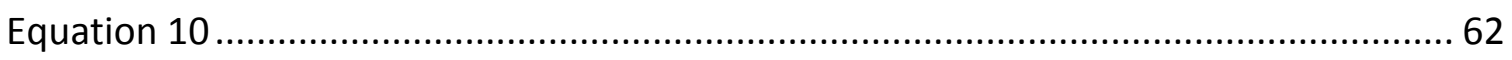

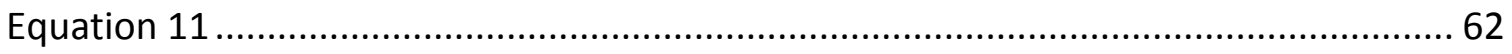

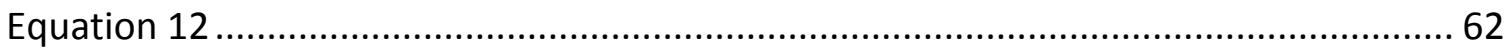

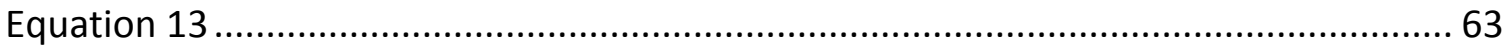

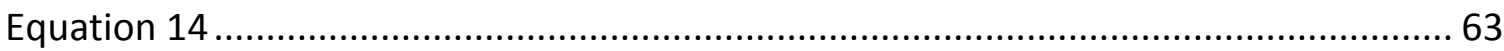

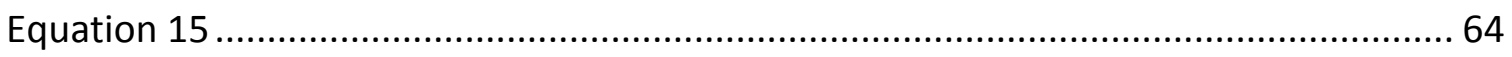

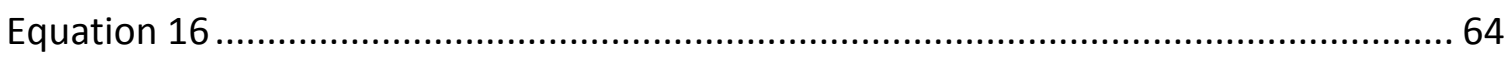

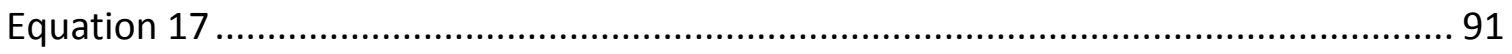

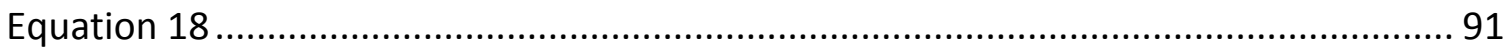

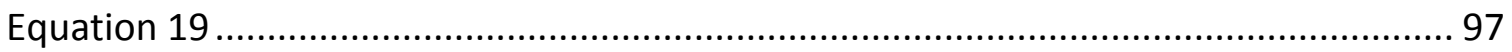

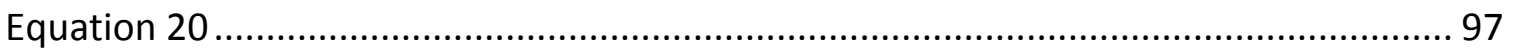

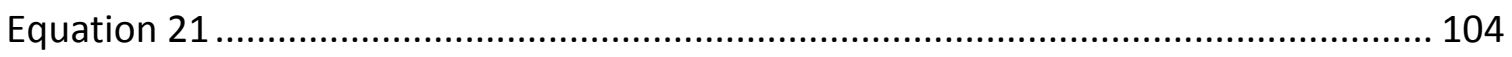

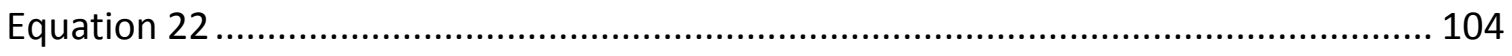

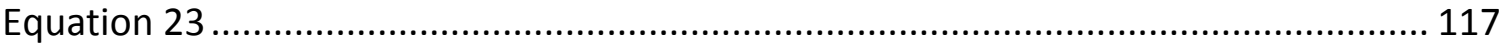


Equation 24 ...

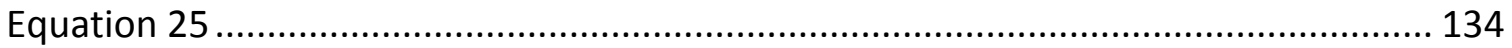




\section{LIST OF ACRONYMS AND NOMENCLATURE}

\begin{tabular}{|c|c|}
\hline AD - anaerobically digested (sludge) & EU - European Union \\
\hline BOD - biochemical oxygen demand & FSS - fixed suspended solids \\
\hline $\mathrm{BOD}_{5}-5$-day BOD & HAA - haloacetic acids \\
\hline BPA - bisphenol A & HPC - heterotrophic plate count \\
\hline CEC - Council of the European & $\mathbf{H R L}$ - high risk limit \\
\hline Communities & MCE - mixed cellulose ester \\
\hline CFU - colony forming units & MOE - Ministry of the Environment \\
\hline CL - confidence limits (95\%) & (Ontario) \\
\hline $\mathbf{C M}$ - criteria for metals & MPN - most probable number \\
\hline COD - chemical oxygen demand (total) & MTF - multiple tube fermentation \\
\hline $\mathbf{C P}$ - criteria for pathogens & NASM - non-agricultural source material \\
\hline CST - capillary suction time & $\mathbf{N H}_{\mathbf{3}}-\mathbf{N}$ - ammonia measured as $\mathbf{N}$ \\
\hline DOC - dissolved organic carbon & $\mathbf{N O}_{3}{ }^{-}-\mathbf{N}$ - nitrate measured as $\mathrm{N}$ \\
\hline DS - dry solids & OC-odour categories \\
\hline E1 - estrone & PBS - phosphate buffered saline \\
\hline E2 - 17ß-estradiol & PCB - polychlorinated biphenyl \\
\hline E3 - estriol & PPCPs - pharmaceuticals and personal care \\
\hline EE2 - 17 $\alpha$-ethynilestradiol & products \\
\hline E. coli-Escherichia coli & PS - primary sludge \\
\hline EDC - endocrine disrupting compound & PST - primary settling tank \\
\hline EOC - emerging organic compound & RBC - rotating biological contactor \\
\hline EQ - except & RCF- relative centrifugal force \\
\hline
\end{tabular}


sCOD - soluble COD

SDL - specific detection limit

THM - trihalomethanes

TKN - total Kjeldahl nitrogen

$\mathbf{T N}$ - total nitrogen

TOC - total organic carbon

TP - total phosphorus

TS - total solids
TSS - total suspended solids

VS - volatile solids

VSS - volatile suspended solids

WAS - waste activated sludge

WTP - water treatment plant

WWT - wastewater treatment

WWTP - wastewater treatment plant 


\section{INTRODUCTION}

Treatment and disposal of wastewater sludges typically represents approximately $40 \%$ of the operating budget for a wastewater treatment plant (Lee et al., 2010). Sludge treatment refers to the thickening, stabilisation (reduction of pathogens and odours), conditioning and dewatering of sludge. Once treated, sludge is placed in a landfill, incinerated and the ash is placed in a landfill, or where regulations permit, applied to land. Land application of biosolids is an effective sludge disposal option which recycles nutrients and organic matter back into the environment. However, proper measures must be taken to ensure the protection of human health and the environment.

To achieve biosolids suitable for land application, the sludge must be stabilised to reduce the concentrations of pathogens and indicator organisms and eliminate the potential for putrefaction (MOE, 2014). Prior to sludge stabilisation, the sludge is generally thickened, by removing some of the excess water, to reduce the sludge volume and facilitate subsequent treatment processes. Following sludge treatment, the stabilised sludge is conditioned using chemicals and again dewatered to reduce transportation and disposal costs. Sludge dewatering has been one of the most expensive and least understood processes for wastewater treatment plants (Bruus et al., 1992). Large mechanical equipment such as centrifuges and filter-presses are generally used, however these require large amounts of chemicals, and skilled personnel to operate and maintain the equipment. In cold climates, mechanical sludge 
dewatering is particularly difficult because the equipment ices up regularly in sub-zero temperatures. Furthermore, chemicals and dewatering aides can be expensive.

There has been considerable interest in freeze-thaw technology to help solve the dewatering problem in cold climates. Freeze-thaw dewatering works on the principle that when sludge freezes, water molecules crystallise together forcing the solids and other impurities to the boundary of the ice crystal, where they become compressed or dehydrated (Metcalf and Eddy, 2003). During the thaw period, meltwater drains away freely through the network of channels between the consolidated particles, leaving a dewatered sludge. Simple gravity meltwater drainage during the thawing stage can reduce the volume of sludge by 85 to $96 \%$, leaving a sludge cake ranging from $20 \%$ up to 82\% solids (Diak et al., 2011, Martel, 1993, Martel and Diener 1991a, Martel and Diener, 1991b). Sludge freeze-thaw beds together with a storage facility, such as a lagoon, tank, or digester to store the sludge in summer, can be used as the sole method of dewatering in cold regions. In temperate climates, freeze-thaw beds can double as drying beds during the summer (Hellström and Kvarnström, 1997).

Freeze-thaw can also reduce the concentration of pathogens and indicator organisms in wastewater sludges (Gao et al., 2009, Gao et al., 2006, Chu et. al., 1999, Sanin et al., 1994). Mazur (1986) postulated that cell injury and death caused by freeze-thaw is the result of two predominant mechanisms: osmotic effects resulting in cell dehydration, and intracellular ice formation, resulting in cell damage or rupture. Cell dehydration occurs when the extracellular solution is gradually frozen, causing an increase in the 
solute concentration directly outside the cell. This causes the cells to dehydrate, which can increase the intracellular solute concentration to lethal levels. On the other hand, intracellular ice nucleation occurs when the rate of freezing is fast, and the cells do not have enough time to balance osmotically. When the intracellular water freezes, expanding ice crystals can damage or even rupture the cell membrane, causing the release of intracellular materials. Örmeci and Vesilind (2001) observed an increase in the DNA concentration of waste activated sludge (WAS) supernatant following freeze-thaw suggesting that cell lysis did occur at a relatively warm freezing temperature of $-8^{\circ} \mathrm{C}$. This suggests that cell-lysis may occur even at low freezing rates. Damage to cells can also be assessed by the elution of proteins and carbohydrates (Wang et al., 2001), however proteins and carbohydrates are also present in the exocellular polymeric substances of the sludge floc, and are released as a result of sludge floc disruptions during ice formation. Chu et al. (1999) studied the effects of freezing rates ranging from 1.8 to 50 $\mathrm{mm} / \mathrm{hr}$ on WAS, and noticed that freezing rates $<8 \mathrm{~mm} / \mathrm{hr}$ resulted in greater inactivation of total coliform, achieving Class B sludge according to EPA sludge disposal regulations. Experiments by Gao et al. $(2009,2006)$ also saw greater levels of coliform inactivation using warmer freezing temperatures, which generated slower freezing rates. This suggests that slow freezing rates may also result in both cell lysis and cell dehydration.

Studies by Sanin et al. (1994) demonstrated that the pathogen reduction capabilities of freeze-thaw vary depending on the type of microorganism. For example, the concentration of the protozoa Cryptosporidium parvum was reduced by $>8$ - $\log$, whereas 
the helminth Ascaris suum ova was unaffected by freeze-thaw. This suggests that standalone freeze-thaw may be insufficient for the inactivation of pathogens. Furthermore, wastewater sludges can vary significantly depending on the source of the influent wastewater, the type of wastewater treatment employed and the age of the sludge (Metcalf and Eddy, 2003). Municipal sludges, whether sludge from a primary settling tank (PST), activated sludge reactor or anaerobic digester, are typically produced from a mixture of domestic and industrial or commercial sewage. On the other hand, rural wastewater systems, particularly household on-site wastewater treatment systems, such as the septic tank and soil adsorption system, typically do not receive industrial or commercial sewage. This means that sludge or septage from a household septic tank is considerably different from the sludges generated at a centralised wastewater treatment plant (WWTP). Similarly, the sludges generated WWTPs during the various unit operations and processes also vary significantly. For example, primary sludge is raw sludge, which has not undergone any form of controlled treatment and is considered pathogenic, whereas digested sludge, whether anaerobically or aerobically digested, has undergone treatment to stabilise the sludge, thereby reducing its potential for putrefaction. Therefore, digested sludges have lower fecal coliform concentrations than primary sludge, and their odour is considerably less offensive. Different types of sludge also have different physical characteristics which affect their settleability, dewaterability and solids concentrations. This variability can make sludge stabilisation and dewatering processes difficult to operate and maintain, particularly at the small-scale. 
A flexibility sludge stabilisation approach is important to maintain a consistent level of sludge treatment and good quality biosolids, suitable for land application. Furthermore, increasingly strict sludge and WWT regulations are eventually expected to require a reduction in the concentration of pharmaceutically active compounds, to limit their spread and effect on human health and the environment. While uni-directional freezing has been used to remove pharmaceutically active compounds from water by concentrating them in the un-frozen liquid (Gao and Shao, 2009), it is not expected that freeze-thaw can degrade these compounds. An additional treatment operation is required ensure a sufficient level of sludge stabilisation, and to reduce the concentration of pharmaceutically active compounds in sludge.

Ferrate(VI) $\left(\mathrm{FeO}_{4}^{-2}\right)$ is a very strong oxidant, which is capable of degrading emerging organic compounds (EOCs), such as pharmaceuticals and personal care products (PPCPs) and other endocrine disrupting compounds (EDCs) (Li et al., 2008, Sharma et al., 2006, Sharma and Mishra, 2006). Ferrate(VI) has also been used to inactivate a range of pathogens and indicator organisms (Jiang et al., 2007, Schuck et al., 2006,), and reduce sludge odours (de Luca et al., 1996). Odorous compounds in sludge generally contain reduced forms of nitrogen, such as ammonia and amines, and reduced forms of sulphur, such as sulphides and mercaptans (Chen et al., 2011, de Luca et al., 1996). Ferrate(VI) has the strength to oxidise these reduced forms of nitrogen and sulphur to nitrate and sulphate, representing changes of 9 and 10 in their respective oxidation numbers (He et al., 2009, de Luca et al., 1996). 
The strength of ferrate(VI) as an oxidant depends on the $\mathrm{pH}$. Under near-neutral and mildly acidic conditions $(\mathrm{pH}=4.5-7.5)$, the ferrate $(\mathrm{VI})$ ion has an oxidation-reduction potential $\left(E^{0}\right)$ of $2.20 \mathrm{~V}$ (Wood, 1957), making it a stronger oxidant than ozone (Jiang, 2007). However in alkaline solutions ( $\mathrm{pH}>9.5)$, ferrate $(\mathrm{VI})$ is particularly more stable, with an oxidation potential of $0.72 \mathrm{~V}$ (Wood, 1957), which is similar to chlorine (0.84 V) (Jiang, 2007). Although chlorination is the most commonly used potable water disinfection technique, the potential for disinfection byproducts (DBPs) and the added costs associated with the removal of DBPs has created a desire to find alternative disinfection technologies.

Ferrate(VI) is a proven disinfectant which is also capable of oxidizing organic and inorganic micro-pollutants. Furthermore, in oxidizing contaminants, $\mathrm{Fe}(\mathrm{VI})$ is reduced to Fe(III), which results in the formation of ferric hydroxide. This acts as a coagulant, aiding the removal of heavy metals and other suspended solids (Bartzatt et al., 1992, Murmann and Robinson, 1974, Sharma et al., 2008). However, ferrate(VI) is not yet common in water and wastewater treatment operations because ferrate solutions are unstable at room temperature, which limits storability, and the preparation of ferrate salts requires additional reagents and processing time, making it expensive (Alsheyab et al., 2009).

In recent years there has been considerable interest and progress in the use of on-line electrochemical generation of ferrate(VI) (Yang et al., 2012a, Ding et al., 2012, Alsheyab et al., 2010a, Alsheyab et al., 2010b, Jiang et al., 2009, Alsheyab et al., 2009). Advancements in ferrate technology have also made it an attractive addition to the 
freeze-thaw sludge stabilisation and dewatering process. The combined treatment effects of freeze-thaw with ferrate(VI) oxidation have the potential to stabilise and dewater sludge, producing biosolids suitable for land application. The co-treatment technology would facilitate sludge treatment and dewatering for remote facilities, and reduce transportation and disposal costs. In particular, the use of ferrate(VI) as a chemical oxidant provides a high level of flexibility which can be potentially applied to all sludge types, from raw primary sludge to anaerobically digested sludge. However, ferrate(VI) technology may be best suited in a rural decentralised setting where only 1 or 2-log inactivation of fecal coliform in sludge is required, prior freeze-thaw and final disposal on land. Similarly, domestic sludge from settling ponds and surrounding septic tanks can be collected seasonally and stored on site, followed by spreading on a freezethaw bed, when the local weather permits. These sludges will have undergone a certain level of stabilisation, and therefore small doses of ferrate(VI) may be sufficient to achieve biosolids that are suitable for land application when the ferrate(VI) treatment is used in combination with freeze-thaw dewatering.

This research will shed light on the ferrate(VI) dose required to achieve a desired level of fecal coliform inactivation in different types of wastewater sludges when used as a pre and post-treatment with freeze-thaw. Different types of sludge and a pure culture $E$. coli sample will be used to evaluate the effect of different ferrate( $\mathrm{VI})$ doses, and also the effects of different freezing rates (freezing temperatures) and time spent frozen. In addition, bench-scale drainage beds will be used to separate meltwater from the sludge cake during the thawing stage. This will allow the meltwater and sludge cake to be 
characterised separately, similar to a full-scale freeze-thaw sludge dewatering unit. In addition, the natural drainability of sludge (via gravity) during the sludge thawing stage will be evaluated by measuring the rate at which water thaws and drains and the final volume expelled during the thawing process.

This thesis has begun with an introduction describing the potential of freeze-thaw and ferrate $(\mathrm{VI})$ oxidation technology to provide a simple and effective means to treat and dewater wastewater sludges in remote cold regions. The following chapter (Chapter 2) provides a detailed literature review related to the treatment and disposal of wastewater sludges, with special focus on freeze-thaw sludge conditioning and ferrate(VI) oxidation. Chapter 3 states the objectives and hypotheses of the research, and Chapter 4 presents the detailed materials and methods used for the experiments, while a condensed version is included in each of the research chapters. This thesis contains five research articles, presented as Chapters 5 through 9. The first research article (Chapter 5) is a published study on the stabilisation and dewatering of sludge from a rotating biological contactor (RBD) using freeze-thaw, in a pilot-scale freezing bed with meltwater drainage. The second research article (Chapter 6) studied the combined and stand-alone effects of freeze-thaw and ferrate(VI) oxidation on a pure culture of $E$. coli in a sterilised phosphate buffered saline (PBS) solution. The third research article, presented in Chapter 7, studied the combined and stand-alone effects of freeze-thaw and ferrate(VI) addition on a variety of parameters using primary sludge and anaerobically digested sludge. The fourth research article, presented in Chapter 8, evaluated the potential of ferrate( $\mathrm{VI})$ oxidation and freeze-thaw dewatering to be used 
as a stand-alone treatment technology for raw wastewater sludges in remote cold regions. The fifth research article, presented in Chapter 9, evaluated the ability of low doses of ferrate(VI) to reduce the concentration of hormones in sludge. Chapter 10 provides an example of freeze-thaw sludge dewatering using ferrate(VI) oxidation, which includes a detailed discussions. And, the final chapter is a summary of the major conclusions drawn from the research.

While freeze-thaw has been shown to improve sludge dewaterability, the technology is not widely applied in Canada. This is mainly due to concerns over the putrescibility of wastewater sludges. Furthermore, despite its wide range of applications for wastewater treatment, ferrate(VI) treatments have not been widely adopted, mainly due to the instability of the ferrate(VI) ion, and the high cost to produce ferrate(VI) salts. However, interest in ferrate $(\mathrm{VI})$ for environmental remediation has been renewed due to recent advancements in on-line electrochemical ferrate(VI) generation and a major cost decrease in the production of ferrate(VI).

Ferrate(VI) oxidation treatments have the ability to reduce the potential for putrefaction in sludge by oxidising reduced forms of nitrogen (ammonia and amines) and sulphur (sulphides and mercaptans), and inactivating pathogens and other organisms. This would complement the natural freeze-thaw sludge dewatering process, and potentially inactivate fecal coliform to below the CP1 regulated limits for land application of biosolids in Ontario (MOE, 2014). In addition, the high-strength chemical oxidant has the potential to reduce the concentration of hormones and other EDCs in sludge, which are 
of increasing concern, particularly where biosolids are applied to land. Ferrate(VI) and freeze-thaw co-treatment technology would simplify sludge treatment and disposal for remote communities in cold climates, and offers large degree of process flexibility which can be tailored the particular design requirements.

This research was the first to evaluate the combination of ferrate(VI) oxidation and freeze-thaw sludge stabilisation and dewatering, and among the first to examine the oxidation of EDCs in sludge using ferrate(VI). In addition, freeze-thaw dewatering was achieved using pilot-scale and bench-scale sludge dewatering beds, and several process operational parameters such as ferrate(VI) dose, freezing temperature, freezing rate and time frozen, were varied to evaluate the impacts on sludge stabilisation and dewatering. The research also characterised in detail, the individual and combined effects of ferrate(VI) oxidation and freeze-thaw treatments on the physical, chemical and biological characteristics of various sludge types when simulated drainage beds were used to separate meltwater from the sludge cake during the thawing process. The effects of the operational parameters were evaluated on both meltwater and the sludge cake remaining following freeze-thaw dewatering.

This research will offer new insight into the treatment of sludge using ferrate(VI), and evaluate the combined effects when ferrate(VI) oxidation is combined with freeze-thaw sludge dewatering. Sludge co-treatment using ferrate(VI) and freeze-thaw would be a convenient way for remote communities in cold climates to stabilise and dewater wastewater sludges on-site, allowing for safe disposal of the treated biosolids on land. 
The following chapter presents a review of literature pertinent to this study, including an overview of sludge treatment and disposal, with special focus and land application regulations, followed by a detailed review on freeze-thaw sludge conditioning, followed by a review of common chemical stabilisation techniques, with particular focus on ferrate(VI) oxidation. 


\section{LITERATURE REVIEW}

\subsection{SLUDGE TREATMENT AND DISPOSAL}

Sludge generated during wastewater treatment processes must undergo treatment and disposal. For cities with a large wastewater treatment plant (WWTP), sludge treatment typically involves sludge thickening, stabilisation, conditioning and dewatering. Sludge thickening reduces the volume of sludge by removing some of the excess water. Sludge from primary clarifiers or PSTs, known as primary sludge (PS), typically has a solids content of 2 to $5 \%$, with readily settleable solids and can be effectively thickened to 5 to $10 \%$ using gravity thickeners. On the other hand, waste activated sludge (WAS) is lighter and typically has 0.5 to $2.5 \%$ solids. As a result, mechanical thickeners such as centrifuges, gravity belts or sometimes air flotation thickeners are often used (Metcalf and Eddy, 2003).

Thickened sludge is then stabilised to reduce pathogens, odours and the potential for putrefaction. Popular sludge stabilisation processes include alkaline stabilisation (ex: lime stabilisation), anaerobic digestion, aerobic digestion and composting. Many largescale WWTPs use anaerobic digesters to digest wastewater sludges (PS and WAS) and generate usable methane gas.

Following anaerobic digestion, the sludge is referred to as anaerobically digested (AD) sludge. AD sludge is then conditioned to improve sludge dewatering, which has been one of the most expensive and least understood processes in wastewater treatment 
operations (Bruus et al., 1992). Polymers are added to the sludge to improve the agglomeration of negatively charged sludge particles through charge neutralisation and inter-particle bridging, allowing the free water to be removed more easily (Bohm and Kulicke, 1997). Mechanical dewatering equipment such as centrifuges and filter presses are generally used in urban areas to conserve space. However, in rural areas, where the large foot print required is available at low cost, a variety of sludge drying beds, including lagoons and reed beds, have also been used (Northcott et al., 2005, Hedström and Hanaeus, 1999, Hellström and Kvarnström, 1997, Penman and Van Es, 1973).

Following the stabilisation and dewatering processes, sludge is referred to as biosolids, and depending on the level of treatment, and a few other considerations such as heavy metal concentrations, the treated biosolids may be suitable for land application (CCME, 2010, U.S. EPA, 2003). In certain scenarios, such as with sludge generated from industrial wastewater treatment processes, incineration of the dewatered sludge may be favourable. Or in certain cases, biosolids are disposed of in a landfill.

\subsubsection{Land Application Regulations}

Land application of treated sludge is an effective and sustainable sludge disposal option which provides a variety of nutrients and organic materials to receiving soils. It is a particularly well-suited disposal option for decentralised regions, and in the European Union (EU) is considered the Best Practicable Environmental Option over incineration and landfilling (Gendebien et al., 2010). In Canada, approximately 43\% of biosolids generated are land applied (Hamid and Eskicioglu, 2013), and it is expected that this 
percentage will increase as municipalities continue to move away from incineration and landfilling (Apedaile, 2001). To ensure proper protection of human health and the environment, land application of biosolids is a highly regulated process with increasingly stringent regulations.

\subsubsection{Canada}

In Canada, sludge management practices are regulated at the provincial or territorial level. In Ontario, as of January 2011, sewage biosolids are classified as a Category 3 nonagricultural source material (NASM) in Ontario Regulation (O. Reg.) 267/03 (last amendment: O. Reg. 284/13) under the Nutrient Management Act of 2002 (Ministry of Environment (MOE) 2014, Ontario Ministry of Agriculture, Food and Rural Affairs (OMAFRA, 2013). To be suitable for land application, biosolids must meet criteria for metals (CM) and criteria for pathogens (CP). For each of these criteria, there are two sub-categories. CM1 and CP1 biosolids are considered good-quality since they have lower concentration requirements for metals and pathogens. These biosolids can be applied to land with fewer restrictions, than the lower grade CM2 and CP2 biosolids, in terms of proximity to wells, groundwater and surface water, and waiting periods prior to crop harvest or grazing. Furthermore, based on the type of sludge stabilisation treatments used, biosolids are categorised based on their potential for odours. Three odour categories (OC) exist: OC1, OC2 and OC3. These categories restrict the transportation, storage and application methods, as well as proximity of biosolids applications to dwellings, residential areas and institutions (OMAFRA, 2013). 
Additionally, a NASM plan must be registered and approved along with the required sample testing, and biosolids application rates are restricted (OMAFRA, 2013).

Heavy metals in sludge are generally the result of certain upstream commercial or industrial facilities, and so their concentrations in sludge depend entirely on the presence of these facilities. In many rural communities, and particularly with septage pumped out of household septic tanks, heavy metal concentrations would be very low. For this research, the scope of the sludge analysis for land application is only in terms of the pathogen indicator: fecal coliform. The criteria for CP1 and CP2 biosolids in Ontario are presented in Table 2-1.

Table 2-1: Ontario criteria pollutants for land application of biosolids (MOE, 2014).

\begin{tabular}{|c|c|c|c|c|}
\hline \multirow[b]{2}{*}{ Pathogen } & \multicolumn{2}{|c|}{ CP1 } & \multicolumn{2}{|c|}{ CP2 } \\
\hline & $\begin{array}{c}\text { Level in Aqueous } \\
\text { Material }^{1} \\
\end{array}$ & $\begin{array}{c}\begin{array}{c}\text { Level in Non-Aqueous } \\
\text { Material }^{2}\end{array} \\
\end{array}$ & $\begin{array}{c}\text { Level in Aqueous } \\
\text { Material }\end{array}$ & $\begin{array}{c}\text { Level in Non-Aqueous } \\
\text { Material }\end{array}$ \\
\hline E. coli & $1,000 \mathrm{CFU} / \mathrm{mL}$ & $\begin{array}{l}1,000 \text { CFU/g total } \\
\text { solids, dry weight }\end{array}$ & $2,000,000 \mathrm{CFU} / \mathrm{mL}$ & $\begin{array}{c}2,000,000 \text { CFU/g total } \\
\text { solids, dry weight }\end{array}$ \\
\hline Salmonella & $3 \mathrm{CFU}$ or $\mathrm{MPN} / \mathrm{mL}$ & $\begin{array}{c}3 \text { CFU or MPN/4 g total } \\
\text { solids, dry weight }\end{array}$ & -- & -- \\
\hline \begin{tabular}{|l|} 
Viable Helminth \\
ova
\end{tabular} & $\begin{array}{c}\text { No detectable level } \\
\text { in } 100 \mathrm{~mL} \\
\end{array}$ & $\begin{array}{c}\text { No detectable level in } \\
4 \mathrm{~g} \text { total solids, dry }\end{array}$ & -- & -- \\
\hline $\begin{array}{l}\text { Total culturable } \\
\text { enteric virus }\end{array}$ & $\begin{array}{c}\text { No detectable level } \\
\text { in } 100 \mathrm{~mL}\end{array}$ & $\begin{array}{c}\text { No detectable level in } \\
4 \mathrm{~g} \text { total solids, dry }\end{array}$ & -- & -- \\
\hline
\end{tabular}

Nutrients levels in sludge make sludge addition to agricultural crops beneficial in terms of plant growth and soil quality, however over-fertilization will harm vegetative crops Therefore, regular monitoring of the nitrogen (ammonium + nitrate) levels must be conducted, and 5-year application rates of $200 \mathrm{~kg}$ nitrogen/ha must not be exceeded (MOE, 2014). The phosphorus content of the sludge also must be measured. Phosphorus is a common fertilizer for plants, and approximately $40 \%$ of the phosphorus in sludge 
will be available for plant uptake. Potassium is another nutrient for plants, however biosolids from sewage sludges generally have low potassium concentrations, and are only measured on a case by case basis (MOE, 1996). Biosolids from sewage sludges also contain low sodium levels; however food wastes and wastes from the food processing industry often have high sodium levels. High level of sodium can cause severe and irreversible damage to soil, therefore sodium application rates are restricted to between 200 and $500 \mathrm{~kg} / \mathrm{ha} / \mathrm{yr}$ based on soil type (MOE, 2014). Similarly, fats, oils and grease (FOG) are limited to 2500 and $5000 \mathrm{~kg} / \mathrm{ha}$ depending on soli type, and boron, which is harmful to most crops, is limited to $1 \mathrm{~kg}$ boron/ha/yr (MOE, 2014).

In addition to the tests and criteria for metals, nutrients, and other elements, the proponent also has the right to request additional analytical information regarding other metals, elements and organic pollutants not specifically covered by the Regulation (MOE, 2014). As our understanding of the fate of EDCs improves, standards related to these compounds will be applied which limit the concentrations in sludge and the receiving soils, and the biosolids application rates. The increasing number of new compounds and their increasing concentrations in waste streams are a major concern. Therefore, amendments to the regulations are increasingly stringent and often include additional criteria pollutants.

Provinces in Atlantic Canada strongly encourage recycling of biosolids on land, but have strict regulations and guidelines in place. Depending on the levels of particular metals and pathogens, biosolids are classified as Exceptional Quality (EQ), Class A or Class B 
biosolids (Nova Scotia Environment, 2010, Environment Canada, 2006). Recent biosolids guidelines for Nova Scotia only use Class A and B categories (Nova Scotia Environment, 2010), however Class A contaminant concentration criteria are stricter than the previously used EQ category from 2006 Atlantic Canada guidelines (Environment Canada, 2006). British Columbia, Quebec, Northwest Territories, Nunavut and Saskatchewan also have similar standards for pathogens and pathogen indicators in biosolids (CCME, 2010), and it is assumed other provinces will soon adopt similar, more stringent, criteria for contaminant concentrations in biosolids used on agricultural lands.

\subsubsection{United States}

The U.S. EPA also has limits for pathogens and indicator organisms (fecal coliform, Salmonella, parasites and enteric viruses) in biosolids for land application (U.S. EPA, 2003). The values originally set out by the U.S. EPA have since been adopted in many parts of Canada (Table 2-1), however the regulated limits for parasites and enteric viruses in biosolids are both listed as $<1$ per 4 grams of total solids, dry weight, rather than undetected. Similar to the regulations in Canada, metals in land-applied sewage sludges are restricted in the United States based on the concentrations in the sewage sludge, the loading rate to soil, and the existing concentration in the receiving soil. When biosolids meet the criteria for treatment and pollutant criteria of Part 503.13, then they are often applied as a fertilizer to improve or maintain productive soils and encourage plant growth. As of 2002 , roughly $60 \%$ of biosolids generated are land applied, however regulations or requirements to test for organic pollutants are still being investigated (Harrison et al., 2006). 


\subsubsection{European Union}

Land application of sludges in the EU is considered the Best Practicable Environmental Option for sludge disposal, compared to alternatives such as incineration and landfilling (Gendebien et al., 2010). Recent estimates suggest approximately $36 \%$ of sludge generated in the EU are applied to land, however there is significant variation by country (Gendebien et al., 2010). In the UK, approximately $77 \%$ of biosolids (treated sludges) were recycled to agricultural land (WATER UK, 2010), whereas Germany moved away from land application in favour of incineration from 2000 to 2005 . In $2000,33 \%$ of municipal sludge were applied to agricultural lands, but in 2005 , only $22 \%$ was land applied (Klaerschlamm, 2005). A number of directives, such as Directive 2000/76/EC are in place which set standards and technical requirements in terms of air emissions, water discharges contamination and plant designs for incineration of waste. Similarly, Directive $99 / 31 /$ EC is aimed to limit the amount of biodegradable waste sent to landfills, by restricting 2006 values to 35\% of 1995 levels (Gendebien et al., 2010), essentially eliminating landfilling as an option for sludge disposal.

In the EU, sludge application to land is regulated by 1986 EU Sludge Directive (86/278/EEC), which was set up to encourage sludge disposal on agricultural lands, while protecting surrounding soil, vegetation, animals and humans (WATER UK, 2010). The EU Directive sets limits for concentrations of heavy metals in sludge and soil similar to those for Canada and the United States, and also restricts application of untreated sludges unless they are injected or worked into the soil (Council of the European Communities (CEC), 1986). The term "treated sludge" is rather loosely defined as: 
Sludge which has undergone biological, chemical or heat treatment, long-term storage or any other appropriate process so as significantly reduce its fermentatability and the health hazards resulting from its use. (CEC, 1986)

Other aspects of sludge disposal on land may also be controlled under certain national waste management regulations or agricultural regulations. For instance, in Scotland, Waste Management Amendment Regulations 2003, 2004 and 2006 are applicable, and throughout the rest of the UK, the UK Sludge (Use in Agriculture) Regulations, supported by a Code of Practice and a Hazard Analysis and Critical Control Points (HACCP) regulate land application of sludges (WATER UK, 2010). Furthermore, the EU is now also considering appropriate limits for certain organic pollutants in biosolids for land application, and in the near future it is expected that only sludges with very low concentrations of criteria pollutants will be applied to agricultural lands (Abad et al., 2005).

\subsubsection{Emerging Organic Compounds (EOCs)}

EOCs is a term used to represent a wide range of synthetic or naturally occurring compounds, which are not commonly monitored but have the ability to enter the environment, and cause known or suspected adverse ecological or human health effects (U.S. Geological Survey, 2011). They include pesticides, veterinary medicines, coatings such as fire retardants and other industrial chemicals, natural and synthetic hormones, and other PPCPs (Tremblay et al., 2011). Inevitably, these compounds end up at the WWTP, and many leave the plant either in the liquid effluent or in the treated biosolids 
(Lind et al., 2010, Ternes et al., 2002). It is generally understood that current biological treatments, such as conventional activated sludge and anaerobic digestion, are only capable of removing small percentages of a few of these commonly occurring EDCs from wastewater (Li et al., 2008, Ternes et al., 1999). Studies by Stumpf et al. (1999) revealed the presence of a variety of drug residues from lipid regulators and anti-inflammatories, and certain drug metabolites in raw sewage, treated wastewater and river water in the state of Rio de Janeiro, Brazil. Raw wastewaters from 10 WWTPs contained on average $1.2 \mu \mathrm{g} / \mathrm{L}$ of the lipid regulator bezafibrate, and wastewater effluents contained between 0.6 to $1 \mu \mathrm{g} / \mathrm{L}$. In downstream river waters bezafibrate concentrations were below detection limits $(0.01 \mu \mathrm{g} / \mathrm{L})$, however clofibric acid, diclofenac and naproxen were often detected at concentrations between 0.02 to $0.04 \mu \mathrm{g} / \mathrm{L}$. Furthermore, $\mathrm{Xu}$ et al (2009) detected a variety of PPCPS and EDCs in the runoff water from a potato field that was irrigated with treated wastewater.

A number of these compounds are known to interfere with the endocrine systems (normal function of hormones) of humans and animals, and are therefore referred to as endocrine disrupting compounds (EDCs). Steroidal hormones of interest include estrone

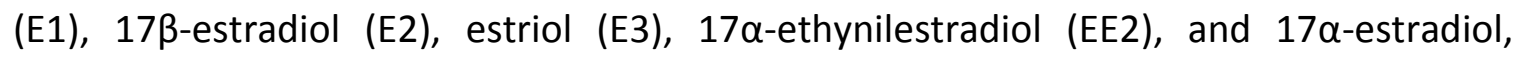
because these compounds are known to have the highest estrogenicity (Silva et al., 2012, Ning et al., 2007). Similarly, Bisphenol A (BPA) is a synthetic EDC that was used in polycarbonate and polysulfone plastics and epoxy resins, which has an estrogenicity about 500 times that of octylphenol (Nagel et al., 1997). Estrone, 17 $\beta$-estradiol and estriol are all natural hormones, while $17 \alpha$-ethynilestradiol, norethindrone, norgestrel 
and mestranol are synthetic hormones used in oral contraceptives for women. Other common EDCs include equilin, equilenin and testosterone, which are estrogenic steroids used in hormone replacement therapy (Furlong et al., 2010.

During wastewater treatment, the fate of these compounds varies (Hamid and Eskicioglu, 2012). Gabet-Giraud et al. (2010) showed that many of the conventional biological wastewater treatment process such as activated sludge, rotating biological contactors (RBCs), reed-bed filters and trickling filters removed approximately $90 \%$ of dissolved estrogens. Gabet-Giraud also investigated the effects of the various biological treatments on different beta blockers, and while acebutolol and nadolol were reduced by $80 \%$, sotalol and propranolol were reduced by less than $20 \%$. Furthermore, while conventional wastewater treatment processes may lead to the partial degradation of certain estrogenic compounds, many EDCs are adsorbed onto the sludge solids, and removed with the PS and WAS (Hamid and Eskicioglu, 2012, Silva et al., 2012, Li et al., 2011). The resulting EDC concentrations in the sludge matrix can be high. Ternes et al. (2002) detected estrone and $17 \beta$-estradiole in activated and digested sludge at concentrations of up to 37 and $49 \mathrm{ng} / \mathrm{g}$ respectively.

The degree of partitioning of individual EDCs onto sludge solids can be estimated by their respective octanol-water partition coefficients $\left(\mathrm{K}_{\mathrm{ow}}\right)$. Estrogenic hormones generally have log $\mathrm{K}_{\mathrm{ow}}$ values between 2.5 and 4.0, suggesting that these compounds are moderately hydrophobic, and are more likely to be adsorbed onto the sludge solids (Li and Zhang, 2014, Hamid and Eskicioglu, 2012, Jones-Lepp and Stevens, 2007). However, 
due to the complex nature of the sludge matrix, and the difficulties in hormone extraction for LC-MS/MS analysis, there are few studies related to the fate of EDCs during full-scale sludge treatment processes (Hamid and Eskicioglu, 2013, Clark and Smith, 2011).

Kimura et al. (2010) studied the removal of various pharmaceuticals in activated sludge and MBR sludge samples diluted with a phosphate buffered saline (PBS) solution, and spiked with $1.0 \mu \mathrm{g} / \mathrm{L}$ of each pharmaceutical. Studies demonstrated greater removal of acidic pharmaceuticals under slightly acidic conditions $(\mathrm{pH}=6)$ compared to neutral $\mathrm{pH}$. Studies also revealed that removal of selected pharmaceuticals was achieved primarily via biodegradation, which led to a variety of subsidiary compounds, generated from the partial mineralisation of their respective parent compounds. For example, Ternes et al. (1999b), reported that $17 \beta$-estradiol biodegraded to estrone, which subsequently biodegraded to estriol. In the study by Barontri et al. (2000), estrone levels at full-scale WWTP were higher in the effluent than the influent, suggesting that retention times were too low for complete biodegradation. Additionally, while Muller et al. (2010) measured between 20 and 30\% removal of E1, E2 and E3 during full-scale anaerobic digestion of PS and WAS (65:35 v/v), increases in the concentration of EE2, from below the detection limit to $>2 \mathrm{ng} / \mathrm{d}$ dry weight, were recorded. Similarly, Hamid and Eskicioglu (2013) noticed an accumulation of estrone, progesterone and androstenedione in anaerobic digesters due to low biodegradation efficiency and potential microbial transformations. 
A very detailed study by Furlong et al. (2010) evaluated the fate of several estrogenic compounds during municipal sludge stabilisation and dewatering at four different WWTPs. The study concluded that there was significant variability in the effectiveness of various sludge digestion operations (aerobic digestion, anaerobic digestion, lime) for reducing the estrogenicity of biosolids. Aerobic digestion reduced the estrogenicity of the sludge by $18 \%$, however mesophilic and thermophilic anaerobic digestion resulted in an increase in the estrogenicity of the sludge (Hamid and Eskicioglu, 2013).

Beginning in the 1970's, several studies have revealed that significant exposures to persistent human-made chemicals have led to a variety of reproductive disorders in molluscs, fish, birds, reptiles and mammals (Janex-Habibi et al., 2009). Studies by Purdom et al. (1994) revealed the feminisation of male trout placed downstream from a WWTP due to the presence of sterols in the WWTP effluent, and Sumpter (2007) confirmed that nonylphenols can cause partial or complete feminisation of male fish.

Furthermore, Lind et al., 2010 demonstrated that adult ewes exposed to EDCs through land application of biosolids showed an anti-estrogenic effect, suggesting that human exposure to multiple EDCs may have adverse effects on human health and bone structure. And in a similar study by Rhind et al. (2011), while tissue concentrations of EDCs in ewes and lambs increased with duration on pastures treated with sewage sludge, the increases differed significantly and were not easily linked to the increases in duration of exposure. 


\subsubsection{Discussion on the Land Application of Treated Biosolids}

Throughout Canada, the United States and Europe, land application of biosolids is used as a sludge disposal technique, which also provides organics and a variety of nutrients to soils. Based on the concentrations of certain metals, nutrients, and pathogens and indicator organisms, biosolids are classified and their use is restricted. High quality biosolids, listed as Class A in the United States and many parts of Canada, has the fewest restrictions. In the U.S., biosolids which meet the treatment and pollutant criteria of the Federal standards for the use and disposal of sewage sludge, Part 503.13, are considered Class A biosolids, and can be sold as fertiliser with very limited restrictions (U.S. EPA, 2003). Lower-grade biosolids, known as Class B biosolids, in the U.S. and some parts of Canada have restrictions in terms of application rate, type of land, and proximity to objects like water wells, water bodies, drainage ditches, buildings and property lines. Class B biosolids also have restrictions on waiting periods prior to harvest when used as an agricultural soil additive (Nova Scotia Environment, 2010). This means that high-quality biosolids can be used by the general public on lawns and gardens, including food crops for human consumption, whereas lower-grade biosolids are better suited for animal-feed crops and non-agricultural lands.

Despite many decades using biosolids as an agricultural fertiliser and very few instances of any personal injury, the increasing number of EOCs in domestic wastewater sludges requires modifications to current sludge management practices in terms of treatment, monitoring and disposal. At present, Nova Scotia is the only Province which has placed limits on the concentrations of certain organic pollutants (polychlorinated biphenyls 
(PCBs), dioxins and furans) in biosolids, however it is expected that the rest of Canada and the U.S. will soon adopt similar criteria. The EU is also considering appropriate limits for selected EOCs in sludges to be used on agricultural lands (Abad et al., 2005). This will increase the demands on preceding sludge treatment processes, and ultimately redefine sludge stabilisation. It is important for sludge treatment technologies to continue to improve with our increased knowledge of the effects of organic pollutants on humans and the environment. Effective sludge stabilization will mean that land application of biosolids continues to be a safe and sustainable sludge disposal option in the future.

\subsection{FREEZE-THAW SLUDGE CONDITIONING}

Efficient sludge stabilisation requires sludge dewatering. Dewatered sludge has a reduced volume which facilitates treatment processes and reduces treatment costs. Depending on the type of sludge, the solids concentration typically varies from 1 to $5 \%$ solids (Metcalf and Eddy, 2003). As described by Vesilind and Martel (1990), the distribution of water in sludge can be categorised into four groups: free or bulk water, interstitial water, surface water and intracellular or bound water, as shown in Figure $2-1$. Free water accounts for $70-75 \%$ of the total water in sludge. This water is not bound or absorbed, it surrounds the sludge flocs and moves freely, unaffected by capillary forces amongst the particles. Interstitial is trapped within the sludge floc or is held to the sludge particles by capillary forces. It is termed interstitial water because it is bound within the interstice of the sludge particles and microorganisms within the floc (Kopp \& Dichtl, 2001). This water can be removed through mechanical dewatering, but only when the sludge floc is broken (Yin et al., 2004). Surface water and vicinal water is the 
water that covers the entire surface of the of the sludge particles, often several layers thick. Intracellular water or bound water (or water of hydration (Vesilind, 1994)) is chemically bound to the individual particles, and can only be released by destroying the particle.

(a)

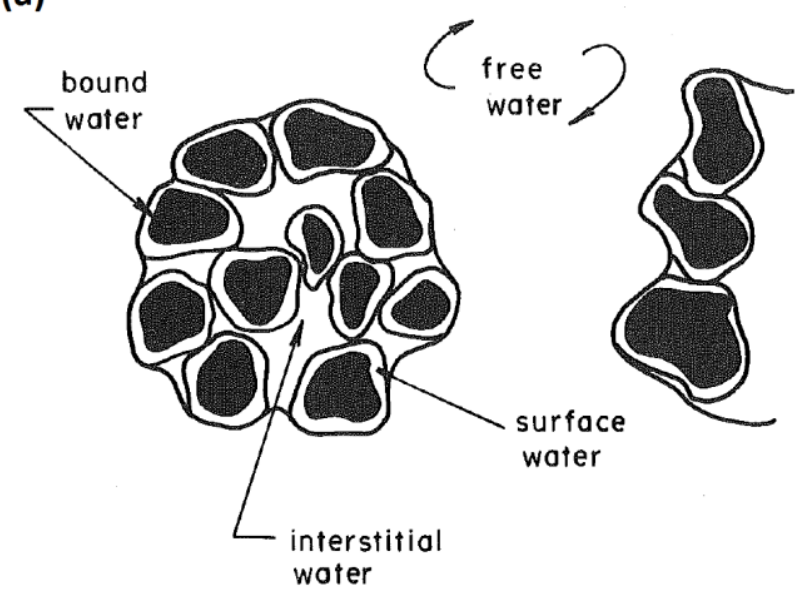

(b)

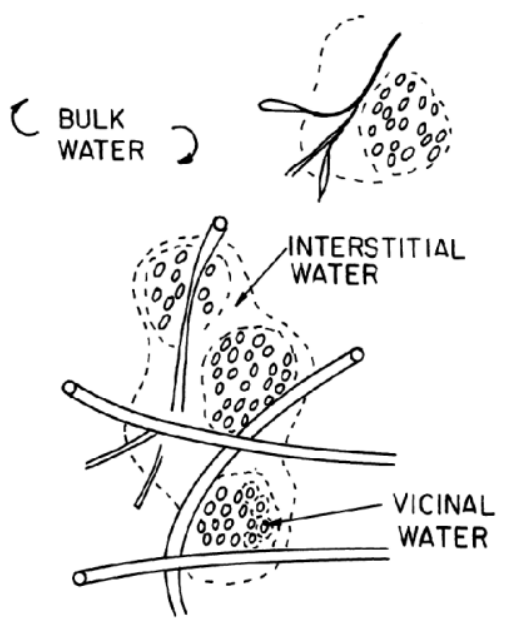

Figure 2-1: Postulated distribution of water in sludge by (a) Vesilind and Martel (1990), and (b) Vesilind (1994).

Freeze-thaw treatment works on the principal that ice crystals grow by incorporating water molecules only (Örmeci and Vesilind, 2001). Taber (1930) conducted early studies on the mechanics of frost heaving in soils, and noticed the separation of water from solids into "masses of more or less pure ice". Taber proposed that a thin film of water between the ice and the soil particles pulled water molecules from the surrounding soils with the advancing ice front.

Corte (1962) was one of the first to actively study particle migration during freezing and found that the thin layer of surface water surrounding the sludge particles allows them to move along the growing ice front. Corte found that small particles are easily pushed 
aside by the advancing ice front, since the film of water is more easily replenished, whereas larger particles are more likely to become trapped by the ice. Corte also noticed that slower freezing rates improved the migration of the larger and less spherical particles. Hoekstra and Miller (1967) studied the relationship between freezing rate and size of particles which can be expelled by the growing ice front. They used the term transition layer to describe the critical zone between the advancing ice front and the surface of solid particles, where mobility of water molecules takes place.

As sludge freezes, ice crystals grow by projecting ice needles throughout the free water, forcing the solids and other impurities to the boundary of the ice crystal where they become compressed and dehydrated (Reed et al., 1986). When sludge is gradually frozen, the slow growing ice crystals create a network of continuous channels throughout the consolidated sludge particles. Once all of the free water has frozen, interstitial water is drawn from the sludge flocs via diffusion, and added to the network of ice crystals (Vesilind and Martel 1990). The removal of interstitial water has an important effect on the dewaterability of sludges, particularly biological sludges which have highly hydrated sludge flocs (Erdincler and Vesilind, 2003). The viscous or gelatinous consistency of sludge is converted to a more granular and free-draining material (Flint, 1972). Parker and Collins (1999) developed a conceptual model to explain how the removal of surface water from colloidal particles within the sludge floc could bring particles into close contact, causing the formation of new bonds amongst particles, leading to granular and readily dewaterable sludge. During thaw, the meltwater (effluent) drains freely, leaving a dewatered sludge cake. 
Freeze-thaw treatments can also inactivate certain pathogenic microorganisms (Gao et al., 2009, Gao et al., 2006, Kato et al., 2002, Sanin et al., 1994,). During freezing, the formation of sharp ice-crystals can damage or rupture cell membranes, causing the release of intracellular materials. Mazur (1986) hypothesized that pathogen attenuation due to ice nucleation is predominant for high freezing rates, and that long curing times can lead to cell dehydration. Hu et al. (2011) later demonstrated the role of curing time, on floc dehydration and the crystallisation of inter-aggregate moisture causing damage to cell membranes and the release of intra-cellular materials.

\subsubsection{Laboratory Studies}

The following sections provide a summary of the laboratory studies on freeze-thaw treatments related to the effects of freezing rate, solids content and particle size, curing time, curing temperature and freeze-thaw cycling.

\subsubsection{Freezing Rate}

Early studies by Clements (1950) revealed that freezing rate is a critical parameter for the optimisation of freeze-thaw sludge dewatering. High freezing rates can reduce the dewaterability of sludge by entrapping particles within the ice front. Knocke and Trahern (1989) also determined that rapid freezing can cause the generation of fine particles, thus reducing sludge dewaterability. Logsdon and Edgerley (1971) found critical freezing rates from 43 to $65 \mathrm{~mm} / \mathrm{hr}$ for chemical sludges, and Lee and Hsu (1994) determined that a freezing rate of $40 \mathrm{~mm} / \mathrm{hr}$ caused the densification of WAS flocs and improved filterability. They also noticed that the biochemical oxygen demand (BOD) and 
chemical oxygen demand (COD) of the supernatant increased significantly, and proposed that some extracellular polymers and interstitial water were released from the sludge floc, which decreased the bound water and increased the BOD and COD of the supernatant.

Halde (1980) used a freezing rate of $30 \mathrm{~mm} / \mathrm{hr}$ on various sewage sludges, and achieved greatest solids separation with PS and WAS. Hung et al. (1996a) employed unidirectional freezing on WAS to test various freezing rates, and determined that freezing rates as high as $250 \mathrm{~mm} / \mathrm{hr}$ could be used to improve filterability as long as the sludge was completely frozen. However, freezing rates less than $10 \mathrm{~mm} / \mathrm{hr}$ were most effective in terms of settleability and reduction of bound water.

Hung et al. (1996b) also studied the corresponding changes in chemical composition of sludge for various freezing rates, and found that low freezing rates, which resulted in mass particle migration, also resulted in the largest changes in chemical composition.

Hung et al. (1997) later went on to investigate differences between in unidirectional freezing and radial freezing on gross particle migration. Using freezing temperatures of $20^{\circ} \mathrm{C}$, unidirectional freezing rates varied from 4.2 to $167.4 \mathrm{~mm} / \mathrm{hr}$, while radial freezing rates varied from 2.6 to $15.7 \mathrm{~mm} / \mathrm{hr}$, as a result of the different vessel and freezing configurations. Experiments confirmed that, similar to unidirectional freezing, particle migration due to radial freezing is strongly influenced by freezing rate. For both freezing methods, low freezing rates resulted in almost complete particle migration whereas high freezing rates resulted in almost no migration. 
The slow advancing ice front during freezing also rejects dissolved impurities. Gao and Shao (2009) employed uni-directional downward freezing to remove pharmaceutically active compounds (ibuprofen, gemfibrozil, acetylsalicylic acid, metoprolol and sulfamethoxazole) from water. The advancing ice front concentrated the pharmaceuticals in the unfrozen portion of the sample, and reduced the concentrations in the frozen portion by $84-92 \%$. Furthermore, when the same treatment was reapplied on the previously unfrozen portion, the net reduction was increased to $99 \%$.

Mazur (1986) postulated that freezing rate can also have an effect on the mechanism of pathogen attenuation. For low freezing rates, extracellular water gradually freezes causing the solute concentrations in the surface water surrounding the cell increase. The resulting difference in osmotic pressure between the water in cell and the surrounding surface water generates a transfer of water out of the cell, which causes the cell to dehydrate, which can generate lethally high solute concentrations inside the cell. For high freezing rates, the water inside the cell freezes before the cell has time to equilibrate, resulting in ice nucleation (Silvares et al., 1975). These sharp ice crystals can damage or rupture cell membranes, causing the release of intracellular materials (Mazur, 1986). Cell damage can be assessed by measuring the elution of proteins and carbohydrates (Wang et al., 2001), however sludge floc disruptions may also lead to an increase in proteins and carbohydrate concentrations in the sludge supernatant. Örmeci and Vesilind (2001) measured increases in the DNA concentration of sludge supernatant to confirm that freeze-thaw resulted in cell lysis, while others have used microscopy to study floc deformations during sludge freezing (Parker et al., 1998, Hung et al., 1996a). 
Chu et al. (1999) studied the effects of freezing rates ranging from 1.8 to $50 \mathrm{~mm} / \mathrm{hr}$ on the filterability (CST) and microbial density of WAS. While CST was reduced for all freezing rates, maximum particle migration occurred with lower freezing rates (1.8 $\mathrm{mm} / \mathrm{hr}$ ). Freezing rates $<8 \mathrm{~mm} / \mathrm{hr}$ were also found to be most effective at reducing the microbial densities of heterotrophic plate count (HPC) bacteria and total coliform, achieving Class B sludge according to EPA sludge disposal regulations. Similarly, experiments by Gao et al. $(2009,2006)$ also saw greater levels of coliform inactivation using warmer freezing temperatures of -5 and $-7^{\circ} \mathrm{C}$, compared to freezing temperatures of -35 and $-80^{\circ} \mathrm{C}$. This suggests slower freezing rates are favorable for the inactivation of fecal coliform, and while slow freezing rates are believed to result in cell dehydration, there is evidence that cell lysis can also result when relatively warm freezing temperatures $\left(-7^{\circ} \mathrm{C}\right)$ are used.

\subsubsection{Sludge Solids Content and Particle Size}

Sludge solids content is an important parameter in freeze-thaw treatment since free water provides facilitated growth of ice crystals. Particularly with unidirectional directional freezing, the build-up of particles rejected by the growing ice front creates a layer of debris at the ice front interface. As this layer of particles thickens, the flow of water to the growing ice crystal can be restricted, leading to particle entrapment as the growing ice front over-takes the layer of debris in seek of more water. Once the ice has overtaken the layer of particles, the process repeats itself. This cycling between particle rejection and particle entrapment is known as rhythmic banding (Halde, 1980), and often occurs even at constant freezing temperatures (Parker et al., 1998). 
Particle size has long been known to have a critical role on the effectiveness of freezethaw treatments since smaller particles are more easily rejected from the growing ice crystal, whereas larger particles are more likely to become entrapped (Corte, 1962). This means that sludge with small particles can be frozen faster than sludge with larger particles. Similarly, the irregularity of certain sludge flocs increases the drag forces, and thus increases its chances of being entrapped in the advancing ice front.

Chung et al. (2003) studied the hydrodynamic drag force exerted on activated sludge flocs with diameters around $1888 \mu \mathrm{m}$ during. When sludge samples were frozen very quickly, using a liquid nitrogen bath, there were no differences in the hydrodynamic drag coefficient and no consolidation of particles. On the other hand, when sludge samples were frozen at $-17^{\circ} \mathrm{C}$ for 24 hours, there was considerable consolidation of particles and resulting in sludge flocs with compact interiors, shown in a series microphotographs.

Chang and Lee (1998) froze WAS samples in stainless steel vessels $(25 \mathrm{~cm}$ diameter, 25 $\mathrm{cm}$ high, $0.15 \mathrm{~cm}$ thick), immersed in a pool at $-15^{\circ} \mathrm{C}$ for 48 hours. The resulting average freezing rate was determined to be $2.6 \mathrm{~mm} / \mathrm{hr}$, by dividing the radius of freezing cylinder by the time required to completely freeze. They studied the ternary consolidation of WAS flocs and proposed a model to describe the relation between void ratio and compressive pressure of the sludge cake.

Tao et al. (2006a) measured forces exerted by an advancing ice front on individual sludge flocs. Studies employed unidirectional freezing rates of 18 and $28.5 \mathrm{~mm} / \mathrm{hr}$ on 
sludge flocs with diameters of 2170 and $2240 \mu \mathrm{m}$. They also monitored the deformation of the sludge floc as it was engulfed by the growing ice front.

\subsubsection{Freezing/Curing Temperature, Curing Time \& Freeze-Thaw Cycles}

Sludge freezing temperature will have direct implications on the sludge freezing rate, however the sludge freezing rate can also be heavily influenced by other factors such as the method of freezing, the geometry of the freezing apparatus and the direction of applied freezing. In certain laboratory experiments the freezing rate and curing temperature were controlled separately (Sanin et al., 1994), whereas other experiments used a constant temperature for freezing and curing (Gao et al., 2006, Gao et al., 2009, Chang et al., 2004, Örmeci and Vesilind, 2001). Warm freezing temperatures will result in low freezing rates, suitable for particle migration, whereas colder curing temperatures will cause the surface water to eventually freeze, further compacting individual sludge particles (Chang et al, 2004, Vesilind and Martel, 1990).

Gao et al. (2009) studied the effect of freezing temperature $\left(-7,-15,-30\right.$ and $\left.-80{ }^{\circ} \mathrm{C}\right)$, number of freeze-thaw cycles ( 1 to 5 cycles) and sample volume (100 $\mathrm{mL}$ and $100 \mu \mathrm{L}$ ) on E. Coli and Enterococcus faecalis. For each freeze-thaw cycle, samples were frozen for 24 hours, then thawed at room temperature. Freeze-thaw treatments resulted in 3.3 to 4.3 log reductions in E. Coli and 1.5 to 2.4 log reductions in Enterococcus faecalis for $100 \mathrm{ml}$ bottles frozen at $-7,-15$ and $-30^{\circ} \mathrm{C}$. However, coldest freezing temperature of $-80^{\circ}$ was found to be least effective at inactivating the bacteria tested. Studies also found that 
repeated freeze-thaw cycling improved inactivation of two different $E$. coli and Enterococcus strains.

In the experiments by Gao et al. (2006), inactivation capacity of $E$. coli in water via freezing was studied using freezing temperatures of $-5,-15$ and $-30^{\circ} \mathrm{C}$. The study also examined the effects of curing time and number of freeze thaw cycles. In general, warmer temperatures and longer curing times resulted in greater inactivation of E. coli. Freeze-thaw cycling did not seem to be beneficial since approximately $98 \%$ of the bacteria were killed after the first cycle for a freezing temperature of $-5^{\circ} \mathrm{C}$. Similarly, for freezing temperature of $-15^{\circ} \mathrm{C}, 75 \%$ were killed after the first freezing cycle, and after two freezing cycles, injury to cells decreased dramatically.

In the experiments by Tao et al. (2006b), up to 6 cyclic freeze thaw tests were carried out on 2 different wastewater sludge flocs from a food processing industry (floc 1 diameter $=2170 \mu \mathrm{m} \&$ floc 2 diameter $=2240 \mu \mathrm{m})$. During the first freezing cycle, both flocs were pulled apart by the growing ice, and during thawing, floc shapes were only partially restored. Subsequent freezing cycles generally did not exert the same amount of force on the sludge flocs, meaning that sludge dewaterability may not be improved by repeated freeze-thaw cycles.

Chang et al. (2004) studied freeze-thaw treatments on "hard-to-dewater" sludge from an industrial park manufacturing high-tech commodities in Taiwan. Sludge samples were frozen at $-16.5^{\circ} \mathrm{C}$ for 24 hours then thawed at room temperature for 12 hours. After an 
additional 24 hours for gravity settling, the layer of clear supernatant occupied $83 \%$ of the vessel height, while the settled solids occupied the remaining height of only $17 \%$.

In the experiments by Chu et al. (2004), WAS with initial TS of $0.935 \%$ was frozen in small (65 mL) sample vessels at $-16.5^{\circ} \mathrm{C}$ for 24 hours. Samples took 2 hours to freeze, meaning samples cured for 22 hours. Microphotographs revealed that freeze-thawed biomass contained granules of size $80 \mu \mathrm{m}$, and became very compact, with pore diameters less than $5 \mu \mathrm{m}$. Compact granules indicate good floc dehydration, and improved dewaterability.

Curing time is the time at which the frozen mass is kept completely frozen. Extra curing time allows extra time for sludge to fully dehydrate. In the experiments by Parker et al., 1998, entrapped sludge flocs following unidirectional freezing at an average freezing rate of $3 \mathrm{~mm} / \mathrm{hr}$ showed different characteristics as a result of different curing times exerted on the sludge flocs throughout the $16 \mathrm{~cm}$ freezing cylinder. Sludge flocs which had been cured for only short periods were "clayey and soft", whereas sludge flocs cured for up to 53 hours were granular.

In the study by Hu et al. (2011), the role of curing time during freeze-thaw of WAS was investigated. The experiments revealed that while bulk freezing was responsible for the gross improvements in sludge dewaterability, the curing stage, during which time "tiny unfrozen regimes in the ice matrix were continuously dehydrated", was responsible for the solubilisation of COD release of ammonia. 
In the studies by Sanin et al. (1994), freeze-thaw tests on sludge samples were carried out in two phases. First, aerobically digested sludge was used to examine the effect of freezing temperature (or freezing rate), curing time and curing temperature on the reduction of fecal coliform, Salmonella, and virus survival (measured by plaque forming units). Freezing temperatures of $-7,-18$ and $-25^{\circ} \mathrm{C}$, curing temperatures of $-7,-18$ and $25^{\circ} \mathrm{C}$, and curing times of 1,7 and 28 days were used. The freezing temperature, or freezing rate, was found to be an important factor in the reduction of fecal coliform and, to a lesser extent, Salmonella. Faster freezing rates were found to improve the reduction of fecal coliform and Salmonella, but did not improve the reductions in plaque forming units. Curing temperature did not improve reductions in fecal coliform and Salmonella for curing times greater than 1 day. Curing time was found to be an important parameter for the optimisation of freeze thaw treatments. Sludge samples which were frozen for only 1 day had low pathogen reductions, while longest curing time of 28 days did not show have a benefit over the intermediate storage time of 7 days.

The second phase of experiments by Sanin et al (1994) examined the effect of freeze thaw treatments on the reduction of fecal coliform, fecal streptococci, plaque forming units, Salmonella, poliovirus, Ascaris ova, and Cryptosporidium parvum oocysts, in aerobically and AD sludges. A pathogenic bacteria (Salmonella), a virus (poliovirus), and two parasites (Ascaris suum ova and Cryptosporidium parvum oocysts) were chosen as sample microorganisms from a list of "Principal Pathogens of Concern in Municipal wastewater and Sludge" prepared by the US EPA (EPA, 1989). From the results of the 
first phase, the high freezing temperature $\left(-25^{\circ} \mathrm{C}\right)$, intermediate curing time (7 days), and low curing temperature $\left(-25^{\circ} \mathrm{C}\right)$ were chosen for the second phase of experiments. Similar to the first phase of experiments, fast freezing rates and low curing temperatures reduced plaque forming units (poliovirus), fecal coliform, and to a lesser extent, Salmonella. However fecal streptococci were reduced by only $0.2-\log$, indicating that not all bacteria are affected by freeze thaw treatments. Similarly, the parasites Cryptosporidium parvum oocysts (protozoa) were reduced by $>8$-log, while Ascaris suum ova were unaffected by the freeze thaw treatments.

Wang et al. (2001) conducted freeze thaw experiments to measure the effect of different freezing temperatures $\left(-80,-20,-10^{\circ} \mathrm{C}\right)$ on dewaterability and viable cell counts. Plate counts revealed that warmer freezing temperatures had greater reduction in bacteria. Similarly, while elution of proteins and carbohydrates were observed for all freezing temperatures, elution was greater at warmer freezing temperatures $\left(-10^{\circ} \mathrm{C}\right)$ compared to the coldest freezing temperature $\left(-80^{\circ} \mathrm{C}\right)$. Slower freezing rates were also found to be more effective on dewaterability.

Kouloumbos et al. (2008) looked at the effect of freeze-thaw conditioning, and several other dewatering techniques, on the fate of nonylphenol in liquid $A D$ sludge and thickened sludge using freezing temperatures of $-21^{\circ} \mathrm{C}$ for 48 hours, and thawing at room temperature. Nonylphenol was chosen as an indicator of the general fate of organic pollutants. Freeze-thaw increased extractable nonylphenol by $6 \%$ compared to 
non-treated samples, and decreased humin-bound residues by $2.1 \%$ and mineralisation by $2.3 \%$.

\subsubsection{Discussion of the Critical Parameters for the Optimisation of}

\section{Freeze-Thaw Sludge Stabilisation and dewatering}

Critical parameters for optimisation of freeze-thaw treatments include freezing rate or freezing temperature, curing time, and curing temperature. Freeze-thaw treatments are generally most effective using low freezing rates (warm freezing temperatures), long curing times and low curing temperatures (Wang et al., 2001, Sanin et al., 1994, Gao et al., 2009, Gao et al., 2006, Tao et al., 2006b). Under certain conditions, repeated freezethaw cycles can increase reduction of pathogens, however freeze-thaw treatments generally kill the majority of bacteria during the first freezing cycle (Gao et al., 2006). Low freezing rates achieve maximum particle migration due to the slow growing ice front, resulting in consolidation of sludge flocs and maximum dewaterability. Sludge that is frozen too fast will lead to particle entrapment and reduced dewaterability (Chung et al., 2003, Hung et al., 1996a, Halde, 1980). Hung et al., 1997 found that radial freezing rates of $2.6 \mathrm{~mm} / \mathrm{hr}$ and unidirectional rates of $4.2 \mathrm{~mm} / \mathrm{hr}$ resulted in complete migration of WAS flocs. Low freezing rates can also cause large changes in chemical composition (Hung et al., 1996b) and are also more effective on the reduction of pathogenic microorganisms and other bacteria (Sanin, et al., 1994, Wang et al., 2001). As postulated by Mazur (1965), cause of injury to cells during fast freezing is from ice nucleation which can damage or rupture cell membranes, causing the release of 
intracellular polymers to the supernatant. Wang et al. (2001) measured the elution of proteins and carbohydrates to assess cell damage.

The solubilisation of sludge components via freeze-thaw, has also been used as a pretreatment to improve subsequent stabilisation and biogas generation during anaerobic digestion. Montusiewicz et al. (2010) used freeze-thaw to increase the concentrations of soluble COD (sCOD) and volatile fatty acids (VFA) as a pre-treatment to anaerobic digestion, resulting in increased biogas production. The authors went on to point out that freeze-thaw of the mixed sewage sludge, followed by anaerobic digestion, could be considered as a "double phase digestion" rather than a simple pre-treatment method.

From the review of laboratory studies, it was determined that freezing temperatures from -30 to $-10^{\circ} \mathrm{C}$, using freezing vessels containing 150 to $500 \mathrm{~mL}$ of sludge result in freezing rates of less than $10 \mathrm{~mm} / \mathrm{hr}$, and typically range from 1.5 to $7 \mathrm{~mm} / \mathrm{hr}$. Freezing rates within this range are very effective in terms of particle migration and pathogen attenuation (Hung, 1996a, Sanin et al., 1994). To achieve the desired freezing rate, a range of vessel sizes and dimensions, and freezing temperatures can be used.

Curing time is another important factor which should be optimised during freeze-thaw sludge treatment. Incomplete freezing significantly decreases the effectiveness (Kinsley et al., 2012), while long curing times offer few to no advantages over intermediate curing times of 5 to 15 days (Sanin et al., 1994). In the experiments by Chang et al. (2004) and Örmeci and Vesilind (2001), curing times as short as 24 and 36 hours were used for small samples, and achieved great results in terms of dewaterability. In the 
experiments by Martel (1989), curing times of 7, 14 and 84 days reduced typhoid bacilli by 50,90 and $100 \%$ respectively, indicating that the majority of kills are achieved in the first few weeks of freezing. In the study by Sanin, et al. (1994), it was shown that short curing times (<24 hours) are insufficient in for pathogen reduction, while curing times of 28 days offer no advantage over a 7-day curing period in terms of a reduction in fecal coliform and Salmonella.

During the curing stage, after all of the free water has frozen, intracellular water migrates out of the cell by diffusion causing the cell to dehydrate (Mazur, 1965). As postulated by Mazur (1965), this movement of water from inside the cell can result in lethal solute concentrations inside the cell. In the study by Sanin et al., (1994), 28-day curing period resulted in greater than 4.81 log reduction in plaque forming units, regardless of the curing temperature. This suggests that extended curing times can have beneficial effects in terms of reduction of certain, more resilient, pathogenic microorganisms.

For effective and efficient laboratory experiments, repeatability should be considered as well as the expected lifespan of the test microorganisms being used. While Cryptosporidium parvum is known for its resistance to inactivation by conventional treatment methods, complete inactivation was achieved using freeze-thaw treatments. On the other hand, freeze-thaw treatments had no effect on the viability of Ascaris suum ova parasites (Sanin et al., 1994). A reduction in fecal coliform was observed as a result of freeze-thaw treatment, but this reduction was not improved when the curing 
time was increased from 7 to 28 days (Sanin et al., 1994). Based on the review of laboratory experiments, curing times from 1,8 and 15 days were chosen for this study, due to their effectiveness in terms of dewaterability and overall pathogen attenuation, and because extended curing times ( $>15$ days) did not seem to offer any significant advantages.

Curing temperature can also influence the level of inactivation of certain pathogens during freeze-thaw treatments. Although microorganisms such as fecal coliform and Salmonella are killed at both warm $\left(-7^{\circ} \mathrm{C}\right)$ and cold $\left(-25^{\circ} \mathrm{C}\right)$ curing temperatures, colder curing temperatures of -18 and $-25^{\circ} \mathrm{C}$ increased the inactivation of plaque forming units, compared to a curing temperature of $-7^{\circ} \mathrm{C}$. On the other hand, in the experiments by Gao et al. (2006) and Gao et al. (2009), warmer curing temperatures of -5 and $-15^{\circ} \mathrm{C}$ were more effective on inactivation of $E$. coli, compared to colder curing temperatures of $-30^{\circ} \mathrm{C}$ and $-80^{\circ} \mathrm{C}$. In the experiments by Sanin et al. (1994), inactivation of plaque forming units was heavily influenced by curing temperature and curing time. Using long curing times of 28 days, plaque forming units were reduced by greater than 4.8 log, regardless of curing temperature. However, with a shorter curing time of 7 days, plaque forming units in samples cured at $-7^{\circ} \mathrm{C}$ were inactivated by only $0.9 \mathrm{log}$, whereas samples cured at -18 and $-25^{\circ} \mathrm{C}$ were inactivated by greater than $5.1 \mathrm{log}$.

From the review of laboratory experiments, freeze-thaw sludge treatments using curing temperatures from -10 to $-30^{\circ} \mathrm{C}$ where chosen for this study due to their effectiveness in terms of dewaterability and pathogen attenuation. Furthermore, these temperatures 
are in the range of winter temperatures for many parts of Canada, where freeze-thaw treatments may be applied. Similarly, this study did not differentiate between freezing temperature and curing temperature, since temperature control during natural freezethaw treatments is not easily achievable in practice.

Although Jean et al. (2001) have had success conditioning a variety of sludges using dry ice, mechanical or other non-natural freezing techniques are generally not economically feasible. On the other hand, natural freeze-thaw cycles in cold climates can be used to effectively condition sludge. The following sections will present some pilot and full-scale studies.

\subsubsection{Pilot and Field-Scale Freezing Beds}

Early freeze-thaw treatment units by Benn \& Doe (1969) \& Benn et al., (1965) worked well, however they were troubled by design flaws with the freezing bed (Ezekwo et al., 1980). The bulk storage tanks had a tendency to rupture after only a few cycles due to the extreme force of the expanding sludge mass (Farrell, 1970). Other freezing beds have been used successfully for a number of decades now (MANROT, 2010, Reed et al., 1986, Martel and Diener, 1991b). Ivanov and Ivanov (2010), Gruber (1970), and Wood and Allanson (1970) have patented processes involving freeze-thaw treatment. And in Quebec, Canada, full-scale freeze-thaw operations are used by 7 WWTPs. Natural freeze-thaw has also been used in eastern and western parts of Canada, northern United States, Antarctica and Sweden (Desjardins and Brière, Penman and Van Es, 1973, Martel 1993, Martel 2001, Hedström and Hanaeus, 1999). Natural freeze thaw 
treatments have also been used in existing lagoons and drying beds (Penman and Van Es, 1973, Canfield and Sutphen, 1982, Schleppenbach, 1984), however a properly designed sludge freezing bed like the ones shown in Figure 2-2 and Figure 2-3 offer several advantages (Hellström and Kvarnström, 1997, Martel, 2001).

\subsubsection{Design and Operation of Freezing Beds}

Natural freeze-thaw sludge treatment is a very simple technology, applicable at any scale and many levels of design complexity. The earliest and simplest example of freezethaw treatment of human refuse is an early "out-house", which were commonly found in provincial and national parks throughout Canada. During use, solids build up and partially dry, then in winter months the solids freeze, causing the consolidation of solids and eventually the release of water during thaw. Slightly more advanced freezing beds are constructed directly into the surrounding soil creating a ditch or a pond, however proper lining should be used to contain the waste. Furthermore, eventual removal of sludge cake and excess water after thawing must be considered. More advanced sludge freezing beds are equipped with drainage or meltwater collection systems to collect draining water as it is released from the thawing sludge mass. Meltwater collection systems offer several advantages.

During thawing, meltwater drains to the base of the freezing bed and unless removed, can lead to septic conditions and potential for putrefaction. To remove meltwater effectively the base of most freezing beds is angled, or sometimes in a saw-tooth configuration, to help the flow of meltwater to a more centralised collection zone where 
it can be pumped from the freezing bed and subsequently treated. Figure 2-2 shows a ditch-shaped freezing bed equipped with three meltwater collection pipes. To maximise the flow of meltwater to the drainage pipes while preventing the migration of solids, the drainage pipes are encased in a layer of gravel and the entire base, including the collection pipes, are covered with sand.

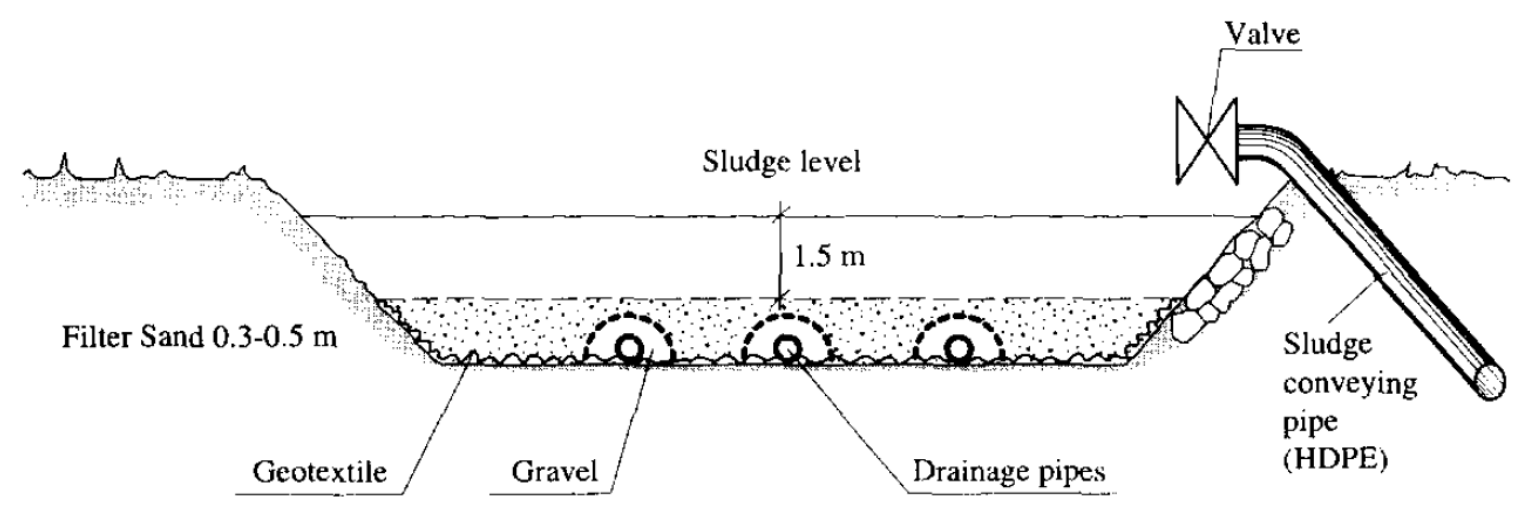

Figure 2-2: Sludge freezing bed used in full-scale tests by Hellström and Kvarnström (1997).

It is also advantageous to add water to the base of the freezing bed, and provide sufficient time for this water to freeze before adding sludge. This will reduce the risks of over-compaction of the sand layer, and will also prevent immediate sludge drainage when the unfrozen sludge is initially added to the freezing bed. If liquid sludge infiltrates the sand layer and drainage collection system, porosity of the sand will be reduced and the flow of water to the meltwater collection system will be restricted.

Another important design consideration is whether or not to incorporate a surrounding structure, such as a roof and/or side walls. While some have insisted a roof is required to keep rain and snow off of the sludge freezing bed, others have demonstrated that snow accumulation does not affect the performance of the freeze-thaw bed since each 
added layer of sludge melted any snow which had accumulated on the previous sludge layer. Furthermore, the presence of a roof can make applying liquid sludge and removing dewatered sludge cake difficult by restricting machine accessibility. The freezing bed in Figure 2-3 has transparent roof to prevent snow and rain from entering the unit, without restricting added benefits of solar drying. Similarly, air circulation from wind can improve sludge drying, however snow drifts entering the unit may be undesirable. One option is to use partial walls along the base on the unit, or as with the freezing bed in Figure 2-3, use louvered side walls.

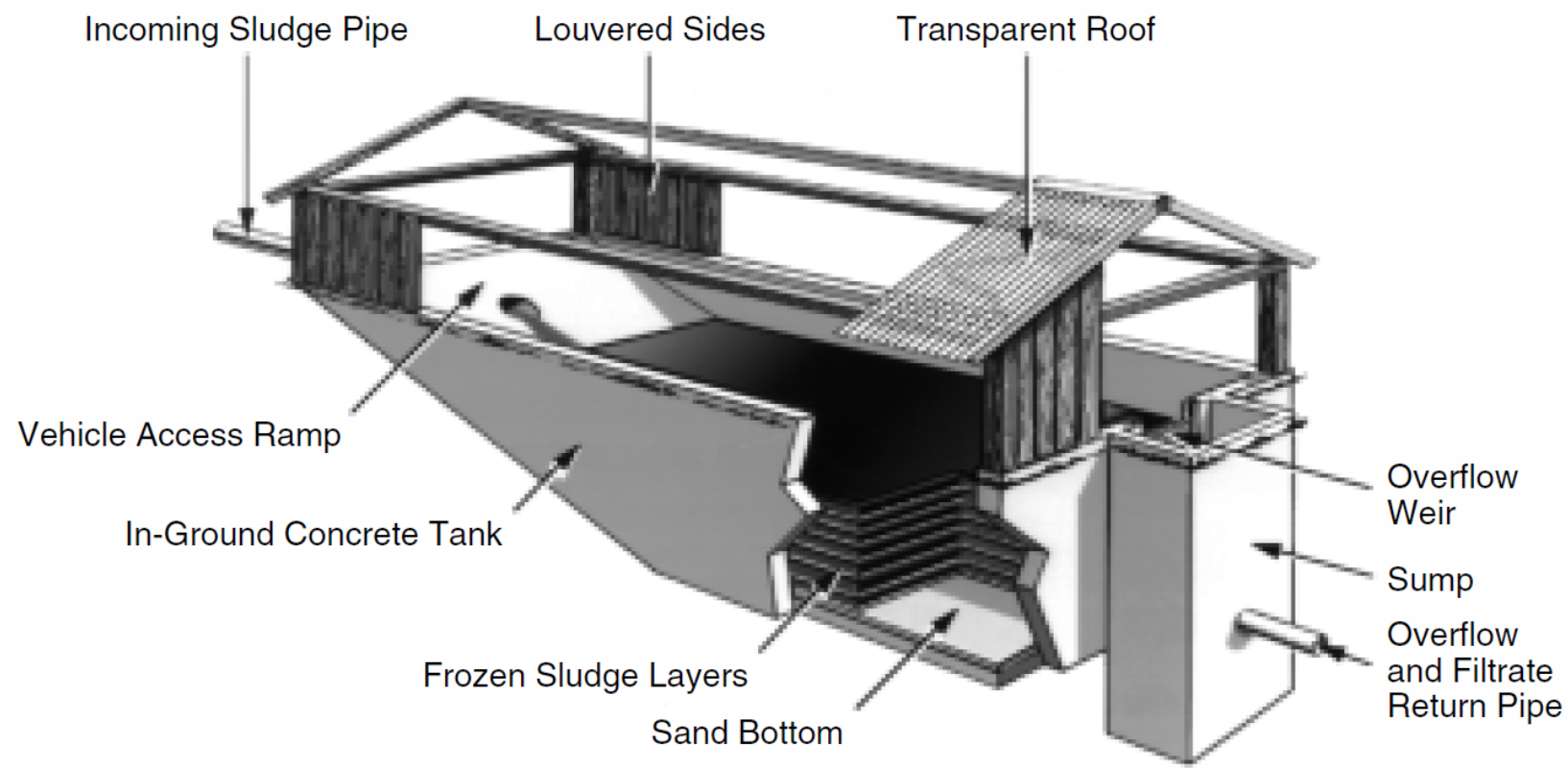

Figure 2-3: Conceptual drawing of a sludge freezing bed (Martel, 2001).

Finally, the most critical design and operational considerations for the application of freeze-thaw technology are how the liquid sludge will be added to the freezing bed, and how the dewatered sludge cake will be removed. Sludge addition can be accomplished in any number of ways, with varying complexity. The most suitable techniques depend 
on how sludge is delivered to the site (i.e. hauling truck or sludge conveyance pipe), and as mentioned earlier, the presence of any physical restraints such as a roof or side walls. The simplest option is the direct application of sludge from a sewage hauling truck or other machinery, however for on-site applications at a WWTP, or where sludge storage is available, a sludge conveyance pipe may be suitable. Figure $2-2$ shows a sludge conveyance pipe used to add sludge to the freezing bed, while Figure 2-3 shows a builtin sub-terrain sludge feed pipe as well as the option for access and unloading of sewage hauling trucks.

When adding sludge to freezing beds it is important to have a good understanding of the average daily temperature, because this will govern how fast sludge will freeze and therefore how much or how often sludge can be applied. Reed et al. (1986), stress that the same factors and therefore the same equations used to determine frost penetration or ice formation on ponds and lakes are applicable for natural sludge freezing. Equation 1 is of the Neumann- or Stephan-form, and can be used to predict freezing or thawing in for a giving location.

$$
\begin{array}{ll}
\mathrm{x}=\mathrm{m} \sqrt{\Delta \mathrm{T} \cdot \mathrm{t}} & \text { Equation } 1
\end{array}
$$

where $\quad \mathrm{x}$ : depth of freezing or thawing $(\mathrm{cm})$

$\mathrm{m}$ : proportionality coefficient $\left(\mathrm{cm} \cdot\left[{ }^{\circ} \mathrm{C} \cdot \mathrm{day}\right]^{-1 / 2}\right)$

$\Delta \mathrm{T}$ : difference between the freezing temp. and the average daily temp. $\left({ }^{\circ} \mathrm{C}\right)$

t: time period (days)

According to Reed et al., (1986), the proportionality coefficient, m, ranges from 1.7 for fast flowing streams or ponds with a heavy snow cover, to 3.07 for ideal freezing 
conditions. For liquid sludge, with less than $8 \%$ solids, Reed et al. (1986) determined m to range between 2.01 and $2.14 \mathrm{~cm}\left({ }^{\circ} \mathrm{C} \cdot \mathrm{d}\right)^{-1 / 2}$.

In the study by Kinsley et al. (2012) using septage pumped from septic tanks, there was a strong bias in the calculated proportionality coefficient with the depth of septage frozen. To correct for this, the authors modified Equation 1 to account for the time taken for the water in the septage to cool to the freezing point. The modified form, presented as Equation 2, includes terms for the initial septage sludge temperature and thickness of the applied sludge layer, and the resulting proportionality coefficient determined for the septage was $1.45 \mathrm{~cm}\left({ }^{\circ} \mathrm{C} \cdot \mathrm{d}\right)^{-1 / 2}$ (Kinsley et al., 2012). It should be noted that this equation was intended to determine the total thickness of septage $(x)$ with a temperature, $T_{0}$, that can be frozen in successive layers of thickness, $h_{0}$, at an average daily negative temperature, $\Delta \mathrm{T}$.

$$
\mathrm{h}=\mathrm{m} \sqrt{\Delta \mathrm{T} \cdot \mathrm{t}+\mathrm{aT}_{0} h_{0}} \quad \text { Equation } 2
$$

where $\quad \mathrm{h}$ : depth of sludge freezing $(\mathrm{cm})$

$\mathrm{m}$ : proportionality coefficient $\left(\mathrm{cm} \cdot\left[{ }^{\circ} \mathrm{C} \cdot \mathrm{day}\right]^{-1 / 2}\right)$

$\Delta \mathrm{T}$ : difference between the freezing temp. and the average daily temp. $\left({ }^{\circ} \mathrm{C}\right)$

$\mathrm{t}$ : time period (days)

a: constant ( $0.119 \mathrm{day} / \mathrm{cm})$

$\mathrm{T}_{0}$ : sludge temperature $\left({ }^{\circ} \mathrm{C}\right)$

$h_{0}:$ depth of the applied sludge layer $(\mathrm{cm})$

Using this value of $\mathrm{m}=1.45 \mathrm{~cm}\left({ }^{\circ} \mathrm{C} \cdot \mathrm{d}\right)^{-1 / 2}$ and an ambient air temperature of $-10^{\circ} \mathrm{C}$, Equation 2 suggests that $60 \mathrm{~cm}$ of liquid sludge at $5^{\circ}$ would take about 175 days to completely freeze, which represents a uni-directional freezing rate of $0.14 \mathrm{~mm} / \mathrm{hr}$. On 
the other hand, if sludge was sequentially applied in layers $2 \mathrm{~cm}$ thick, then it would take less than 7.5 hours to freeze each layer (freezing rate $=2.7 \mathrm{~mm} / \mathrm{hr}$ ), and less than 10 days to freeze $60 \mathrm{~cm}$. These simple calculations clearly demonstrate that daily temperature, thickness of each applied sludge layer, the temperature of the applied sludge, and the amount of time between each successive sludge application are critical to ensure complete freezing and efficient sludge treatment (Kinsley et al., 2012).

Kinsley et al. 2012 also developed a sludge thawing model to estimate the depth of sludge that can be thawed at a given degree days of thawing, as shown by Equation 3.

$$
\mathrm{x}=\mathrm{b}(\Delta \mathrm{T} \times \mathrm{t})
$$

Equation 3

where $\quad \mathrm{x}$ : depth of sludge thawing $(\mathrm{cm})$

b: parameter coefficient $\left(\mathrm{cm} /\left[{ }^{\circ} \mathrm{C} \cdot\right.\right.$ day $\left.]\right)$

$\Delta \mathrm{T}$ : average daily temperature $\left({ }^{\circ} \mathrm{C}\right)$

t: time period (days)

Martel (1998a, b) also presented equations for the maximum sludge depth which can be frozen in a given winter (Equation 4, Martel, 1998a), or thawed in a given summer (Equation 5, Martel, 1998b), based on specific climatic data. These equations should be used in the design of sludge freezing beds because sludge depth and sludge loading rate will govern the footprint required for sludge freezing. These equations are also useful for operators to know when to stop adding sludge, since climates with limited days above zero will have a limited thawing period, as is the case with the sludge freezing beds used at McMurdo, Antarctica, where sludge heating may be required to thaw the sludge (Martel, 2001). 


$$
\mathrm{D}_{\mathrm{f}}=\frac{\mathrm{P}_{\mathrm{f}}\left(\mathrm{T}_{\mathrm{f}}-\overline{\mathrm{T}}_{\mathrm{af}}\right)}{\rho \mathrm{L}\left(\frac{1}{\overline{\mathrm{h}}_{\mathrm{c}}}+\frac{\varepsilon}{2 \mathrm{~K}_{\mathrm{fs}}}\right)}
$$

where $\quad D_{f}$ : freezing depth $(m)$

$\mathrm{P}_{\mathrm{f}}$ : freezing period $(\mathrm{hr})$

$\mathrm{T}_{\mathrm{f}}$ : freezing point temperature $\left({ }^{\circ} \mathrm{C}\right)$

$\overline{\mathrm{T}}_{\mathrm{af}}$ : average ambient temperature during freezing $\left({ }^{\circ} \mathrm{C}\right)$

$\rho_{\mathrm{f}}$ : density of frozen sludge $\left(917 \mathrm{~kg} / \mathrm{m}^{3}\right)$

$\mathrm{L}$ : latent heat of fusion $(93 \mathrm{~W} \cdot \mathrm{hr} / \mathrm{kg})$

$\overline{\mathrm{h}}_{\mathrm{c}}$ : convection coefficient $\left(7.5 \mathrm{~W} / \mathrm{m}^{2}{ }^{\circ} \mathrm{C}\right)$

$\varepsilon$ : thickness of each sludge layer $(\mathrm{m})$

$\mathrm{K}_{\mathrm{fs}}$ : conductivity coefficient of frozen sludge $\left(2.21 \mathrm{~W} / \mathrm{m} \cdot{ }^{\circ} \mathrm{C}\right)$

$$
D_{t}=\left[\left(\frac{K_{s s}}{\theta \bar{h}_{c}}\right)^{2}+\frac{2 K_{s s} P_{t h}}{\theta \beta}\right]^{1 / 2}-\frac{K_{s s}}{\theta \bar{h}_{c}}
$$

where $\beta=\rho_{\mathrm{f}} \mathrm{L} / \overline{\mathrm{T}}_{\mathrm{at}}\left(\overline{\mathrm{T}}_{\mathrm{at}}-\mathrm{T}_{\mathrm{f}}+\alpha \tau \overline{\overline{\mathrm{I}}} / \overline{\mathrm{h}}_{\mathrm{c}}\right)$

$\mathrm{D}_{\mathrm{t}}$ : thawing design depth $(\mathrm{m})$

$\mathrm{K}_{\mathrm{ss}}$ : thermal conductivity of settled sludge $\left(0.35 \mathrm{~W} / \mathrm{m} \cdot{ }^{\circ} \mathrm{C}\right)$

$\theta$ : fraction of settled sludge per unit depth of thawed sludge (0.15 for $A D)$

$\mathrm{P}_{\text {th }}$ : thawing period $(\mathrm{hr})$

$\overline{\mathrm{T}}_{\mathrm{at}}$ : average ambient air temperature during thaw $\left({ }^{\circ} \mathrm{C}\right)$

$\alpha$ : solar absorbance of the sludge (0.9)

$\tau$ : transmittance of the roof material ( 0.9 for fibre reinforced polyester roof)

$\overline{\overline{\mathrm{I}}}$ : average insulation during thawing period $\left(\mathrm{W} / \mathrm{m}^{2}\right)$

Reed et al. (1986) compiled temperature data for a number of locations throughout the United States, and calculated the potential depth of sludge which could be frozen in 8 $\mathrm{cm}$ layers during the warmest winter between 1972 and 1983 . The results were then plotted on a map and contour lines were drawn (lines of equal-depth were drawn, as 
shown in Figure 2-4). The figure suggests that freeze-thaw treatments can be applied in many regions of the United States.

Kinsley et al., 2012 produced a similar figure for Canada, also assuming $8 \mathrm{~cm}$ layers of sludge. Their figure is based on experiments with septage, and used a proportionality coefficient value of $1.45 \mathrm{~cm}\left({ }^{\circ} \mathrm{C} \cdot \mathrm{d}\right)^{-1 / 2}$. Kinsley et al. (2012) proposed that freeze-thaw can be applied in many parts of Canada, with the exception of coastal regions and southern Ontario.

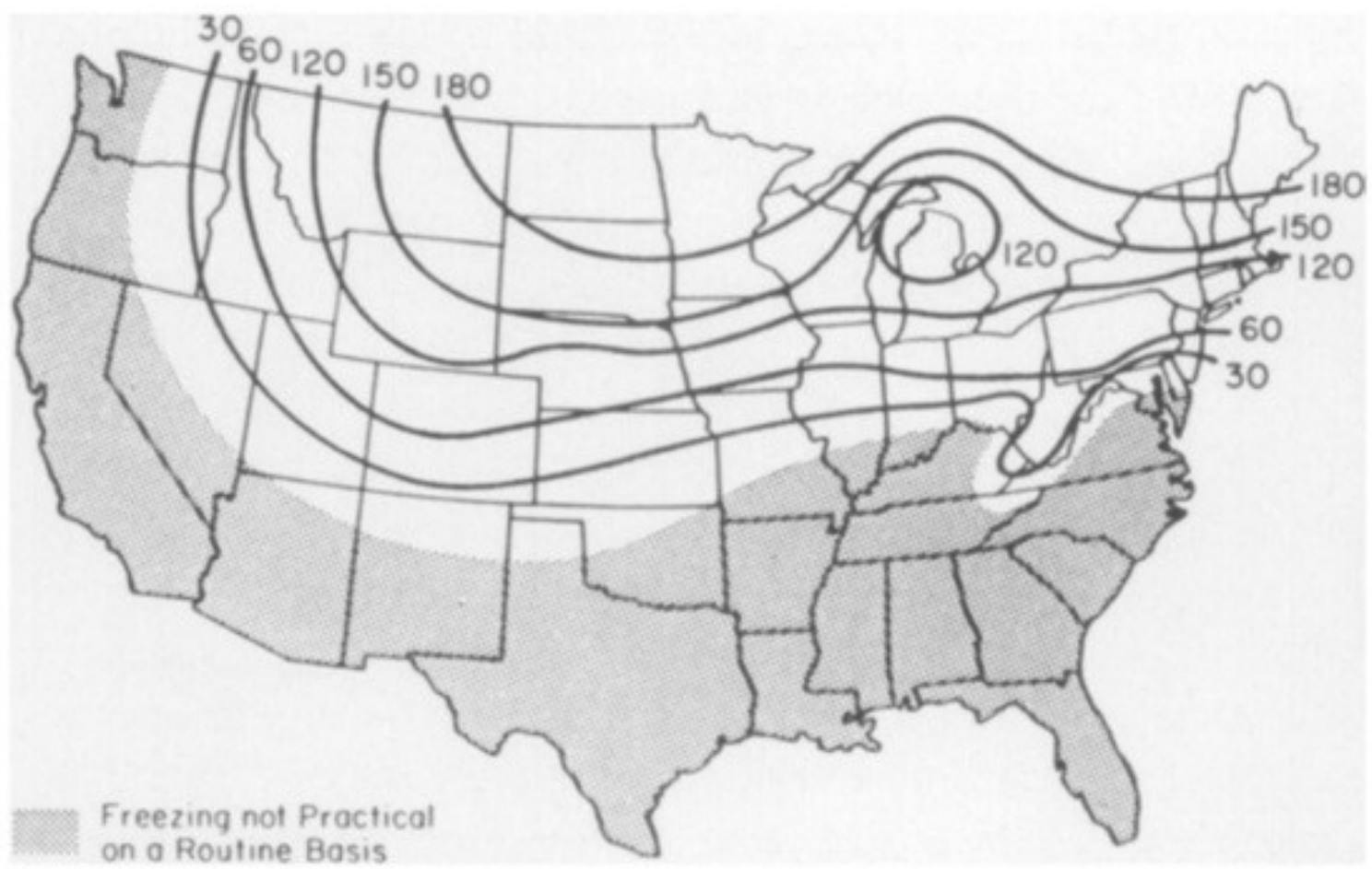

Figure 2-4: Potential depth of sludge $(\mathrm{cm})$ that could be frozen in the United States, if applied in sequential layers, $8 \mathrm{~cm}$ thick (Reed et al., 1986). 


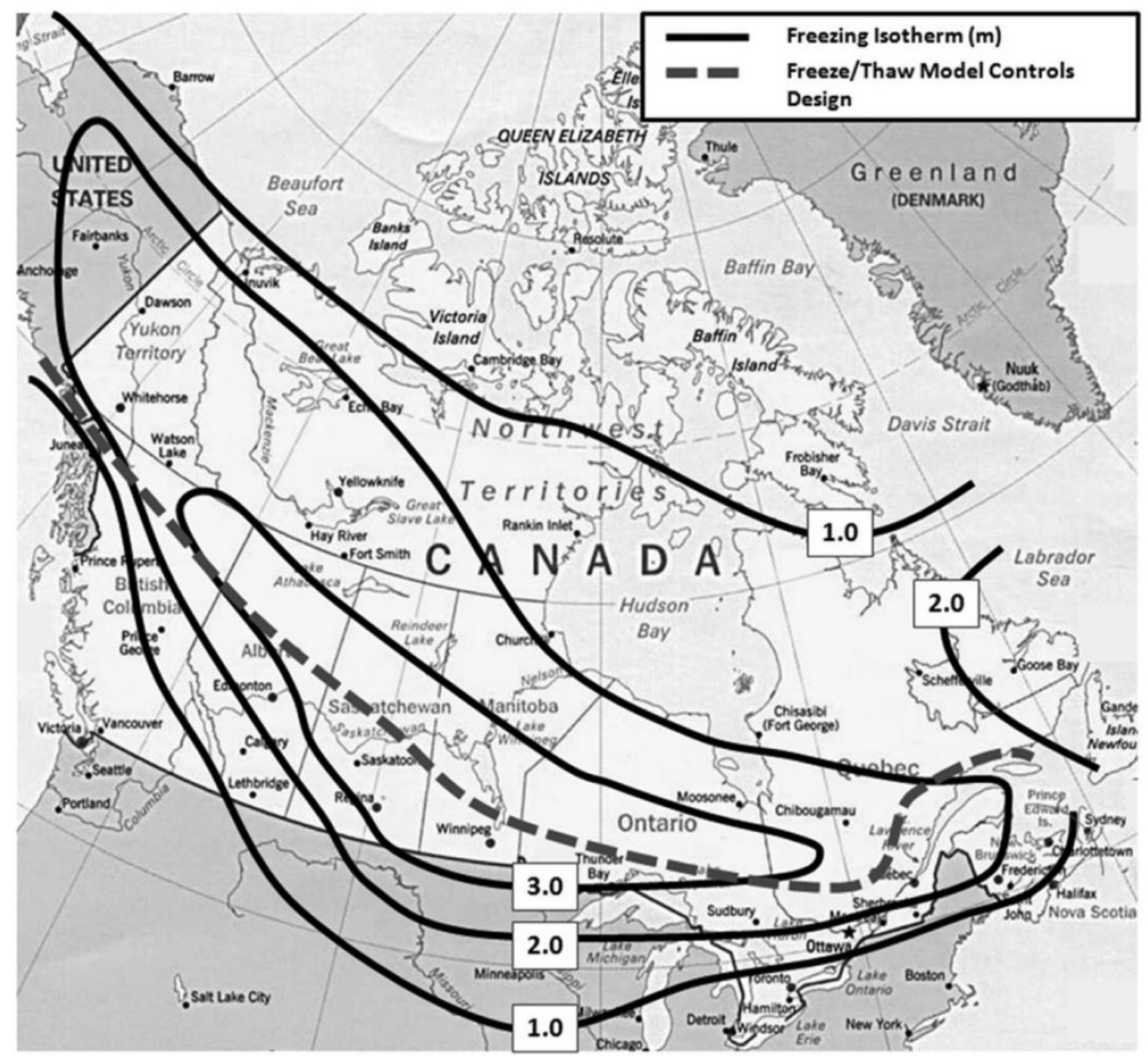

Figure 2-5: Potential depth of sludge $(\mathrm{m})$ that could be frozen in Canada and Alaska, if applied in sequential layers, $8 \mathrm{~cm}$ thick (Kinsley et al., 2012).

\subsubsection{Pilot and Field Studies}

Martel and Diener (1991b) constructed a pilot-scale freezing bed in Hanover NH (USA), and tested natural freeze-thaw dewatering with both anaerobically and aerobically digested sludges. In the first year, AD sludge was frozen in six layers, ranging from 2.5 to $14 \mathrm{~cm}$ thick, to a total depth of $58 \mathrm{~cm}$. During the thawing period, meltwater drained easily, eventually increasing the solids content from $6.7 \%$ to $39.3 \%$ solids. The third year 
of tests used aerobically digested sludge, and increased the solids content from $1.1 \%$ to 24.5\%. Meltwater grab samples collected from the thawing aerobically digested sludge had average $B O D=333 \mathrm{mg} / \mathrm{L}, \mathrm{COD}=740 \mathrm{mg} / \mathrm{L}$, total suspended solids $(\mathrm{TSS})=96 \mathrm{mg} / \mathrm{L}$, Turbidity = $116 \mathrm{NTU}$ and $\mathrm{pH}=6.9$.

Desjardins and Brière (1996) conducted a series of laboratory column experiments using biological sludge and chemical (alum) sludge from phosphorus removal both with and without polymer addition. They also compared two types of drainages systems: conventional sand and gravel, and a plastic Wedgewater system (Gravity Flow Systems). Although the two drainage systems behaved similarly in terms of meltwater drainage rate and TSS concentrations, the sand and gravel system was deemed more effective in terms of maximisation of final cake solids content.

In one of the sets of experiments by Desjardins and Brière (1996), sludge was added to the $15 \mathrm{~cm}$ diameter columns to a height of $200 \mathrm{~cm}$, for a total of $34.9 \mathrm{~L}$ of sludge. The columns were then moved to the outdoors and allowed to freeze and cure in the natural climate for a total of six days. The columns were then brought back in and allowed to thaw for 3 days. Biological sludge samples with an initial TS of $5.1 \%$ increased to $30.8 \%$, while the TS of the chemical sludge increased from 2.7 to $33.7 \%$. Meltwater samples generally had high COD and BOD but were low in TSS, as shown in Table 2-2. 
Table 2-2: Concentrations of TSS, COD and BOD in the meltwater collected during column tests by Desjardin and Brière (1996).

\begin{tabular}{lcccccc} 
Sludge & \multicolumn{7}{c}{ Meltwater Concentration $(\mathrm{mg} / \mathrm{L})$} \\
Type & TSS & COD & SCOD & BOD $_{5}$ & BOD $_{7}$ & BOD $_{\text {u }}$ \\
Biological & 88 & 849 & 772 & 271 & 559 & 666 \\
Chemical & 69 & 393 & 331 & 90 & 163 & 282
\end{tabular}

The experiments by Desjardins and Brière (1996) demonstrated the ability of freezethaw treatments to dewater both biological and chemical sludges, noting the changes in the gelatinous nature of chemical sludge to a granular sludge. They also noticed that the application of sludge in thin layers and complete freezing were essential to the performance of the sludge dewatering experiments.

Hellström and Kvarnström (1997) used full-scale natural freeze-thaw treatments to dewater sewage sludge using the freezing bed shown in Figure 2-2. Prior to sludge addition, a layer of water was added to the freezing bed to cover the sand layer and prevent immediate sludge drainage, which could clog the sand filter or other part of the meltwater collection system, and later restrict drainage. Once the layer of water was frozen, sludge layers approximately $10 \mathrm{~cm}$ thick were applied to the freezing bed. In the first year of testing, between 110 and $130 \mathrm{~m}^{3}$ of sludge with a TS ranging from 6 to $10 \%$ solids were added to the freezing bed during the winter months. In the spring, the sludge thawed and throughout the summer months, the sludge cake dried. In August, the final volume was between 50 and $60 \mathrm{~m}^{3}$, and the TS ranged from 20 to $72 \%$ solids, with an average of $53 \%$ solids. In the second year, two sludges were tested: one treated with a polymer, and another untreated sludge sample. Different climatic conditions, and different operating strategies lead to poorer results than the first year. However, final TS 
of the sludge cake following drying reached $26 \%$ solids, and interestingly the polymer did not have an effect on the final TS of the sludge cake following freeze-thaw treatment.

Hedström and Hanaeus (1999) experimented with full-scale freeze-thaw treatment of septic tank sludge (septage), followed by drying and composting. $500 \mathrm{~L}$ of septage with TS from 4 to $5 \%$ solids was added to a lined freezing bed as a single layer ranging from 2 to $15 \mathrm{~cm}$ thick in November. Sludge thawed the in the middle of May, and proceeded to dry, reducing to a volume of $180 \mathrm{~L}$, and TS ranging from 25 to $95 \%$ solids. During the summer months (June to August), the remaining sludge cake was co-composted with kitchen waste, exceeding temperatures $65^{\circ} \mathrm{C}$ after only two weeks.

Kinsley et al. (2012) used 12 pilot-scale freeze-thaw beds for treatment of septage from septic tanks. Following natural freeze-thaw, the sludge cake had a TS of $25 \%$, and E. coli

concentrations $<2.0 \times 10^{6} \mathrm{CFU} / \mathrm{g}$ dry solids (DS). Furthermore, the study demonstrated that snow accumulation on the freeze-thaw bed was not an issue since the addition of successive layers of sludge melted any snow accumulation on the previously frozen layer of sludge.

\subsubsection{Discussion on the Application of Full-Scale Freeze-Thaw Sludge}

\section{Stabilisation and dewatering}

Pilot and full scale sludge freezing beds are able to dewater both water and wastewater sludges to $20 \%$ solids through simple gravity drainage, and by incorporating sludge drying, anywhere up to $80 \%$ solids can be achieved under natural freezing conditions 
(Martel, 1993, Desjardins and Brière, 1996, Hellström and Kvarnström, 1997). Keys to a successful operation include foremost a properly designed sludge freezing bed. Although, sludge freezing has been successful in lined freezing beds (Hedström and Hanaeus, 1999), built-in drainage systems offer several important advantages. First, the relatively quick transition from frozen sludge to drained or partially dried sludge eliminates the generation of septic conditions resulting in odours. Second, a simple gravity drainage system immediately reduces the volume of sludge requiring disposal. Third, several studies have demonstrated that a variety of properly designed drainage or meltwater collection systems are capable of producing readily draining and easy-topump effluent with low suspended solids. And finally, while many researchers agree, the changes in floc structure caused by expanding ice crystals are irreversible unless significant shear forces are applied, it is also believed that the continuous network of channels created throughout the sludge mass facilitate subsequent meltwater drainage during thawing if the sludge mass is left undisturbed.

Another important parameter for the successful operation of freezing beds is the rate and method of sludge application. Several researches have stressed the importance of successive thin sludge layers, approximately 5 to $10 \mathrm{~cm}$ thick, and intermittent sludge applications to allow enough time for each applied layer to completely freeze before the next layer is added. Sludge application rates will be governed by climatic conditions, most obviously temperature, however high wind speeds will increase freezing rates if there is sufficient air flow to the freezing bed (Martel, 1998a). A sludge freezing bed in Duluth MN, successfully freezes sludge in layers $23 \mathrm{~cm}$ thick, and in Fairbanks AL, $36 \mathrm{~cm}$ 
layers have been used (Reed et al., 1986). Local climate will also govern the maximum depth of sludge that can be frozen and thawed in a normal annual cycle (Martel, 1998a, Martel, 1998b, Reed et al., 1986).

For regions with cold climates, natural freeze-thaw dewatering combined with sludge stabilisation, using an oxidation process or lime stabilisation, could provide a simple and cost effective sludge management approach. Furthermore, with the increasing concerns over the presence of EOCs in biosolids, the use of a strong oxidant to treat sludge may provide a level of treatment that cannot be achieved with conventional sludge stabilisation techniques.

\subsection{CHEMICAL STABILISATION TECHNIQUES}

Sludge stabilisation can be achieved in various ways, as mentioned in section 2.1. In older texts, the term stabilisation referred to the reduction of odours and general potential for putrefaction (Bruce and Fisher, 1984), while disinfection referred to the destruction of pathogenic organisms (Havelaar, 1984). In more recent years, the two terms have been used interchangeably since the same treatment methods are often used to achieve both reduction of odours and putrescibility, and reduction of pathogens. The following section will present some research on chemical oxidation, and focus on more recent studies involving the use of ferrate(VI) as a chemical oxidant in wastewater and sludge treatment processes. Following the review of chemical oxidation techniques, conventional lime stabilisation will be presented since lime is cheap and 
readily available, and it can effectively inactivate pathogens and reduce sludge odours (Pritchard et al., 2010, Reinthaler et al., 2010, Wong and Selvam, 2009).

\subsubsection{Chemical Oxidation}

Chemical oxidation of sludge results in sludge solubilisation, including the destruction of cell walls and the release of intracellular materials (Bougrier et al., 2006a, Bougrier et al., 2006b, Saktaywin et al., 2005). Chemical oxidation treatments have been widely used as a pre-treatment for anaerobic digestion, increasing biogas yield and VSS destruction (Wu et al., 2014). However, sludge oxidation treatments have also been used to inactivate pathogens, and reduce odours, heavy metals and EOCs in sludge (de Luca et al., 1996, Devai and Delaune, 2002, Lumetta and Rapko, 1999, Jiang, 2007). Common oxidants include hydrogen peroxide and Fenton's reagent, ozone, permanganate and more recently, ferrate(VI). Chlorination is unfavourable for wastewater and sludge applications due to the formation of disinfection by-products (DBP) such as trihalomethanes (THM) and haloacetic acids (HAA) (Bruce and Fisher, 1984).

The strength of a particular oxidant is given by reaction potential, also known as the oxidation potential, $\mathrm{E}^{\circ}(\mathrm{V})$. Table $2-3$ lists the oxidation potential and the reactions involved for common chemical oxidants used for sludge treatment. 
Table 2-3: Oxidation potential of common oxidants used in wastewater and sludge treatment processes (Jiang, 2007).

\begin{tabular}{|l|c|c|}
\hline Oxidant & Reaction & $\mathbf{E}^{\circ}(\mathbf{V})$ \\
\hline Fenton's reagent & $\mathrm{Fe}^{+2}+\mathrm{H}_{2} \mathrm{O}_{2} \Leftrightarrow \mathrm{Fe}^{+3}+\mathrm{OH}^{*}+\mathrm{OH}^{-}$ & 2.76 \\
\hline Ferrate(VI) & $\mathrm{FeO}_{4}^{-2}+8 \mathrm{H}^{+}+3 \mathrm{e}^{-} \Leftrightarrow \mathrm{Fe}^{+3}+4 \mathrm{H}_{2} \mathrm{O}-$ & 2.2 \\
\hline Ozone & $\mathrm{O}_{3}+2 \mathrm{H}^{+}+2 \mathrm{e}^{-} \Leftrightarrow \mathrm{O}_{2}+\mathrm{H}_{2} \mathrm{O}$ & 2.076 \\
\hline Hydrogen Peroxide & $\mathrm{H}_{2} \mathrm{O}_{2}+2 \mathrm{H}^{+}+2 \mathrm{e}^{-} \Leftrightarrow 2 \mathrm{H}_{2} \mathrm{O}$ & 1.776 \\
\hline \multirow{2}{*}{ Permanganate } & $\mathrm{MnO}_{4}^{-}+4 \mathrm{H}^{+}+3 \mathrm{e}^{-} \Leftrightarrow \mathrm{MnO}_{2}+2 \mathrm{H}_{2} \mathrm{O}$ & 1.679 \\
\cline { 2 - 3 } & $\mathrm{MnO}_{4}^{-}+8 \mathrm{H}^{+}+5 \mathrm{e}^{-} \Leftrightarrow \mathrm{Mn}^{+2}+4 \mathrm{H}_{2} \mathrm{O}$ & 1.507 \\
\hline Dissolved Oxygen & $\mathrm{O}_{2}+4 \mathrm{H}^{+}+3 \mathrm{e}^{-} \Leftrightarrow 2 \mathrm{H}_{2} \mathrm{O}$ & 1.229 \\
\hline
\end{tabular}

\subsubsection{Ozone, Hydrogen Peroxide, Fenton's Reagent and Permanganate}

Hydrogen peroxide has been widely used as a chemical oxidant in wastewater treatment applications due to its highly oxidative and destructive properties. However, it is not nearly as effective when used alone due to the presence of certain refractory compounds, which reduce reaction rates (Neyens and Baeyens, 2003). Most often, metal salts such as ferrous sulphate are used with hydrogen peroxide, in a process called Fenton's reagent. This process leads to the generation of highly reactive hydroxyl radicals $(* \mathrm{OH})$, as shown in Equation 7 , and is therefore very effective for the oxidation of organic compounds (Li and Zhang, 2014, Valo et al., 2004).

$$
\mathrm{Fe}^{+2}+\mathrm{H}_{2} \mathrm{O}_{2} \rightarrow \mathrm{Fe}^{+3}+\mathrm{HO}^{*}+\mathrm{OH}^{-} \quad \text { Equation } 7
$$

Fenton's oxidation has also been used to oxidise the EPS in WAS and improve filterability and dewaterability (Ye et al., 2014). Furthermore, recent studies have used Fenton oxidation for the removal of EDCs in WAS (Li and Zhang, 2014, Zhang and Li, 2014). In the study by Li and Zhang (2014), WAS samples were spiked hormones (estrone, 17b-estradiol, estriol, and 17a-ethinylestradiol), and treated using various 
dosages of $\mathrm{H}_{2} \mathrm{O}_{2}$ and $\mathrm{Fe}(\mathrm{II})$, at various $\mathrm{pH}$, with various contact times. Under optimal conditions $\left(\mathrm{H}_{2} \mathrm{O}_{2}\right.$ dose $=15.62 \mathrm{mmol} / \mathrm{g}$, initial $\mathrm{pH}=3$, reaction time $=60 \mathrm{~min}, \mathrm{Fe}(\mathrm{II})$ to $\mathrm{H}_{2} \mathrm{O}_{2}$ molar ratio $\left.=0.167\right), 70,90,84$ and $98 \%$ reductions in estrone, $17 \mathrm{~b}$-estradiol, estriol, and 17a-ethinylestradiol were achieved. The authors noticed that increasing the $\mathrm{Fe}(\mathrm{II})$ to $\mathrm{H}_{2} \mathrm{O}_{2}$ ratio increased the generation of $* \mathrm{OH}$ radicals, however ratios greater than 0.167 may led to excess Fe(II) which likely consumed some of the $* \mathrm{OH}$ radicals.

Hydroxyl radicals can also be generated from hydrogen peroxide using ozone, as shown by Equation 8, or using ultraviolet light, which breaks one hydrogen peroxide molecule into two hydroxyl radicals, as shown by Equation 9 (Neyens and Baeyens, 2003).

$$
\begin{array}{cr}
\mathrm{O}_{3}+\mathrm{H}_{2} \mathrm{O}_{2} \rightarrow \mathrm{HO}^{*}+\mathrm{O}_{2}+\mathrm{HO}_{2}^{*} & \text { Equation } 8 \\
\mathrm{H}_{2} \mathrm{O}_{2}+\mathrm{h} v \rightarrow 2 \mathrm{HO}^{*} & \text { Equation } 9
\end{array}
$$

Direct ozonation of sludge also results in the generation of hydroxyl radical due to the wide variety of complexes present in sludge. The highly reactive hydroxyl radicals are non-selective, reacting with all components in the sludge, including soluble and particulate components, both organic and inorganic (Bougrier et al., 2006a). Ozonation and UV cause the destruction of cell walls, and the release of intracellular materials, resulting in an increase in SCOD and a reduction in TSS (Bougrier et al., 2006a, Bougrier et al., 2006b, Saktaywin et al., 2005). However, ozone is highly toxic, corrosive and very unstable, therefore it must be generated on site using an ozone generator. Typically ozone generators use a high voltage discharge to convert oxygen gas in air to ozone gas (Seol et al., 2003). 
Permanganate can also be used to oxidise sludge, causing the disintegration of sludge flocs and a release of EPS into the supernatant (Wu et al., 2014). A $500 \mathrm{mg} / \mathrm{L}$ dose of potassium permanganate increased the SCOD of WAS from below $100 \mathrm{mg} / \mathrm{L}$ to approximately $1600 \mathrm{mg} / \mathrm{L}$ after 15 minutes, and to approximately $2000 \mathrm{mg} / \mathrm{L}$ after 60 minutes. The increase in SCOD coincided with increases in supernatant proteins $(490 \%)$ and polysaccharides (141\%).

Devai and Delaune (2002) tested the ability of a variety of oxidants (ferric chloride, sodium hypochlorite, potassium permanganate, hydrogen peroxide and potassium nitrate) to suppress or eliminate reduced sulphurous gas emissions, such as hydrogen sulphide, from trickling filter sludge. Doses of 0, 30, 300 and $1000 \mathrm{ppm}$ of each oxidant were used, and sulphurous emissions were tested at $0.5,1,2,4$ and 24 hours. Experiments showed that potassium permanganate and hydrogen peroxide were most effective at reducing hydrogen sulphide emissions. $1000 \mathrm{ppm}$ of potassium permanganate eliminated hydrogen sulphide emissions in all cases, and the sample treated with 300 ppm only had very small hydrogen sulphide emissions, and only after 24 hours.

Ferrous salts have been used to reduce hydrogen sulphide emissions from sewage sludge by to precipitating sulphide (Bruce and Fisher, 1984), and are also used in conjunction with lime for coagulation and flocculation of solids in wastewater treatment (El-Gohary et al., 2010). Furthermore, ferrate(VI), in which iron exists at the +6 oxidation 
state, first acts as an oxidant, until it is reduced to ferric species, at which point they act as a coagulant (Jiang et al., 2006a, Jiang and Lloyd, 2002).

\subsubsection{Ferrate(VI)}

Ferrate(VI) is a very strong oxidant capable of inactivating, degrading and oxidising harmful microorganisms, and organic and inorganic micro-pollutants (Li et al., 2008, Jiang et al., 2007, Jiang, 2007, Zhu et al., 2006, Schuck et al., 2006). Many of the odours in sludge are the result of sulphur compounds such as hydrogen sulphide, mercaptans, and organic sulphides, and nitrogen compounds such as ammonia and organic nitrogen, organic acids and other organic compounds such as aldehydes and ketones (He et al., 2009, de Luca et al., 1996, Read et al. 2000, Read et al., 2003). Ferrate(VI) can be used to oxidise highly reduced substances, for example ammonia, which is the fully reduced form of nitrogen, to nitrate, which represents 8 numbers of oxidation $(-3$ to +5$)$ (de Luca et al., 2996). Furthermore, the use of ferrate(VI) does not result in the formation of any known harmful DBPs (Gombos et al., 2013, Jiang et al., 2007, Schuck et al. 2006).

\section{Ferrate(VI) Chemistry}

The ferrate(VI) ion can exist as the protonated $\left(\mathrm{H}_{3} \mathrm{FeO}_{4}{ }^{+}, \mathrm{H}_{2} \mathrm{FeO}_{4}, \mathrm{HFeO}_{4}{ }^{-}\right)$or non-

protonated $\left(\mathrm{FeO}_{4}^{-2}\right)$ forms depending on the $\mathrm{pH}$, as shown by Figure 2-6. In strong alkali, the unprotonated $\mathrm{FeO}_{4}{ }^{-2}$ ion is the predominant species, whereas under mildly acidic conditions, the protonated ion, $\mathrm{HFeO}_{4}{ }^{-}$, is the predominant species (Delaude and Laszlo, 1996). Figure 2-6 shows the fractional speciation of ferrate(VI) under various $\mathrm{pH}$. 


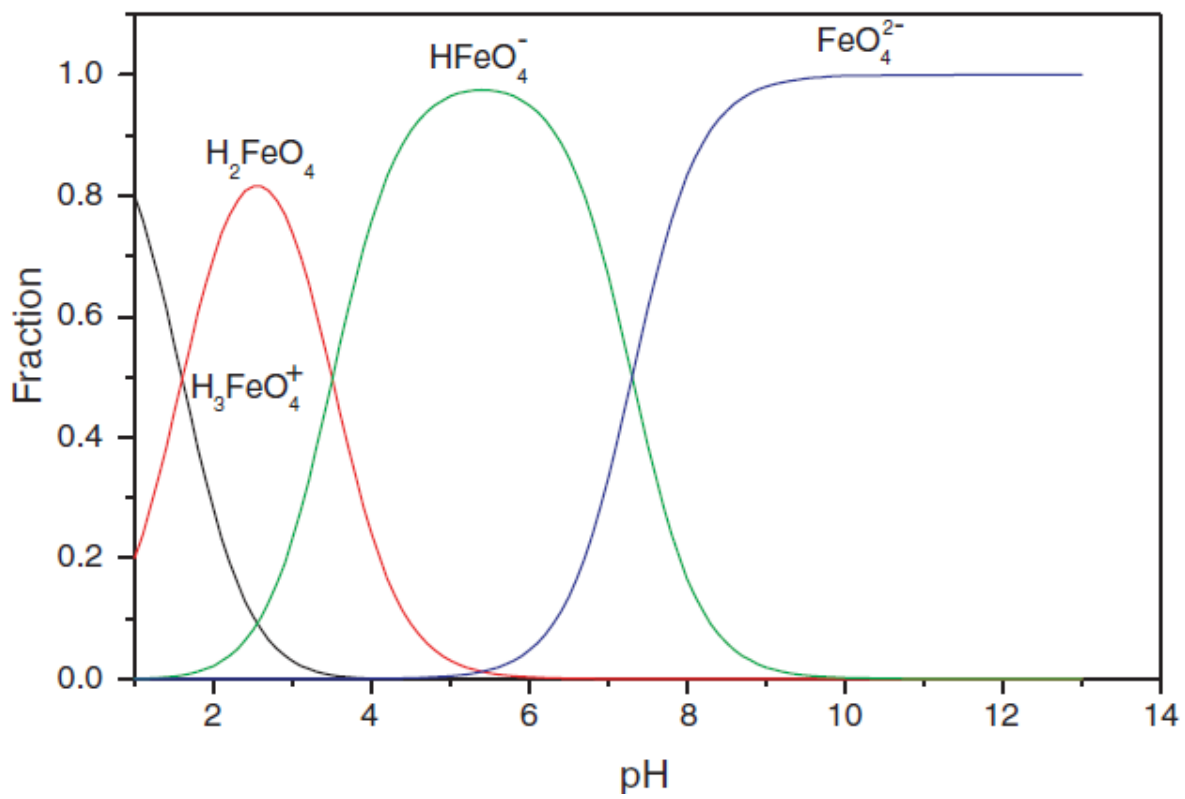

Figure 2-6: Speciation of ferrate(VI) for various pH (Delaude and Laszlo, 1996).

The dissociation of the tri-, di-, and mono-protonated forms of the ferrate(VI) ion are also shown by Equation 10, Equation 11 and Equation 12 respectively.

$$
\begin{array}{lll}
\mathrm{H}_{3} \mathrm{FeO}_{4}^{+} \leftrightarrow \mathrm{H}^{+}+\mathrm{H}_{2} \mathrm{FeO}_{4} & p K_{a, \mathrm{H}_{3} \mathrm{FeO}_{4}^{+}}=1.6 & \text { Equation } 10 \\
\mathrm{H}_{2} \mathrm{FeO}_{4} \leftrightarrow \mathrm{H}^{+}+\mathrm{HFeO}_{4}^{-} & p K_{a, \mathrm{H}_{2} \mathrm{FeO}_{4}}=3.5 & \text { Equation } 11 \\
\mathrm{HFeO}_{4}^{-} \leftrightarrow \mathrm{H}^{+}+\mathrm{FeO}_{4}^{-2} & p K_{a, \mathrm{HFOO}_{4}^{-}}=7.3 & \text { Equation } 12
\end{array}
$$

As shown by Figure 2-6, for $\mathrm{pH}>5$, concentrations of $\mathrm{H}_{3} \mathrm{FeO}_{4}{ }^{+}$and $\mathrm{H}_{2} \mathrm{FeO}_{4}$ are negligible, therefore only the speciation between the mono-protonated, $\mathrm{HFeO}_{4}^{-}$, and the nonprotonated, $\mathrm{FeO}_{4}{ }^{-2}$, species are applicable for the proposed sludge treatment applications. The $\mathrm{pK}_{\mathrm{a}}$ for this reaction is 7.3 , which means that for $\mathrm{pH}>7.3$ the nonprotonated, $\mathrm{FeO}_{4}{ }^{-2}$, is the dominant species, whereas under neutral and mildly acidic conditions, the protonated, $\mathrm{HFeO}_{4}^{-}$, is dominant. 
The strength of ferrate(VI) as an oxidant and the stability of the ferrate(VI) ion are highly dependent on $\mathrm{pH}$. Under acidic conditions $(\mathrm{pH}=10)$, ferrate( $\mathrm{VI})$ decomposes rapidly according to Equation 13, with an oxidation potential of $2.20 \mathrm{~V}$, and under basic conditions, ferrate is more stable, with an oxidation potential of $0.72 \mathrm{~V}$ (Equation 14) (Wood, 1958).

$$
\begin{array}{cr}
\mathrm{FeO}_{4}^{-2}+8 \mathrm{H}^{+}+3 e^{-} \rightarrow \mathrm{Fe}^{+3}+4 \mathrm{H}_{2} \mathrm{O} & \text { Equation } 13 \\
\mathrm{FeO}_{4}^{-2}+4 \mathrm{H}_{2} \mathrm{O}+3 e^{-} \rightarrow \mathrm{Fe}(\mathrm{OH})_{3}+5 \mathrm{OH}^{-} & \text {Equation } 14
\end{array}
$$

From Table 2-3 we see that ferrate(VI) is a stronger oxidant than most commonly used chemical oxidants including ozone, permanganate and hydrogen peroxide. The only oxidant with a higher oxidation potential is Fenton's reagent, which uses metal salts such as ferrous iron to generate hydroxyl radicals from hydrogen peroxide.

\section{Ferrate(VI) Synthesis}

Common forms of solid ferrate( $\mathrm{VI})$ are sodium ferrate $\left(\mathrm{Na}_{2} \mathrm{FeO}_{4}\right)$ and potassium ferrate $\left(\mathrm{K}_{2} \mathrm{FeO}_{4}\right)$. Potassium ferrate $(\mathrm{VI})$ is a crystalline purple to black solid, stable at room temperatures. Solid potassium ferrate can be generated at the electrode during electrolytic oxidation of crude iron in a solution with 20 to $50 \%$ sodium hydroxide (Murmann and Robinson, 1974). However, according to Madarász et al. (2006), the yield for this method is usually less than $50 \%$. Another way to prepare potassium ferrate( $\mathrm{VI})$ is according to the method by Thompson et al. (1951), where ferric nitrate is added to an aqueous alkaline sodium hypochlorite solution resulting in the formation of soluble potassium ferrate(IV). Potassium hydroxide is then added to the solution to precipitate 
potassium ferrate(VI), and then the crystals are dried using ethanol. Madarász et al. (2006) produced $>98 \%$ pure potassium ferrate using this method. A recent article in American Laboratory ${ }^{\circledR}$ reported that powdered potassium ferrate (VI) cost approximately USD $\$ 80 / \mathrm{kg} \mathrm{K}_{2} \mathrm{FeO}_{4}$ (Waite, 2012).

When added to water, potassium ferrate decomposes according to Equation 15 (Sharma et al 2008). The decomposition can be observed by the changing solution colour, from violet to yellow, and eventually to a colourless liquid with some yellowish precipitate, once all of the ferrate(VI) has been reduced to ferric hydroxide. Under neutral or mildly acidic conditions, this reaction proceeds very quickly, whereas under alkali conditions the ferrate(VI) ion is more stable (Li et al., 2005).

$$
2 \mathrm{FeO}_{4}^{-2}+5 \mathrm{H}_{2} \mathrm{O} \rightarrow 2 \mathrm{Fe}(\mathrm{OH})_{3}+1.5 \mathrm{O}_{2}+4 \mathrm{OH}^{-} \quad \text { Equation } 15
$$

Concentrated solutions of ferrate(VI) can also be prepared directly, however ferrate(VI) solutions are very unstable and must be generated on-site. One method of generating ferrate(VI) solutions is by oxidising solutions of ferric hydroxide using hypochlorite, in a concentrated alkali, as shown in Equation 16 (Sharpe, 1986). This method is referred to as the wet chemical synthesis method (Mácová et al., 2009).

$$
\mathrm{Fe}_{2} \mathrm{O}_{3}+3 \mathrm{OCl}^{-}+4 \mathrm{OH}^{-} \rightarrow 2 \mathrm{FeO}_{4}^{-2}+3 \mathrm{Cl}^{-}+2 \mathrm{H}_{2} \mathrm{O} \quad \text { Equation } 16
$$

Another method is the thermo-chemical method, in which iron is oxidised to ferrate(VI), under extreme temperatures $\left(500-650^{\circ} \mathrm{C}\right)$ (Alsheyab et al., 2009). One example of this method is the oxidation of $\mathrm{Fe}^{+3}$ salts using molten $\mathrm{KOH}$ (Mácová et al., 2009). 
Chemical and thermo-chemical synthesis of ferrate( $\mathrm{VI})$ has been used to produce a high purity product (Madarász et al., 2006), however these methods require multiple steps and reagents to obtain a high yield with good purity (Alsheyab et al., 2009).

On the other hand, electrochemical ferrate( $\mathrm{VI})$ generation is relatively simple, and has the potential to be used on-site, which has attracted considerable attention recently (Ding et al., 2012, Yang et al., 2012a, Alsheyab et al., 2010a, Alsheyab et al., 2010b, Stanford et al., 2010, Alsheyab et al., 2009, Jiang et al., 2009, Mácová et al., 2009). One way to generate ferrate(VI) electrochemically is with a sacrificial (dissolving) iron anode in a strongly alkaline solution as part of an electrolysis cell. When a current is applied to the electrolysis cell, the iron anode is oxidised to ferrate(VI) (Alsheyab et al., 2009). The chemicals required are $12 \%$ bleach, $40 \%$ ferric chloride and $50 \%$ caustic solutions, which are commonly present at most treatment plants (Waite, 2012). The cost to produce ferrate(VI) using this method is approximately $11 / \mathrm{kg} \mathrm{FeO}_{4}^{-2}$ (Waite, 2012). Another similar method is with an inert anode, in an iron(III) solution, however this method has only recently gained attention (Mácová et al., 2009).

Regardless of how ferrate(VI) is generated, salts or solutions can be used for environmental remediation, and particularly water, wastewater and sludge treatment. The following section describes some of the research using ferrate(VI) for such purposes. 


\section{Ferrate(VI) for Water, Wastewater and Sludge Treatment}

Early experiments using ferrate(VI) for water treatment were conducted by Murmann and Robinson (1974). They treated river and well water samples with sodium ferrate and potassium ferrate to reduce metal ion concentrations. Potassium ferrate doses of 20 to $100 \mathrm{mg} / \mathrm{L}$ reduced lead(II) concentrations to below detectable limits. Similarly, ferrate(VI) treatments significantly reduced copper, mercury and chromium(III) concentrations. However, ferrate(VI) was less effective on zinc, chromium(II) and cadmium(II), and not at all effective on chromium(VI). In the same experiments, ferrate(VI) also eliminated the typical sulphurous odours in the well water samples using doses as low as 2 to $5 \mathrm{mg} / \mathrm{L}$, and led to the inactivation of bacteria in the water samples. Non-recombining Pseudomonas concentrations were reduced by $50 \%$ using $1.5 \mathrm{mg} / \mathrm{L}$ potassium ferrate, and completely eliminated using $4 \mathrm{mg} / \mathrm{L}$. Similarly, combining Pseudomonas concentrations were halved using $20 \mathrm{mg} / \mathrm{L}$, and eliminated with $50 \mathrm{mg} / \mathrm{L}$ potassium ferrate, and the Missouri river water bacteria were halved using $2 \mathrm{mg} / \mathrm{L}$, and completely eliminated with $100 \mathrm{mg} / \mathrm{L}$. Furthermore, an improvement in the rate of sedimentation was also observed. This is because ferrate(V) is a strong oxidant, capable of oxidising odorous compounds, pathogens and indicator organisms (Ding et al., 2012, He et al., 2009, Schuck et al., 2006, Read et al., 2003, Schink and Waite, 1980), and furthermore, once the ferrate(VI) ion has been reduced, ferric hydroxide is generated which acts as a coagulant (Gombos et al., 2013, Jiang et al., 2006a). Several studies have investigated ferrate(VI) treatment for the combined disinfection and coagulation of water and wastewater (Gombos et al., 2013, Jiang et al., 2006b, Li et al., 2005). 
Early studies by Waite(1979) used potassium ferrate for disinfection of secondary effluents, achieving 4 log inactivation of fecal coliform after 15 minutes, using $8 \mathrm{mg} / \mathrm{L}$ of ferrate(VI). And following a 4 hour sedimentation period, ammonia concentrations in the effluent were reduced by $70 \%$, phosphate was reduced by 40 to $60 \%$, BOD was reduced by up to $95 \%$, and TSS were reduced by 80 to $85 \%$. Subsequent studies by Schink and Waite (1980) went on to demonstrate that ferrate(VI) is also capable of inactivating $\mathrm{f} 2$ virus in PBS and secondary effluent, and furthermore $\mathrm{f} 2$ virus seemed to be equally or even less resistant to ferrate(VI) treatments than previously studied bacteria, including E. coli.

Jiang et al., (2006a, 2006b) also demonstrated that potassium ferrate(VI) can be used for coagulation and flocculation, and disinfection of municipal water and wastewater. They found that potassium ferrate(VI) was more effective than ferric sulphate and aluminum sulphate for the removal of turbidity, COD, colour (absorbance at $400 \mathrm{~nm}$ ) and bacteria. A ferrate(VI) dose of less than $0.27 \mathrm{mM}$ as Fe resulted in greater than 4-log inactivation of total and fecal coliform, and was more effective than ferric sulphate and aluminum sulphate on the removal of other contaminants, and resulted in smaller sludge volumes (Jiang et al., 2006a). Similar results were obtained in the study by Jiang et al. (2007), demonstrating that ferrate(VI) has a faster rate of disinfection than sodium hypochlorite, and was less affected by solution $\mathrm{pH}$. Furthermore, ferrate(VI) resulted in $30 \%$ greater reduction in COD compared to aluminum sulphate and ferric sulphate, and resulted in 3-log greater inactivation of bacteria, using a similar or even smaller dose. 
Schuck et al. (2006) also compared sodium ferrate(IV) and sodium hypochlorite disinfection of biologically treated wastewater effluents in terms of COD, total organic carbon (TOC), ammonium-nitrogen, combined chlorine, THMs and HAAs. $15 \mathrm{mg} / \mathrm{L}$ of sodium ferrate, and a 7-day reaction period, resulted in almost complete oxidation of ammonium-nitrogen, with final concentrations from 0.3 to $0.8 \mathrm{mg} / \mathrm{L}$, however COD, dissolved organic carbon (DOC) and $\mathrm{pH}$ were only slightly reduced. As expected, disinfection with sodium ferrate did not result in the formation of any THMs or HAAs, whereas disinfection with sodium hypochlorite resulted in THM and HAA concentrations directly proportional to the dose, and reaction time.

Gombos et al. (2013) compared ferrate(VI) and chlorine disinfection for the inactivation of cultivable heterotrophic bacteria in secondary effluents from two different WWTPs, and also compared COD and TOC removals, as well as the generation of absorbable organic haloids (AOX) as disinfection by-products. Results demonstrated that $5 \mathrm{mg} / \mathrm{L}$ of ferrate $(\mathrm{VI})$ resulted in $100 \%$ inactivation of bacteria, greater than $97 \%$ reduction in reactive phosphate, $40 \%$ reduction in COD and a $20 \%$ reduction in TOC, while $3 \mathrm{mg} / \mathrm{L}$ ferrate(VI) resulted in 3-log inactivation. However the ferrate(VI) treatments also resulted in the generation of $\mathrm{AOX}$. Comparing ferrate $(\mathrm{VI})$ and chlorine disinfection, to achieve 3-log bacterial inactivation, $15 \mathrm{mg} / \mathrm{L} \mathrm{Cl}_{2}$ was required, whereas only $3 \mathrm{mg} / \mathrm{L}$ of ferrate was required. Using these doses as points of comparison, the resulting AOX concentrations following disinfection were lower in the samples that were treated with ferrate(VI). Furthermore, $15 \mathrm{mg} / \mathrm{L}$ chlorine did not reduce the concentration of reactive phosphate, and only reduced the concentrations of COD by $32 \%$ and TOC by $2 \%$. 
Ferrate(VI) can also be used to oxidise reduced sulfur and nitrogen species, which largely contribute to odours in wastewater and sludge treatment operations. He et al. (2009) studied the reaction kinetics for the oxidation hydrogen sulphide, thiosemicarbazide and methyl mercaptan using ferrate(IV), and determined the reaction stoichiometry at neutral and alkaline $\mathrm{pH}$. Stoichiometric molar ratios of ferrate to sulphur, at $\mathrm{pH}$ 9.0, for hydrogen sulphide, thiosemicarbazide and methyl mercaptan were $2.5,2.0$ and 4.6 respectively. Read et al. $(2003,2000)$ also studied the reaction kinetics for a variety of sulphurous compounds using ferrate(VI), and determined that there was a rate determining step between the component being oxidised and the nonprotonated, $\mathrm{FeO}_{4}^{-2}$, ferrate $(\mathrm{VI})$ ion. However, the reactions with ferrate( $\left.\mathrm{VI}\right)$ proceeded very quickly, reaching completion within 1 second.

Recent studies have employed on-line electrochemical ferrate( $\mathrm{VI})$ generation to remove odours. Studies by Yang et al. (2012a) used a 99.5\% pure iron sheet as a sacrificial anode, along with a platinum cathode in a sodium hydroxide electrolyte to generate a ferrate(VI) solution. A synthetic odorous gas containing methyl mercaptan $\left(\mathrm{CH}_{3} \mathrm{SH}\right)$ was bubbled through the electrolysis cell, and the headspace gas analysis revealed that the $\mathrm{CH}_{3} \mathrm{SH}$ was quickly oxidised within seconds. Ding et al. (2012) conducted a very similar study, using in-situ electrochemical ferrate(VI) generation to oxidise $\mathrm{CH}_{3} \mathrm{SH}$. They found that a molar ratio of $\mathrm{Fe}(\mathrm{VI})$ to $\mathrm{CH}_{3} \mathrm{SH}$ of 2.2 to 1 was a minimum dosage for the destruction of $\mathrm{CH}_{3} \mathrm{SH}$, and a ratio of 4.53 to 1 , resulted in the complete conversion of $\mathrm{CH}_{3} \mathrm{SH}$ to sulphate. The authors also noted that the reaction rate between ferrate( $\left.\mathrm{VI}\right)$ and $\mathrm{CH}_{3} \mathrm{SH}$ was much faster than the rate of ferrate( $\left.\mathrm{VI}\right)$ self-decomposition in water. 
Other researchers have experimented with on-line electrochemical ferrate(VI) generation for wastewater treatment, primarily for the inactivation of pathogens and the removal of BOD and TSS in secondary effluents (Alsheyab et al., 2010a, 2010b, Jiang et al., 2009). Alsheyab et al (2010a, 2010b) has published some works on parameter optimisation for on-line electrochemical ferrate(VI) generation at WWTPs, such as optimal current density, sodium hydroxide electrolyte concentration, anode carbon content, inter-electrode gap, etc..., while Jiang et al. (2009) has successfully demonstrated a pilot-scale trial.

In the experiments by Jiang et al (2009), a laboratory-scale electrochemical ferrate(VI) generator was built using $2 \mathrm{~mm}$ thick steel electrodes (<98\% Fe, 0.1-0.12\% C). $16 \mathrm{M}$ sodium hydroxide was fed to the electrolysis cell to generate ferrate( $\mathrm{VI})$, which was subsequently added to raw sewage, taken from the influent line to a WWTP. Although the influent flow to the WWTP varied, the influent flow to the pilot-scale ferrate(VI) treatment unit was kept constant at $2000 \mathrm{~L} / \mathrm{hr}$. The optimum current density for the generation of ferrate $(\mathrm{VI})$ was $36 \mathrm{~A} / \mathrm{m}^{2}$, with an average current efficiency of $43 \%$, and an average specific energy consumption of $0.2 \mathrm{kWh} / \mathrm{g} \mathrm{Fe}(\mathrm{VI})$. Using small doses of only 0.04 $\mathrm{mg} / \mathrm{L}$ of ferrate $(\mathrm{VI})$, TSS was reduced by $70 \%$, phosphate was reduced by $40 \%$, COD was reduced by $40 \%$ and BOD was reduced by $20 \%$. The removal efficiencies obtained using $0.03 \mathrm{mg} / \mathrm{L}$ of ferrate(VI) were similar or better than the results obtained using a dose of $37 \mathrm{mg} / \mathrm{L}$ of ferric sulphate. Furthermore, under these two dosing conditions, the residual iron content in the effluent was almost 70 times lower when using ferrate(VI). 
Studies by Stanford et al (2010) also used electrochemical synthesis of ferrate(VI) to treat domestic wastewater, and compare the result to conventional ferric sulphate treatment. Their laboratory-scale electrochemical ferrate(VI) generator and the synthesis methods used were very similar to Jiang et al. (2009). The ferrate(VI) generated was always used within 50 minutes of production due to the instability of the ferrate(VI) ions in solution. Doses of less than $1 \mathrm{mg} / \mathrm{L}$ resulted in approximately $80 \%$ reduction in TSS, $70 \%$ reduction in COD, and $40 \%$ reduction in phosphorus. The ferrate(VI) treatment, using doses of less than $1 \mathrm{mg} / \mathrm{L}$ as Fe(VI), outperformed $35.8 \mathrm{mg} / \mathrm{L}$ doses ferric sulphate, equivalent to $10 \mathrm{mg} / \mathrm{L}$ as Fe(III), in terms of TSS removal, COD removal, and phosphorus removal.

Recent advancements in the use of on-line, or in-situ, electrochemical ferrate(VI) generation is largely due to the need to address concerns over the spread of EOCs in the environment. Several studies have demonstrated that ferrate(VI) can oxidise a range of EOCs, including PPCPs and other EDCs (Jiang and Zhoo, 2013, Yang et al., 2012b, Li et al., 2008, Jiang, 2007, Li et al., 2008, Sharma 2008, Sharma, 2006, Sharma and Mishra, 2006, Read et al., 2003, Read et al., 2000).

Jiang and Zhoo (2013) studied the removal of various drug residues (iopamidol, ammonium diatrizoate, ciprofloxacin, sulphamethoxazole, n-acetyl sulphamethoxazole, erythromycin- $\mathrm{H}_{2} \mathrm{O}$, naproxen, ibuprofen, atenolol, cyclophosphamide, ifosfamide, carbamazepine, bezafibrate and lidocaine) in water using ferrate(VI) doses of 1 to 5 $\mathrm{mg} / \mathrm{L}$. The destruction of the various drug residues was dependant on the physical and 
chemical properties of each pharmaceutical. Certain compounds were more easily oxidised than others. Ciprofloxacin was reduced by $63 \%$, whereas $n$-acetyl sulphamethoxazole was reduced by only $8 \%$.

Yang et al (2012b) studied the removal of 68 EDCs and PPCPs in wastewater using ferrate(VI). Similar to the experiments by Jiang and Zhoo (2013), they found that ferrate $(\mathrm{VI})$ treatment resulted in the selective oxidation of pharmaceuticals. Electron rich organic moieties in select compounds such as phenols, olefins, amines and anilines were consistently oxidised by the ferrate(VI), whereas ferrate(VI) did not react with triclocarban, 3 androgens (epi-androsterone, androsterone, 5a-dihydrotestosterone), 7 acidic pharmaceuticals (clofibric acid, 2,4-dichlorophenoxyacetic acid, 2-methyl-4chlorophenoxyacetic acid, ibuprofen, fenoprofen, gemfibrozil and ketoprofen), 2 neutral pharmaceuticals (primidone, cyclophosphamide) and erythromycin- $\mathrm{H}_{2} \mathrm{O}$. Despite the inability of ferrate $(\mathrm{VI})$ to oxidise certain compounds, the authors point out that ferrate(VI) as a tertiary treatment would oxidise and eliminate most of the EDCs present, and reduce the colour $\left(\mathrm{UV}_{400}\right)$ and $\mathrm{DOC}$ of secondary wastewater effluents.

Li et al. (2008) studied the effect of ferrate(VI) on synthetic EDCs: BPA and EE2, and natural EDCs: E1, E2, and E3. Experiments were carried with $\mathrm{pH}$ ranging from 8.2 to 12, and ferrate(IV) doses from 0.05 to $0.5 \mathrm{mM}$, on samples containing $0.1 \mathrm{mM} \mathrm{BPA}$ and 0.01 $\mathrm{mM}$ of $\mathrm{E} 1, \mathrm{E2}$, E3 and EE2, to determine a kinetic model based on second-order reaction kinetics, incorporating various species equilibria for the particular EDC and ferrate(VI) dose. LC/MS-MS and GC/MS-MS analysis indicated the presence of nine specific 
compounds, suggesting that although BPA was completely degraded in under 5 minutes with a molar ration of 5 moles ferrate(VI) per mole BPA, reaction products ( $p$ isopropylphenol, p-isopropenylphenol, 4-isopropanolphenol) persist and require further treatment. Full-scale applications employing much larger ferrate(VI) doses would result in the destruction of many of these reaction products, however it important to recognise the potential for incomplete oxidation resulting in the generation of various reaction products.

Experiments by $\mathrm{Li}$ et al. (2008) demonstrate the effectiveness of ferrate(VI) for the removal of EDCs, but point out that the extent of treatment depends heavily on $\mathrm{pH}$ due to the nature of the ferrate ion and the extent of dissociation of the particular EDC. Many researchers have demonstrated that the protonated ferrate ion $\left(\mathrm{HFeO}_{4}{ }^{-}\right)$is a much stronger oxidant due to the spontaneous decomposition of $\mathrm{Fe}(\mathrm{VI})$ to $\mathrm{Fe}(\mathrm{III})$ (Sharma and Mishra, 2006, Li et al., 2008, Zhu et al., 2006). The difference in oxidation potential for the decomposition of ferrate(VI) under neutral and alkaline conditions is a clear indication of the stability non-protonated ferrate ion $\left(\mathrm{FeO}_{4}^{-2}\right)$ compared to the protonated ion $\left(\mathrm{HFeO}_{4}^{-}\right)$.

Sharma et al. (2006) determined that the reaction kinetics for the oxidation of sulfamethoxazole with ferrate( $\mathrm{VI})$ are first-order with respect to each reactant and second-order overall, and determined that with an initial sulfamethoxazole concentration of $10 \mu \mathrm{M}$ and excess $\mathrm{Fe}(\mathrm{VI})$, the half-life of the reaction was 2 minutes at $\mathrm{pH}$ 7. However, at $\mathrm{pH} 9$, the half-life of the reaction increased to almost 10 hours. 
Similarly, Sharma et al. (2008) observed that the second-order rate constants for the oxidation of cyanide, thiocyanide and copper(II) cyanide decreased with increasing $\mathrm{pH}$, as a result of the speciation of the ferrate(VI) as either $\mathrm{HFeO}_{4}^{-}$under mildly acidic conditions, or $\mathrm{FeO}_{4}^{-2}$ under alkaline conditions. And in the experiment by Sharma and Mishra (2006), the oxidation rate of ibuprofen in water samples using ferrate(VI) also decreased with increasing $\mathrm{pH}$. Again, the oxidation was described by first order kinetics with respect to each reactant (second order overall), and the increase in reaction rate at near-neutral $\mathrm{pH}$ was attributed to the protonation of ferrate(VI). Conversely, for the purposes of water treatment, Li et al. (2005) suggest an optimum $\mathrm{pH}$ of 9, which balances the stability of the ferrate(VI) and its oxidising strength. However, for the purpose of sludge stabilisation, neutral or mildly acidic conditions may be more favourable due to the increase in reaction rate, allowing for shorter reaction periods.

Zhu et al. (2006) used potassium ferrate to improve biodegradation of the herbicide alachlor in a mixture of PS and WAS. Optimal conditions for ferrate oxidation were at $\mathrm{pH}$ of 7, and with 2 moles of ferrate(VI) per mole of alachlor, resulting in the complete removal of $40 \mathrm{mg} / \mathrm{L}$ of alachlor within 10 minutes. However, at $\mathrm{pH} \mathrm{10,} \mathrm{removal}$ decreased to $81 \%$, even with an extended reaction period of 40 minutes. The experiments also studied the subsequent treatment of ferrate(VI)-treated solutions using an aerobic up-flow bioreactor and determined that short reaction times of 10 minutes had inhibitory effects on subsequent activated sludge treatments, while 20 minute oxidation period resulted in almost the complete removal of all COD in subsequent aerobic treatments. 
Sylvester et al. (2001) used ferrate(VI) to reduce 60 to $70 \%$ of the chromium present in high-level radioactive tank sludge. The tests showed that chromium removal using ferrate( $\mathrm{VI})$ was more efficient in $5 \mathrm{M} \mathrm{NaOH}$ solutions, compared to $3 \mathrm{M} \mathrm{NaOH}$, and that increasing the ferrate dose increased chromium removal to a maximum $\mathrm{Fe}(\mathrm{VII}) / \mathrm{Cr}(\mathrm{III})$ ratio of around 10, at which point chromium removal levelled off with increasing ferrate(VI) doses. The study also revealed that the ferrate-treated leachate solutions did not contain significant amounts of chromium, meaning that the resulting chromate is a stable end-product.

Sharma et al. (2008) used ferrate( $\mathrm{VI})$ and ferrate(V) to oxidise cyanide, thiocyanide and copper(II) cyanide from electroplating rinse water and gold mill effluent. The experiments demonstrated that ferrate( $\mathrm{VI})$ was a much faster treatment than alkaline chlorination, sulphur-based treatments, hydrogen peroxide and ozonation, requiring only seconds to minutes for treatment. Furthermore, the resulting products: cyanate, sulphate, $\mathrm{Fe}(\mathrm{III})$ and molecular oxygen, are environmentally benign, unlike the BDPs associated with chlorination treatments.

In the study by de Luca et al. (1996), conventional sludge stabilisation using lime was compared to stabilisation with potassium ferrate(VI) for the purposes land application of treated biosolids. The study evaluated the sanitary effectiveness of the treatments in terms of pathogen reduction (bacteria, fungi, and helminth eggs), and the resulting concentration of soluble heavy metals to assess the possibility of treated biosolids leaching heavy metals into agricultural soils. 2 L sludge samples were treated with 10,15 
and $30 \mathrm{~g}$ doses of powdered potassium ferrate( $\mathrm{VI})$ per litre of sludge, and mixed at low speeds (150 to 200 RPM) for 10 minutes. Lime-treated samples were treated with enough lime to increase the $\mathrm{pH}$ above 12 for at least 2 hours. Lime doses required for the different sludge types varied from 2 to $75 \mathrm{~g}$ lime per litre of sludge, with an average of $2.7,7.1$ and $16.1 \mathrm{~g} / \mathrm{L}$ for WAS, AD sludge and PS respectively. Relatively high ferrate(VI) doses were used because preliminary tests showed that low ferrate(VI) doses increased bacterial growth, which may have been caused by increased concentrations of nitrate, with little variation in $\mathrm{pH}$ (de Luca et al., 1996), or due to excess oxygen produced by decomposition of potassium ferrate(VI) in water. The experiments confirmed that ferrate(VI) oxidation is capable of oxidising reduced forms of sulphur to sulphate and ammonia to nitrate. Lime treatments resulted in $140 \%$ increase in nitrate, while ferrate(VI) doses of 10, 15 and $30 \mathrm{~g} / \mathrm{L}$ sludge resulted in 1350, 2020 and $3243 \%$ increases in nitrate respectively. Similarly, lime treatments resulted in $18 \%$ increase in sulphate, and ferrate(VI) doses of 10, 15 and $30 \mathrm{~g} / \mathrm{L}$ sludge resulted in 1, 29 and $76 \%$ increases in sulphate respectively.

\subsubsection{Lime Stabilisation}

Lime stabilisation techniques have been used for centuries to reduce odours and putrescibility of substances (Bruce and Fisher, 1984). Sludge stabilisation with lime not only eliminates offensive odours and inhibits, reduces or eliminates the potential for putrefaction, it also destroys pathogens (Wong and Selvam, 2009, Jepsen et al., 1997, Godfree et al., 1984). Lime can be added in pure form as calcium hydroxide $\left(\mathrm{Ca}(\mathrm{OH})_{2}\right)$, known as hydrated or slaked lime or in the form of calcium oxide $(\mathrm{CaO})$, referred to as 
quicklime. In general, liquid sludges $(<10 \%$ solids) are treated with slaked lime, while dewatered sludges ( $>20 \%$ solids) are treated with quicklime. When quicklime is added to sludge, the calcium oxide rapidly reacts with water to form calcium hydroxide. This exothermic reaction can achieve temperatures of 60 to $70^{\circ} \mathrm{C}$ in dewatered sludge, resulting in a pasteurisation effect, and a rapid destruction of pathogens (Havelaar, 1984). For sludges with a high water content, the effects of the exothermic reaction of calcium oxide and water are insignificant, therefore slaked lime is generally used for liquid sludges, prior to the dewatering process. However, lime treatments are rarely used prior to dewatering with centrifuges and belt filter-presses due to the abrasive nature of lime-treated sludges (Metcalf and Eddy, 2003).

When lime is added to sludge a variety of chemical reactions occur which chemically alter the composition and cause an increase in $\mathrm{pH}$. The high $\mathrm{pH}$ environment inhibits or substantially slows down microbial reactions, therefore suppressing the emission of volatile sulphides and fatty acids which lead to odours and vector attraction (Bruce and Fisher, 1984). On the other hand, the high pH will increase the emission of amines and ammonia, which will require collection and treatment in odour control systems (Sawyer and McCarty, 1978). Nonetheless, release of amines and ammonia are less offensive, and release of free ammonia at $\mathrm{pH} 12$ further helps to destroy viruses, in addition to the direct effects of high $\mathrm{pH}$ on virus reduction.

High pH environments also reduce the number of pathogens and indicator organisms in sludge (Wong and Selvam, 2009, Jepsen et al., 1997). In the studies by Jepsen et al. 
(1997), $\mathrm{CaO}$ additions, achieving a $\mathrm{pH}>12$ resulted in a 3-log reduction in Salmonella and fecal Streptococcus after 24 hours of treatment.

In the studies by Godfree et al. (1984), Salmonella were exposed to increasing $\mathrm{pH}$ values, due to increasing lime doses, for 15 minutes, and the percent reductions were plotted (Figure 2-7). The results suggest that $\mathrm{pH}$ of 11.5 or greater can result in greater than $90 \%$ reduction in Salmonella with a 15 minute exposure time. Similarly, the beef tapeworm Taenia saginata showed a reduction in the ability of the ova to hatch in vitro at $\mathrm{pH}>11.5$.

Proper lime treatment designs should maintain a $\mathrm{pH}>12$ for at least 2 hours (Metcalf and Eddy, 2003). The actual dosage required to achieve an appropriate $\mathrm{pH}$ will depend heavily on the characteristics of the sludge. Typical lime doses are based on the sludge TS. Table 2-4 presents the lime doses required to maintain $\mathrm{pH}$ of 12 for 30 minutes, for different sludge types (PS, WAS, AD sludge \& septage). 


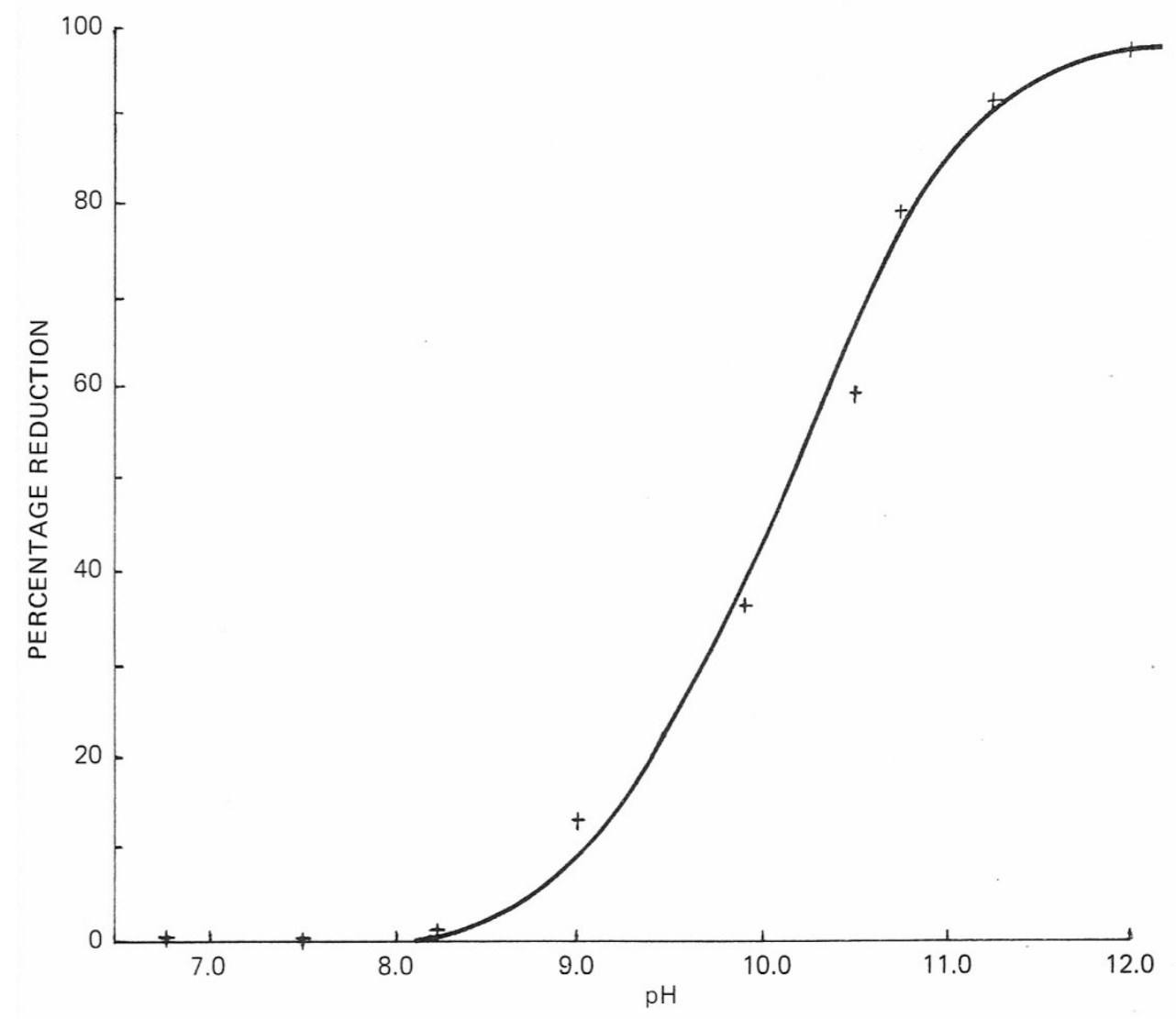

Figure 2-7: Percent reduction in Salmonella, 15 minute exposure time (Godfree et al., 1984).

Table 2-4: Typical lime doses for pre-treatment sludge stabilisation (WEF, 1995).

\begin{tabular}{lcccc} 
Sludge Type & \multicolumn{2}{c}{$\begin{array}{c}\text { Solids } \\
\text { Range }\end{array}$} & Average & Lime Dosage $\left(\mathrm{g} \mathrm{Ca}(\mathrm{OH})_{2} / \mathrm{kg} \mathrm{DS}\right)^{*}$ \\
PS & $3-6$ & 4.3 & $60-170$ & Average \\
WAS & $1-1.5$ & 1.3 & $210-430$ & 120 \\
AD Sludge & $6-7$ & 5.5 & $140-250$ & 300 \\
Septage & $1-4.5$ & 2.7 & $90-510$ & 200 \\
* amount of $\mathrm{Ca}(\mathrm{OH})_{2}$ required to maintain a pH of 12 for 30 minutes &
\end{tabular}

Reinthaler et al. (2010) studied the effect of $\mathrm{Ca}(\mathrm{OH})_{2}$ additions on the reduction of $E$ coli in dewatered and non-dewatered sludges. Results showed that complete inactivation of E coli was only reliably achieved when $\mathrm{Ca}(\mathrm{OH})_{2}$ was used on dewatered sludges. In fact, $\mathrm{Ca}(\mathrm{OH})_{2}$ treatments on non-dewatered sludges resulted in an increase in $E$ coli 
concentrations following stabilisation and dewatering. In Australia, full-scale applications treat sewage sludge from domestic WWTPs with quicklime following dewatering, achieving high levels of pathogen reduction. The stabilised biosolids are then used as a source of nutrients, and to improve soil properties of agricultural land (Pritchard et al., 2010). Alkaline sludge stabilisation has also been accomplished using fly ash, cement kiln dust and carbide lime. Experiments by Wong and Selvam (2009) compared the effects of fly ash and lime additions on the reduction of Salmonella, fecal coliform, E. coli and fecal Streptococcus. Lime and fly ash additions resulted in complete inactivation of pathogens and indicator organisms within 4 days, when the $\mathrm{pH}$ was maintained around 12 . High doses of $4 \%$ lime and $50 \%$ coal fly ash were effective against regrowth, whereas lower levels of treatment resulted in regrowth of all Salmonella to above 3 MPN/4 g DS, which exceeds limits for agricultural land application in the United States and Canada, as discussed in section 2.1.1. For agricultural purposes, application of lime-treated sludges can also be effective for rehabilitation or neutralisation of acidic soils, due to the alkaline nature of the sludge, however the primary benefits to the soil are generally the result of the added nutrients and organic matter (Pritchard et al., 2010).

\subsubsection{Discussion on the Use of Ferrate(VI) as a Chemical Oxidant for Wastewater}

\section{Treatment and Sludge Stabilisation}

Chemical stabilisation treatments can reduce or eliminate many refractory components in sludge, which are not treated biologically in a timely manner. The strength of an oxidant is described by its oxidation potential, and compared to conventional oxidants, 
ferrate(VI) is one of the strongest, as shown Table 2-3. Ferrate(VI) is a multi-purpose chemical for water and wastewater treatment applications. It can be used to remove bacteria and pathogenic microorganisms (Gombos et al., 2013, Jiang et al., 2006a, Murmann and Robinson 1974), heavy metals (Sharma et al., 2008, Sylvester et al., 2001), ammonia and other reduced sulphur compounds (Ding et al., 2012, Yang et al., 2012a, He et al., 2009, de Luca et al., 1996, Read et al. 2000, Read et al. 2003), herbicides (Zhu et al. 2006), PPCPs, EDCs and other EOCs (Jiang and Zhoo, 2013, Yang et al., 2012b, Li et al. 2008, Sharma et al., 2006). Furthermore, ferrate(VI) is also effective for coagulation and flocculation, and would reduce demands on subsequent disinfection processes (Jiang et al., 2009, Jiang et al., 2006b, Jiang and Lloyd, 2002). But probably the most attractive attribute is that disinfection using ferrate( $\mathrm{VI})$ does not result in the formation of the DBPs that are typically associated with chlorination (Jiang, 2007, Schuck et al., 2006), making it one of the most all-around effective and environmentally friendly disinfectants available. Furthermore, recent advancements in the use of on-line electrochemical ferrate $(\mathrm{VI})$ generation has made the use of ferrate $(\mathrm{VI})$ at water and wastewater treatment plants more feasible (Ding et al., 2012, Yang et al., 2012a, Alsheyab et al., 2010a, 2010b, 2009, Jiang et al., 2009).

Oxidation treatments using ferrate( $\mathrm{VI})$ generally use excess ferrate(VI) to ensure complete oxidation (Sharma et al. 2008, Sharma et al. 2006, Sharma and Mishra, 2006). Experiments by Sharma et al. (2008) used 2.5 times the molar amount of cyanide for complete oxidation, due to competing reactions. And He et al. (2009) determined the molar ratios required for ferrate( $\mathrm{VI})$ oxidation of reduced sulphur compounds to be 2.5 
for hydrogen sulphide, 2.0 for thiosemicarbazide and 4.6 for methyl mercaptan. Experiments by Li et al. (2008) used ferrate(VI) doses from 0.05 to $0.5 \mathrm{mM}$ for samples with $0.1 \mathrm{mM} \mathrm{BPA}$ and $0.01 \mathrm{mM}$ of E1, E2, E3 and EE2. Removal of EDCs increased with increasing ferrate $(\mathrm{VI})$ dose, and complete BPA removal was achieved in less than 5 minutes using 5 moles ferrate per mole BPA.

Under optimal conditions, namely mildly acidic $\mathrm{pH}$ and excess ferrate(VI), reaction kinetics are generally quite fast, requiring only minutes for complete removal of a wide range of contaminants (He et al. 2009, Li et al. 2008, Zhu et al. 2006). For sludge treatment applications, and particularly with dewatered sludges, mixing is required to achieve sufficient contact between the oxidant and the sludge. This could be achieved using conventional mixing basins, similar to those used for lime stabilisation.

Ferrate(VI) oxidation is a promising technology for sludge treatment which can target several types of contaminants. However, experimental data is lacking on the effects ferrate(VI) oxidation treatments for EOCs in sludges. More research is required to assess the ability of ferrate(VI) to oxidise EOCs in sludge, to ensure that land application of treated biosolids continues to be a safe and sustainable sludge disposal option.

The proposed research will investigate important freeze-thaw parameters such as freezing rate, curing time and curing temperature, and compare the effects of ferrate( $\mathrm{VI})$ oxidation when used as a pre- and post-treatment to freeze-thaw conditioning. Results from the research will help to design plans for a simple and 
effective sludge management system for regions with cold climates, and offer insight into the ability of ferrate(VI) to oxidise EDCs in sludge.

\subsection{CONCLUSIONS}

Sludge stabilisation and dewatering in remote cold regions can be a significant challenge. Furthermore, due to the increasing concerns over the spread of EDCs in the environment, there is an added demand for a simple and effective sludge treatment technology for remote communities in cold climates.

Freeze-thaw is a proven sludge dewatering technique (Diak et al., 2011, Hu et al., 2011, Northcott et al., 2005), and ferrate(VI) is a strong oxidant, capable of oxidising EDCs (Jiang and Zhoo, 2013, Yang et al., 2012b, Li et al., 2008, Jiang, 2007, Zhu et al. 2006), inactivating pathogens and indicator organisms (Jiang et al., 2007, Schuck et al., 2006), and reducing the concentrations of odour causing compounds (Ding et al., 2012, He et al., 2009, de Luca et al., 1996). The combined effects of freeze-thaw dewatering and ferrate(VI) oxidation have the potential to provide a simple and effective sludge treatment alternative for remote cold regions.

The literature review presented above revealed that there has not been any research into the treatment of sewage sludge using ferrate(VI) oxidation and freeze-thaw conditioning. Furthermore, there is very limited research on the oxidation of EDCs in sludge using ferrate(VI). These knowledge gaps and the applicability of the technology for cold and remote regions in Canada were the motivation behind the thesis. 
This research was the first to evaluate the combination of ferrate(VI) oxidation and freeze-thaw sludge dewatering, and among the first to examine the oxidation of EDCs in sludge using ferrate(VI). 


\section{OBJECTIVES AND HYPOTHESES}

The purpose of this study was to treat and dewater wastewater sludges using freezethaw with ferrate( $\mathrm{VI})$ oxidation to inactivate pathogens and indicator organisms and reduce the concentration of PPCPs, to achieve good quality biosolids, suitable for land application.

The research investigated freeze-thaw parameters such as freezing temperature, freezing rate and time frozen on the properties of wastewater sludges, and evaluated the effects of ferrate(VI) oxidation when used as a pre- and post-treatment to freezethaw.

The following hypotheses were the starting point for the research:

- Stand-alone freeze-thaw will inactivate pathogens and indicator bacteria, and consolidate sludge particles during freezing, allowing the meltwater to drain away freely during the thawing stage. (Chapters: 5-8)

- Faster freezing rates and longer time frozen will increase the level of inactivation of pathogens and indicator bacteria, whereas slower freezing rates will improve particle consolidation during freezing and improve sludge drainability during thaw. (Chapters: 6, 8)

- Ferrate(VI) additions will oxidise sludge constituents, causing a reduction of pathogens and indicator bacteria, PPCPs and EDCs, and odour-causing 
compounds. Furthermore, ferrate(VI) additions will solubilise organic matter, which will decrease the filterability of the sludge. Chapters: 7-9

- The effects of freeze-thaw and ferrate(VI) oxidation treatments on fecal coliform inactivation will be additive when ferrate( $\mathrm{VI})$ is used as a pre-and post-treatment with freeze-thaw. In other words, the inactivation resulting from the combined (freeze-thaw and ferrate(VI)) treatments will be the sum of the inactivations resulting from the individual treatments. (Chapters: 6-7)

- When ferrate $(\mathrm{VI})$ is used as a pre-treatment with freeze-thaw, the solubilisation of organic matter and reduced sludge filterability caused by the ferrate additions will not reduce the drainability (filterability) of the sludge during thaw, due to the particle consolidation caused by the advancing ice front during freezing. (Chapters: 7-8)

- It is expected that freeze-thaw and ferrate(VI) oxidation co-treatments can treat and dewater sewage sludges to below the CP1 limits for land application of biosolids in Ontario. (Chapters: 7-8)

- Ferrate(VI) additions will reduce the potential for regrowth of pathogens once the sludge is applied to land. (Chapter 11: Future Work) 


\section{MATERIALS AND METHODS}

The first experiment was a pilot scale freeze-thaw sludge dewatering study to evaluate freeze-thaw sludge dewatering and sludge volume reduction (Chapter 5). The research also characterised the physical, chemical and biological characteristics of the initial sludge and the final cake and meltwater collected following freeze-thaw. The second set of experiments evaluated the effects of freezing temperature (freezing rate) and time frozen on a sample of pure E. coli (Chapter 6). In addition, the effects of ferrate(VI) pre and post-treatment were evaluated as a co-treatment technology. The following set of experiments evaluated the co-treatment of primary sludge using ferrate( $\mathrm{VI})$ pre- and post-treatments with freeze-thaw, followed by a set of co-treatment tests involving a simulated drainage bed setup for dewatering anaerobically digested (Chapter 7). The fourth set of experiments evaluated the effects of the ferrate(VI) and freeze-thaw cotreatments on primary sludge, using various freezing temperatures to evaluate the drainability of sludge following various freezing rates, and the level of fecal coliform inactivation. In addition, the physical, chemical and biological characteristics of the meltwater samples were characterised.

The following sections provide detailed materials and methods used for each of the research chapters. 


\subsection{FREEZE-THAW TREATMENT OF RBC SLUDGE FROM A REMOTE MINING EXPLORATION FACILITY IN SUBARCTIC CANADA (CHAPTER 5)}

\subsubsection{Summary}

Freeze-thaw dewatering of RBC sludge was tested using a pilot-scale freeze-thaw bed placed in a mobile freezer unit operated at $-10^{\circ} \mathrm{C}$. Sludge samples from a remote mining exploration facility were flown in, and added to the freezing bed in 8 layers, each $10 \mathrm{~cm}$ thick. Approximately 4 months after the first layer of sludge was added, the pilot unit was removed from the freezer and thawed at ambient temperatures. The characteristics of the initial sludge, the dewatered sludge cake and the meltwater samples collected during the thawing stage were analysed.

\subsubsection{Sludge Samples}

The RBC sludge used was from 5 different sludge batches. The initial characteristics of each sludge batch are presented in Table 4-1. Since one of the sludge batches was used for 3 consecutive layers of sludge, and another batch was used for 2 sludge layers, the quoted averages at the bottom of Table 4-1 list a weighted average.

Table 4-1: Characteristics of the RBC sludge used in the freeze-thaw pilot study.

\begin{tabular}{|c|c|c|c|c|c|c|c|c|}
\hline Sludge & TS & VS & COD & sCOD & \multicolumn{2}{|c|}{ Fecal Coliforms } & \multicolumn{2}{|c|}{ Salmonella } \\
\hline Layer(s) & $\%$ & $\%$ & $\mathrm{mg} / \mathrm{L}$ & $\mathrm{mg} / \mathrm{L}$ & MPN/mL & MPN/g DS & MPN/mL & MPN/g DS \\
\hline 1 & 2.2 & 2.0 & 35,233 & 2490 & 33 & 1471 & 1.6 & 516 \\
\hline 2 & 2.7 & 2.4 & 39,933 & 2863 & 5 & 169 & 1.6 & 240 \\
\hline 3 & 2.1 & 1.9 & 45,900 & 3850 & 130 & 6245 & 1.6 & 308 \\
\hline $4,5,6$ & 2.7 & 2.4 & 53,217 & 3850 & 56 & 2115 & 1.6 & 241 \\
\hline 7,8 & 3.0 & 2.7 & 51,217 & 3400 & 5 & 149 & 1.6 & 213 \\
\hline Average & 2.6 & 2.3 & 47,894 & 3444 & 43 & 1816 & 1.6 & 277 \\
\hline
\end{tabular}




\subsubsection{Pilot-Scale Freeze-Thaw Bed}

The pilot-scale freezing bed (Figure 4-1) measured $60 \mathrm{~cm} \mathrm{X} 60 \mathrm{~cm}$ X $100 \mathrm{~cm}$ (LxWxH), with a large underlying meltwater collection box. The base of the freezing bed was angled towards an open slot, and was lined with a synthetic drainage fabric (screen) followed by a layer of sand, approximately $10 \mathrm{~cm}$ thick (36 L). To prevent early clogging, approximately $10 \mathrm{~L}$ of water was also added to the base of the pilot unit to fully saturate the sand. The water was then allowed to freeze prior to sludge addition. When the RBC sludge samples arrived from the remote site, the samples were mixed vigorously, and then $36 \mathrm{~L}$ of the sludge was added to the pilot unit, creating a layer of sludge approximately $10 \mathrm{~cm}$ thick (deep). The remaining sludge sample was then sent to the lab, where it was analysed. This process was repeated whenever a new sludge batch was received from the remote site. Due to difficulties with the transportation of sludge from the remote site, the fourth sludge batch was used for three consecutive layers (4, $5,6)$, and the fifth sludge batch was used for 2 consecutive layers $(7,8)$. For these cases, approximately 24 hours was provided for the previous layer of sludge to fully freeze, before the subsequent layer was applied. After the addition of all 8 sludge layers, each $10 \mathrm{~cm}$ thick, the total volume of sludge in the pilot unit was approximately $290 \mathrm{~L}$ and the average TS content of the sludge was $2.6 \%$. 


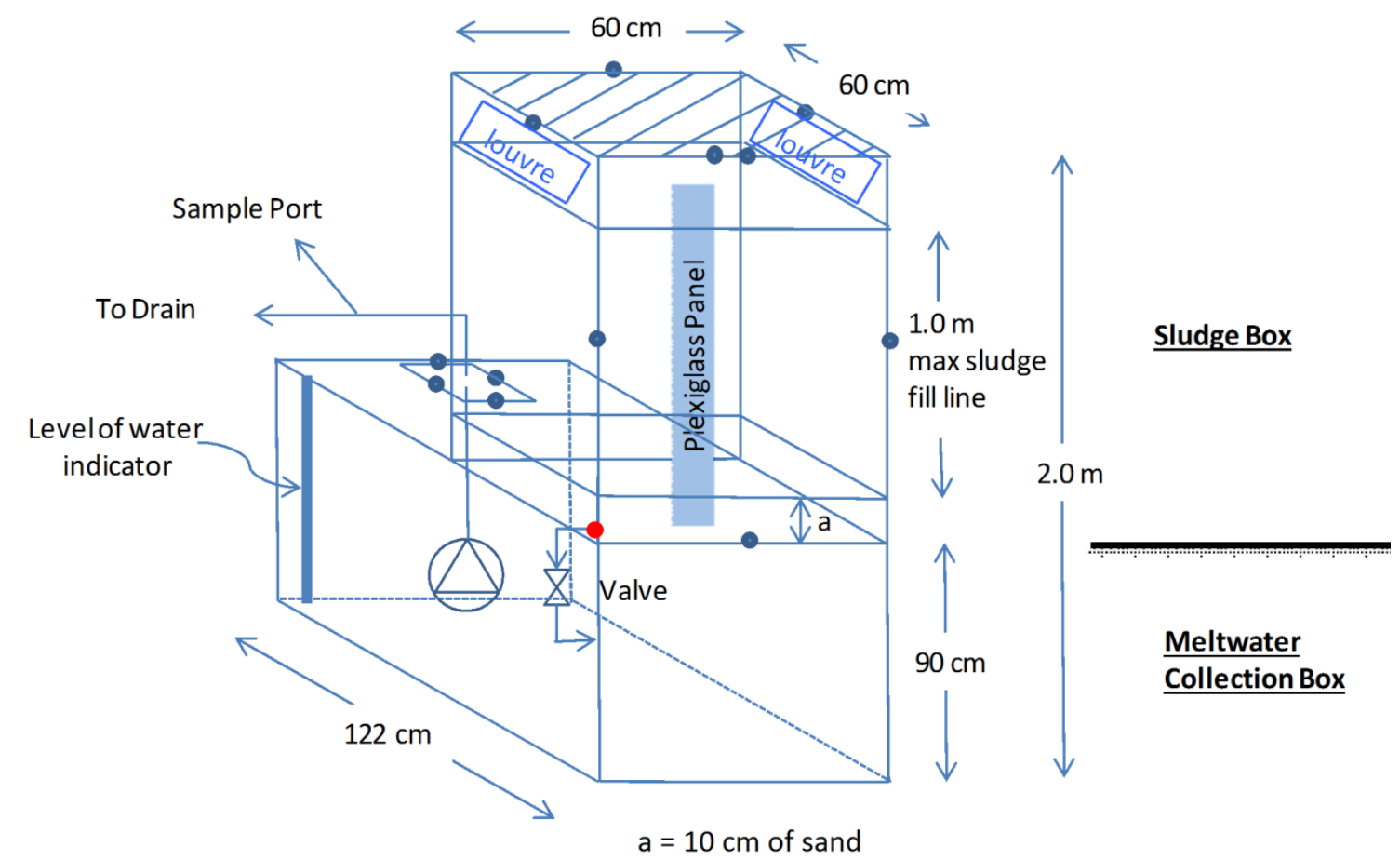

Figure 4-1: Schematic of the pilot freezing bed used in preliminary freeze-thaw experiments with RBC sludge (Seprotech Systems Inc.).

Approximately 4 months after the first layer of sludge was added, the pilot unit was removed from the freezer and thawed at ambient outdoor temperatures which varied diurnally between 17.5 and $26^{\circ} \mathrm{C}$. During the 10-day thawing period, meltwater drained from the sludge via gravity, and was removed from the unit for analysis. After the 10day thawing period, the remaining sludge cake was also sampled and analysed.

The methods used to analyse the initial sludge, the final cake, and the meltwater collected are summarised below. 


\subsubsection{Sample Analysis: Initial Sludge and Sludge Cake Following Freeze-Thaw}

\subsubsection{TS and VS}

TS and VS were measured using Standard Method 2540 G (APHA, 2005). Approximately $30 \mathrm{~g}$ of the sludge sample was placed in a dried and weighed aluminum dish, the wet sample and dish were weighed, and the samples were placed in a drying oven set to $107^{\circ} \mathrm{C}$ for 24 hours. The dried samples were placed in a desiccator for approximately 30 minutes to cool, and weighed again. The samples were then ignited in a muffle furnace at $550^{\circ} \mathrm{C}$ for 4 hours, cooled in a desiccator and weighed. The TS and VS were calculated according to Equation 17 and Equation 18 respectively.

All TS and VS measurements were performed in triplicate. Data points on the figures in Chapter 5 and subsequent chapters represent the average of three replicates, and the error bars represent the standard deviation.

$$
\begin{array}{lr}
\mathrm{TS}(\%)=100 \times \frac{\mathrm{C}-\mathrm{A}}{\mathrm{B}-\mathrm{A}} & \text { Equation } 17 \\
\operatorname{VS}(\%)=100 \times \frac{\mathrm{C}-\mathrm{D}}{\mathrm{B}-\mathrm{A}} & \text { Equation } 18
\end{array}
$$

where A: mass of dish (mg)

B: mass of wet sample and dish $(\mathrm{mg})$

C: mass of dried $\left(105^{\circ} \mathrm{C}\right)$ residue and dish $(\mathrm{mg})$

D: mass of ignited $\left(550^{\circ} \mathrm{C}\right)$ residue and dish $(\mathrm{mg})$ 


\subsubsection{Fecal Coliform}

Fecal coliform were quantified by multiple tube fermentation (MTF) using A-1 medium, according to EPA method 1681 (U.S. EPA 2005a). Reagents required include sterilised PBS, A-1 medium, $1 \mathrm{~N}$ sodium hydroxide solution and $1 \mathrm{~N}$ hydrochloric acid solution.

\section{Sodium Hydroxide and Hydrochloric Acid Solutions}

$1 \mathrm{~N}$ sodium hydroxide and $1 \mathrm{~N}$ hydrochloric acid solutions were used for $\mathrm{pH}$ adjustments during microbial enumeration techniques. $2.0 \mathrm{~g}$ of $>98 \%$ pure sodium hydroxide (BioShop Canada Inc., Burlington, ON) was added to $50.0 \mathrm{~mL}$ of deionised water to make the $1 \mathrm{~N}$ sodium hydroxide solution, and $4.1 \mathrm{~mL}$ of a $12.2 \mathrm{M}(37.5 \% \mathrm{w} / \mathrm{w})$ hydrochloric acid solution (Anachemia Canada Inc., Montreal, QC) was added to $50 \mathrm{~mL}$ of deionised water to make the $1 \mathrm{~N}$ hydrochloric acid solution. The solutions were stored in dark brown dropper bottles at room temperature.

\section{Phosphate Buffered Saline (PBS) Solution}

A PBS solution was used as dilution water for all microbial enumeration techniques. To prepare sterilised PBS solution, first a stock phosphate buffer solution and a stock magnesium chloride solution were required. To prepare the stock phosphate buffer solution, 34.0 g of $\geq 98 \%$ monopotassium phosphate (Sigma-Aldrich Co., St. Louis, MO, USA) was added to $500 \mathrm{~mL}$ of deionised water, the $\mathrm{pH}$ was adjusted to 7.2 using $1 \mathrm{~N}$ sodium hydroxide solution, and the volume was brought up to the $1.0 \mathrm{~L}$ mark with deionised water. The solution was then loosely sealed with a screw on-cap, wrapped in aluminum foil, and autoclaved at $121^{\circ} \mathrm{C}$ and 15 psi for 15 minutes. The stock magnesium 
chloride solution was prepared in a similar way, using $81.1 \mathrm{~g}$ of $\geq 99.0 \%$ magnesium chloride hexahydrate (BioShop Canada Inc., Burlington, ON) per $1.0 \mathrm{~L}$ of deionised water. The working PBS solution used for all sample dilutions during the MPN tests was prepared accordingly. $1.25 \mathrm{~mL}$ of the stock phosphate buffer solution and $5.0 \mathrm{~mL}$ of the stock magnesium chloride solution were added to $1.0 \mathrm{~L}$ of deionised water, and the solution was autoclaved $\left(121^{\circ} \mathrm{C}, 15 \mathrm{psi}, 15\right.$ minutes). The resulting concentrations of phosphate and magnesium chloride in the PBS solution, used for all dilutions, were 0.3 $\mathrm{mM}$ phosphate and $2.0 \mathrm{mM}$ magnesium chloride. All stock solutions and prepared PBS solutions were stored in the refrigerator until required, and were warmed to room temperature before used.

\section{A1 Medium}

To prepare A-1 medium, $30.5 \mathrm{~g}$ of dried A1-medium (EMD Chemicals Inc., Gibbstown, NJ, USA) was added to $1.0 \mathrm{~L}$ of deionised water, and the solution was heated and stirred on a magnetic stirrer/hot plate until the powdered reagent was completely dissolved. The $\mathrm{pH}$ was adjusted to $6.9 \pm 0.1$ using either $1.0 \mathrm{~N}$ hydrochloric acid or $1.0 \mathrm{~N}$ sodium hydroxide, if necessary. Racks of tests tubes were prepared, where each rack contained $4 \times 10$ test tubes, each tube with a $16 \mathrm{~mm}$ diameter and $150 \mathrm{~mm}$ height. Subsequently, an inverted vial (10 mm diameter, $75 \mathrm{~mm}$ height) was placed in each tube, and $10 \mathrm{~mL}$ aliquots of the prepared A1 medium were dispensed into each test tube. The test tubes containing the A1 medium and inverted vials were capped loosely with a screw-on cap, wrapped in aluminum foil, and autoclaved at $151^{\circ} \mathrm{C}$ and $15 \mathrm{psi}$, for 10 minutes. After being autoclaved, the A1 medium filled the inverted tubes leaving no air bubbles. The 
racks of tests tubes containing $\mathrm{A} 1$ medium were cooled to room temperature, and stored in a refrigerator, for up to 7 days. Prior to be being used, the media was removed from the refrigerator and warmed to room temperature.

\section{Sample Preparation}

When coliform were enumerated in the initial (liquid) sludge, approximately $300 \mathrm{~mL}$ of sludge was homogenised using a blender on high speed for 1 minute, then $\mathrm{pH}$ was checked to ensure that it was between 7.0 and 7.5. If the $\mathrm{pH}$ was out of range, then either hydrochloric acid or sodium hydroxide was used to adjust it. $100 \mathrm{~mL}$ of the homogenised, $\mathrm{pH}$-adjusted sample was then placed in a $150 \mathrm{~mL}$ sterilised Erlenmeyer flask, representing the raw, undiluted, sludge sample. $10 \mathrm{~mL}$ of the undiluted sample was placed in a second flask containing $90 \mathrm{~mL}$ of PBS, representing the $10^{-1}$ dilution, and the flask was stirred using a magnetic stirrer. This process was repeated to create serial dilutions up to $10^{-10}$, if the concentration of fecal coliform was completely unknown, or to a dilution level that was 2 or 3 levels of magnitude greater than the expected fecal coliform concentration.

When coliform enumerations were performed on the dewatered sludge cake samples, $30 \mathrm{~g}$ of the sludge cake was added to $270 \mathrm{~mL}$ of PBS, the mixture was homogenised using a blender on high speed for 1 minute, and the $\mathrm{pH}$ was adjusted (if necessary) to between 7.0 and 7.5. This prepared sample represented the $10^{-1}$ dilution, and was used in subsequent serial dilutions, as described in the paragraph above. 


\section{Inoculation and Incubation}

Once the appropriate dilutions sets were prepared, $1.0 \mathrm{~mL}$ of the last dilution (most dilute) was added to the first series of ten test tubes containing the A1 media and inverted vials, then $1.0 \mathrm{~mL}$ of the second-last dilution was added to the second series of ten tubes, and so on. This process was repeated until the all samples within the desired range of dilutions inoculated a series of ten tests tubes. U.S. EPA Method 1681 requires 5 replicates per dilution, however for these experiments 10 test tubes were used, resulting in duplicate readings for each sample, which were then averaged.

Following inoculation of all test tubes containing A1 media, the tubes were incubated in an oven set to $35.0^{\circ} \mathrm{C} \pm 0.5^{\circ} \mathrm{C}$ for 3 hours \pm 15 minutes, then transferred to a water bath at $44.5^{\circ} \mathrm{C} \pm 0.2^{\circ} \mathrm{C}$, and incubated for an additional $21 \pm 2$ hours (total incubation time not to exceed $24 \pm 2$ hours).

\section{Results and Enumeration}

After the incubation period, the racks of tubes were removed from the incubator and each tube was inspected carefully by gently swirling the tube, and noticing the formation of gas bubbles or growth, if any. Figure 4-2 shows two test tubes containing A1 media, following inoculation and incubation. The test tube on the left is cloudy, which indicates coliform growth, and shows gas generation, which has been collected in the inverted vial and displaced the A1 medium. This tube represents a positive result, whereas the tube on the right does not show any growth or gas generation, and is therefore a negative result. 


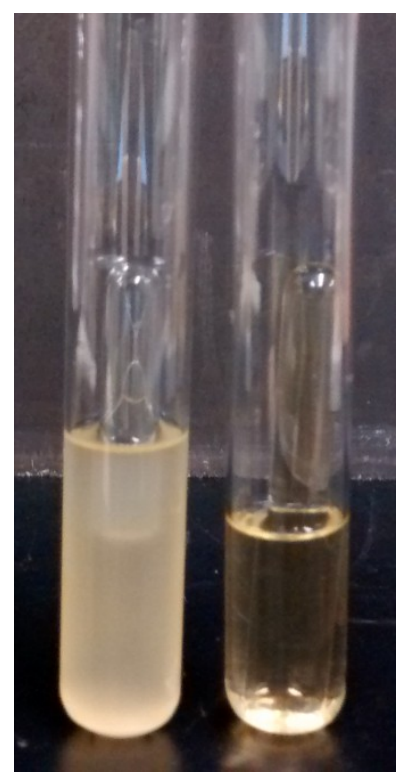

Figure 4-2: Fecal coliform enumeration using the MTF method, demonstrating a positive result (left), and a negative result (right).

Positive or negative results for each tube were recorded, and the three significant dilutions were selected according to the criteria presented in Appendix A1 on page 375. Table B1 in Appendix A1 also provides some examples of positive results combinations and the resulting significant dilutions.

Based on the significant dilutions and the combination of positive results, the MPN index was determined using the MPN index table, which is included in Appendix A2 on page 376. From the MPN index and the largest volume of sample used in the significant dilution series, the MPN/mL was calculated using Equation 19, and Equation 20 was used to convert the MPN/mL to a dry weight basis (U.S. EPA, 2005a). Built-in with the MPN index values in Appendix A2 on page 376, are 95\% confidence limits associated with the measurement. Equation 19 and Equation 20 were also used to adjust the 95\% 
confidence limits according to the significant dilution factor used. The $95 \%$ confidence limits are represented on the corresponding figures as error bars on the data points.

$$
\begin{array}{ll}
\mathrm{MPN} / \mathrm{mL}=\frac{\text { MPN Index from Table B2 }}{\text { Largest volume tested in the dilution series }} & \text { Equation } 19 \\
\text { MPN/g dry solids }=\frac{\mathrm{MPN} / \mathrm{mL}}{\% \mathrm{TS}(\text { espressed as a decimal) }} & \text { Equation } 20
\end{array}
$$

\subsubsection{Salmonella}

Salmonella enumeration was performed using U.S. EPA Method 1682, with the Modified Semisolid Rappaport-Vassiliadis (MSRV) medium. Reagents required include sterilised PBS, tryptic soy broth (TSB), MSRV medium, xylose-lysine desoxycholate (XLD) agar, $1 \mathrm{~N}$ sodium hydroxide solution and $1 \mathrm{~N}$ hydrochloric acid solution. The PBS solution was used for all sample dilutions, and the sodium hydroxide and hydrochloric acid solutions were used adjust the $\mathrm{pH}$ of the reagents, if necessary. The preparation of these solutions has been described on page 92 . The methods used to prepare the additional reagents required for the enumeration of Salmonella are presented below.

\section{TSB}

TSB is a non-selective growth media which was used in the first of three incubation steps. To prepare TSB, $30.0 \mathrm{~g}$ of Bacto ${ }^{\mathrm{Tm}}$ brand (Becton, Dickinson and Co., Sparks, MD, USA) powdered TSB media was added to $1.0 \mathrm{~L}$ of deionised water, and the media was stirred and heated using a magnetic stirrer and hot plate, until completely dissolved. The $\mathrm{pH}$ was adjusted to $7.3 \pm 0.2$ with $1.0 \mathrm{~N}$ hydrochloric acid or $1.0 \mathrm{~N}$ sodium 
hydroxide, if necessary. For solid and semi-solid samples, triple-strength (3X) TSB was required to ensure that inoculation volume did not excessively dilute the media. To prepare 3X TSB, $90.0 \mathrm{~g}$ of Bacto ${ }^{\mathrm{Tm}}$ powdered TSB media was added to $1.0 \mathrm{~L}$ of deionised water, and the same procedure followed as described above. Following the preparation of the $1 \mathrm{X}$ TSB and $3 \mathrm{X}$ TSB, the media were dispensed into test tubes. $10 \mathrm{~mL}$ volumes of 3X TSB were dispensed into forty $16 \times 150 \mathrm{~mm}$ test tubes, $5 \mathrm{~mL}$ volumes of $3 X$ TSB were dispensed into another forty $16 \times 150 \mathrm{~mm}$ test tubes, and $10 \mathrm{~mL}$ volumes of $1 \mathrm{X}$ TSB were dispensed into forty $25 \times 150 \mathrm{~mm}$ test tubes. All test tubes were capped loosely, wrapped in aluminum foil, and autoclaved at $121^{\circ} \mathrm{C}$ and $15 \mathrm{PSI}$ for 15 minutes. To store the prepared media, the racks of test tubes were wrapped in aluminum foil and stored at room temperature until required.

\section{MSRV Medium}

MSRV medium was used for the second incubation step to inhibit non-Salmonella species, while allowing Salmonella to grow. To prepare the MSRV medium, $31.6 \mathrm{~g}$ of Difco $^{\text {tm }}$ brand (Becton, Dickinson and Co., Sparks, MD, USA) was added to $1.0 \mathrm{~L}$ of deionised water, and the media was stirred and heated until boiling to dissolve completely. The $\mathrm{pH}$ was adjusted to $5.2 \pm 0.2$ with $1.0 \mathrm{~N}$ hydrochloric acid or $1.0 \mathrm{~N}$ sodium hydroxide, if necessary, and cooled to $50^{\circ} \mathrm{C}$.

A $2 \%$ novobiocin stock solution was prepared by dissolving $1.0 \mathrm{~g}$ of Promega brand $\geq 95 \%$ sodium novobiocin (Promega Biosciences, San Luis Obispo, CA, USA) into $50 \mathrm{~mL}$ of deionised water. The solution was filtered through a Millex ${ }^{\circledR}$-GV $0.22 \mu \mathrm{m}$ syringe filter 
(Merck Millipore Ltd., Tullagreen, Carrigtwohill, County Cork, IRL), and $1.1 \mathrm{~mL}$ volumes were dispensed into $2 \mathrm{~mL}$ cryovials. The cryovials were stored in the freezer at $-20^{\circ} \mathrm{C}$ until required, and were thawed and warmed to room temperature prior to use.

For every litre of freshly-prepared (warm) MSRV media, $1.0 \mathrm{~mL}$ of the $2 \%$ sodium novobiocin solution was added. The media was mixed thoroughly, and $25 \mathrm{~mL}$ volumes were dispensed into sterile $15 \times 100 \mathrm{~mm}$ petri plates. The plates were cooled to room temperature, and the media solidified. The prepared petri dishes containing the MSRV media were wrapped in aluminum foil, and stored (not inverted) at room temperature for up to 2 days.

\section{$X L D$ Agar}

XLD agar was used in the third incubation step to isolate presumptive Salmonella species. To prepare XLD agar $55.0 \mathrm{~g}$ of Difco $^{\mathrm{Tm}}$ brand (Becton, Dickinson and Co., Sparks, MD, USA) XLD agar was added to $1.0 \mathrm{~L}$ of deionised water, and the media was stirred and heated just until boiling to dissolve completely. The $\mathrm{pH}$ was adjusted to $7.4 \pm 0.2$ with $1.0 \mathrm{~N}$ hydrochloric acid or $1.0 \mathrm{~N}$ sodium hydroxide, if necessary, and cooled to approximately 45 or $50^{\circ} \mathrm{C}$. While the media was war, $12 \mathrm{~mL}$ volumes were dispensed into sterile $15 \times 100 \mathrm{~mm}$ petri plates. The plates were cooled to room temperature, and the media solidified. The prepared petri dishes containing the XLD agar were wrapped in aluminum foil and stored (inverted) in the refrigerator for up to 2 weeks. Prior to use, the desired number of petri plates were removed from the refrigerator and warmed to room temperature. 


\section{Sample Preparation}

Enumeration of Salmonella was performed on the initial sludge samples and the final sludge cake remaining following freeze-thaw dewatering. The sample preparation procedures for the detection and enumeration of Salmonella (EPA method 1682) are identical to the procedures used for the enumeration of fecal coliform using MTF (EPA method 1681), therefore the same prepared samples were used for each of these enumeration techniques. The detailed sample preparation procedures used were presented earlier on page 94.

\section{Inoculation and Incubation}

Figure 4-3 shows a summary of the incubation steps used for the detection and enumeration of Salmonella using EPA method 1682. First, $20.0 \mathrm{~mL}$ of the prepared sample was added to each of the first series of 5 test tubes containing $10 \mathrm{~mL}$ of $3 X$ TSB, $10.0 \mathrm{~mL}$ of the prepared sample was added to each of the second series of 5 test tubes containing $5 \mathrm{~mL}$ of $3 X \mathrm{TSB}$, and $1.0 \mathrm{~mL}$ of the prepared sample was added to each of the third series of 5 test tubes containing $10 \mathrm{~mL}$ of $1 \mathrm{X}$ TSB. The racks of test tubes were then incubated at $24 \pm 2$ hours. Following the first incubation, turbid (cloudy) tubes were recorded as positives, however due to the non-inhibitory nature of the TSB used as enrichment medium, generally all of the tubes following the first incubation step were positive for (non-specific) growth.

The second incubation step used MSRV medium to inhibit non-Salmonella species while allowing Salmonella to grow. To do this, 6 discrete $30-\mu \mathrm{L}$ drops from each TSB tube were 
applied to a plate containing the MSRV medium, and the plates were left out at room temperature for approximately one hour, to allow the inoculations (drops of TSB) to soak into the MSRV medium. The plates were then incubated (not inverted) in an oven at $42^{\circ} \mathrm{C} \pm 0.5^{\circ} \mathrm{C}$ for 16 to 18 hours in an incubator containing a large pan of water at the bottom, to maintain sufficient humidity throughout the incubation process. Following the incubation period, the plates were removed from the incubator and examined for "whitish halos" surrounding the inoculations. If present, the halos represent Salmonella motility in the MSRV medium. A sterile inoculating loop was used to stab into the outer edge of the halo to collect a sample presumptively containing Salmonella.

The third incubation step used XLD agar to isolate Salmonella colonies. The samples collected from the outer edge of the halos on the MSRV medium were streaked onto the XLD plate, changing loops with every streaking direction. The plates were then incubated for 18 to 24 hours at $36^{\circ} \mathrm{C} \pm 1.5^{\circ} \mathrm{C}$. Following the incubation period, the plates were removed from the incubator and examined for pink to red colonies with black centres, which are considered Salmonella (U.S. EPA, 2005b). The final stage of the detection and enumeration process requires three separate incubations for biochemical confirmation of the presence of Salmonella in the pink to red colonies produced on the XLD agar. However, due to time restraints and the number of samples, the biochemical confirmation step was omitted, and the ability to publish the results was forfeited. 


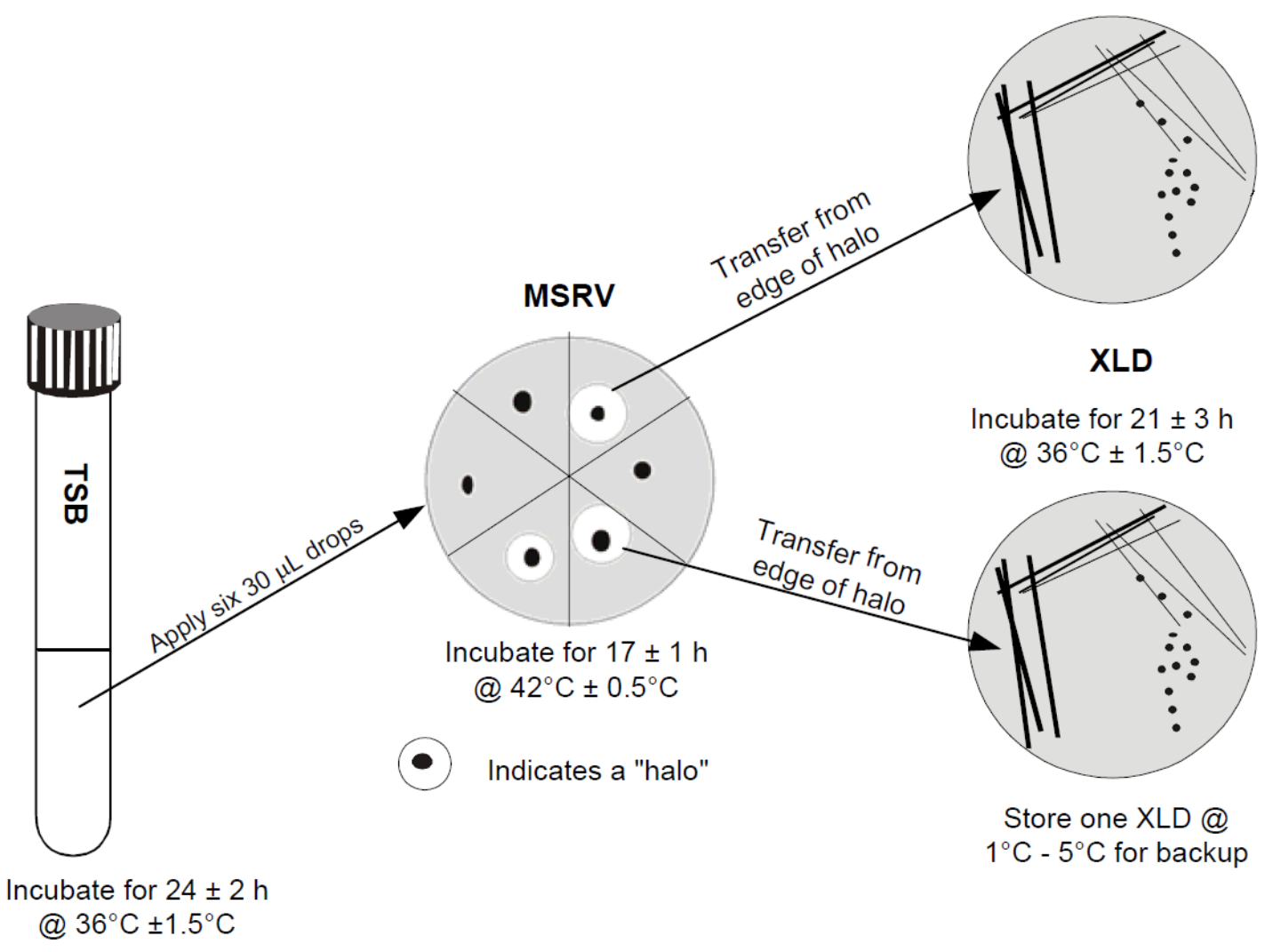

Figure 4-3: Summary of the incubation procedures used for the detection and enumeration of Salmonella using EPA method 1682 (U.S. EPA 2005b).

\section{Results and Enumeration}

The enumeration of Salmonella was based on the combination of positive tubes from the first incubation step. In order for one of the original TSB tubes to be considered positive for Salmonella, the TSB tube must be positive for growth (cloudy), the associated inoculations on the MSRV medium must be positive for Salmonella motility (halos present), and the subsequent incubation of motile Salmonella on the XLD agar must produce pink to red colonies with black centres.

Based on the combination of positive results for the 3 sets of 5 tubes from each sample, the MPN index for the sample was determined using the MPN table included in 
Appendix A3 on page 379. For liquid sludge samples, since there was no dilution, the MPN index was equal to the MPN/mL, whereas for solid samples there was a dilution factor of 10 during the sample preparation and homogenisation procedure, therefore the MPN index value was multiplied by 10 to get the MPN/mL.

\subsubsection{Sample Analysis: Meltwater Collected During Sludge Thaw}

During the thawing stage, meltwater drained via gravity into a collection box, beneath the freeze-thaw bed. The meltwater was removed from the basin using a pump, whenever there was sufficient volume for the pump to self-prime. These meltwater grab-samples were characterised in terms of TSS, VSS, pH, turbidity, COD (total and soluble), nitrogen (total, nitrate, ammonia) and phosphorus. The detailed procedures used are described below.

\subsubsection{TSS and VSS}

The TSS and VSS of the meltwater samples were measured according to Standard Methods 2540 D and 2540 E respectively (APHA, 2005). For each sample, a minimum of three replicates were used, and for each replicate at least $500 \mathrm{~mL}$ of meltwater was vacuum-filtered through a $70 \mathrm{~mm}$ diameter Whatman grade 934-AH glass fibre filter paper with a $1.5 \mu \mathrm{m}$ retention (Sigma-Aldrich Canada Ltd., Oakville, ON). The filter papers were first prepared by placing them in clean and dry labelled crucibles. The crucibles and filter papers were ignited in a muffle furnace at $550^{\circ} \mathrm{C}$ for 30 minutes, cooled in a desiccator, and the filter papers were weighed. The prepared filter papers were placed in a Buchner funnel apparatus, wetted with deionised water, and the 
sample was filtered. Following the filtration of the entire sample through the funnel, the sample container and funnel apparatus were rinsed three times with deionised water. The wet filter papers containing the samples were carefully removed from the filtration apparatus and replaced in their appropriately labelled crucibles. The crucibles containing the wet filter papers and filtered samples were then placed in a drying oven at $105^{\circ} \mathrm{C} \pm 2^{\circ} \mathrm{C}$ for 24 hours, and cooled in a desiccator before being weighed. The dried sample and filter paper were then ignited at $550^{\circ} \mathrm{C}$ for 4 hours, cooled in a desiccator and weighed. The TSS and VSS were calculated according to Equation 21 and Equation 22 respectively.

$$
\begin{aligned}
\operatorname{TSS}(\mathrm{mg} / \mathrm{L})=\frac{\mathrm{G}-\mathrm{F}}{\mathrm{E}} & \text { Equation 21 } \\
\operatorname{VSS}(\mathrm{mg} / \mathrm{L})=\frac{\mathrm{G}-\mathrm{H}}{\mathrm{E}} & \text { Equation 22 }
\end{aligned}
$$

where E: sample volume (L)

F: mass of filter paper $(\mathrm{mg})$

G: mass of dried $\left(105^{\circ} \mathrm{C}\right)$ residue and filter paper $(\mathrm{mg})$

$\mathrm{H}$ : mass of ignited $\left(550^{\circ} \mathrm{C}\right)$ residue and filter paper $(\mathrm{mg})$

\subsubsection{Turbidity and $\mathrm{pH}$}

The turbidity was measured using a HACH Model 2100AN Turbidimeter (Hach Company, Loveland CO, USA). Meltwater samples were mixed thoroughly by hand, and $20 \mathrm{~mL}$ of each sample was placed in a labelled $25 \mathrm{~mm}$ sample cell. The sample cells were capped with a screw-on cap, wiped with a Kimwipe, and placed in the turbidity meter. Following 
the turbidity measurement, the screw cap was removed and the $\mathrm{pH}$ was measured using a Thermo Orion 5-star bench-top meter kit and ROSS ultra pH electrode (Thermo Fisher Scientific Inc., Rockford, IL, USA). The samples were then discarded, the sample cells and $\mathrm{pH}$ probe were rinsed, and the process was repeated two more times to get a total of three replicates for each measurement. Data points on the figures represent the average of the three replicates, and the error bars represent the standard deviation.

\subsubsection{Total and Soluble COD}

The COD of the initial (liquid) sludge was measured using $\mathrm{HACH}$ method 8000 with the high range plus COD digestion reagent $(200-15,000 \mathrm{mg} / \mathrm{L} \mathrm{COD})$, and the COD of the meltwater was measured using $\mathrm{HACH}$ method 8000 with the high range reagent (20$1500 \mathrm{mg} / \mathrm{L} \mathrm{COD}$ ). For the total COD measurements, $20 \mathrm{~mL}$ of raw sample (sludge or meltwater) was added to $80 \mathrm{~mL}$ of deionised water (dilution factor $=5$ ) and the mixture was stirred using a magnetic stirrer for 5 minutes.

To obtain the soluble component, $50 \mathrm{~mL}$ of the raw sample (sludge or meltwater) was centrifuged at $3400 \mathrm{rpm}$ (relative centrifugal force, RCF = $1200 \mathrm{~g}$ ) for 15 minutes, then a $10 \mathrm{~mL}$ portion of the supernatant was withdrawn from the centrifuge tube, and filtered using a $0.45 \mu \mathrm{m}$ mixed cellulose ester (MCE) syringe filter (Shanghai Derian Instrument Co., Ltd., Shanghai, China). In some cases, due to the low RCF, the supernatant obtained from the first centrifugation process was centrifuged a second time, prior to being filtered through the MCE syringe filter. For the SCOD measurements, no dilution factor was used. 
For the high range plus tests, $0.2 \mathrm{~mL}$ of the prepared sample was added to each COD digestion reagent vial, whereas the high range tests require $2.0 \mathrm{~mL}$ of the prepared sample for each replicate. Following the addition of the each sample to the COD digestion reagent vials, the vials were tightly capped, inverted several times, and placed in a HACH DRB 200 digital block reactor preheated to $150^{\circ} \mathrm{C}$, for two hours. Following the two hour reaction period, the vials were cooled to room temperature, and analysed using program 435 (COD HR) on a HACH DR 2800 spectrophotometer. All COD tests were performed in triplicate. The data points on the figures show the averages of the three replicates, and the error bars represent the standard deviations.

\subsubsection{Nitrogen (Total, Nitrate, Ammonia) and Phosphorus}

Total nitrogen (TN) was measured using $\mathrm{HACH}$ method 10072 (high range: 10-150 mg/L as N) with a dilution factor of $3.10 \mathrm{~mL}$ of sample was added to $20 \mathrm{~mL}$ of deionised water, and the samples were mixed using a vortex mixer (Baxter Diagnostics Inc., IL, USA). $0.5 \mathrm{~mL}$ of the diluted sample was added to each hydroxide reagent vial, the vials were capped and mixed vigorously, and placed in a DRB200 preheated to $105^{\circ} \mathrm{C}$ for 30 minutes. Following the 30 minute reaction period, the vials were immediately removed from the reactor and cooled to room temperature. Each vial was analysed using program 394 (N, Total HR TNT) on a HACH DR 2800 spectrophotometer, and the diluted TN concentration was recorded.

Nitrate was measured using $\mathrm{HACH}$ method 10020 (high range: 0.2-30.0 mg/L NO${ }_{3}^{-} \mathrm{N}$ ) with no dilution factor. Each vial was analysed using program 344 (N, Nitrate HR TNT) on a HACH DR 2800 spectrophotometer. $1.0 \mathrm{~mL}$ of raw sample was added to a NitraVer $\mathrm{X}$ 
Reagent A Test ' $N$ Tube vial, the vial was capped and inverted ten times to mix. This sample was inserted into the spectrophotometer and used as a blank. The contents of a NitraVer X Reagent B Powder Pillow were added to the vial. The vial was inverted ten times, and allowed 5 minutes for reaction at room temperature. Following the 5 -minute reaction period, the samples were inserted back into the spectrophotometer and the nitrate concentration of the sample was measured.

Ammonia was measured using $\mathrm{HACH}$ method 10031 (high range: 0.4-50.0 mg/L NH a dilution factor of $10.5 \mathrm{~mL}$ of sample was added to $45 \mathrm{~mL}$ of deionised water, and the samples were mixed using a vortex mixer. $0.1 \mathrm{~mL}$ of the diluted sample was added to an AmVer ${ }^{\text {Tm }}$ Diluent Reagent Test ' $N$ Tube vial, then the contents of one Ammonia Salicylate Reagent Powder Pillow were added, and contents of one Ammonia Cyanurate Reagent Powder Pillow were added. The vials were capped and shaken vigorously, and allowed 20 minutes for reaction at room temperature. Following the 20 minute reaction period, the vials were analysed using program 343 (N, Ammonia HR TNT) on a HACH DR 2800 spectrophotometer, and the diluted ammonia concentrations were recorded.

Total phosphorus (TP) was measured using $\mathrm{HACH}$ method 10127 (high range: 1.0-100.0 $\mathrm{mg} / \mathrm{L} \mathrm{PO}_{4}^{-3}$ ) with a dilution factor of $3.10 \mathrm{~mL}$ of sample was added to $20 \mathrm{~mL}$ of deionised water, and the samples were mixed using a vortex mixer. $5.0 \mathrm{~mL}$ of the diluted sample was added to a Total Phosphorus Test ' $N$ Tube vial, and the contents of one Potassium Persulfate Powder Pillow was added. Each vial was capped tightly, and shaken to dissolve the powdered reagent. The vials were then placed in a DRB200 reactor, preheated to $150^{\circ} \mathrm{C}$, for 30 minutes. Following the 30 -minute reaction period, the vials were removed from the 
reactor, and cooled to room temperature. $2.0 \mathrm{~mL}$ of $1.54 \mathrm{~N}$ sodium hydroxide was added to each vial, the vials were capped and inverted several times to mix, and then $0.5 \mathrm{~mL}$ of Molybdovanadate Reagent was added to each vial, and the vials were capped and inverted to mix. Following the addition the reagents, a 7-minute reaction period occurred, and then the samples were analysed using program 542 (P Total HR TNT), and the TP concentrations of the diluted samples were recorded.

All nitrogen (total, nitrate, ammonia) and phosphorus measurements were carried out in triplicate. Data points on the figures represent the averages of the three measurements, and the error bars represent the standard deviations.

\subsection{COMBINED FREEZE-THAW AND FERRATE(VI) OXIDATION TREATMENT ON E. COLI IN A PBS SOLUTION (CHAPTER 6)}

\subsubsection{Summary}

This study evaluated the individual and combined effects of potassium ferrate(VI) and freeze-thaw treatment on the reduction of E. coli in a PBS solution. A low dose (LD) of $0.5 \mathrm{mg} / \mathrm{L}$ and a high dose (HD) of $1.0 \mathrm{mg} / \mathrm{L}$ of potassium ferrate were used as a pre- and post-treatment to freeze-thaw, at temperatures of $-10,-20$ and $-30^{\circ} \mathrm{C}$, for 1,8 and 15 days.

\subsubsection{Sample (E. coli) Preparation}

E. coli (ATCC 23621), from Cedarlane Laboratories (Hornby, ON), was used as the indicator organism for all tests. The bacteria were revived according to the methods suggested by the main supplier (ATCC). To summarise the ATCC method: The primary $E$. 
coli stock, in pellet-form, was rehydrated using $0.5 \mathrm{~mL}$ of TYG broth (ATCC Medium \#603), transferred into a tube containing $6.0 \mathrm{~mL}$ TYG, and incubated at $37^{\circ} \mathrm{C}$ for $24 \mathrm{~h}$. This primary stock was divided into $1 \mathrm{~mL}$ aliquots and stored in a deep freezer at a temperature of $-20^{\circ} \mathrm{C}$.

The test sample for each set of experiments was a freshly prepared $E$. coli culture in sterilised PBS containing $0.3 \mathrm{mM} \mathrm{PO}_{4}^{-3}$ and $2.0 \mathrm{mM} \mathrm{Ca}^{+2}$. The PBS solution was used as a dilution medium because it is isotonic and non-toxic to $E$. coli (and most other) cells. This means that the concentration of solute inside the cell is the same as the surrounding solute concentration, and there is no osmotic pressure gradient across the cell membrane. As a result, the cells do not shrink or swell, which can be potentially lethal when the rate of transfer into or out of the cell is too fast. A fast flow of water into the cell can cause the cell to swell and burst, while a fast flow of water out of the cell can increase the solute concentration inside the cells to lethal levels. The PBS solution was prepared according to the method described in section 4.1.4.2, on page 92 . Fresh test samples of $E$. coli culture and PBS were prepared accordingly: A $1.0 \mathrm{~mL}$ aliquot of E. coli was removed from the freezer, and allowed to thaw and warm to room temperature. The sample was added to $100 \mathrm{~mL}$ of sterilised Bacto ${ }^{\mathrm{TM}}$ tryptic soy broth (TSB) from BD Biosciences (Mississauga, ON) and revived overnight in an incubator at $37^{\circ} \mathrm{C}$, without shaking. The next morning, $1.0 \mathrm{~mL}$ of the revived stock was added to another $100 \mathrm{~mL}$ of sterilised TSB, and incubated at $37^{\circ} \mathrm{C}$ for 5 -hours with periodic shaking. Preliminary experiments showed that after 4-5 $\mathrm{h}$ of incubation, the bacteria were at their late exponential growth or early stationary phase. After incubation was 
complete, the cells were removed from the broth and washed with PBS as follows: Two $20 \mathrm{~mL}$ samples of $E$. coli and broth were centrifuged at 10,000 rpm (relative centrifugal force, $\mathrm{rcf}=15,317 \mathrm{~g}$ ) for 10 minutes. The excess broth was poured off, and the cells were rinsed with two $20 \mathrm{~mL}$ aliquots of PBS and resuspended using a vortex mixer (Baxter Diagnostics Inc., IL, USA). The samples were centrifuged again at 10,000 rpm for 10 minutes, the excess PBS and trace amounts of broth were poured off, and the samples were rinsed with two $20 \mathrm{~mL}$ aliquots of PBS and resuspended using a vortex mixer. This "cell washing" process with PBS was repeated a total of three times to ensure that no broth was remaining amongst the cells that would be the cause of regrowth later. After the washing process, the cells and PBS were added to $2.0 \mathrm{~L}$ of sterilised PBS, and the initial E. coli concentration was measured immediately. The resulting initial concentration of the test samples used for the experiments was approximately $1 \times 10^{7}$ E. coli colony forming units (CFU) per $\mathrm{mL}$, expressed as the most probable number (MPN) per $\mathrm{mL}$.

\subsubsection{Freeze-Thaw Treatments}

$150 \mathrm{~mL}$ aliquots of the test sample (PBS and E. coli) were dispensed into $500 \mathrm{~mL}$ autoclavable polypropylene Nalgene ${ }^{\circledR}$ bottles, and placed in a freezer (VWR FORMA ${ }^{\circledR}$ $40^{\circ} \mathrm{C}$ Lab Freezer Model 5722, VWR International, Mississauga, ON). Freezer temperatures of $-10,-20$ or $-30^{\circ} \mathrm{C}\left(+/-1^{\circ} \mathrm{C}\right)$ were used to represent a range of temperatures that occur in the natural environment where freeze-thaw is most applicable. Also, preliminary tests demonstrated that these freezer temperatures 
generated freezing rates below $10 \mathrm{~mm} / \mathrm{hr}$, which is favourable for pathogen attenuation and particle migration.

The freezing rate was determined based on the geometry of the liquid portion of sample and the time taken for the liquid to completely freeze. The temperature at the centre of a $150 \mathrm{~mL}$ test sample and the temperature of the freezer were monitored using a Traceable ${ }^{\circledR}$ dual-channel thermometer with type $\mathrm{K}$ thermocouples and computer output (Model 4137, Control Company, Friendswood, TX, USA). Temperatures were recorded on a PC every minute using the data acquisition software (DAS ${ }^{\mathrm{TM}}$, Control Company, Friendswood, TX, USA). When the centre of the sample was cooled to the freezing point, generally around $-0.7^{\circ} \mathrm{C}$, the temperature did not change as the liquid changed to ice. Once the entire sample was frozen, the temperature at the centre of the sample began to decrease, until reaching the ambient temperature inside the freezer. During this phase change, when the temperature was stable, it was assumed that the ice front approached the centre of the sample radially and vertically inwards. The elapsed time for the phase change to occur and the radius of the sample was then used to determine the velocity of the advancing ice-front, also known as the freezing rate. The resulting freezing rates generated using freezer temperatures of $-10,-20$ and $-30^{\circ} \mathrm{C}$ were approximately 3,5 and $7 \mathrm{~mm} / \mathrm{hr}$ in the $150 \mathrm{~mL}$ samples respectively. Plots of the temperature logs used to estimate the freezing rates are presented in Appendix B1 on page 380. These freezing rates are within the optimal range in terms of pathogen reduction and particle migration, leading to consolidated particles and good dewaterability (Wang et al., 2001, Sanin et al., 1995, Hung et al., 1997). Once the 
samples were completely frozen, the samples were kept under the freezing conditions for an additional 1,8 or 15 days. It was presumed that 1 day frozen would reveal the effects of the freezing and thawing processes, while longer periods of 8 and 15 days would show any additional effects caused by prolonged freezing periods. After either 1 , 8 or 15 days completely frozen, the samples were removed from the freezer, and thawed at room temperature $\left(22-23^{\circ} \mathrm{C}\right)$. It took between 350 and 400 minutes for the samples to completely thaw and warm up to room temperature before any further treatments or analysis was performed.

\subsubsection{Pre- and Post-Treatment with Potassium ferrate(VI)}

Whenever potassium ferrate was used, a fresh ferrate(VI) stock solution was prepared by adding $5.0 \mathrm{mg}$ of Ferratec Brand ${ }^{\mathrm{TM}}>90 \%$ pure potassium ferrate (Sigma-Aldrich Canada Ltd., Oakville, ON) to $10.0 \mathrm{~mL}$ of deionised water. Resulting potassium ferrate stock solution was $0.5 \mathrm{mg} / \mathrm{mL}$ of potassium ferrate salt, equivalent to approximately $0.27 \mathrm{mg} \mathrm{FeO}_{4}^{-2} / \mathrm{mL}$.

Addition of ferrate(VI) prior to freeze-thaw treatment (ferrate(VI) pre-treatment) was conducted in the $500 \mathrm{~mL}$ sample bottles, which were placed on magnetic stirrers. For the low dose (LD) pre-treatments, $0.15 \mathrm{~mL}$ of the stock solution containing $0.5 \mathrm{mg}$ $\mathrm{K}_{2} \mathrm{FeO}_{4} / \mathrm{mL}$ was added to each of the designated $150 \mathrm{~mL}$ samples, and the samples were stirred for 15-minutes before being placed in the freezer for subsequent freeze-thaw treatment. The resulting $L D$ was $0.5 \mathrm{mg} / \mathrm{L}$ of potassium ferrate equivalent to $0.273 \mathrm{mg}$ $\mathrm{FeO}_{4}{ }^{-2} / \mathrm{L}$. High dose (HD) pre-treatments were conducted the same way, however 0.30 
$\mathrm{mL}$ of the stock solution was added to each of the designated sample bottles, and the resulting $\mathrm{HD}$ was $1.0 \mathrm{mg} / \mathrm{L}$ of potassium ferrate equivalent to $0.545 \mathrm{mg} \mathrm{FeO}_{4}^{-2} / \mathrm{L}$.

Post treatment with the LD and HD was conducted in a similar fashion. After the designated freezing period, samples were removed from the freezer and thawed at room temperature. This process took between 350 and 400 minutes. Once the samples were completely thawed, and warmed to room temperature, $0.15 \mathrm{~mL}$ of the potassium ferrate stock solution containing $0.5 \mathrm{mg} / \mathrm{mL}$ of potassium ferrate was added to the bottles designated for LD post-treatment, and $0.30 \mathrm{~mL}$ of the stock solution was added the bottles designated for HD post-treatment. The bottles were stirred for 15 minutes using a magnetic stirrer. After the 15 -minute contact time, $10 \mathrm{~mL}$ of the sample was immediately removed from the bottles and added to $90 \mathrm{~mL}$ of PBS, and subsequently serial-diluted with more PBS as part of the bacterial enumeration technique.

\subsubsection{Sample Analysis: E. coli Enumeration}

For all experiments, multiple tube fermentation (MTF) with A1 medium (EMD Chemicals Inc., Gibbstown, NJ, USA) was used to enumerate the E. coli according to U.S. EPA Method 1681 (U.S. EPA, 2005a). For some experiments, membrane filtration (MF) with Difco $^{\mathrm{TM}} \mathrm{m}$-Endo Agar LES medium (Becton, Dickson \& Company, MD, USA) was performed according to Standard Method 9222 B (APHA et al., 2005).

\subsubsection{Multiple Tube Fermentation (MTF)}

The MTF procedure used to enumerate $E$. coli in the PBS samples was the same procedure used to enumerate fecal coliform in the RBC sludge samples during the pilot- 
scale study, which was described in detail in section 4.1.4.2, on page 92. The only difference in the procedure used was with the sample preparation. Since the test samples for these experiments were a pure culture of $E$. coli in a sterilised PBS solution, the raw sample contained in the bottles was used as the initial undiluted sample. The $\mathrm{pH}$ of all raw samples were checked to ensure that they were between 7.0 and 7.5 , and similar to the previous experiments, $10 \mathrm{~mL}$ of the undiluted samples was placed in a flask containing $90 \mathrm{~mL}$ of PBS, representing the $10^{-1}$ dilution, and the flask was stirred using a magnetic stirrer. This process was repeated to create serial dilutions up to $10^{-10}$, if the concentration of $E$. coli was completely unknown, or to a dilution level that was 2 or 3 levels of magnitude greater than the expected $E$. coli concentration. All inoculation, incubation, and enumeration procedures were the same as those described in section 4.1.4.2, on page 95 , however since the sample was a liquid sample, free of solids, the $E$. coli concentrations were reported as $\mathrm{MPN} / \mathrm{mL}$, on a wet basis, only.

\subsubsection{Membrane Filtration (MF)}

A few of the preliminary tests also used MF according to Standard Method 9222 B (APHA et al., 2005) for comparison purposes. The detailed MF procedure is described below.

\section{LES M-Endo Agar}

MF was performed using Difco $^{\mathrm{TM}}$ M-Endo Agar LES medium (Becton, Dickson \& Company, MD, USA). The dried agar was rehydrated by adding $51.05 \mathrm{~g}$ to $1.0 \mathrm{~L}$ of deionised water containing $20 \mathrm{~mL}$ of $90 \%$ ethanol, and the media was stirred and heated 
using a magnetic stirrer and hotplate until near boiling to completely dissolve the agar. Once completely dissolved, the media was immediately removed from the heat, cooled to between 45 and $50^{\circ} \mathrm{C}$, and the final $\mathrm{pH}$ was adjusted to $7.2 \pm 0.2$ using either $1 \mathrm{~N}$ sodium hydroxide or $1 \mathrm{~N}$ hydrochloric acid solutions. $7 \mathrm{~mm}$ volumes of the warm agar were dispensed into the lower section of $60-\mathrm{mm}$ diameter plastic petri dishes, and cooled further at room temperature until the agar solidified. The prepared petri dishes were placed in a sealed bag and stored in the refrigerator until required, and prior to use, the dishes were removed from the refrigerator and warmed to room temperature.

\section{Sample Preparation}

As discussed earlier, in section 4.2.5.1 on page 113 , because the samples used in these experiments were a pure culture of $E$. coli in a sterilised PBS solution, the raw sample following treatments was used as the undiluted sample, in which subsequent serial dilutions were made using sterilised PBS. PBS was also used as rinse water during the vacuum filtration procedure.

\section{Vacuum Filtration Procedures}

A standard vacuum filtration apparatus was used for all MF tests. This included an Air Cadet $^{\circledR}$ vacuum/pressure pump (15 L/min, $68 \mathrm{kPa}$ maximum), a $300 \mathrm{~mL}$ Fisherbrand $^{\mathrm{TM}}$ borosilicate glass funnel with a $47 \mathrm{~mm}$ diameter vacuum filter paper holder and funnel

clamp, a $1.0 \mathrm{~L}$ waste filtrate collection flask and a $1.0 \mathrm{~L}$ secondary flask used as a water trap, pre-sterilised $47 \mathrm{~mm}$ diameter, $0.45 \mu \mathrm{m}$ pore size, Whatman ${ }^{\mathrm{TM}}$ type $\mathrm{WCN}$ cellulose nitrate membrane filters, and sterile forceps. Prior to commencing a filtration series, all 
reusable filtration equipment was wrapped in aluminum foil, and sterilised by autoclave at $151^{\circ} \mathrm{C}$ and $15 \mathrm{psi}$, for 15 minutes. During the vacuum filtration procedure, forceps were sterilised in between samples by dipping in 95\% ethyl alcohol and flaming.

Filtration procedure began by placing a membrane filter on the filter holder, and securing the funnel using the clamp. $25 \mathrm{~mL}$ of the rinse water was poured into the funnel, and the vacuum pump was turned on, filtering the rinse water through the membrane filter. The interior of the funnel was rinsed using three $25 \mathrm{~mL}$ portions, then the vacuum pump was shut off, the funnel was unclamped from the membrane filter holder, and the filter paper was removed using the sterile forceps. The membrane filter was then placed on the agar using a rolling motion to avoid air entrapment, the lid was replaced on the petri dish, and the dish was inverted and labelled.

As described earlier, for each test sample, a set of serial dilutions were prepared. For each dilution, three MF replicates were performed. For each replicate, $25 \mathrm{~mL}$ of the sample was filtered through the membrane filter, and the interior of the funnel was rinsed using three $25 \mathrm{~mL}$ portions of sterile PBS. Similar to the procedure with the blanks, the funnel was unclamped from the membrane filter holder, the filter paper was removed using the sterile forceps and placed on the agar, then the lid was replaced on the petri dish, and the dish was inverted and labelled. This process was repeated 3 times for each of the serial dilutions, for each of the test samples. 
Throughout the vacuum filtrations blanks were conducted using rinse water only, as described above, to ensure no cross-contamination, and following the vacuum filtrations another set of blanks were performed.

\section{Incubation, Analysis and Enumeration}

Following the vacuum filtrations, all petri dishes, including blanks, were incubated inverted for 20 to 22 hours at $35 \pm 0.5^{\circ} \mathrm{C}$. Following the incubation period, the petri dishes were removed from the incubator, all blanks were also checked to ensure no growth, and the appropriate sample dilutions were selected for counting, while overgrown plates were discarded. Ideal plates for counting contained 20 to 80 colonies on the membrane surface, while plates containing $>200$ colonies were considered overgrown. The red colonies with a golden metallic sheen were counted using a Reichart $^{\mathrm{TM}}$ Darkfield Quebec ${ }^{\mathrm{TM}}$ electronic register colony counter (Reichart Inc., Depew, NY, USA). Once all results were recorded, the original sample $E$. coli concentrations were calculated using Equation 23. For example, a plate containing 27 colonies from the filtration of $25 \mathrm{~mL}$ of a $10^{-7}$ dilution sample would contain $27 /\left(25 \times 10^{-7}\right)=10.8 \times 10^{6}$ colonies/mL, or 7-log.

Coliform Conc. $($ Coliform $/ \mathrm{mL})=\frac{\text { Colonies Counted }}{\text { Volume Filtered }(\mathrm{mL})} \quad$ Equation 23

\subsubsection{Statistical Analysis}

A two-tailed t-test was used to determine the $p$ values and statistical significance $(p<$ 0.05) of the results. All MF tests were carried out in triplicate, using at least three 
dilutions. All MTF tests were carried out in duplicate, using at least four dilutions. Error bars on all figures show the $95 \%$ confidence interval, which is built-in to the MTF test, as described earlier in section 4.1.4.2 on page 95. When the measured concentration of a particular test sample was below the detection limit, the error bars for the particular data point in the figures were replaced with a downward arrow.

\subsection{INDIVIDUAL AND COMBINED EFFECTS OF FREEZE-THAW AND FERRATE(VI) OXIDATION FOR THE STABILISATION AND DEWATERING OF WASTEWATER SLUDGES (CHAPTER 7)}

\subsubsection{Summary}

The first part of the experiments, using primary sludge, compared potassium ferrate(VI) additions prior to freeze-thaw treatment (pre-treatment) versus potassium ferrate(VI) additions following freeze-thaw treatment (post-treatment). These tests were also compared to stand-alone freeze-thaw, without potassium ferrate(VI). The second part of the study, using anaerobically digested sludge, evaluated the effects of potassium ferrate(VI) pre-treatment using simulated drainage beds to separate the meltwater from the sludge cake samples during the sludge thawing period.

\subsubsection{Sludge Samples}

The sludge used for part I (sludge bottle experiments) was primary sludge, obtained from the Robert O. Pickard Environmental Centre (ROPEC) in Ottawa, Ontario, Canada. The sludge used for part II (sludge drainage experiments) was anaerobically digested 
sludge from ROPEC. The initial characteristics of the two sludges are presented in Table

4-2.

Table 4-2: Characteristics of the primary and anaerobically digested sludge samples used for part I and part II respectively of the combined freeze-thaw and ferrate(VI) oxidation experiments.

\begin{tabular}{|c|c|c|c|c|c|}
\hline \multirow{2}{*}{$\frac{\text { Parameter }}{\text { Total Solids (TS) }}$} & \multirow{2}{*}{$\begin{array}{c}\text { Units } \\
\%\end{array}$} & \multicolumn{2}{|c|}{$\begin{array}{c}\text { Primary Sludge } \\
\text { AVERAGE }( \pm 95 \% \mathrm{CL})\end{array}$} & \multicolumn{2}{|c|}{$\begin{array}{l}\begin{array}{c}\text { Anaerobically Digested } \\
\text { Sludge }\end{array} \\
\text { AVERAGE }( \pm 95 \% \mathrm{CL}) \\
\end{array}$} \\
\hline & & 4.7 & $( \pm 0.3)$ & 2.0 & $( \pm 0.0)$ \\
\hline Volatile Solids (VS) & $\%$ & 4.0 & $( \pm 0.3)$ & 1.2 & $( \pm 0.0)$ \\
\hline Fecal Coliforms & $\log (\mathrm{MPN} / \mathrm{mL})$ & 4.7 & $(+0.4,-0.6)$ & 3.9 & $(+0.4,-0.5)$ \\
\hline Fecal Coliforms & $\log (\mathrm{MPN} / \mathrm{g} \mathrm{DS})$ & 6.0 & $(+0.4,-0.6)$ & 5.6 & $(+0.4,-0.5)$ \\
\hline Capillary Suction Time (CST) & s & 912 & $( \pm 11)$ & & - \\
\hline Ammonia & $\mathrm{mg} / \mathrm{L} \mathrm{NH}_{3}-\mathrm{N}$ & 210 & $( \pm 11)$ & & - \\
\hline Sulfide & $\mathrm{mg} / \mathrm{L} \mathrm{s}^{-2}$ & 39 & $( \pm 1)$ & & - \\
\hline $\mathrm{pH}$ & & & - & 7.41 & $( \pm 0.03)$ \\
\hline Soluble Proteins & $\mathrm{mg} / \mathrm{L}$ & & - & 12 & $( \pm 1)$ \\
\hline Soluble Carbohydrates & $\mathrm{mg} / \mathrm{L}$ & & - & 28 & $( \pm 4)$ \\
\hline Soluble Chemical Oxygen Demand (sCOD) & $\mathrm{mg} / \mathrm{L}$ & & - & 568 & $( \pm 13)$ \\
\hline
\end{tabular}

\subsubsection{Part I: Freeze-Thaw Treatment in Bottles}

$250 \mathrm{~mL}$ primary sludge samples were dispensed into $500 \mathrm{~mL}$ autoclavable polypropylene Nalgene bottles, and placed in a freezer (VWR FORMA ${ }^{\circledR}-40^{\circ} \mathrm{C}$ Lab Freezer Model 5722, VWR International, Mississauga, ON) set to $-20^{\circ} \mathrm{C} \pm 1^{\circ} \mathrm{C}$. The temperature at the centre of a $250 \mathrm{~mL}$ test sample and the temperature inside the freezer were monitored using a Traceable ${ }^{\circledR}$ dual-channel thermometer with type $\mathrm{K}$ thermocouples and computer output (Model 4137, Control Company, Friendswood, TX, USA). Temperatures were recorded on a PC every minute using the data acquisition software (DAS ${ }^{\mathrm{TM}}$, Control Company, Friendswood, TX, USA). When the samples were placed in the freezer, the temperature at the centre of the test sample decreased at a steady rate of $1^{\circ} \mathrm{C}$ every 10 minutes until 
reaching the sludge freezing temperature, which was approximately $-0.3^{\circ} \mathrm{C}$. At this point, the temperature of the sample remained constant for approximately 7 hours while the sample froze. During this freezing period, it was assumed that the direction of the advancing ice front was radially and vertically inward, since the sample bottles were not insulated. Once the samples were completely frozen, the temperature of the sample began to decrease again, until eventually reaching the freezer temperature of $-20^{\circ} \mathrm{C}$. Plots of the temperature logs used to determine the freezing rates are presented in Appendix B2 on page 381. The 7 hour elapsed time for the sludge to freeze, and the 31 $\mathrm{mm}$ depth to the centre of the sample were used to estimate the freezing rate of approximately $4 \mathrm{~mm} / \mathrm{hr}$, which is within the optimal range for pathogen reduction and particle migration leading to improved dewaterability (Wang et al., 2001, Hung et al., 1997). After the samples were completely frozen, they were kept frozen at $-20^{\circ} \mathrm{C}$ for an additional 1, 8 or 15 days. After the designated period in the freezer, the samples were removed and thawed at room temperature $\left(22-23^{\circ} \mathrm{C}\right)$ for 12 hours.

\subsubsection{Pre- and Post-Treatment with Potassium Ferrate(VI)}

Primary sludge samples that were pre-treated with potassium ferrate(VI), prior to freeze-thaw, were given a low dose $(L D=1.0 \mathrm{~g} / \mathrm{L}$ ) or a high dose $(\mathrm{HD}=10.0 \mathrm{~g} / \mathrm{L})$ of Ferratec Brand $^{\mathrm{TM}}>90 \%$ pure potassium ferrate( $\left.\mathrm{VI}\right)$. Following the addition of potassium ferrate(VI), the samples were stirred using a magnetic stirrer for 15 minutes and subsequently placed into the freezer. 
Post-treatment with potassium ferrate(VI) was conducted in a similar fashion. After the designated time in the freezer, the samples were removed and thawed at room temperature $\left(22-23^{\circ} \mathrm{C}\right)$. Once the samples were completely thawed and warmed to room temperature, $250 \mathrm{mg}$ of potassium ferrate(VI) was added to the bottles designated for LD post-treatment $(1.0 \mathrm{~g} / \mathrm{L})$, and $2.5 \mathrm{~g}$ was added to the bottles designated for HD post-treatment $(10.0 \mathrm{~g} / \mathrm{L})$. After the addition of potassium ferrate(VI), the bottles were stirred using a magnetic stirrer for 15 minutes prior to being analysed.

\subsubsection{Sample Analysis: Primary Sludge}

Preliminary tests on the primary sludge evaluated the filterability using capillary suction time (CST), and ammonia and sulphide concentrations of the sludge before and after the addition of potassium ferrate(VI). The fecal coliform, TS and VS concentrations of the primary sludge were measured prior to the addition of potassium ferrate( $\mathrm{VI})$ and freezethaw treatment, following the addition of potassium ferrate(VI) prior to freeze-thaw treatment, following the freeze-thaw treatment, and following the addition of potassium ferrate(VI) after the freeze-thaw treatment. The methods used to measure each of these parameters are presented below.

\section{TS and VS}

Following freeze-thaw, the samples were stirred by hand, and 3 samples of approximately $20 \mathrm{~g}$ each were used as replicates to determine the TS and VS of the sludge samples according to Standard Method 2540 G (APHA, 2005). The remaining procedures used were the same as those presented earlier in section 4.1.4.1, on page 
91. All TS and VS measurements were performed in triplicate, and data points on the figures represent the average of the three replicates, and the error bars represent the 95\% confidence limits.

\section{CST}

The CST is a commonly used test to quantify the rate of release of water from sludges. As shown in Figure 4-4, the apparatus consists of an upright cylindrical sludge reservoir resting on a piece of chromatography paper, placed on the block containing electrical contacts at a radially outwards distance of 15.9 and $22.2 \mathrm{~mm}$ from the centre of the sample reservoir. When the sludge is added to the reservoir, the chromatography paper draws out water from the sludge via capillary action. When the water reaches the first electrical contact, the change in conductivity automatically starts a timer, and when the water reaches the second electrical contact, the timer is automatically stopped. The elapsed time for the water to travel from the inner contact to the outer contact is measured in seconds, and is referred to as the CST.

The CST was measured using a Triton Electronics Type 319 multi-purpose CST apparatus (Triton Electronics Ltd., Great Dunmow, Essex, England), which contained three CST setups connected to a single timer, allowing the simultaneous measurement of the three sample replicates. All CST measurements were performed according to Standard Method 2710 G (APHA, 2005). Prior to each measurement, the CST timer was rest, the sample reservoir and the test block were rinsed and dried, and a new CST paper was placed on the block, with the rough side of the paper facing upwards. Then, a $10-\mathrm{mL}$ 
pipette, with a widened tip, was used to pipette $6.4 \mathrm{~mL}$ of sludge into each sample well. Following each measurement, the elapsed time was recorded, and the procedure was repeated. All CST sample measurements were conducted in triplicate.

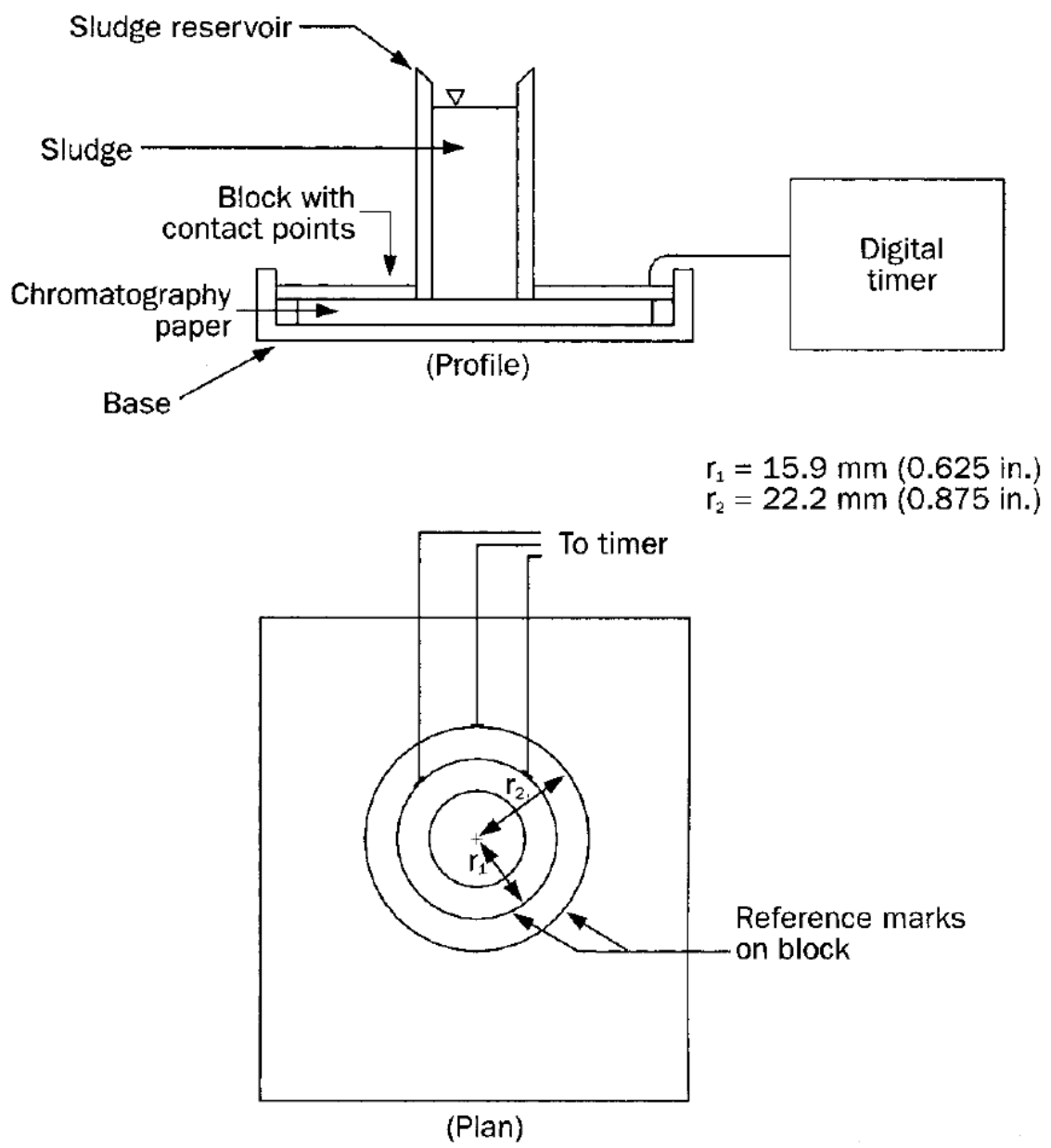

Figure 4-4: CST apparatus used to quantify the rate of release of water from sludge (APHA et al., 2005).

\section{Ammonia}

The total ammonia concentration $\left(\mathrm{NH}_{3}+\mathrm{NH}_{4}{ }^{+}\right)$was measured using $\mathrm{HACH}$ method 10031 (high range: $0.4-50.0 \mathrm{mg} / \mathrm{L} \mathrm{NH}_{3}-\mathrm{N}$ ) with a dilution factor of $10.5 \mathrm{~mL}$ of sample was added to $45 \mathrm{~mL}$ of deionised water, and the samples were mixed using a vortex mixer. 0.1 
$\mathrm{mL}$ of the diluted sample was added to an AmVer ${ }^{\mathrm{Tm}}$ Diluent Reagent Test ' $\mathrm{N}$ Tube vial, then the contents of one Ammonia Salicylate Reagent Powder Pillow were added, and contents of one Ammonia Cyanurate Reagent Powder Pillow were added. The vials were capped and shaken vigorously, and a 20-minute reaction proceeded at room temperature. First, ammonia compounds combine with chlorine to form monochloramine, which reacts with salicylate to for 5-aminosalicylate, which is then oxidised in the presence of a catalyst called sodium nitroprusside to form a blue-coloured compound, which is masked by the yellow colour of the excess reagent, resulting in a green-coloured solution. Following the reaction period, the absorbances of the vials were measured at a wavelength of $655 \mathrm{~nm}$ using program 343 (N, Ammonia HR TNT) on a HACH DR 2800 spectrophotometer, and the diluted ammonia concentrations were recorded. Actual ammonia concentrations of the original samples were then calculated based on the dilution factor of 10 .

\section{Sulphide}

The sulphide concentration was measured using $\mathrm{HACH}$ method 8131 (methylene blue method, 5-800 $\mu \mathrm{g} / \mathrm{L} \mathrm{S}^{-2}$ ). The primary sludge sample was diluted with deionised water by a factor of $100.1 .0 \mathrm{~mL}$ of the test sample was added to $99.0 \mathrm{~mL}$ of deionised water, to create the $10^{-2}$ dilution. Despite the large dilution factor, there was visible turbidity in the test samples, therefore as outlined in the $\mathrm{HACH}$ method 8131, a test blank was adjusted for turbidity using a combination of bromine water $(30 \mathrm{~g} / \mathrm{L})$ and a phenol solution ( $30 \mathrm{~g} / \mathrm{L})$. First, $25 \mathrm{~mL}$ of the test sample was placed in a $50-\mathrm{mL}$ Erlenmeyer flask, and bromine water was added one drop at a time, with constant swirling, until a constant yellow color appeared. Then, the phenol solution was added one drop at a 
time, with constant swirling, until the yellow color just disappeared. This sulphide-free blank was then used in the test procedure, along with the test samples.

$10 \mathrm{~mL}$ of the diluted test sample was placed into the $10 \mathrm{~mL}$ sample cell using a pipette. Similarly, $10 \mathrm{~mL}$ of the prepared test blank, described above, was placed in a $10 \mathrm{~mL}$ sample cell. A calibrated dropper was used to add $0.5 \mathrm{~mL}$ of Sulfide 1 Reagent to each cell, and the cells were gently swirled to mix, then a dropper was used to add $0.5 \mathrm{~mL}$ of Sulfide 2 Reagent to each cell, and the sample cells were immediately capped and inverted to mix. A pink colour developed immediately, and during the 5-minute reaction period at room temperature, the sample turned blue in the presence of sulphide. This is because hydrogen sulphide and other acid-soluble metal sulphides present in the sample react with the $\mathrm{N}, \mathrm{N}$-dimethyl-p-phenylenediamine sulphate to form methylene blue. The intensity of the blue colour in therefore proportional to the amount of sulphide present in the original sample. Following the reaction period, the cells were wiped clean, and placed in the spectrophotometer, which read the sample absorbances at a wavelength of $665 \mathrm{~nm}$. In all cases, special care was taken to avoid excess agitation, as this seemed to re-suspend colloids, which skewed absorbance measurements and the subsequent concentration readings. The concentration readings for all diluted samples were recorded, and the actual sulphide concentrations of the original samples were calculated based on the dilution factor of 100 . 


\section{Fecal Coliform}

The enumeration of fecal coliform in the sludges before and after was performed using MTF with A-1 medium, according to EPA method 1681 (U.S. EPA 2005a). All reagents required were prepared according to the procedures previously outlined in section 4.1.4.2, on page 92. $300 \mathrm{~mL}$ of the liquid sludge samples were blended on high for 2 minutes, the $\mathrm{pH}$ of the sample was adjusted to between 7.0 and 7.5 , and $10.0 \mathrm{~mL}$ of the homogenised sample was added to $90 \mathrm{~mL}$ of sterilised PBS, representing the $10^{-1}$ dilution. Subsequent serial dilutions were made to ensure that each set of incubation tubes, for each sample, contained both positive and negative results following the 24hour incubation. MTF results for the liquid sludge samples are reported on a wet basis $(\mathrm{MPN} / \mathrm{mL})$, and a dry basis (MPN/g DS) using the measured TS of the associated sludge samples.

\subsubsection{Part II: Freeze-Thaw Treatment in Simulated Drainage Beds}

For Part II experiments using the simulated drainage beds, $200 \mathrm{~mL}$ samples of anaerobically digested sludge were placed in $500 \mathrm{~mL}$ bottles. The bottles were sealed tightly with a wide-mouth lid, and the bottles were inverted as shown in Figure 4-5a. The inverted bottles were then placed in the freezer, which was set to $-20^{\circ} \mathrm{C}$. The freezing rate generated was approximately $6 \mathrm{~mm} / \mathrm{hr}(4.5 \mathrm{hrs}$ to travel $25 \mathrm{~mm})$. A plot of the temperature log used to estimate the freezing rate is presented in Appendix B3 on page 382 . Once the samples were completely frozen, they were kept frozen at $-20^{\circ} \mathrm{C}$ for an additional 1, 8 or 15 days. After the designated time in the freezer, the samples were removed, the solid lid was replaced with a perforated lid lined with drainage fabric, and 
the tape sealing the air hole was removed. The frozen samples were then placed upside down in a funnel, which rested in the opening of a $50 \mathrm{~mL}$ graduated cylinder, as shown in Figure 4-5b. The samples thawed at room temperature $\left(22-23^{\circ} \mathrm{C}\right)$ for 12 hours and the meltwater was collected via gravity drainage. The experiments using simulated drainage beds did not use ferrate(VI) as a post-treatment to freeze-thaw due to the separation of solids and meltwater. Only ferrate(VI) pre-treatment was used for these experiments, along with stand-alone freeze-thaw treatment.
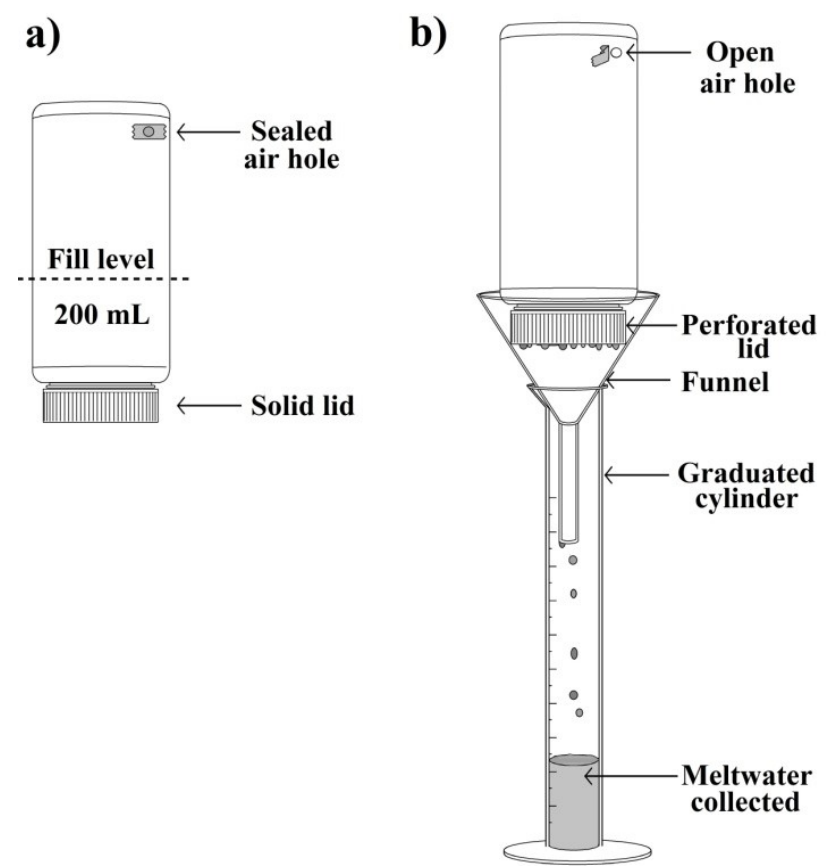

Figure 4-5: Schematic of (a) the freezing vessel and (b) the simulated drainage bed with meltwater collection, used for the freeze-thaw experiments with anaerobically digested sludge.

\subsubsection{Pre-Treatment with Potassium Ferrate(VI)}

Anaerobically digested sludge samples that were pre-treated with potassium ferrate(VI), prior to freeze-thaw, were given $L D=0.5 \mathrm{~g} / \mathrm{L}$ or $H D=5.0 \mathrm{~g} / \mathrm{L}$ of potassium ferrate $(\mathrm{VI})$. Following the addition of the potassium ferrate(VI) doses, the samples were stirred 
using a magnetic stirrer for 15 minutes, and then a $10 \mathrm{~mL}$ portion was immediately withdrawn and diluted in $90 \mathrm{~mL}$ of sterilised PBS. This $10^{-1}$ dilution was used later for fecal coliform enumeration.

\subsubsection{Sample Analysis: Initial Sludge and Ferrate(VI)-Treated Sludge}

The TS, VS, fecal coliform, SCOD, soluble proteins and soluble carbohydrates concentrations of the sludge were measured before and after the addition of potassium ferrate. Below is a description of the methods used for each of these test.

\section{TS and VS}

The TS and VS of the initial sludge and following pre-treatment with potassium ferrate(VI) were measured according to Standard Method 2540 G (APHA, 2005), as outlined earlier in section 4.1.4.1, on page 91. Three replicates of approximately $40 \mathrm{~g}$ of sludge were used for each TS and VS measurement. The data points on the figures represent the average of the three replicates, and the error bars represent the $95 \%$ confidence limits

\section{Fecal Coliform}

The enumeration of fecal coliform in the initial sludge and in the sludge samples following ferrate(VI) treatment was performed using MTF with A-1 medium, according to EPA method 1681 (U.S. EPA 2005a). All reagents required were prepared according to the procedures previously outlined in section 4.1.4.2, on page 92. Because the sample were liquid sludge samples, $300 \mathrm{~mL}$ of each test sample was blended on high for 2 minutes, then $10 \mathrm{~mL}$ was diluted in $90 \mathrm{~mL}$ of PBS to begin the serial dilution process. 
The sCOD was measured using HACH method 8000 (high range: $20-1500 \mathrm{mg} / \mathrm{L} \mathrm{COD),}$ with a dilution factor of 2. First, to obtain the soluble component from the liquid sludge, the samples were centrifuged at 10,000 rpm (relative centrifugal force, $\mathrm{RCF}=15,317$ ) for 10 minutes, then the supernatant was filtered using a $0.45 \mu \mathrm{m}$ mixed cellulose ester (MCE) syringe filter (Shanghai Derian Instrument Co., Ltd., Shanghai, China). The soluble component of the sludge samples were also used to measure the concentrations of soluble proteins and soluble carbohydrates, which are presented below. For the sCOD measurements, $2.0 \mathrm{~mL}$ of the 1:1 diluted sample was added to a COD digestion reagent vial, the vial was tightly capped, inverted several times, and placed in a HACH DRB 200 digital block reactor preheated to $150^{\circ} \mathrm{C}$, for two hours. Following the two hour reaction period, the vials were cooled to room temperature, and analysed using program 435 (COD HR) on a HACH DR 2800 spectrophotometer. All sCOD tests were performed in triplicate. The data points on the figures show the averages of the three replicates, and the error bars represent the standard deviations.

\section{Soluble Proteins}

The concentration of soluble proteins was measured using Coomassie brilliant blue G250 reagent (Pierce Protein Biology Products, Thermo Fisher Scientific Inc., Rockford, IL, USA) with the Bradford method (Bradford, 1976). First, this method required a calibration curve to relate the absorbance of the test samples, using a wavelength of $595 \mathrm{~nm}$, to the protein concentration of the test sample. A Coomassie (Bradford) brilliant blue G-250 reagent kit (Pierce Protein Biology Products, Thermo Fisher Scientific 
Inc., Rockford, IL, USA) contained $10 \times 1.0 \mathrm{~mL}$ ampoules of a $2000 \mathrm{mg} / \mathrm{L}$ Albumin standard, Bovine Serum Albumin (BSA). The Albumin standard was diluted using deionised water to create a series of standards containing $0,25,125,250,500,750$, 1000,1500 and $2000 \mathrm{mg} / \mathrm{L}$ of protein, and the absorbance of the samples was measured accordingly. A series of clean $10 \mathrm{~mL}$ test tubes with tight-fitting screw-on caps were labelled and filled with $5.0 \mathrm{~mL}$ of the Coomassie reagent. For each standard, $0.1 \mathrm{~mL}$ was added to each test tube containing $5.0 \mathrm{~mL}$ of Coomassie. All test tubes were subsequently capped, and inverted several times to mix, then left to incubate at room temperature for 10 minutes. During this time, the HACH DR 2800 spectrophotometer was turned on, set to a wavelength of $595 \mathrm{~nm}$, and zeroed using a test tube filled with deionised water. Following the 10 minute reaction period, the absorbance of each standard was measured using the spectrophotometer at a wavelength of $595 \mathrm{~nm}$. The absorbance of the sample blank containing $0 \mathrm{mg} / \mathrm{L}$ of the BSA was subtracted from all other samples. Two replicate samples were analysed for each of the standards, and the blank-corrected absorbance values for each standard were plotted to generate the calibration curve shown in Figure 4-6. The data resulted in a linear trend for concentrations $<1000 \mathrm{mg} / \mathrm{L}$ (absorbance $<1.5$ ), therefore this was chosen as the upper limit for the quantification method. The resulting equation of the linear trendline within the working range was rearranged to give an equation for the protein concentration as a function of the measured absorbance at $595 \mathrm{~nm}$, as shown by Equation 24. This equation was used to convert absorbance readings for the unknown samples. 


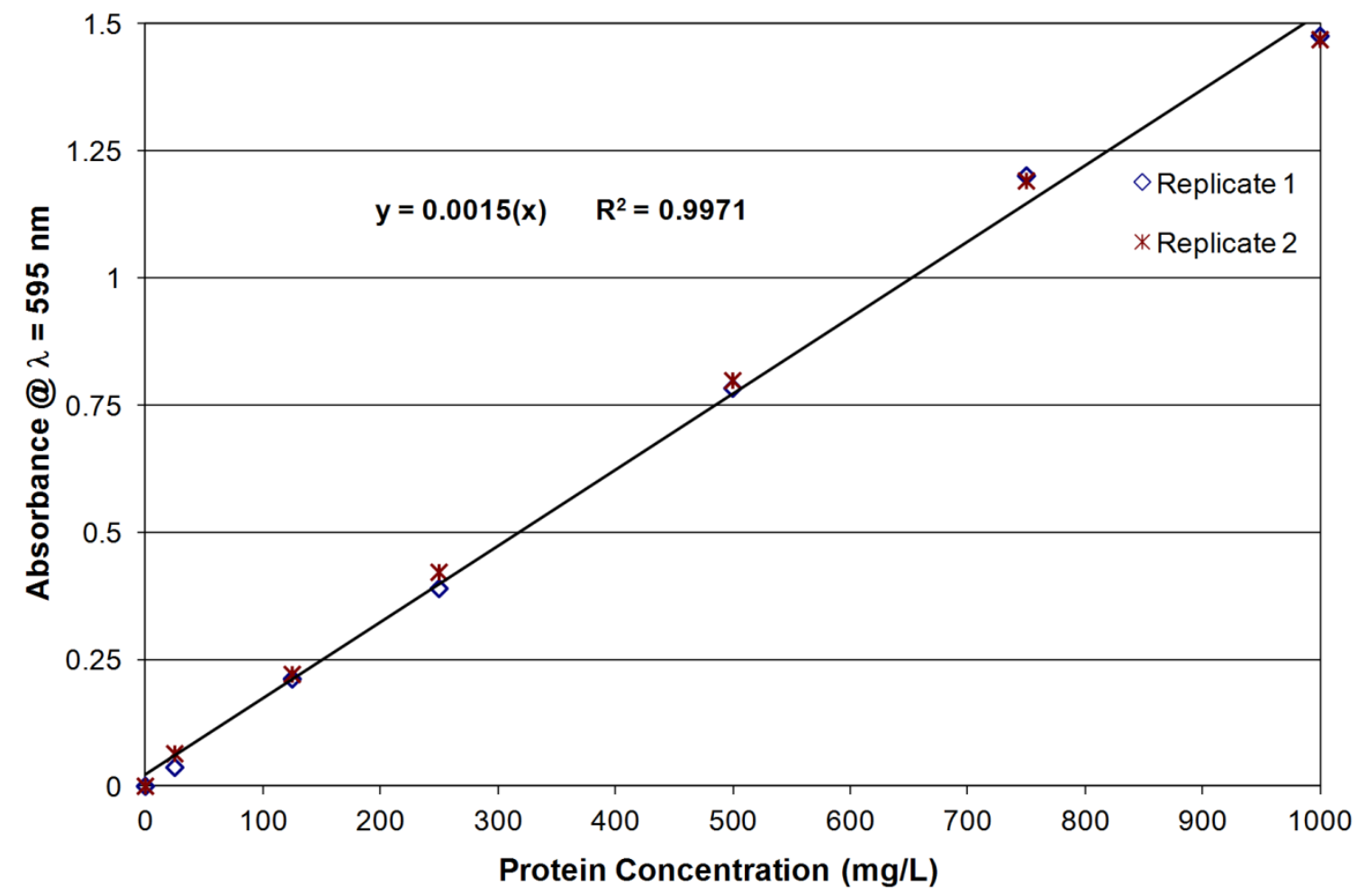

Figure 4-6: Protein calibration curve.

Protein Conc. $\left(\frac{\mathrm{mg}}{\mathrm{L}}\right)=\frac{\text { Absorbance } @ \lambda=595 \mathrm{~nm}}{0.0015} \quad$ Equation 24

As described in the previous section, the soluble component of each test sample was obtained by centrifuging the samples at $10,000 \mathrm{rpm}$ (relative centrifugal force, $\mathrm{RCF}=$ 15,317) for 10 minutes, then filtering the supernatants using a $0.45 \mu \mathrm{m}$ MCE syringe filter. A series of labelled test tubes and caps containing $5.0 \mathrm{~mL}$ of the Coomassie reagent were prepared, including three replicate test tubes for each unknown sample, and an extra test tube used as a blank. $0.1 \mathrm{~mL}$ volumes of each undiluted, soluble test sample were added to the appropriately labelled test tube containing the Coomassie reagent. For the blank sample, $0.1 \mathrm{~mL}$ of deionised water was added to a test tube 
containing $5.0 \mathrm{~mL}$ of the Coomassie reagent. The test tubes were then capped, inverted several times to mix, and left to incubate at room temperature for 10 minutes. During this time, the spectrophotometer was turned on, set to $595 \mathrm{~nm}$, and zeroed using a test tube filled with deionised water. Following the 10 minute reaction period, the absorbances of blank and the unknown test samples were measured, then the absorbance of the blank was subtracted from the absorbances of all test samples, and Equation 24 was used to convert the blank-corrected absorbances of the test samples to a concentration, expressed as $\mathrm{mg} / \mathrm{L}$ of soluble proteins.

\section{Soluble Carbohydrates}

The concentration of soluble carbohydrates was measured using the Anthrone method (Morris, 1948). The working reagent was prepared by dissolving $2.0 \mathrm{~g}$ of $95 \%$ pure Anthrone (Across Organics, Thermo Fisher Scientific Inc., Rockford, IL, USA) in 1.0 L of a 95\% pure sulfuric acid solution. A fresh solution of the Anthrone reagent was prepared weekly, and when not in use, the reagent was stored in an opaque bottle, shielded from light. First a calibration curve was generated to relate the absorbance of the sample, at a wavelength of $620 \mathrm{~nm}$, to the carbohydrate concentration of the sample. To do this, a series of standards were prepared using 98\% pure galactose powder (Across Organics, Thermo Fisher Scientific Inc., Rockford, IL, USA. Stock solutions containing 0, 25, 50, 75, $100,150,200$ and $300 \mathrm{mg} / \mathrm{L}$ of galactose were prepared, and the absorbances of the samples were measured as follows. A series of clean $10 \mathrm{~mL}$ test tubes with tight-fitting screw-on caps were labelled and filled with $6.0 \mathrm{~mL}$ of the Anthrone reagent. For each standard, $3.0 \mathrm{~mL}$ was added to the appropriate test tube containing the Anthrone 
reagent, and the tube was caped and inverted several times to mix. A 10-minute reaction period proceeded, during which time the spectrophotometer was turned on, set to a wavelength of $620 \mathrm{~nm}$, and zeroed using a test tube filled with deionised water. Following the 10 minute reaction period, the absorbance of each standard was measured, and the absorbance of the blank sample, containing $3 \mathrm{~mL}$ of deionised water in $6.0 \mathrm{~mL}$ of the Anthrone reagent, was subtracted from the absorbance of all other standards. Two replicate samples were analysed for each of the standards, and the blank-corrected absorbance were plotted to generate the calibration curve shown in Figure 4-7. The data resulted in a linear trend for carbohydrate concentrations $<200$ $\mathrm{mg} / \mathrm{L}$, therefore the upper limit for the method was set at $200 \mathrm{mg} / \mathrm{L}$. The resulting equation of the linear trendline within the working range was rearranged to give an equation for the carbohydrate concentration as a function of the measured absorbance at $620 \mathrm{~nm}$, as shown by Equation 25. This equation was used to convert absorbance readings for the unknown samples. 


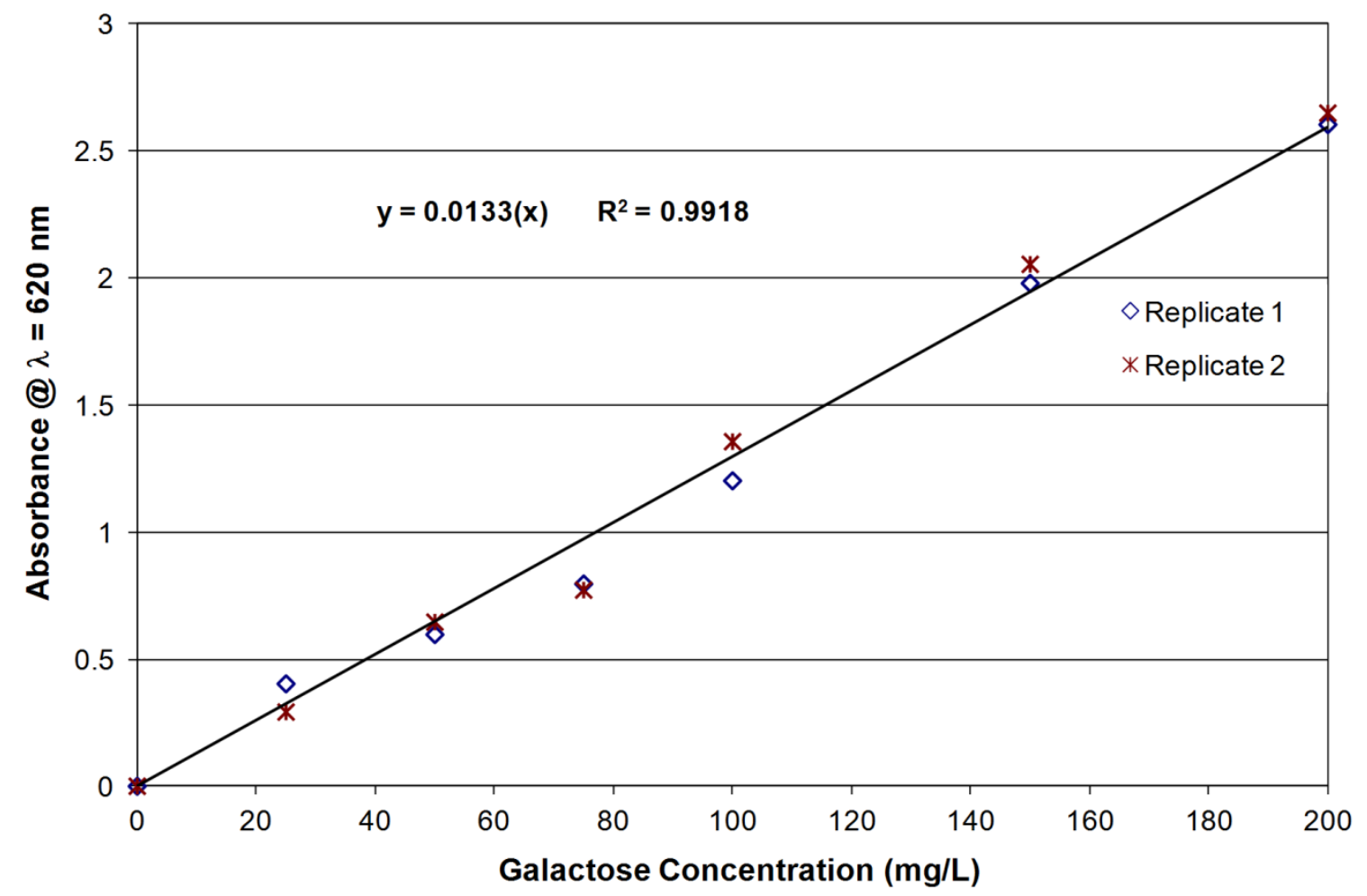

Figure 4-7: Carbohydrate calibration curve.

Carbohydrate Conc. $\left(\frac{\mathrm{mg}}{\mathrm{L}}\right)=\frac{\text { Absorbance @ } \lambda=620 \mathrm{~nm}}{0.0133} \quad$ Equation 25

The unknown samples were the soluble component of their representative test samples, isolated through centrifugation and subsequent filtration, as described in the previous section. To measure the concentration of soluble carbohydrates in the unknown samples, a series of labelled test tubes with caps were prepared, each containing $6.0 \mathrm{~mL}$ of the Anthrone reagent. Three replicate test tubes were prepared for each unknown sample, and an extra test tube was prepared as a blank. $3.0 \mathrm{~mL}$ of volumes from each unknown sample were added to the appropriately labelled test tubes containing the Anthrone reagent, and the test tubes were capped, inverted several times to mix, and 
allowed 10 minutes for reaction at room temperature. During this time, the spectrophotometer was turned on, set to $620 \mathrm{~nm}$, and zeroed using a test tube filled with deionised water. Following the 10 minute reaction period, the absorbances of blank and the unknown samples were measured, then the absorbance of the blank was subtracted from the absorbances of all test samples, and Equation 25 was used to convert the blank-corrected absorbances of the test samples to a concentration, expressed as $\mathrm{mg} / \mathrm{L}$ of soluble carbohydrates.

\subsubsection{Sample Analysis: Sludge Cake Following Freeze-Thaw}

Following freeze-thaw dewatering, the TS, VS and fecal coliform concentrations of the sludge cake were measured. The methods used to measure each of these parameters are presented below.

\section{TS and VS}

Following freeze-thaw with gravity meltwater drainage, the TS and VS of the sludge cake remaining was measured according to Standard Method 2540 G (APHA, 2005), as outlined earlier in section 4.1.4.1, on page 91 . However, due to $>85 \%$ reduction in sludge volume following freeze-thaw with meltwater drainage, there was only approximately $30 \mathrm{~g}$ of sludge cake remaining for analysis. Since $10.0 \mathrm{~g}$ of the sludge cake was required for fecal coliform enumeration, the mass of sludge cake used for each TS/VS replicate varied from approximately 6 to $8 \mathrm{~g}$, which is less than the recommended $25 \mathrm{~g}$ minimum. However, the results for these tests were generally quite consistent, with only normal variation among the sample replicates. The data points on the figures 
represent the average of the three replicates, and the error bars represent the $95 \%$ confidence limits.

\section{Fecal Coliform}

The enumeration of fecal coliform in the sludge cake remaining following freeze-thaw with meltwater drainage was performed using MTF with A-1 medium, according to EPA method 1681 (U.S. EPA 2005a). All reagents required were prepared according to the procedures previously outlined in section 4.1.4.2, on page 92. However, as mentioned in the previous section, following freeze-thaw with meltwater drainage, the mass of sludge cake remaining in each of the sample bottles was approximately $30 \mathrm{~g}$. Since a portion of the mass remaining was required to determine the TS and VS of the samples, only $10.0 \mathrm{~g}$ was used to enumerate fecal coliform, instead of the recommended $30 \mathrm{~g}$. For each of the sludge cake samples, $10.0 \mathrm{~g}$ was added to a blender containing $90 \mathrm{~mL}$ of sterilised PBS. The sample was blended on high for 2 minutes, and poured into a flask

representing the $10^{-1}$ dilution. Subsequent serial dilutions were prepared from this homogenised and diluted sample, as outlined previously, and the remaining steps used to enumerate the fecal coliform in the sludge cake samples was the same as the method outlined earlier in section 4.1.4.2, on page 92.

\subsubsection{Sample Analysis: Meltwater Collected During Sludge Thaw}

Following the designated freezing periods, the samples were removed from the freezer and thawed at room temperature for 12 hours, as described earlier. During the 12-hour thaw period, the entire volume of meltwater from each sample was collected. Following 
the 12-hour thaw period, the meltwater samples were inverted several times to mix, then grab samples were withdrawn for subsequent analysis. The concentrations of fecal coliform, sCOD, soluble proteins, soluble carbohydrates, $\mathrm{pH}$ and turbidity were measured for all meltwater samples. Below is a summary of the methods used.

\section{Fecal Coliform}

Enumeration of fecal coliform was performed using MTF with A-1 medium, according to EPA method 1681 (U.S. EPA 2005a), as outlined in section 4.1.4.2, on page 92. Since the meltwater samples were liquid, the undiluted samples were homogenised in a blender for 2 minutes, and a $100 \mathrm{~mL}$ portion was poured into a flask representing the undiluted sample ( $10^{\circ}$ dilution). $10 \mathrm{~mL}$ of this sample was then added to $90 \mathrm{~mL}$ of sterilised PBS, representing the $10^{-1}$ sample, and a subsequent set of serial dilutions were performed as previously described. The remaining procedures used for fecal coliform enumeration were presented earlier in section 4.1.4.2, on page 92.

\section{sCOD, Soluble Proteins and Soluble Carbohydrates}

Each of the methods used to measure the concentrations of SCOD, soluble proteins and soluble carbohydrates were previous described in section 4.3.4.2 on pages 129,129 and 132 respectively. The soluble component of the meltwater sample, obtained through centrifugation and subsequent filtration, was diluted at a ratio of 1:1 with deionised water, whereas the undiluted soluble samples were used for the soluble protein and soluble carbohydrate tests. 


\section{Turbidity and $\mathrm{pH}$}

The turbidity was measured using a HACH Model 2100AN Turbidimeter (Hach Company, Loveland $\mathrm{CO}, \mathrm{USA}$ ), and the $\mathrm{pH}$ was measured using a Thermo Orion 5-star bench-top meter kit and ROSS ultra pH electrode (Thermo Fisher Scientific Inc., Rockford, IL, USA). When not in use, the $\mathrm{pH}$ probe was stored in an electrode storage solution, and prior to each set of tests, the probe was calibrated using 3-point calibration with pH 4.01, 7.00 and 10.01 calibration standards.

Meltwater samples were inverted several times to mix, and $20 \mathrm{~mL}$ of each sample was placed in a labelled $25 \mathrm{~mm}$ sample cell. The sample cells were capped with a screw-on cap, wiped with a Kimwipe, placed in the turbidity meter, and the sample measurements were recorded. Following the turbidity measurement, the screw cap was removed and the $\mathrm{pH}$ was measured using a Thermo Orion 5-star bench-top meter kit and ROSS ultra $\mathrm{pH}$ electrode (Thermo Fisher Scientific Inc., Rockford, IL, USA). The samples were then discarded, the sample cells and $\mathrm{pH}$ probe were rinsed, and the process was repeated two more times to get a total of three replicates for each meltwater sample. Data points on the figures represent the average of the three replicates, and the error bars represent the $95 \%$ confidence interval. However, replicate $\mathrm{pH}$ measurements on the individual samples were generally very consistent, therefore in many cases the error bars on the figures are very small, and difficult to notice. 


\subsubsection{Statistical Analysis}

A two-tailed t-test was used to determine the $p$ values and statistical significance $(p<$ 0.05) of all results. All tests (except fecal coliform enumeration using MTF) were performed in triplicate. The data points on the figures represent the average of the three replicates and the error bars represent the $95 \%$ confidence limits. All MTF tests were carried out in duplicate, using at least four dilutions. Error bars on all figures show the $95 \%$ confidence interval, which is built-in to the MTF test, as described earlier in section 4.1.4.2 on page 95. When the measured fecal coliform concentration of a particular test sample was below the detection limit, the error bars on the particular data point in the figures were replaced with a downward arrow.

\subsection{STABILISATION AND DEWATERING OF PRIMARY SLUDGE USING FERRATE(VI) PRE-TREATMENT FOLLOWED BY FREEZE-THAW IN SIMULATED DRAINAGE BEDS (CHAPTER 8)}

\subsubsection{Summary}

These experiments examined the individual and combined effects of potassium ferrate(VI) additions and freeze-thaw conditioning for the stabilisation and dewatering of primary sludge. Potassium ferrate(VI) additions of 0.5 and $5.0 \mathrm{~g} / \mathrm{L}$ were used as a pretreatment, prior to freeze-thaw. Samples were frozen at $-10,-20$ and $-30^{\circ} \mathrm{C}$, and were kept frozen for 1, 8 and 15 days. After the designated freezing period, the samples were removed from the freezer and thawed at room temperature in a setup which allowed meltwater to be separated from the sludge cake via gravity drainage. 


\subsubsection{Sludge Samples}

The primary sludge used for these experiments was obtained from ROPEC. A different batch of sludge was used for each set of experiments with a different freezing temperature $\left(-10,-20,-30^{\circ} \mathrm{C}\right)$. The initial characteristics of the three sludge batches are presented in Table 4-3. A separate set of follow-up experiments with higher ferrate(VI) concentrations were also conducted using a fourth batch of primary sludge obtained from ROPEC. The initial TS and VS of this sludge batch were 4.6 and $3.5 \%$ respectively, and the concentration of fecal coliform was $9.6 \times 10^{6} \mathrm{MPN} / \mathrm{mL}$ (7.0-log), equivalent to $2.0 \times 10^{8} \mathrm{MPN} / \mathrm{g}$ DS (8.3-log).

Table 4-3: Initial characteristics of the primary sludge samples.

\begin{tabular}{|c|c|c|c|c|}
\hline \multirow[b]{2}{*}{ Parameter } & \multirow[b]{2}{*}{ Units } & \multicolumn{3}{|c|}{ Primary Sludge Batch } \\
\hline & & $\begin{array}{c}\text { Sample A }\left(-10^{\circ} \mathrm{C}\right) \\
\text { AVERAGE }( \pm 95 \% \mathrm{CL})\end{array}$ & $\begin{array}{c}\text { Sample B }\left(-20^{\circ} \mathrm{C}\right) \\
\text { AVERAGE }( \pm 95 \% \mathrm{CL})\end{array}$ & $\begin{array}{c}\text { Sample C }\left(-30^{\circ} \mathrm{C}\right) \\
\text { AVERAGE }( \pm 95 \% \mathrm{CL}) \\
\end{array}$ \\
\hline TS & $\%$ & $2.9 \quad( \pm 0.0)$ & $( \pm 0.1)$ & $( \pm 0.1)$ \\
\hline VS & $\%$ & $( \pm 0.0)$ & $( \pm 0.1)$ & $( \pm 0.1)$ \\
\hline Fecal Coliforms & $\log (\mathrm{MPN} / \mathrm{mL})$ & $( \pm 0.7)$ & $5.3(+0.6,-0.7)$ & $4.5 \quad(+0.5,-0.6)$ \\
\hline Fecal Coliforms & $\log (\mathrm{MPN} / \mathrm{g} \mathrm{DS})$ & $( \pm 0.7)$ & $6.6(+0.6,-0.7)$ & $6.0 \quad(+0.5,-0.6)$ \\
\hline Soluble COD & $\mathrm{g} / \mathrm{L}$ & $( \pm 0.2)$ & $( \pm 0.1)$ & $( \pm 0.1)$ \\
\hline Soluble Proteins & $\mathrm{mg} / \mathrm{L}$ & $( \pm 3)$ & $( \pm 3)$ & $( \pm 2)$ \\
\hline Soluble Carbohydrates & $\mathrm{mg} / \mathrm{L}$ & $( \pm 5)$ & $( \pm 7)$ & $( \pm 2)$ \\
\hline
\end{tabular}

\subsubsection{Pre-Treatment with Potassium Ferrate(VI)}

Primary sludge samples that were pre-treated with potassium ferrate $(\mathrm{VI})$, prior to freeze-thaw, were given $\mathrm{LD}=0.5 \mathrm{~g} / \mathrm{L}$ or $\mathrm{HD}=5.0 \mathrm{~g} / \mathrm{L}$ of potassium ferrate(VI). Following the addition of the potassium ferrate(VI) doses, the samples were stirred using a 
magnetic stirrer for 15 minutes, and then a $10 \mathrm{~mL}$ portion was immediately withdrawn and diluted in $90 \mathrm{~mL}$ of sterilised PBS. This $10^{-1}$ dilution was used later for fecal coliform enumeration.

\subsubsection{Freeze-Thaw Treatment}

$200 \mathrm{~mL}$ primary sludge samples were dispensed into the same $500 \mathrm{~mL}$ wide-mouth bottles used in the previous research chapter. The bottles were sealed using the screwon lids and inverted as shown previously in Figure 4-5a. The inverted bottles were then placed in a freezer which was set to either $-10,-20$ or $-30^{\circ} \mathrm{C}$. The temperature inside the freezer and the temperature at the centre of a $200 \mathrm{~mL}$ test sample were monitored using a Traceable ${ }^{\circledR}$ dual-channel thermometer with type $\mathrm{K}$ thermocouples and computer output, and the temperatures were recorded on a PC every minute using the data acquisition software. The resulting average freezing rates generated using freezer temperatures of $-10,-20$ and $-30^{\circ} \mathrm{C}$ were approximately 3,6 and $10 \mathrm{~mm} / \mathrm{hr}$ respectively. Plots of the temperature logs used to estimate the freezing rates are presented in Appendix B4 on page 383. These are within the optimal range for pathogen reduction and particle migration leading to improved dewaterability (Wang et al., 2001, Sanin et al., 1995, Hung et al., 1997). After the samples were completely frozen, they were kept under the freezing conditions for an additional 1,8 or 15 days. After the designated freezing period, the samples were removed from the freezer, the solid lid was replaced with a perforated lid that was lined with a geotextile drainage fabric, and the tape sealing the air hole was removed. The frozen samples were then placed upside down in a funnel, which rested in the opening of a $50 \mathrm{~mL}$ graduated cylinder, as shown in Figure 
4-5b. All samples thawed at room temperature $\left(22-23^{\circ} \mathrm{C}\right)$ for 12 hours, and the meltwater was collected via gravity drainage.

\subsubsection{Sample Analysis: Initial Sludge and Ferrate(VI)-Treated Sludge}

The TS, VS, fecal coliform, SCOD, soluble proteins and soluble carbohydrates concentrations of the sludge were measured before and after the addition of potassium ferrate.

\subsubsection{TS, VS and Fecal Coliform}

The TS, VS and fecal coliform concentrations of the initial sludge, and the samples following ferrate(VI) treatment, were determined using the same methods as the previous research chapter, which were described earlier in section 4.3.4.2, on page 128.

\subsubsection{2 sCOD, Soluble Proteins and Soluble Carbohydrates}

Similarly, each of the methods used to measure the concentrations of sCOD, soluble proteins and soluble carbohydrates were previous described in section 4.3.4.2 on pages 129,129 and 132 respectively. For sCOD measurements, the soluble component of the meltwater sample, obtained through centrifugation and subsequent filtration, was diluted by a factor of 10 with deionised water, whereas the undiluted soluble samples were used for the soluble protein and soluble carbohydrate tests.

\subsubsection{Sample Analysis: Sludge Cake Following Freeze-Thaw}

Following freeze-thaw dewatering, the TS, VS and fecal coliform concentrations of the sludge cake were measured. 


\subsubsection{TS, VS and Fecal Coliform}

The TS, VS and fecal coliform concentrations of the sludge cake following freeze-thaw treatment were determined using the same methods as the part II experiments in the previous research chapter, and are described in section 4.3.4.3 on pages 135, 135 and 136 respectively.

\subsubsection{Sample Analysis: Meltwater Collected During Sludge Thaw}

Following the 12-hour thaw period, the meltwater samples collected were inverted several times to mix, then grab samples were withdrawn for subsequent analysis. The concentrations of fecal coliform, sCOD, soluble proteins, soluble carbohydrates, $\mathrm{pH}$ and turbidity were measured for all meltwater samples.

\subsubsection{Fecal Coliform}

Similar to previous chapters, enumeration of fecal coliform was performed using MTF with A-1 medium, according to EPA method 1681 (U.S. EPA 2005a), as outlined in section 4.1.4.2, on page 92. Since the meltwater samples were liquid, the undiluted samples were homogenised in a blender for 2 minutes, and a $100 \mathrm{~mL}$ portion was poured into a flask representing the undiluted sample, from which a subsequent set of serial dilutions were prepared as previously described. The remaining procedures used for fecal coliform enumeration were presented earlier in section 4.1.4.2, on page 92.

\subsubsection{2 sCOD, Soluble Proteins and Soluble Carbohydrates}

Each of the methods used to measure the concentrations of SCOD, soluble proteins and soluble carbohydrates were previous described in section 4.3.4.2 on pages 129,129 and 
132 respectively. The soluble component of the meltwater sample, obtained through centrifugation and subsequent filtration, was diluted by a factor of 10 with deionised water, whereas the undiluted soluble samples were used for the soluble protein and soluble carbohydrate tests.

\subsubsection{Turbidity and $\mathrm{pH}$}

The turbidity and $\mathrm{pH}$ of the meltwater samples were measured using the same procedures as the previous research chapter, and are presented in section 4.3.4.4, on page 138.

\subsubsection{Statistical Analysis}

A two-tailed t-test was used to determine the $p$ values and statistical significance $(p<$ 0.05) of the results. All tests (except fecal coliform enumeration using MTF) were performed in triplicate. The data points on the figures represent the average of the three replicates and the error bars represent the $95 \%$ confidence limits. All MTF tests were carried out in duplicate, using at least four dilutions. Error bars on all figures show the $95 \%$ confidence interval, which is built-in to the MTF test, as described earlier in section 4.1.4.2 on page 95. When the measured fecal coliform concentration of a particular test sample was below the detection limit, the error bars for the particular data point in the figures were replaced with a downward arrow. 


\subsection{OXIDATION OF SELECTED HORMONES IN ANAEROBICALLY DIGESTED (AD) SLUDGE (CHAPTER 9)}

\subsubsection{Summary}

The purpose of the study was to evaluate the ability of ferrate(VI) to reduce the concentration of selected hormones in sludge. Two $100 \mathrm{~mL}$ anaerobically digested sludge samples were spiked with ten different hormones. The first sludge sample was spiked with a low concentration range of hormones $(3-75 \mathrm{ng} / \mathrm{mL})$, and the second sludge sample was spiked with a high concentration range (12-300 ng/L). Sub-samples from each of these were then treated with either 0.5 or $1.0 \mathrm{~g} / \mathrm{L}$ of potassium ferrate(VI).

\subsubsection{Hormone Stock Solutions}

The hormones used in these experiments were seven estrogens: estrone (E1) ( $\geq 99)$, $17 \alpha$-estradiol ( $\geq 98), 17 \beta$-estradiol (E2) ( $\geq 98 \%), 17 \alpha$-ethinylestradiol (EE2) ( $\geq 98 \%$ ), estriol (E3) ( $\geq 97 \%)$, mestranol (99\%), and equilin ( $\geq 99)$, the progestins (synthetic progestogens): norethindrone ( $\geq 98 \%)$ and norgestrel $(\geq 98 \%)$, and the natural androgen: testosterone ( $\geq 99 \%)$. All hormones were purchased from Sigma-Aldrich, Co. (St. Louis, MO, USA).

To prepare the hormone stock solutions, $0.5 \mathrm{mg}$ of each hormone was weighed using a Sartorius MC21S digital balance (Sartorius Canada Inc., Mississauga, ON), and placed in $0.5 \mathrm{~mL}$ sample vials. $0.5 \mathrm{~mL}$ of methanol was added to each vial, and the resulting 1 $\mathrm{mg} / \mathrm{mL}$ solutions were mixed using a vortex mixer (Baxter Diagnostics Inc., IL, USA). Most of the hormone solutions were diluted a second time. For these samples, $0.05 \mathrm{~mL}$ portions of the $1 \mathrm{mg} / \mathrm{mL}$ solution were added to $0.45 \mathrm{~mL}$ of methanol; to create the 
$100,000 \mathrm{ng} / \mathrm{mL}$ stock solutions. All stock solutions were stored in the freezer until required.

\subsubsection{Sludge Sample and Hormone Spiking}

Anaerobically digested sludge, obtained from ROPEC, was used in these experiments. Since conventional biological treatment processes, such as anaerobic digestion, are not reliably effective at removing EDCs (Li and Zhang, 2014, Hamid and Eskicioglu, 2013 and 2012), these experiments aimed to determine whether hormones in sludge can be specifically targeted and removed using small amounts of ferrate(VI) following biological sludge treatment. The initial TS and VS concentrations of the sludge were 20.3 and 11.7 $\mathrm{g} / \mathrm{L}$, equivalent to 2.0 and $1.2 \%$ respectively. Two $100 \mathrm{~mL}$ sludge samples were placed in $250 \mathrm{~mL}$ Erlenmeyer flasks, and stirred using a magnetic stirrer. Individually prepared stock solutions containing the hormones were removed from the freezer and thawed at room temperature. Once the vials of stock solutions were completely thawed and warmed to room temperature, a vortex mixer was used to thoroughly mix the stock solutions. The concentration of each hormone stock solution was $10^{5} \mathrm{ng} / \mathrm{mL}$, except for norgestrel, which was $10^{6} \mathrm{ng} / \mathrm{mL}$. The spiked initial concentration of each hormone in the low concentration and the high concentration sludge samples are presented in Table 4-4. Following the addition of the selected hormones, the sludge samples were stirred using a magnetic stirrer for 4 hours. Three subsets from each of the two sludge samples were removed. The first subset was used to measure the initial concentration of hormones, and the other two were used for subsequent ferrate(VI) treatments. 
Table 4-4: Spiked concentrations of the selected hormones in the low concentration and high concentration sludge samples used for subsequent tests with potassium ferrate(VI).

\begin{tabular}{|l|c|c|}
\hline \multicolumn{1}{|c|}{ Hormone } & \multicolumn{2}{|c|}{ Spiked Concentration $(\mathbf{n g} / \mathbf{m L})^{*}$} \\
\hline Equilin & Low Concentration Range & High Concentration Range $^{*}$ \\
\hline Estrone (E1) & 15 & 120 \\
\hline $17 ß-E s t r a d i o l(E 2)$ & 15 & 60 \\
\hline $17 \alpha$-Estradiol & 15 & 60 \\
\hline $17 \alpha$-Ethinylestradiol (EE2) & 15 & 60 \\
\hline Estriol (E3) & 60 & 60 \\
\hline Mestranol & 75 & 240 \\
\hline Norethindrone & 15 & 300 \\
\hline Norgestrel & 15 & 60 \\
\hline Testosterone & 3 & 60 \\
\hline
\end{tabular}

${ }^{*} \mathrm{ng} / \mathrm{mL}$ is equivalent to parts per billion ( $\left.\mathrm{ppb}\right)$

\subsubsection{Ferrate(VI) Treatment}

Ferrate(VI) treatments were conducted using Ferratec Brand ${ }^{\mathrm{TM}}>90 \%$ pure potassium ferrate salt $\left(\mathrm{K}_{2} \mathrm{FeO}_{4}\right)$. Two levels of ferrate( $\left.\mathrm{VI}\right)$ treatment were used: a low dose (LD) of $0.5 \mathrm{~g} / \mathrm{L}$ and a high dose (HD) of $1.0 \mathrm{~g} / \mathrm{L}$ of potassium ferrate(VI). The equivalent ferrate(VI) ion added was approximately $0.3(>0.27)$ and $0.6(>0.55) \mathrm{mg} / \mathrm{L} \mathrm{of} \mathrm{FeO}_{4}{ }^{-2} .40$ $\mathrm{mL}$ sludge samples were treated with 20 and $40 \mathrm{mg}$ of potassium ferrate(VI) salt, and the samples were mixed using a vortex mixer for 5 minutes. The samples were then prepared for over-night shipment to the analytical laboratory.

\subsubsection{Sample Analysis}

All samples were placed in designated sample bottles (provided by AXYS), and into a well-insulated cooler that was filled with frozen cooler packs. The cooler was then shipped overnight to Sidney, BC, Canada. Axys Analytical Services Ltd. in Sidney, BC, Canada performed the extraction of hormones from the sludge samples, and measured 
the concentrations of hormones using AXYS Method MLA-072 on a high performance liquid chromatograph coupled to a triple quadrupole mass spectrophotometer (LCMS/MS). The reporting limits for the method range from 0.3 to $150 \mathrm{ng} / \mathrm{g}$ based on a $1 \mathrm{~g}$ solid sample, and from 0.3 to $150 \mathrm{ng} / \mathrm{L}$ for a $1 \mathrm{~L}$ aqueous sample. A summary of the method is presented below. All information related to the analysis of hormones including hormone extraction and clean-up, sample analysis via LC-MS/MS, and the quantification of hormones has been taken from the Method Summary of AXYS Method MLA-072 Revision 03 Version 02 (AXYS, 2012) and is proprietary.

\subsubsection{Extraction and Cleanup}

Prior to hormone extraction and clean-up procedures, the $\mathrm{pH}$ of each sample was adjusted to 2.0, and each sample was spiked with the labelled surrogates $\left(d_{6^{-}}\right.$ norethindrone, $d_{6}$-norgestrel, $d_{9}$-progesterone, $d_{4}-17 \beta$-estradiol, $d_{4}$-ethinylestradiol) associated with each of the hormones.

The aqueous phase of each sample was obtained through filtration, to remove all particulates. The solid phase of each sample was extracted by sonication with aqueous buffered acetonitrile and pure acetonitrile, then concentrated using rotary evaporation, and diluted to a volume of $200 \mathrm{~mL}$ with ultra-pure water. The aqueous samples and the diluted extracts from the solid phase were cleaned-up using solid phase extraction (SPE), and the recovery standards $\left({ }^{13} \mathrm{C}_{6}{ }^{13} \mathrm{C}_{3}\right.$-atrazine, 2,4,5-trichlorophenooxyacetic acid) were added to the extracts. After the addition of the recovery standards, the samples were 
filtered, then analysed using a high performance liquid chromatograph coupled to a triple quadrupole mass spectrophotometer (LC-MS/MS).

\subsubsection{Instrumental Analysis}

The analysis was performed in positive and negative electrospray ionisation (ESI) modes, requiring a total of two runs on the liquid chromatograph to analyze the complete set of hormones. Positive ESI hormones analysed include estriol (E3), mestranol, norethindrone, norgestrel and testosterone, which were spiked, as well as allyl trembolone, androstenedione, androsterone, desogestrel and progesterone, which were not spiked. Negative ESI hormones analysed include equilin, estrone, 17 $\beta$ estradiol, $17 \alpha$-estradiol and $17 \alpha$-ethinylestradiol, which were spiked, as well as $17 \alpha$ dihyroequilin and equilenin, which were not spiked.

Instrument calibration was performed using a series of calibration standards to generate six data points for each of the hormones, including the labelled surrogate standards, covering the working range of the instrument $(0.2-3000 \mathrm{ng} / \mathrm{mL})$. The calibration runs were used to determine the mean retention time and percent recovery for each of the target hormones. The LC-MS/MS was run in multiple reaction monitoring (MRM) mode, using an injection volume of $10 \mu \mathrm{L}$. Quantification was performed by converting the peak areas of the applicable daughter ions for each of the specified parent ion to daughter ion transitions, from each of the sample chromatograms. 


\subsubsection{Hormone Identification}

Positive identification of each target hormone, surrogate standard and recovery standard required a measured concentration above the concentration of the lowest calibration standard used, and a signal to noise ratio $\geq 3: 1$ for the parent ion to daughter ion transition. In addition, the retention time for the particular target hormone had to fall within 0.4 minutes ( 24 seconds) of the mean retention time determined using the calibration standards, and for the labelled surrogates, the retention time had to fall within 0.1 minutes ( 6 seconds) of the mean retention time determined during calibration.

\subsubsection{Reported Results}

The specific detection limits $(S D L)$ for each sample were calculated using 3 times the signal of the noise recorded, and converting it to an equivalent concentration. The reporting limit for each target compound was defined as the concentration of the lowest calibration standard used, which was also greater than the method detection limit and the SDL.

The results for each of the samples were reported on a dry weight (ng/g dry solids) basis, so the results were converted to a wet basis using the total solids (TS) concentrations of the representative samples. The TS and volatile solids (VS) concentrations were measured according to standard method 2540 G (APHA, 2005). 


\section{FREEZE-THAW TREATMENT OF ROTATING BIOLOGICAL CONTACTOR (RBC) SLUDGE FROM A REMOTE MINING EXPLORATION FACILITY IN SUBARCTIC CANADA}

James Diak ${ }^{*}$, Banu Örmeci ${ }^{*}$ and Corinne Proux ${ }^{* *}$

*Department of Civil and Environmental Engineering, Carleton University, 1125 Colonel By Drive, Ottawa, ON K1S 5B6, Canada.

${ }^{* *}$ Seprotech Systems Incorporated, 2378 Holly Lane, Ottawa, ON K1V 7P1, Canada.

\subsection{ABSTRACT}

Freeze-thaw conditioning of RBC (Rotating Biological Contactor) sludge was tested using a pilot-scale freezing bed placed in a mobile freezer operated at $-10^{\circ} \mathrm{C}$. Sludge samples from a remote mining exploration facility were flown in every 2 weeks, and added to the freezing bed in 8 layers of $10 \mathrm{~cm}$ thick. Approximately 4 months after the first layer of sludge was added, the pilot unit was removed from the freezer and thawed at ambient temperatures. After one day of thawing, the solids concentration increased from $2.6 \%$ to $16.2 \%$. The final cake solids concentration was $21 \%$. Meltwater had increasing turbidity, COD, TSS, VSS, nitrogen and phosphorus concentrations during the thawing period. Freeze-thaw conditioning also decreased the initial densities of fecal coliform 
and Salmonella in sludge. The results of this study showed that freeze-thaw technology successfully dewatered RBC sludge without the need for mechanical equipment, and is a sustainable option for sludge dewatering in cold and remote regions.

KEYWORDS: Freeze-thaw, land application, RBC (rotating biological contactor), sludge, treatment.

\subsection{INTRODUCTION}

Sludge dewatering is one of the most challenging processes during sludge treatment. Particularly in cold climates, mechanical sludge dewatering becomes a major problem for treatment plants because the equipment is difficult to maintain and ices up frequently in subfreezing temperatures. In addition, supplying chemicals and dewatering aids can be expensive particularly for treatment plants in remote locations.

Attention has been placed on freeze-thaw technology in an attempt to solve the dewatering problem in cold regions. Freeze-thaw conditioning is a simple, effective, and low-cost technology when freezing is achieved naturally. Freeze-thaw technology works on the principal that ice crystals grow by incorporating water molecules only. Because the structure of ice crystal is highly organized and symmetrical, it cannot accommodate any other atoms or molecules. Each ice crystal continues to grow as long as water 
molecules are available. All other impurities and solids are forced to the boundaries of the ice crystal, where they become compressed or dehydrated (Chalmers, 1959).

Hoekstra and Miller (1967) hypothesized that the layer of surface water surrounding the sludge particles allows them to move along the growing ice front. This means that smaller particles are more easily forced from the ice crystal, whereas larger particles are more likely to become entrapped in the ice crystal. Similarly, high freezing rates can reduce the effectiveness of freeze-thaw dewatering by entrapping particles within the ice front (Corte, 1962). Therefore, freezing temperatures greater than $-10^{\circ} \mathrm{C}$ are generally used to generate slow-growing ice crystals, capable of moving sludge solids along the ice front. Slow growing ice crystals result in a network of continuous channels throughout the consolidated sludge particles (Hung et al., 1996). During thaw, the meltwater drains freely throughout the network of channels, leaving a dewatered sludge.

Sludge characteristics play a role in determining the effectiveness of freeze-thaw conditioning. Freeze-thaw conditioning dramatically converts alum sludge from a fine particle suspension to a mixture of clear water and granular particles (Martel and Diener, 1991). Presence of dissolved ions and extracellular polymers in activated sludge adversely affects the freezing process (Örmeci and Vesilind, 2002; Örmeci, 2004). Removal of these constituents from activated sludge substantially improves the effectiveness of freeze-thaw resulting in better dewaterability and meltwater quality (Örmeci and Vesilind, 2001). 
Previous research has shown that freeze-thaw technology can be effective at reducing the quantity of pathogens and indicator bacteria in sludge (Sanin et al., 1994). Freezing temperature, storage time, and freeze-thaw cycles affected the survival of Escherichia coli and Enterococcus faecalis, and greater inactivation efficiencies were achieved under longer storage time and higher freezing temperatures (Gao et al., 2006; Gao et al, 2009). It was suggested that longer freezing times result in cell dehydration (Mazur, 1965).

Sludge freezing beds together with a storage facility, such as a lagoon, tank, or digester to store the sludge in summer, can be used as the sole method of dewatering in cold regions. In temperate climates, freezing beds can be used in combination with drying beds to freeze the sludge in winter and dry it in summer. Most of the United States and all of Canada can potentially use freezing beds. Freeze-thaw technology is particularly a viable and sustainable option for facilities located in remote and cold regions.

The goal of this study was to evaluate the effectiveness of freeze-thaw treatment on RBC (rotating biological contactor) sludge generated at a remote mining exploration facility, and determine the improvements observed in physical, chemical and biological sludge characteristics after the freeze-thaw. The mining exploration facility is located near Matoush, which is located $950 \mathrm{~km}$ north of Montreal, Quebec in Canada. Freezethaw conditioning was considered as a low-cost and low-maintenance option that can solve the dewatering problem on site. The site is only accessible by air, and there is very limited opportunity for transportation both in summer and winter which limits the treatment options. The site has a subarctic climate with winter temperatures averaging - 
$10^{\circ} \mathrm{C}$ but ranging down to $-40^{\circ} \mathrm{C}$. Summer temperatures are around $15^{\circ} \mathrm{C}$ but can reach $30^{\circ} \mathrm{C}$, allowing the thawing of frozen sludge in summer.

\subsection{MATERIALS AND METHODS}

Wastewater collected from the living areas and eating facilities of the personnel is treated using a portable, containerized wastewater treatment system (Seprotech Systems Incorporated, Canada) which achieves primary and secondary treatment. RBCs (Rotating Biological Contactor) are used for biological treatment in the containerized unit. Figure 5-1(a) shows the remote site in Matoush where the facility is located.

Sludge samples from the RBC unit were flown in every two weeks during the summer months and added to a pilot-scale freezing bed. There were a total of 5 shipments of sludge. Figure 5-1(b) shows the pilot-scale freezing bed, which consists of a vertical freezing bed, a drainage system and underlying meltwater collection basin. The freezing bed was made of stainless steel and had a Plexiglas window to see the layers of frozen sludge. The pilot unit was kept in a mobile freezer at $-10^{\circ} \mathrm{C}$ throughout the freezing period. To prevent clogging, approximately $10 \mathrm{~cm}$ of medium-coarse sand and $10 \mathrm{~L}$ of water were added to the base of the drainage bed and frozen prior to sludge addition. Sludge was frozen in layers approximately $10 \mathrm{~cm}$ thick to ensure complete freezing. The thickness of each frozen sludge layer was measured through the Plexiglas window. A total of 8 layers of sludge were added to the pilot unit over 3 months.

Approximately 4 months after the first layer of sludge was applied, the pilot unit was removed from the freezer and thawed at ambient temperatures varying from 17.5 to 
$26^{\circ} \mathrm{C}$ for 2 weeks. Meltwater was analyzed for total suspended solids (TSS), volatile suspended solids (VSS), total dissolved solids (TDS), turbidity, $\mathrm{pH}$, chemical oxygen demand (COD), nitrogen (total, nitrate and ammonia), phosphorus (total), fecal coliform and Salmonella. Sludge cake samples were analyzed for total solids (TS), volatile solids (VS), COD, fecal coliform and Salmonella.

The following methods were used to analyze the sludge feed and sludge cake: TS and VS using Standard Method 2540 G (APHA, 2005); COD using HACH method 8000 High Range Plus; and fecal coliform and Salmonella using EPA methods 1681 and 1682 respectively (U.S. EPA, 2005a, 2005b). All measurements were carried out in triplicate.

Methods used to analyze the meltwater were: TSS and VSS using Standard Methods 2540 D and 2540 E (APHA, 2005); turbidity using HACH Chemical Model 2100AN turbidity meter; pH using a Thermo Orion 5-Star Benchtop Meter Kit and a Ross Ultra pH electrode; total nitrogen (TN), nitrate and ammonia using $\mathrm{HACH}$ methods 10072,10020 , and 10031 respectively, and total phosphorus (TP) using $\mathrm{HACH}$ method 10127. All measurements were carried out in triplicate. 

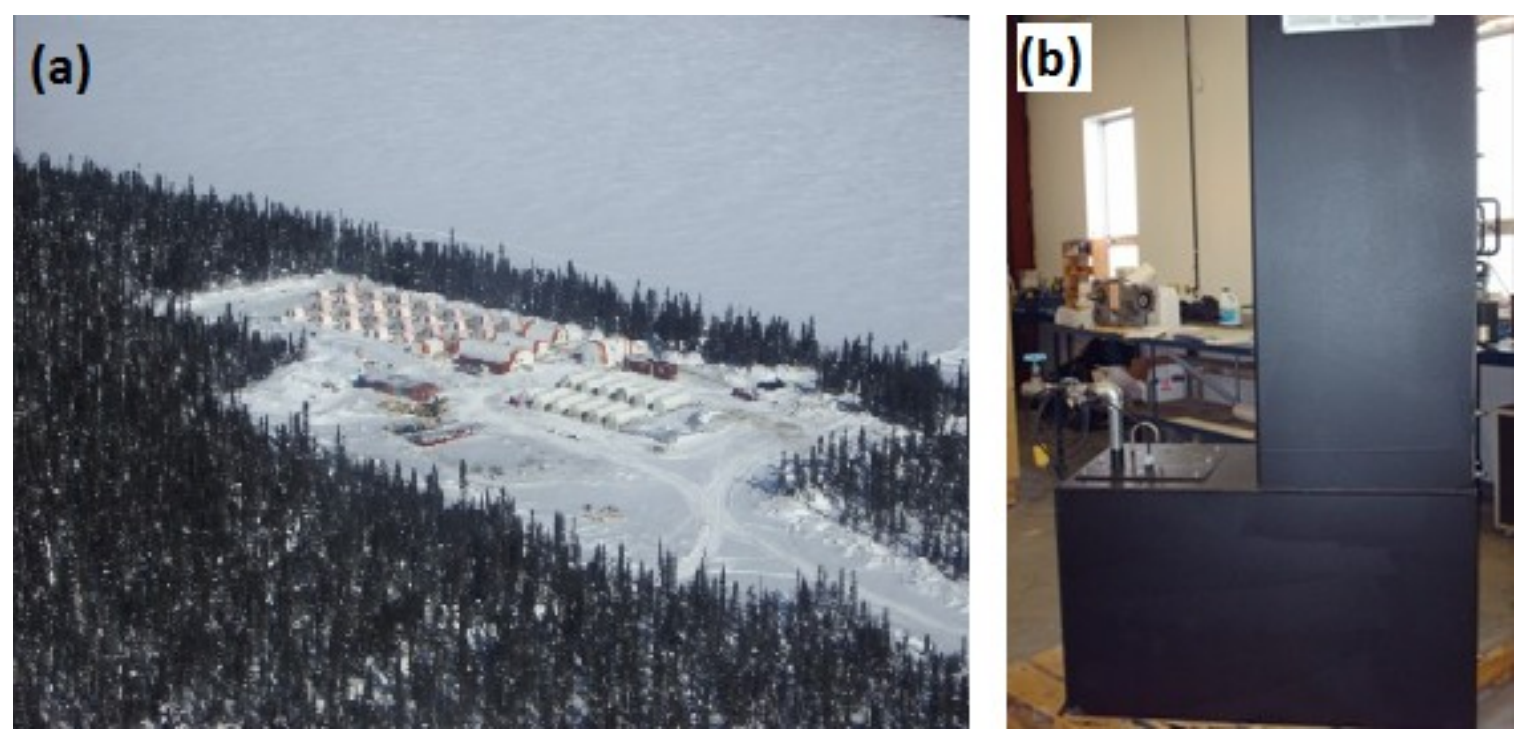

Figure 5-1: (a) Remote mining exploration facility in Matoush, Quebec (Strateco Resources Inc.), (b) Pilot-scale freezing bed used for freeze-thaw conditioning of RBC sludge.

\subsection{RESULTS AND DISCUSSION}

Initial TS of the five shipments of sludge samples from the site varied from 2 to $3 \%$ solids (average $=2.6 \%$ ), initial fecal coliform varied from 150 to $6250 \mathrm{MPN} / \mathrm{g}$ dry solids (average $=1816 \mathrm{MPN} / \mathrm{g}$ dry solids), and the initial Salmonella counts varied from 213 to $308 \mathrm{MPN} / 4 \mathrm{~g}$ dry solids (average $=242 \mathrm{MPN} / 4 \mathrm{~g}$ dry solids). It should be noted that the initial sludge bacteria numbers were lower than expected possibly due to the long transportation time (typically 3-5 days) of the samples from the remote site to the laboratory facility at Carleton University.

Overall, $290 \mathrm{~L}$ of liquid sludge was added to the freezing bed during the study. After 10 days of thawing period at ambient temperatures, $250 \mathrm{~L}$ of meltwater was collected through simple drainage. Figure 5-2 shows the cumulative volume of meltwater collected and ambient temperature during the thawing period. During this time, the average total solids increased from $2.6 \%$ to $19 \%$, and average volatile solids increased 
from $2.3 \%$ to $17.3 \%$. Figure $5-3$ shows TS and VS concentrations of the sludge before and during the thawing period. After an additional month, the cake solids content increased to $21 \%$, and the final cake volume was approximately $35 \mathrm{~L}$, which indicates that roughly $5 \mathrm{~L}$ of water evaporated.

The meltwater collected periodically during the 2 week thawing period and was analyzed for several parameters.

Figure 5-4 shows that TSS and VSS concentrations of the meltwater gradually increased over time. This was expected since the first few meltwater samples (thaw time $=1-2$ days) contained some of the extra water which was added to the base of the freezing bed to prevent clogging, and secondly fines from upper sludge layers were displaced from the sludge cake by the draining meltwater. Two sets of data points on Figure 5-4 (day 7 and 8 ) do not show error bars because the sample replicates were contaminated during sample filtration and drying. 


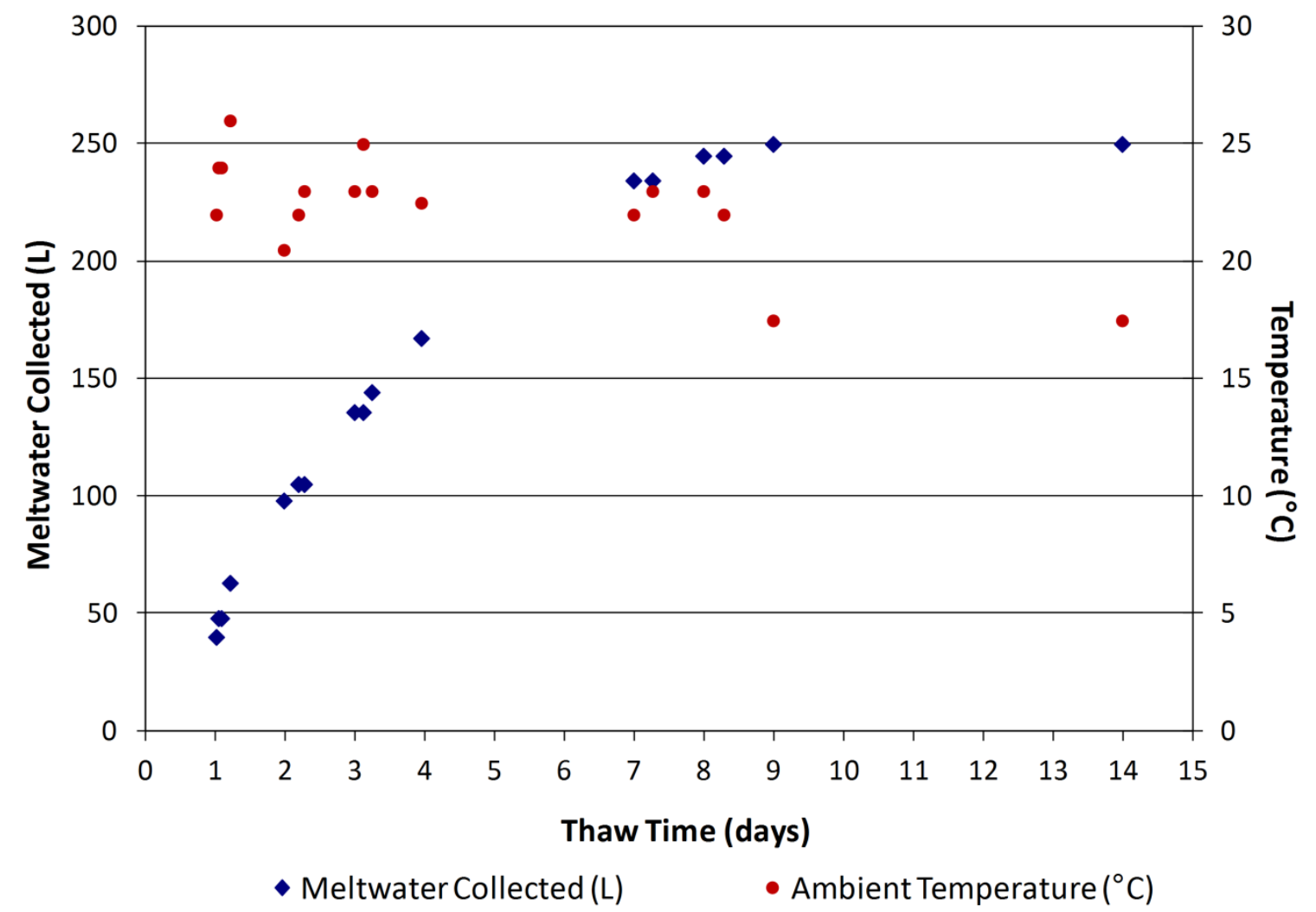

Figure 5-2: Volume of meltwater collected during thaw at ambient temperatures. 


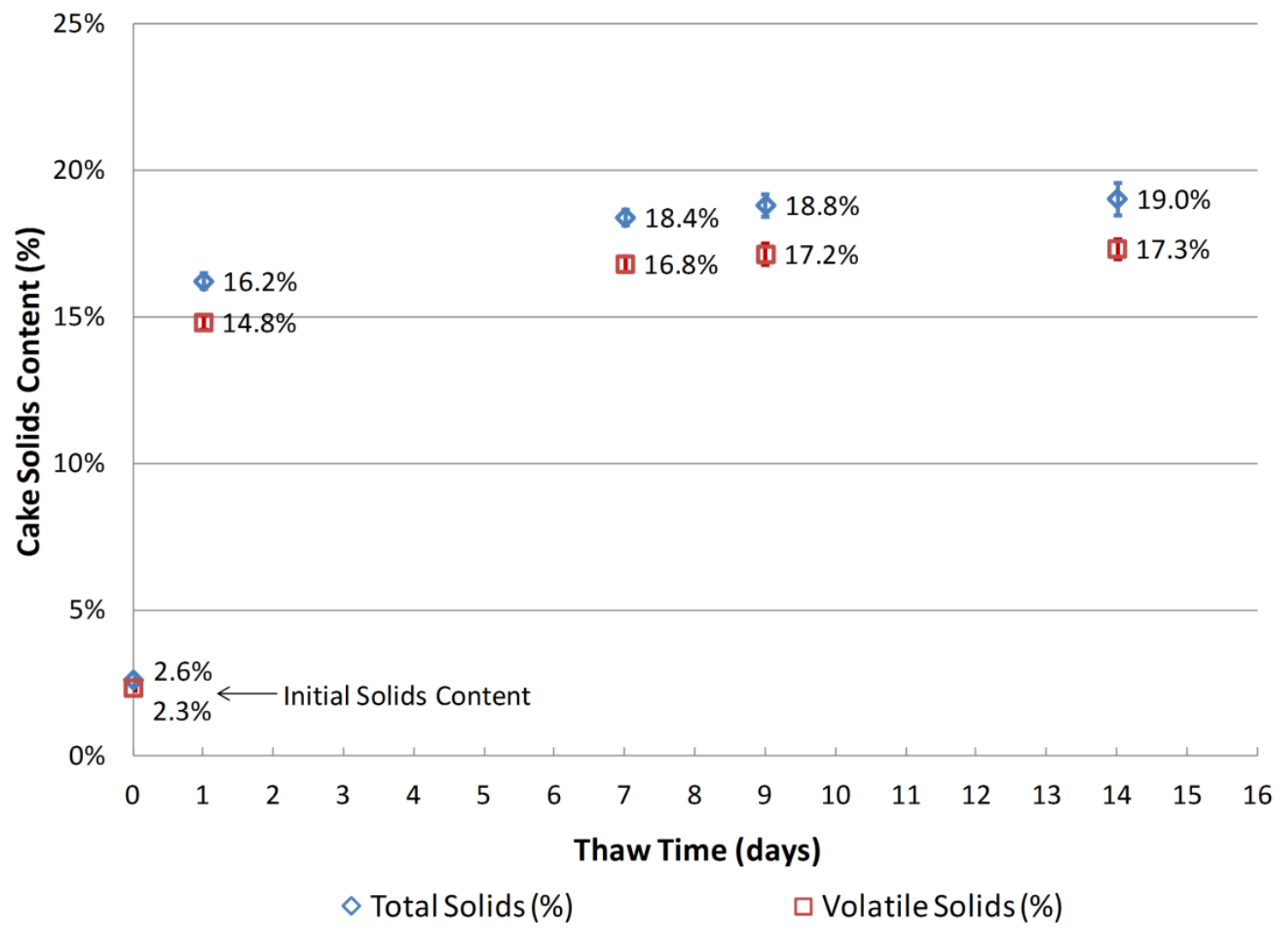

Figure 5-3: Total solids and volatile solids content of the sludge cake during thaw. 


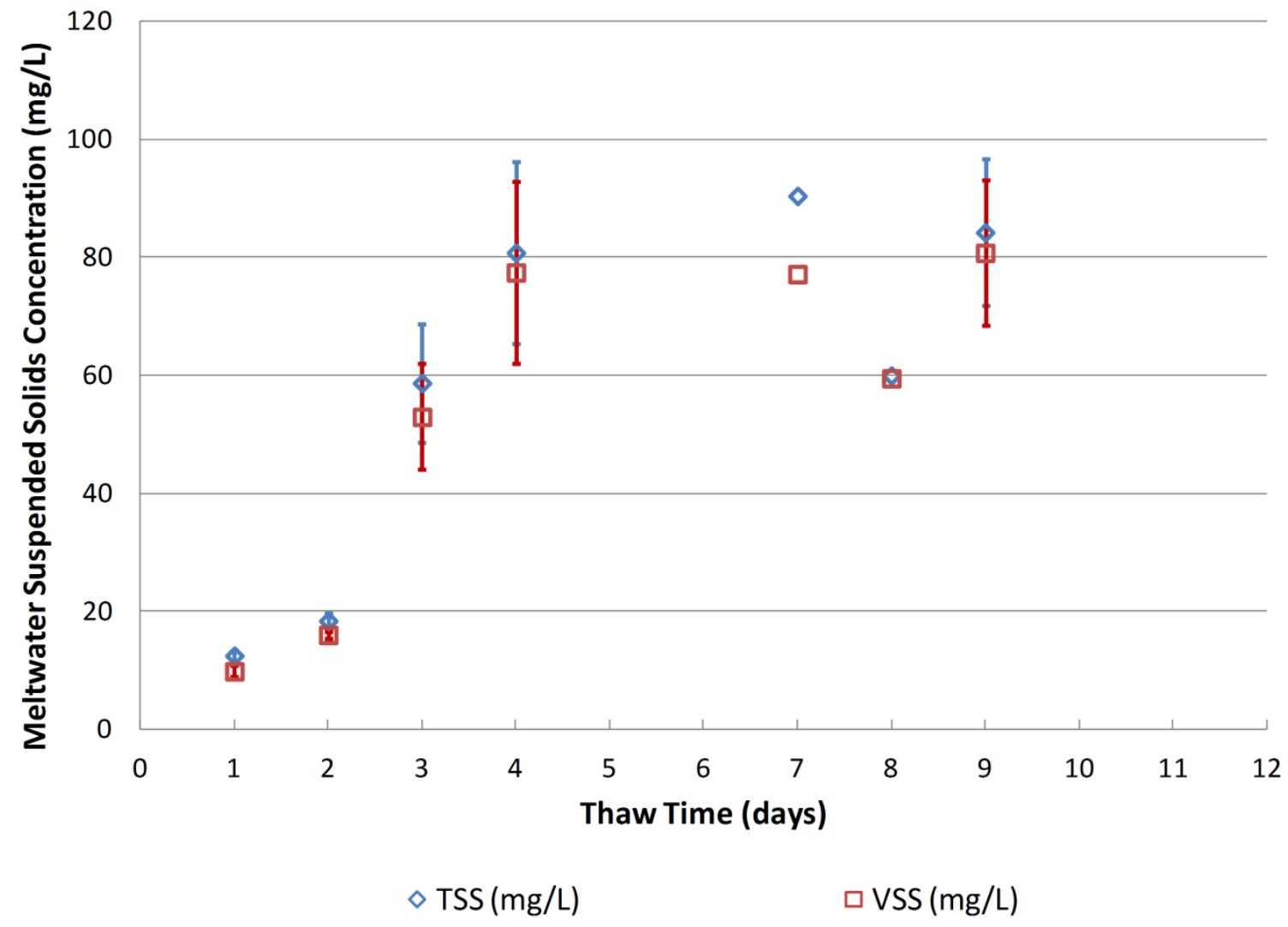

Figure 5-4: TSS and VSS concentrations of the meltwater during thaw.

Figure 5-5 shows the COD concentrations in the meltwater. The average COD concentration was calculated as $3,100 \mathrm{mg} / \mathrm{L}$. Similar to the TSS concentrations, the COD of the meltwater increased during the first 4 days of the thawing period. After the first day of thawing, $50 \mathrm{~L}$ of meltwater was collected with an average COD of $1300 \mathrm{mg} / \mathrm{L}$. The following day, another $15 \mathrm{~L}$ of meltwater was collected with a COD around $3000 \mathrm{mg} / \mathrm{L}$. Peak COD concentrations in the meltwater occurred after 2-3 days of thawing, and were around $5000 \mathrm{mg} / \mathrm{L}$. In the following days, the meltwater COD concentrations decreased, and after 8-9 days of thawing, the COD of the meltwater was around $2000 \mathrm{mg} / \mathrm{L}$. In addition to the meltwater CODs, the total COD of the feed sludge and the total COD of the cake were also measured ( $1.84 \pm 0.3$ and $1.76 \pm 0.1 \mathrm{~g} / \mathrm{g}$ TS respectively), and showed 
that majority of the organic substances remained in the cake during thawing and did not pass into the meltwater.

Figure 5-6 shows the measured TP, TN, nitrate and ammonia concentrations of the meltwater collected during the thawing period. TP, TN and ammonia concentrations increased in the first week but then levelled off or slightly decreased in the following days. As expected, majority of the total nitrogen was contributed by ammonia, and the nitrate concentrations were close to zero. The highest TP concentration was $90 \mathrm{mg} / \mathrm{L}$ and the highest TN concentration was $465 \mathrm{mg} / \mathrm{L}$.

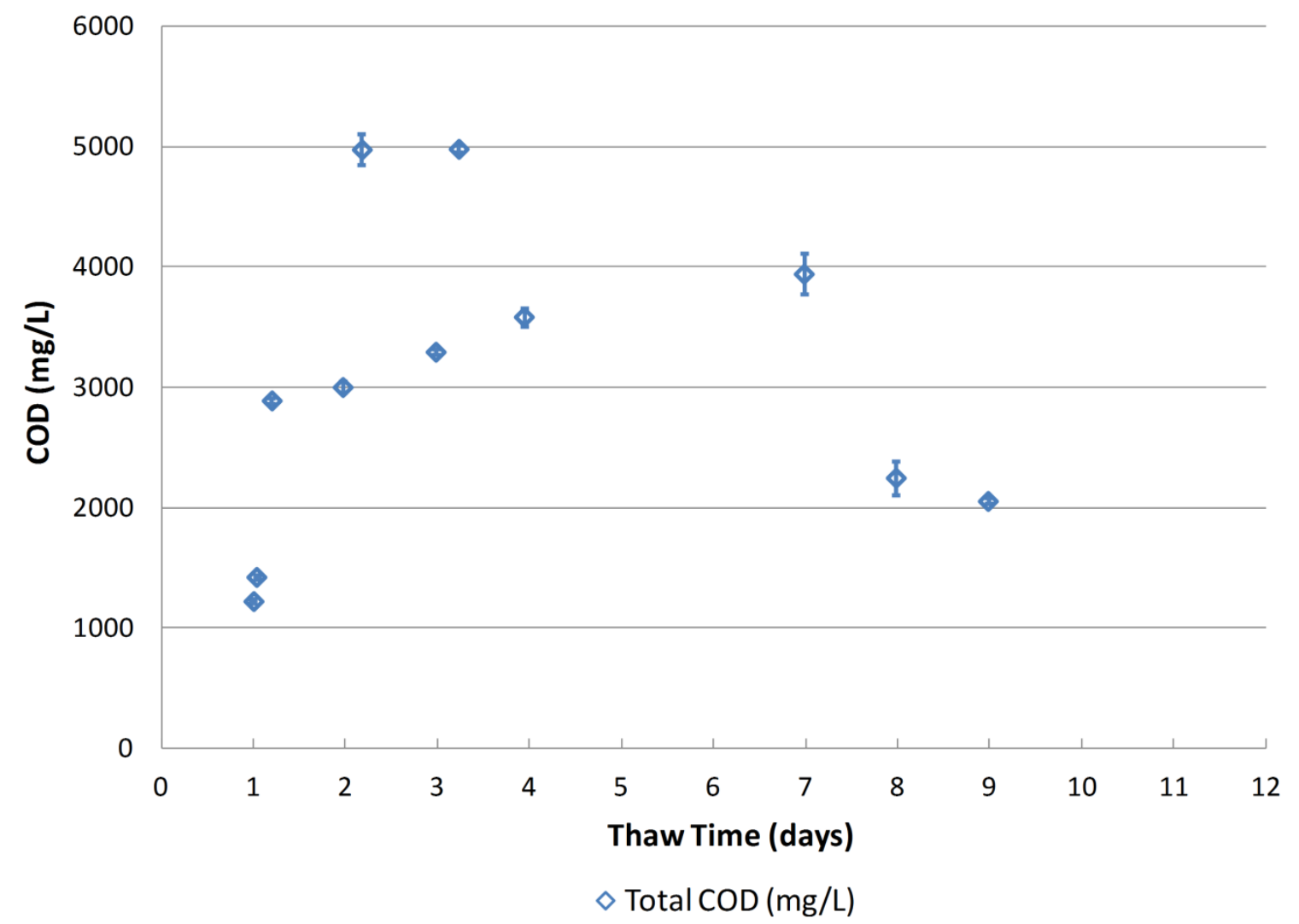

Figure 5-5: Total COD concentrations of the meltwater during thaw. 


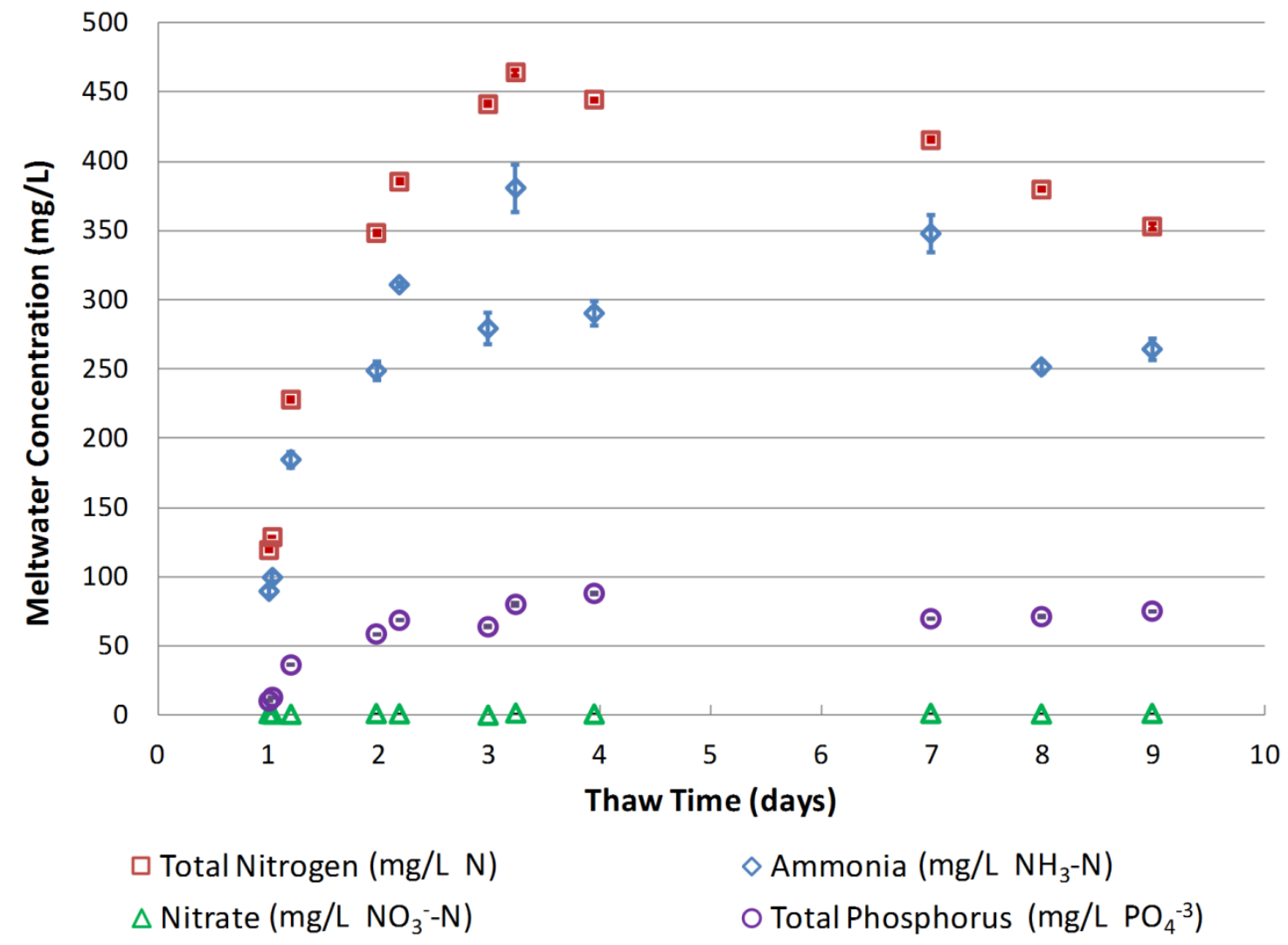

Figure 5-6: TP, TN, ammonia and nitrate concentrations of the meltwater during thaw.

Figure 5-7 shows the turbidity and $\mathrm{pH}$ of the meltwater during the thawing period. Turbidity measurements on the meltwater samples collected during the first day were all less than 30 NTU. After 2 days, the turbidity increased slightly to 40 NTU and after 3 days the turbidity was around 60 NTU. After 4 days however, the turbidity of the meltwater increased to $125 \mathrm{NTU}$, and eventually reached a maximum of $340 \mathrm{NTU}$ after 8 days of thawing. The gradual increase in turbidity is due to fines being washed down with the meltwater, with microbial growth also potentially playing a role. The average $\mathrm{pH}$ of the meltwater collected during the first week of thawing was 5.7, and 6.5 during the second week. The standard deviation for each $\mathrm{pH}$ measurement was less than 0.3. 


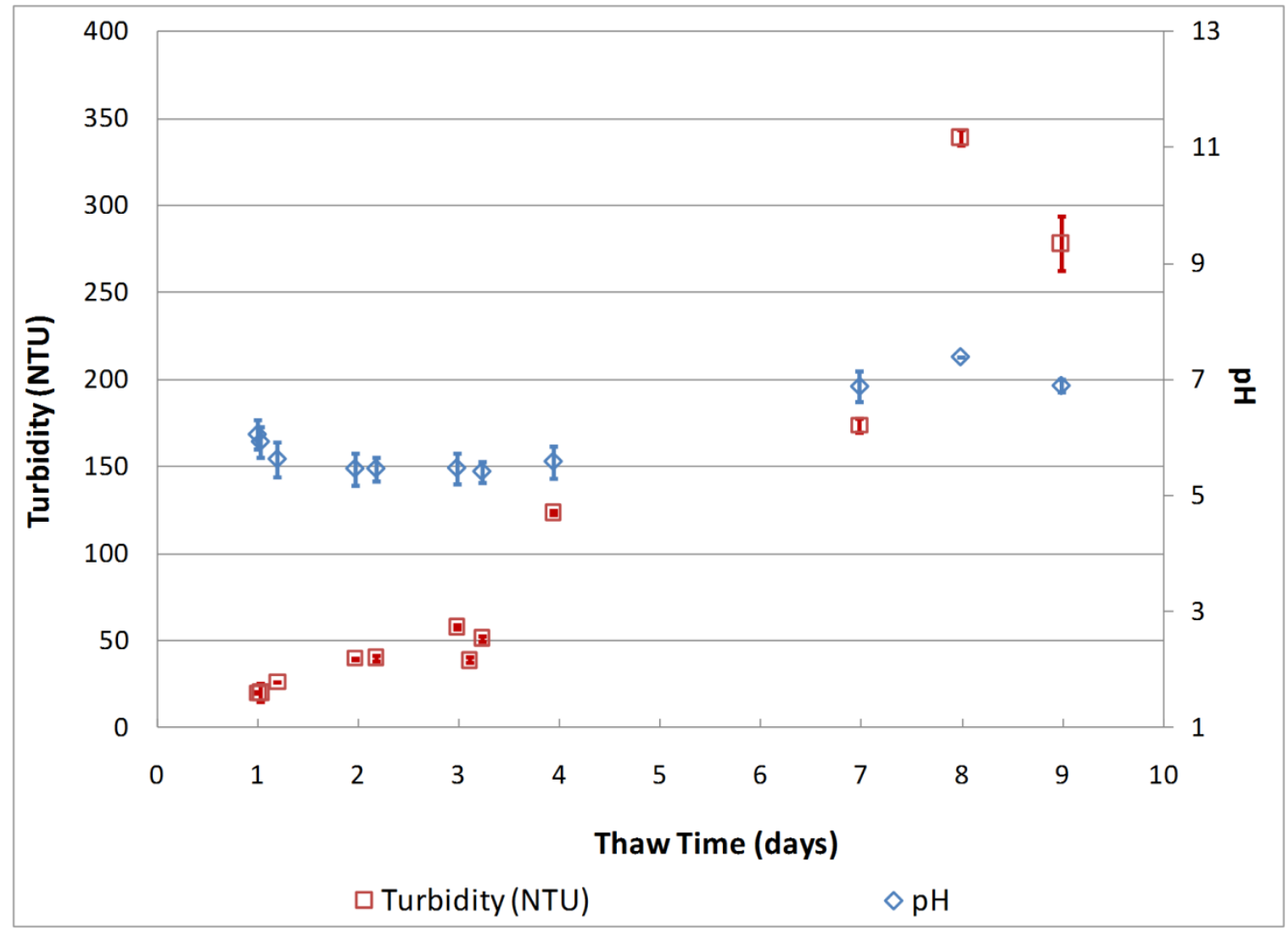

Figure 5-7: Turbidity and $\mathrm{pH}$ of the meltwater during thaw.

A decrease was also measured in the fecal coliform and Salmonella counts in sludge after the freeze-thaw treatment. The fecal coliform decreased from 1816 MPN/g dry solids to $102 \mathrm{MPN} / \mathrm{g}$ dry solids, and the Salmonella decreased from $242 \mathrm{MPN} / 4 \mathrm{~g}$ dry solids to $14 \mathrm{MPN} / 4 \mathrm{~g}$ dry solids. Ice crystals that form inside and outside the cells can rupture the cell membrane and cause significant injury or death to the cells. In this study, approximately 1-log reduction was observed in fecal coliform and Salmonella counts. It should again be noted that the initial sludge bacteria numbers were lower than expected possibly due to long transportation times. The findings of this research are in agreement with previously reported decreases in sludge microbial densities after freeze-thaw treatment (Sanin et al., 1994; Chu et al., 1999; Gao et al., 2009). Sludge 
characteristics, freezing and storage temperatures, and number of freeze-thaw cycles are expected to affect the inactivation rates of microorganisms during freeze-thaw treatment.

The results of this study show that freeze-thaw treatment achieves excellent sludge dewatering and major sludge volume reduction in a simple and cost-effective way. During the thawing period, $>50 \%$ of the initial sludge volume was collected in the form of meltwater within the first 4 days of thawing at temperatures which varied diurnally from 17 to $25{ }^{\circ} \mathrm{C}$. The meltwater had high COD, ammonia and phosphorus concentrations, and therefore require treatment before disposal. This can be achieved on-site by recirculating the meltwater to the headworks of the RBC treatment system. Due to the high strength of the meltwater, it should be fed into the RBC unit at a rate of $<5 \%$ of the normal average daily wastewater flow rate to prevent overloading the biological system. Depending on the thawing conditions, the volume of meltwater generated may be too much for continuous flow into the RBC unit, therefore meltwater storage may be required. However, continuous removal of meltwater from the freezethaw dewatering unit is favourable to avoid the potential for odours.

Odour generation during thawing is another potential problem that might be encountered in the field. In this study, all of the meltwater was collected within a week and odours were not detected until day 3 when sludge was still partially frozen. Unless the meltwater or sludge cake are stored for extended periods under anaerobic 
conditions after thawing, odour generation is not expected to be a significant problem, especially in remote regions.

\subsection{CONCLUSIONS}

Freeze-thaw conditioning successfully dewatered RBC sludge and increased the solids content from $2.6 \%$ to $21 \%$ when sludge was frozen at $-10^{\circ} \mathrm{C}$. Meltwater quickly drained after thawing, and the solids concentration increased to $16.2 \%$ after only one day of thawing. Increases in TSS, VSS, COD, turbidity, TN, TP and ammonia were observed in the meltwater during the thawing period. Overall, the meltwater had high concentrations of COD, nitrogen and phosphorus and required treatment which can be achieved on site by circulating the drainage waters back to the wastewater treatment system. Freeze-thaw conditioning also decreased the densities of fecal coliform and Salmonella in sludge by 1 -log. The results of this study indicate that freeze-thaw technology is an effective, low-cost and low-tech method of sludge treatment, which is ideal for small treatment facilities located in cold and remote regions.

\subsection{ACKNOWLEDGEMENTS}

This work was funded by Natural Sciences and Engineering Research Council of Canada (NSERC) under the Discovery program. The authors would like to thank Strateco Resources Inc. personnel for collecting and shipping the sludge samples to our laboratory, and Seprotech Systems Inc. for providing the pilot scale freezing bed. 


\subsection{REFERENCES}

American Public Health Association (APHA), American Water Works Association and Water Pollution Control Federation, 2005. Standard Methods for the Examination of Water and Wastewater. APHA / AWWA, Washington DC.

Chalmers, B. (1959) How water freezes. Scientific American, 200, 114-122.

Chu, C. P., Feng, W. C., Chang, B. V., Chou, C. H., and Lee, D. J. (1999) Reduction of Microbial Density Level in Wastewater Sludge via Freezing and Thawing. Water Research, 33 (16), 3532-3535.

Corte, A. (1962) Vertical migration of particles in front of a moving freezing plane. Journal of Geophysical Research, 67 (3), 1085-1090.

Gao, W., Smith, D. W., and Li, Y. (2006) Natural freezing as a wastewater treatment method: E. coli inactivation capacity. Water Research, 40(12), 2321-2326.

Gao, W., Leung, K., and Hawdon, N. (2009) Freezing inactivation of Escherichia coli and Enterococcus Faecalis in Water: Response of different strains. Water Environment Research, 81 (8), 824-830.

Hoekstra P., Miller, R.D. (1967). On the ability of water molecules in the transition layer between ice and solid surface. Journal of Colloid and Interface Science. 25 (2), 166-173

Hung, W.T., Chang, I.L., Lin, W.W., Lee D.J. (1996) Unidirectional freezing of wasteactivated sludges: Effects of freezing speed. Environmental Science \& Technology, 30(7) 2391-2396 
Martel, C. J. and Diener, C. J. (1991) A pilot scale study of alum sludge dewaterability in a freezing bed. Journal of American Water Works Association, 83 (12), 51-55.

Mazur, P. (1965). Causes of injury in frozen and thawed cells. Federation Proceedings of the Federation of American Societies for Experimental Biology 24, Suppl. 15, S175-S182

Örmeci, B. (2004) Freeze-thaw conditioning of activated sludge: Effect of monovalent, divalent, and trivalent cations. Journal of Residuals Science and Technology, 1 (2), 103110.

Örmeci, B. and Vesilind, P. A. (2001) Effect of dissolved organic material and cations on freeze-thaw conditioning of activated and alum sludges. Water Research, 35 (18), 42994306.

Örmeci, B. and Vesilind, P. A. (2002) Effect of extracellular polymers on freeze-thaw conditioning of activated sludge. Water Science and Technology, 46 (10), 269-275.

Sanin, F.D., Vesilind, P.A., Martel C.J. (1994). Pathogen reduction capabilities of freeze/thaw sludge conditioning. Water Research, 28 (11), 2393-2398.

United States Environmental Protection Agency (U.S. EPA) (2005a). Method 1681: Fecal Coliforms in Sewage Sludge (Biosolids) by Multiple Tube Fermentation using A-1 Medium. Washington DC, USA.

U.S. EPA (2005b). Method 1682: Salmonella in Sewage Sludge (Biosolids) by Modified Semisolid Rappaport-Vassiliadis (MSRV) Medium. Washington DC, USA. 


\section{COMBINED FREEZE-THAW AND FERRATE(VI) OXIDATION TREATMENT ON E. COLI IN A PHOSPHATE BUFFERED SALINE (PBS) SOLUTION}

James Diak and Banu Örmeci

Department of Civil and Environmental Engineering, Carleton University, 1125 Colonel By Drive, Ottawa, ON K1S 5B6, Canada.

\subsection{ABSTRACT}

The study examined the individual and combined effects of potassium ferrate(VI) and freeze-thaw treatment on the reduction of Escherichia coli (E. coli) in a phosphate buffered saline (PBS) solution. A low dose (LD) of $0.5 \mathrm{mg} / \mathrm{L}$ and a high dose (HD) of 1.0 $\mathrm{mg} / \mathrm{L}$ of potassium ferrate(VI) were used as a pre- and post-treatment to freeze-thaw, at temperatures of $-10,-20$ and $-30^{\circ} \mathrm{C}$, for 1,8 and 15 days.

The LD did not have a significant effect on E. coli concentrations following a 15 minute contact time. However, when the LD was used as a pre-treatment to freeze-thaw, E. coli concentrations were reduced by more than 6-log, to less than $0.18 \mathrm{MPN} / \mathrm{mL}$, regardless of the freezing temperature or the duration of the freezing conditions. When used as a post-treatment to freeze-thaw, LD improved E. coli reductions compared to stand-alone 
freeze-thaw treatments; however, LD was much more effective when used as a pretreatment to freeze-thaw.

The HD resulted in up to 6.4-log inactivation of $E$. coli following a 15-minute contact time. When used as a pre-treatment to freeze-thaw, E. coli concentrations were reduced by more than 6-log regardless of the freezing temperature or the duration of the freezing conditions, similar to the pre-treatment tests with LD.

Freeze-thaw treatments at temperatures of $-10,-20$ and $-30^{\circ} \mathrm{C}$, for 1,8 and 15 days resulted in 1.4 to $3-\log$ inactivation of E. coli, with no significant difference among the various freezing temperatures and freezing times.

The study confirmed the effectiveness of potassium ferrate(VI) and freeze-thaw treatments for the $E$. coli inactivation, and also demonstrated that relatively low doses of potassium ferrate( $\mathrm{VI})$ are extremely effective when used as a pre-treatment, prior to freeze-thaw.

\subsection{INTRODUCTION}

Freeze-thaw treatment is a proven sludge dewatering technology (Hu et al., 2011, Diak et al., 2011, Northcott et al., 2005), and has also been shown to inactivate pathogens and indicator bacteria (Gao et al., 2009, Gao et al., 2006, Kato et al., 2002, Sanin et al., 1994). In practice, natural freeze-thaw can be applied at any scale, and is therefore particularly attractive in northern rural environments. Freeze-thaw beds are essentially 
drying beds or drainage beds, and can be constructed in a variety of ways. Simple sand and tile systems, and inclined sand and drain systems, have been used successfully in pilot-scale and full-scale applications (Diak et al., 2011, Martel 2001, Hedström and Hanaeus, 1999, Hellström, D., Kvarnström, E., 1997, Desjardins and Brière, 1996, Martel 1993, Martel and Diener, 1991). Natural freeze thaw treatments have also been used in existing lagoons and drying beds (Canfield and Sutphen, 1982, Penman and Van Es, 1973), and new materials such as geosynthetic dewatering fabrics offer another attractive design alternative.

When applying liquid sludge to a freeze-thaw bed, during thaw, and when finally removing the sludge cake, odours and pathogens may be a concern. Furthermore, when transporting storing, and eventually disposing of the dewatered sludge cake on land, regrowth of pathogens are also a concern. Although pathogen inactivation can be achieved during freeze-thaw, and can even be enhanced by a combination of multiple freeze-thaw cycles, air drying and solar desiccation, the potential for regrowth and odours remains.

Ferrate $(\mathrm{VI})$ is a very strong oxidant $\left(\mathrm{E}^{0}=2.2 \mathrm{~V}\right)$ and an attractive compliment to the natural freeze-thaw stabilisation and dewatering process. Ferrate(VI) is an effective coagulant and a proven disinfectant (Gombos et al., 2013, Jiang et al., 2007, Jiang et al., 2006, Schuck et al. 2006). It is also capable of oxidising a variety of compounds such as ammonia and reduced sulphur compounds which contribute to odours (Ding et al., 2012, Yang et al., 2012a, He et al., 2009, Schuck et al. 2006). Ferrate(VI) can also be used 
to oxidise a variety of pharmaceuticals and personal care products (PPCPs) (Jiang and Zhoo, 2013, Yang et al., 2012b, Li et al., 2008, Jiang, 2007, Zhu et al. 2006).

The purpose of this research was to evaluate the effect of potassium ferrate(VI) in combination with freeze-thaw treatment for the inactivation of $E$. coli in a phosphate buffered solution. The study examined the use of potassium ferrate(VI) both as a pretreatment, prior to freeze-thaw treatment, and as a post-treatment, following freezethaw treatment. Freezing temperatures of $-10,-20$ and $-30^{\circ} \mathrm{C}$, and freezing periods of 1 , 8 and 15 days were used. The effects of potassium ferrate(VI) dose and contact time were also evaluated, and a freeze-thaw repeatability test, and a comparison of the multiple tube fermentation (MTF) and membrane filtration (MF) enumeration techniques were conducted.

\subsection{MATERIALS AND METHODS}

\subsubsection{Sample (E. Coli) Preparation}

E. coli (ATCC 23621), from Cedarlane Laboratories (Hornby, ON), was used as the indicator organism for all tests. The bacteria were revived according to the methods suggested by the main supplier (ATCC). To summarise the ATCC method: The primary E. coli stock, in pellet-form, was rehydrated using $0.5 \mathrm{~mL}$ of TYG broth (ATCC Medium \#603), transferred into a tube containing $6.0 \mathrm{~mL}$ TYG, and incubated at $37^{\circ} \mathrm{C}$ for $24 \mathrm{~h}$. This primary stock was divided into $1 \mathrm{~mL}$ aliquots and stored in a deep freezer at a temperature of $-20^{\circ} \mathrm{C}$. 
The test sample for each set of experiments was a freshly prepared $E$. coli culture in sterilised phosphate buffered saline (PBS) solution containing $0.3 \mathrm{mM} \mathrm{PO}_{4}^{-3}$ and $2.0 \mathrm{mM}$ $\mathrm{Ca}^{+2}$. The PBS solution was used as a dilution medium because it is isotonic and nontoxic to E. coli (and most other) cells. This means that the concentration of solute inside the cell is the same as the surrounding solute concentration, and there is no osmotic pressure gradient across the cell membrane. As a result, the cells do not shrink or swell, which can be potentially lethal when the rate of transfer into or out of the cell is too fast. A fast flow of water into the cell can cause the cell to swell and burst, while a fast flow of water out of the cell can increase the solute concentration inside the cells to lethal levels.

Fresh test samples of $E$. coli culture and PBS were prepared accordingly: A $1.0 \mathrm{~mL}$ aliquot of E. coli was removed from the freezer, and allowed to thaw and warm to room temperature. The sample was added to $100 \mathrm{~mL}$ of sterilised Bacto ${ }^{\mathrm{Tm}}$ tryptic soy broth (TSB) from BD Biosciences (Mississauga, ON) and revived overnight in an incubator at $37^{\circ} \mathrm{C}$, without shaking. The next morning, $1.0 \mathrm{~mL}$ of the revived stock was added to another $100 \mathrm{~mL}$ of sterilised TSB, and incubated at $37^{\circ} \mathrm{C}$ for 5 -hours with periodic shaking. Preliminary experiments showed that after 4-5 $\mathrm{h}$ of incubation, the bacteria were at their late exponential growth or early stationary phase. After incubation was complete, the cells were removed from the broth and washed with PBS as follows: Two $20 \mathrm{~mL}$ samples of $E$. coli and broth were centrifuged at 10,000 rpm (relative centrifugal force, $r c f=15,317 \mathrm{~g})$ for 10 minutes. The excess broth was poured off, and the cells were rinsed with two $20 \mathrm{~mL}$ aliquots of PBS and resuspended using a vortex mixer 
(Baxter Diagnostics Inc., IL, USA). The samples were centrifuged again at 10,000 rpm for 10 minutes, the excess PBS and trace amounts of broth were poured off, and the samples were rinsed with two $20 \mathrm{~mL}$ aliquots of PBS and resuspended using a vortex mixer. This "cell washing" process with PBS was repeated a total of three times to ensure that no broth was remaining amongst the cells that would be the cause of regrowth later. After the washing process, the cells and PBS were added to $2.0 \mathrm{~L}$ of sterilised PBS, and the initial E. coli concentration was measured immediately. The resulting initial concentration of the test samples used for the experiments was approximately $1 \times 10^{7}$ E. coli colony forming units (CFU) per $\mathrm{mL}$, expressed as the most probable number (MPN) per $\mathrm{mL}$. Throughout the experiments there was some variability in the initial concentration of the E. coli sample; therefore, all tests used for direct comparisons were performed on the same batch of $E$. coli.

\subsubsection{Freeze-Thaw Treatments}

$150 \mathrm{~mL}$ aliquots of the test sample (PBS and E. coli) were dispensed into $500 \mathrm{~mL}$ autoclavable polypropylene Nalgene ${ }^{\circledR}$ bottles, and placed in a freezer (VWR FORMA ${ }^{\circledR}$ $40^{\circ} \mathrm{C}$ Lab Freezer Model 5722, VWR International, Mississauga, ON). Freezer temperatures of $-10,-20$ or $-30^{\circ} \mathrm{C}\left(+/-1^{\circ} \mathrm{C}\right)$ were used to represent a range of temperatures that occur in the natural environment where freeze-thaw is most applicable. Also, preliminary tests demonstrated that these freezer temperatures generated freezing rates below $10 \mathrm{~mm} / \mathrm{hr}$, which is favourable for pathogen attenuation and particle migration. 
The freezing rate was determined based on the geometry of the liquid portion of sample and the time taken for the liquid to completely freeze. The temperature at the centre of a $150 \mathrm{~mL}$ test sample and the temperature of the freezer were monitored using a Traceable ${ }^{\circledast}$ dual-channel thermometer with type $\mathrm{K}$ thermocouples and computer output (Model 4137, Control Company, Friendswood, TX, USA). Temperatures were recorded on a PC every minute using the data acquisition software (DAS ${ }^{\mathrm{m}}$, Control Company, Friendswood, TX, USA). When the centre of the sample was cooled to the freezing point, generally around $-0.7^{\circ} \mathrm{C}$, the temperature did not change as the liquid changed to ice. Once the entire sample was frozen, the temperature at the centre of the sample began to decrease, until reaching the temperature inside the rest of the freezer. During this phase change, when the temperature was stable, it was assumed that the ice front approached the centre of the sample radially and vertically inwards. The elapsed time for the phase change to occur and the geometry of the sample was then used to determine the freezing rate. The resulting freezing rates generated using freezer temperatures of $-10,-20$ and $-30^{\circ} \mathrm{C}$ were approximately $3.5,4.7$ and $6.6 \mathrm{~mm} / \mathrm{hr}$ in the $150 \mathrm{~mL}$ samples respectively. Plots of the temperature logs used to estimate the freezing rates are presented in Appendix B1 on page 380. These freezing rates are within the optimal range in terms of pathogen reduction and particle migration, leading to consolidated particles and good dewaterability (Wang et al., 2001, Sanin et al., 1995, Hung et al., 1997). Once the samples were completely frozen, the samples were kept under the freezing conditions for an additional 1, 8 or 15 days. It was presumed that 1 day frozen would reveal the effects of the freezing and thawing processes, while longer 
periods of 8 and 15 days would show any additional effects caused by prolonged freezing periods. After either 1,8 or 15 days completely frozen, the samples were removed from the freezer, and thawed at room temperature $\left(22-23^{\circ} \mathrm{C}\right)$. It took between 350 and 400 minutes for the samples to completely thaw and warm up to room temperature before any further treatments or analysis was performed.

\subsubsection{Pre-and Post-Treatments with Potassium Ferrate(VI)}

Whenever potassium ferrate(VI) was used, a fresh ferrate(VI) stock solution was prepared by adding $5.0 \mathrm{mg}$ of Ferratec Brand ${ }^{\mathrm{TM}}>90 \%$ pure potassium ferrate(VI) (SigmaAldrich Canada Ltd., Oakville, ON) to $10.0 \mathrm{~mL}$ of deionised water. Resulting potassium ferrate(VI) stock solution was $0.5 \mathrm{mg} / \mathrm{mL}$ of potassium ferrate(VI) salt, equivalent to approximately $0.27 \mathrm{mg} \mathrm{FeO}_{4}^{-2} / \mathrm{mL}$.

Addition of ferrate(VI) prior to freeze-thaw treatment (ferrate(VI) pre-treatment) was conducted in the $500 \mathrm{~mL}$ sample bottles, which were placed on magnetic stirrers. For the low dose (LD) pre-treatments, $0.15 \mathrm{~mL}$ of the stock solution containing $0.5 \mathrm{mg}$ $\mathrm{K}_{2} \mathrm{FeO}_{4} / \mathrm{mL}$ was added to each of the designated $150 \mathrm{~mL}$ samples, and the samples were stirred for 15-minutes before being placed in the freezer for subsequent freeze-thaw treatment. The resulting $L D$ was $0.5 \mathrm{mg} / \mathrm{L}$ of potassium ferrate(VI) equivalent to 0.273 $\mathrm{mg} \mathrm{FeO}_{4}^{-2} / \mathrm{L}$. High dose (HD) pre-treatments were conducted the same way, however $0.30 \mathrm{~mL}$ of the stock solution was added to each of the designated sample bottles, and the resulting $\mathrm{HD}$ was $1.0 \mathrm{mg} / \mathrm{L}$ of potassium ferrate(VI) equivalent to $0.545 \mathrm{mg} \mathrm{FeO}_{4}^{-2} / \mathrm{L}$. 
Post treatment with the LD and HD was conducted in a similar fashion. After the designated freezing period, samples were removed from the freezer and thawed at room temperature. This process took between 350 and 400 minutes. Once the samples were completely thawed, and warmed to room temperature, $0.15 \mathrm{~mL}$ of the potassium ferrate(VI) stock solution containing $0.5 \mathrm{mg} / \mathrm{mL}$ of potassium ferrate(VI) was added to the bottles designated for LD post-treatment, and $0.30 \mathrm{~mL}$ of the stock solution was added the bottles designated for HD post-treatment. The bottles were stirred for 15 minutes using a magnetic stirrer. After the 15 -minute contact time, $10 \mathrm{~mL}$ of the sample was immediately removed from the bottles and added to $90 \mathrm{~mL}$ of PBS, and subsequently serial-diluted with more PBS as part of the bacterial enumeration technique.

As a reference for the remainder of this paper, Table 6-1 below lists the sample labels used in subsequent figures along with a description of the various treatments applied to each of the samples. 
Table 6-1: Description of the treatments applied to the test samples and their representative abbreviations used in subsequent figures.

\begin{tabular}{|l|l|}
\hline Label & Treatment \\
\hline Initial & No treatment \\
\hline LD & Low Dose, LD $=0.5 \mathrm{mg} / \mathrm{L}$ potassium ferrate and 15 minute contact time \\
\hline HD & High Dose, HD $=1.0 \mathrm{mg} / \mathrm{L}$ potassium ferrate and 15 minute contact time \\
\hline F1 & Stand-alone freeze-thaw, with 1 day completely frozen \\
\hline F8 & Stand-alone freeze-thaw, with 8 days completely frozen \\
\hline F15 & Stand-alone freeze-thaw, with 15 days completely frozen \\
\hline LD-F1 & LD pre-treatment, followed by freeze-thaw, with 1 day completely fozen \\
\hline LD-F8 & LD pre-treatment, followed by freeze-thaw, with 8 day completely fozen \\
\hline LD-F15 & LD pre-treatment, followed by freeze-thaw, with 15 day completely fozen \\
\hline F1-LD & Freeze-thaw, with 1 day completely frozen, followed by LD post-treatment \\
\hline F8-LD & Freeze-thaw, with 8 day completely frozen, followed by LD post-treatment \\
\hline F15-LD & Freeze-thaw, with 15 day completely frozen, followed by LD post-treatment \\
\hline HD-F1 & HD pre-treatment, followed by freeze-thaw, with 1 day completely fozen \\
\hline HD-F8 & HD pre-treatment, followed by freeze-thaw, with 8 day completely fozen \\
\hline HD-F15 & HD pre-treatment, followed by freeze-thaw, with 15 day completely fozen \\
\hline F1-HD & Freeze-thaw, with 1 day completely frozen, followed by HD post-treatment \\
\hline F8-HD & Freeze-thaw, with 8 day completely frozen, followed by HD post-treatment \\
\hline F15-HD & Freeze-thaw, with 15 day completely frozen, followed by HD post-treatment \\
\hline
\end{tabular}

\subsubsection{E. Coli Enumeration}

For all experiments, multiple tube fermentation (MTF) with A1 medium (EMD Chemicals Inc., Gibbstown, NJ, USA) was used to enumerate the E. coli according to U.S. EPA Method 1681 (U.S. EPA, 2005). For some experiments, membrane filtration (MF) with Difco $^{\text {TM }}$ m-Endo Agar LES medium (Becton, Dickson \& Company, MD, USA) was performed according to Standard Method 9222 B (APHA et al., 2005). 


\subsubsection{Statistical Analysis}

All MTF tests were carried out in duplicate, using at least four dilutions. All MF tests were carried out in triplicate, using at least three dilutions. Error bars on all figures show the $95 \%$ confidence interval which is a built-in function of the MTF test. Generally the error-bars represent approximately \pm 1 -log, however, in some cases where the sample replicates had large variability, the $95 \%$ confidence limits increased to up $1.4-\log$. When the measured concentration of a particular test sample was below the detection limit, the error bars on the data point were replaced with a downward arrow. A two-tailed ttest was used to determine the $p$ values and statistical significance $(p<0.05)$ of the results.

\subsection{RESULTS AND DISCUSSION}

\subsubsection{Effect of Ferrate(VI) Dose and Contact Time}

Figure 6-1(a) shows decreasing $E$. coli concentrations in the two test samples with increasing contact time, following the addition of $0.5 \mathrm{mg} / \mathrm{L}$ of potassium ferrate( $\mathrm{VI})$. For both samples, a 5 minute contact time was not effective; however, a 15 minute contact time resulted in a 1.2-1.4 log reduction ( $p=0.053)$. The results for a 10 min contact time showed some variance among the two test samples; however, some variance is expected with the prepared E. coli sample and the MTF tests used for enumeration. For subsequent tests, a 15 minute contact time was used. Additionally, due to some variation with the initial concentration of the prepared E. coli samples, all tests and comparisons with the pure culture of $E$. coli were performed on the same batch of $E$. coli that was prepared on the day of testing. Any comparisons between $E$. coli samples were 
to the relative log inactivations of the test samples and their representative initial (blank) E. coli samples.

Figure 6-1(b) shows decreasing $E$. coli concentrations with increasing ferrate(VI) dose, following a 15 minute contact time. Data for Sample $C$ shows that $0.1 \mathrm{mg} / \mathrm{L}$ of potassium ferrate(VI) was not effective; however, $0.5 \mathrm{mg} / \mathrm{L}$ resulted in a $2.4 \log$ reduction in $E$. coli concentration, and $1.0 \mathrm{mg} / \mathrm{L}$ resulted in a 5 - $\log$ reduction for sample $\mathrm{C}$ and a $3.2 \mathrm{log}$ reduction for sample $D(p=0.011)$. Doses greater than $2.0 \mathrm{mg} / \mathrm{L}$ reduced $E$. coli concentrations to the detection limit. Although every E. coli sample was prepared the same way, there were slight variations in the initial concentration, and in the response of the test sample to similar treatments with potassium ferrate(VI). Despite these discrepancies, an appropriate working range of potassium ferrate(VI) doses was determined to be from 0.5 to $1.0 \mathrm{mg} / \mathrm{L}$. This range was chosen to allow the effects of the freeze-thaw treatments on $E$. coli to be observed when both treatments are applied to the same test sample. For all combined treatment tests using potassium ferrate(VI) as either a pre- or post-treatment to freeze-thaw, LD was set to $0.5 \mathrm{mg} / \mathrm{L}$ and HD was set to $1.0 \mathrm{mg} / \mathrm{L}$. 


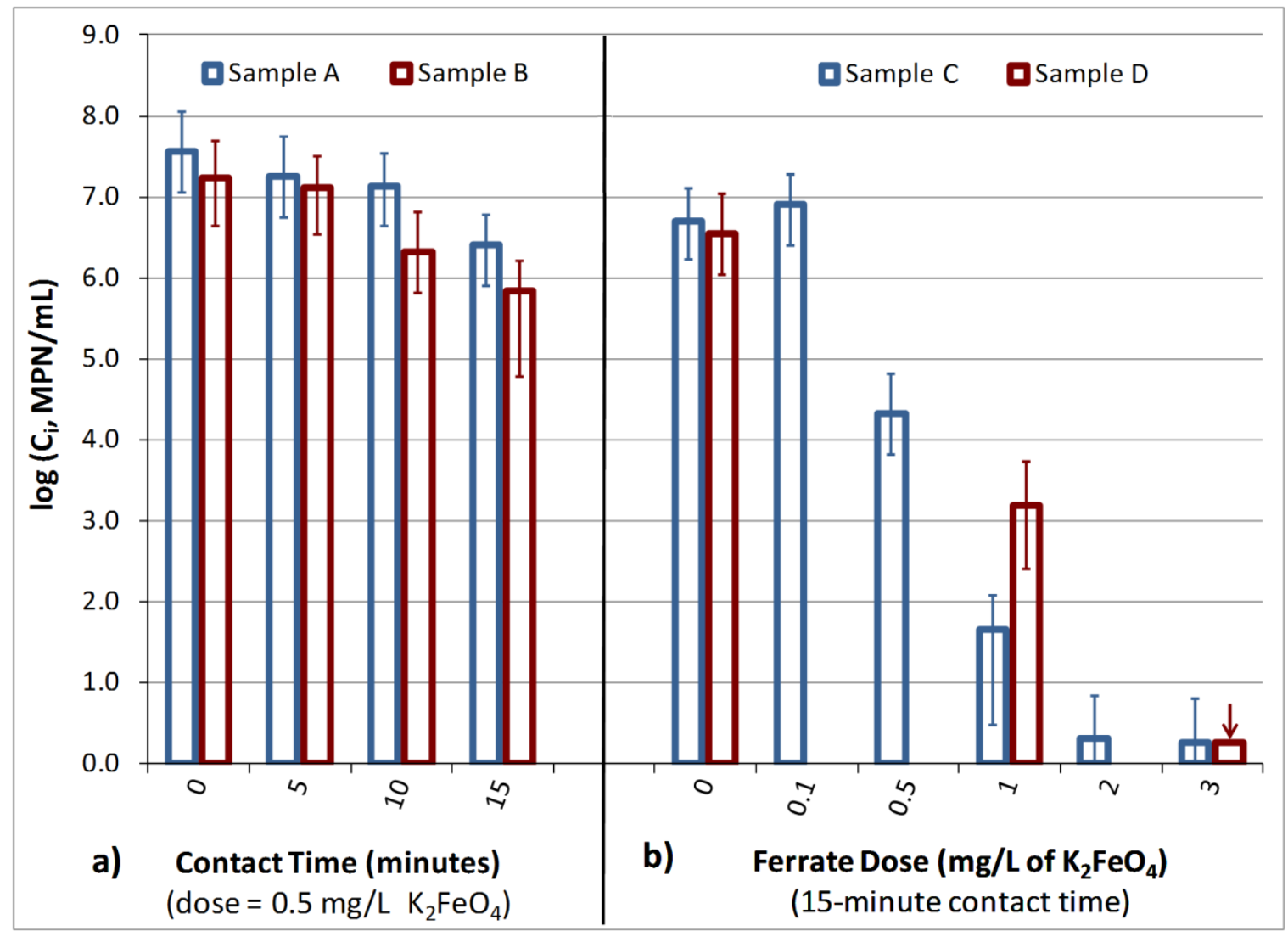

Figure 6-1: E. coli concentration following (a) various contact times using a $0.5 \mathrm{mg} / \mathrm{L} \mathrm{of} \mathrm{K}_{2} \mathrm{FeO}_{4}$, (b) various potassium ferrate(VI) doses using a 15-minute contact time.

\subsubsection{Comparison of MTF and MF Enumeration Techniques, and 7-day Freeze-}

\section{Thaw Reproducibility Test}

Figure 6-2 shows the reproducibility of freeze-thaw treatment on the reduction of $E$. coli. Five $150 \mathrm{~mL}$ aliquots of the same test sample (Sample A) were placed in separate bottles and frozen at $-20^{\circ} \mathrm{C}$ for 7 days, then thawed at room temperature. This procedure was repeated using a second, freshly prepared sample (Sample B). The initial concentration of $E$. coli in both samples, prior to being frozen, was measure using MTF and MF enumeration techniques to compare these two popular methods. For each sample, two sets of tests were conducted for each enumeration technique. 
The average initial concentration of E. coli in Sample A was 7.2-log measured using MTF, and 7.5-log measured using MF. Similarly, the initial concentration of Sample B was 7.2log using MTF, and 7.7-log using MF. Results show that the MTF and MF enumeration techniques produce similar results, with no significant differences $(p>0.05)$ for each of the test samples $\left(p_{A}=0.439, p_{B}=0.09\right)$. Since MTF is the U.S. EPA approved method for enumeration of coliform in sludge (U.S. EPA, 2005), MTF was used to enumerate $E$. coli in subsequent tests. It should be noted that the error bars from MTF are built-in $95 \%$ confidence limits $(\mathrm{CL})$ values, based on 5 replicates of 3 dilutions, whereas the error bars for MF are the calculated $95 \% \mathrm{CL}$ based on 3 replicates of one dilution, picked from a set of 3 dilutions.

Following the 7-day freezing period, the samples were removed from the freezer and thawed at room temperature. The E. coli concentrations in all 5 Sample A and Sample B bottles were measured using the MTF method, and the results were compared to the initial MTF concentrations describe above. The results show that some variance in E. coli reduction can be expected after freeze thaw treatment. For sample $A$, the initial E. coli concentration was 7.2-log, and following the 7-day freezing period, the average E. coli concentration of all 5 bottles was $6.5-\log (95 \% \mathrm{CL}=+/-0.3-\log )$, representing a 0.7- $\log$ inactivation. However, there was considerable variation among the 5 bottles, with a maximum of $1.2-\log$ inactivation, and a minimum inactivation of $0.3-\log$. Even more variation was observed with the Sample B tests. The initial concentration of 7.2-log, was reduced to $6-\log$ on average $(95 \% \mathrm{CL}=+/-0.5-\log )$, with a maximum inactivation of 2-log, and a minimum of $0.7-\log$. 


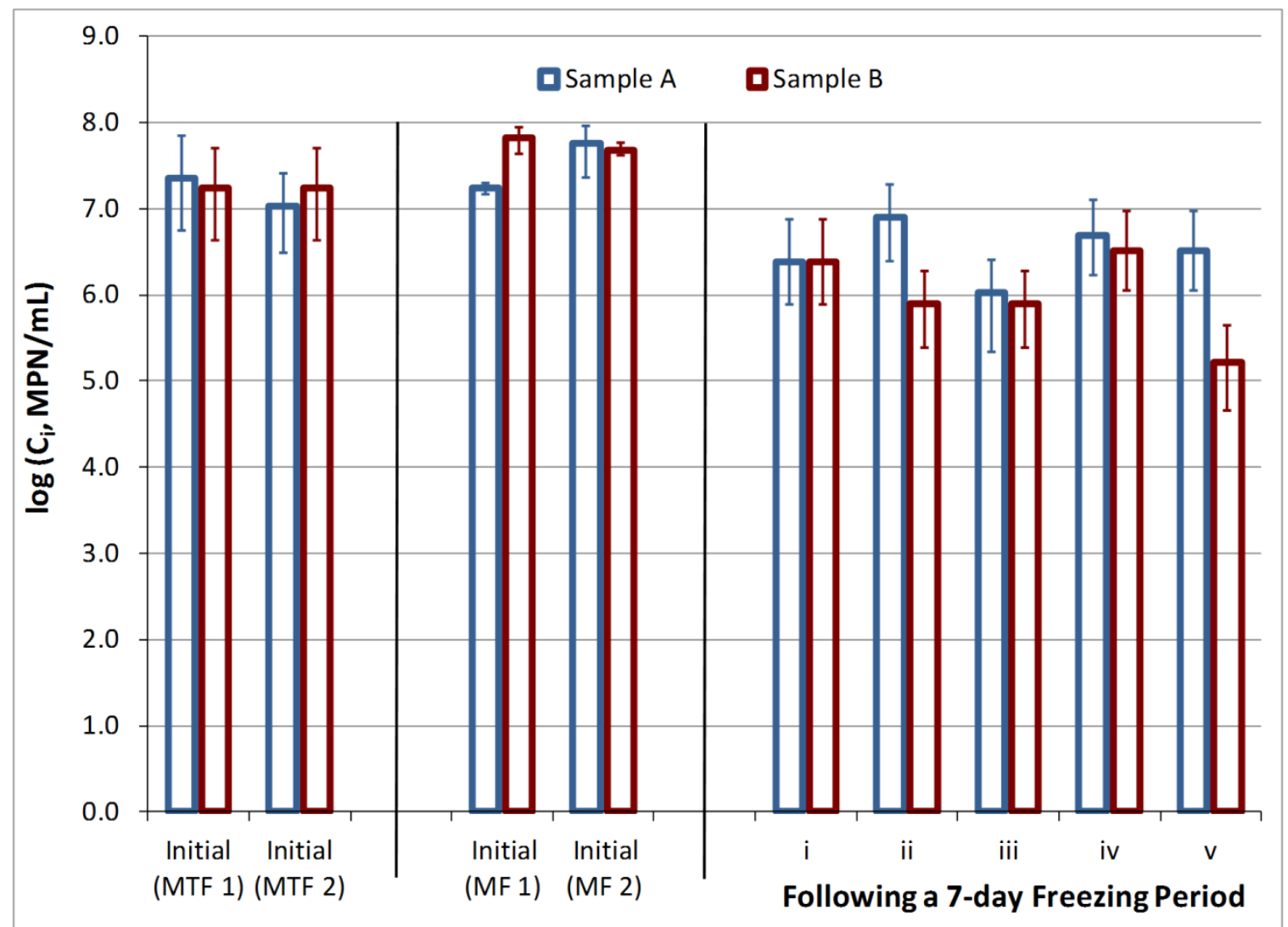

Figure 6-2: A comparison of the Multiple Tube Fermentation (MTF) and the Membrane Filtration (MF) methods for the enumeration of E. coli, and a reproducibility test on freezethaw treatments for the reduction of $E$. coli concentrations.

\subsubsection{Freezer Temperature $=-10^{\circ} \mathrm{C}$}

Figure 6-3 shows the effect of the low dose of potassium ferrate(VI) (LD $=0.5 \mathrm{mg} / \mathrm{L}$ $\left.\mathrm{K}_{2} \mathrm{FeO}_{4}\right)$ when used alone, with a 15 -minute contact time, and as a pre- and posttreatment to freeze-thaw with a freezing temperature of $-10^{\circ} \mathrm{C}$. Results suggest that the LD had no significant effect on $E$. coli concentrations after 15 minutes $(p=0.2)$. However following 1,8 or 15 days of $-10^{\circ} \mathrm{C}$ freezing temperatures, greater than 7 -log reduction was achieved, reducing $E$. coli concentrations to the detection limit ( $<0.18 \mathrm{MPN} / \mathrm{mL}$ ) or close (LD-F15 = $0.45 \mathrm{MPN} / \mathrm{mL})$. LD-F1, LD-F8 and LD-F15 imply a low dose $(0.5 \mathrm{mg} / \mathrm{L}$ $\left.\mathrm{K}_{2} \mathrm{FeO}_{4}\right)$ of potassium ferrate( $\left.\mathrm{VI}\right)$ and then 1,8 or 15 day(s) completely frozen, 
respectively. In addition to the designated freezing times, samples took 340 minutes to freeze at temperatures of $-10^{\circ} \mathrm{C}$, which provided additional time for the potassium ferrate $(\mathrm{VI})$ to inactivate $E$. coli prior to freeze-thaw. Furthermore, samples took approximately 360 minutes to thaw and warm to room temperature, however this was unlikely to have contributed to any further reaction time with the short-lived ferrate(VI) ion.

LD post-treatment improved the log reduction of freeze-thaw treatments by an additional $0.86-1.89$ compared to stand-alone freeze-thaw treatments, however no significant improvement was achieved $\left(p_{1}=0.19, p_{8}=0.13, p_{15}=0.43, p>0.05\right)$. Comparing the LD pre- and post-treatments, it was clear that pre-treatment was far superior to post-treatment.

Stand-alone freeze-thaw resulted in 1.4 to 2.7-log inactivation of $E$. coli, with no significant difference $(p>0.05)$ among the samples that were frozen for 1,8 or 15 days. This suggests that the effects of freeze-thaw mainly occurred during the first day of the freezing conditions, and additional days spent frozen did not result in a significant improvement. 


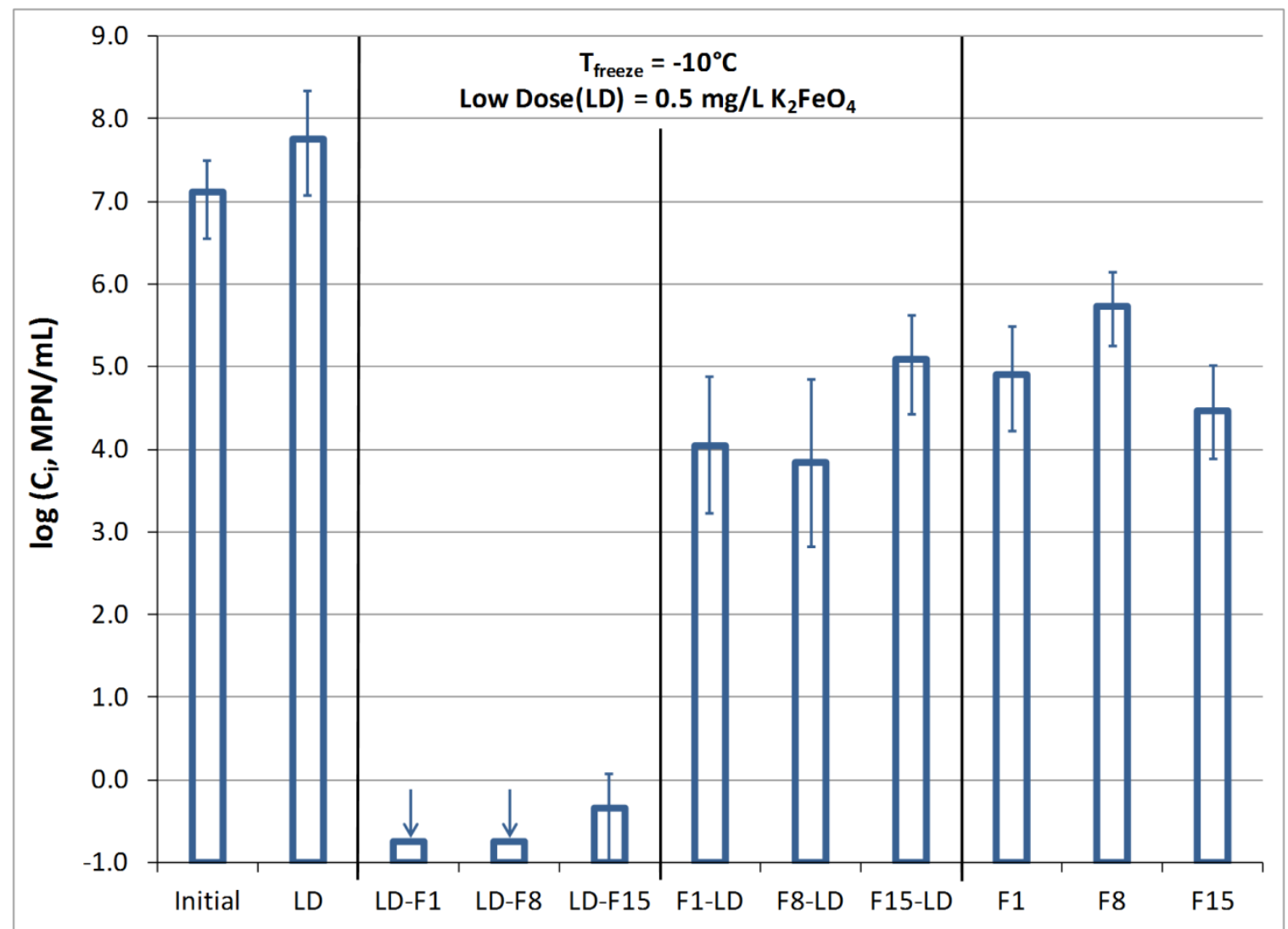

Figure 6-3: Effect of low ferrate(VI) dose ( $\left.\mathrm{LD}=0.5 \mathrm{mg} / \mathrm{L} \mathrm{K}_{2} \mathrm{FeO}_{4}\right)$ when used alone (15-min contact time), and as a pre- and post-treatment to freeze-thaw with $\mathrm{T}_{\text {Freeze }}=-10^{\circ} \mathrm{C}$.

Figure 6-4 shows the effect of the high dose of potassium ferrate(VI) (HD $=1.0 \mathrm{mg} / \mathrm{L}$ $\mathrm{K}_{2} \mathrm{FeO}_{4}$ ) when used alone, and as a pre- and post-treatment to freeze-thaw, with a freezing temperature of $-10^{\circ} \mathrm{C}$. HD resulted in a 6.4-log inactivation of $E$. coli after 15 minutes contact time, and similar to LD pre-treatments, HD pre-treatment followed by freeze-thaw, reduced $E$. coli concentrations to the detection limit $(<0.18 \mathrm{MPN} / \mathrm{mL})$, regardless of the duration of the freezing period. However, when HD with a 15-minute reaction period was used as a post-treatment, following freeze-thaw, E. Coli inactivation was only 4.1, 5.2 and 3.9-log for samples that were frozen for 1,8 and 15 days respectively. Although there is a high level of inactivation, the samples following freeze- 
thaw were less sensitive to the ferrate $(\mathrm{VI})$ treatments than the initial samples that were only treated with ferrate( $\mathrm{VI})$. This was most likely because these samples, following freeze-thaw, did not fully warm up to room temperature prior to the addition of the HD post-treatment, resulting in a more stable ferrate(VI) solution and slower reaction rates. Despite the presumably colder sample temperatures, potassium ferrate(VI) additions following freeze-thaw resulted in an additional 1.8, 3.7 and 1.3-log inactivation of E. coli in samples that were frozen for 1,8 and 15 days, and similar to previous tests, the duration of the freezing conditions did not have a significant effect on E. coli.

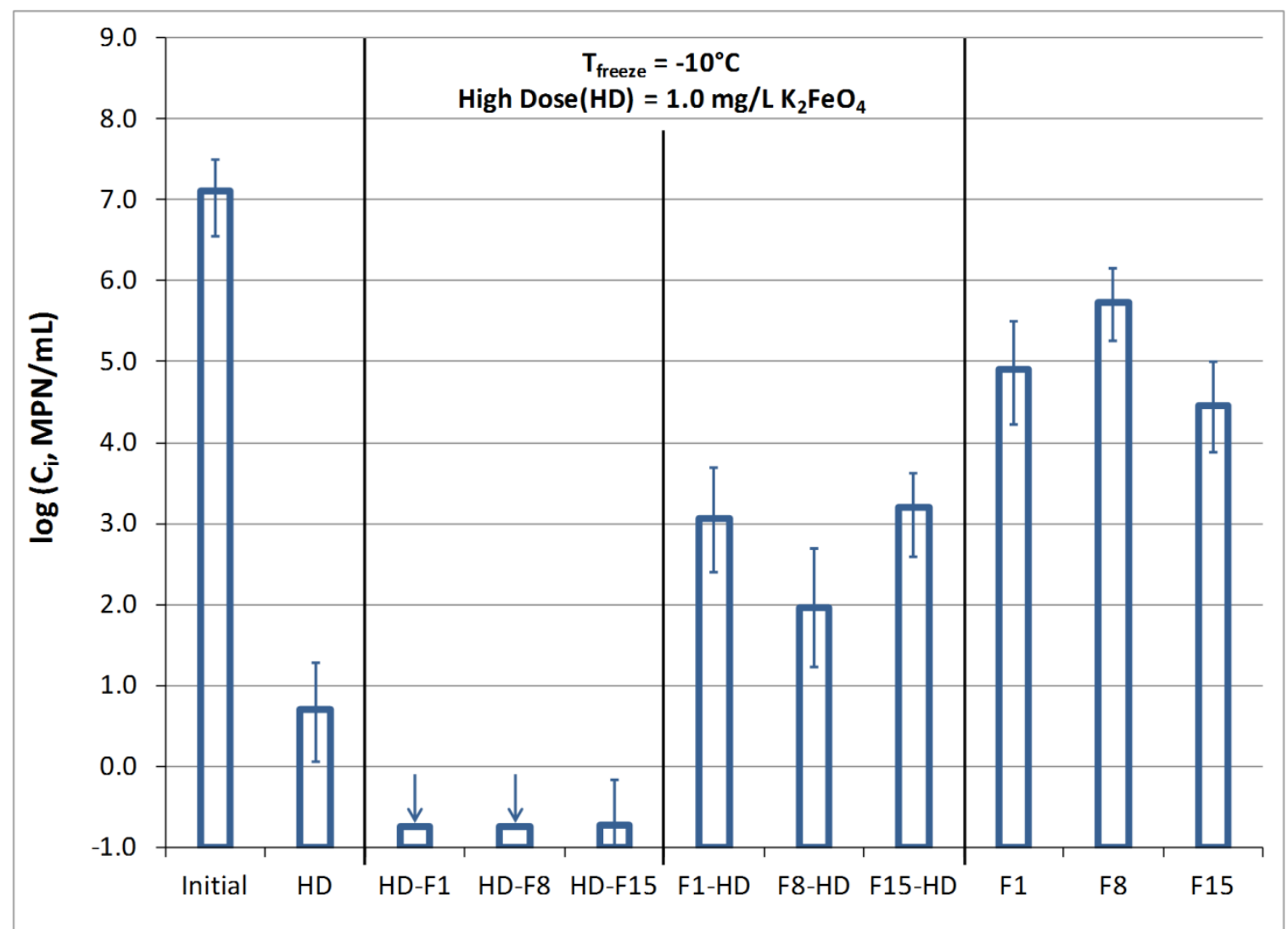

Figure 6-4: Effect of high ferrate(VI) dose ( $\left.\mathrm{HD}=1.0 \mathrm{mg} / \mathrm{L} \mathrm{K}_{2} \mathrm{FeO}_{4}\right)$ when used alone (15-min contact time), and as a pre- and post-treatment to freeze-thaw with $\mathrm{T}_{\text {Freeze }}=-10^{\circ} \mathrm{C}$. 


\subsubsection{Freezer Temperature $=-20^{\circ} \mathrm{C}$}

Figure 6-5 shows the effect of the LD when used alone, and as a pre- and post-treatment to freeze-thaw with a freezing temperature of $-20^{\circ} \mathrm{C}$. Similar to the $-10^{\circ} \mathrm{C}$ tests, $\mathrm{LD}$ did not have a significant effect on the concentration of $E$. coli after 15 minutes $(p=0.36)$, however following 1 day of freezing at $-20^{\circ} \mathrm{C}$, a 7-log-reduction was achieved, and again increased freezing time did not result in a significant improvement in the reduction of $E$. coli.

LD post-treatments improved the log-reduction of stand-alone freeze-thaw treatments by $0.38,1.07$ and $1.64-\log$, for freezing times of 1,8 and 15 days respectively. However, similar to the tests at $-10^{\circ} \mathrm{C}$, pre-treatment with ferrate(VI) resulted in several orders of magnitude greater reduction in E. coli.

Similar to the tests at $-10^{\circ} \mathrm{C}$, stand-alone freeze-thaw treatments resulted in $1.7,2.5$ and 1.4 log-reductions in $E$. coli for samples frozen for 1,8 and 15 days at $-20^{\circ} \mathrm{C}$ respectively, with no significant difference among samples. This suggests that additional freezing time did not improve the reduction of E. coli. 


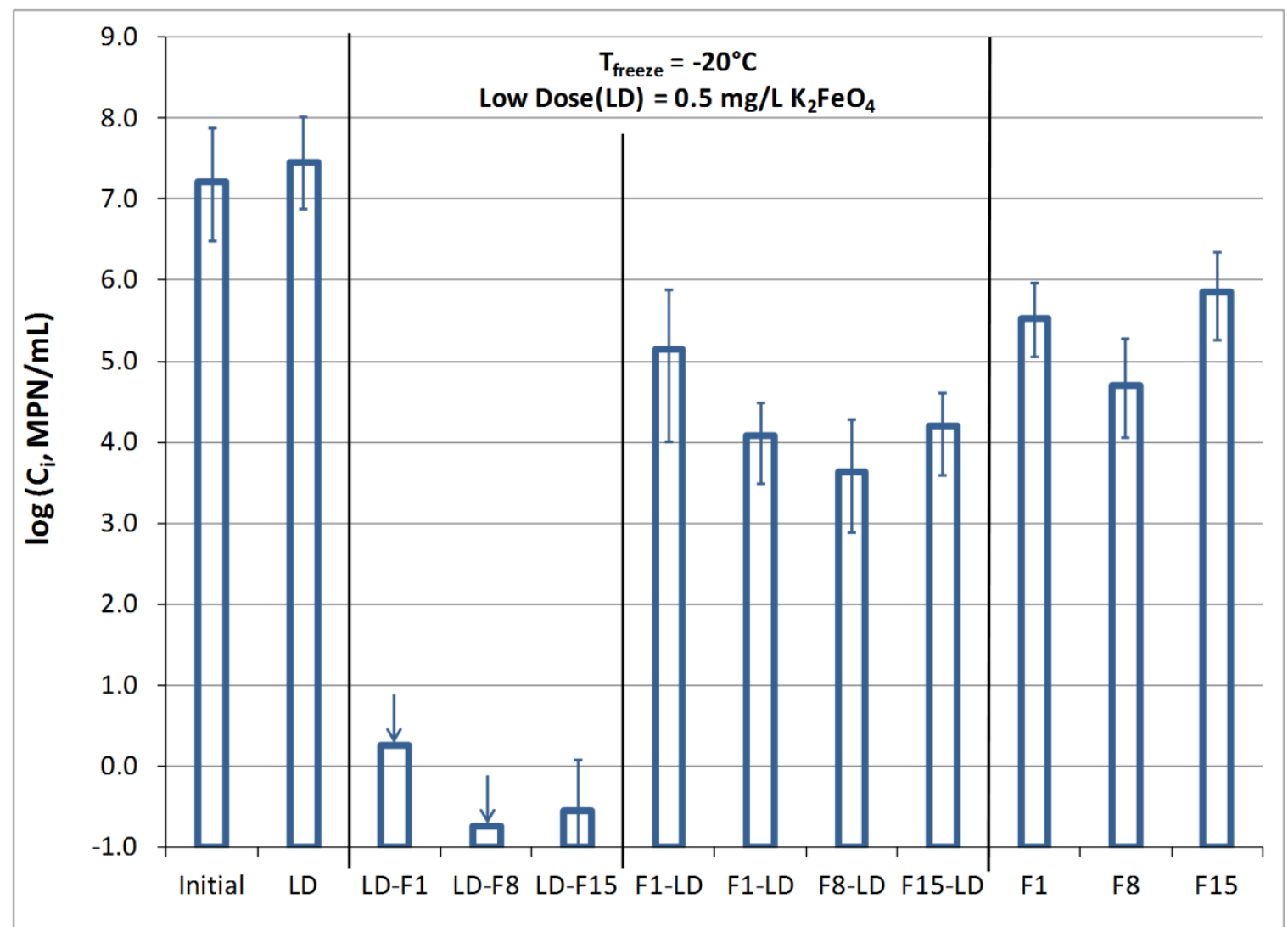

Figure 6-5: Effect of low ferrate(VI) dose (LD=0.5 mg/L K $\mathrm{FeO}_{4}$ ) when used alone (15-min contact time), and as a pre- and post-treatment to freeze-thaw with $\mathrm{T}_{\text {Freeze }}=-20^{\circ} \mathrm{C}$.

Figure 6-6 shows the effect of the HD when used alone, and as a pre- and posttreatment to freeze-thaw, with a freezing temperature of $-20^{\circ} \mathrm{C}$. HD resulted in a 5 -log reduction in $E$. coli after 15 minutes contact time, and when followed by freeze-thaw at a freezing temperature of $-20^{\circ} \mathrm{C}, \mathrm{E}$. coli concentrations were reduced to the detection limit $(<0.18 \mathrm{MPN} / \mathrm{mL})$ for freezing periods of 1,8 and 15 days.

Compared to stand-alone freeze-thaw treatments, HD post-treatment significantly improved the reduction of $E$. coli by an additional 2.1, 2.3 and 3.2-log for freezing periods of 1,8 and 15 days respectively $\left(p_{1}=0.04, p_{8}=0.05, p_{15}=0.002\right)$, and similar to previous tests, the duration of the freezing conditions did not have a significant effect 
on the reduction of $E$. coli. However, also similar to previous tests, the combined effects of freeze-thaw and ferrate( $\mathrm{VI})$ post-treatment was less effective than the same dose of ferrate(VI) and a 15-minute reaction period.

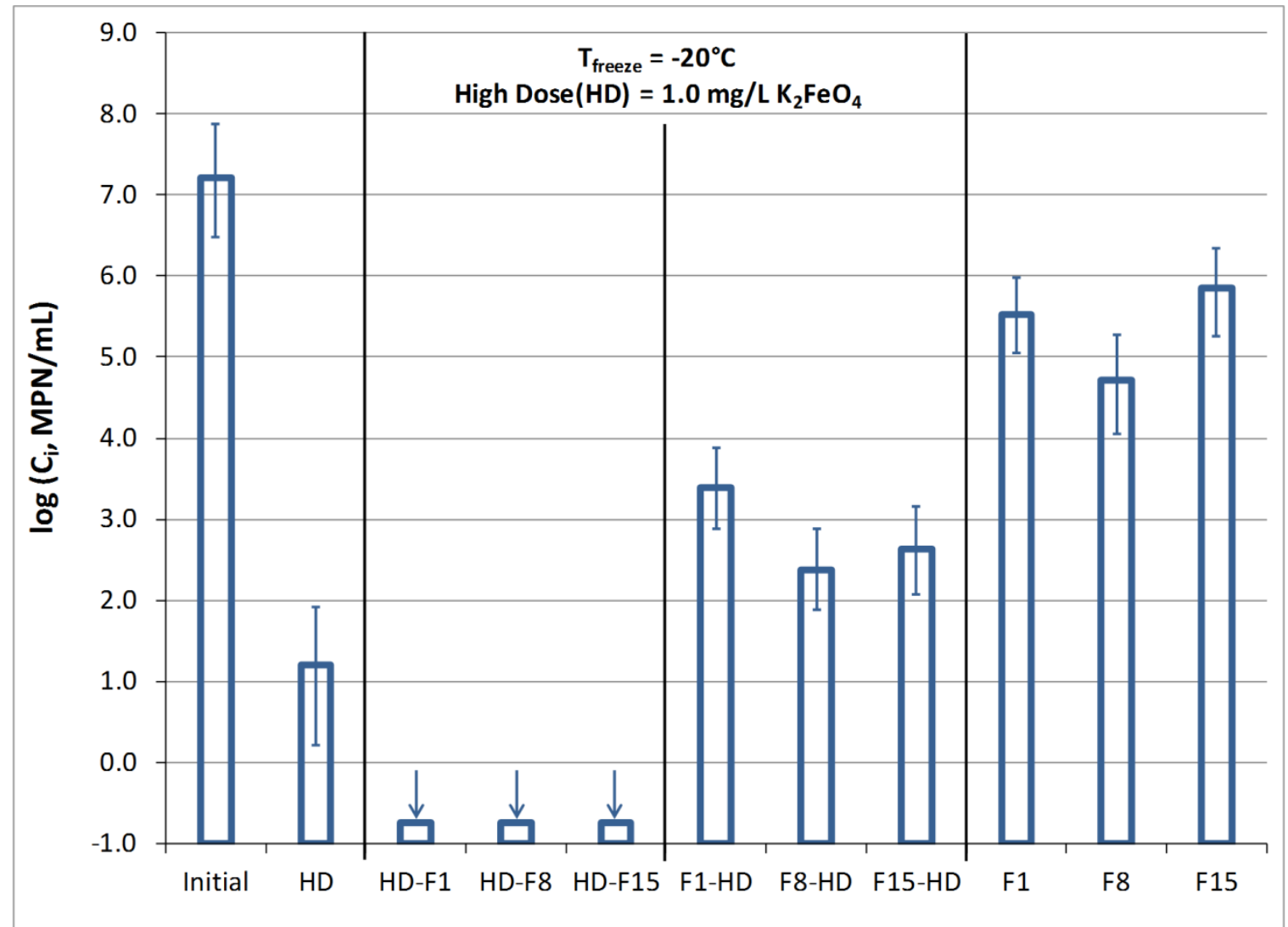

Figure 6-6: Effect of high ferrate(VI) dose ( $\left.\mathrm{HD}=1.0 \mathrm{mg} / \mathrm{L} \mathrm{K}_{2} \mathrm{FeO}_{4}\right)$ when used alone (15-min contact time), and as a pre- and post-treatment to freeze-thaw with $\mathrm{T}_{\text {Freeze }}=-20^{\circ} \mathrm{C}$.

\subsubsection{Freezer Temperature $=-30^{\circ} \mathrm{C}$}

Figure 6-7 shows the effect of the LD when used alone, and as a pre- and post-treatment to freeze-thaw with a freezing temperature of $-30^{\circ} \mathrm{C}$. Although the results following the LD show a 1.7-log reduction in E. coli after 15 minutes contact time, there was a large variance in the sample replicates, particularly for the initial enumeration of $E$. coli. Therefore, no significant reduction in E. coli was achieved (following the LD with a 15 
minute contact time) $(p=0.35)$, similar to the previous LD experiments. However, when followed by either 1,8 or 15 days freezing at $-30{ }^{\circ} \mathrm{C}$, LD pre-treatments reduced $E$. coli concentrations to the detection limit $(<0.18 \mathrm{MPN} / \mathrm{mL})$, as with the previous LD pretreatment experiments using freezing temperatures of -10 and $-20^{\circ} \mathrm{C}$.

Stand-alone freeze-thaw treatments using a freezing temperature of $-30^{\circ} \mathrm{C}$ resulted in 2 , 3 and 1.4-log reductions in E. coli following 1, 8 and 15 days frozen, with no significant difference among the freezing periods. Post-treatment with LD improved the reduction of $E$. coli caused by stand-alone freeze-thaw treatments by an additional 1.6, 1.4 and 1.7-log for the samples frozen for 1,8 and 15 days respectively, however there was no significant difference among the samples with and without LD post-treatment $\left(p_{1}=0.14\right.$, $\left.\mathrm{p}_{8}=0.26, \mathrm{p}_{15}=0.10\right)$. These results are again similar to the -10 and $-20^{\circ} \mathrm{C}$ experiments. 


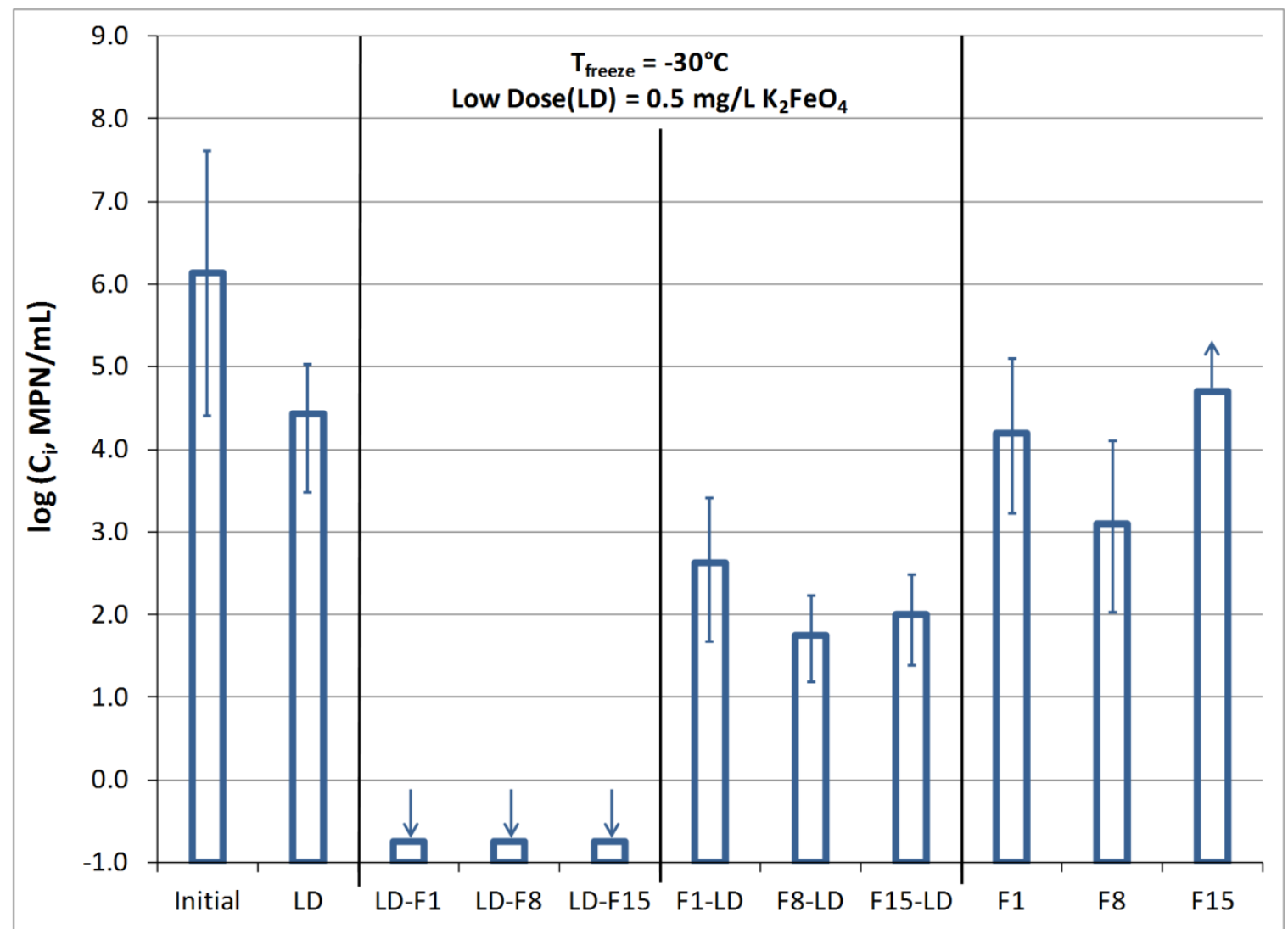

Figure 6-7: Effect of low ferrate(VI) dose ( $\left.\mathrm{LD}=0.5 \mathrm{mg} / \mathrm{L} \mathrm{K}_{2} \mathrm{FeO}_{4}\right)$ when used alone (15-min contact time), and as a pre- and post-treatment to freeze-thaw with $\mathrm{T}_{\text {Freeze }}=-30^{\circ} \mathrm{C}$.

Figure 6-8 shows the effect of HD when used alone, and as a pre- and post- treatment to freeze-thaw, with a freezing temperature of $-30^{\circ} \mathrm{C}$. HD resulted in a $3.4-\log$ reduction in E. coli after 15 minutes, which was a lower reduction than those achieved in previous tests, as shown in Figure 6-4 and Figure 6-6. However, similar to previous tests, HD pretreatment followed by 1,8 and 15 days at a freezing temperature of $-30^{\circ} \mathrm{C}$ reduced $E$. coli concentrations to the detection limit $(<0.18 \mathrm{MPN} / \mathrm{mL})$.

When used as a post-treatment, following freeze-thaw at a temperature of $-30^{\circ} \mathrm{C}, \mathrm{HD}$ significantly improved the reduction of $E$. coli compared to stand-alone freeze-thaw treatments, resulting in an additional 2.5 to $3.2-\log$ reduction in E. coli. 


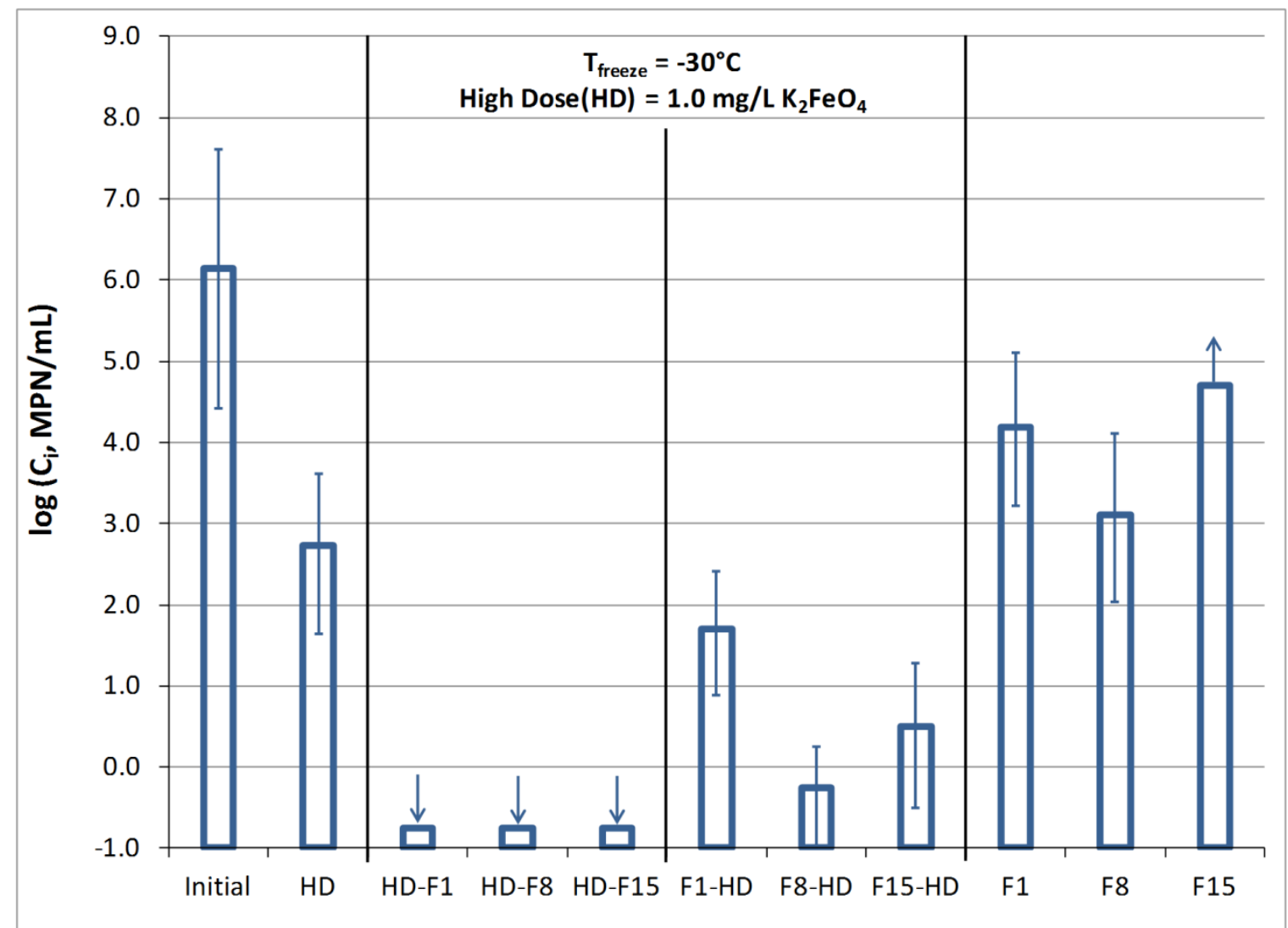

Figure 6-8: Effect of high ferrate(VI) dose ( $\left.\mathrm{HD}=1.0 \mathrm{mg} / \mathrm{L} \mathrm{K}_{2} \mathrm{FeO}_{4}\right)$ when used alone (15-min contact time), and as a pre- and post-treatment to freeze-thaw with $\mathrm{T}_{\text {Freeze }}=-30^{\circ} \mathrm{C}$.

\subsubsection{Effect of Freezing Time and Freezing Temperature (Freezing Rate)}

Experiments examined the effects of 1,8 and 15-day freezing periods on the reduction of $E$. coli using freezing temperatures of $-10,-20$ and $-30^{\circ} \mathrm{C}$. In addition to the designated freezing periods, samples took 400, 310 and 235 minutes to freeze using temperatures of $-10,-20$ and $-30^{\circ} \mathrm{C}$ respectively, and approximately 350 to 400 minutes to thaw and warm to room temperature. For freezing periods of 1,8 and 15 days, results suggest that the duration of the freezing conditions does not have a significant effect on the inactivation of $E$. coli in PBS. However, freezing periods greater than 15 days may result in additional reductions in E. coli. Kato et al., 2002, suggested that prolonged freezing 
periods led to higher C. parvum oocyst inactivation. Furthermore, Gao et al., 2006, did see slightly greater $E$. coli inactivation when the freezing period was increased from 10 to 20 days, however the majority of the inactivation occurred in the first few days of being frozen. Sanin et al., 1994, also noticed that the 0.6 to $0.7-\log$ inactivation of fecal coliform achieved during freeze-thaw was independent of the duration of the freezing conditions and also the storage temperature.

Freezer temperatures of $-10,-20$ and $-30^{\circ} \mathrm{C}$ generated freezing rates of $3.5,4.7$ and 6.6 $\mathrm{mm} / \mathrm{hr}$. These freezing rates fall within the optimal range for freeze-thaw treatments in terms of pathogen reduction and particle migration, leading to consolidated particles and good dewaterability (Wang et al., 2001, Sanin et al., 1995, Hung et al., 1997). Inactivation of E. coli ranged from 1.4 to $3-\log$, when the samples were frozen at -10, 20 and $-30^{\circ} \mathrm{C}$, for either 1,8 or 15 days. However, there were no significant differences among the various freezing temperatures. Similarly, the study by Gao et al., 2009, found that the levels of inactivation of two strains of $E$. coli and two strains of $E$. faecalis using freezing temperatures of $-7,-15$ and $-30^{\circ} \mathrm{C}$ were not statistically different from one another. However, previously Gao et al. (2006) found that a $-5^{\circ} \mathrm{C}$ freezing temperature resulted in greater inactivation in E. coli than -15 and $-35^{\circ} \mathrm{C}$.

\subsection{CONCLUSION}

The study examined the individual and combined effects of potassium ferrate(VI) and freeze-thaw treatment on the inactivation of $E$. coli in PBS. A low dose $(0.5 \mathrm{mg} / \mathrm{L})$ and a 
high dose $(1.0 \mathrm{mg} / \mathrm{L})$ of potassium ferrate $(\mathrm{VI})$ were used as a pre- and post-treatment to freeze-thaw at temperatures of $-10,-20$ and $-30^{\circ} \mathrm{C}$, for 1,8 and 15 days.

A (low) dose of $0.5 \mathrm{mg} / \mathrm{L}$ of potassium ferrate( $\mathrm{VI})$ and a 15 minute contact time did not have a significant effect on the concentration of $E$. coli. However, when the low dose was used as a pre-treatment, followed by freeze-thaw treatment for either 1,8 or 15 days at temperatures of $-10,-20$ or $-30^{\circ} \mathrm{C}$, greater than 6 -log inactivation of $E$. coli was achieved, and the concentrations were reduced to the detection limit $(<0.18 \mathrm{MPN} / \mathrm{mL})$.

$\mathrm{A}$ (high) dose of $1.0 \mathrm{mg} / \mathrm{L}$ of potassium ferrate $(\mathrm{VI})$ and a 15 minute contact time reduced E. coli concentrations by up to 6.4-log and when the high dose was used as a pretreatment, followed by freeze-thaw, E. coli concentrations were reduced to the detection limit $(<0.18 \mathrm{MPN} / \mathrm{mL})$. However $1.0 \mathrm{mg} / \mathrm{L}$ of potassium ferrate(VI) was excessive when used as a pre-treatment to freeze-thaw, since a dose of only $0.5 \mathrm{mg} / \mathrm{L}$ followed by freeze-thaw, also reduced $E$. coli concentrations to the detection limit.

As a post-treatment, potassium ferrate( $\mathrm{VI})$ additions resulted in greater inactivation of E. coli compared to stand-alone freeze-thaw treatments; however, the use of potassium ferrate $(\mathrm{VI})$ as a pre-treatment to freeze-thaw was much more effective.

Freezing temperatures of $-10,-20,-30^{\circ} \mathrm{C}$, and freezing periods of 1,8 and 15 days resulted in 1.4 to $3-\log$ inactivation of $E$. coli, with no significant difference among the various freezing temperatures and freezing times. 


\subsection{REFERENCES}

Canfield, C., Sutphen, R.L. (1982) Mother nature as sludge thickener. Water Eng Manag $129(13): 22-24$

Desjardins, M.-A., Brière, F.G. (1996) Conditionnement et déshydration de boues d'étangs facultatifs à l'aide du gel - dégel naturel: résultats d'essais. Can J Civ Eng $23(2): 323-339$

Ding, L., Liang, H.-C., Li, X.-Z. (2012) Oxidation of CH3SH by in situ generation of ferrate(VI) in aqueous alkaline solution for odour treatment. Sep Purif Technol 91 117124

Diak, J., Örmeci, B., Proux, C., (2011) Freeze-thaw treatment of RBC sludge from a remote mining exploration facility in subarctic Canada. Water Sci Technol 63(6):13091313

Gombos, E., Barkács, K., Felföldi, T., Vértes, C., Makó, M., Palkó, G., Záray, C. (2013) Removal of organic matters in wastewater treatment by ferrate(VI)-technology. Microchem J 107:115-120

He, C., Li, X.-Z., Sharma, V.K., Li, S.-Y. (2009) Elimination of sludge door by oxidizing sulphur-containing compounds with ferrate(VI). Environ Sci Technol 43:5890-5895

Hedström, A., Hanaeus, J. (1999) Natural freezing, drying, and composting for treatment of septic sludge. J Cold Reg Eng December 1999 
Hellström, D., Kvarnström, E., (1997) Natural sludge dewatering. I: Combination of freezing, thawing, and drying as dewatering methods. J Cold Reg Eng March 1997 Hu, K., Jiang, J.-Q., Zhao, Q.-L., Lee, D.-J., Wang, K., Qiu, W. (2011) Conditioning of wastewater sludge using freezing and thawing: Role of curing. Water Res 45(18):59695976

Hung, W.T., Feng, W.H., Tsai, I.H., Lee, D.J., Hong, S.G. (1997) Uni-directional freezing of waste activated sludges: vertical freezing versus radial freezing. Water Res 31(9):22192228

Jiang, J.-Q., Wang, S., Panagoulopoulos, A. (2007) The role of potassium ferrate(VI) in the inactivation of Escherichia coli and in the reduction of COD for water remediation. Desalination 210(1-3):266-273

Jiang, J.-Q., Zhoo, Z. (2013) Removal of pharmaceutical residues by ferrate(VI). PLoS ONE 8(2): e55729. doi:10.1371/journal.pone.0055729

Jiang, J.-Q. (2007) Research progress in the use of ferrate(VI) for the environmental remediation. J Haz Mat 146(3):617-623

Jiang, J.-Q., Panagoulopoulos, A., Bauer, M., Pearce, P. (2006) The application of potassium ferrate for sewage treatment. J Environ Manag 79(2):215-220

Kato, S., Jenkins, M.B., Fogarty, E.A., Bowman, D.D. (2002) Effects of freeze-thaw events on the viability of Cryptosporidium parvum oocysts in soil. J Parasitol 88(4):718-722 
Li, C., Li, X.Z., Graham, N., Gao, N.Y. (2008) The aqueous degradation of bisphenol A and steroid estrogens by ferrate. Water Res 42(1-2):109-120

Martel, C.J. (2001) Design of freezing bed for sludge dewatering at McMurdo, Antarctica. US Army Corps of Engineers - Cold Regions Research \& Engineering Laboratory. Technical Report ERDC/CRREL TR, prepared for National Science Foundation Martel, C.J. (1993) Fundamentals of sludge dewatering in freezing beds. Water Sci Technol 28(1):29-35

Martel, C.J., Diener, C.J. (1991) Pilot-scale studies of sludge dewatering in a freezing bed. Can J Civ Eng 18(4):681-689

Mazur, P. (1986). Causes of injury in frozen and thawed cells. Fedn Proc Fedn Am Socs Exp Biol 24, Suppl. 15, S175-S182

Northcott, K.A., Snape, I., Scales, P.J., Stevens, G.W. (2005) Contaminated water treatment in cold region: an example of coagulation and dewatering modelling in Antarctica. Cold Reg Sci Technol 41(1):61-72

Penman, A., Van Es, D.W. (1973) Winnipeg freezes sludge, slashes disposal costs 10 fold. Civ Eng ASCE 43(11):65-67

Sanin, F.D., Vesilind, P.A., Martel, C.J. (1994) Pathogen reduction capabilities of freeze/thaw sludge conditioning. Water Res 28(11):2393-2398 
Schuck, C.A., De Luca, S.J., Peralba, M., De Luca, M.A. (2006) Sodium ferrate(IV) and sodium hypochlorite in disinfection of biologically treated effluents. Ammonium nitrogen protection against THMs and HAAs. J Environ Sci Health A41(10):2329-2343 United States Environmental Protection Agency (U.S. EPA) (2005). Method 1681: Fecal Coliforms in Sewage Sludge (Biosolids) by Multiple Tube Fermentation using A-1 Medium. Washington DC, USA.

Wang, Q., Fujisaki, K., Ohsumi, Y., Ogawa, H.I. (2001) Enhancement of dewaterability of thickened waste activated sludge by freezing and thawing treatment. J Environ Sci Health A36(7):1361-1371

Yang, E.-L., Shi, J.-J., Liang, H.-C. (2012a) On-line electrochemical production of ferrate(VI) for odor control. Electrochim Acta 63:369-374

Yang, B., Ying, G.-G., Zhao, J,-L, Liu, S., Zhou, L.-J., Chen, F. (2012b) Removal of selected endocrine disrupting chemicals (EDCs) and pharmaceuticals and personal care products (PPCPs) during ferrate(VI) treatment of secondary wastewater effluents. Water Res 46(7):2194-2204

Zhu, J.-H., Yan, X.-L., Liu, Y., Zhang, B. (2006) Improving alachlor biodegradability by ferrate oxidation. J Haz Mat 135(1-3):94-99 


\section{INDIVIDUAL AND COMBINED EFFECTS OF FREEZE-THAW AND FERRATE(VI) OXIDATION FOR THE STABILISATION AND DEWATERING OF WASTEWATER SLUDGES}

James Diak and Banu Örmeci

Department of Civil and Environmental Engineering, Carleton University, 1125 Colonel By Drive, Ottawa, ON K1S 5B6, Canada.

\subsection{ABSTRACT}

The study examined the individual and combined effects of potassium ferrate(VI) additions and freeze-thaw conditioning for the stabilisation and dewatering of sludge samples. The first part of the experiments, using primary sludge, compared potassium ferrate(VI) additions prior to freeze-thaw treatment (pre-treatment) versus potassium ferrate(VI) additions following freeze-thaw treatment (post-treatment). These tests were also compared to stand-alone freeze-thaw, without potassium ferrate(VI) additions. A low dose (LD) of $1.0 \mathrm{~g} / \mathrm{L}$ and a high dose (HD) of $10.0 \mathrm{~g} / \mathrm{L}$ of potassium ferrate(VI) were used as either a pre- or post-treatment to freezing, at a temperature of $-20^{\circ} \mathrm{C}$, for 1,8 and 15 days. Following the designated freezing period, the samples were removed from the freezer and thawed at room temperature for 12 hours. Sludge samples were characterised in terms of fecal coliform, total solids (TS), volatile solids (VS), capillary suction time (CST), ammonia and sulphide. 
The second part of the study, using anaerobically digested sludge, evaluated the effects of potassium ferrate $(\mathrm{VI})$ pre-treatment, prior to freeze-thaw at $-20^{\circ} \mathrm{C}$, using $\mathrm{LD}=0.5 \mathrm{~g} / \mathrm{L}$ and $H D=5.0 \mathrm{~g} / \mathrm{L}$. The study used simulated drainage beds to separate the meltwater from the sludge cake samples during the sludge thawing period. The effluent meltwater was characterised in terms of fecal coliform, soluble proteins, soluble carbohydrates, soluble chemical oxygen demand (sCOD), $\mathrm{pH}$ and turbidity. The sludge cake was characterised in terms of fecal coliform, TS and VS.

The study demonstrated that stand-alone freeze-thaw can reduce fecal coliform by >3log after being frozen for only 1 day, and pre-treatment with potassium ferrate(VI) can be used to improve the effects of freeze-thaw on fecal coliform inactivation in sludge. Furthermore, the drainability of the sludge following freeze-thaw was not significantly deteriorated when potassium ferrate( $\mathrm{VI})$ was added to the sludge prior to freezing, despite greater than 4-fold increases in the concentrations of soluble proteins and soluble carbohydrates.

The meltwater collected during the sludge thawing was approximately $85 \%$ of the initial sludge volume. When $5 \mathrm{~g} / \mathrm{L}$ of potassium ferrate(VI) was added to the sludge prior to freezing, the meltwater collected had $<0.28 \mathrm{MPN} / \mathrm{mL}$ fecal coliform, the turbidity was $<10 \mathrm{NTU}$, and the $\mathrm{pH}$ was 9.1. Pre-treatment with potassium ferrate(VI) also reduced the concentration of fecal coliform in the sludge cake, suggesting that freeze-thaw coupled with potassium ferrate(VI) additions can be used to stabilise sludge, and reduce sludge volume. 


\subsection{INTRODUCTION}

Freeze-thaw sludge conditioning can be used to dewater sludge (Hu et al., 2011, Diak et al., 2011, Northcott et al., 2005, Parker and Collins, 1999) and reduce pathogens and indicator bacteria (Gao et al., 2009, Gao et al., 2006, Kato et al., 2002, Sanin et al., 1994). When the sludge freezes, ice crystals grow which consolidate sludge solids and create a continuous network of ice (Tao et al., 2006, Vesilind and Martel, 1990). When the sludge thaws, the meltwater drains freely leaving a dewatered cake (Diak et al., 2011, Martel, 1993, Martel and Diener 1991a, Martel and Diener, 1991b). Despite the obvious attractiveness of using natural freeze-thaw dewatering in rural cold climates, there are concerns which need to be addressed. A major concern is the potential for reactivation, regrowth and odour generation, particularly during the thawing stage. Another is the quantity, quality, management and disposal of the meltwater and remaining sludge cake.

In recent years, there has been considerable interest in the reactivation and regrowth of indicator organisms during the dewatering and storage of anaerobically digested biosolids (Chen et al., 2011, Gardner et al., 2010, Higgins et al., 2007). Reactivation and regrowth is not only a health and safety concern, it will also lead to odour generation. Odorous compounds in sludge generally contain reduced forms of nitrogen, such as ammonia and amines, and reduced forms of sulphur, such as sulphides and mercaptans (Chen et al., 2011, de Luca et al., 1996). 
Ferrate $(\mathrm{VI})$ is a very strong oxidant $\left(\mathrm{E}^{0}=2.2 \mathrm{~V}\right)$, capable of oxidising reduced sulphur to sulphate and ammonia to nitrate (de Luca et al., 1996), and it is an effective coagulant and a proven disinfectant, which produces no known harmful disinfection by-products (Gombos et al., 2013, Jiang et al., 2007, Jiang et al., 2006, Schuck et al. 2006, Murmann and Robinson 1974). It is also capable of oxidising a variety of pharmaceuticals and personal care products (PPCPs) (Jiang and Zhoo, 2013, Yang et al., 2012b, Li et al., 2008, Jiang, 2007, Zhu et al. 2006). Ferrate(VI) oxidation treatments can be used in conjunction with freeze-thaw to potentially reduce the risks of regrowth and odour generation, and improve the quality of the meltwater and sludge cake.

Oxidation treatments disintegrate sludge flocs resulting in smaller particle sizes and the release of extracellular polymeric substances (EPS) (Wu et al., 2014). Sludge oxidation can also result in cell lysis, and the release of intracellular organic substances (Saktaywin et al., 2005). The solubilisation of EPS and intracellular constituents increases the concentration of proteins and carbohydrates in the supernatant (Örmeci and Vesilind, 2001). This alters the sludge surface charge and increases the polymer demand during sludge conditioning (Apul et al., 2010).

A decrease in particle size increases the amount of water bound to the surface of the sludge particles, which typically reduces sludge dewaterability via conventional means. However during freeze-thaw, the smaller particle sizes improve particle migration during freezing (Corte, 1962), which will improve the formation of ice-channels and improve sludge drainability. 
The meltwater collected during the thawing stage can amount to more than $85 \%$ of the initial sludge volume (Diak et al., 2011). A well-designed sludge drainage bed will result in a meltwater that is free of solids, and easily collected for subsequent treatment and disposal. Land application of the dewatered sludge cake is an economical way to dispose of sludge, and an efficient strategy to recycle nutrients and organic matter.

The purpose of this research was to evaluate the individual and combined effects of potassium ferrate(VI) additions and freeze-thaw conditioning for the stabilisation and dewatering of wastewater sludges. The first part of the experiments compared potassium ferrate(VI) additions prior to freeze-thaw (pre-treatment) and following freeze-thaw (post-treatment). These tests were also compared to stand-alone freezethaw. The second part of the study used freeze-thaw treatment with bench-scale simulated drainage beds to separate the meltwater from the sludge cake via gravity during the sludge thawing period to be able to study the cake and meltwater characteristics individually.

\subsection{MATERIALS AND METHODS}

\subsubsection{Sludge samples}

The sludge used for part I (sludge bottle experiments) was primary sludge, obtained from the Robert O. Pickard Environmental Centre (ROPEC) in Ottawa, Ontario, Canada. The sludge used for part II (sludge drainage experiments) was anaerobically digested sludge from ROPEC. The initial characteristics of the sludges are presented in Table 7-1. 
Table 7-1: Characteristics of the primary and anaerobically digested sludge samples used for part I and part II respectively.

\begin{tabular}{|c|c|cc|cc|}
\hline & & \multicolumn{2}{|c|}{$\begin{array}{c}\text { Primary Sludge } \\
\text { Parameter }\end{array}$} & Units & $\begin{array}{c}\text { Anaerobically Digested } \\
\text { Sludge } \\
\text { AVERAGE } \pm 95 \% \mathrm{CL})\end{array}$ \\
AVERAGE $( \pm 95 \% \mathrm{CL})$
\end{tabular}

\subsubsection{Part I: Freeze-thaw treatment in bottles}

$250 \mathrm{~mL}$ primary sludge samples were dispensed into $500 \mathrm{~mL}$ autoclavable polypropylene Nalgene bottles, and placed in a freezer (VWR FORMA ${ }^{\circledR}-40^{\circ} \mathrm{C}$ Lab Freezer Model 5722, VWR International, Mississauga, ON) set to $-20^{\circ} \mathrm{C} \pm 1^{\circ} \mathrm{C}$. The temperature at the centre of a $250 \mathrm{~mL}$ test sample and the temperature inside the freezer were monitored using a Traceable ${ }^{\circledR}$ dual-channel thermometer with type $\mathrm{K}$ thermocouples and computer output (Model 4137, Control Company, Friendswood, TX, USA). Temperatures were recorded on a PC every minute using the data acquisition software (DAS ${ }^{\mathrm{TM}}$, Control Company, Friendswood, TX, USA). When the samples were placed in the freezer, the temperature at the centre of the test sample decreased at a steady rate of $1^{\circ} \mathrm{C}$ every 10 minutes until reaching the sludge freezing temperature, which was approximately $-0.3^{\circ} \mathrm{C}$. At this point, the temperature of the sample remained constant for approximately 7 hours while the sample froze. During this freezing period, it was assumed that the direction of 
the advancing ice front was radially inward, since the sample bottles were not insulated. Once the samples were completely frozen, the temperature of the sample began to decrease again, until eventually reaching the freezer temperature of $-20^{\circ} \mathrm{C}$. The 7 hour elapsed time for the sludge to freeze, and the $31 \mathrm{~mm}$ depth to the sample centre were used to estimate the freezing rate of approximately $4.4 \mathrm{~mm} / \mathrm{hr}$, which is within the optimal range for pathogen reduction and particle migration leading to improved dewaterability (Wang et al., 2001, Hung et al., 1997). A plot of the temperature log used to estimate the freezing rate is present in Appendix B2 on page 381. After the samples were completely frozen, they were kept frozen at $-20^{\circ} \mathrm{C}$ for an additional 1,8 or 15 days. After the designated period in the freezer, the samples were removed and thawed at room temperature $\left(22-23^{\circ} \mathrm{C}\right)$.

\subsubsection{Pre- and Post-Treatment with Potassium Ferrate(VI) $\left(\mathrm{K}_{2} \mathrm{FeO}_{4}\right)$}

Primary sludge samples that were pre-treated with potassium ferrate(VI), prior to freeze-thaw, were given a low dose $(L D=1.0 \mathrm{~g} / \mathrm{L})$ or a high dose $(\mathrm{HD}=10.0 \mathrm{~g} / \mathrm{L})$ of Ferratec Brand ${ }^{\mathrm{TM}}>90 \%$ pure potassium ferrate(VI) (Sigma-Aldrich Canada Ltd., Oakville, $\mathrm{ON})$. Following the addition of potassium ferrate(VI), the samples were stirred using a magnetic stirrer for 15 minutes and subsequently placed into the freezer.

Post-treatment with potassium ferrate(VI) was conducted in a similar fashion. After the designated time in the freezer, the samples were removed and thawed at room temperature $\left(22-23^{\circ} \mathrm{C}\right)$. Once the samples were completely thawed and warmed to room temperature, $250 \mathrm{mg}$ of potassium ferrate(VI) was added to the bottles 
designated for LD post-treatment $(1.0 \mathrm{~g} / \mathrm{L})$, and $2.5 \mathrm{~g}$ was added to the bottles designated for HD post-treatment $(10.0 \mathrm{~g} / \mathrm{L})$. After the addition of potassium ferrate(VI), the bottles were stirred using a magnetic stirrer for 15 minutes prior to being analysed.

A brief description of the various treatments performed on the primary sludge samples is presented in, along with an abbreviated sample label that is used in the subsequent figures.

Table 7-2: Description of the treatments applied to the primary sludge samples and their representative abbreviation.

\begin{tabular}{|c|c|}
\hline Label & Description of the treatments applied to the primary sludge samples \\
\hline Initial & Initial sludge sample prior to any treatment \\
\hline LD & Low dose $(\mathrm{LD}=1.0 \mathrm{~g} / \mathrm{L})$ of $\mathrm{K}_{2} \mathrm{FeO}_{4}$ and a 15 -min contact time \\
\hline HD & High dose $(\mathrm{HD}=10.0 \mathrm{~g} / \mathrm{L})$ of $\mathrm{K}_{2} \mathrm{FeO}_{4}$ and a 15 -min contact time \\
\hline LD-F1 & Low-dose $\left(\mathrm{LD}=1.0 \mathrm{~g} / \mathrm{L} \mathrm{K}_{2} \mathrm{FeO}_{4}\right)$ pre-treatment followed by 1 day frozen and a 12 -hr thaw \\
\hline LD-F8 & Low-dose $\left(\mathrm{LD}=1.0 \mathrm{~g} / \mathrm{L} \mathrm{K}_{2} \mathrm{FeO}_{4}\right)$ pre-treatment followed by 8 days frozen and a 12 -hr thaw \\
\hline LD-F15 & Low-dose $\left(\mathrm{LD}=1.0 \mathrm{~g} / \mathrm{L} \mathrm{K}_{2} \mathrm{FeO}_{4}\right)$ pre-treatment followed by 15 days frozen and a 12 -hr thaw \\
\hline F1-LD & 1 day frozen and a $12-\mathrm{hr}$ thaw followed by a low dose (LD=1.0 g/L) of $\mathrm{K}_{2} \mathrm{FeO}_{4}$ and 15 -min contact time \\
\hline F8-LD & 8 days frozen and a $12-\mathrm{hr}$ thaw followed by a low dose $\left(\mathrm{LD}=1.0 \mathrm{~g} / \mathrm{L}\right.$ ) of $\mathrm{K}_{2} \mathrm{FeO}_{4}$ and 15 -min contact time \\
\hline F15-LD & 15 days frozen and a 12 -hr thaw followed by a low dose (LD=1.0 g/L) of $\mathrm{K}_{2} \mathrm{FeO}_{4}$ and 15 -min contact time \\
\hline HD-F1 & High-dose $\left(\mathrm{HD}=10.0 \mathrm{~g} / \mathrm{L} \mathrm{K}_{2} \mathrm{FeO}_{4}\right)$ pre-treatment followed by 1 day frozen and a 12 -hr thaw \\
\hline HD-F8 & High-dose $\left(\mathrm{HD}=10.0 \mathrm{~g} / \mathrm{L} \mathrm{K}_{2} \mathrm{FeO}_{4}\right)$ pre-treatment followed by 8 days frozen and a 12 -hr thaw \\
\hline HD-F15 & High-dose $\left(\mathrm{HD}=10.0 \mathrm{~g} / \mathrm{L} \mathrm{K}_{2} \mathrm{FeO}_{4}\right)$ pre-treatment followed by 15 days frozen and a 12 -hr thaw \\
\hline F1-HD & 1 day frozen and a $12-\mathrm{hr}$ thaw followed by a high dose $(\mathrm{HD}=10.0 \mathrm{~g} / \mathrm{L})$ of $\mathrm{K}_{2} \mathrm{FeO}_{4}$ and a 15 -min contact time \\
\hline F8-HD & 8 days frozen and a $12-\mathrm{hr}$ thaw followed by a high dose $(\mathrm{HD}=10.0 \mathrm{~g} / \mathrm{L})$ of $\mathrm{K}_{2} \mathrm{FeO}_{4}$ and a 15 -min contact time \\
\hline F15-HD & 15 days frozen and a 12 -hr thaw followed by a high dose $(\mathrm{HD}=10.0 \mathrm{~g} / \mathrm{L})$ of $\mathrm{K}_{2} \mathrm{FeO}_{4}$ and a 15 -min contact time \\
\hline F1 & 1 day frozen and a $12-\mathrm{hr}$ thaw \\
\hline F8 & 8 days frozen and a $12-\mathrm{hr}$ thaw \\
\hline F15 & 15 days frozen and a $12-\mathrm{hr}$ thaw \\
\hline
\end{tabular}

\subsubsection{Part II: Freeze-thaw treatment in simulated drainage beds}

$200 \mathrm{~mL}$ samples of anaerobically digested sludge were placed in $500 \mathrm{~mL}$ bottles. The bottles were sealed tightly with a wide-mouth lid, and the bottles were inverted as shown in Figure 7-1a. The inverted bottles were then placed in the freezer, which was 
set to $-20^{\circ} \mathrm{C}$. The freezing rate generated was approximately $5.6 \mathrm{~mm} / \mathrm{hr}$ ( $4.6 \mathrm{hrs}$ to travel $25 \mathrm{~mm}$ ). A plot of the temperature log used to estimate the freezing rate is presented in Appendix B3 on page 382. Once the samples were completely frozen, they were kept frozen at $-20^{\circ} \mathrm{C}$ for an additional 1,8 or 15 days. After the designated time in the freezer, the samples were removed, the solid lid was replaced with a perforated lid lined with drainage fabric, and the tape sealing the air hole was removed. The frozen samples were then placed upside down in a funnel, which rested in the opening of a $50 \mathrm{~mL}$ graduated cylinder, as shown in Figure $7-1 \mathrm{~b}$. The samples thawed at room temperature $\left(22-23^{\circ} \mathrm{C}\right)$ and the meltwater was collected via gravity drainage. The experiments using simulated drainage beds did not use ferrate(VI) as a post-treatment to freeze-thaw due to the separation of solids and meltwater. Only ferrate(VI) pre-treatment was used for these experiments, along with stand-alone freeze-thaw treatment. 
a)

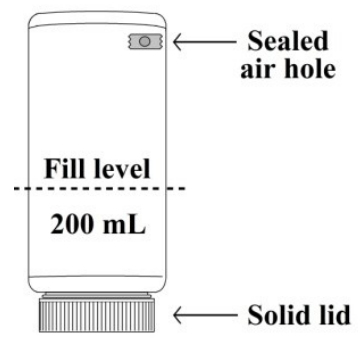

b)

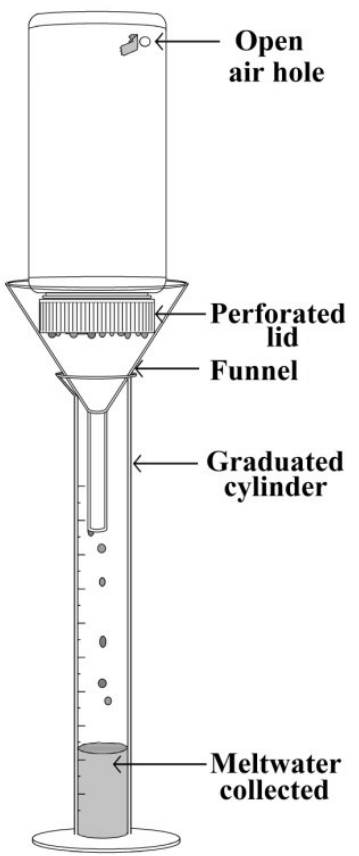

Figure 7-1: Schematic of (a) the freezing vessel and (b) the simulated drainage bed with meltwater collection, used for the freeze-thaw experiments with anaerobically digested sludge.

\subsubsection{Pre-Treatments with Potassium Ferrate(VI) $\left(\mathrm{K}_{2} \mathrm{FeO}_{4}\right)$}

Anaerobically digested sludge samples that were pre-treated with potassium ferrate(VI), prior to freeze-thaw, were given $L D=0.5 \mathrm{~g} / \mathrm{L}$ or $H D=5.0 \mathrm{~g} / \mathrm{L}$ of potassium ferrate $(\mathrm{VI})$. Following the addition of potassium ferrate(VI), the samples were stirred using a magnetic stirrer for 15 minutes and subsequently placed into the freezer.

\subsubsection{Sample analysis}

Preliminary tests on the primary sludge evaluated the filterability using capillary suction time (CST), and ammonia and sulphide concentrations of the sludge before and after the addition of potassium ferrate(VI). The fecal coliform, Total Solids (TS) and Volatile Solids (VS) concentrations of the primary sludge were measured prior to the addition of potassium ferrate( $\mathrm{VI})$ and freeze-thaw treatment, following the addition of potassium 
ferrate(VI) prior to freeze-thaw treatment, following the freeze-thaw treatment, and following the addition of potassium ferrate(VI) after the freeze-thaw treatment.

For the freeze-thaw sludge dewatering experiments using anaerobically digested sludge, the fecal coliform, TS and VS, soluble chemical oxygen demand (sCOD), soluble proteins and soluble carbohydrates concentrations of the sludge were measured before and after the addition of potassium ferrate(VI). Following freeze-thaw dewatering, the fecal coliform, TS and VS concentrations of the sludge cake were measured, and the concentration of fecal coliform, sCOD, soluble proteins, soluble carbohydrates, $\mathrm{pH}$ and turbidity of the meltwater were measured. The methods used to measure each of these parameters are presented below.

The concentration of fecal coliform was measured using multiple tube fermentation (MTF) with A1 medium (EMD Chemicals Inc., Gibbstown, NJ, USA) according to U.S. EPA Method 1681 (U.S. EPA, 2005). All MTF tests were carried out in duplicate using a minimum of 4 dilutions, with 5 replicates per dilution. All other tests were conducted in triplicate. The TS and VS concentrations were measured according to Standard Method 2540 G (APHA, 2005). The CST was measured using a Triton Electronics Type 319 multipurpose CST apparatus (Triton Electronics Ltd., Great Dunmow, Essex, England) according to Standard Method 2710 G (APHA, 2005). The turbidity was measured using a HACH Model 2100AN Turbidimeter (Hach Company, Loveland CO, USA).

Colorimetric tests were conducted using a HACH DR 2800 spectrophotometer, and for COD tests, a HACH DRB 200 digital reactor block was also used. The sulphide 
concentration was measured using HACH method 8131 (methylene blue method, 5-800 $\mu \mathrm{g} / \mathrm{L} \mathrm{S}^{-2}$ ). The primary sludge sample was diluted with deionised water by a factor of 100. The test blank was adjusted for turbidity using a combination of bromine water and phenol, as outlined in the $\mathrm{HACH}$ method. The total ammonia concentration $\left(\mathrm{NH}_{3}+\mathrm{NH}_{4}{ }^{+}\right)$ was measured using $\mathrm{HACH}$ method 10031 (high range: 0.4-50.0 mg/L NH$-\mathrm{N}$ ) with a dilution factor of 10. The $\mathrm{pH}$ was measured using a Thermo Orion 5-star bench-top meter kit and ROSS ultra pH electrode (Thermo Fisher Scientific Inc., Rockford, IL, USA).

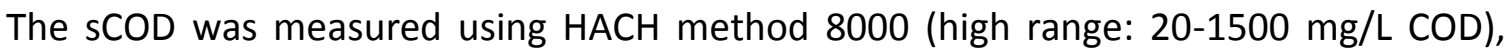
with a dilution factor of 2 . The concentration of soluble proteins was measured using Coomassie brilliant blue G-250 reagent (Pierce Protein Biology Products, Thermo Fisher Scientific Inc., Rockford, IL, USA) with the Bradford method (Bradford, 1976) and the concentration of soluble carbohydrates was measured using the Anthrone method (Morris, 1948). To obtain the soluble component, the samples were centrifuged at $10,000 \mathrm{rpm}$ (relative centrifugal force, $\mathrm{RCF}=15,317$ ) for 10 minutes, then the supernatant was filtered using a $0.45 \mu \mathrm{m}$ mixed cellulose ester (MCE) syringe filter (Shanghai Derian Instrument Co., Ltd., Shanghai, China).

\subsubsection{Statistical analysis}

Error bars on all figures show the $95 \%$ confidence interval. In cases where the measurement is below the detection limit, a downward arrow is used in place of the error bars. When comparing the data, a two-tailed t-test was used to determine the $p$ values and statistical significance $(p<0.05)$ of the results. 


\subsection{RESULTS AND DISCUSSION}

\subsubsection{Part I: Freeze-thaw treatment in bottles}

Experiments in part I evaluated the effects of LD and HD on the CST, ammonia and sulphide concentrations in primary sludge, following a 15-minute reaction time. Experiments also compared the effects of stand-alone freeze-thaw sludge conditioning using a freezer temperature of $-20^{\circ} \mathrm{C}$. Samples were kept frozen for 1,8 and 15 days, then thawed at room temperature. The use of potassium ferrate( $\mathrm{VI})$ as a pre- and a post-treatment to freeze-thaw conditioning was also compared. All samples were characterised in terms of fecal coliform, TS and VS concentrations.

\subsubsection{Effect of Potassium Ferrate(VI) on the Capillary Suction Time (CST)}

Figure 7-2a shows the effect of potassium ferrate(VI) additions on the CST of the primary sludge. Chemical oxidation of sludge generally results in the disintegration of sludge flocs and the destruction of cell walls, causing the release of intra- and extracellular biopolymers such as proteins and carbohydrates, and colloids (Wu et al., 2014). This may reduce the measured filterability of the sludge by clogging the filter papers used in conventional sludge filterability tests such as the CST and the specific resistance to filtration (SRF) (Ye et al., 2014). In this study, LD decreased the CST from 912 to 575 seconds following a 15-minute contact time. This was likely due to the oxidation of free biopolymers in sludge. However, HD increased the CST from 912 to 2648 seconds. This suggests that HD led to the solubilisation of sludge flocs and the destruction cell walls, causing the release of intracellular and extra cellular materials to the supernatant. Pretreatment techniques which result in sludge solubilisation are generally unfavourable in 
terms of sludge dewaterability. However, during freezing, sludge solids are consolidated by the advancing ice-front as the free water is drawn away from the particles and added to the growing network of ice crystals (Hoekstra and Miller, 1967). Furthermore, Corte (1962) demonstrated that smaller particles are more easily pushed away from the advancing ice front, and less likely to be engulfed by the growing ice crystals. This means that pre-treatment with potassium ferrate(VI), prior to freeze-thaw, may improve particle consolidation during freezing and increase the separation of meltwater from the sludge solids during the thawing stage.

According to Vesilind and Örmeci (2000), the escape of water from the sludge following freeze-thaw conditioning is too fast for the CST test, making the movement of water through the filter paper the rate-limiting step, rather than the release of water from the sludge. Therefore, the CST test was not used to measure the rate of movement of water through the sludge following freeze-thaw conditioning. In Part II of this study, a benchscale freeze-thaw apparatus, equipped with meltwater collection via gravity drainage, was used to measure the release of water from the sludge following freeze-thaw.

\subsubsection{Effect of Potassium Ferrate(VI) on the Concentration of Ammonia and Sulphides}

Odorous compounds in sludge generally contain reduced forms of nitrogen and sulphur. These include ammonia and amines, sulphides and mercaptans (Metcalf and Eddy, 2003). The addition of a chemical oxidant, such as potassium ferrate(VI), can oxidise the reduced nitrogen and sulphur, causing a decrease in the concentration of ammonia, 
amines, sulphides and mercaptans, and a reduction in sludge odour. Figure 7-2b shows the effect of potassium ferrate( $\mathrm{VI})$ additions on the concentrations of ammonia and sulphide in the primary sludge.

The ammonia concentration decreased by $11 \%$, from 210 to $187 \mathrm{mg} / \mathrm{L} \mathrm{NH}_{3}-\mathrm{N}$, due to the addition of $10.0 \mathrm{~g} / \mathrm{L}$ of potassium ferrate $(\mathrm{VI})$ with a 15 -minute contact time $(\mathrm{p}=0.0249)$; however, $1.0 \mathrm{~g} / \mathrm{L}$ did not have a significant effect on the concentration of ammonia $(p=0.1161)$. The concentration of sulphides decreased by approximately $72 \%$, from 39.3 $\mathrm{mg} / \mathrm{L}$ to $11.1 \mathrm{mg} / \mathrm{L}$, using $1.0 \mathrm{~g} / \mathrm{L}$ of potassium ferrate $(\mathrm{VI})$, and by $89 \%$, from $39.3 \mathrm{mg} / \mathrm{L}$ to $4.2 \mathrm{mg} / \mathrm{L}$, using $10.0 \mathrm{~g} / \mathrm{L}$. The authors also noted that following the addition of $1.0 \mathrm{~g} / \mathrm{L}$ of potassium ferrate( $\mathrm{VI})$, the odour of the primary sludge was considerably less offensive, and following the addition of $10.0 \mathrm{~g} / \mathrm{L}$, the odour of the primary sludge resembled an earthy rust-water. 

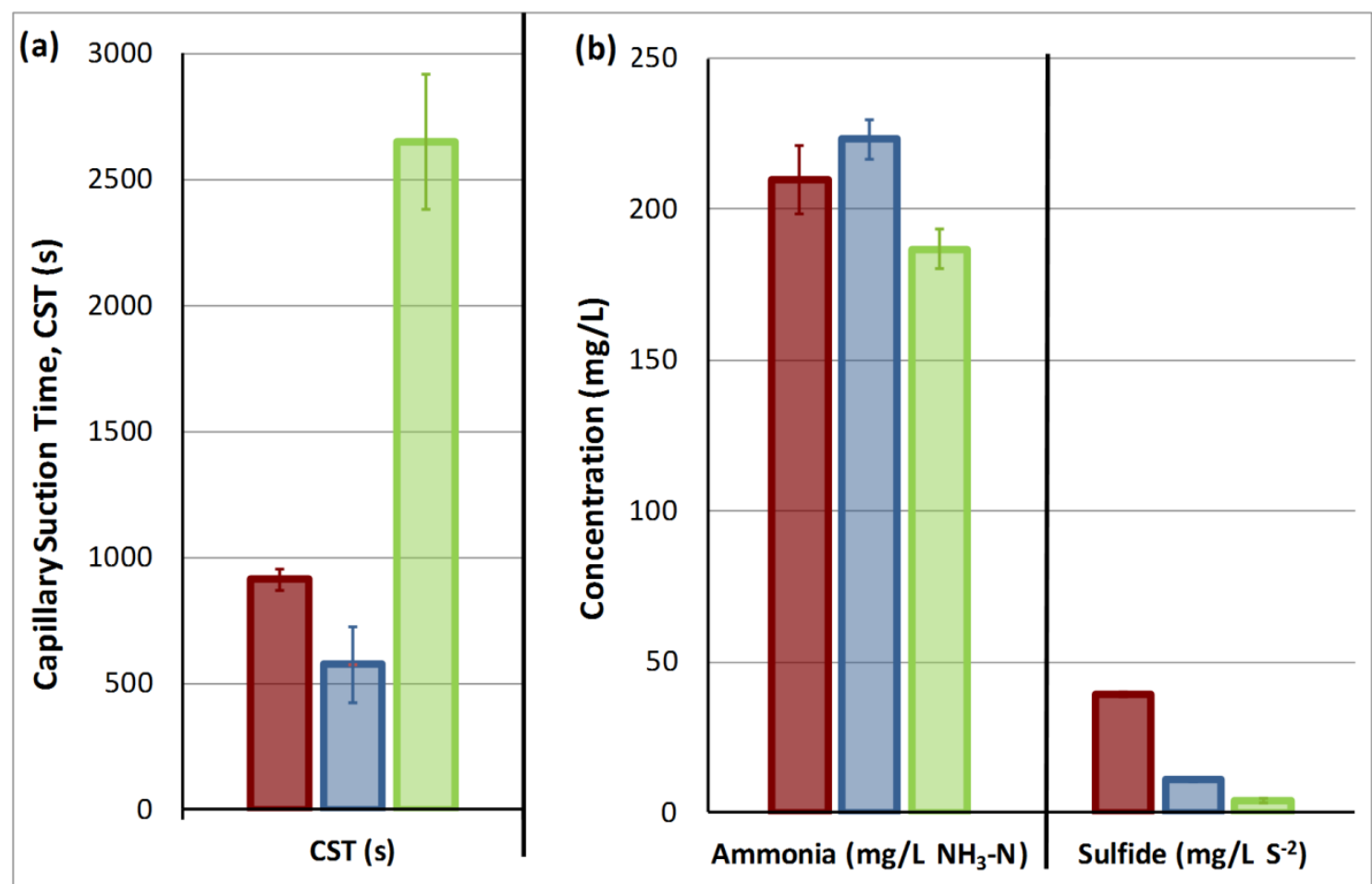

Initial Sludge $\square$ Low Dose (LD) $=1.0 \mathrm{~g} / \mathrm{L} \mathrm{K}_{2} \mathrm{FeO}_{4} \square \mathrm{High}$ Dose (HD) $=10 \mathrm{~g} / \mathrm{L} \mathrm{K}_{2} \mathrm{FeO}_{4}$ (15-minute contact time)

(15-minute contact time)

Figure 7-2: Effect of LD and HD on the (a) CST and (b) ammonia and sulphide concentrations in the primary sludge, following a 15 -minute contact time.

\subsubsection{Pre- and Post-Treatment with LD - Fecal Coliform}

Figure 7-3 shows the effect of LD on the concentration of fecal coliform when used as a pre- and post-treatment to freeze-thaw. The figure also shows the effects of standalone freeze-thaw for 1,8 and 15 days frozen. The initial concentration of fecal coliform in the primary sludge was $1 \times 10^{6} \mathrm{MPN} / \mathrm{g}$ dry solids (DS). Following a 15-minute reaction with $L D=1.0 \mathrm{~g} / \mathrm{L}$ potassium ferrate $(\mathrm{VI})$, there was no significant change in concentration $(p=0.9237)$. Stand-alone freeze-thaw resulted in a 3.4 to $3.9-\log$ reduction, with no significant difference among the samples that were frozen for 1,8 or 15 days (F1 vs. F8: $p=0.0715, F 1$ vs. F15: $p=0.7173, F 8$ vs. F15: $p=0.1858)$. It was expected that samples that 
were pre-treated with potassium ferrate(VI) would have a greater fecal coliform inactivation than stand-alone freeze-thaw; however, LD pre-treatment may have reduced the effectiveness of stand-alone freeze-thaw. Samples that were pre-treated with LD and subsequently frozen resulted in 1.6 to $2.7-\log$ inactivation, approximately 1.3-log less than stand-alone freeze-thaw. de Luca et al. (1996) suspected that low ferrate(VI) doses may stimulate bacterial growth due to an increase in the nitrate concentration, with little change in the $\mathrm{pH}$. Additionally, potassium ferrate(VI) increased the amount of polymers in the sludge supernatant which may have acted as cryoprotectants, shielding bacteria during freezing (Montusiewicz et al., 2010). Furthermore, sludge solubilisation increased the available substrate for surviving bacteria, which may have contributed to regrowth during the thawing period.

Samples treated with LD following freeze-thaw did not have a significantly different level of inactivation compared to stand-alone freeze-thaw. This suggests that $1.0 \mathrm{~g} / \mathrm{L}$ of potassium ferrate( $\mathrm{VI})$ was insufficient for the inactivation of fecal coliform in primary sludge. There are many constituents in sludge which quickly react with the highly reactive ferrate $(\mathrm{VI})$ ions. It is likely that a potassium ferrate( $\mathrm{VI})$ dose of only $1.0 \mathrm{~g} / \mathrm{L}$ was quickly consumed by other components in the sludge, leaving little ferrate(VI) for disinfection. 


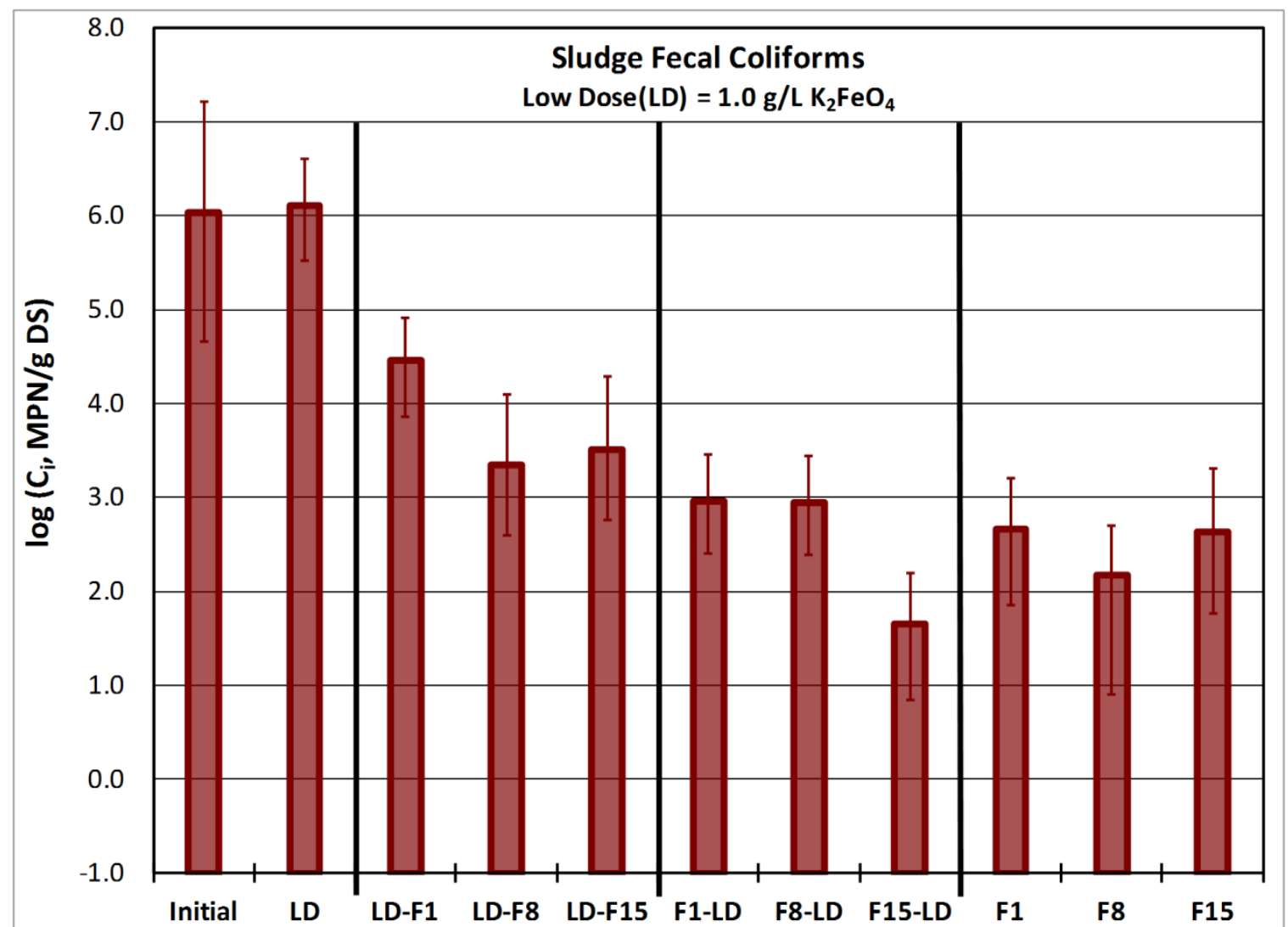

Figure 7-3: Effect of LD on the concentration of fecal coliform in primary sludge when used alone, and as a pre- and post-treatment with freeze-thaw.

\subsubsection{Pre- and Post-Treatment with LD - TS and VS}

Figure 7-4 shows the effect of LD on the TS and VS. The initial TS and VS of the primary sludge was $4.7 \%$ and $4.0 \%$ respectively. Following the addition of LD, there was no significant change in TS and VS ( $p>0.05)$. When LD was used as a pre-treatment, the samples that were frozen for 1,8 and 15 days had TS decreases of $10.6,16.8$ and $16.4 \%$, and VS decreases of $13.4,20.1$ and $22.2 \%$ respectively. Stand-alone freeze-thaw for 1,8 and 15 days resulted in TS and VS decreases of 12 to $23 \%$. The decreases in TS and VS may be the result of biodegradation carried out by the surviving microorganisms during the 12-hour thaw. In the study by Montusiewicz et al. (2010), the TS and VS of mixed 
sludge decreased by $16.1 \%$ and $16.9 \%$ respectively following freeze-thaw. In addition to biodegradation during the thawing stage, the authors also suggested that the decreases in TS and VS may be due to the action of exoenzymes in the system, and endoenzymes released from the cells following intracellular and extracellular ice formation.

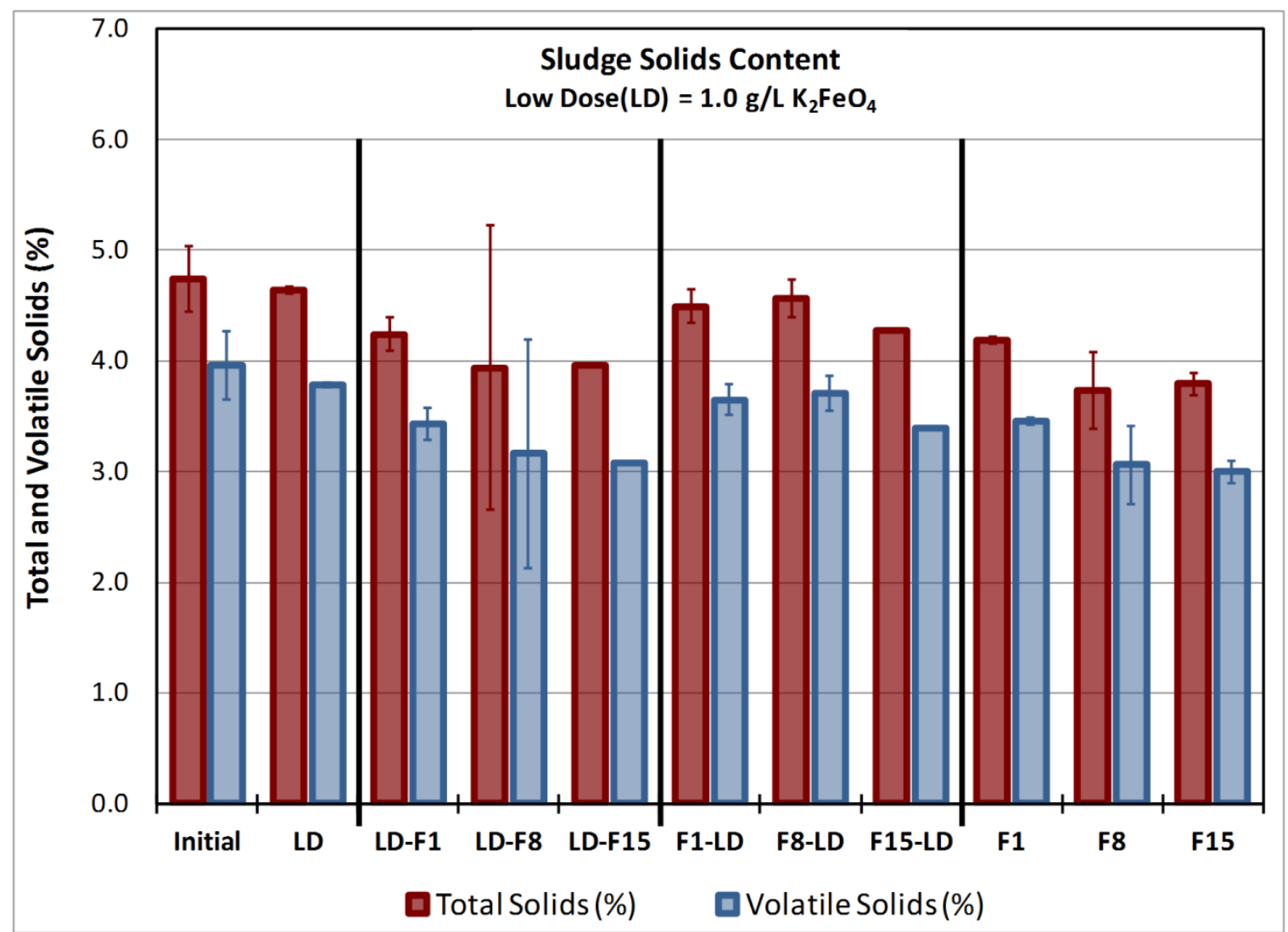

Figure 7-4: Effect of LD on the TS and VS when used alone, and as a pre- and post-treatment with freeze-thaw.

\subsubsection{Pre- and Post-Treatment with HD - Fecal Coliform}

Figure 7-5 shows the effect of HD on the concentration of fecal coliform in primary sludge. Following the 15-minute reaction period, HD resulted in a 2.5 -log inactivation of 
fecal coliform. When used as a pre-treatment with freeze-thaw, overall inactivation ranged from 4.2 to $4.6-\mathrm{log}$, with no significant difference among the samples that were frozen for 1,8 or 15 days. Similarly, when HD was used as a post-treatment, overall inactivation ranged from 4.4 to $5.0-\mathrm{log}$, regardless of the time spent frozen. However, stand-alone freeze-thaw resulted in a 3.4 to $3.9-\log$ reduction in fecal coliform, therefore, HD pre- or post-treatment, increased the fecal coliform inactivation caused by freeze-thaw by approximately $1-\log$, which is less than the 2.5 -log inactivation achieved using HD with a 15-minute contact time. This demonstrates that the level of inactivation caused by the combination of ferrate $(\mathrm{VI})$ treatments and freeze-thaw are not directly additive. Furthermore, while these experiments with sludge did not show a difference between pre- and post-treatment with ferrate(VI) in terms of fecal coliform inactivation, previous experiments with a pure culture E. coli sample (Chapter 6) did demonstrate that pre-treatment with ferrate(VI) was more effective than posttreatment. 


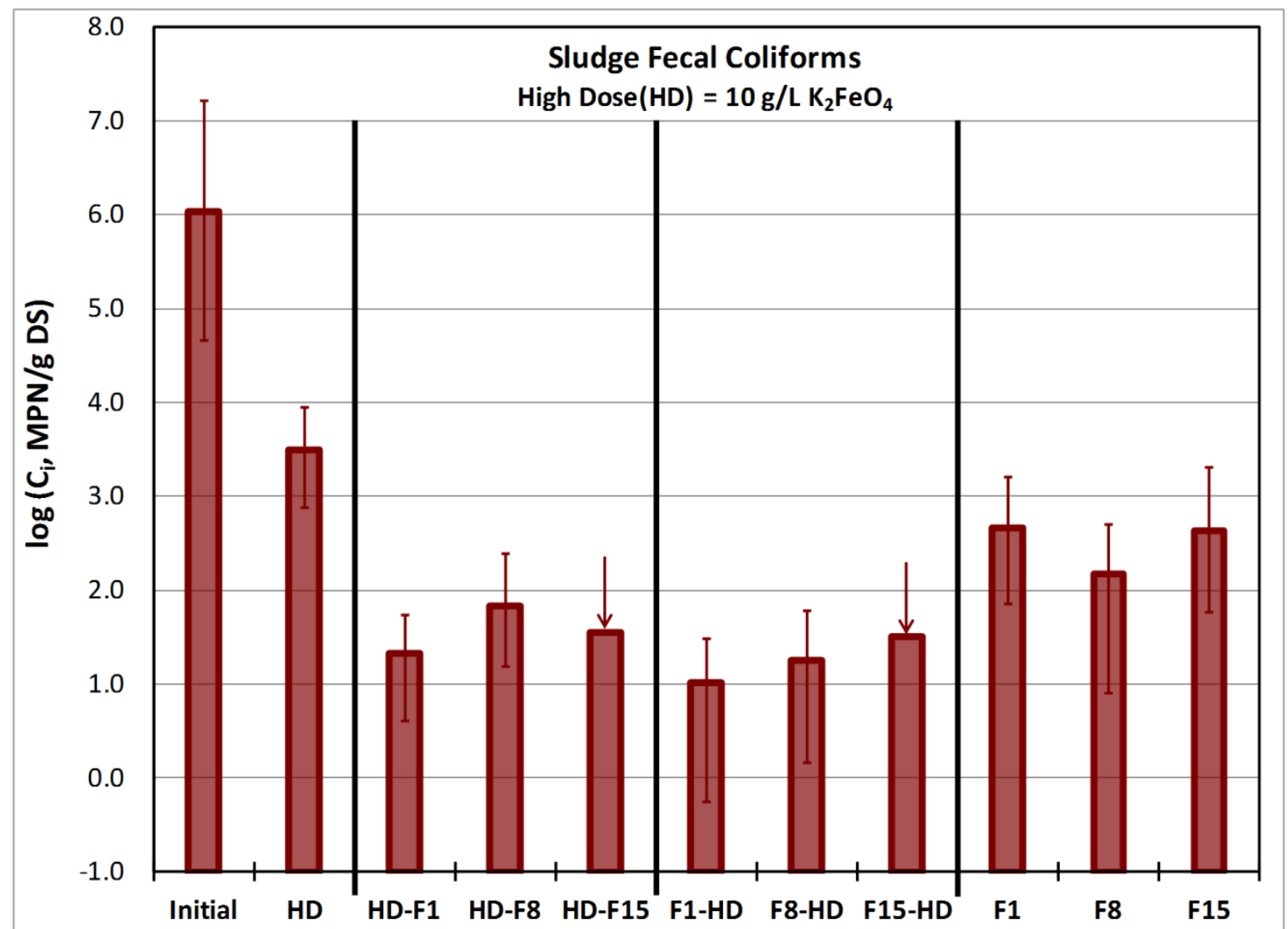

Figure 7-5: Effect of HD on the concentration of fecal coliform in primary sludge when used alone, and as a pre- and post-treatment with freeze-thaw.

\subsubsection{Pre- and Post-Treatment with HD - TS and VS}

Figure 7-6 shows the effect of HD on the TS and VS. Following the 15-minute reaction period, HD increased the TS by $18 \%(8.5 \mathrm{~g} / \mathrm{L})(\mathrm{p}=0.0052)$ with no significant change in VS $(p=0.4754)$. This was likely due to the formation of inorganic solids, such as iron oxides $\left(\mathrm{Fe}_{2} \mathrm{O}_{3} \cdot \mathrm{nH}_{2} \mathrm{O}\right)$ and iron oxide-hydroxides $\left(\mathrm{FeO}(\mathrm{OH}), \mathrm{Fe}(\mathrm{OH})_{3}\right)$, which also gave the sludge a rust colour. All samples treated with HD, pre- or post-freeze-thaw, had increases in TS ranging from $5.8 \%$ to $19.1 \%$, while stand-alone freeze-thaw samples had decreases in TS from $11.6 \%$ to 21.1 . As discussed earlier, the decreases in TS and VS following stand-alone freeze-thaw may be the result of biodegradation during the 12- 
hour thawing stage. Samples that were pre-treated with HD also had VS reductions following freeze-thaw, ranging from 7 to $24 \%$. However, samples that were post-treated with HD had insignificant reductions in VS from $4.2 \%$ to $8.6 \%$, which was also the trend when LD was used as a post-treatment to freeze-thaw.

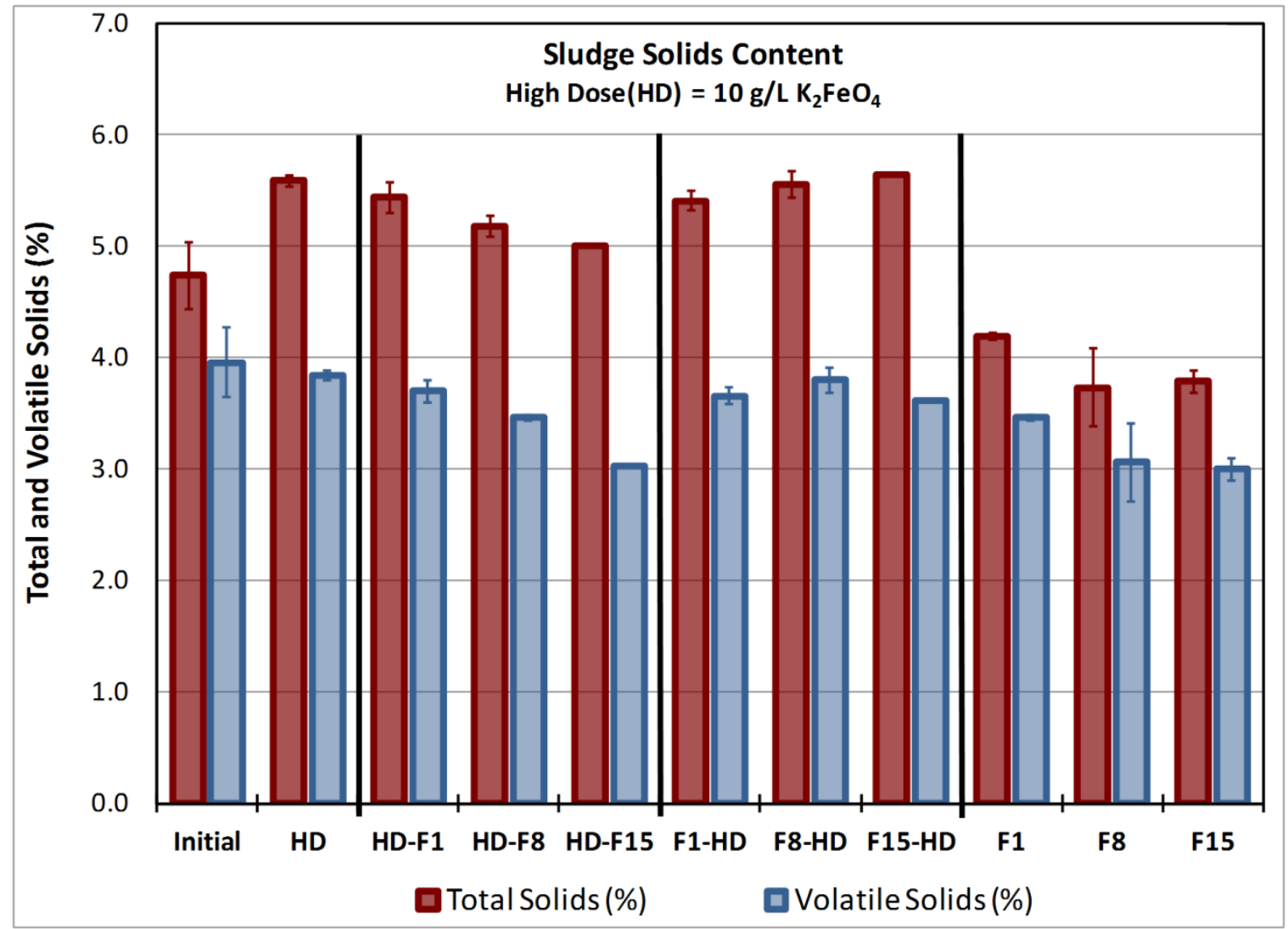

Figure 7-6: Effect of HD on the TS and VS when used alone, and as a pre- and post-treatment with freeze-thaw.

For the previous described set of experiments in Part I, the sludge samples were not dewatered as a result of the freeze-thaw process, therefore the sludge volumes remained constant throughout the testing period, and the pre- and post- treatments with ferrate(VI) were on the same volume of liquid sludge. In Part II of these experiments, a simulated drainage bed setup was used to allow meltwater to drain from 
the sludge during the thawing period, via gravity. This reduced the sludge volume, producing a dewatered cake, which made post-treatment with ferrate(VI) difficult at the practical level due to mixing requirements. Therefore, the effects of ferrate(VI) posttreatment were not evaluated in subsequent experiments using the simulated drainage beds.

\subsubsection{Part II: Freeze-thaw treatment in simulated drainage beds}

The second part of the study evaluated the effects of potassium ferrate(VI) pretreatment, prior to freeze-thaw using a drainage bed set-up. Anaerobically digested sludge samples were pre-treated with either $0.5 \mathrm{~g} / \mathrm{L}(\mathrm{LD})$ or $5.0 \mathrm{~g} / \mathrm{L}$ (HD) of potassium ferrate( $\mathrm{VI})$, frozen at $-20^{\circ} \mathrm{C}$ for 1,8 or 15 days, and thawed at room temperature for 12 hours. Another set of samples underwent stand-alone freeze-thaw, without potassium ferrate(VI) additions. For these tests, two sample bottles were kept frozen at $-20{ }^{\circ} \mathrm{C}$ for each period of 1,8 and 15-days.

The study used simulated drainage beds to separate the meltwater from the sludge cake during the sludge thawing period (Figure 7-7). Following freeze-thaw, all $200 \mathrm{~mL}$ sludge samples released approximately $170 \mathrm{~mL}$ of meltwater via gravity drainage. Remaining in each of the sample bottles following the 12-hour thawing and drainage period was approximately $30 \mathrm{~g}$ of sludge cake. When the initial, un-frozen, sludge sample was placed on the drainage setup, only $10 \%$ of the sample was able to drain before the drainage fabric and perforated lid clogged. Table 7-3 shows the volume of meltwater collected and the corresponding mass of sludge cake remaining for each of the samples. 


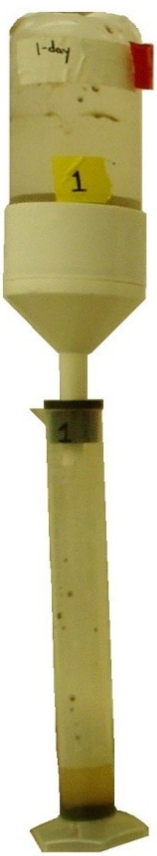

Initial

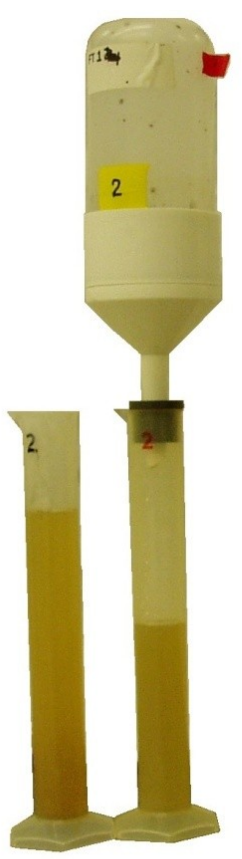

F1

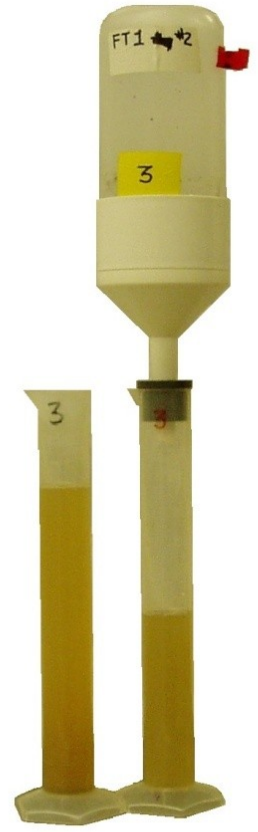

F1 \#2

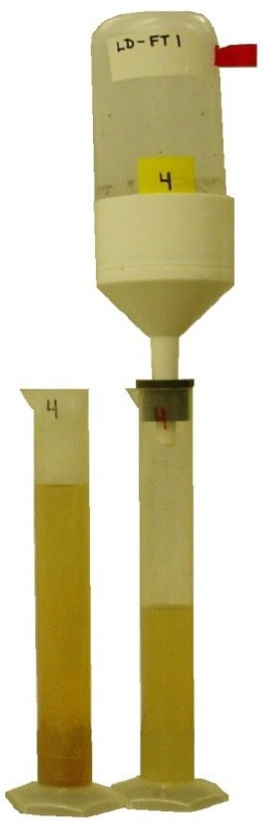

LD-F1

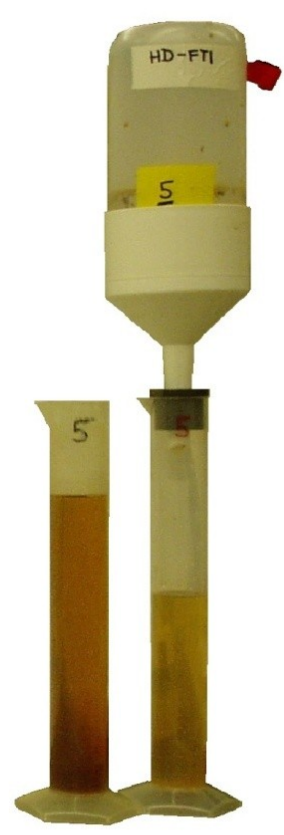

HD-F1

Figure 7-7: Simulated drainage beds with meltwater collection used for the freeze-thaw experiments with anaerobically digested sludge samples.

Table 7-3: Volume of meltwater collected via gravity drainage during the thawing process and the corresponding mass of sludge cake remaining.

\begin{tabular}{|c|c|c|c|c|c|c|c|c|c|c|c|c|c|}
\hline & Initial & LD-F1 & LD-F8 & LD-F15 & HD-F1 & HD-F8 & HD-F15 & F1 & F1\#2 & F8 & F8\#2 & F15 & F15\#2 \\
\hline $\begin{array}{c}\text { Meltwater } \\
\text { Collected (mL) }\end{array}$ & 19 & 170 & 170 & 169 & 169 & 169 & 166 & 172 & 169 & 171 & 170 & 175 & 171 \\
\hline $\begin{array}{c}\text { Sludge Cake } \\
\text { Remaining (g) }\end{array}$ & 179.8 & 29.3 & 28.0 & 27.2 & 33.9 & 33.5 & 34.4 & 29.7 & 30.3 & 30.4 & 27.7 & 26.8 & 27.8 \\
\hline
\end{tabular}

\subsubsection{Meltwater}

The meltwater collected via gravity drainage during the thawing stage was characterised

in terms of fecal coliform, soluble proteins, soluble carbohydrates, sCOD, $\mathrm{pH}$ and turbidity. 


\section{Fecal coliform}

The concentration of fecal coliform in the meltwater samples is presented in Figure 7-8. For comparison, the figure also includes the initial (untreated) sludge sample, and the LD and HD sludge samples following a 15-minute reaction period with the potassium ferrate(VI). The concentration of fecal coliform in the meltwater following stand-alone freeze-thaw for 1 day was approximately $7.6 \times 10^{2} \mathrm{MPN} / \mathrm{mL}, 1-\log$ lower than the initial sludge. Samples that were frozen for 8 and 15 days had additional reductions of approximately $0.5-\log$ and 1 -log respectively. By grouping the replicates for samples F1 with F1 \#2, F8 with F8 \#2 and F15 with F15 \#2, t-tests were performed, which suggest that this decreasing trend is statistically significant (F1 vs. F8: $p=0.0015, F 8$ vs. F15: $p=0.0433)$. Results from another study, suggest that the freezing temperature and the time spent frozen do not influence the level of inactivation of fecal coliform caused by freeze-thaw (Diak et al., to be submitted). Similar results have also been reported for by Gao et al. (2009) and Sanin et al. (1994). However, these experiments, which incorporate meltwater collection, suggest that the fecal coliform, which were captured by the ice, and drained with the meltwater, were further inactivated when the time spent frozen was increased. This trend was also observed in samples that were pretreated with LD prior to freeze-thaw (LD-F1 vs. LD-F8: $p=0.0130$, LD-F8 vs. LD-F15: $p=0.0424)$. In addition, LD pre-treatment did not improve the inactivation of fecal coliform resulting from stand-alone freeze-thaw, after being frozen for 1 day; however, when the time frozen was increased to 8 days, LD did result in greater fecal coliform inactivation compared to stand-alone freeze-thaw for 8 days $(p=0.001)$. This also seems 
to be the case when the time frozen was 15 days, however due to a large amount of variation in the treatment replicates, the results are not statistically significant $(p=0.0694)$. HD pre-treatment, on the other hand, reduced the concentration of fecal coliform in the meltwater to $<0.28 \mathrm{MPN} / \mathrm{mL}$ following freeze-thaw (detection limit = $0.18 \mathrm{MPN} / \mathrm{mL}$ ), regardless of the time spent frozen.

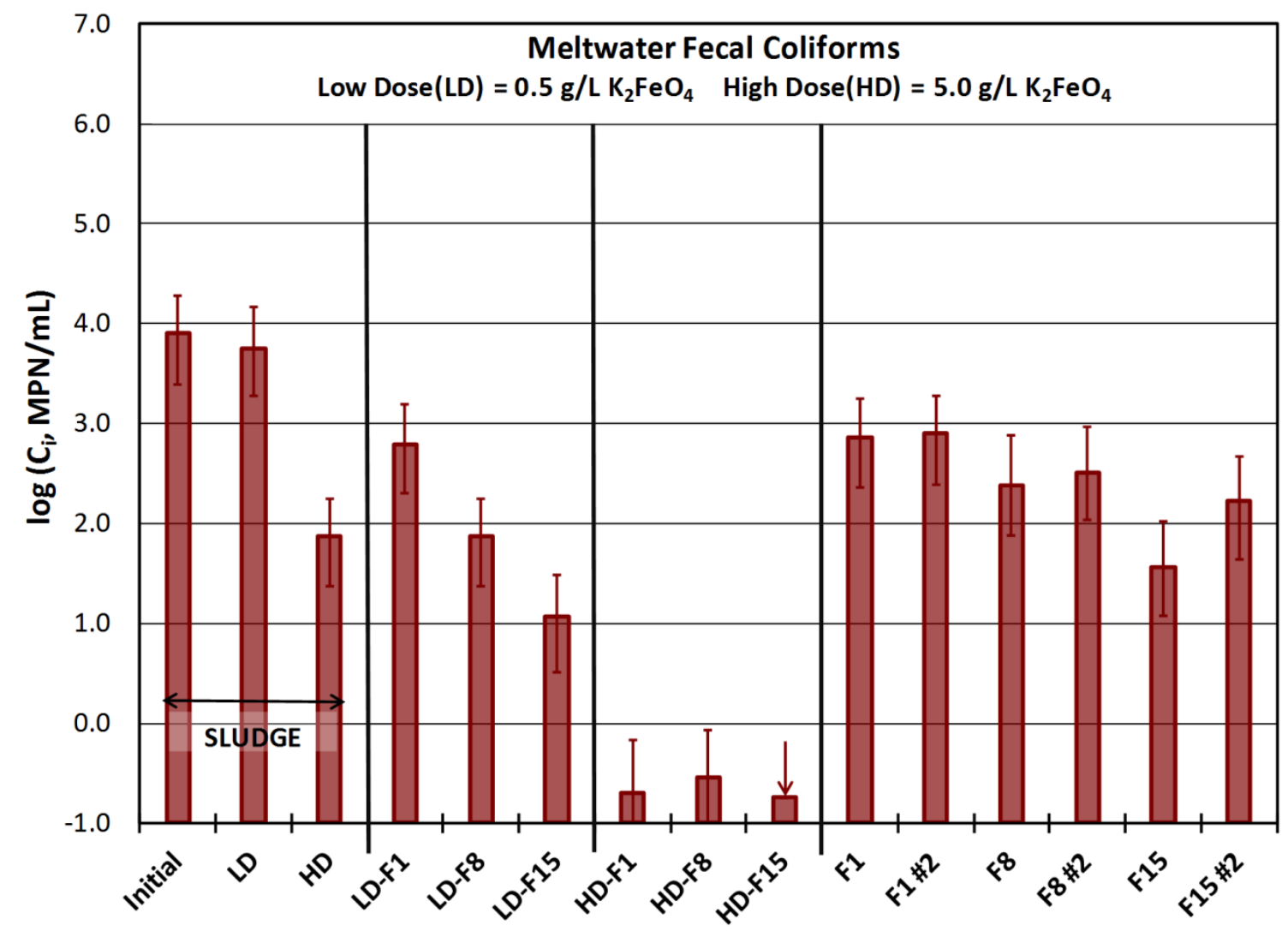

Figure 7-8: Effect of LD and HD on the concentration of fecal coliform in the meltwater collected during the thawing stage.

\section{Soluble proteins, carbohydrates and COD}

Figure 7-9 shows the concentrations of SCOD, soluble carbohydrates and soluble proteins. The initial SCOD of the anaerobically digested sludge was $568 \mathrm{mg} / \mathrm{L}$, with 28 
$\mathrm{mg} / \mathrm{L}$ soluble carbohydrates and $12 \mathrm{mg} / \mathrm{L}$ proteins. Following the addition of $L D$, the SCOD increased to $639 \mathrm{mg} / \mathrm{L}$ ( $13 \%$ increase), and the soluble carbohydrates increased to $40 \mathrm{mg} / \mathrm{L}$ (44\% increase), but the soluble proteins only slightly increased to $14 \mathrm{mg} / \mathrm{L}$. When LD was used as a pre-treatment with freeze-thaw, there were no significant differences in the degree of solubilisation resulting from stand-alone freeze-thaw treatment.

Stand alone freeze-thaw increased the degree of sludge solubilisation. The SCOD increased from 600 to $1300 \mathrm{mg} / \mathrm{L}$, soluble carbohydrates increased from 28 to $60 \mathrm{mg} / \mathrm{L}$, and soluble proteins increased from 10 to $>175 \mathrm{mg} / \mathrm{L}$. These results agree with previous studies which have demonstrated that freeze-thaw disrupts sludge flocs, causing the release of EPS, into the sludge supernatant (Hong et al., 1995, Hung et al., 1997). Furthermore, the largest increases were the increases in soluble proteins, which were also observed in the study by Hu et al., 2011.

Following the addition of $\mathrm{HD}$, the SCOD increased to $1857 \mathrm{mg} / \mathrm{L}$, the soluble carbohydrates increased to $127 \mathrm{mg} / \mathrm{L}$, and the soluble proteins increased to $104 \mathrm{mg} / \mathrm{L}$. When HD was used as a pre-treatment with freeze-thaw, soluble proteins in the meltwater were lower than stand-alone freeze-thaw and LD pre-treated samples, however the SCOD and soluble carbohydrate concentrations were considerably higher. This may be due to direct oxidation of proteins by the ferrate(VI), or perhaps the degradation of proteins during thaw, stimulated by the addition of potassium ferrate(VI). 


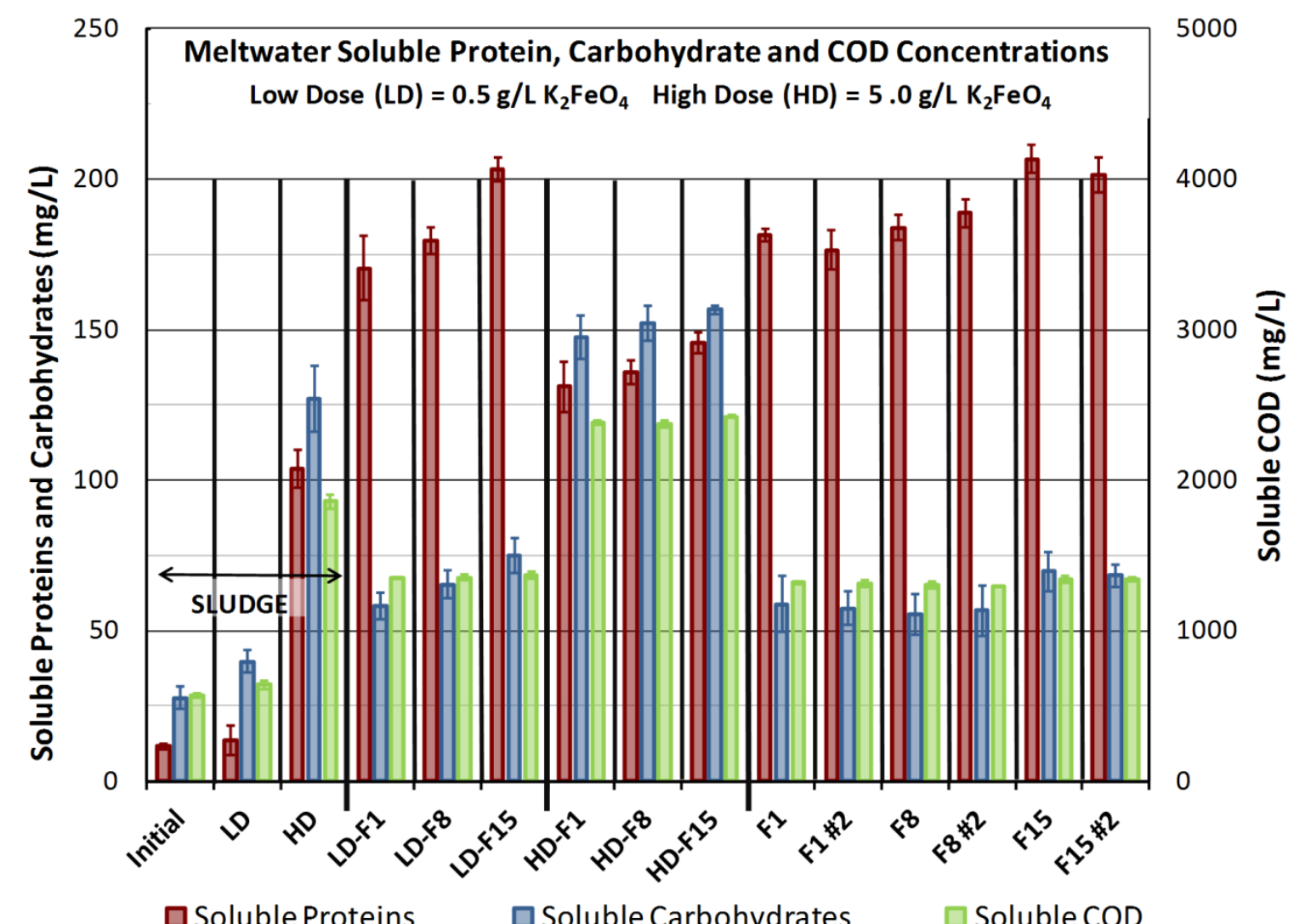

Figure 7-9: Effect of LD and HD on the concentrations of soluble proteins, soluble carbohydrates and SCOD in the meltwater collected during the thawing stage.

\section{Turbidity and $\mathrm{pH}$}

Figure 7-10 shows a sample of the initial sludge, and the meltwater samples collected from sludge samples that were frozen for 1 day. Freeze-thaw resulted in a very significant decrease in turbidity due to the formation of ice within the sludge sample, which rejected sludge solids and consolidated sludge particles. During the thawing stage, the meltwater was able to drain freely via gravity drainage, without resuspending the solids. Furthermore, the addition of potassium ferrate( $\mathrm{VI})$, prior to freeze-thaw, 
seems to have improved the precipitation and coagulation of solids, which further reduced the turbidity of the meltwater.

Figure 7-11 shows the turbidity and $\mathrm{pH}$ of the initial sludge and the meltwater samples collected during the thawing stage. The initial turbidity of the sludge was $>10,000$ NTU (over range). Similarly, the turbidity of LD and HD samples, prior to freeze-thaw, was also over range. Following stand-alone freeze-thaw for 1 day, the meltwater collected had a turbidity of approximately 178 NTU. When the time frozen was increased to 8 and 15 days, turbidity was reduced to 136 NTU, with no significant difference between F8 and F15 samples. When LD pre-treatment was used, the turbidity of the meltwater after 1 day frozen was 46 NTU, almost 75\% lower than stand-alone freeze-thaw (F1 and F1 \#2). After 8 days, the turbidity decreased to 38 NTU, $72 \%$ lower than F8 and F8 \#2 samples, and after 15 days frozen, the turbidity was 30 NTU, 78\% lower than F15 and F15 \#2. When HD pre-treatment was used, the turbidity of the meltwater collected after 1 day and 8 days frozen were 9 NTU. After 15 days frozen, the turbidity of the meltwater was 6.5 NTU. In all cases, the turbidity of the meltwater from samples that were pretreated with HD, were approximately 95\% lower than the stand-alone freeze-thaw samples. It was also shown that increasing the time spent frozen from 1 to 15 days reduces the turbidity of the meltwater samples. However, the drastic reductions in turbidity occur as a result of the freeze-thaw process, and increasing the time spent frozen only slightly reduces the turbidity further. 


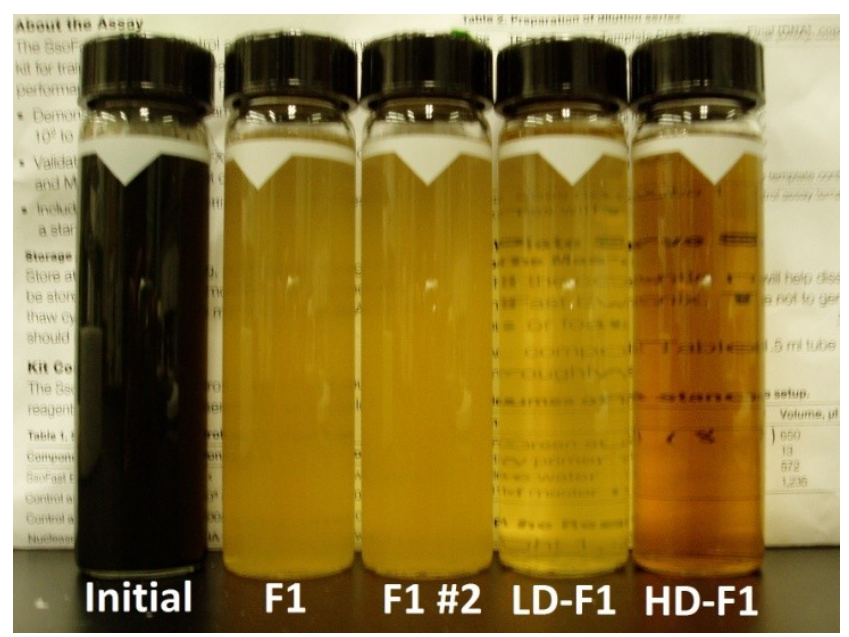

Figure 7-10: Initial sludge and meltwater samples collected following freeze-thaw (1 day frozen). The visible text in the background was used to demonstrate the clarity of the samples following freeze-thaw with ferrate(VI) pre-treatment.

The initial $\mathrm{pH}$ of the anaerobically digested sludge was 7.4. Following a 15-minute reaction with $L D$ and $H D$, the $\mathrm{pH}$ of the sludge increased to 8.0 and 9.1, respectively (Figure 7-11). Increased $\mathrm{pH}$ means that odours from hydrogen sulphide are reduced since the $\mathrm{pK}_{\mathrm{a}}$ of hydrogen sulphide is 7. Therefore, at $\mathrm{pH}>9$ essentially all of the hydrogen sulphide is converted to the non-volatile ionised forms (Haug et al., 1992). Furthermore, when potassium ferrate(VI) dissolves in sludge, the ferrate(IV) ions are reduced to ferric and ferrous iron, which can form precipitates with sulphide, thus reducing the concentration of hydrogen sulphide (de Luca et al., 1996).

When LD was used as a pre-treatment with freeze-thaw, the meltwater had a $\mathrm{pH}$ of approximately 8.1, regardless of the time spent frozen. Similarly, when HD was used as a pre-treatment, the $\mathrm{pH}$ of the meltwater from all 3 samples was 9.1. This suggests that the increases in $\mathrm{pH}$ are due to the addition of potassium ferrate( $\mathrm{VI})$, and very little to do with freeze-thaw. Furthermore, the length of time that the samples were kept frozen 
did not have an effect on $\mathrm{pH}$. This was also the case with stand-alone freeze-thaw. The meltwater collected had a $\mathrm{pH}$ of approximately 7.9 , regardless of the number of days that the samples were kept frozen. The increase in $\mathrm{pH}$ from 7.4, in the initial sludge, to 7.9 in the meltwater following stand-alone freeze-thaw, may be the result of precipitation of buffering salts during freezing. As the water in sludge freezes, the solute concentration increases. This can lead to salt precipitation depending on the concentrations of the salt components and their relative solubilities (Pikal-Cleland et al., 2000). Furthermore, the increases in soluble proteins, carbohydrates and other sludge constituents following freeze-thaw may also affect the $\mathrm{pH}$ of the meltwater.

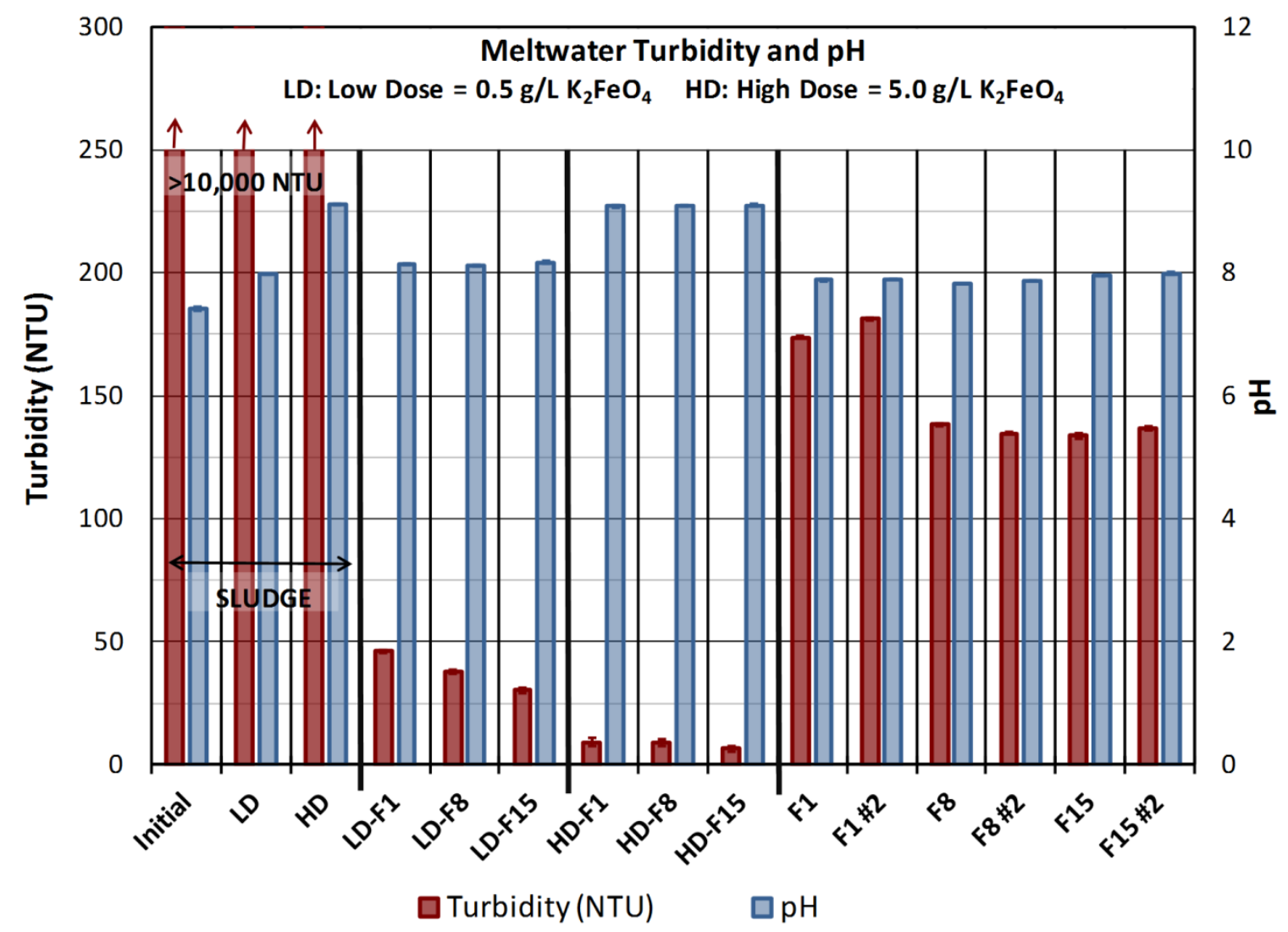

Figure 7-11: Effect of LD and HD on the turbidity and $\mathrm{pH}$ of the meltwater collected during the thawing stage. 


\subsubsection{Sludge Cake}

The sludge cake remaining after meltwater drainage was characterised in terms of fecal coliform, TS and VS.

\section{Fecal coliform}

Figure 7-12 shows the effect of LD and HD on the concentration of fecal coliform in the sludge when used as a pre-treatment, prior freeze-thaw with meltwater separation. The figure also shows the effects of stand-alone freeze-thaw. The initial concentration of fecal coliform in the anaerobically digested sludge was approximately $3.9 \times 10^{5} \mathrm{MPN} / \mathrm{g}$ DS. Following a 15-minute contact time, HD resulted in a 2.1-log fecal coliform inactivation, whereas LD did not have a significant effect $(p=0.3910)$.

When LD was used as a pre-treatment to freeze-thaw with meltwater separation, overall fecal coliform inactivation in the sludge cake ranged from 0.9 to $2.0-\log$. However, stand-alone freeze-thaw resulted in 1.4 to 2.1 -log inactivation, suggesting that LD pre-treatment did not improve the fecal coliform inactivation caused by stand-alone freeze-thaw $(p=0.1354)$. Similar to the experiments with primary sludge, the length of time that the samples were kept frozen $\left(1,8\right.$ or 15 days at $\left.-20^{\circ} \mathrm{C}\right)$ did not have a significant effect on fecal coliform inactivation ( $p>0.05)$.

When HD was used as a pre-treatment with freeze-thaw, the overall reductions in fecal coliform ranged from 3.6 to $3.9-\log$, which is 1.8 to 2.2 -log greater inactivation than stand-alone freeze-thaw. This indicates that pre-treatment using $5 \mathrm{~g} / \mathrm{L}$ of potassium 
ferrate(VI) prior to freeze-thaw increases the level of inactivation of fecal coliform caused by stand-alone freeze-thaw $(\mathrm{p}=0.0016)$.

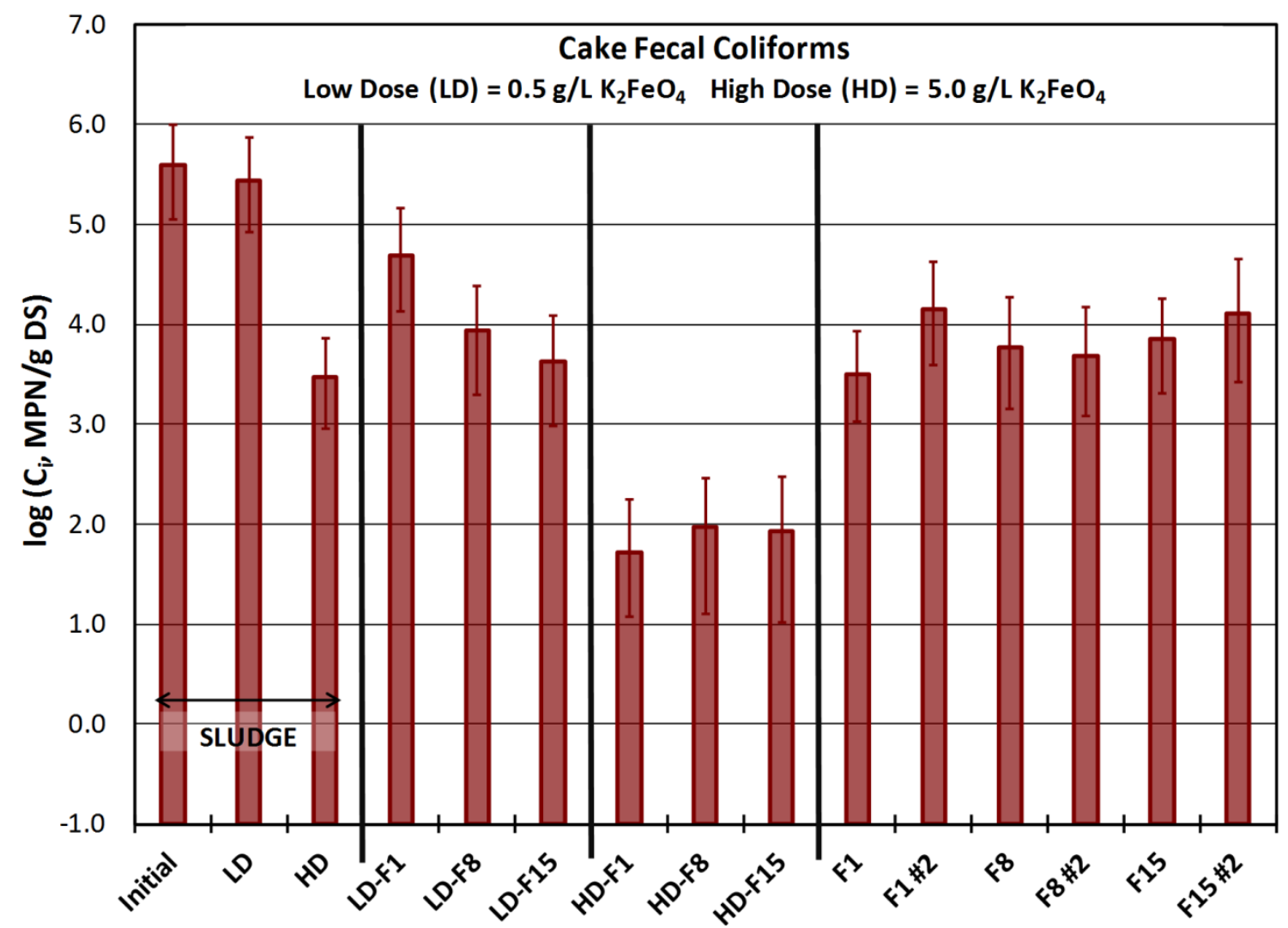

Figure 7-12: Effect of LD and HD on the concentration of fecal coliform in the sludge cake when used alone, and as a pre-treatment to freeze-thaw with meltwater separation.

TS and VS

Figure 7-13 shows the effect of LD and HD on the TS and VS when used alone and as a pre-treatment to freeze-thaw with meltwater separation. The initial TS and VS of the anaerobically digested sludge were $2.0 \%$ and $1.2 \%$ respectively. Following the addition of $5 \mathrm{~g} / \mathrm{L}(\mathrm{HD})$ of potassium ferrate( $\mathrm{VI})$, the TS increased to $2.5 \%$ with no change in VS. Following the addition of $0.5 \mathrm{~g} / \mathrm{L}(\mathrm{LD})$, there was no significant change in TS or VS. 
Freeze-thaw with meltwater collection via gravity drainage reduced the $200 \mathrm{~mL}$ sludge samples to approximately $30 \mathrm{~g}$ of sludge cake, indicating a reduction of approximately $85 \%$ in the sludge mass. The TS of the sludge cakes ranged from $12 \%$ to $14 \%$ solids.

HD pre-treatment may have decreased the drainability of the sludge slightly. During the thawing process, up to $9 \mathrm{~mL}(5 \%)$ less meltwater was collected, and up to $7.6 \mathrm{~g}$ more sludge cake remained (Table 7-3). The extra water in the sludge cake meant that the TS and VS of HD pre-treated samples were slightly lower than the other samples. Comparing the group of HD pre-treatment samples to the group of stand-alone freezethaw samples, there is a statistically significant decrease in the TS when HD pretreatment was used $(p=0.0038)$. This is likely due to the higher concentrations of EPS in the supernatant which interfere with filterability (Ye et al., 2014), or due to the formation of inorganic solids which remained in the sludge cake following drainage.

Furthermore, samples that were frozen for 8 and 15 days may have drained slightly better than the samples that were frozen for only 1 day. Longer freezing periods of 8 and 15 days generally resulted in greater meltwater volume, and reduced mass of sludge cake remaining (Table 7-3). In addition, sludge samples that were frozen for 8 and 15 days consistently had higher TS concentrations than the samples that were kept frozen for only 1 day. This was the case for stand-alone freeze-thaw samples (F1 vs. F8: $p=0.0000$, F1 vs. F15: $p=0.0002$ ), and the samples that were pre-treated with LD (LD-F1 vs. LD-F8: $p=0.0102$, LD-F1 vs. LD-F15: $p=0.0026)$. Vesilind and Martel (1990) believed that prolonged freezing periods at low temperatures allowed time for the ice crystals to 
extract the surface water surrounding the sludge particles, bringing them into contact to create larger particles. Furthermore, while Hu et al. (2011) recognised that the majority of the freeze-thaw sludge dewatering benefits occur during the bulk freezing stage, extended "curing" time allowed biological sludge flocs to be further dehydrated by the surrounding ice.

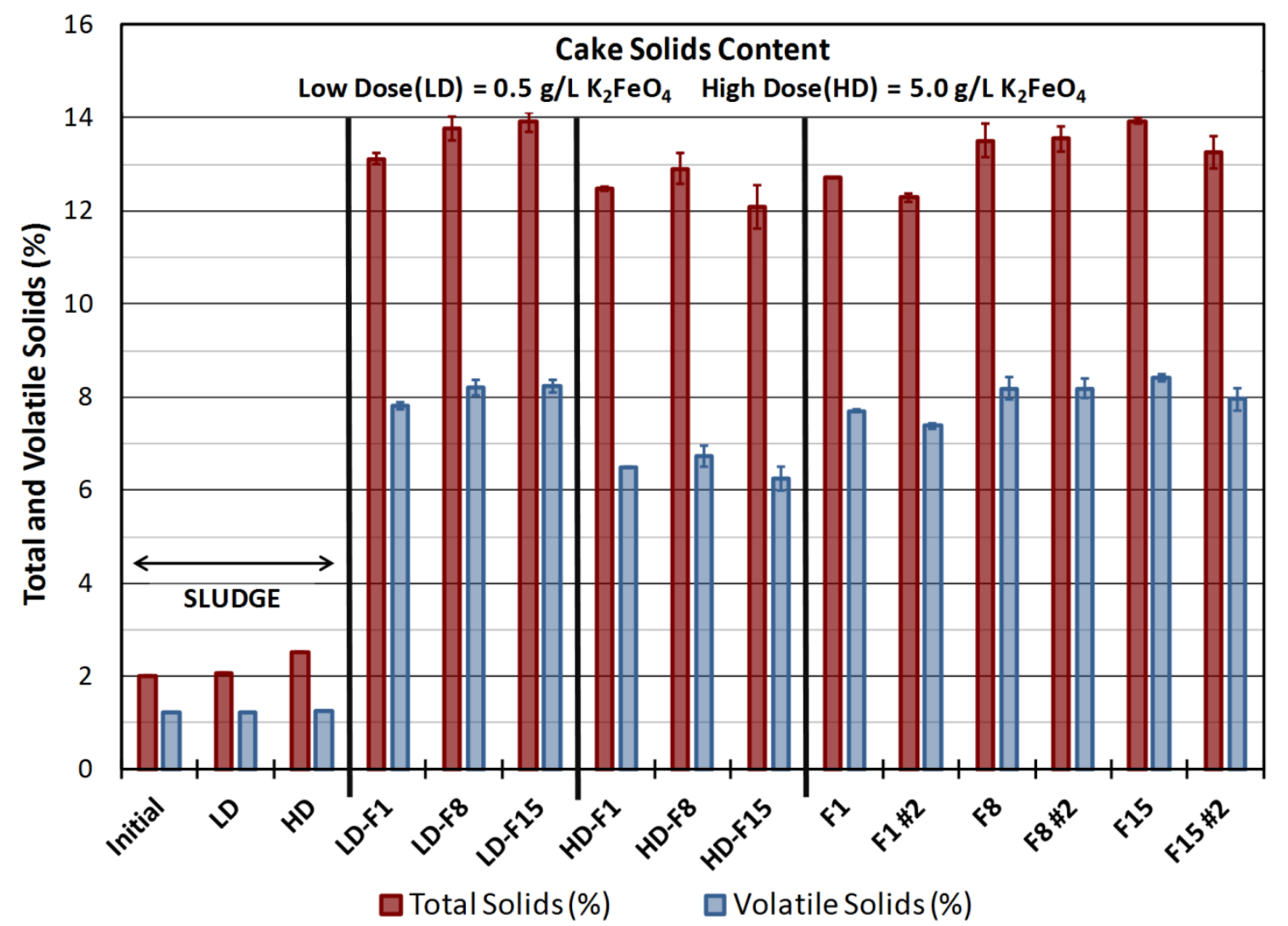

Figure 7-13: Effect of LD and HD on the TS and VS of the sludge cake when used alone and as a pre-treatment to freeze-thaw with meltwater separation.

\subsection{CONCLUSION}

The study examined the individual and combined effects of potassium ferrate(VI) additions and freeze-thaw conditioning for the treatment and dewatering of sludge 
samples. The first part of the experiments, using primary sludge, demonstrated the following:

- Stand-alone freeze-thaw resulted in $>3-\log$ inactivation of fecal coliform after being frozen for 1 day. Increasing the time frozen up to 15 days did not result in any significant differences.

- $\mathrm{LD}=1.0 \mathrm{~g} / \mathrm{L}$ of potassium ferrate(VI) did not have a significant effect on the primary sludge following a 15-minute reaction period, or when used as a pre- or posttreatment to freeze-thaw.

- $\mathrm{HD}=10 \mathrm{~g} / \mathrm{L}$ of potassium ferrate(VI) significantly changed the sludge properties. Following the 15-minute reaction period, the CST increased by 3 fold, the concentrations of ammonia and sulphides were reduced by $11 \%$ and $89 \%$ respectively, the TS increased by approximately by $18 \%(8.5 \mathrm{~g} / \mathrm{L})$, and fecal coliform inactivation was $2.5-\log$. When used as a pre- and post-treatment to freeze-thaw, fecal coliform inactivation caused by stand-alone freeze-thaw was increased by an additional 1-log.

The second part of the study tested freeze-thaw treatment on anaerobically digested sludge using simulated drainage beds, to allow meltwater to drain out of the sludge during the thawing stage, leaving a partially dewatered sludge cake. Below is a summary of the key points from this study.

- Freeze-thaw with simple gravity drainage reduced the mass of sludge by $85 \%$ and increased the TS of the sludge to approximately $13 \%$ solids. Furthermore, stand- 
alone FT with meltwater drainage resulted in 1.6-log inactivation of fecal coliform in the sludge cake.

- Freeze-thaw increased the concentration of soluble components, particularly proteins, and resulted in a meltwater that was $<200$ NTU after 1 day frozen and $<150$ NTU after 8 days. The concentration of fecal coliform in the meltwater following freeze-thaw was reduced to $<10^{3} \mathrm{MPN} / \mathrm{mL}$.

- Pre-treatment with $5 \mathrm{~g} / \mathrm{L}$ potassium ferrate(VI) followed by freeze-thaw with meltwater drainage, reduced the concentration of fecal coliform in the meltwater to below $0.28 \mathrm{MPN} / \mathrm{mL}$, which is very near the detection limit $(0.18 \mathrm{MPN} / \mathrm{mL})$. The meltwater also had lower turbidity and a higher degree of solubilisation than the meltwater from stand-alone freeze-thaw.

- Pre-treatment with $5 \mathrm{~g} / \mathrm{L}$ of potassium ferrate( $\mathrm{VI})$, followed by freeze-thaw with meltwater drainage, reduced the concentration of fecal coliform in the sludge cake to below $100 \mathrm{MPN} / \mathrm{g}$ DS, representing >3.5-log inactivation.

The findings of this study demonstrate that freeze-thaw with simple gravity meltwater drainage can reduce the volume of the sludge by $85 \%$, and cause fecal coliform inactivation in the sludge cake and meltwater. In addition, sludge pre-treatment with 5 $\mathrm{g} / \mathrm{L}$ potassium ferrate(VI) followed by freeze-thaw with meltwater drainage, reduced the fecal coliform in the sludge cake to below Class A requirements (<1000 MPN/g DS) for sewage biosolids in terms of fecal coliform (U.S. EPA, 2003) and many other countries in Europe. However the concentrations of Salmonella, Viable Helminth ova and total enteric virus were not measured. It is recommended that future studies investigate 
ferrate(VI) oxidation treatments on criteria pathogens such as Salmonella, Viable Helminth ova and total enteric virus in wastewater sludges.

\subsection{REFERENCES}

APHA (American Public Health Association), American Water Works Association, Water Pollution Control Federation (2005) Standard Methods for the Examination of Water and Wastewater. APHA/AWWA, Washington DC.

Apul, O.G., Atalar, I., Zorba, T., Sanin, F.D. (2010) The dewaterability of disintegrated sludge samples before and after anaerobic digestion. Dry Technol 28(7):901-909

Bradford, M.M. (1976) A rapid and sensitive method for the quantification of microgram quantities of protein utilizing the principle of protein-dye binding. Anal Biochem 72(12):248-254

Chen, Y.-C., Higgins, M.J., Beightol, S.M., Murthy, S.N., Toffey, W.E. (2011) Anaerobically digested biosolids odor generation and pathogen indicator regrowth after dewatering. Water Res 45(8):2616-2626

Corte, A.E. (1962) The frost behavior of soils. Field and laboratory data for a new concept. Part1: Vertical sorting; Part II: Horizontal sorting. U.S. Army Cold Regions Research and Engineering Laboratory, Corps of Engineers, Research Report 85 de Luca, S., Idle, C.N., Chao, A.C. (1996) Quality improvement of biosolids by ferrate(VI) oxidation of offensive odour compounds. Water Sci Technol 33(3):119-130 
Diak, J., Örmeci, B., Proux, C., (2011) Freeze-thaw treatment of RBC sludge from a remote mining exploration facility in subarctic Canada. Water Sci Technol 63(6):13091313

Gardner, J., Örmeci, B. (2010) Effect of aging, time, and temperature on fecal coliform counts during centrifugal dewatering and role of centrate in growth inhibition. Water Environ Res 82(2):51-61

Gao, W., Leung, K., and Hawdon, N. (2009) Freezing inactivation of Escherichia coli and Enterococcus faecalis in water: Response to different strains. Water Environ Res 81(8):824-830

Gao, W., Smith, D.W., Li, Y. (2006) Natural freezing as a wastewater treatment method: E. Coli inactivation capacity. Water Res 40(12):2321-2326

Gombos, E., Barkács, K., Felföldi, T., Vértes, C., Makó, M., Palkó, G., Záray, C. (2013) Removal of organic matters in wastewater treatment by ferrate(VI)-technology. Microchem J 107:115-120

Haug, R.T., Kuchenrither, R., Oerke, D., Prakasam, T., Soszynski, S., and Zenz, D. (1992) Sludge processing technology. Ch. 6 in Municipal Sewage Sludge Management, editors: C. Lue-Hing, D.R. Zenz, and R. Kuchenrither. Technomic Publishing, Lancaster PA, USA, pp. 223-298. 
Higgins, M.J., Chen, Y., Murthy, S.N., Maas, N.A., Hendrickson, D. (2007) Reactivation and regrowth of non-culturable indicator bacteria in anaerobically digested biosolids after centrifuge dewatering. Water Res 41(3):665-673

Hoekstra, P., Miller, R.D. (1967) On the mobility of water molecules in the transition layer between ice and a solid surface. J Colloid Interface Sci 25(2):166-173

Hong, S.G., Young, Y.D., Chen, G.W., Chang, I.L., Hung, W.T., Lee, D.J. (1995) Freeze/thaw treatment on waste activated sludge: an FTIR spectroscopic study. J Environ Sci Health A30(8):1717-1724

Hu, K., Jiang, J.-Q., Zhao, Q.-L., Lee, D.-J., Wang, K., Qiu, W. (2011) Conditioning of wastewater sludge using freezing and thawing: Role of curing. Water Res 45(18):59695976

Hung, W.T., Feng, W.H., Tsai, I.H., Lee, D.J., Hong, S.G. (1997) Uni-directional freezing of waste activated sludges: vertical freezing versus radial freezing. Water Res 31(9):22192228

Jiang, J.-Q., Zhoo, Z. (2013) Removal of pharmaceutical residues by ferrate(VI). PLoS ONE 8(2): e55729. doi:10.1371/journal.pone.0055729

Jiang, J.-Q., Wang, S., Panagoulopoulos, A. (2007) The role of potassium ferrate(VI) in the inactivation of Escherichia coli and in the reduction of COD for water remediation. Desalination 210(1-3):266-273 
Jiang, J.-Q. (2007) Research progress in the use of ferrate(VI) for the environmental remediation. J Hazard Mater 146(3):617-623

Jiang, J.Q., Panagoulopoulos, A., Bauer, M., Pearce, P. (2006) The application of potassium ferrate for sewage treatment. J Environ Manag 79(2):215-220

Kato, S., Jenkins, M.B., Fogarty, E.A., Bowman, D.D. (2002) Effects of freeze-thaw events on the viability of Cryptosporidium parvum oocysts in soil. J Parasitol 88(4):718-722

Li, C., Li, X.Z., Graham, N., Gao, N.Y. (2008) The aqueous degradation of bisphenol A and steroid estrogens by ferrate. Water Res 42(1-2):109-120

Martel, C.J. (1993) Fundamentals of sludge dewatering in freezing beds. Water Sci Technol 28(1):29-35

Martel, C.J., Diener, C.J. (1991a) A pilot-scale study of alum sludge dewatering in a freezing bed. J Amer Water Works Assn, 83(12):51-55

Martel, C.J., Diener, C.J. (1991b) Pilot-scale studies of sludge dewatering in a freezing bed. Can J Civil Eng 18(4):681-689

Metcalf \& Eddy, Inc. (2003) Wastewater Engineering: Treatment and Reuse, $4^{\text {th }}$ ed., Tata-McGraw-Hill Pub., NY pp. 1557-1559

Montusiewicz, A., Lebiocka, M., Rozej, A., Zacharska, E., Pawlowski, L. (2010) Freezing/thawing effects on anaerobic digestion of mixed sewage sludge. Bioresource Technol 101(10):3466-3473 
Morris, D.L. (1948) Quantitative determination of carbohydrates with Dreywood's anthrone reagent. Science 107(2775):254-255

Murmann, R.K., Robinson, P.R. (1974) Experiments utilising $\mathrm{FeO}_{4}{ }^{2-}$ for purifying water. Water Res 8(8):543-547

Northcott, K.A., Snape, I., Scales, P.J., Stevens, G.W. (2005) Contaminated water treatment in cold region: an example of coagulation and dewatering modelling in Antarctica. Cold Reg Sci Technol 41(1):61-72

Örmeci, B., Vesilind, P.A. (2001) Effect of dissolved organic material and cations on freeze-thaw conditioning of activated and alum sludges. Water Res 35(18):4299-4306 Parker, P.J., Collins, G. (1999) Dehydration of flocs by freezing. Environ Sci Technol 33(3):482-488

Pikal-Cleland, K.A., Rodriguez-Hornedo, N., Amidon, G.L., Carpenter, J.F. (2000) Protein denaturation during freezing and thawing in phosphate buffer systems: Monomeric and tetrameric $\beta$-galactosidase. Arch Biochem Biophys 384(2):398-406

Saktaywin, W., Tsuno, H., Nagare, H., Soyama, T., Weerapakkaroon, J. (2005) Advanced sewage treatment process with excess sludge reduction and phosphorus recovery. Water Res 39(5), 902-910

Sanin, F.D., Vesilind, P.A., Martel, C.J. (1994) Pathogen reduction capabilities of freeze/thaw sludge conditioning. Water Res. 28(11), 2393-2398 
Schuck, C.A., de Luca, S.J., Peralba, M., de Luca, M.A. (2006) Sodium ferrate(IV) and sodium hypochlorite in disinfection of biologically treated effluents. Ammonium nitrogen protection against THMs and HAAs. J Environ Sci Health A41(10):2329-2343

Tao, T., Peng, X.F., Lee, D.J. (2006) Interaction between wastewater-sludge floc and the moving ice front. Chem Eng Sci 61(10):5369-5376

U.S. EPA (United States Environmental Protection Agency) (2005). Method 1681: Fecal Coliforms in Sewage Sludge (Biosolids) by Multiple Tube Fermentation using A-1 Medium. Washington DC, USA.

U.S. EPA (United States Environmental Protection Agency) (2003) Environmental Regulations and Technology - Control of Pathogens and Vector Attraction in Sewage Sludge (Including Domestic Septage). Under 40 CFR Part 503, EPA/625/R-92/013, Revised July 2003

Vesilind, P.A. and Örmeci, B. (2000) A modified capillary suction time apparatus for the measuring the filterability of super-flocculated sludges. Water Sci Technol 42(9):135-139 Vesilind, P.A. and Martel, C.J. (1990) Freezing of water and wastewater sludges. J Environ Eng-ASCE 116(5):854-862

Wang, Q., Fujisaki, K., Ohsumi, Y., Ogawa, H.I. (2001) Enhancement of dewaterability of thickened waste activated sludge by freezing and thawing treatment. J Environ Sci Health A36(7):1361-1371 
Wu, C., Zhang, G., Zhang, P., Chang, C.-C. (2014) Disintegration of excess activated sludge with potassium permanganate: Feasibility, mechanisms and parameter optimization. Chem Eng J 240:420-425

Yang, B., Ying, G.-G., Zhao, J,-L, Liu, S., Zhou, L.-J., Chen, F. (2012) Removal of selected endocrine disrupting chemicals (EDCs) and pharmaceuticals and personal care products (PPCPs) during ferrate(VI) treatment of secondary wastewater effluents. Water Res 46(7):2194-2204

Ye, F.X., Peng, G., Li, Y, (2014) Fenton's oxidation to improve the filterability and dewaterability of excess activated sludge by affecting extracellular polymeric substances. Asian J Chem 26(8):2259-2263

Zhu, J.-H., Yan, X.-L., Liu, Y., Zhang, B. (2006) Improving alachlor biodegradability by ferrate oxidation. J Hazard Mater 135(1-3):94-99 


\section{STABILISATION AND DEWATERING OF PRIMARY SLUDGE USING FERRATE(VI) PRE-TREATMENT FOLLOWED BY FREEZE-THAW IN SIMULATED DRAINAGE BEDS}

James Diak and Banu Örmeci

Department of Civil and Environmental Engineering, Carleton University, 1125 Colonel By Drive, Ottawa, ON K1S 5B6, Canada.

\subsection{ABSTRACT}

The study examined the individual and combined effects of potassium ferrate(VI) additions and freeze-thaw conditioning for the stabilisation and dewatering of primary sludge. Potassium ferrate(VI) additions of 0.5 and $5.0 \mathrm{~g} / \mathrm{L}$ were used as a pre-treatment, prior to freeze-thaw. Samples were frozen at $-10,-20$ and $-30^{\circ} \mathrm{C}$, and were kept frozen for 1,8 and 15 days. After the designated freezing period, the samples were removed from the freezer and thawed at room temperature in a setup which allowed meltwater to be separated from the sludge cake via gravity drainage. The meltwater was characterised in terms of fecal coliform, soluble COD, soluble proteins, soluble carbohydrates, $\mathrm{pH}$ and turbidity. The sludge cake was characterised in terms of fecal coliform, total solids (TS) and volatile solids (VS). 
Freeze-thaw with gravity meltwater drainage reduced the sludge volume by up to $79 \%$. After being frozen for only 1 day, the concentrations of fecal coliform in many of the primary sludge samples were reduced to $<1000 \mathrm{MPN} / \mathrm{g}$ dry solids (DS), representing $>3$ log reduction in some cases. However, pre-treatment of the primary sludge with potassium ferrate $(\mathrm{VI})$ doses $\leq 5.0 \mathrm{~g} / \mathrm{L}$, prior to freeze-thaw, may have reduced the effectiveness of stand-alone freeze-thaw. When potassium ferrate(VI) was added to the sludge, there were significant increases in soluble proteins, soluble carbohydrates and sCOD. The increases in soluble components may have acted as cryoprotectants for the surviving bacteria during freezing and may have stimulated regrowth during the thawing stage. Follow-up experiments using doses ranging from 5.1 to $24.9 \mathrm{~g} / \mathrm{L}$ of potassium ferrate(VI) demonstrated that $<5$-log inactivation of fecal coliform in raw primary sludge can be achieved within 15 minutes, using $15 \mathrm{~g} / \mathrm{L}$ of potassium ferrate(VI). The resulting concentration of fecal coliform in the sludge was 1023 MPN/g DS, which is near the limit for CP1 biosolids in Ontario. Pre-treatment with $22.0 \mathrm{~g} / \mathrm{L}$ of potassium ferrate(VI), followed by freeze-thaw, with only 3 days frozen, reduced the concentration of fecal coliform to below the detection limit in the meltwater and the sludge cake remaining following the 12-hour thawing period. This demonstrates that sludge pre-treatment with potassium ferrate( $\mathrm{VI})$, followed by freeze-thaw could be used as a stand-alone sludge treatment technology.

Potassium ferrate( $\mathrm{VI})$ additions also acted as coagulants once the ferrate( $\mathrm{VI})$ ions were reduced to ferric ions and ferric hydroxide. This increased the $\mathrm{pH}$ and reduced the turbidity of the meltwater. 


\subsection{INTRODUCTION}

Sludge dewatering in remote cold regions can be achieved using natural freeze-thaw (freeze-thaw) cycles (Martel, 1993, Martel and Diener, 1991a, Martel and Diener, 1991b, Reed et al., 1986). During winter months, sludge is spread in successive layers, allowing each layer to freeze before the next layer is applied. Then during spring when the sludge thaws, the meltwater drains, leaving a dewatered cake. Simple gravity drainage can reduce sludge volumes by 85 to 96\%, leaving a sludge cake ranging from 20\% up to $82 \%$ solids (Diak et al., 2011, Martel, 1993, Martel and Diener 1991a, Martel and Diener, 1991b).

During freezing, the advancing ice front forces solids and other impurities to the boundary of the ice crystal where they become compressed and dehydrated (Chalmers, 1959). Parker and Collins (1999) demonstrated how the removal of surface water from the colloidal particles within the sludge floc could bring particles into close contact, allowing the formation of new bonds amongst particles. These coagulated sludge particles are denser and stronger than conventional sludge flocs, and do not break apart easily when agitated (Parker and Collins, 1999).

In addition to the densification of sludge flocs, freeze-thaw also results in an increase in BOD and COD of the supernatant due to the release exocellular polymers from the sludge floc (Hong et al., 1995, Lee and Hsu, 1994). Furthermore, freeze-thaw can cause cell disruptions and the release of intracellular materials, marked by an increase in the DNA concentration of the supernatant (Örmeci and Vesilind, 2001). The release of intra- 
and extra-cellular materials also results in an increase in the concentrations of proteins, carbohydrates and cations in the supernatant (Örmeci and Vesilind, 2001). Hung et al. (1996) used FTIR spectra to demonstrate that the increase in soluble carbohydrates following freeze-thaw was primarily due to the release of cellulose-containing materials. Wang et al. (2001) found strong correlations between the elution of proteins and carbohydrates and a decrease in viable cell count. Chu et al. (1999) also saw a decrease in microbial density following freeze-thaw.

Freeze-thaw can also reduce the concentration of pathogens and indicator organisms in wastewater sludges (Gao et al., 2009, Gao et al., 2006, Chu et. al., 1999, Sanin et al., 1994, Mazur, 1986, Silvares et al., 1975). Sanin et al (1994) studied the effects of freezethaw on the concentration of different bacteria, viruses, and parasites in anaerobically and aerobically digested sludges. In this study, freeze-thaw resulted in at least 0.5 to 1.0-log reduction in the concentrations of bacteria (fecal coliform, Salmonella) and viruses (plaque forming units, poliovirus) except for fecal streptococci, which were only reduced by $0.2-\log$. Furthermore, the concentration of Cryptosporidium parvum (protozoa) was reduced by $>8-\log$, whereas the concentration of Ascaris suum ova (helminth) was unaffected by freeze-thaw. Results of the study suggest that the combination of freeze-thaw and sludge digestion can easily reduce the concentration of fecal coliform to below $2 \times 10^{6} \mathrm{MPN} / \mathrm{g}$ dry solids, and meet U.S. Class B requirements for sludge disposal on land (U.S. EPA, 2003). Chu et al. (1999) also used freeze-thaw to reduce total coliform in WAS (waste activated sludge) to below Class B limits. 
Land application of treated sludge is an effective sludge disposal option which provides a variety of nutrients and organic materials to soils. Regulations exist to ensure the protection of human health and the environment. The regulated parameters and limits vary by jurisdiction, however primary concerns include the concentrations of heavy metals, pathogens and indicator organisms (CCME 2010, U.S. EPA, 2003). Additionally, organic chemicals are of increasing concern, and are likely to be regulated in the future (Harrison et al., 2006, Abad et al., 2005).

Ferrate(VI) is a very strong oxidant capable of reducing sludge odours (de Luca et al., 1996), inactivating pathogens and indicator organisms (Jiang et al., 2007, Schuck et al., 2006, Murmann and Robinson, 1974), removing heavy metals (Sylvester et al. 2001, Murmann and Robinson, 1974) and oxidising organic chemicals (Li et al., 2008, Jiang, 2007, Zhu et al. 2006). Furthermore, when ferrate(VI) is used as an oxidant, it is reduced to Fe(III) ions and ferric hydroxide, which act as a coagulant (Jiang and Lloyd, 2002). Therefore, sludge pre-treatment with potassium ferrate( $\mathrm{VI})$ prior to freeze-thaw may improve the freeze-thaw process. From an operational stand-point, the most obvious benefit would be a reduction in pathogens and sludge odours, which would make the application and removal of sludge to and from the freeze-thaw bed safer and less offensive. Ferrate(VI) pre-treatment may also improve the dewaterability of the sludge and reduce the possibility of fecal coliform and pathogenic bacteria regrowth following dewatering and during storage. Reactivation and regrowth of indicator organisms during the dewatering and storage of anaerobically digested biosolids have gained considerable interest in recent years (Chen et al., 2011, Gardner et al., 2010, Higgins et 
al., 2007). Ferrate(VI) pre-treatment may even reduce the concentrations of emerging organic compounds in sludge, such as bisphenol-A (BPA), ethinyl estradiol (EE2) and alachlor (Li et al., 2008, Jiang, 2007, Zhu et al. 2006).

Depending on the characteristics of the initial sludge, freeze-thaw co-treatment with potassium ferrate(VI) may render the dewatered cake suitable for land application. This would dramatically simplify septage treatment and disposal in rural communities which use a conventional septic tank and soil adsorption system. Septage is the sludge pumped from the bottom of septic tanks. The characteristics of septage depend heavily on the feed to the septic tank and the age of the sludge, which typically varies from 2 to 5 years (Kinsley et al., 2012). Septic tanks essentially act as passive anaerobic digesters, operating under ambient or uncontrolled conditions. Therefore septage can have elevated fecal coliform concentrations if the septage is relatively fresh ( $<1 \mathrm{yr})$, whereas older septage may have a reduced level of fecal coliform (3-5 yr). Similarly, sludges pumped from holding tanks and dredged from wastewater lagoons will have undergone a certain level of passive anaerobic stabilisation. Therefore, additional stabilisation using ferrate(VI) oxidation and freeze-thaw co-treatments may be able to provide a sufficient level of pathogen inactivation for the treated biosolids to be applied to land.

To test the ability of potassium ferrate( $\mathrm{VI})$ and freeze-thaw to treat and dewater septage, primary sludge was used for these experiments because it has relatively high concentration of fecal coliform. 
The purpose of this study was to examine the individual and combined effects of potassium ferrate(VI) additions and freeze-thaw conditioning for the stabilisation and dewatering of primary sludge. Potassium ferrate(VI) additions of 0.5 and $5.0 \mathrm{~g} / \mathrm{L}$ were added to the sludge, and the samples were frozen at $-10,-20$ or $-30{ }^{\circ} \mathrm{C}$, for 1,8 or 15 days. Following the designated freezing period, the samples were removed from the freezer and thawed at room temperature in a setup which allowed meltwater to drain out of the sludge cake via gravity. The meltwater was characterised in terms of fecal coliform, soluble chemical oxygen demand (COD), soluble proteins, soluble carbohydrates, $\mathrm{pH}$ and turbidity, and the sludge cake was characterised in terms of fecal coliform, total solids (TS) and volatile solids (VS).

\subsection{MATERIALS AND METHODS}

\subsubsection{Sludge samples}

Primary sludge was obtained from the Robert O. Pickard Environmental Centre (ROPEC) in Ottawa, Ontario, Canada. A different batch of sludge was used for each set of experiments with a different freezing temperature $\left(-10,-20,-30^{\circ} \mathrm{C}\right)$. The initial characteristics of the three sludge batches are presented in Table 8-1. A separate set of follow-up experiments with higher ferrate(VI) concentrations were conducted using a fourth batch of primary sludge obtained from ROPEC. The initial TS and VS of this sludge batch were 4.6 and $3.5 \%$ respectively, and the concentration of fecal coliform was $9.6 \mathrm{x}$ $10^{6} \mathrm{MPN} / \mathrm{mL}$ (7.0-log), equivalent to $2.0 \times 10^{8} \mathrm{MPN} / \mathrm{g} \mathrm{DS}$ (8.3-log). 
Table 8-1: Initial characteristics of the primary sludge samples.

\begin{tabular}{|c|c|c|c|c|}
\hline \multirow[b]{2}{*}{ Parameter } & \multirow[b]{2}{*}{ Units } & \multicolumn{3}{|c|}{ Primary Sludge Batch } \\
\hline & & $\begin{array}{c}\text { Sample } \mathrm{A}\left(-10^{\circ} \mathrm{C}\right) \\
\text { AVERAGE }( \pm 95 \% \mathrm{CL})\end{array}$ & $\begin{array}{c}\text { Sample B }\left(-20^{\circ} \mathrm{C}\right) \\
\text { AVERAGE }( \pm 95 \% \mathrm{CL})\end{array}$ & $\begin{array}{c}\text { Sample C }\left(-30^{\circ} \mathrm{C}\right) \\
\text { AVERAGE }( \pm 95 \% \mathrm{CL})\end{array}$ \\
\hline TS & $\%$ & $( \pm 0.0)$ & $( \pm 0.1)$ & $( \pm 0.1)$ \\
\hline VS & $\%$ & $( \pm 0.0)$ & $( \pm 0.1)$ & $( \pm 0.1)$ \\
\hline Fecal Coliforms & $\log (\mathrm{MPN} / \mathrm{mL})$ & $( \pm 0.7)$ & $5.3(+0.6,-0.7)$ & $4.5 \quad(+0.5,-0.6)$ \\
\hline Fecal Coliforms & $\log (\mathrm{MPN} / \mathrm{g} \mathrm{DS})$ & $( \pm 0.7)$ & $6.6(+0.6,-0.7)$ & $6.0(+0.5,-0.6)$ \\
\hline Soluble COD & $\mathrm{g} / \mathrm{L}$ & $( \pm 0.2)$ & $( \pm 0.1)$ & $( \pm 0.1)$ \\
\hline Soluble Proteins & $\mathrm{mg} / \mathrm{L}$ & $( \pm 3)$ & $( \pm 3)$ & $( \pm 2)$ \\
\hline Soluble Carbohydrates & $\mathrm{mg} / \mathrm{L}$ & $( \pm 5)$ & $( \pm 7)$ & $( \pm 2)$ \\
\hline
\end{tabular}

\subsubsection{Pre-treatment with potassium ferrate(VI) $\left(\mathrm{K}_{2} \mathrm{FeO}_{4}\right)$}

Samples designated for pre-treatment with potassium ferrate(VI) were given a low dose $(\mathrm{LD}=0.5 \mathrm{~g} / \mathrm{L})$ or a high dose $(\mathrm{HD}=5.0 \mathrm{~g} / \mathrm{L})$ of Ferratec Brand ${ }^{\mathrm{TM}}>90 \%$ pure potassium ferrate(VI) (Sigma-Aldrich Canada Ltd., Oakville, ON), and stirred using a magnetic stirrer for 15 minutes.

\subsubsection{Freeze-thaw stabilisation and dewatering}

$200 \mathrm{~mL}$ sludge samples were dispensed into $500 \mathrm{~mL}$ wide-mouth bottles. The bottles were sealed using the screw-on lids and inverted as shown in Figure 8-1a. The inverted bottles were then placed in a freezer (VWR FORMA ${ }^{\circledR}-40^{\circ} \mathrm{C}$ Lab Freezer Model 5722, VWR International, Mississauga, ON) which was set to either $-10,-20$ or $-30^{\circ} \mathrm{C}$. The temperature inside the freezer and the temperature at the centre of a $200 \mathrm{~mL}$ test sample were monitored using a Traceable $^{\circledR}$ dual-channel thermometer with type $\mathrm{K}$ thermocouples and computer output (Model 4137, Control Company, Friendswood, TX, USA). Temperatures were recorded on a PC every minute using the data acquisition software (DAS ${ }^{\mathrm{TM}}$, Control Company, Friendswood, TX, USA). The resulting average 
freezing rates generated using freezer temperatures of $-10,-20$ and $-30^{\circ} \mathrm{C}$ were approximately 3.0, 7.1 and $9.0 \mathrm{~mm} / \mathrm{hr}$ respectively. A plot of the temperature logs used to estimate the freezing rates is presented in Appendix B4 on page 383, and a summary of the various sample media, freezing geometries, freezing temperatures and freezing rates used for all experiments is presented as Appendix B5 on page 384. The freezing rates generated were within the optimal range for pathogen reduction and particle migration leading to improved dewaterability (Wang et al., 2001, Sanin et al., 1995, Hung et al., 1997). After the samples were completely frozen, they were kept under the freezing conditions for an additional 1,8 or 15 days. After the designated freezing period, the samples were removed from the freezer, the solid lid was replaced with a perforated lid that was lined with a geotextile drainage fabric, and the tape sealing the air hole was removed. The frozen samples were then placed upside down in a funnel, which rested in the opening of a $50 \mathrm{~mL}$ graduated cylinder, as shown in Figure 8-1b. All samples thawed at room temperature $\left(22-23^{\circ} \mathrm{C}\right)$ for 12 hours, and the meltwater was collected via gravity drainage. 
a)

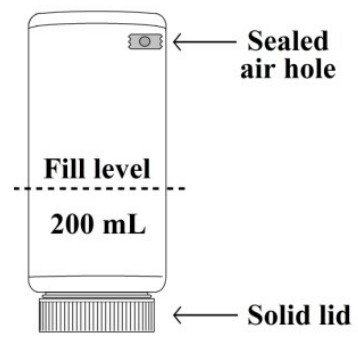

b)

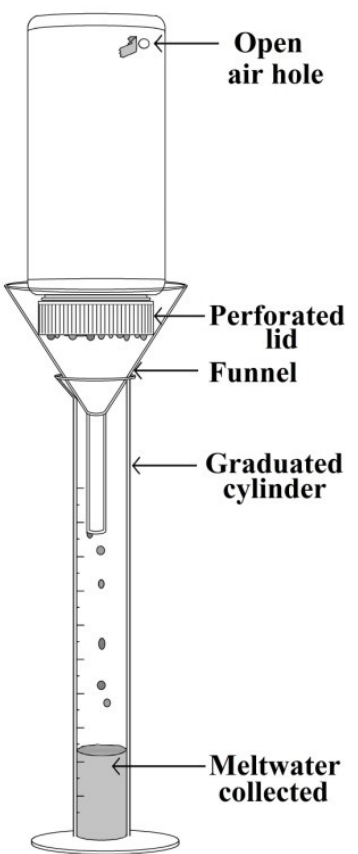

Figure 8-1: Schematic of the (a) freezing vessel and (b) thawing/meltwater collection apparatus used in the freeze-thaw sludge dewatering experiments with primary sludge.

\subsubsection{Sample analysis}

The fecal coliform, TS, VS, soluble COD, soluble proteins and soluble carbohydrates concentrations of the sludge were measured before and after the addition of potassium ferrate(VI). Following freeze-thaw dewatering, the fecal coliform, TS and VS concentrations of the sludge cake were measured, and the concentration of fecal coliform, soluble COD, soluble proteins, soluble carbohydrates, $\mathrm{pH}$ and turbidity of the meltwater were measured. The methods used to measure each of these parameters are presented below.

The concentration of fecal coliform was measured using multiple tube fermentation (MTF) with A1 medium (EMD Chemicals Inc., Gibbstown, NJ, USA) according to U.S. EPA Method 1681 (U.S. EPA, 2005). All MTF tests were carried out in duplicate using a 
minimum of 4 dilutions, with 5 replicates per dilution. All other tests were conducted in triplicate. The TS and VS concentrations were measured according to Standard Method $2540 \mathrm{G}$ (APHA, 2005). The turbidity was measured using a HACH Model 2100AN Turbidimeter (Hach Company, Loveland CO, USA). Colorimetric tests were conducted using a HACH DR 2800 spectrophotometer, and for COD tests, a HACH DRB 200 digital reactor block was also used. The $\mathrm{pH}$ was measured using a Thermo Orion 5-star benchtop meter kit and ROSS ultra pH electrode (Thermo Fisher Scientific Inc., Rockford, IL, USA). The soluble COD was measured using HACH method 8000 (high range: 20-1500

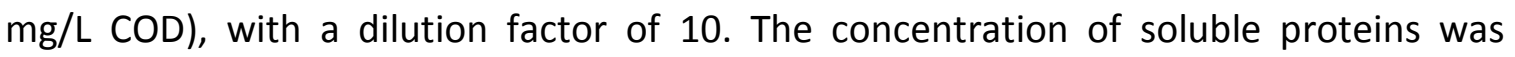
measured using Coomassie brilliant blue G-250 reagent (Pierce Protein Biology Products, Thermo Fisher Scientific Inc., Rockford, IL, USA) with the Bradford method (Bradford, 1976) and the concentration of soluble carbohydrates was measured using the Anthrone method (Morris, 1948). To obtain the soluble component, the samples were centrifuged at $10,000 \mathrm{rpm}$ (relative centrifugal force, $\mathrm{RCF}=15,317 \mathrm{~g}$ ) for 10 minutes, then the supernatant was filtered using a $0.45 \mu \mathrm{m}$ mixed cellulose ester (MCE) syringe filter (Shanghai Derian Instrument Co., Ltd., Shanghai, China).

\subsubsection{Statistical analysis}

Error bars on all figures show the $95 \%$ confidence interval. A two-tailed t-test was used to determine the $p$ values and statistical significance $(p<0.05)$ of the results. 


\subsection{RESULTS AND DISCUSSION}

As a reference for the remainder of this paper, Table 8-2 lists the sample labels used in subsequent figures along with a description of the various treatments applied to the samples.

Table 8-2: Description of the treatments applied to the primary sludge samples and their representative abbreviation.

\begin{tabular}{|c|c|}
\hline Label & Description of the treatments applied to the primarty sludge samples \\
\hline Initial & Initial sludge sample prior to any treatment \\
\hline LD & Low dose $(\mathrm{LD}=0.5 \mathrm{~g} / \mathrm{L})$ of $\mathrm{K}_{2} \mathrm{FeO}_{4}$ and a 15 -min contact time \\
\hline HD & High dose $(\mathrm{HD}=5.0 \mathrm{~g} / \mathrm{L})$ of $\mathrm{K}_{2} \mathrm{FeO}_{4}$ and a 15 -min contact time \\
\hline LD-F1 & Low-dose $\left(\mathrm{LD}=0.5 \mathrm{~g} / \mathrm{L} \mathrm{K}_{2} \mathrm{FeO}_{4}\right)$ pre-treatment, 1 day frozen and a 12 -hr thaw with meltwater separation \\
\hline LD-F8 & Low-dose $\left(\mathrm{LD}=0.5 \mathrm{~g} / \mathrm{L} \mathrm{K}_{2} \mathrm{FeO}_{4}\right)$ pre-treatment, 8 days frozen and a 12-hr thaw with meltwater separation \\
\hline LD-F15 & Low-dose $\left(\mathrm{LD}=0.5 \mathrm{~g} / \mathrm{L} \mathrm{K}_{2} \mathrm{FeO}_{4}\right)$ pre-treatment, 15 days frozen and a 12-hr thaw with meltwater separation \\
\hline HD-F1 & High-dose $\left(\mathrm{HD}=5.0 \mathrm{~g} / \mathrm{L} \mathrm{K}_{2} \mathrm{FeO}_{4}\right)$ pre-treatment, 1 day frozen and a 12-hr thaw with meltwater separation \\
\hline HD-F8 & High-dose $\left(\mathrm{HD}=5.0 \mathrm{~g} / \mathrm{L} \mathrm{K}_{2} \mathrm{FeO}_{4}\right)$ pre-treatment, 8 days frozen and a 12 -hr thaw with meltwater separation \\
\hline HD-F15 & High-dose $\left(\mathrm{HD}=5.0 \mathrm{~g} / \mathrm{L} \mathrm{K}_{2} \mathrm{FeO}_{4}\right)$ pre-treatment, 15 days frozen and a 12-hr thaw with meltwater separation \\
\hline F1a & 1 day frozen and a $12-\mathrm{hr}$ thaw with meltwater separation \\
\hline F1b & 1 day frozen and a 12 -hr thaw with meltwater separation \\
\hline F8a & 8 days frozen and a 12 -hr thaw with meltwater separation \\
\hline $\mathrm{F} 8 \mathrm{~b}$ & 8 days frozen and a 12 -hr thaw with meltwater separation \\
\hline F15a & 15 days frozen and a 12 -hr thaw with meltwater separation \\
\hline F15b & 15 days frozen and a 12 -hr thaw with meltwater separation \\
\hline
\end{tabular}

Following the designated freezing periods, the $200 \mathrm{~mL}$ sludge samples were removed

from the freezer and thawed at room temperature in the apparatus shown in Figure 8-1b. Table 8-3 shows the volumes of meltwater collected and the mass of sludge cake remaining for each of the samples, and Table 8-4 shows the corresponding percent reductions in sludge volume following freeze-thaw with meltwater drainage. Freezethaw with meltwater drainage reduced the volume of sludge by 70 to $79 \%$ in sludge samples $A$ and $C$, however the volume of sludge B was only reduced by 46 to $56 \%$. This may be due to the higher initial solids concentration in sludge $B$ compared to sludges $A$ 
and $C$, which may have impeded particle migration and reduced the drainability of the sludge. This is discussed in more detail in the following sections.

Table 8-3: Volume of meltwater collected via gravity drainage during the thawing process and the corresponding mass of sludge cake remaining.

\begin{tabular}{|c|c|c|c|c|c|c|c|c|c|c|c|c|c|}
\hline & & F1a & F1b & F8a & $\mathrm{F} 8 \mathrm{~b}$ & F15a & F15b & LD-F1 & LD-F8 & LD-F15 & HD-F1 & HD-F8 & HD-F15 \\
\hline \multirow{2}{*}{ A } & Meltwater Collected (mL) & 148 & 148 & 149 & 148 & 149 & 146 & 142 & 146 & 149 & 144 & 144 & 142 \\
\hline & Sludge Cake Remaining (g) & 50 & 49 & 50 & 51 & 51 & 51 & 55 & 55 & 53 & 58 & 59 & 56 \\
\hline \multirow{2}{*}{ B } & Meltwater Collected (mL) & 102 & 102 & 99 & 90 & 104 & 104 & 101 & 93 & 105 & 100 & 92 & 105 \\
\hline & Sludge Cake Remaining (g) & 95 & 93 & 98 & 103 & 89 & 88 & 96 & 102 & 91 & 100 & 109 & 97 \\
\hline \multirow{2}{*}{ C } & Meltwater Collected (mL) & 129 & 126 & 138 & 137 & 144 & 136 & 125 & 138 & 140 & 127 & 142 & 140 \\
\hline & Sludge Cake Remaining (g) & 41 & 47 & 44 & 42 & 49 & 48 & 53 & 49 & 51 & 56 & 42 & 50 \\
\hline
\end{tabular}

Table 8-4: Percent reduction in sludge volume following freeze-thaw with gravity meltwater drainage.

\begin{tabular}{|c|c|c|c|c|c|c|c|c|c|c|c|c|}
\hline Volume Reduction (\%) & F1a & F1b & F8a & F8b & F15a & F15b & LD-F1 & LD-F8 & LD-F15 & HD-F1 & HD-F8 & HD-F15 \\
\hline Sample A $\left(-10^{\circ} \mathrm{C}\right)$ & 75 & 76 & 75 & 74 & 75 & 75 & 72 & 73 & 73 & 71 & 70 & 72 \\
\hline Sample B $\left(-20^{\circ} \mathrm{C}\right)$ & 53 & 53 & 51 & 49 & 55 & 56 & 52 & 49 & 54 & 50 & 46 & 51 \\
\hline Sample C $\left(-30^{\circ} \mathrm{C}\right)$ & 79 & 77 & 78 & 79 & 76 & 76 & 73 & 75 & 75 & 72 & 79 & 75 \\
\hline
\end{tabular}

\subsubsection{Sludge Cake}

Figure 8-2 shows the effects of LD and HD pre-treatment with freeze-thaw on the TS and VS of the sludge cake following meltwater drainage. For each of the sludge samples shown, the shaded bars represent the VS, and the outlined bars represent the TS, meaning that the fixed solids for each sample are shown by the "hollow" portion of each bar. The initial TS of the sludge batches varied from 2.9 to $4.2 \%$ solids, with an organic fraction of 0.81 to 0.84 . Following a 15 -minute contact time, LD did not have a significant effect on the TS and VS of the sludge, whereas HD increased the TS of the sludge, but did not have a significant effect on the VS. The increase in TS was likely due to the formation of inorganic solids, such as iron oxides and iron oxide-hydroxides. The 
increases in TS for all three samples were approximately $5 \mathrm{~g} / \mathrm{L}$, which coincides with the $5 \mathrm{~g} / \mathrm{L}$ of potassium ferrate( $\mathrm{VI})$ which was added to these samples.

Stand-alone freeze-thaw with meltwater drainage reduced the volume of the sludge by up to $79 \%$, with no significant differences among the various freezing periods (Table 8-3). However, the volumes of meltwater from sludge B samples were considerably less than the $A$ and $C$ sludge samples. This may be due to the higher solids concentration in the sample B sludge, which may have impeded particle migration during freezing (Halde, 1980), and therefore reduced solids separation and sludge drainability. However, in the follow-up experiments (described below), the volume of sludge with an initial TS of $4.6 \%$ was reduced by approximately $68 \%$ following freeze-thaw with gravity meltwater drainage. It was expected that increased water content (lower TS) would facilitate the formation of ice crystals during freezing, and lead to a continuous network of ice channels throughout the sludge, which would allow meltwater to drain freely during the thawing stage. Supporting this theory is that the sludges with the lowest initial TS (highest water content) demonstrated the greatest drainability during thawing, and the greatest percent reductions in sludge volume (sludge samples A and C). However, the sludge with the poorest drainability was not the sludge with the highest initial TS, therefore it is likely that additional factors are contributing to the drainability of the sludge in addition to the solids concentration.

When potassium ferrate(VI) was added to the sludge prior to freeze-thaw, the sludge drainability may have been reduced. In most cases, samples treated with LD and HD 
generated less meltwater and left a greater mass of sludge cake compared to standalone freeze-thaw with meltwater drainage (Table 8-3). However, the differences are relatively small and, in most cases, are statistically insignificant. Furthermore, LD pretreatment did not have a significant effect on the TS and VS of the sludge samples compared to stand-alone freeze-thaw. On the other hand, HD pre-treatment reduced the organic fraction of solids in the sludge cake. This seems to be the result of a combination of an increase in the inorganic solids and a decrease in the organic solids. As mentioned earlier, HD resulted in an increase in TS following a 15-minute reaction period, with no change in VS. However, when used as a pre-treatment, HD resulted in a decrease in VS compared to stand-alone freeze-thaw. As suggested in the study by Montusiewicz et al. (2010), the decrease in VS may be the result of biodegradation carried out by the surviving microorganisms during the thawing process, or the action of enzymes released from cells and sludge flocs during freezing. 


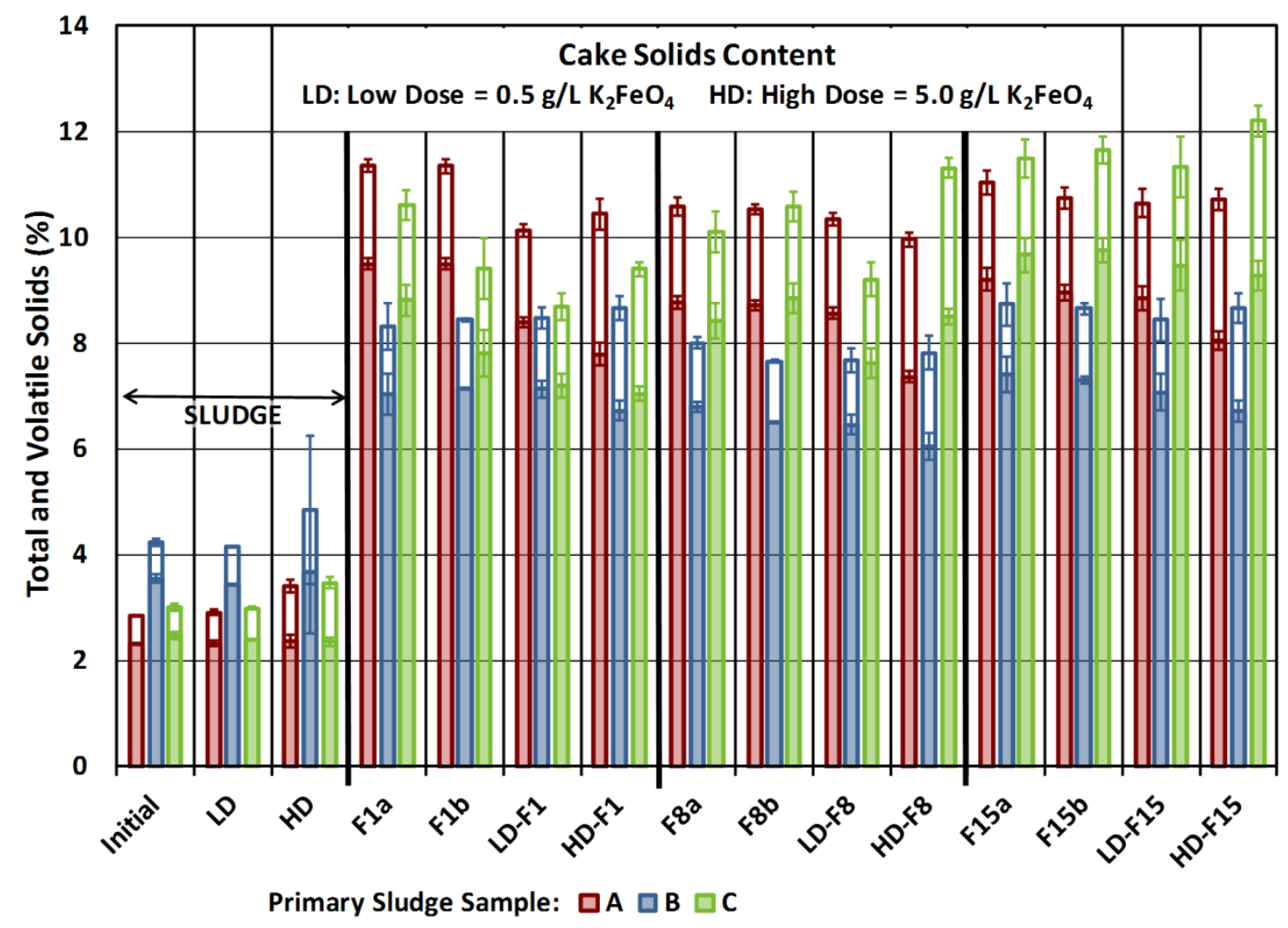

Figure 8-2: Effect of LD and HD pre-treatment with freeze-thaw on the TS and VS of the sludge cake following meltwater drainage.

Figure 8-3 shows the effect of LD, HD and freeze-thaw on the concentration of fecal coliform in sludge. The initial concentration of fecal coliform in the primary sludge was $10^{3.5}, 10^{6.6}$ and $10^{6} \mathrm{MPN} / \mathrm{g}$ DS for the $-10,-20$ and $-30^{\circ} \mathrm{C}$ sludge samples, respectively. Following a 15-minute contact time, LD did not have a significant effect on the concentration of fecal coliform, and HD resulted in only $0.5,1.0$ and $0.6-\log$ inactivation of fecal coliform in sludge batches $A, B$ and $C$ respectively, which suggests that $5.0 \mathrm{~g} / \mathrm{L}$ of potassium ferrate(VI) was insufficient for the stabilisation of primary sludge.

Stand-alone freeze-thaw for periods of 1,8 and 15 days resulted in 0.75 to 3.5 -log reduction in fecal coliform (average $=2.5-\log$ reduction). However, after only 1 day 
frozen, samples A, B and C had log reductions of 1.0, 2.3 and 3.2-log (average $=2.2-\log$ reduction). This suggests that the majority of fecal coliform inactivation occurs during the freeze-thaw process, and increasing the duration of time frozen from 1 day to 8 and 15 days does not significantly increase the level of inactivation.

When LD pre-treatment was used, there were no significant differences in the level of inactivation of fecal coliform compared to stand-alone freeze-thaw, suggesting that the $0.5 \mathrm{~g} / \mathrm{L}$ dose of potassium ferrate $(\mathrm{VI})$ was quickly consumed by other components in the primary sludge, leaving no ferrate(VI) for disinfection.

However, HD pre-treatment may have reduced the effectiveness of freeze-thaw by protecting certain fecal coliform during sludge freezing or perhaps stimulating regrowth in some of the samples during the thawing period. This was also observed in the meltwater collected from HD pre-treated samples, suggesting that $5.0 \mathrm{~g} / \mathrm{L}$ of potassium ferrate(VI) was insufficient for the treatment of primary sludge, despite the action of freeze-thaw with meltwater separation. 


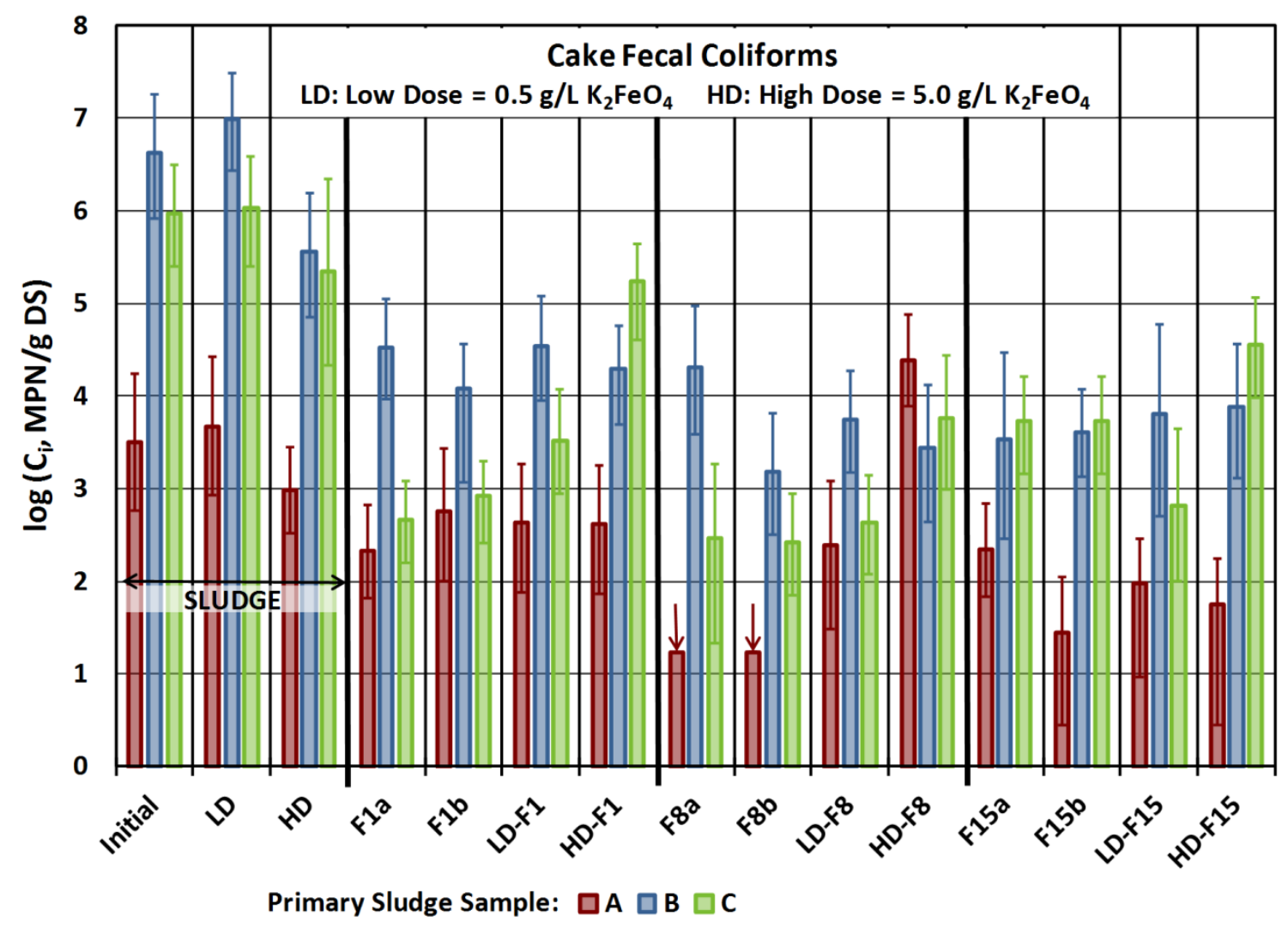

Figure 8-3: Effect of LD and HD pre-treatment with freeze-thaw on the concentration of fecal coliform in the sludge cake following meltwater drainage.

\subsubsection{Meltwater}

Stand-alone freeze-thaw reduced the concentration of fecal coliform in the meltwater to below the detection limit $(<0.18 \mathrm{MPN} / \mathrm{mL})$ in ten of the samples, while the other eight stand-alone freeze-thaw samples had an average of 7 fecal coliform per $\mathrm{mL}$ of meltwater (Figure 8-4). However, when HD pre-treatment was used, the meltwater contained up to 4-log more fecal coliform ( $<1600 \mathrm{MPN} / \mathrm{mL}$ ), with an average of 328 $\mathrm{MPN} / \mathrm{mL}$. In some cases, LD pre-treatment also resulted in higher concentrations of fecal coliform in the meltwater compared to the stand-alone freeze-thaw samples. This may be the result of the following mechanisms. First, the addition of potassium 
ferrate $(\mathrm{VI})$ caused the release of polymers, such as proteins and carbohydrates, to the supernatant, which may have acted as cryoprotectants, protecting cells during freezing (Montusiewicz et al., 2010). Second, the addition of potassium ferrate(VI) created an affluent environment for the surviving bacteria, rich in food (sCOD) and nutrients (nitrates), which may have lead to regrowth during the thawing process. This was observed by de Luca et al. (1996), who reported that "low dosages" of ferrate(VI) created a "luxuriant environment" for surviving bacteria, which stimulated growth in sludge samples. To inhibit fecal coliform regrowth, de Luca et al. (1996) used higher doses of 10,15 and $30 \mathrm{~g} / \mathrm{L}$ of potassium ferrate(VI). However, previous experiments (presented in the previous chapter) demonstrated that $10 \mathrm{~g} / \mathrm{L}$ of potassium ferrate(VI) followed by freeze-thaw, without meltwater drainage, resulted in 4.2 to $4.6-\log$ inactivation of fecal coliform in primary sludge. Therefore, these experiments attempted smaller doses of $5.0 \mathrm{~g} / \mathrm{L}$ potassium ferrate $(\mathrm{VI})$ since it was expected that meltwater separation during the thawing stage would further reduce the concentration of fecal coliform.

HD resulted in 23,69 and $20 \%$ increases in the sCOD of sludge samples A, B and C respectively, following a 15-minute reaction period (Figure 8-5). This coincided with 640, 520 and $140 \%$ increases in soluble proteins (Figure 8-6) and 150, 400 and $190 \%$ increases in soluble carbohydrates (Figure 8-7) in sludge samples A, B and C respectively. This means that the surviving fecal coliform were surrounded by a supernatant that was rich in substrate and biopolymers during freezing which likely contributed to regrowth during the 12-hour thawing period. 
A mass balance on the MPN of fecal coliform throughout the ferrate( $\mathrm{VI})$ and freeze-thaw co-treatment process show net reductions, however the potential for regrowth during was further investigated using potassium ferrate(VI) doses of 5.1, 8.0, 15.0, 22.0 and $24.9 \mathrm{~g} / \mathrm{L}$ with a fourth batch of primary sludge. No signs of regrowth were observed in the follow-up experiments, which are presented in detail following the current discussion on the first set of experiments.

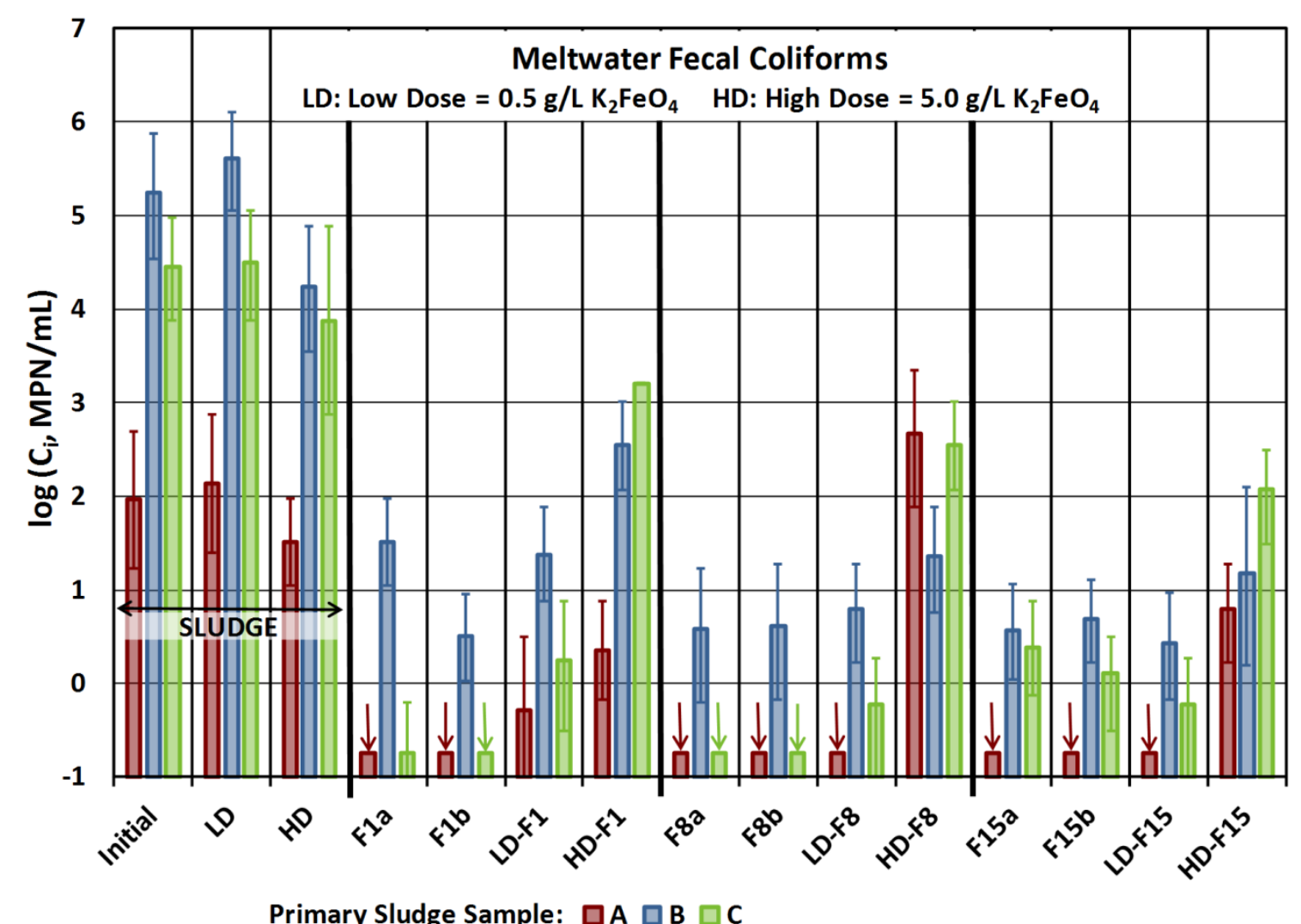

Figure 8-4: Effect of LD and HD pre-treatment with freeze-thaw on the concentration of fecal coliform in meltwater. 
The initial SCOD of the primary sludge batches ranged from 3.6 to $4.8 \mathrm{~g} / \mathrm{L}$ (Figure 8-5). Following the addition of LD with a 15-minute contact time, there was no significant change in the SCOD. Stand-alone freeze-thaw increased the SCOD by approximately $25 \%$ regardless of the duration of the freezing period, and also increased the concentrations of soluble proteins (Figure 8-6) and carbohydrates (Figure 8-7). Hung et al. (1996) demonstrated that the growing ice layer during freezing caused the release of cellulosebased materials from the sludge floc. Örmeci and Vesilind (2001) also saw increases in the concentrations proteins, carbohydrates and cations in the supernatant, caused by the release of intra- and extra-cellular materials following freeze-thaw.

When LD pre-treatment was used, there were no significant differences in the SCOD of the meltwater compared to stand-alone freeze-thaw (Figure 8-5). This was also the case with soluble carbohydrates (Figure 8-7); however, LD pre-treatment did result in a $30 \%$ (average) increase in soluble proteins compared to stand-alone freeze-thaw (Figure 8-6).

Following $\mathrm{FT}$, the SCOD of HD pre-treated samples increased by an additional $30 \%$, along with additional increases in the soluble carbohydrates. However, only sludge sample C had additional increases in the soluble protein concentrations when samples were pretreated with HD followed by FT. 


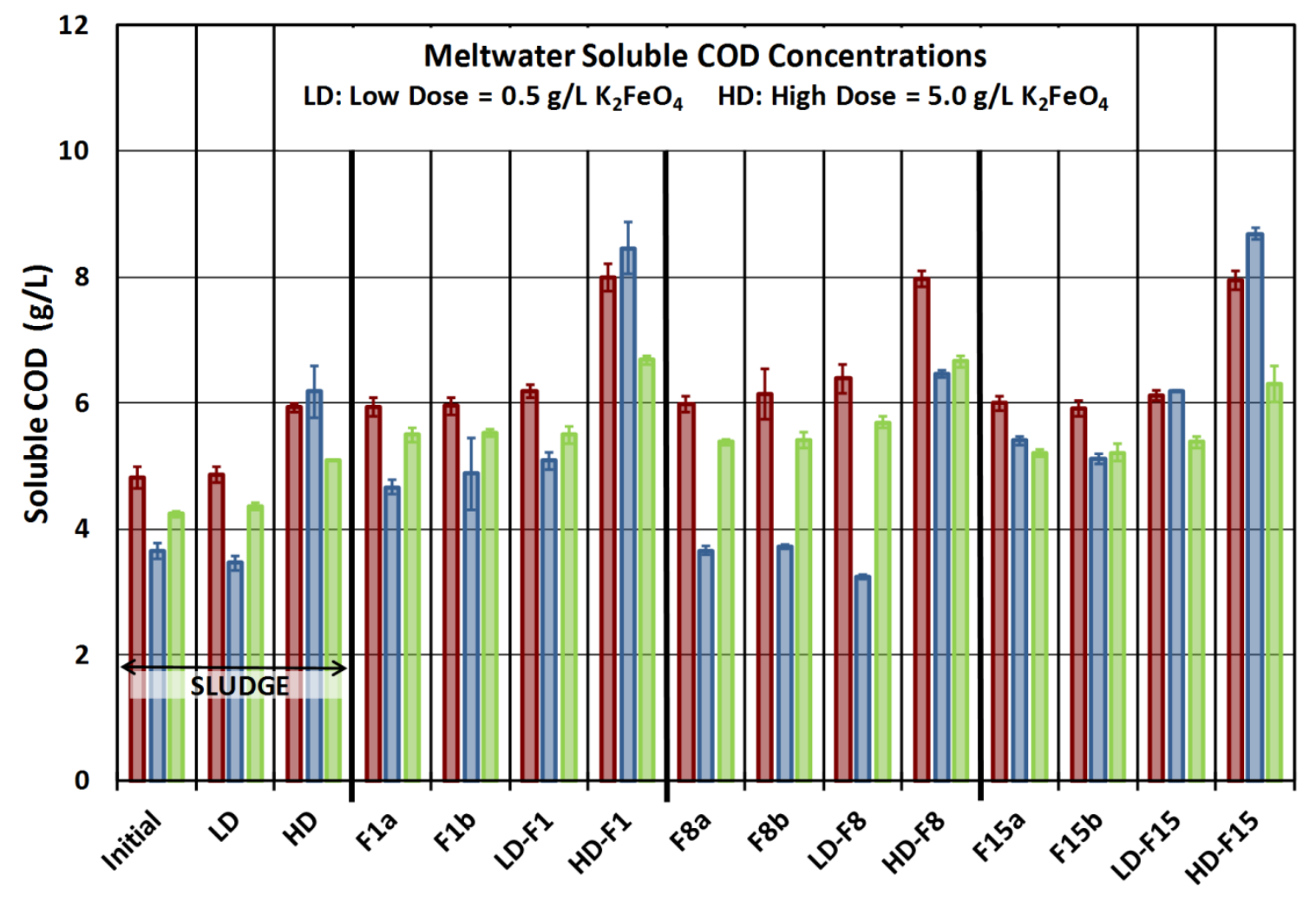

Primary Sludge Sample: $\square$ A $\square$ B $\square$ C

Figure 8-5: Effect of LD and HD pre-treatment with freeze-thaw on the SCOD of the meltwater. 


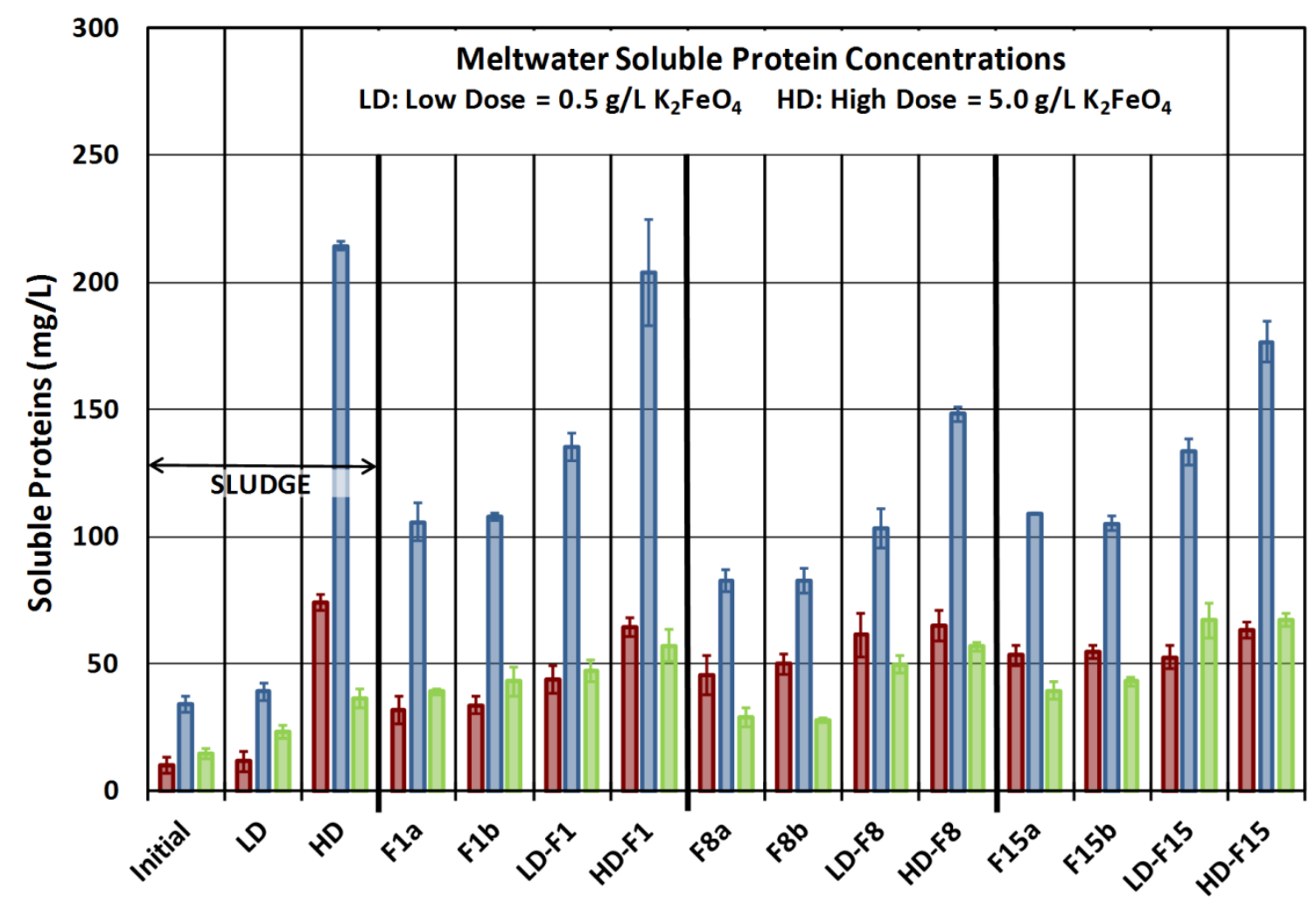

Primary Sludge Sample: $\square$ A $\square$ B $\square$ C

Figure 8-6: Effect of LD and HD pre-treatment with freeze-thaw on the concentration of soluble proteins in the meltwater. 


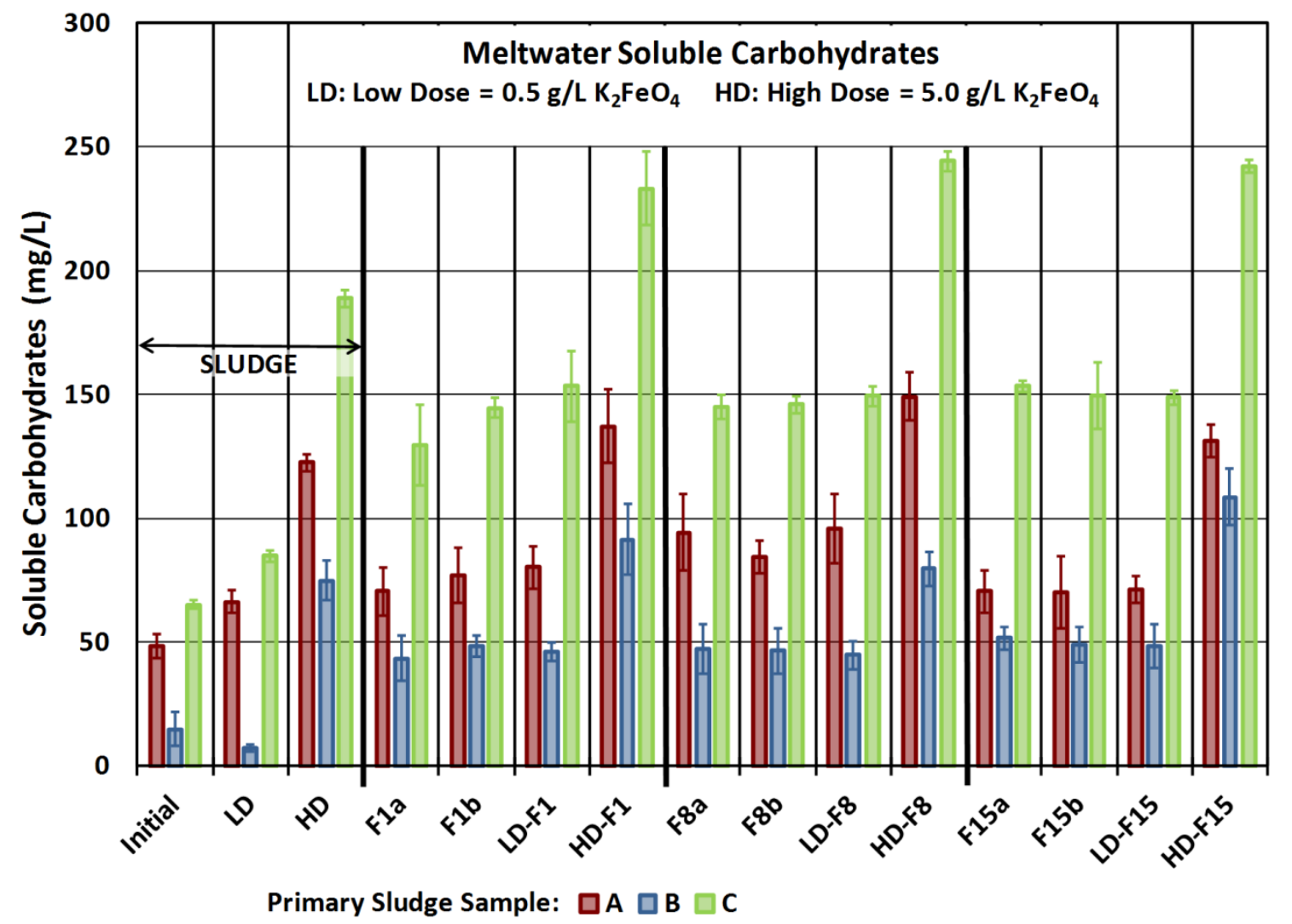

Figure 8-7: Effect of LD and HD pre-treatment with freeze-thaw on the concentration of soluble carbohydrates in the meltwater.

The turbidity of the meltwater collected from each of the sludge samples during the thawing stage is presented in Figure 8-8. The maximum meltwater turbidity was 276 NTU, collected from sludge sample $\mathrm{C}$ after being frozen for 1 day. Meltwater collected from sludge C generally had a higher turbidity than sludge samples A and B. Furthermore, the turbidity of the meltwater from sludge $\mathrm{C}$ decreased when the freezing period was increased. This was also the case for sludge A, when the time frozen was increased from 1 day to 8 days. It is possible that the longer freezing periods provided the additional time required to further dehydrate sludge flocs and remove surface water from the colloids. Parker and Collins (1999) believed that sludge floc dehydration and 
the removal of surface water from the colloids within a sludge floc can bring sludge particles in contact with one another, allowing the formation of new bonds amongst particles. In this study, the coagulated sludge particles following freeze-thaw were granules which allowed meltwater to drain freely out of the sludge.

When HD pre-treatment was used, the meltwater contained significantly lower turbidity than the stand-alone freeze-thaw samples. This is due to the coagulating action of the spent ferrate $(\mathrm{VI})$ ions. When ferrate $(\mathrm{VI})$ oxidises the sludge constituents it is reduced to Fe(III) ions and ferric hydroxide which act as coagulants (Jiang and Lloyd, 2002). Figure 8-9 shows a picture of the meltwater samples collected from sludge A C, after being frozen for 1 day. This picture shows the decreases in turbidity resulting from LD and HD pre-treatments.

When LD pre-treatment was used, the meltwater also contained lower turbidity than the stand-alone freeze-thaw samples, with the exception of sludge $B$, which had a higher initial TS and VS than the other sludge samples (Table 7-1, Figure 8-2) and following freeze-thaw released less meltwater (Table 8-3). It is possible that LD generated fines in sludge B that did not subsequently coagulate, and therefore resulted in a higher concentration of solids in the meltwater, and higher turbidity. 


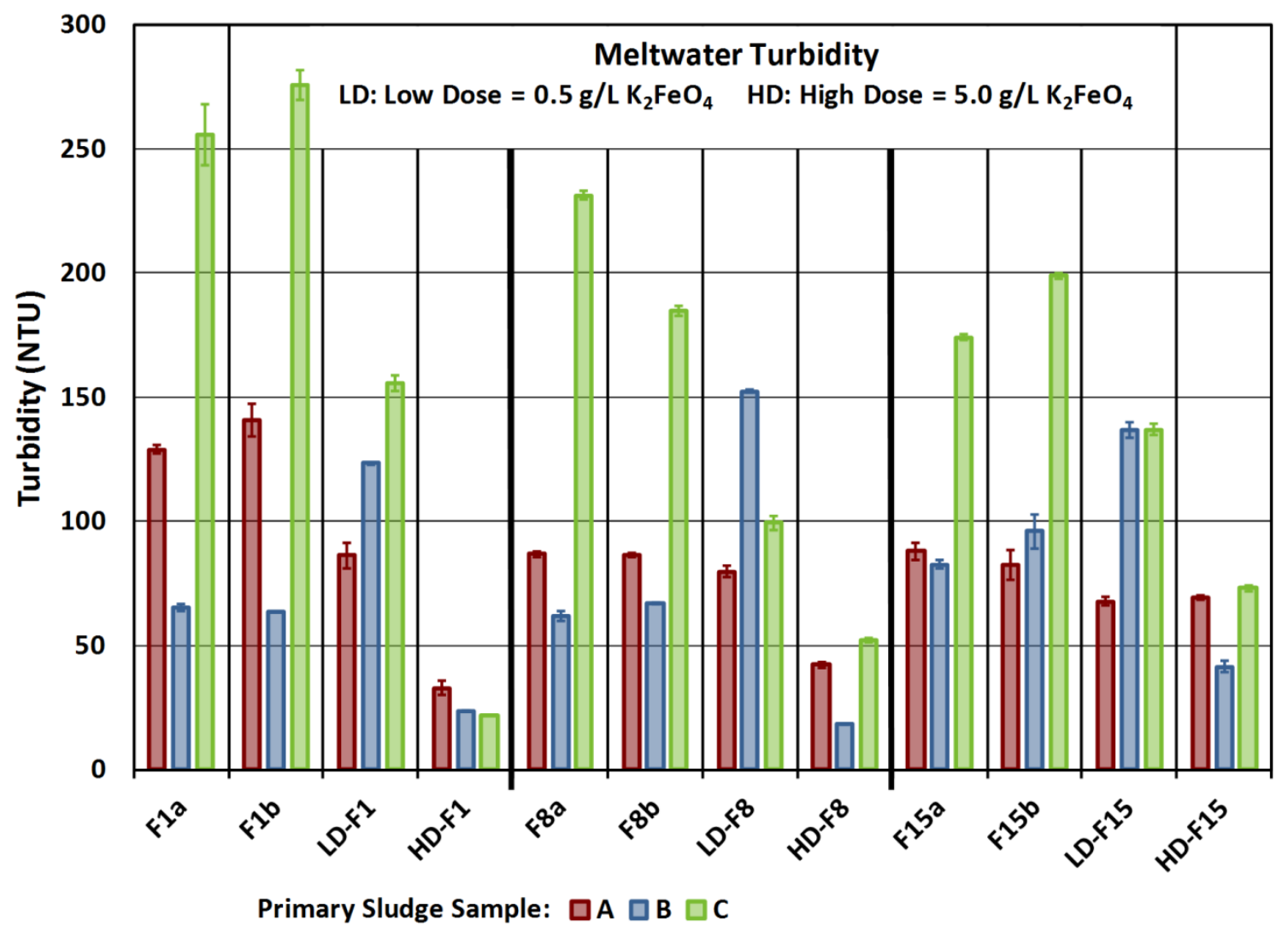

Figure 8-8: Effect of LD and HD pre-treatment with freeze-thaw on the turbidity of the meltwater.

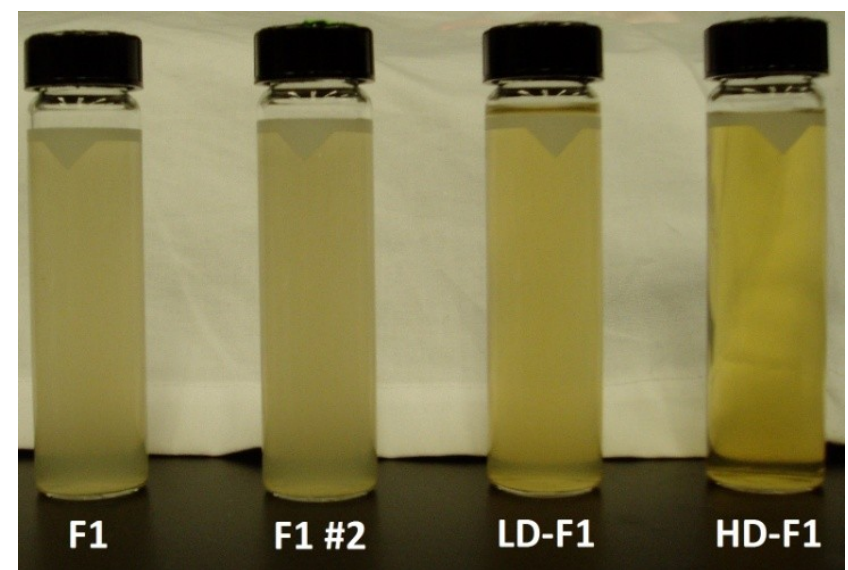

Figure 8-9: Meltwater samples collected from sludge batch A, after being frozen for 1 day.

The $\mathrm{pH}$ of the meltwater samples collected during the thawing stage is presented in

Figure 8-10. The $\mathrm{pH}$ of the meltwater samples following stand-alone freeze-thaw were 
between 5.0 and 5.5, and increasing the duration of the freezing conditions did not have an effect on the $\mathrm{pH}$. However, when potassium ferrate(VI) was used as a pre-treatment, the $\mathrm{pH}$ of the meltwater increased. LD pre-treatment increased the $\mathrm{pH}$ of the meltwater to between 5.5 and 6, and HD pre-treatment increased the $\mathrm{pH}$ to between 7.7 and 8.0.

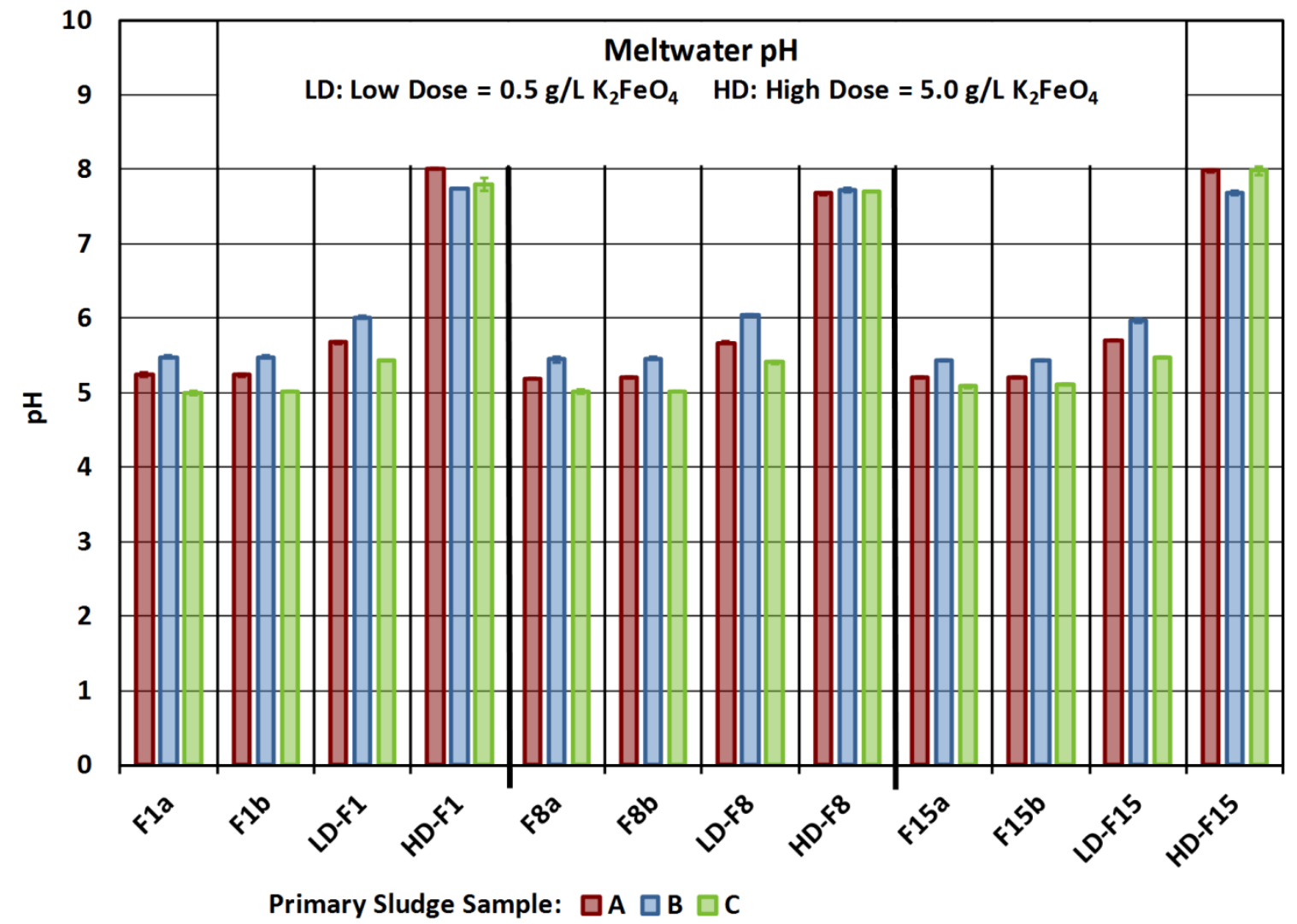

Figure 8-10: Effect of LD and HD pre-treatment with freeze-thaw on the $\mathrm{pH}$ of the meltwater.

\subsection{FOLLOW-UP EXPERIMENTS WITH HIGHER DOSES OF FERRATE(VI)}

A set of follow-up experiments were conducted using higher potassium ferrate(VI) doses of $5.1,8.0,15.0,22.0$ and $24.9 \mathrm{~g} / \mathrm{L}$ on a different sample of primary sludge. These doses were chosen to satisfy the criteria for a 2-factor 2-level fully-rotatable central composite (circumscribed) design, to generate a response surface (not presented). 
The initial TS and VS of the sludge were 4.6 and $3.5 \%$ respectively, and the concentration of fecal coliform was $9.6 \times 10^{6} \mathrm{MPN} / \mathrm{mL}(7.0-\log )$, equivalent to $2.0 \times 10^{8}$ MPN/g TS (8.3-log).

$200 \mathrm{~mL}$ sludge samples were frozen in a freezer set to $-20^{\circ} \mathrm{C}$, and kept frozen for periods of $1,3,8,13$ and 15 days. Following the designated freezing period, the samples were removed from the freezer and thawed at room temperature for 12 hours, in the same simulated drainage bed setup used in the previous set of experiments. However, for this set of experiments, the graduated cylinder used to collect the meltwater was wrapped with aluminum foil to shield the samples from light and keep the samples cool.

Cake samples were characterised in terms of fecal coliform, TS and VS, and the meltwater samples were characterised in terms of fecal coliform. The purpose of this set of experiments was to investigate the potential for regrowth during the thawing period.

\subsubsection{Potassium Ferrate(VI) Only}

Samples that were treated with potassium ferrate(VI) were stirred using a magnetic stirrer for 15 minutes following the addition of the potassium ferrate(VI), and then a portion of the sample was immediately enumerated for fecal coliform. Figure 8-11 shows the resulting concentration of fecal coliform, TS and VS following the 15-minute reaction period with various potassium ferrate(VI) doses. The concentration of fecal coliform is expressed as log (MPN/mL) by the shaded bars, and log (MPN/g DS) by the corresponding outlined bars. Similarly, the TS concentrations are represented by the outlined bars, while the VS concentrations are represented by the shaded bars. 
The initial TS and VS of the sludge were 4.6 and $3.5 \%$ respectively. Similar to the previous experiments using $\mathrm{HD}=5.0 \mathrm{~g} / \mathrm{L}$ of potassium ferrate $(\mathrm{VI}), 5.1 \mathrm{~g} / \mathrm{L}$ of potassium ferrate(VI) increased the TS of the sludge with no significant effect on the VS. Furthermore, a clear trend of increasing TS with increasing potassium ferrate(VI) dose was observed, and the measured increase in TS was within $10 \%$ of the mass of potassium ferrate(VI) added. For example, when $5.1 \mathrm{~g} / \mathrm{L}$ of potassium ferrate(VI) was added to the sludge, the TS increased from $4.6 \%$ solids to $5.1 \%$ solids, representing an increase of $0.5 \%$ solids or $4.7 \mathrm{~g} / \mathrm{L}$, and when $24.9 \mathrm{~g} / \mathrm{L}$ of potassium ferrate $(\mathrm{VI})$ was added to the sludge, the TS increased to $7.1 \%$ solids, representing an increase of $24.5 \mathrm{~g} / \mathrm{L}$. In addition, while $5.1 \mathrm{~g} / \mathrm{L}$ of potassium ferrate(VI) did not have a significant effect on the VS $(p=0.2147)$, higher doses seem to have resulted in a decrease in VS, however only a dose of $24.9 \mathrm{~g} / \mathrm{L}$ resulted in significantly lower VS than the initial concentration $(p=0.0379)$. This suggest that high doses of potassium ferrate(VI) may oxidise a small portion of the organic matter in sludge. At the same dose of $24.9 \mathrm{~g} / \mathrm{L}$ of potassium ferrate(VI), the measured fecal coliform concentration was at the detection limit of the MTF test, with only 1 positive result out of the 5 tubes, for both replicates, in the undiluted primary sludge sample. The resulting concentration was $-0.7-\log$, equivalent to $0.2 \mathrm{MPN} / \mathrm{mL}$ or $0.45 \mathrm{MPN} / \mathrm{g} \mathrm{DS}$, representing approximately $7.7-\log$ inactivation of fecal coliform.

The initial fecal coliform concentration of the primary sludge was $9.6 \times 10^{6} \mathrm{MPN} / \mathrm{mL}$ (7.0-log), equivalent to $2.0 \times 10^{8} \mathrm{MPN} / \mathrm{g}$ DS (8.3-log), which was higher than the previous three batches of primary sludge used in the first set of experiments. However, similar to 
the previous set of experiments, $5.1 \mathrm{~g} / \mathrm{L}$ of potassium ferrate $(\mathrm{VI})$ resulted in a 1.1-log reduction in fecal coliform, following a 15-minute reaction period. Increasing the dose to $8.0 \mathrm{~g} / \mathrm{L}$ resulted in $2.4-\log$ inactivation, and $15.0 \mathrm{~g} / \mathrm{L}$ resulted in approximately $5.2-\log$ inactivation and a final concentration of $1023 \mathrm{MPN} / \mathrm{g} \mathrm{DS}$, which is close to the fecal coliform limit for CP1 or Class A biosolids. $22.0 \mathrm{~g} / \mathrm{L}$ reduced the concentration of fecal coliform to $1.0 \mathrm{MPN} / \mathrm{mL}$ (0- $\mathrm{log})$, equivalent to $15.3 \mathrm{MPN} / \mathrm{g}$ DS $(1.2-\mathrm{log})$, and $24.9 \mathrm{~g} / \mathrm{L}$ reduced the fecal coliform concentration to $0.2 \mathrm{MPN} / \mathrm{mL}$ or $0.45 \mathrm{MPN} / \mathrm{g}$ DS.

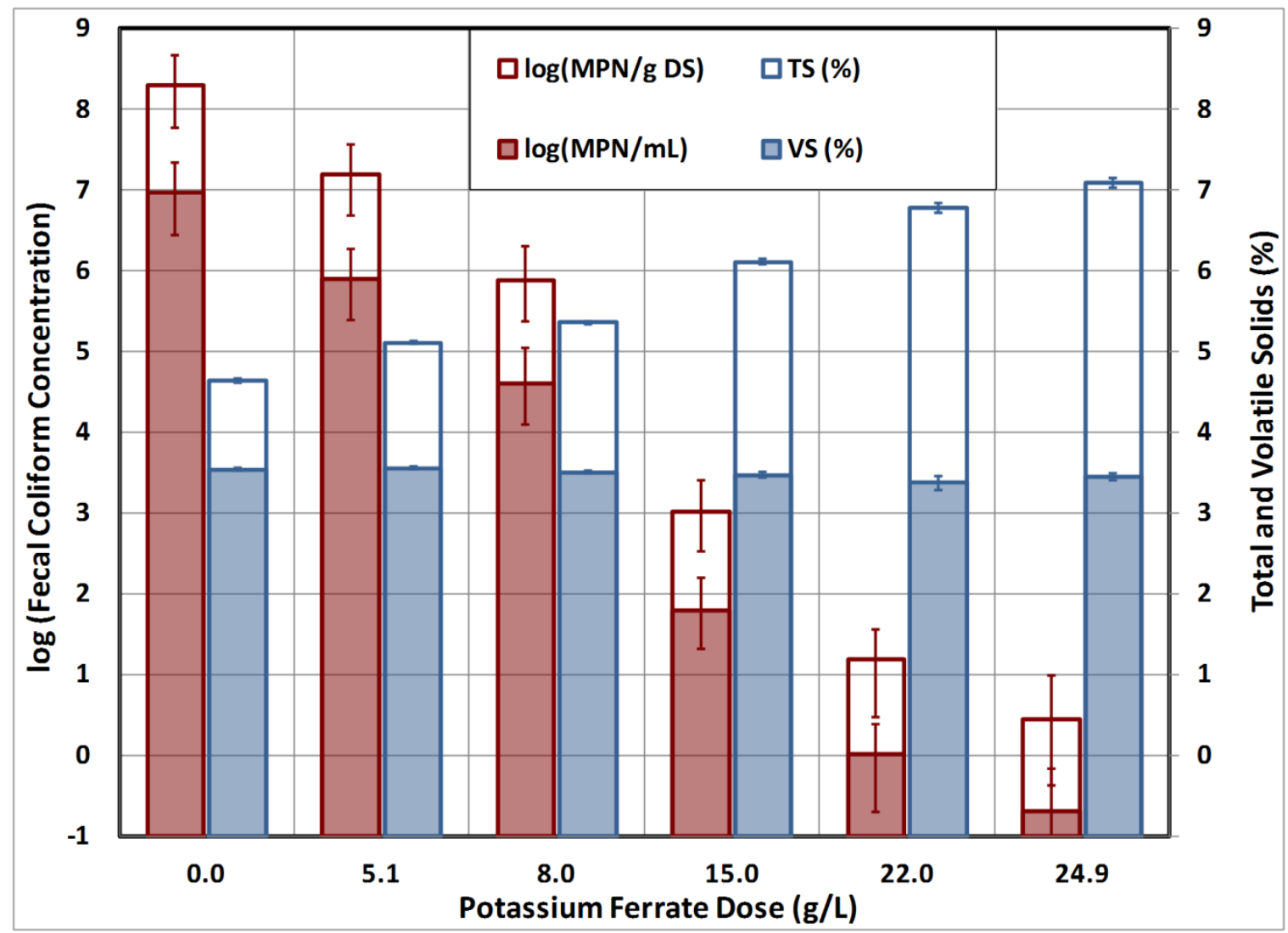

Figure 8-11: Effect of potassium ferrate(VI) dose on the concentration of fecal coliform, TS and VS in the primary sludge. 


\subsubsection{Freeze-thaw with potassium ferrate(VI) pre-treatment}

Pre-treated sludge samples were frozen at a temperature of $-20^{\circ} \mathrm{C}$, and kept frozen for periods of $1,3,8,13$ and 15 days. Following the designated frozen period, the samples were removed from the freezer and thawed at room temperature for 12 hours, using the simulated drainage bed setup described earlier, which allowed meltwater to drain freely from the sludge cake via gravity. For this set of experiments however, the graduated cylinder used to collect the meltwater was wrapped in aluminum foil to shield the collected meltwater from light throughout the 12-hour thawing and meltwater collection period.

Following freeze-thaw treatment, the concentration of fecal coliform was further reduced in both the sludge cake (Figure 8-12) and the meltwater samples (Figure 8-13), with no indication of regrowth during the 12-hour thawing period. In all cases, increasing the potassium ferrate( $\mathrm{VI})$ dose and increasing the duration of time frozen, increased the level of inactivation of fecal coliform.

$5.1 \mathrm{~g} / \mathrm{L}$ of potassium ferrate(VI) resulted in 1.1-log inactivation after 15 minutes, and following freeze-thaw, with 8 days frozen, the concentration of fecal coliform in the sludge cake was reduced by an additional $1.7-\log$, to $10^{5.5} \mathrm{MPN} / \mathrm{g}$ DS (Figure 8-12), which meets the criteria for $\mathrm{CP} 2$, or Class B, biosolids. Meanwhile, the concentration of fecal coliform in the meltwater was reduced to $3.3-\log$, equivalent to approximately $2000 \mathrm{MPN} / \mathrm{mL}$ (Figure 8-13). Both samples that were pre-treated with $5.1 \mathrm{~g} / \mathrm{L}$ of potassium ferrate( $\mathrm{VI})$ resulted in greater fecal coliform inactivation than the stand-alone 
freeze-thaw samples, unlike some of the samples from the first set of experiments that were pre-treated with $5.0 \mathrm{~g} / \mathrm{L}$ of potassium ferrate(VI). This suggests that potential regrowth that was observed in the first set of experiments can be prevented or inhibited by shielding the meltwater collection vessel from light, and keeping the samples cool.

$8.0 \mathrm{~g} / \mathrm{L}$ of potassium ferrate(VI) reduced the concentration of fecal coliform in the sludge by 2.4-log after the 15-minute reaction period. Following freeze-thaw treatment, the concentrations of fecal coliform in the sludge cakes were further reduced to $10^{5.2}$ and $10^{4.5} \mathrm{MPN} / \mathrm{g}$ DS, after being frozen for 3 and 13 days respectively, representing a reduction of 3.1- and 3.8-log respectively (Figure 8-12). Similarly, pre-treatment with 8.0 $\mathrm{g} / \mathrm{L}$ of potassium ferrate(VI) reduced the concentration of fecal coliform in the meltwater to $7.9 \mathrm{MPN} / \mathrm{mL}$ for the sample that was frozen for 3 days, and $1.8 \mathrm{MPN} / \mathrm{mL}$ in the sample that was frozen for 13 days (Figure 8-13). This represents approximately 6.1 and 6.8-log inactivation for the 3-day and 13-day samples respectively, with no signs of regrowth during the 12 -hour thawing period. As mentioned earlier, $15.0 \mathrm{~g} / \mathrm{L}$ of potassium ferrate(VI) resulted in approximately 5.2-log inactivation after the 15-minute reaction period, and a concentration of $62.5 \mathrm{MPN} / \mathrm{mL}$, equivalent to $1023 \mathrm{MPN} / \mathrm{g}$ DS. However, due to limitations on the amount of potassium ferrate(VI) available, no samples were frozen following pre-treatment with $15.0 \mathrm{~g} / \mathrm{L}$ of potassium ferrate(VI).

Following pre-treatment with $22.0 \mathrm{~g} / \mathrm{L}$, the concentration of fecal coliform in the sludge was reduced to $1.0 \mathrm{MPN} / \mathrm{mL}$ (15.3 MPN/g DS) after the 15 -minute reaction period, representing approximately 7-log inactivation. Following freeze-thaw, the concentration 
was reduced to below the detection limit in both the sludge cake $(<12.0 \mathrm{MPN} / \mathrm{g}$ DS or $<1.8 \mathrm{MPN} / \mathrm{mL})$ and the meltwater samples $(<0.18 \mathrm{MPN} / \mathrm{mL})$. Similarly, $24.9 \mathrm{~g} / \mathrm{L}$ of potassium ferrate $(\mathrm{VI})$ reduced the concentration of fecal coliform to $0.2 \mathrm{MPN} / \mathrm{mL}$, equivalent to $0.45 \mathrm{MPN} / \mathrm{g}$ DS, which was the detection limit for the undiluted sludge sample. This represents approximately 7.7-log inactivation of fecal coliform in primary sludge. Following freeze-thaw treatment, no fecal coliform were detected in the sludge cake (Figure 8-12) or the meltwater (Figure 8-13) following the 12-hour thawing period, which shows that no regrowth of fecal coliform occurred.

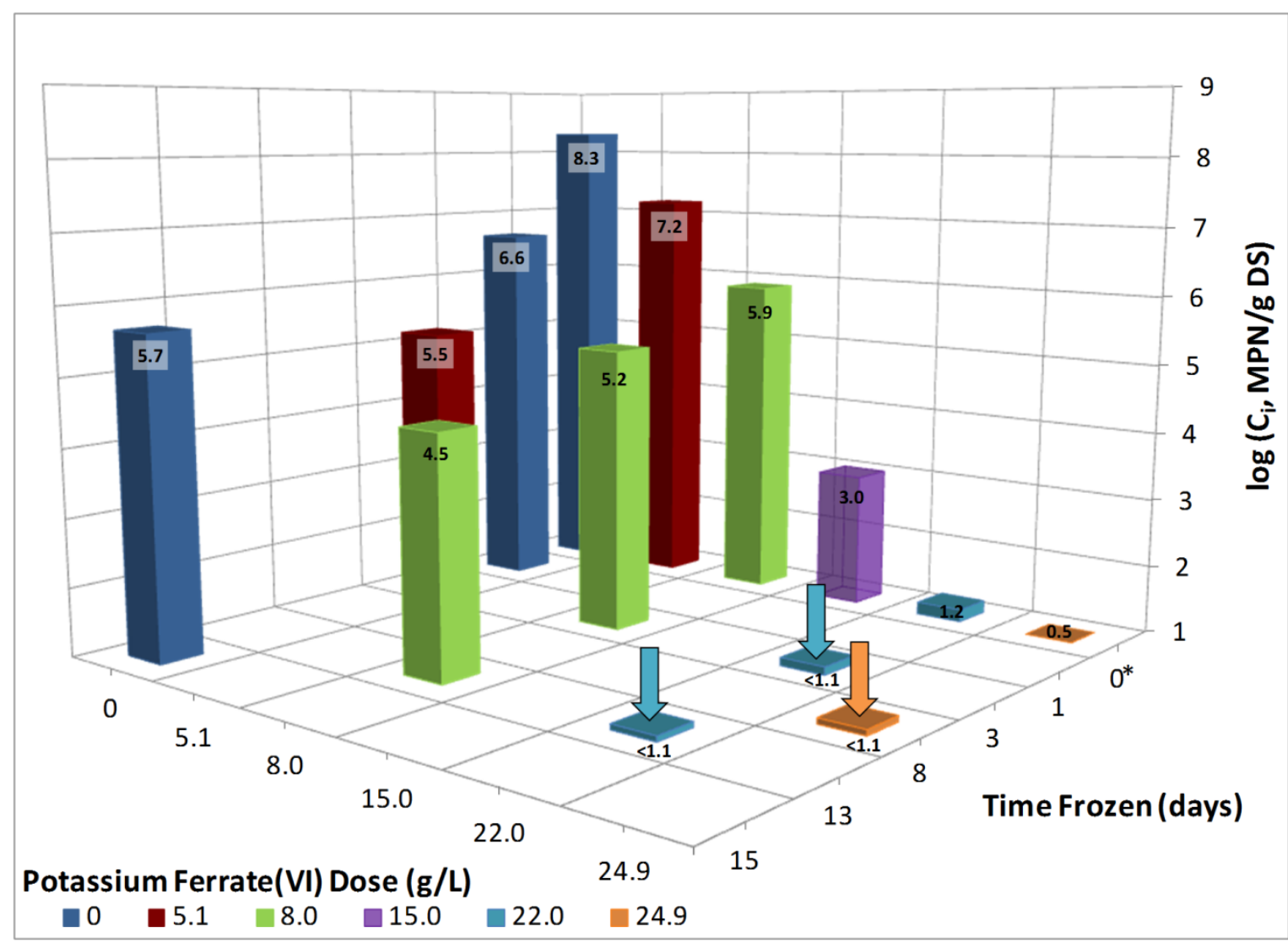

Figure 8-12: Concentration of fecal coliform in the sludge cake remaining following pretreatment with potassium ferrate(VI), and freeze-thaw using a freezer temperature of $-20^{\circ} \mathrm{C}$, and a 12 hour thaw period at room temperature, in a simulated drainage bed setup $\left(0^{*}\right.$ represents samples following a 15 -minute reaction period with the potassium ferrate(VI), without any freeze-thaw or cake-meltwater separation, and the downward arrows are to indicate that the measured concentration was at the lower method detection limit). 


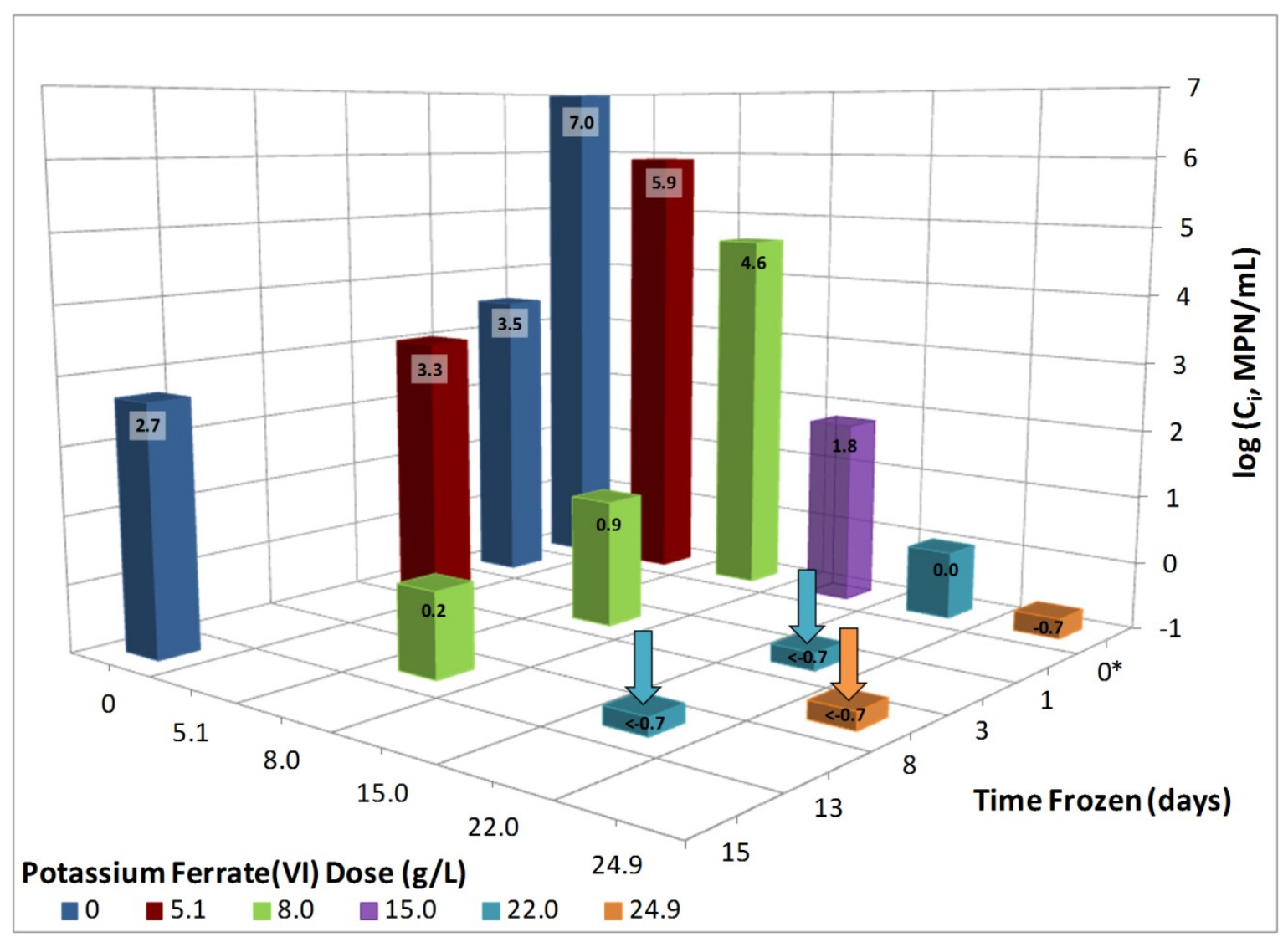

Figure 8-13: Concentration of fecal coliform in the meltwater samples collected following pretreatment with potassium ferrate $(\mathrm{VI})$, and freeze-thaw using a freezer temperature of $-20^{\circ} \mathrm{C}$, and a 12 hour thaw period at room temperature, in a simulated drainage bed setup $\left(0^{*}\right.$ represents samples following a 15-minute reaction period with the potassium ferrate(VI), without any freeze-thaw or cake-meltwater separation, and the downward arrows are to indicate that the measured concentration was at the lower method detection limit).

\subsubsection{Freeze-Thaw Only}

Increasing the freezing time from 1 to 15 days did not have an effect on the drainability of the sludge. The two $200 \mathrm{~mL}$ samples that were frozen for 1 day generated 133 and $135 \mathrm{~mL}$ of meltwater, and the two samples that were frozen for 15 days generated 136 and $134 \mathrm{~mL}$ of meltwater. Similarly, the resulting solids concentrations of the sludge cakes were between 13 and 14\% TS, and 10 to 11\% VS (Figure 8-14), and did not differ significantly $\left(p_{T S}=0.1475, p_{V S}=0.1422\right)$. However, increasing the time frozen from 1 to 15 
days did result in an increase in fecal coliform inactivation in both the sludge cake and meltwater samples ( $\left.p_{\text {Cake }}=0.0075, p_{\text {Meltwater }}=0.0001\right)$. By increasing the time frozen from 1 to 15 days, the concentration of fecal coliform in the sludge cake was reduced from $10^{6.6} \mathrm{MPN} / \mathrm{g}$ DS to $10^{5.7} \mathrm{MPN} / \mathrm{g} \mathrm{DS}$, representing an additional 0.9-log inactivation. Similarly, the concentration of fecal coliform in the meltwater was reduced from $10^{3.5}$ $\mathrm{MPN} / \mathrm{mL}$ to $10^{2.7} \mathrm{MPN} / \mathrm{mL}$, representing an additional 0.8 -log inactivation. However, similar to the observations from the first set of experiments, the majority of the fecal coliform inactivation occurred during the freeze-thaw process.

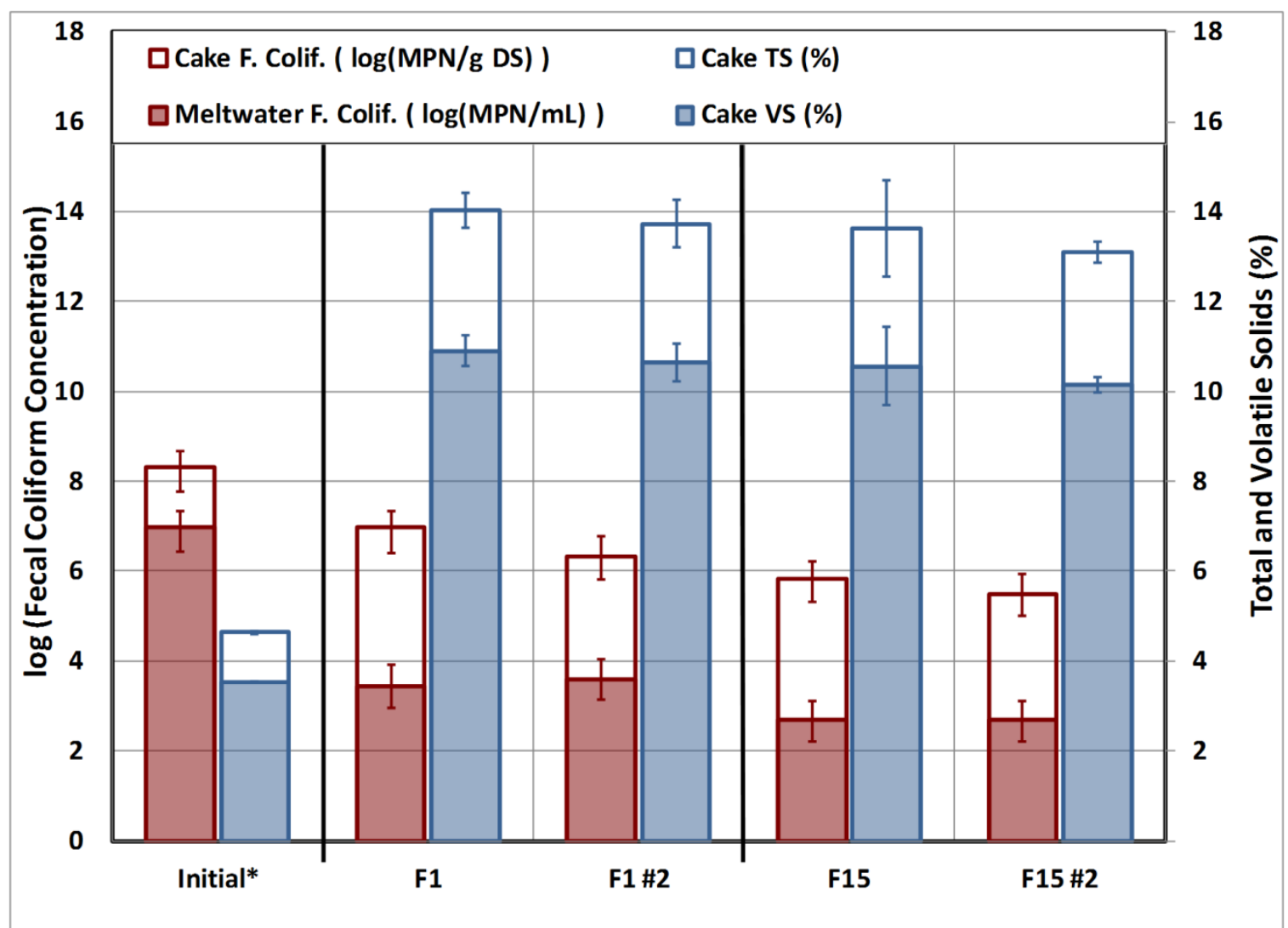

Figure 8-14: Effect of stand-alone freeze-thaw treatment on the concentration of TS, VS and fecal coliform in the sludge cake, and the concentration of fecal coliform in the meltwater (Initial* represents the raw primary sludge without any meltwater-cake separation, the units are quoted on a wet and dry basis). 


\subsection{CONCLUSION}

Following a 15 -minute reaction period, LD $(0.5 \mathrm{~g} / \mathrm{L}$ potassium ferrate $(\mathrm{VI}))$ did not have a significant effect on the concentration of fecal coliform in sludge, and HD (5.0 g/L) only resulted in $0.5,1.0$ and 0.6 -log inactivation of fecal coliform in sludge samples $A\left(T_{\text {Freeze }}=\right.$ $\left.-10^{\circ} \mathrm{C}\right), \mathrm{B}\left(\mathrm{T}_{\text {Freeze }}=-20^{\circ} \mathrm{C}\right)$ and $\mathrm{C}\left(\mathrm{T}_{\text {Freeze }}=-30^{\circ} \mathrm{C}\right)$ respectively. This suggests that potassium ferrate $(\mathrm{VI})$ doses of $5 \mathrm{~g} / \mathrm{L}$ were insufficient for the stabilisation of raw primary sludge. However, $5.0 \mathrm{~g} / \mathrm{L}$ of potassium ferrate(VI) did result in sludge solubilisation. The sCOD increased by 23, 69 and 20\%, soluble proteins increased by 640,520 and 140\%, and soluble carbohydrates increased by 150,400 and $190 \%$ in sludge samples A, B and C respectively. This may have created an affluent environment for the surviving bacteria, which may have stimulated regrowth during the 12-hour thawing period. As well, the increase in soluble components may have acted as cryoprotectants, shielding bacteria during freezing. In 3 of the samples, HD pre-treatment resulted in higher cake fecal coliform concentrations than the stand-alone freeze-thaw treatments (For the other 6 samples, there was either no difference, or HD resulted in a lower fecal coliform concentration). Similarly, meltwater collected from the samples that were pre-treated with HD consistently had a higher fecal coliform concentration than the stand-alone freeze-thaw samples. Although, meltwater from a full-scale freeze-thaw bed could be treated easily in any municipal or other on-site (decentralised) wastewater treatment system, a series of follow-up experiments were conducted using potassium ferrate(VI) doses of $5.1,8.0,15.0,22.0$ and $24.9 \mathrm{~g} / \mathrm{L}$, to evaluate the potential for regrowth during the 12-hour thawing potential. 
Follow-up experiments using $5.1 \mathrm{~g} / \mathrm{L}$ of potassium ferrate(VI) resulted in greater fecal coliform inactivation than the stand-alone freeze-thaw treatments, suggesting that potassium ferrate $(\mathrm{VI})$ did not contribute to any regrowth during the 12-hour thawing period when the meltwater collection vessel was shielded from light. Similarly, pretreatment with $8.0 \mathrm{~g} / \mathrm{L}$ of potassium ferrate(VI) improved fecal coliform inactivation caused by stand-alone freeze-thaw by $>1-\log$ in the sludge cake and $>2.5-\log$ in the meltwater, while pre-treatment with $22.0 \mathrm{~g} / \mathrm{L}$ reduced the concentration of fecal coliform to $<12.0 \mathrm{MPN} / \mathrm{g}$ DS in the sludge cake, and $<0.18 \mathrm{MPN} / \mathrm{mL}$ in the meltwater. This demonstrated that pre-treatment with potassium ferrate(VI), prior to freeze-thaw, can be used to improve fecal coliform inactivation caused by freeze-thaw, and can also be used to reduce the concentration of fecal coliform in primary sludge to below the detection limit of the MTF test (U.S. EPA, 2005a). Therefore, co-treatment of sewage sludge using freeze-thaw with potassium ferrate(VI) pre-treatment can be tailored to the design requirements of a particular application. The process can be used to compliment an existing sewage or sludge treatment process, or sludge pre-treatment using potassium ferrate( $\mathrm{VI})$ followed by freeze-thaw conditioning can be used as a complete process to dewater sludge and reduce the fecal coliform concentrations in the sludge cake and meltwater to below detectable levels.

Freeze-thaw with gravity drainage reduced the volume of primary sludge by up to $79 \%$ when the initial TS was around 3\% solids; however, when the initial TS of the sludge was $4.2 \%$ solids, the sludge volume was reduced by only 46 to $56 \%$. This suggests that freeze-thaw with gravity meltwater drainage is more effective at reducing the volume of 
sludges with lower solids concentration, which may be due to facilitated particle migration during freezing, and the generation of continuous networks of ice channels throughout the sludge, which drain when thawed. However, in the follow-up experiments using primary sludge with an initial TS of $4.6 \%$, the volume of sludge following freeze-thaw, with 12 hours of gravity drainage during thawing, was reduced by approximately $68 \%$. This suggests that other characteristics of the sludge, in addition to the solids concentration, contribute to the sludge drainability following freeze-thaw treatment.

Following stand-alone freeze-thaw, with 1-day frozen, many of the dewatered sludge cakes had fecal coliform concentrations <1000 MPN/g dry solids, representing >3-log reduction in some cases. Furthermore, meltwater collected from the simulated freezethaw beds had a low turbidity and could be easily pumped to the headworks of a passive biological wastewater treatment system such as a lagoon or septic tank and soil adsorption system.

\subsection{REFERENCES}

Abad, E., Martínez, K., Planas, C., Palacios, O., Caixach, J., Rivera, J. (2005) Priority organic pollutant assessment of sludges for agricultural purposes. Chemosphere 61(9): $1358-1369$

APHA (American Public Health Association), American Water Works Association, Water Pollution Control Federation (2005) Standard Methods for the Examination of Water and Wastewater. APHA/AWWA, Washington DC. 
Bradford, M.M. (1976) A rapid and sensitive method for the quantification of microgram quantities of protein utilizing the principle of protein-dye binding. Analytical Biochemistry 72(1-2):248-254

Canadian Council of Ministers of the Environment (CCME) (2010) A review of current Canadian legislative framework for wastewater biosolids. PN 1446 ISBN 978-1-89699795-7 PDF

Chalmers, B. (1959) How water freezes. Sci Am 200, 114-122

Chen, Y.-C., Higgins, M.J., Beightol, S.M., Murthy, S.N., Toffey, W.E. (2011) Anaerobically digested biosolids odor generation and pathogen indicator regrowth after dewatering. Water Res 45(8):2616-2626

Chu, C.P., Feng, W.C., Chang, B.-V., Chou, C.H., Lee, D.J. (1999) Reduction of microbial density level in wastewater activated sludge via freezing and thawing. Water Res 33(16):3532-3535

de Luca, S., Idle, C.N., Chao, A.C. (1996) Quality improvement of biosolids by ferrate(VI) oxidation of offensive odour compounds. Water Sci Technol 33(3):119-130

Diak, J., Örmeci, B., Proux, C., (2011) Freeze-thaw treatment of RBC sludge from a remote mining exploration facility in subarctic Canada. Water Sci Technol 63(6):13091313 
Gao, W., Leung, K., and Hawdon, N. (2009) Freezing inactivation of Escherichia coli and Enterococcus faecalis in water: Response to different strains. Water Environ Res 81(8):824-830

Gao, W., Smith, D.W., Li, Y. (2006) Natural freezing as a wastewater treatment method: E. Coli inactivation capacity. Water Res 40(12), 2321-2326

Gardner, J., Örmeci, B. (2010) Effect of aging, time, and temperature on fecal coliform counts during centrifugal dewatering and role of centrate in growth inhibition. Water Environ Res 82(2):51-61

Halde, R. (1980) Concentration of impurities by progressive freezing. Water Res 14:575580

Harrison, E.Z., Oakes, S.R., Hysell, M., Hay, A. (2006) Organic chemicals in sewage sludges. Science Total Environ 367:481-497

Higgins, M.J., Chen, Y., Murthy, S.N., Maas, N.A., Hendrickson, D. (2007) Reactivation and regrowth of non-culturable indicator bacteria in anaerobically digested biosolids after centrifuge dewatering. Water Res 41(3):665-673

Hong, S.G., Young, Y.D., Chen, G.W., Chang, I.L., Hung, W.T., Lee, D.J. (1995) Freeze/thaw treatment on waste activated sludge: an FTIR spectroscopic study. J Environ Sci Health A30(8):1717-1724 
Hung, W.T., Feng, W.H., Tsai, I.H., Lee, D.J., Hong, S.G. (1997) Uni-directional freezing of waste activated sludges: vertical freezing versus radial freezing. Water Res 31(9):22192228

Hung, W.T., Chang, I.L., Lee, D.J., Hong, S.G. (1996) Sludge chemical composition changes under uni-directional freezing. Water Sci Technol 34(3-4):525-531

Jiang, J.Q., Wang, S., Panagoulopoulos, A. (2007) The role of potassium ferrate(VI) in the inactivation of Escherichia coli and in the reduction of COD for water remediation. Desalination 210(1-3):266-273

Jiang, J.Q. (2007) Research progress in the use of ferrate(VI) for the environmental remediation. J Haz Mat 146(3):617-623

Jiang, J.Q., Lloyd, B. (2002) Progress in the development and use of ferrate (vi) salt as an oxidant and coagulant for water and wastewater treatment. Water Res 36:1397-1408 Lee, D.J., Hsu, Y.H. (1994) Fast freeze/thaw treatment on excess activated sludges: Floc structure and sludge dewaterability. Environ Sci Technol 28(8):1444-1449

Li, C., Li, X.Z., Graham, N., Gao, N.Y. (2008) The aqueous degradation of bisphenol A and steroid estrogens by ferrate. Water Res 42(1-2):109-120

Martel, C.J. (1993) Fundamentals of sludge dewatering in freezing beds. Water Sci Technol 28(1):29-35 
Martel, C.J., Diener, C.J. (1991a) A pilot-scale study of alum sludge dewatering in a freezing bed. J Amer Water Works Assn, 83(12):51-55

Martel, C.J., Diener, C.J. (1991b) Pilot-scale studies of sludge dewatering in a freezing bed. Can J Civ Eng 18(4):681-689

Mazur, P. (1986). Causes of injury in frozen and thawed cells. Fedn. Proc. Fedn Am. Socs. Exp. Biol. 24(15):S175-S182

Montusiewicz, A., Lebiocka, M., Rozej, A., Zacharska, E., Pawlowski, L. (2010)

Freezing/thawing effects on anaerobic digestion of mixed sewage sludge. Bioresource

Technol. 101(10):3466-3473

Morris, D.L. (1948) Quantitative determination of carbohydrates with Dreywood's anthrone reagent. Science 107(2775):254-255

Murmann, R.K., Robinson, P.R. (1974) Experiments utilising $\mathrm{FeO}_{4}{ }^{2-}$ for purifying water. Water Res 8(8):543-547

Örmeci, B., Vesilind, P.A. (2001) Effect of dissolved organic material and cations on freeze-thaw conditioning of activated and alum sludges. Water Res 35(18):4299-4306 Parker, P.J. and Collins, G. (1999) Dehydration of flocs by freezing. Environ Sci Technol 33(3):482-488

Reed, S., Bouzoun, J., Medding, W. (1986) A rational method for sludge de-watering via freezing. J WPCF 58(9):911-916 
Sanin, F.D., Vesilind, P.A., Martel, C.J. (1994) Pathogen reduction capabilities of freeze/thaw sludge conditioning. Water Res. 28(11), 2393-2398

Schuck, C.A., de Luca, S.J., Peralba, M., de Luca, M.A. (2006) Sodium ferrate(IV) and sodium hypochlorite in disinfection of biologically treated effluents. Ammonium nitrogen protection against THMs and HAAs. J Environ Sci Health A41(10):2329-2343

Silvares, O.M., Cravalho, E.G., Toscano, W.M., Higgins, C.E. (1975) The thermodynamics of water transport from biological cells during freezing. J Heat Transf 97:582-590 Sylvester, P., Rutherford, L.A., Gonzalez-Martin, A., Kim, J., Rapko, B.M, Lumetta, G.J. (2001) Ferrate treatment for removing chromium from high-level radioactive tank waste. Environ Sci Technol 35, 216-221

U.S. EPA (United States Environmental Protection Agency) (2005). Method 1681: Fecal Coliforms in Sewage Sludge (Biosolids) by Multiple Tube Fermentation using A-1 Medium. Washington DC, USA.

U.S. EPA (2003) Environmental Regulations and Technology - Control of Pathogens and Vector Attraction in Sewage Sludge (Including Domestic Septage). Under 40 CFR Part 503, EPA/625/R-92/013, Revised July 2003

Wang, Q., Fujisaki, K., Ohsumi, Y., Ogawa, H.I. (2001) Enhancement of dewaterability of thickened waste activated sludge by freezing and thawing treatment. J Environ Sci Health A36(7):1361-1371 
Zhu, J.-H., Yan, X.-L., Liu, Y., Zhang, B. (2006) Improving alachlor biodegradability by ferrate oxidation. J Haz Mat 135(1-3):94-99 


\section{OXIDATION OF SELECTED HORMONES IN ANAEROBICALLY DIGESTED SLUDGE}

James Diak and Banu Örmeci

Department of Civil and Environmental Engineering, Carleton University, 1125 Colonel By Drive, Ottawa, ON K1S 5B6, Canada.

\subsection{ABSTRACT}

The purpose of the study was to evaluate the ability of ferrate(VI) to oxidise selected hormones in sludge. Two $100 \mathrm{~mL}$ anaerobically digested sludge samples were spiked with ten different hormones: estrone (E1), 17 $\alpha$-estradiol, 17ß-estradiol (E2), estriol (E3), 17a-ethinylestradiol (EE2), equilin, mestranol, testosterone, norethindrone and norgestrel The first sludge sample was spiked with a low concentration range of hormones (3-75 ng/mL), and the second sludge sample was spiked with a high concentration range $(12-300 \mathrm{ng} / \mathrm{L})$. Sub-samples from each of these were then treated with either 0.5 or $1.0 \mathrm{~g} / \mathrm{L}$ of potassium ferrate(VI).

The study demonstrated that potassium ferrate(VI) additions as low as $1.0 \mathrm{~g} / \mathrm{L}$ can reduce the concentration of hormones, particularly estrogens, in wastewater sludge. A $1.0 \mathrm{~g} / \mathrm{L}$ dose of potassium ferrate( $\mathrm{VI})$ reduced the concentration E1 by 15 and 23\%, E3 
by 36 and $42 \%$, and EE2 by 11 and $13 \%$ in the low concentration and high concentration sludge samples, respectively. E2 was spiked in the low and high concentration sludge samples at a concentration of 15 and $60 \mathrm{ng} / \mathrm{mL}$, respectively; however, the measured concentrations were all below the sample specific detection limit (SDL). While some of the E2 may have been lost due to adsorption onto the walls of the sample vessels, it is possible that some of the E2 may have degraded to E1 in the presence of the anaerobically digested sludge, since the measured concentrations of E1 were higher than the spiked concentrations. Similarly, the equine estrogen, equilin, may have biotransformed into equilenin once added to sludge. However, when treated with 1.0 $\mathrm{g} / \mathrm{L}$ of potassium ferrate( $\mathrm{VI})$, the concentration of equilin remaining was reduced by 20 and $23 \%$ in the low concentration and high concentration sludge samples respectively.

Potassium ferrate(VI) additions of 0.5 and $1.0 \mathrm{~g} / \mathrm{L}$ were less effective at reducing the concentrations of measured androgens and progestogens, however most of these hormones were measured at concentrations which were near or below the SDL for the specific target compound.

KEYWORDS: Ferrate(VI), Chemical Oxidation, Hormones, Endocrine Disrupting Compounds (EDCs), Sludge 


\subsection{INTRODUCTION}

The ferrate $(\mathrm{VI})$ ion, $\mathrm{FeO}_{4}{ }^{-2}$, is a very strong oxidant (oxidation reduction potential, $\mathrm{E}^{0}=2.2$ $\mathrm{V}$ in acidic conditions, $0.72 \mathrm{~V}$ in alkaline), in which iron exist at a +6 oxidation state (Mácová et al., 2009, Li et al., 2005). It is an attractive chemical oxidant for environmental remediation since the spent ferrate(VI) is reduced to environmentally benign Fe(III) species (Yang et al., 2012a, Jiang, 2007).

Early experiments using ferrate(VI) for water treatment were conducted by Murmann and Robinson (1974). They added sodium ferrate $\left(\mathrm{N}_{2} \mathrm{FeO}_{4}\right)$ to river water and well water samples, and saw a reduction in bacteria, odours and metal ions, and an improvement in the rate of sedimentation. Ferrate(V) is capable of oxidising odorous compounds, pathogens and indicator organisms (Ding et al., 2012, He et al., 2009, Schuck et al., 2006, Read et al., 2003, Schink and Waite, 1980), and furthermore, once the ferrate ion has been reduced, ferric hydroxide is generated which acts as a coagulant (Gombos et al., 2013). Several studies have investigated ferrate(VI) treatment for the combined disinfection and coagulation of water and wastewater (Gombos et al., 2013, Jiang et al., 2006, Li et al., 2005, Bartzatt et al., 1992).

Despite the range of applications, ferrate $(\mathrm{VI})$ has not been widely adopted. This is mainly because solutions of the ferrate(VI) ion are very unstable, decomposing rapidly at room temperature, and the production of ferrate(VI) salts, such as sodium ferrate and potassium ferrate, is quite costly (Alsheyab et al., 2009, Jiang et al., 2009). Stable ferrate(VI) salts are expensive to produce because several chemicals and multiple 
chemical processes are required, and the synthesis and processing time is long (Jiang et al., 2009). However, recent developments in the on-line generation of ferrate has brought down the cost substantially and increased its applicability. There has also been renewed interest in ferrate( $\mathrm{VI})$ for water, wastewater and sludge treatment due to increasing concerns surrounding endocrine disrupting compounds (EDCs) such as pharmaceuticals and personal care products (PPCPs) and other emerging organic compounds (EOCs), in potable water intakes, treated biosolids and effluents from wastewater treatment plants (WWTPs) (Yang et al., 2012b, Xu et al., 2009).

The fate of EDCs during wastewater treatment (WWT) has been studied in recent years due to the increasing concerns over their effect on the natural environment (Huang et al., 2014, Hamid and Eskicioglu, 2012, Janex-Habibi et al., 2009). During WWT operations, EDCs are partitioned between the aqueous phase (water) and the suspended phase (sludge) depending primarily on whether the particular compound is hydrophilic or hydrophobic. The partitioning of EDCs means that certain EDCs will undergo a different series of treatment operations. Conventional physical and biological WWT operations are capable of reducing $>90 \%$ of influent EDCs, however there is considerable variability in the performance of similar WWTPs (Hamid and Eskicioglu, 2012, Janex-Habibi et al., 2009, Auriol et al., 2006), and even with a high percent removal, the total mass of EDCs released to the environment from many WWTPs can be very high (Huang et al., 2014). The average mass of total EDCs from 6 WWTPs in the study by Huang et al. (2014) was $660 \mathrm{~g} /$ day in the effluent and over $490 \mathrm{~g} /$ day in the excess sludge. Many EDCs, including hormones and alkylphenolic compounds, are 
partially degraded during activated sludge and anaerobic digestion processes, whereas primary sedimentation has little effect (Huang et al., 2014). The destruction of EDCs during conventional WWT is primarily due to biodegradation, however the complex mechanisms involved result in a series of biotransformations, where the concentrations of certain EDCs may actually increase (Liu et al., 2010, Janex-Habibi et al., 2009). For example, several studies have demonstrated that biodegradation of $17 \beta$-estradiol (E2) under both aerobic and anaerobic conditions generates estrone (E1), and estriol (E3) is a metabolite of E1 and E2 (Liu et al., 2009a, Hamid and Eskicioglu, 2012, Auriol et al., 2006). In the study by Janex-Habibi et al. (2009), the removal of E2 during anaerobic digestion varied from 0 to $80 \%$ in the 7 WWTPs employing anaerobic digestion, while the concentration of E1 increased by $130 \%$ to $204 \%$. Similarly, degradation of longchained alkylphenol polyethoxylates generates octylphenol and nonylphenol, with the 1 and 2 ethoxylates as intermediates. In the same study, anaerobic digestion was found to increase the nonylphenol concentration by 100 to $463 \%$. Furthermore, nonylphenol is considered one of the most toxic alkylphenols, capable of causing the partial or even complete feminisation of male fish (Sumpter, 2007). This suggests that partial degradation of alkylphenols can have an adverse effect on the overall estrogenicity of the treated biosolids.

Layton et al. (2000) studied the mineralisation of ${ }^{14} \mathrm{C}$-labelled hormones using biosolids from 4 municipal WWTPs and an industrial system, and demonstrated that the adapted microbial populations present in the municipal biosolids were largely responsible for the biodegradation of estrogens. Yoshimoto et al. (2004) later isolated and identified 
Rhodococcus zopfii (strain Y 50158) from activated sludge which completely and rapidly degraded $100 \mathrm{mg} / \mathrm{L}$ of E1, E2, E3 and 17 $\alpha$-ethinylestradiol (EE2) in 24 hours. Three other Rhodococcus equi (strains Y 50155, Y 50156 and Y 50157) were also isolated, which showed comparable levels of biodegradation activity. A similar study by Liu et al. (2009b) isolated Acinetobacter sp. (strain XA05) and Sphingomonas sp (strain FG03) from waste activated sludge, and achieved greater than $95 \%$ reduction in phenol after 35 hours, when the initial phenol concentration was $800 \mathrm{mg} / \mathrm{L}$. These studies suggest that the removal of hormones in conventional activated sludge systems can be improved by maintaining healthy populations of specific microorganisms in the aeration basin, and may also explain why there is so much variability in the performance of WWTPs regarding the destruction of EDCs.

Other studies have also attempted to improve the destruction of EDCs in sludge during anaerobic digestion. Hamid and Eskicioglu (2013) used microwave hydrolysis as pretreatment to anaerobic digestion to improve the degradation of 16 steroidal hormones, but still found an accumulation of E1, progesterone and androstenedione in the digesters. Again this suggests that there are a series of microbial transformations taking place amongst the hormones during the anaerobic digestion process. Another study by Li and Zhang (2014) used Fenton oxidation to reduce the concentration of steroidal hormones in waste activated sludge, obtaining a removal efficiency of 33, 59, 89 and $49 \%$ for E1, E2, E3 and EE2 respectively. 
Although several studies have demonstrated that ferrate(VI) is capable of oxidising a variety of EDCs in water and wastewater (Jiang and Zhoo, 2013, Yang et al., 2012b, Li et al., 2008, Jiang, 2007, Li et al., 2008, Sharma, 2006, Sharma and Mishra, 2006, Read et al., 2003), very few studies have investigated the ability of ferrate(VI) to oxidise EDCs in wastewater sludges. There are a variety of oxidisable components in sludge, meaning that there are countless competing reactions with the highly reactive (and short-lived) ferrate(VI) ions. As a result, the ferrate(VI) may be quickly consumed by the other sludge constituents, leaving very little or no oxidising strength for the EDCs in the sludge.

However, recent interest surrounding ferrate(VI) treatments, and particularly the use of on-line (in situ) electrochemical ferrate(VI) generation (Ding et al., 2012, Yang et al., 2012a) has made sludge treatment with ferrate(VI) more feasible. On-line ferrate(VI) generation eliminates the issue of ferrate( $\mathrm{VI})$ instability, since the solution is generated on-site, and used immediately. Ferrate(VI) can be generated using wet chemical, thermal chemical and electrochemical synthesis techniques (Mácová et al., 2009). Electrochemical synthesis seems to be attracting the most attention for in-situ applications (Ding et al., 2012, Yang et al., 2012a, Alsheyab, et al., 2009, Jiang et al., 2009, Mácová et al., 2009). Electrochemical production of ferrate(VI) is generally achieved using a sacrificial iron anode in a strongly alkaline solution as part of an electrolysis cell. When a current is applied to the electrolysis cell, the iron anode is oxidised to ferrate(VI) (Alsheyab et al., 2009). In the present study however, potassium ferrate(VI) salt was used because it was more easily attainable, and very suitable for these preliminary tests. 
The purpose of the study was to evaluate the ability of potassium ferrate(VI) to oxidise selected hormones in anaerobically digested sludge using relatively low doses of potassium ferrate $(\mathrm{VI})$. The experiments aim to determine whether the low doses of ferrate(VI) are quickly consumed by the readily oxidisable matter present in the sludge, or whether the ferrate(VI) can oxidise the hormones present. Doses of 0.5 and $1.0 \mathrm{~g} / \mathrm{L}$ of potassium ferrate(VI) were used to test the ability of ferrate(VI) oxidation to reduce the concentration of hormones in sludge. Oxidation of hormones using small doses of potassium ferrate(VI) could be used in conjunction with conventional sludge stabilisation processes to improve the reduction of hormones and other EDCs.

\subsection{MATERIALS AND METHODS}

\subsubsection{Hormone Stock Solutions}

The hormones used in these experiments were seven estrogens: estrone (E1) ( $\geq 99)$,

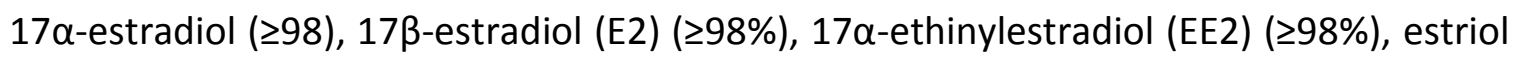
(E3) ( $\geq 97 \%)$, mestranol (99\%), and equilin ( $\geq 99)$, the progestins (synthetic progestogens): norethindrone ( $\geq 98 \%)$ and norgestrel $(\geq 98 \%)$, and the natural androgen: testosterone ( $\geq 99 \%$ ). All hormones were purchased from Sigma-Aldrich, Co. (St. Louis, MO, USA).

To prepare the hormone stock solutions, $0.5 \mathrm{mg}$ of each hormone was weighed using a Sartorius MC21S digital balance (Sartorius Canada Inc., Mississauga, ON), and placed in $0.5 \mathrm{~mL}$ sample vials. $0.5 \mathrm{~mL}$ of methanol was added to each vial, and the resulting 1 
$\mathrm{mg} / \mathrm{mL}$ solutions were mixed using a vortex mixer (Baxter Diagnostics Inc., IL, USA). Most of the hormone solutions were diluted a second time. For these samples, $0.05 \mathrm{~mL}$ portions of the $1 \mathrm{mg} / \mathrm{mL}$ solution were added to $0.45 \mathrm{~mL}$ of methanol; to create the $100,000 \mathrm{ng} / \mathrm{mL}$ stock solutions. All stock solutions were stored in the freezer until required.

\subsubsection{Sludge Sample and Hormone Spiking}

Anaerobically digested sludge, obtained from the Richard O. Pickard Environmental Centre (ROPEC) wastewater treatment plant in Ottawa, Canada, was used in these experiments. Since conventional biological treatment processes, such as anaerobic digestion, are not reliably effective at removing EDCs (Li and Zhang, 2014, Hamid and Eskicioglu, 2013 and 2012), these experiments aimed to determine whether hormones in sludge can be specifically targeted and removed using small amounts of ferrate(VI) following biological sludge treatment. The initial total solids (TS) and volatile solids (VS) concentrations of the sludge were 20.3 and $11.7 \mathrm{~g} / \mathrm{L}$ respectively. Two $100 \mathrm{~mL}$ sludge samples were placed in $250 \mathrm{~mL}$ Erlenmeyer flasks, and stirred using a magnetic stirrer. Individually prepared stock solutions containing the hormones were removed from the freezer and thawed at room temperature. Once the vials of stock solutions were completely thawed and warmed to room temperature, a vortex mixer was used to thoroughly mix the stock solutions. The concentration of each hormone stock solution was $10^{5} \mathrm{ng} / \mathrm{mL}$, except for norgestrel, which was $10^{6} \mathrm{ng} / \mathrm{mL}$. The spiked initial concentration of each hormone in the low concentration and the high concentration sludge samples are presented in Table 4-4. Following the addition of the selected 
hormones, the sludge samples were stirred using a magnetic stirrer for 4 hours. Three subsets from each of the two sludge samples were removed. The first subset was used to measure the initial concentration of hormones, and the other two were used for subsequent ferrate(VI) treatments.

Table 9-1: Spiked concentrations of the selected hormones in the low concentration and high concentration sludge samples used for subsequent tests with potassium ferrate(VI).

\begin{tabular}{|l|c|c|}
\hline & \multicolumn{2}{|c|}{ Spiked Concentration $(\mathbf{n g} / \mathbf{m L})^{*}$} \\
\hline \multicolumn{1}{|c|}{ Hormone } & Low Concentration Range & High Concentration Range $^{*}$ \\
\hline Equilin & 30 & 120 \\
\hline Estrone (E1) & 15 & 60 \\
\hline $17 ß$-Estradiol (E2) & 15 & 60 \\
\hline $17 \alpha$-Estradiol & 15 & 60 \\
\hline 17a-Ethinylestradiol (EE2) & 15 & 60 \\
\hline Estriol (E3) & 60 & 240 \\
\hline Mestranol & 75 & 300 \\
\hline Norethindrone & 15 & 60 \\
\hline Norgestrel & 15 & 60 \\
\hline Testosterone & 3 & 12 \\
\hline
\end{tabular}

* $\mathrm{ng} / \mathrm{mL}$ is equivalent to parts per billion ( $\mathrm{ppb}$ )

\subsubsection{Ferrate(VI) Treatment}

Ferrate(VI) treatments were conducted using Ferratec Brand ${ }^{\mathrm{TM}}>90 \%$ pure potassium ferrate salt $\left(\mathrm{K}_{2} \mathrm{FeO}_{4}\right)$ from Sigma-Aldrich Canada Ltd., in Oakville, ON, Canada. Two levels of ferrate(VI) treatment were used: a low dose (LD) of $0.5 \mathrm{~g} / \mathrm{L}$ and a high dose (HD) of $1.0 \mathrm{~g} / \mathrm{L}$ of potassium ferrate. The equivalent ferrate(VI) ion added was approximately 0.3 $(>0.27)$ and $0.6(>0.55) \mathrm{mg} / \mathrm{L}$ of $\mathrm{FeO}_{4}{ }^{-2} .40 \mathrm{~mL}$ sludge samples were treated with 20 and $40 \mathrm{mg}$ of potassium ferrate salt, and the samples were mixed using a vortex mixer for 5 minutes. The samples were then prepared for over-night shipment to the analytical laboratory. 


\subsubsection{Sample Analysis}

Axys Analytical Services Ltd. in Sidney, BC, Canada performed the extraction of hormones from the sludge samples, and measured the concentrations of hormones using AXYS Method MLA-072 (AXYS, 2012) on a high performance liquid chromatograph coupled to a triple quadrupole mass spectrophotometer (LC-MS/MS). All samples were placed in designated sample bottles, and into a well-insulated cooler that was filled with frozen cooler packs. The cooler was then shipped overnight to Sidney, BC, Canada. The reporting limits for the Method MLA-072 range from 0.3 to $150 \mathrm{ng} / \mathrm{g}$ based on a $1 \mathrm{~g}$ solid sample, and from 0.3 to $150 \mathrm{ng} / \mathrm{L}$ for a $1 \mathrm{~L}$ aqueous sample. A summary of the method is presented below.

Prior to hormone extraction and clean-up procedures, the $\mathrm{pH}$ of each sample was adjusted to 2.0 , and each sample was spiked with the labelled surrogates $\left(d_{6}{ }^{-}\right.$ norethindrone, $d_{6}$-norgestrel, $d_{9}$-progesterone, $d_{4}$-17ß-estradiol, $d_{4}$-ethinylestradiol) associated with each of the hormones.

The aqueous phase of each sample was obtained through filtration, to remove all particulates. The solid phase of each sample was extracted by sonication with aqueous buffered acetonitrile and pure acetonitrile, then concentrated using rotary evaporation, and diluted to a volume of $200 \mathrm{~mL}$ with ultra-pure water. The aqueous samples and the diluted extracts from the solid phase were cleaned-up using solid phase extraction (SPE), and the recovery standards $\left({ }^{13} \mathrm{C}_{6}{ }^{13} \mathrm{C}_{3}\right.$-atrazine, 2,4,5-trichlorophenooxyacetic acid) were added to the extracts. After the addition of the recovery standards, the samples were 
filtered, then analysed using a high performance liquid chromatograph coupled to a triple quadrupole mass spectrophotometer (LC-MS/MS).

The analysis was performed in positive and negative electrospray ionisation (ESI) modes, requiring a total of two runs on the liquid chromatograph to analyze the complete set of hormones. Positive ESI hormones analysed include estriol (E3), mestranol, norethindrone, norgestrel and testosterone, which were spiked, as well as allyl trembolone, androstenedione, androsterone, desogestrel and progesterone, which were not spiked. Negative ESI hormones analysed include equilin, estrone, 17 $\beta$ estradiol, $17 \alpha$-estradiol and $17 \alpha$-ethinylestradiol, which were spiked, as well as $17 \alpha$ dihyroequilin and equilenin, which were not spiked.

Instrument calibration was performed using a series of calibration standards to generate six data points for each of the hormones, including the labelled surrogate standards, covering the working range of the instrument $(0.2-3000 \mathrm{ng} / \mathrm{mL})$. The calibration runs were used to determine the mean retention time and percent recovery for each of the target hormones. The LC-MS/MS was run in multiple reaction monitoring (MRM) mode, using an injection volume of $10 \mu \mathrm{L}$. Quantification was performed by converting the peak areas of the applicable daughter ions for each of the specified parent ion to daughter ion transitions, from each of the sample chromatograms. First however, positive identification of each target hormone, surrogate standard and recovery standard required a measured concentration above the concentration of the lowest calibration standard used, and a signal to noise ratio $\geq 3: 1$ for the parent ion to daughter 
ion transition. In addition, the retention time for the particular target hormone had to fall within 0.4 minutes ( 24 seconds) of the mean retention time determined using the calibration standards, and for the labelled surrogates, the retention time had to fall within 0.1 minutes (6 seconds) of the mean retention time determined during calibration.

The specific detection limits (SDL) for each sample were calculated using 3 times the signal of the noise recorded, and converting it to an equivalent concentration. The reporting limit for each target compound was defined as the concentration of the lowest calibration standard used, which was also greater than the SDL and the method detection limit.

The results for each of the samples were reported on a dry weight (ng/g dry solids) basis, so the results were converted to a wet basis using the total solids (TS) concentrations of the representative samples. The TS and volatile solids (VS) concentrations were measured according to standard methods $2540 \mathrm{~B}$ and $2540 \mathrm{E}$ respectively (APHA, 2005).

\subsection{RESULTS AND DISCUSSION}

\subsubsection{Estrogens}

E1, E2 and E3 are natural free estrogens, present in human urine, while EE2 is a synthetic estrogen, commonly used in oral contraceptives (Belhaj, et al., 2014, Cajthaml et al., 2009, Yoshimoto et al., 2004). Together these estrogenic hormones are the major contributor to the overall estrogenicity of waterways (Hamid and Eskicioglu, 2012), and 
are some of the most commonly detected compounds in WWTPs (Liu et al., 2009). Furthermore, the synthetic estrogen, EE2, is one of the most estrogenic hormones, and is generally the most resistant to biodegradation (Liu et al., 2009, Cajthaml et al., 2009, Czajka and Londry, 2006).

Another commonly detected estrogen in wastewater is mestranol, which is also a synthetic estrogen used in oral contraceptives (Auriol et al., 2006). It is the 3-methyl ether of EE2, and a large contributor to the estrogenicity measured in WWTPs since it is typically demethylated to EE2 in the liver with $70 \%$ conversion efficiency (Faigle and Schenkel, 1998). 17 $\alpha$-estradiol is another estrogenic hormone detected at WWTPs, which can be generated through microbial transformations of E1 and E2 under anaerobic conditions (Czajka and Londry, 2006). Equilin and equilenin are equine estrogens, derived from the urine of pregnant mares, and are used in human hormone replacement therapy. These estrogens, and $17 \alpha$-dihydroequilin, the metabolite of equilenin, have been linked to estrogenic responses in fish at concentrations as low as 0.6 to $4.2 \mathrm{ng} / \mathrm{L}$ (Tyler et al., 2009).

The estrogens used in this study were selected due to their general persistence and adverse effects in the environment. Furthermore, the log $\mathrm{K}_{\mathrm{ow}}$ values for all spiked estrogens range from 2.45 to 4.68 , which means they have a high affinity for the organic phase, and are likely to bind with the organic solids in wastewater sludges. Other estrogens, which were not spiked, were also measured due to the potential for transformations among the compounds. 
Two different sludge samples were used in this study: a sample that was spiked with a low concentration range of estrogens $(15-75 \mathrm{ng} / \mathrm{mL})$, and a sample that was spiked with a high concentration range of estrogens $(60-300 \mathrm{ng} / \mathrm{mL})$. Figure $9-1(\mathrm{a})$ shows the spiked and measured initial concentrations of selected estrogens in the low concentration (15$75 \mathrm{ng} / \mathrm{mL}$ ) sludge sample, and the effects of LD and HD treatments on the selected estrogens, and Figure 9-1(b) shows the spiked and measured initial concentrations of selected estrogens in the high concentration $(60-300 \mathrm{ng} / \mathrm{mL})$ sludge sample, and the effects of LD and HD treatments on the measured estrogens.

The measured concentrations of $17 \beta$-estradiol (E2) were below the reporting limits in all of the samples, when the spiked concentration was $15 \mathrm{ng} / \mathrm{mL}$ in the low concentration sludge samples, and $60 \mathrm{ng} / \mathrm{mL}$ in the high concentration samples. This may be due to adsorption onto the walls of the container following spiking, or perhaps due to transformation of E2 to E1. Biotransformation of E2 to E1 has been shown to readily occur in the presence of sewage bacteria (Hamid and Eskicioglu, 2012, Layton et al. 2000), therefore the transformation may have occurred during the 24-hour period prior to sample preparation and analysis. Furthermore, the measured E1 concentration of the initial (untreated) sample was $25 \%$ higher than the spiked concentration in the low concentration sludge sample, and $21 \%$ higher in the high concentration sludge, which further suggests that biotransformation may have occurred.

In the case of equilenin, which was not spiked in the sludge, the measured concentrations of the initial, LD, and HD samples from the low concentration sludge 
were all $2.7 \mathrm{ng} / \mathrm{mL}$. However, for the high concentration sludge, the measured concentrations of the initial, LD, and HD samples were $10.2,9.9$ and $7.7 \mathrm{ng} / \mathrm{mL}$ respectively. Because equilenin was not added to the sludge, and both sludge batches originated from the same sludge sample prior to spiking, this suggests that equilenin was generated in the sludge due to the transformation of the spiked hormones. The most likely transformation is that of equilin to equilenin. Although these equine estrogens have been used for decades in hormone replacement therapy, due to an understanding that there has been a declining trend in their use, few studies have been published related to fate of these compounds at WWTPs and in the environment (Liu et al., 2015, Hamid and Eskicioglu, 2012, Tyler et al., 2009). Biotransformation of equilin would also account for a portion of the missing spiked mass, since the measured initial concentrations of equilin were 57 and $60 \%$ lower than the spiked concentrations of the low concentration and high concentration sludge samples respectively. However, the conversion of equilin to equilenin would only account for 17 and $14 \%$ of the missing mass in the low range and high range sludge samples respectively. $17 \alpha$-dihydroequilin is another potential metabolite of equilin, however the measured concentrations of $17 \alpha-$ dihydroequilin were below the SDL in all samples, for both the low concentration and the high concentration sludge samples.

In most cases, the measured concentration of a particular hormone was lower than the spiked concentration, again this may be due to adsorption onto the walls of the sample container, biotransformations to other (measured and not measured) compounds, or perhaps due to variability in the sampling and measurements. It is very difficult to 
extract and quantify low concentrations of hormones in a biosolids (sludge) matrix. During the LC-MS/MS calibration runs, the percent recovery of some analytes varied from as low as $65 \%$, and up to $136 \%$ and during the LC-MS/MS sample analysis runs, the percent recovery of the labelled surrogates varied from 68 to $114 \%$ (average $=90 \%$, standard deviation $=17 \%)$ over the 12 runs.

For all comparisons, the measured initial concentrations were used to evaluate the effects of the ferrate(VI) treatments. Table 9-2 shows the percent change in the concentrations of estrogens in the low concentration and the high concentration sludge samples following the addition of $0.5 \mathrm{~g} / \mathrm{L}(\mathrm{LD})$ and $1.0 \mathrm{~g} / \mathrm{L}(\mathrm{HD})$ of potassium ferrate(VI).

The measured initial concentrations of E1 were reduced by 15 and $24 \%$ following HD treatment on the low concentration and high concentration sludge samples respectively. Similarly, HD treatment on the low and high concentration sludge samples reduced the concentration of E3 by 36 and 42\%, EE2 by 11 and 13\%, $17 \alpha$-estradiol by 15 and $24 \%$, mestranol by 11 and 7\%, equilin by 20 and $23 \%$, and equilenin by 1 and $24 \%$ respectively. $17 \alpha$-dihydroequilin was consistently detected at very small concentrations $(\leq 0.9 \mathrm{ng} / \mathrm{mL})$ in all samples; however, the measured values were all less than the $\mathrm{SDL}$, as indicated by the downwards arrows in the figures. Excluding the readings for E2 and 17a-dihydroequilin which were below the SDL, HD decreased the concentrations of measured estrogens (E1, E3, EE2, 17 $\alpha$-estradiol, mestranol, equilin and equilenin) by an average of $16 \%$ and $22 \%$ in the low concentration and high concentration sludge samples respectively. 
However, LD treatment with $0.5 \mathrm{~g} / \mathrm{L}$ of potassium ferrate(VI) had mixed effects on the measured estrogens. Overall, LD resulted in a 5\% average reduction in the concentration of estrogens in both the low concentration and the high concentration sludge samples, however generally the change in concentration following LD treatment was negligible. Estriol (E3) and mestranol, on the other hand, were reduced by 14 and $29 \%$ respectively, in the low concentration sludge sample, and by 15 and $8 \%$ respectively, in the high concentration sludge sample. 

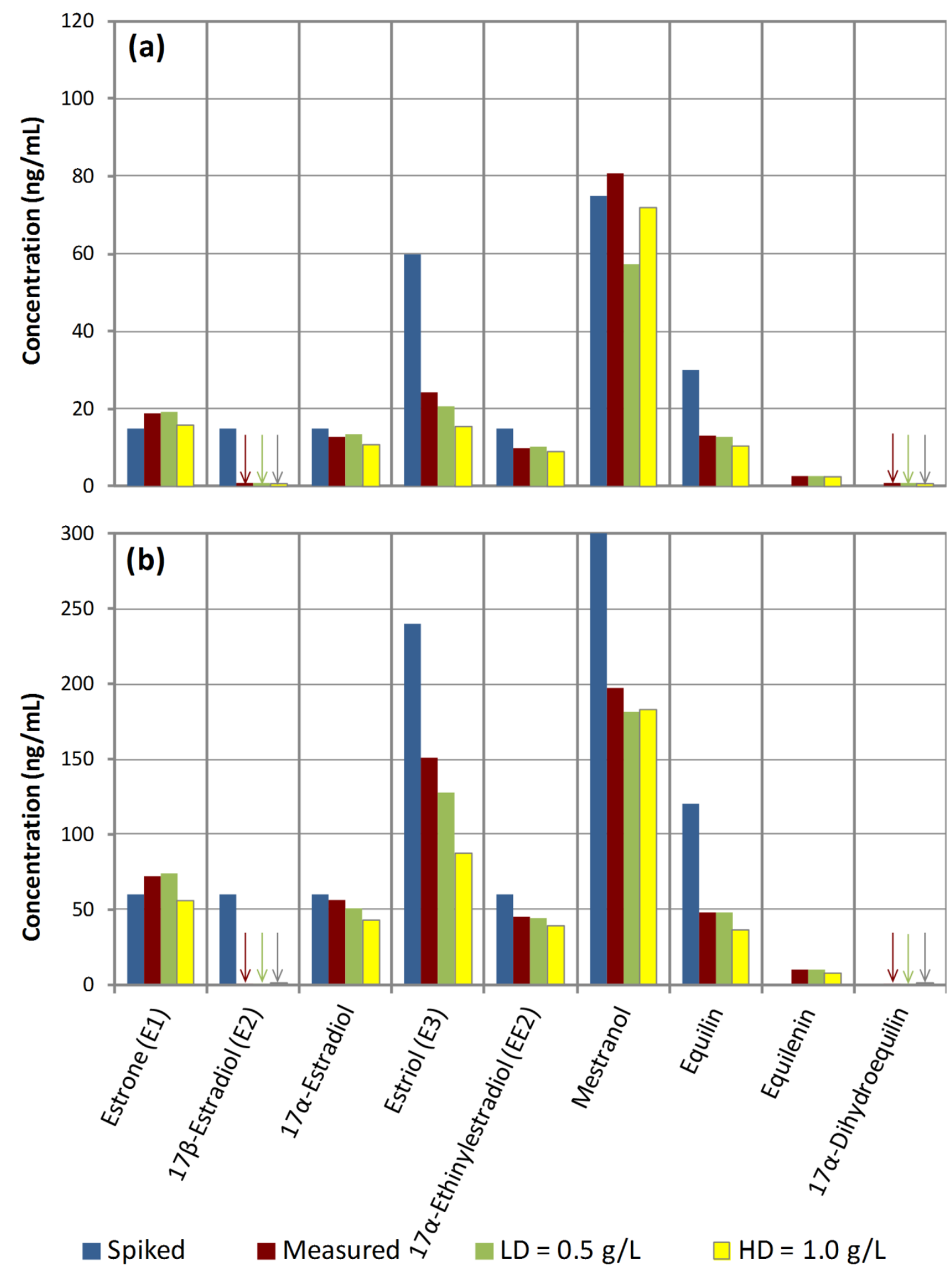

Figure 9-1: Concentrations of estrogens in the samples that were spiked with (a) the low concentration $(15-75 \mathrm{ng} / \mathrm{mL})$, and (b) the high concentration $(60-300 \mathrm{ng} / \mathrm{mL})$ of selected estrogens, and following the subsequent addition of $0.5 \mathrm{~g} / \mathrm{L}$ (LD) and $1.0 \mathrm{~g} / \mathrm{L}$ (HD) of potassium ferrate(VI). 
Table 9-2: Percent change in the concentrations of the measured estrogens in the low concentration $(15-75 \mathrm{ng} / \mathrm{mL})$ and the high concentration $(60-300 \mathrm{ng} / \mathrm{mL})$ sludge samples, following the addition of $0.5 \mathrm{~g} / \mathrm{L}$ (LD) and $1.0 \mathrm{~g} / \mathrm{L}$ (HD) of potassium ferrate(VI).

\begin{tabular}{|l|c|c|c|c|}
\hline \multirow{2}{*}{\multicolumn{1}{c|}{ Estrogen }} & \multicolumn{4}{|c|}{ Change in Concentration (\%) } \\
\cline { 2 - 5 } \multicolumn{1}{c|}{ Low Concentration Range } & \multicolumn{2}{c|}{ High Concentration Range } \\
\hline Estrone (E1) & LD & HD & LD & HD \\
\hline $17 \beta$-Estradiol (E2) & 3.0 & -15.2 & 1.7 & -23.4 \\
\hline $17 \alpha$-Estradiol & $1.2^{*}$ & $-0.5^{*}$ & $1.4^{*}$ & $0.1^{*}$ \\
\hline Estriol (E3) & 5.8 & -15.2 & -10.1 & -23.5 \\
$17 \alpha$-Ethinylestradiol (EE2) & -14.2 & -36.2 & -15.4 & -42.1 \\
\hline Mestranol & 2.9 & -11.4 & -0.6 & -13.3 \\
\hline Equilin & -28.7 & -11.0 & -7.9 & -7.1 \\
\hline Equilenin & -2.5 & -20.2 & 1.4 & -23.1 \\
\hline $17 \alpha$-Dihydroequilin & -1.0 & -1.4 & -3.1 & -24.1 \\
\hline
\end{tabular}

* indicates concentration measurements that were below the reporting limit

\subsubsection{Androgens and Progestogens}

In addition to the seven estrogens (natural and synthetic) that were added to the sludge, a natural androgen (testosterone), and two synthetic progestogens or progestins (northindrone and norgestrel) were also added. Testosterone is a naturally excreted androgen in urine, with high androgenic potency, which can contribute to $>55 \%$ of the total excretion rate of testosterone equivalent (TEQ) from males (Liu et al., 2009). Northindrone is a progestin, used in some of the earliest oral contraceptives (Djerassi et al., 1954), and norgestrel is also a progestin used for contraception (Liu et al., 2013). The other androgens measured were androstenedione and androsterone. These compounds are closely related to testosterone through the endogenous androgen metabolism in humans, along with progesterone, a natural progestogen (Liu et al., 2009). Although testosterone is generally the largest contributor to the overall androgenicity of wastewater (TEQ), androstenedione and particularly androsterone are commonly 
detected at WWTPs and contribute to the TEQ of WWTP influents and effluents (Huang et al., 2014, Liu et al., 2009). Similarly, progesterone has been detected at WWTPS, however removal rates are generally reported to be quite high (Huang et al., 2014, Liu et al., 2009).

Figure 9-2a shows the spiked and measured initial concentrations of androgens and progestogens in the low concentration $(3-15 \mathrm{ng} / \mathrm{mL})$ sludge sample, and the effects of LD and HD treatments on the selected hormones. Figure 9-2b shows the spiked and measured initial concentrations of androgens and progestogens in the high concentration (12-60 ng/mL) sludge sample, and the effects of LD and HD treatments on the selected hormones. In addition to the three hormones that were spiked (testosterone, northindrone and norgestrel), androstenedione and androsterone (androgens), progesterone and allyl trenbolone (progestogens) and desogestrel (progestin) were also measured due to the potential for transformations among the hormones.

Similar to the analysis of estrogens, the measured initial concentrations of testosterone, northindrone and norgestrel were significantly lower than the spiked concentrations in both the low concentration and the high concentration sludge samples. Testosterone was detected at concentrations $\leq 0.7 \mathrm{ng} / \mathrm{mL}$ when the spiked concentration was $3 \mathrm{ng} / \mathrm{mL}$ (Figure 9-2a), and $\leq 1.2 \mathrm{ng} / \mathrm{mL}$ when the spiked concentration was $12 \mathrm{ng} / \mathrm{mL}$ (Figure 9-2b). Similarly, the spiked concentration of northindrone and norgestrel were both 15 $\mathrm{ng} / \mathrm{L}$ in the low concentration sludge sample, and $60 \mathrm{ng} / \mathrm{mL}$ in the high concentration 
sample, however the measured concentrations of northindrone were $\leq 3.3 \mathrm{ng} / \mathrm{mL}$ and $\leq 19.8 \mathrm{ng} / \mathrm{mL}$, and the measured concentrations of norgestrel were $\leq 0.9$ and $\leq 2.0 \mathrm{ng} / \mathrm{mL}$, in the low concentration and high concentration sludge samples respectively. As discussed earlier, this may be due to adsorption onto the sample container, variability in the sampling and measurements, or due to biotransformations in the presence of the sludge biomass.

Androstenedione and progesterone were consistently detected in all sludge samples, however these two compounds were not spiked. While some of the testosterone may have contributed to the measured progesterone, the spiked concentration of $3 \mathrm{ng} / \mathrm{mL}$ in the low concentration sludge sample does not account for the measured progesterone concentrations of 20.5, 20.1 and $20.7 \mathrm{ng} / \mathrm{mL}$ in the initial, LD and HD samples respectively (Figure 9-2a). Similarly, when the spiked testosterone concentration was 12 $\mathrm{ng} / \mathrm{mL}$ in the high concentration sludge sample, the measured initial progesterone concentration was $16.9 \mathrm{ng} / \mathrm{mL}$, and following $\mathrm{LD}$ and $\mathrm{HD}$ ferrate $(\mathrm{VI})$ treatments, the progesterone concentrations were 18.6 and $16.9 \mathrm{ng} / \mathrm{mL}$ respectively (Figure 9-2b).

Androstenedione is another potential transformation product from testosterone. However, the concentration of androstenedione was $8.3,8.4$ and $8.1 \mathrm{ng} / \mathrm{mL}$ in the initial, LD and HD sludge samples respectively, when the spiked concentrations of testosterone was only $3 \mathrm{ng} / \mathrm{mL}$ in the low concentration sludge samples (Figure 9-2a). This suggests that there was some background androstenedione present in the sludge samples. Furthermore, when the spiked testosterone concentration was $12 \mathrm{ng} / \mathrm{mL}$ in the 
high concentration sludge, the measured concentration of androstenedione was 9.3, 8.9 and $8.9 \mathrm{ng} / \mathrm{mL}$ in the initial, LD and HD samples respectively (Figure 9-2b). While there is a slight increase in androstenedione when the spiked concentration of other hormones was increased, the increases are relatively small (12\%), and do not account for all of the androstenedione present in the sludge samples. Again, this suggests that there was some background androstenedione present in the anaerobically digested primary sludge samples. This agrees with the experiments by Hamid and Eskicioglu (2013), which used microwave hydrolysis as a pre-treatment for anaerobic digestion to improve the degradation of 16 steroidal hormones. Despite the success of the pre-treatment on most of the compounds, there was an accumulation of E1, progesterone and androstenedione in the digesters. Androsterone was also detected in all samples, at roughly the same concentration in both the low concentration and high concentration sludge samples, suggesting that its presence was independent of the sludge spiking. However, in all 6 samples, the measured concentrations were below the SDL, meaning that the signal of the noise in the target compound channel was greater than one-third of the recorded target compound signal, expressed as representative concentrations (AXYS, 2012). Desogestrel is another compound that was consistently detected in all samples at relatively high concentrations, however due to elevated noise in the input channel of the target hormone, the sample measurements were all below the stated SDL. Downwards arrows are used in the figures to represent that the measured concentration was below the SDL or method detection limit (MDL). 
In general, the LD $(0.5 \mathrm{~g} / \mathrm{L})$ and $\mathrm{HD}(1.0 \mathrm{~g} / \mathrm{L})$ treatments with potassium ferrate $(\mathrm{VI})$ did not have a large effect on the concentration of androgens and progestogens in the primary sludge. This is likely due to the competing reactions taking place in the sludge, and the relatively low doses of potassium ferrate(VI) doses used. The percent change in the concentrations of all measured androgens and progestogens following the addition of LD and HD ferrate(VI) treatments are presented in Table 9-3. The shaded values with an asterisk $\left({ }^{*}\right)$ are values that were below the individual SDLs for the target compounds.

In the case testosterone, the measured concentrations increased following the addition of potassium ferrate(VI) in some cases, however as discussed earlier, the measured concentrations of testosterone were all $\leq 1.2 \mathrm{ng} / \mathrm{mL}$, and very near the SDL, which increased the potential for variability among the sample measurements. In addition, certain biotransformations may have taken place, which converted the spiked testosterone to other compounds, and these transformations may have been impeded slightly by the ferrate(VI). Furthermore, some testosterone may have been adsorbed onto the walls of the sample container, and the ferrate(VI) may have affected the amount of adsorption.

Similarly, the measured concentrations of norgestrel increased following the addition of potassium ferrate(VI) in the high concentration sludge sample, however the measured concentrations were near the SDL, and all norgestrel measurements were below the SDL in the low concentration sludge sample. When the initial concentration of northindrone was $3.3 \mathrm{ng} / \mathrm{mL}$ in the low concentration sludge sample, LD and HD treatments reduced 
the concentration by only 3 and $1 \%$ respectively, but when the initial concentration of northindrone was $19.8 \mathrm{ng} / \mathrm{mL}$ in the high concentration sludge, LD and HD treatments both reduced the concentration of northindrone by $9 \%$. 

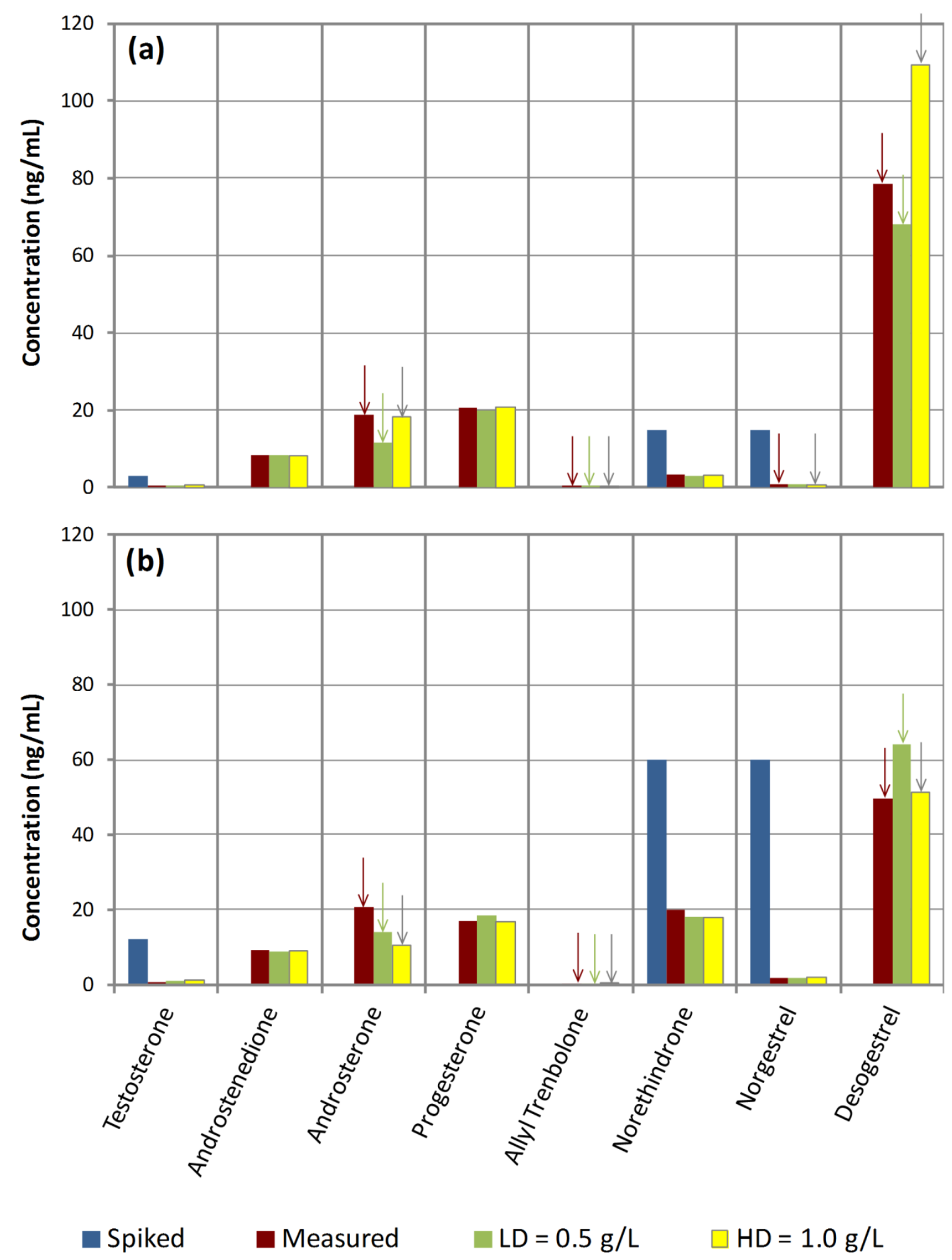

Figure 9-2: Concentrations of androgens and progestogens in the sludge samples that were spiked with (a) the low concentration $(3-12 \mathrm{ng} / \mathrm{mL})$, and (b) the high concentration (12-60 $\mathrm{ng} / \mathrm{mL}$ ) of androgens and progestins, and following the subsequent addition of $0.5 \mathrm{~g} / \mathrm{L}$ (LD) and $1.0 \mathrm{~g} / \mathrm{L}(\mathrm{HD})$ of potassium ferrate(VI). 
Table 9-3: Percent change in the concentrations of the measured androgens and progestogens in the low concentration $(3-15 \mathrm{ng} / \mathrm{mL})$, and the high concentration $(12-60 \mathrm{ng} / \mathrm{mL})$ sludge samples, following the addition of $0.5 \mathrm{~g} / \mathrm{L}$ (LD) and $1.0 \mathrm{~g} / \mathrm{L}$ (HD) of potassium ferrate(VI).

\begin{tabular}{|l|c|c|c|c|}
\hline \multirow{2}{*}{ Androgen/Progestogen } & \multicolumn{4}{|c|}{ Change in Concentration (\%) } \\
\cline { 2 - 5 } & \multicolumn{3}{|c|}{ Low Concentration Range } & \multicolumn{1}{c|}{ High Concentration Range } \\
\hline Testosterone & LD & HD & LD & HD \\
\hline Androstenedione & -6.9 & 21.7 & 32.9 & 70.8 \\
\hline Androsterone & 1.7 & -2.4 & -4.1 & -4.7 \\
\hline Progesterone & $-37.8^{*}$ & $-2.0^{*}$ & $-32.4^{*}$ & $-49.6^{*}$ \\
\hline Allyl Trenbolone & -2.2 & 0.7 & 10.1 & 0.2 \\
\hline Norethindrone & $1.7^{*}$ & $-2.4^{*}$ & $-4.1^{*}$ & $-4.7^{*}$ \\
\hline Norgestrel & -3.0 & -1.0 & -8.7 & -9.0 \\
\hline Desogestrel & $-0.8^{*}$ & $-7.6^{*}$ & 17.6 & 9.1 \\
\hline
\end{tabular}

* indicates concentration measurements that were below the reporting limit

\subsection{CONCLUSION}

Excluding the readings for E2 and 17a-dihydroequilin which were below the SDL, a 1.0 $\mathrm{g} / \mathrm{L}$ dose of potassium ferrate $(\mathrm{VI})$ reduced the concentration of estrogens by an average of 16 and $22 \%$ in the sludge samples that were spiked with a low concentration range $(15-75 \mathrm{ng} / \mathrm{mL})$ and a high concentration range $(60-300 \mathrm{ng} / \mathrm{mL})$ of selected estrogens respectively. Estrone (E1) was reduced by 15 and 23\%, and estriol (E3) was reduced by 36 and $42 \%$ in the low concentration and high concentration sludge samples respectively. The equine estrogen, equilin, was also reduced by 20 and $23 \%$ in the low concentration and high concentration sludge samples respectively. However, treatment using $0.5 \mathrm{~g} / \mathrm{L}$ of potassium ferrate $(\mathrm{VI})$ only resulted in a $5 \%$ (average) reduction of estrogens in both the low and high concentration sludge samples.

Potassium ferrate $(\mathrm{VI})$ additions of 0.5 and $1.0 \mathrm{~g} / \mathrm{L}$ were less effective at reducing the concentrations of measured androgens and progestogens, however most of these 
hormones were measured at concentrations nearing the SDL, which increased the potential for sampling error and variations due to the sample extraction and analysis procedures.

The spiked concentration of E2 was 15 and $60 \mathrm{ng} / \mathrm{mL}$ in the low concentration and high concentration sludge samples respectively, however E2 was not detected in either sample. While adsorption of E2 onto the sample container is possible, it also appears that some of the E2 was degraded to E1 in the presence of the sewage bacteria, since the measured E1 concentrations in the sludge samples were consistently higher than the spiked concentrations. Previous research has demonstrated that E2 is rapidly degraded to E1 in the presence of municipal sewage sludge (Hamid and Eskicioglu, 2012, Janex-Habibi et al., 2009). Another possible explanation is that there was already E1 present in the anaerobically digested sludge., or that there were error during analyte extraction and quantification.

Androstenedione and progesterone may have also been present in the sludge. These two hormones were not spiked, however the measured concentrations of androstenedione was 8.3 and $9.3 \mathrm{ng} / \mathrm{mL}$, and progesterone was 20.5 and $16.9 \mathrm{ng} / \mathrm{mL}$ in the low concentration and the high concentration sludge samples respectively. While the addition of testosterone may have contributed to the measured concentrations of these compounds, the mass of testosterone added does not account for the measured concentrations of androstenedione and progesterone. 
Equilenin seems to have been generated through the transformation of equilin. When the spiked concentration of equilin was $30 \mathrm{ng} / \mathrm{mL}$, the measured concentration of equilenin was $2.7 \mathrm{ng} / \mathrm{mL}$, and when the spiked concentration of equilin was $120 \mathrm{ng} / \mathrm{mL}$, the measured concentration of equilenin was $10.2 \mathrm{ng} / \mathrm{mL}$.

All tests using spiked hormones employed a control sample, in which no treatments were performed, to verify the actual initial concentration of the spiked hormones. In many cases, the actual measured concentration was significantly lower than the spiked concentration. This was likely due to adsorption of the hormones onto the walls of the sample containers, biotransformations in the presence of the sewage bacteria, sampling errors, or poor analyte recovery during sample extraction and quantification via LCMS/MS. The discrepancies identify the need for researchers to use blank samples when analysing the treatment of spiked micro-contaminants in aqueous samples, and particularly in biosolids matrices such as wastewater sludges, since percent recovery of test analytes often show considerable variability.

The study demonstrated that although various spiked hormones undergo biotransformations in the presence of sewage sludge, potassium ferrate(VI) additions as low as $1.0 \mathrm{~g} / \mathrm{L}$ can reduce the concentration of hormones, particularly estrogens. While these experiments aimed to keep the cost of the ferrate(VI) treatment low, higher doses of ferrate(VI) would likely result in more substantial decreases in the concentrations of a majority of these compounds. 


\subsection{REFERENCES}

Alsheyab, M., Jiang, J.Q., Stanford, C. (2009) On-line production of ferrate with an electrochemical method and its potential application for wastewater treatment - A review. J Environ Manag 90(3):1350-1356

APHA (American Public Health Association), American Water Works Association, Water Pollution Control Federation (2005) Standard Methods for the Examination of Water and Wastewater. APHA/AWWA, Washington DC.

Auriol, M., Filali-Meknassi, Y., Tyagi, R.D., Adams, C.D., Surampalli, R.Y. (2006) Endocrine disruptin compounds removal from wastewater, a new challenge. Process Biochem 41(3):525-539

AXYS, 2012 Method Summary of Axys Method MLA-072 Rev. 03 Ver 02: Analysis of Hormones in Solid and Aqueous Samples by LC-MS/MS. (proprietary)

Bartzatt, R., Cano, M., Johnson, L., Nagel, D. (1992) Removal of toxic metals and nonmetals from contaminated water. J Toxicol Environ Health 35(4):205-210

Belhaj, D., Jaabiri, I., Kallel, M., Zhou, J.L. (2014) Occurrence and removal of steroidal estrogens in Centre Eastern Tunisia municipal sewage treatment plant. Desalination Water Treatment 52(10-12):2330-2339

Cajthaml, T., Křesinová, Z., Svobodová, K., Sigler, K., Řezanka, T. (2009) Microbial transformation of synthetic estrogen 17a-ethinylestradiol. Environ Pollut 157(12):33253335 
Czajka, C.P., Londry, K.L. (2006) Anaerobic biotransformation of estrogens. Sci Total Environ 367:932-941

Ding, L., Liang, H.-C., Li, X.-Z. (2012) Oxidation of $\mathrm{CH}_{3} \mathrm{SH}$ by in situ generation of ferrate(VI) in aqueous alkaline solution for odour treatment. Sep Purif Technol 91:117124

Djerassi, C., Miramontes, L., Rosenkranz, G., Sondheimer, F. (1954) Steroids. LIV. ${ }^{1}$ Synthesis of 19-Nov-17 $\alpha$-ethynyltestosterone and $19-$ Nor-17 $\alpha$-methyltestosterone ${ }^{2} . J$ Am Chem Soc 76(16):4092-4094

Faigle, J.W.; Schenkel, L. (1998) Pharmacokinetics of estrogens and progestogens. In:

Fraser, I.S., Jansen, R.P.S., Lobo, R.A., Whitehead, M.I. (editors) Estrogens and Progestogens in Clinical Practice. London: Churchill Livingstone, pp 273-294.

Gombos, E., Barkács, K., Felföldi, T., Vértes, C., Makó, M., Palkó, G., Záray, C. (2013) Removal of organic matters in wastewater treatment by ferrate(VI)-technology. Microchem J 107:115-120

Hamid, H., Eskicioglu, C. (2013) Effect of microwave hydrolysis on transformation of steroidal hormones during anaerobic digestion of municipal sludge cake. Water Res 47:4966-4977

Hamid, H., Eskicioglu, C. (2012) Fate of estrogenic hormones in wastewater and sludge treatment: A review of properties and analytical detection techniques in sludge matrix. Water Res 46, 5813-5833 
He, C., Li, X.-Z., Sharma, V.K., Li, S.-Y. (2009) Elimination of sludge door by oxidizing sulphur-containing compounds with ferrate(VI). Environ Sci Technol 43:5890-5895

Huang, B., Li, X., Sun, W., Ren, D., Li., X., Li., X., Liu, Y., Li, Q., Pan, W. (2014) Occurrence, removal, and fate of preogestogens, androgens, estrogens, and phenols in six sewage treatment plants around Dianchi Lake in China. Environ Sci Pollut R 21:12898-12908

Janex-Habibi, M.L., Huyard, A., Esperanza, M., Bruchet., A. (2009) Reduction of endocrine disruptor emissions in the environment: The benefit of wastewater treatment. Water Res 43:1565-1576

Jiang, J.Q., Stanford, C., Alsheyab, M. (2009) The online genereation and application of ferrate for sewage treatment - A pilot scale trial. Sep Purif Technol 68:227-231

Jiang, J.Q., Panagoulopoulos, A., Bauer, M., Pearce, P. (2006) The application of potassium ferrate for sewage treatment. J Environ Manag 79(2):215-220

Jiang, J.Q., Lloyd, B. (2002) Progress in the development and use of ferrate (vi) salt as an oxidant and coagulant for water and wastewater treatment. Water Res 36:1397-1408

Layton, A.C., Gregory, B.W., Seward, J.R., Schultz, T.W., Sayler, G.S. (2000) Mineralization of steroidal hormones by biosolids in wastewater treatment systems in Tennesse U.S.A.. Environ Sci Technol 34:3925-3931

Li, C., Li, X.Z., Graham, N., (2005) A study of the preparation and reactivity of potassium ferrate. Chemosphere 42:109-120 
Li, Y., Zhang, A. (2014) Removal of steroid estrogens from waste activated sludge using Fenton oxidation: Influencing factors for degradation intermediates. Chemosphere 105:24-30

Liu, S.S., Ying, G.G., Liu, Y.S., Yang, Y.Y., He, L.Y., Chen, J., Liu, W.R., Zhao, J.L. (2015) Occurrence and removal of progestagens in two representative swine farms: Effectiveness of lagoon and digester treatment. Water Res 77:146-154

Liu, S., Ying, G.G., Liu, Y.S., Peng, F.Q., He, L.Y. (2013) Degradation of norgestrel by bacteria from activated sludge: comparison to progesterone. Environ Sci Technol 47:10266-10276

Liu, Z.H., Kanjo, Y., Mizutani, S. (2011) Removal of natural free estrogens and their conjugates in a municipal wastewater treatment plant. Clean - Soil, Air, Water 39(2):128-135

Liu, Z.H., Hashimoto, T., Okumura, Y., Kanjo, Y., Mizutani, S. (2010) Simultaneous analysis of natural free estrogens and their conjugates in wastewater by GC-MS. Clean 38(2):181-188

Liu, Z.H., Kanjo, Y., Mizutani, S. (2009a) Urinary excretion rates of natural estrogens and adrogens from humans, and their occurrence and fate in the environment: A review. Sci Total Environ 407:4975-4985 
Liu, J.L., Nikolausz, M., Wang, X.C. (2009b) Biodegradation and detoxification of phenol using free and immobilized cells of Acinetobacter sp. XA05 and Shingomonas sp. FG03. J Environ Sci Heal A 44:130-136

Liu, Z.H., Kanjo, Y., Mizutani, S. (2009c) Removal mechanisms for endocrine disrupting compounds (EDCs) in wastewater treatment - physical means, biodegradation, and chemical advanced oxidation: A review. Sci Total Environ 407:731-748

Mácová, Z., Bouzek, K., Híveš, J., Sharma, V.K., Terryn, R.J., Baum, J.C. (2009) Research progress in the electrochemical synthesis of ferrate(VI). Electrochim Acta 54:2673-2683

Murmann, R.K., Robinson, P.R. (1974) Experiments utilising $\mathrm{FeO}_{4}{ }^{2-}$ for purifying water. Water Res 8(8):543-547

Read, J.F., Graves, C.R., Jackson, E. (2003) The kinetics and mechanism of the oxidation of the thiols 3-mercapto-1-propane sulfonic acid and 2-mercaptonicotinic acid by potassium ferrate. Inorganica Chimica Acta 348:41-49

Schink, T., Waite, T.D. (1980) Inactivation of $\mathrm{f} 2$ virus with ferrate(VI). Water Res 14:17051717

Schuck, C.A., De Luca, S.J., Peralba, M., De Luca, M.A. (2006) Sodium ferrate(IV) and sodium hypochlorite in disinfection of biologically treated effluents. Ammonium nitrogen protection against THMs and HAAs. J Environ Sci Health A41(10):2329-2343 Sumpter, J.P. (2007) The ecotoxicology of hormonally active micropollutants. Water Sci Technol 57(1), 125-130 
Tyler, C.R., Filby, A.L., Bickley, L.K., Cumming, R.I., Gibson, R., Labadie, P., Katsu, Y., Liney, K.E., Shears, J.A., Silva-Castro, V., Urushitani, H., Lange, A., Winter, M.J., Iguchi, T., Hill, E.M. (2009) Environmental health impacts of equine estrogen derived from hormone replacement therapy. Environ Sci Technol 43:3897-3904

Xu, J., Wu, L., Chen, W., Jiang, P., Chang, A.C.-S. (2009) Pharmaceuticals and Personal Care Products in Runoff from potato field irrigated with wastewater treated in Southern California. J Health Sci 55(2):306-310

Yang, E.-L., Shi, J.-J., Liang, H.-C. (2012a) On-line electrochemical production of ferrate(VI) for odor control. Electrochim Acta 63:369-374

Yang, B., Ying, G.-G., Zhao, J,-L, Liu, S., Zhou, L.-J., Chen, F. (2012b) Removal of selected endocrine disrupting chemicals (EDCs) and pharmaceuticals and personal care products (PPCPs) during ferrate(VI) treatment of secondary wastewater effluents. Water Res 46(7):2194-2204

Yoshimoto, T., Nagai, F., Fujimoto, J., Watanabe, K., Mizukoshi, H., Makino, T., Kimura, K., Saino, H., Sawada, H., Omura, H. Degradation of estrogens by Rhodococcus zopfii and Rhodococcus equi isolates from activated sludge in wastewater treatment plants. Appl Environ Microb 70(9):5283-5289 


\section{APPLICATION OF FERRATE(VI) OXIDATION AND FREEZE- THAW SLUDGE DEWATERING AT A REMOTE FACILITY IN NORTHERN CANADA}

To demonstrate the application of ferrate(VI) and freeze-thaw sludge dewatering, a remote facility in northern Canada will be used as a model for the design and operation of a freeze-thaw sludge dewatering bed, employing ferrate(VI) oxidation treatment. The sludge generated at this remote facility and the associated sludge management issues initiated the pilot-scale sludge dewatering study presented in Chapter 5.

The exploration facility is situated in the Otish mountains, approximately $275 \mathrm{~km}$ north of Chibougamau, Quebec, and is home to approximately 100 people. Transportation to and from the site is via ice road, for approximately 2-3 months per year, and via helicopter. Wastewater generated on-site is treated using a CLEMENTINE $^{\circledR}$ wastewater treatment (WWT) system, which includes a primary settling tank (PST), a rotating biological contactor (RBC) followed by secondary settling, and effluent UV disinfection. The ROTORDISK ${ }^{\circledR}$ RBC unit has approximately $504 \mathrm{~m}^{2}$ of media surface area for attached-growth biological treatment, which treats approximately $14 \mathrm{~m}^{3} /$ day of wastewater. The sludge generated during WWT is difficult to remove from the site, which led to the desire to stabilise and dewater the sludge to below the P1 regulated limits for land application of biosolids in Quebec. 
The subsequent sections will provide some discussion and details on sludge generation rates and sludge storage, ferrate $(\mathrm{VI})$ treatment, the design and operation of a freezethaw sludge dewatering bed, meltwater generation, collection and management, and sludge cake disposal.

\subsection{SLUDGE GENERATION AND STORAGE}

The sludge generated at the facility is a combination of the sludge collected in the primary and secondary settling tanks, and the biomass sloughed from the RBC. All sludges generated and collected in the WWT unit can be left to accumulate for 6 to 9 months prior to being removed, treated and disposed. This is due to high VSS/TSS ratio $(\approx 90 \%)$ of the incoming solids, and the ability of the 4-stage ROTORDISK ${ }^{\circledast}$ RBC unit to digest organic solids, thereby reducing sludge accumulation. To estimate the sludge generation rate, we need to estimate the amount of solids removed from the influent wastewater, the amount of solids generated during biological decomposition of organic matter in the RBC unit, and the amount of solids that are digested within the WWT unit. Assuming the influent wastewater flow of $14 \mathrm{~m}^{3} /$ day has $500 \mathrm{mg} / \mathrm{L}$ of TSS and BOD ${ }_{5}$, and the effluent has $20 \mathrm{mg} / \mathrm{L} \mathrm{TSS}$ and $\mathrm{BOD}_{5}$, the total mass of dry solids (DS) produced per day was estimated to be $5.6 \mathrm{~kg}$ DS/day. Detailed calculations are presented in Appendix C1 on page 385 .

During the pilot-scale study (presented in Chapter 5) sludge removed from the WWT unit had an average TS concentration of $2.6 \%$, and minimum and maximum TS of 2.1 and $3.0 \%$ respectively (Table $4-1$ ). Therefore the total volume of sludge generated 
annually would be approximately $78 \mathrm{~m}^{3} / \mathrm{yr}$. However the $3-5$ day travel period for the sludge samples to be sent from the remote facility to the laboratory facility at Carleton University seems to have resulted in a sludge solubilisation and a decrease in TS. Generally sludge removed from CLEMENTINE ${ }^{\circledR}$ WWT units has a TS $>3 \%$, which would reduce the annual sludge volume to $68 \mathrm{~m}^{3}$ or less. Assuming the sludge generated is allowed to accumulate in the WWT unit for 7 or 8 months prior to being removed, a sludge storage volume of 22 to $26 \mathrm{~m}^{3}$ would be required, in addition to the storage provided by the WWT unit.

\subsection{SLUDGE TREATMENT USING FERRATE(VI)}

Due to the extended sludge storage period within the CLEMENTINE $^{\circledR}$ WWT unit, the concentration of fecal coliform in the sludge removed was relatively low, with an average of 1816 MPN/g DS, and peak concentration of only 6245 MPN/g DS (Table 4-1, page 88). For sludge with such a low concentration of fecal coliform, ferrate(VI) treatments would not be required for fecal coliform inactivation since stand-alone freeze-thaw dewatering can result in approximately 1-3 log inactivation. This would make the remaining sludge cake following thawing and dewatering suitable for land application, in terms of fecal coliform, under P1 regulations in Quebec, which are similar to CP1 regulations in Ontario (MOE, 2014, CCME, 2010). However it was assumed that the 3-5 day travel period for the sludge samples to be sent from the remote facility to the laboratory facility for analysis, may have led to a certain level of fecal coliform inactivation. Therefore, for the purposes of this study, an initial sludge fecal coliform concentration of 100,000 MPN/g DS (5-log) was assumed. 


\subsubsection{Ferrate(VI) Uses for Sludge Treatment}

Ferrate $(\mathrm{VI})$ is a high-strength and environmentally benign chemical oxidant, which can be used inactivate fecal coliform and other pathogens (Jiang et al., 2007, Schuck et al., 2006), reduce sludge odours (de Luca et al., 1996) and oxidise pharmaceuticals and personal care products (PPCPs) and other organic chemicals (Li et al., 2008, Jiang, 2007, Zhu et al. 2006). Furthermore, when ferrate(VI) is used as an oxidant, it is reduced to Fe(III) ions and ferric hydroxide, which act as coagulants and reduce the turbidity of wastewater (Jiang and Lloyd, 2002). Therefore, sludge treatment with potassium ferrate(VI) can be used to improve sludge quality by decreasing the concentration of odours, pathogens and indicator organisms, and by oxidising emerging organic compounds (EOCs) such as PPCPs or other organic chemicals.

\subsubsection{Sludge Pre- and Post-treatment with Ferrate(VI)}

Post-treatment of wastewater sludge following freeze-thaw dewatering was effective for the inactivation of fecal coliform, however ferrate(VI) additions were performed on liquid sludge, not the dewatered sludge cake. In full-scale applications, ferrate(VI) additions to the sludge cake remaining following freeze-thaw would be difficult due to the high solids content of the sludge cake, and the inherent difficulties in sludge mixing following chemical addition to achieve uniform sludge-ferrate(VI) contact. In the event that an adequate mixing device is available, ferrate(VI) additions on the dewatered cake offer several potential advantages. First, freeze-thaw sludge dewatering was shown to reduce the sludge volume by up to $88 \%$, which would reduce ferrate(VI) requirements and costs by the same amount. Second, the ferrate(VI) doses could be chosen more 
efficiently since the ferrate( $\mathrm{VI})$ treatment would act as a polishing treatment following freeze-thaw, and the target organism (or chemical compound) concentration remaining in the sludge cake following freeze-thaw dewatering could be determined prior to ferrate(VI) treatment. Third, ferrate(VI) additions following freeze-thaw dewatering would further stabilise the sludge cake, and thereby reduce the potential for putrefaction during storage, transportation and application of biosolids to land.

However for these experiments, ferrate(IV) post-treatments on the sludge cake remaining following freeze-thaw dewatering was not tested. Ferrate(VI) additions were only tried on liquid sludges, since the chemical additions and sludge mixing at the fullscale would be facilitated. For example, ferrate(VI) additions to liquid sludge could be accomplished when the sludge is pumped into a storage tank prior to being applied on a freeze-thaw bed. Furthermore, sludge pre-treatment with ferrate(VI) would inactivate pathogens and reduce odours prior to the sludge being applied to the freeze-thaw bed. This would be particularly favourable when treating raw sludges, which have greater concentrations of pathogens and greater potential to generate offensive odours.

\subsubsection{Pre-Treatment of RBC Sludge using Ferrate(VI)}

The sludge removed from the CLEMENTINE ${ }^{\circledR}$ WWT unit is a mixture of the sludge collected from the primary and secondary settling tanks, and the biological sludge which is sloughed occasionally from the RBC. As mentioned earlier, the sludge generated in the WWT unit slowly accumulates for 6 to 9 months prior to being removed. Therefore a 
significant level of sludge digestion occurs within the unit, which reduces the sludge accumulation rates and the concentration of fecal coliform.

Sludge samples taken from the RBC unit had an average fecal coliform concentration of only $1816 \mathrm{MPN} / \mathrm{g} \mathrm{DS}$, which make ferrate(VI) additions unnecessary, since stand-alone freeze-thaw is capable of stabilising and dewatering the sludge to below P1 requirements for land application of biosolids in Quebec (CCME, 2010). However, for the purposes of this study, it was assumed that the sludge removed from the WWT unit had an initial fecal coliform concentration of $1 \times 10^{5} \mathrm{MPN} / \mathrm{g} \mathrm{DS}$, which would make standalone freeze-thaw inadequate for the stabilisation of sludge to below P1 limits. Therefore, due sludge transportation limitations and the need to stabilise and dewater sludge on-site, ferrate(VI) additions will be used to provide a minimum 1-log inactivation of fecal coliform, followed by freeze-thaw stabilisation and dewatering. The combined treatments are expected to provide $>2$-log inactivation of fecal coliforms in the sludge cake, which would allow for land application of the treated sludge cake.

\subsubsection{Ferrate(VI) Dose and Target Level of Inactivation}

A potassium ferrate( $\mathrm{VI})$ dose of $5.0 \mathrm{~g} / \mathrm{L}$ was selected for sludge treatment to provide approximately 1-log inactivation of fecal coliform prior to freeze-thaw stabilisation and dewatering. Experimental results demonstrated that following a 15-minute contact time, $5.0 \mathrm{~g} / \mathrm{L}$ of potassium ferrate $(\mathrm{VI})$ resulted in approximately 2.1-log inactivation of fecal coliform in anaerobically digested sludge, while $5.1 \mathrm{~g} / \mathrm{L}$ resulted in 1.1-log inactivation in raw primary sludge. Therefore, it is expected that $5.0 \mathrm{~g} / \mathrm{L}$ will provide at 
least 1-log inactivation of fecal coliform in the sludge generated in the WWT unit, prior to freeze-thaw stabilisation and dewatering. In addition to fecal coliform inactivation, sludge pre-treatment using $5.0 \mathrm{~g} / \mathrm{L}$ of potassium ferrate( $\mathrm{VI})$ would also result in a decrease in the concentration of sulphides, as demonstrated in Chapter 7, which would decrease sludge odours. Furthermore, the concentration of estrogenic hormones in anaerobically digested, was decreased by $26 \%$ using only $1.0 \mathrm{~g} / \mathrm{L}$ of potassium ferrate(VI), therefore $5.0 \mathrm{~g} / \mathrm{L}$ would likely have a more significant effect. The equivalent dose expressed as the ferrate $(\mathrm{VI})$ ion, $\mathrm{FeO}_{4}{ }^{-2}$, is $3.0 \mathrm{~g} / \mathrm{L}$.

\subsubsection{Ferrate (VI) Cost}

Advancements in electrochemical ferrate(VI) generation have reduced production costs to approximately CAD $\$ 15 / \mathrm{kg} \mathrm{FeO}_{4}^{-2}$ (USD $\$ 5 / \mathrm{lb}$ from Waite, 2012). Therefore, a $3.0 \mathrm{~g} / \mathrm{L}$ dose of ferrate(VI) would cost approximately $\$ 44 / \mathrm{m}^{3}$ of sludge, putting the annual cost of ferrate(VI) treatments between $\$ 3000$ and $\$ 3500$, depending on the actual sludge volume requiring treatment. A figure showing the annual cost of electrochemical ferrate(VI) as a function of sludge volume and ferrate(VI) dose is presented as Figure $\mathrm{C} 1$, in Appendix C2 on page 386. As discussed earlier, electrochemical ferrate(VI) generation requires a sacrificial iron anode, a $12 \%$ bleach solution, $40 \%$ ferric chloride solution and a $50 \%$ caustic solution contained in an electrolysis cell (Waite, 2012). Although these chemicals are commonly present at most treatment plants, electrochemical ferrate(VI) generation may be impractical at the remote facility, due to the equipment, chemicals and electricity required to generate the ferrate(VI). In addition, ferrate(VI) solutions would increase the sludge volume. 
Therefore, solid potassium ferrate(VI) additions may be a more favourable approach, however recent estimates put the cost of ferrate(VI) salts around $\$ 176 / \mathrm{kg} \mathrm{FeO}_{4}^{-2}$ (USD $\$ 60 / \mathrm{lb}$ from Waite, 2012), which would increase ferrate(VI) treatments to between $\$ 36,000$ and $\$ 42,000$ per year. A figure showing the annual cost of solid ferrate(VI) as a function of sludge volume and ferrate(VI) dose is presented as Figure $\mathrm{C} 2$, in Appendix C2 on page 386 . Current sludge management at the facility required the periodic removal and transportation of sludge from the facility to an appropriate sludge treatment facility, representing a large operational cost. Furthermore, due to inconsistent winter temperatures near the facility, the ice-roads used to transport gear and supplies to and from the facility were only available for approximately 2 months per year. During warmer months, a helicopter was required to travel to and from the site, which could only transport $1 \mathrm{~m}^{3}$ of liquid sludge per trip. Under such circumstances, the cost of powdered ferrate(VI) treatment, which would enable on-site disposal of the stabilised sludge cake following freeze-thaw dewatering, may be a relatively simple and costeffective sludge management approach.

\subsection{DESIGN AND OPERATION OF A FREEZE-THAW SLUDGE DEWATERING BED}

\subsubsection{Sizing the Freeze-Thaw Bed}

The annual sludge generation at the facility was estimated to be between 68 and $78 \mathrm{~m}^{3}$, assuming sludge solids concentrations of 3.0 and $2.6 \%$ respectively. A freeze-thaw bed measuring $7 \mathrm{~m} \times 5 \mathrm{~m} \times 3 \mathrm{~m}(\mathrm{~L} \times \mathrm{W} \times \mathrm{D})$ would provide $105 \mathrm{~m}^{3}$, allowing for a $0.5 \mathrm{~m}$ deep meltwater collection system, and $>10 \%$ sludge expansion volume. A $3.0 \mathrm{~m}$ total depth is also convenient since a backhoe could be used for excavations. 


\subsubsection{Meltwater Collection System}

The meltwater collection system at the base of the freeze-thaw bed contains, from bottom up, a geotextile ground liner, perforated meltwater collection pipes encased in approximately $10-15 \mathrm{~cm}$ of gravel, followed by a layer of coarse land, approximately 0.3 to $0.5 \mathrm{~m}$ deep. Figure 2-2 shows a sketch of a freeze-thaw dewatering bed with meltwater collection. The meltwater collection pipes should be spaced approximately $0.3 \mathrm{~m}$ from side walls, and no more than $0.75 \mathrm{~m}$ apart from one another, and a minimum $1 \mathrm{~cm}$ drop for every $48 \mathrm{~cm}$ length drain pipe should be used to promote good drainage and prevent excessive meltwater build-up at the base of the freeze-thaw bed. Assuming the network of pipes is run lengthwise, the minimum total drop in elevation required for the drain pipes is $15 \mathrm{~cm}$. Additional grade should also be considered where elbows are used to combine the flows from the individual pipe runs into a centralised meltwater collection, storage and pumping well. More detail related to meltwater collection and management is provided in section 10.3.5 on page 333 .

\subsubsection{Preparation of the Freeze-Thaw Bed}

Application of sludge to the freeze-thaw bed should begin when the average daily temperature is below $-10^{\circ} \mathrm{C}$ to ensure complete freezing of the sludge within 2 or 3 days. This will minimise odours and the migration of fines into the underlying sand drainage layer prior to free zing. Furthermore, the sand drainage layer should be protected from early sludge infiltration by adding successive layers of water, prior to the addition of any sludge. 
For natural freeze-thaw applications without any cover, periods of freezing rain or snow will help to soak the sand drainage layer under freezing conditions. This will fill the voids of the sand layer with ice and restrict sludge from entering the sand prior to freezing. For the pilot-scale test, approximately $10 \mathrm{~L}$ of water effectively protected the $36 \mathrm{~L}$ sand drainage layer. Therefore up to $25 \%$ of the sand volume (used for drainage) in water can be used to prepare the frozen sand layer, prior to sludge additions. However this will contribute to the meltwater volume collected during the thawing period, and must be considered when estimating meltwater generation and sizing meltwater storage. If water applications are performed manually, 3 or more layers, approximately $1 \mathrm{~cm}$ deep, should be added to the sand once the average ambient air temperature is below $-10^{\circ} \mathrm{C}$. Sufficient time should also be provided for the previous layer of water to freeze prior to the addition of the subsequent layer. Once the base of the freeze-thaw bed is a solid base of sand and ice, application of sludge layers can begin.

\subsubsection{Application of Sludge Layers to the Freeze-Thaw Bed}

During pilot-scale tests, sludge layers approximately $10 \mathrm{~cm}$ thick appeared to freeze completely within 1 or 2 days at temperatures of $-10^{\circ} \mathrm{C}$, resulting in good particle consolidation and drainability during the thawing process. However, the estimated sludge freezing period is over 5 days, for a $10 \mathrm{~cm}$ sludge layer with an initial temperature of $5^{\circ} \mathrm{C}$, when the sludge freezing equation and proportionality coefficient, $\mathrm{m}=1.45 \mathrm{~cm}\left({ }^{\circ} \mathrm{C} \cdot \text { day }\right)^{-1 / 2}$, presented by Kinsley et al., 2012 are used (Equation 2, page 47). The main reason for the differences are likely due to the small dimensions of the pilot unit $(60 \mathrm{~cm} \times 60 \mathrm{~cm})$, which allowed frost penetration to occur radially inward from all 
directions. This likely accelerated sludge freezing, or made sludge layers appear completely frozen. Furthermore, the high-powered industrial mobile freezer unit used to freeze the sludge may have produced temperatures lower than $-10^{\circ} \mathrm{C}$ for periods when the compressor was active.

During natural freeze-thaw sludge dewatering applications, diurnal temperature fluctuations can play a significant role. The average daily temperatures used to estimate sludge freezing times should be used with caution, since daily high temperatures may reduce the rate of sludge freezing, particularly during sunny days. Due to the northern location of this particular facility, and the relatively small volume of sludge requiring treatment, the number of days with sufficiently low temperatures for sludge freezing is not a concern.

Equation 2 was used to estimated that an $8 \mathrm{~cm}$ layer of sludge, with an initial temperature of $5^{\circ} \mathrm{C}$, would take approximately 3.5 days to completely freeze at an average temperature of $-10^{\circ} \mathrm{C}$, and as long as 7 days at $-5^{\circ} \mathrm{C}$. However, winter temperatures at the facility in northern Canada consistently reach $-20^{\circ} \mathrm{C}$, which would reduce the freezing time for an $8 \mathrm{~cm}$ sludge layer to $<2$ days, and temperatures can reach as low as $-40^{\circ} \mathrm{C}$, which would reduce the freezing time to $<1$ day. This offers a significant amount of potential process variability. A figure showing the estimated freezing times required for various sludge layer depths and average daily freezing temperatures is presented as Figure C3 in Appendix C3 on page 387. 
Assuming an average freezing temperature of $-10^{\circ} \mathrm{C}$ and a 4-day period between successive $8 \mathrm{~cm}$ sludge layers, it would take a total 112 days to freeze all 28 layers of sludge, totalling the annual volume of sludge generated at the facility $\left(78 \mathrm{~m}^{3}\right)$. However, during peak winter months, sludge layers can be added every other day $\left(T=-20^{\circ} \mathrm{C}\right)$, and even every day $\left(T=-40^{\circ} \mathrm{C}\right)$, in which case the total number of days for sludge application would be much lower.

\subsubsection{Meltwater Collection and Management}

The meltwater volume collected during the pilot-scale freeze-thaw study (presented in Chapter 5) was $86.8 \%$ of the initial sludge volume, and was a high-strength wastewater which required biological treatment. Assuming $78 \mathrm{~m}^{3}$ of sludge was added to the freezethaw bed during winter months, approximately $68 \mathrm{~m}^{3}$ of meltwater would be generated during the thawing period.

According to Equation 3 (page 48), the $2.2 \mathrm{~m}$ depth of sludge would take 93 days to thaw at an average daily temperature of $15^{\circ} \mathrm{C}$, and 70 days at $20^{\circ} \mathrm{C}$. Despite the relatively long thawing times predicted, the results from the pilot-scale study suggest that a peak factor should be used to estimate the meltwater storage volume required for adequate equalisation of the return meltwater flow back into the WWT unit.

Flow equalisation is required for the return meltwater flow to avoid hydraulically overloading the existing WWT unit, and more importantly, the high-strength meltwater could shock the biological treatment system due to sudden increases in organic $\left(\mathrm{BOD}_{5}\right.$ or COD) and nutrient loading (Nitrogen and Phosphorous) if the return flow rate is too 
high. Therefore, meltwater storage is required so that peak daily meltwater volumes can be stored and gradually pumped to the headworks of the WWT unit.

Assuming a $15^{\circ} \mathrm{C}$ thaw temperature and resulting 93 day thaw period, the average daily flow of meltwater would be $0.7 \mathrm{~m}^{3} /$ day, which is $5 \%$ of the average daily wastewater flow. This would increase the $\mathrm{BOD}_{5}, \mathrm{NH}_{3}-\mathrm{N}, \mathrm{TN}$ and TP loadings to the $\mathrm{RBC}$ by $29,27,21$ and $21 \%$ respectively, neglecting any effects of the PST. It is expected that primary settling would have very little effect on the organic and nutrient loadings because the meltwater from the pilot unit had an average TSS of only $51 \mathrm{mg} / \mathrm{L}$ without the use of ferrate(VI) (Chapter 5), and lab-scale experiments with ferrate(VI) further reduced the turbidity of the meltwater samples, suggesting that the highly solubilised meltwater will not be affected by primary settling (Chapters 7,8 ). Details related to the calculation of meltwater collection rates, and organic loadings are presented in Appendix C4 (p. 388).

Fluctuations in the organic and nutrient loadings occur regularly throughout the year (Henderson and Atwater, 1995), therefore fluctuations $<30 \%$ in the average loadings is not a significant concern. However, despite the relatively small increases in the $\mathrm{NH}_{3}-\mathrm{N}$ loading, because the daily loading from the wastewater was already high, the added $\mathrm{NH}_{3}-\mathrm{N}$ would increase the total loading to $1.8 \mathrm{~g} \mathrm{NH}_{3}-\mathrm{N} / \mathrm{m}^{2}$ day, which is above the recommended maximum design loading of $1.5 \mathrm{~g} \mathrm{NH}_{3}-\mathrm{N} / \mathrm{m}^{2}$ day for RBCs (Metcalf and Eddy, 2003). Studies by Henderson and Atwater (1995) demonstrated that while $\mathrm{NH}_{3}-\mathrm{N}$ loadings $<1.5 \mathrm{~g} \mathrm{NH}_{3}-\mathrm{N} / \mathrm{m}^{2}$ day resulted in $>95 \%$ reduction of $\mathrm{NH}_{3}-\mathrm{N}$ in $\mathrm{RBCs}$, loadings ranging from 1.5 to $3.0 \mathrm{~g} \mathrm{NH}_{3}-\mathrm{N} / \mathrm{m}^{2}$ day resulted in 80 to $90 \%$ reduction of $\mathrm{NH}_{3}-\mathrm{N}$, 
indicating some effects of overloading, however removal rates remained high. This suggests that for thawing temperatures of $15^{\circ} \mathrm{C}$, the meltwater generation rate is low enough that the average daily volume of meltwater can be recirculated to the headworks of the WWT unit at a rate of approximately $30 \mathrm{~L} / \mathrm{hr}$ throughout a 24 hour period, without overloading the RBC unit.

However, an average daily temperature of $20^{\circ} \mathrm{C}$ would increase the average daily meltwater collection rate to $1.0 \mathrm{~m}^{3} /$ day. A similar analysis using an average thawing temperature of $20^{\circ} \mathrm{C}$ suggests that the organic and nutrient loading on the RBC unit would increase by $38,36,28$, and $28 \%$ for $\mathrm{BOD}_{5}, \mathrm{NH}_{3}-\mathrm{N}$, TN and TP respectively. Although this would increase the net organic and nutrient loading to the high end of typical design values, the added $\mathrm{BOD}_{5}$, TN and TP would not overload the RBC unit. On the other hand, the net $\mathrm{NH}_{3}-\mathrm{N}$ loading would increase to $1.9 \mathrm{~g} \mathrm{NH}_{3}-\mathrm{N} / \mathrm{m}^{2}$ day, which is $>25 \%$ higher than the maximum recommended $\mathrm{NH}_{3}-\mathrm{N}$ loading for RBCs. A

High $\mathrm{NH}_{3}-\mathrm{N}$ loadings can be lethal to suspended growth systems due to the increased oxygen demands and limited availability. To compensate for increased oxygen requirements, RBCs can be rotated at a faster rate, which has been successful for the treatment of landfill leachate with $\mathrm{NH}_{3}-\mathrm{N}$ concentrations $>2000 \mathrm{mg} / \mathrm{L}$ (Henderson and Atwater, 1995). However, increased loading can also be reduced by limiting the flow rate of meltwater back into the WWT unit. To demonstrate this, a 7-day period with an average daily temperature of $20^{\circ} \mathrm{C}$ was used to generate a peak volume of meltwater that would require flow control when recirculated to the headworks of the WWT unit. 
At peak meltwater generation rates, daily recirculation of the meltwater would reach and estimated $1 \mathrm{~m}^{3} /$ day (42 L/hr), which would potentially overload the RBC, therefore peak meltwater generated would need to be throttled by up to $45 \%$ during a 10 day equalisation period, and an additional storage volume of approximately $500 \mathrm{~L}$ would be required.

\subsubsection{Collection and Disposal of the Final Sludge Cake}

Once the sludge has thawed and drained completely, the remaining sludge cake will continue to dry, depending on local weather conditions. In the event of rain, additional time may be warranted to reduce the mass and volume of the sludge requiring transportation and disposal. For large-scale applications, concrete lined drainage systems may allow heavy machinery to remove the sludge cake, however smaller freeze-thaw beds with sand and drainage pipes should be excavated without driving on the freezing bed.

Follow-up experiments must be conducted to ensure that the biosolids are suitable for land application. For low-grade biosolids, only sufficient levels of inactivation of fecal coliform must be achieved, generally <2, 000, $000 \mathrm{MPN} / \mathrm{g}$ DS, whereas the high-grade biosolids, require additional levels of treatment, which vary by jurisdiction.

RBC sludge from the remote facility following ferrate(VI) oxidation and freeze-thaw stabilisation and dewatering, would require fecal coliform $<1000$ MPN/g DS to meet P1 criteria, which have the fewest restrictions for land application of biosolids in Quebec. The combined inactivation resulting from the ferrate $(\mathrm{VI})$ oxidation and freeze-thaw 
stabilisation and dewatering have been shown to result in up to 7.7-log inactivation, which makes the co-treatment technology highly variable depending on the particular design requirements. 


\section{CONCLUSIONS}

\subsection{FREEZE-THAW SLUDGE STABILISATION AND DEWATERING}

Freeze-thaw with gravity meltwater drainage is a simple and effective means to dewater wastewater sludges, thereby reducing sludge volumes. Freeze-thaw experiments with RBC sludge resulted in an $88 \%$ reduction in sludge volume during the pilot-unit study, and bench-scale tests, using the simulated drainage beds, reduced the volumes of primary and anaerobically digested sludges by up to 79 and $87 \%$ respectively.

Furthermore, freeze-thaw treatment also reduced the concentrations of pathogens and indicator organisms. In some samples, freeze-thaw resulted in $>3$-log inactivation of fecal coliform, however generally freeze-thaw resulted in approximately 2.5-log inactivation of fecal coliform in the primary sludge samples. Similarly, freeze-thaw resulted in approximately 1-log inactivation of Salmonella during the pilot-unit study with RBC sludge.

However, there was no clear trend of increasing level of inactivation with increasing time frozen for the samples that were kept frozen for 1, 8 and 15 days, which suggests that when the time frozen is $\leq 15$ days, the inactivation of fecal coliform caused by freeze-thaw is primarily the result of the freezing process. Similarly, for the range of freezing rates generated, which varied from 3 to $9 \mathrm{~mm} / \mathrm{hr}$, there was no trend in the level of inactivation of fecal coliform, or the drainability of the sludge during the 12-hour thawing process. 


\subsection{FERRATE(VI) TREATMENT ON SLUDGE}

The following section describes the effects of (stand-alone) potassium ferrate(VI) treatments on sludge, following a 15 -minute reaction period. Low doses $(0.5 \mathrm{~g} / \mathrm{L})$ of potassium ferrate(VI) generally did not have a significant effect on any of the measured parameters of the primary sludge. This was likely due to the presence of various oxidisable materials in the sludge and the resulting competing reactions, which quickly consumed the unstable ferrate(VI) ions. Similarly, while $1.0 \mathrm{~g} / \mathrm{L}$ of potassium ferrate(VI) did result in sludge solubilisation and a decrease in the concentration of sulphide, inactivation of fecal coliform was insignificant. However, potassium ferrate(VI) doses as low as $1.0 \mathrm{~g} / \mathrm{L}$ did result in 16 and 22\% reductions (average and overall) in the concentrations of estrogens in the anaerobically digested sludge samples that were spiked with a low concentration range $(15-75 \mathrm{ng} / \mathrm{mL})$ and a high concentration range $(60-300 \mathrm{ng} / \mathrm{mL})$ of estrogens, respectively.

Potassium ferrate(VI) doses of $5.0 \mathrm{~g} / \mathrm{L}$ resulted in significant degree of sludge solubilisation, increasing the sCOD by up to $69 \%$, which coincided with 520 and $400 \%$ increases in the concentrations of soluble proteins and soluble carbohydrates respectively, and a $190 \%$ increase in the CST of the sludge. Furthermore, the concentration of sulphide was reduced by $89 \%$. However, inactivation of fecal coliform using $5.0 \mathrm{~g} / \mathrm{L}$ of potassium ferrate(VI) in primary sludge was only 0.5 to $1.0-\mathrm{log}$, whereas 2-log inactivation achieved using $5.0 \mathrm{~g} / \mathrm{L}$ on anaerobically digested sludge. 
Higher doses of potassium ferrate(VI) were capable of reducing the concentration of fecal coliform to below detectable levels, representing $>8$-log inactivation in some cases. However despite recent advancements in electrochemical ferrate(VI) generation, the cost of ferrate(VI) is high compared to other common chemical oxidants. Therefore, depending on the initial concentration of fecal coliform in the sludge and the target concentration required for disposal, potassium ferrate( $\mathrm{VI})$ doses in excess of $10 \mathrm{~g} / \mathrm{L}$ were unnecessary, particularly when the ferrate(VI) treatments were combined with freezethaw treatment.

\subsection{FREEZE-THAW AND FERRATE(VI) CO-TREATMENTS}

Preliminary co-treatment experiments using potassium ferrate( $\mathrm{VI})$ as a pre- and posttreatment with freeze-thaw conditioning of primary sludge in bottles, demonstrated that while ferrate $(\mathrm{VI})$ and freeze-thaw treatments both contribute to the inactivation of fecal coliform, the inactivation resulting from the combined treatments was not the sum of the individual treatments. Furthermore, in most cases, pre-treatment with potassium ferrate( $\mathrm{VI})$ was more effective than post-treatment, for the overall inactivation of fecal coliform. This was most likely due to the additional contact time for the ferrate(VI) pretreatments to react with the sludge while it was freezing, as opposed to the ferrate(VI) post-treatment tests, which were sampled and diluted immediately following a 15minute reaction period. In addition, some experiments with the pure culture E. coli sample suggested that ferrate post-treatment following freeze-thaw may be less effective at lower temperatures, when the sample has recently thawed. 
Subsequent experiments using the simulated drainage beds also demonstrated that ferrate(VI) pre-treatment did not significantly reduce the drainability of the sludge during the thawing process, despite a large degree of sludge solubilisation marked by increases in SCOD, soluble proteins and soluble carbohydrates. Furthermore, the meltwater had a lower turbidity than the meltwater samples collected from the standalone freeze-thaw samples, and when the ferrate(VI) pre-treated sludge cake samples were diluted following freeze-thaw with meltwater drainage, the sludge particles were compact sludge granules which readily settled. This suggests that the spent ferrate(VI) acted as a coagulant and improved particle consolidation, in addition to the effects of freezing.

Pre-treatment with $5.0 \mathrm{~g} / \mathrm{L}$ of potassium ferrate(VI) followed by freeze-thaw also reduced the concentration of fecal coliform in anaerobically digested sludge following freeze-thaw dewatering to below CP1 regulated limits for land application of biosolids in Ontario. For primary sludge, $15.0 \mathrm{~g} / \mathrm{L}$ reduced the concentration of fecal coliform to below CP1 limits, and $22.0 \mathrm{~g} / \mathrm{L}$ of potassium ferrate(VI) followed by freeze-thaw reduced the concentration of fecal coliform in the meltwater $(<0.18 \mathrm{MPN} / \mathrm{mL})$ and sludge cake (<12.0 MPN/g DS) to below the detection. This demonstrates that potassium ferrate(VI) additions and freeze-thaw dewatering can be used to achieve a desired level of fecal coliform inactivation, and produce dewatered sludge cake suitable for land application under the regulated limits for either CP1 or CP2 sludge categories. 
However for some of the primary sludge samples, pre-treatment with $5.0 \mathrm{~g} / \mathrm{L}$ of potassium ferrate(VI) followed by freeze-thaw with meltwater drainage, may have stimulated fecal coliform regrowth in the meltwater samples collected during the 12hour thawing process. Martel and Diener (1991b) also made a similar observation in that the meltwater became odorous when it was not removed from the collection reservoir periodically, thus indicating the potential for putrefaction and the need for treatment and disposal. The meltwater was a high-strength, low-turbidity wastewater which could be treated either by recirculation to the headworks of an existing WWT system, or to an on-site engineered wetland or other biological treatment unit. Regardless, a follow-up set of experiments were performed to evaluate the potential for regrowth, and also the effect increasing the ferrate(VI) dose further.

The follow-up experiments using $5.1 \mathrm{~g} / \mathrm{L}$ of potassium ferrate(VI) resulted in greater fecal coliform inactivation than the stand-alone freeze-thaw treatments, suggesting that potassium ferrate(VI) did not contribute to any regrowth during the 12-hour thawing period. Similarly, pre-treatment with $8.0 \mathrm{~g} / \mathrm{L}$ of potassium ferrate(VI) improved fecal coliform inactivation caused by stand-alone freeze-thaw by $>1-\log$ in the sludge cake and $>2.5-\log$ in the meltwater, while pre-treatment with $22.0 \mathrm{~g} / \mathrm{L}$ reduced the concentration of fecal coliform to below the detection limit in the sludge cake $(<12.0$ MPN/g DS) and meltwater $(<0.18 \mathrm{MPN} / \mathrm{mL})$. This suggests that ferrate(VI) can also be used to reduce the potential for regrowth of pathogens once the biosolids are applied to land, however no regrowth experiments were conducted on cake samples applied to land. 
In most cases however, reducing the concentration of fecal coliform to below the detection limit is not required and not cost effective, particularly for meltwater. Instead, more conventional on-site biological wastewater treatment systems could be used. For small-scale applications, an engineered wetland, trickling filter, RBC or septic tank and soil adsorption system may suffice, while larger freeze-thaw dewatering applications may require a more conventional biological treatment system such as activated sludge.

\subsection{RECOMMENDATIONS AND GUIDELINES}

Co-treatment of sewage sludge using natural freeze-thaw with potassium ferrate(VI) pre-treatment can be tailored to the design requirements of a particular application. The process can be used to compliment an existing sewage or sludge treatment process, or sludge pre-treatment with ferrate( $\mathrm{VI})$ followed by freeze-thaw can be used as a complete process to dewater sludge and inactivate fecal coliform in the sludge cake and meltwater. For instance, anaerobically digested sludge was treated and dewatered to $<100 \mathrm{MPN} / \mathrm{g}$ DS at $>12 \%$ TS using $5.0 \mathrm{~g} / \mathrm{L}$ of potassium ferrate, which is below the regulated limits for CP1 biosolids in Ontario. Furthermore, raw primary sludge was treated to below CP2 requirements for fecal coliform using $5.1 \mathrm{~g} / \mathrm{L}$ of potassium ferrate(VI) followed by freeze-thaw, and to below CP1 requirements using $<15 \mathrm{~g} / \mathrm{L}$ of potassium ferrate(VI) followed by freeze-thaw.

On the other hand, stand-alone freeze-thaw sludge treatment was not only very effective at dewatering sludge and reducing the sludge volume by up to $88 \%$, there was also approximately $2.5-\log$ inactivation of fecal coliform achieved in the sludge cake. 
This suggests that for cases where the concentration of fecal coliform is already low, stand-alone freeze-thaw may be sufficient for the stabilisation and dewatering of wastewater sludge for land application of the biosolids. Furthermore, while controlled experiments demonstrated that the freezing process causes fecal coliform inactivation, natural (seasonal) freeze-thaw will introduce combinations of a variety of environmental factors such as multiple freeze-thaw cycles, solar desiccation and air-drying, which are known to increase pathogen inactivation (Gao et al., 2006) and sludge volume reduction (Hedström and Hanaeus, 1999, Hellström and Kvarnström, 1997).

In certain circumstances, stabilised sludge may require additional treatment to satisfy the requirements for land application of biosolids. This may be due to a variety of circumstances such as poorly functioning upstream treatment infrastructure, the presence of a particular (group of) persistent, high-concentration or highly toxic compound(s), or due to the environmentally sensitive nature of the sludge disposal site or sludge disposal regulations. As discussed earlier, the physical, biological and chemical nature of sludge depends heavily of the characteristics of the source wastewater. For example, wastewater and sludge samples from 2 swine farms contained high levels of hormones used for animal breeding control and growth promotion (Liu et al., 2015), while sludge from a radioactive waste storage tank contained high levels of metals (Al, $\mathrm{Bi}, \mathrm{Cr}, \mathrm{Fe}, \mathrm{Mn}$, Si and $\mathrm{U})$ and mixed fission products $\left({ }^{137} \mathrm{Cs},{ }^{90} \mathrm{Sr},{ }^{99} \mathrm{TC}\right.$, uranium and transuranic elements) (Sylvester et al., 2001). In such circumstances, ferrate(VI) treatment can be used to target these chemical and/or biological properties of sludge, and improve the quality of the biosolids. 
However, despite a decrease in the cost of ferrate(VI) in recent years, it remains to be a relatively expensive chemical oxidant, which would increase the cost and complexity of natural freeze-thaw sludge dewatering. Therefore, ferrate(VI) additions should only be used as a polishing agent, if needed, to compliment natural freeze-thaw sludge stabilisation and dewatering, or used to target a certain high level of target compounds such as pathogens and odours, PPCPs, heavy metals, and other organic chemicals that may be present in sludge.

\subsection{CONTRIBUTIONS AND FUTURE WORK}

This research was the first to evaluate the combination of ferrate(VI) oxidation and freeze-thaw sludge stabilisation and dewatering, and among the first to examine the oxidation of EDCs in sludge using ferrate(VI). In addition, freeze-thaw dewatering was achieved using pilot-scale and bench-scale sludge dewatering beds, and several process operational parameters such as ferrate(VI) dose, freezing temperature, freezing rate and time frozen, were varied to evaluate the impacts on sludge stabilisation and dewatering. The research also characterised in detail, the individual and combined effects of ferrate(VI) oxidation and freeze-thaw treatments on the physical, chemical and biological characteristics of various sludge types when simulated drainage beds were used to separate meltwater from the sludge cake during the thawing process. The effects of the operational parameters were evaluated on both meltwater and the sludge cake remaining following freeze-thaw dewatering. 
This research offers new insight into ferrate(VI) treatment for sludge for stabilisation, and the ability of the combined effects of ferrate(VI) oxidation and freeze-thaw sludge dewatering. Sludge treatments using ferrate( $\mathrm{VI})$ and freeze-thaw is an alternative way for communities in cold climates to stabilise and dewater wastewater sludges on-site, allowing for safe disposal of the treated biosolids on land. Furthermore, the cotreatment technology has the potential to remove particular recalcitrant target compounds such as hormones or other EDCs, which warrant further investigation.

However, the sludge matrix is a particularly difficult sample matrix for analysis. Furthermore, the physical, chemical and biological characteristics of sludge depend heavily on a variety of consistently variable factors, which means that the characteristics of the same sludge from the same treatment operations will change considerably throughout a given year. In particular, the micro- and even nano-concentrations of EDCs present in sludge are difficult to extract, identify and quantify. While extraction methods have improved considerably, more work is required in this area to generate consistent and reliable results related to the removal of EDCs and other micro-pollutants in sludge.

Future work related to this research should involve natural freeze-thaw applications which evaluate the effects of seasonal factors and variability such as sunshine, wind, rain, snow and temperature. Additionally, research related to a high-rate aerobic treatment system to degrade the high-strength, highly-solubilised and low TSS meltwater would offer additional process stability. Furthermore, additional research related to sludge post-treatment with ferrate(VI), following freeze-thaw stabilisation 
and dewatering, would fill knowledge gaps related to ferrate(VI) treatment of dewatered sludges. Treatment of dewatered sludges, with a reduced volume, would reduce ferrate(VI) demands and costs significantly.

Land application of biosolids is an effective way to dispose of wastewater sludges while recycling nutrients and organic matter back into the soil. However, proper measures must be taken to ensure the protection of human health and the environment. This means that more research should be conducted to ensure that there is no regrowth of pathogens in the sludge cake remaining following freeze-thaw, once it is applied to agricultural lands, particularly when ferrate( $\mathrm{VI})$ is used. Furthermore, research into the spread and effect of EDCs due to land application of biosolids must be investigated. 


\section{REFERENCES}

Abad, E., Martínez, K., Planas,C., Palacios, O., Caixach, J., Rivera, J. (2005) Priority organic pollutant assessment of sludges for agricultural purposes. Chemosphere 61(9), 13581369

Alsheyab, M., Jiang, J.Q., Stanford, C. (2010a) Engineering aspects of electrochemical generation of ferrate: A step towards it full scale application for water and wastewater treatment. Water Air Soil Poll 210(1-4), 203-210 doi 10.1007/s11270-009-0242-2

Alsheyab, M., Jiang, J.Q., Stanford, C. (2010b) Electrochemical generation of ferrate (VI): Determination of optimum conditions. Desalination 254(1-3), 175-178

Alsheyab, M., Jiang, J.Q., Stanford, C. (2009) On-line production of ferrate with electrochemical method and its potential application for wastewater treatment - A review. J Environ Manage 90(3), 1350-1356

Apedaile, E. (2001) A perspective on biosolids management. Can J Infect Dis 12(4), $202-$ 204

APHA (American Public Health Association), American Water Works Association, Water Pollution Control Federation (2005) Standard Methods for the Examination of Water and Wastewater. APHA / AWWA, Washington DC.

Apul, O.G., Atalar, I., Zorba, T., Sanin, F.D. (2010) The dewaterability of disintegrated sludge samples before and after anaerobic digestion. Dry Technol 28(7):901-909 
Auriol, M., Filali-Meknassi, Y., Tyagi, R.D., Adams, C.D., Surampalli, R.Y. (2006) Endocrine disruptin compounds removal from wastewater, a new challenge. Process Biochem 41(3):525-539

AXYS, 2012 Method Summary of Axys Method MLA-072 Rev. 03 Ver 02: Analysis of Hormones in Solid and Aqueous Samples by LC-MS/MS. (proprietary)

Bartzatt, R., Cano, M., Johnson, L., Nagel, D. (1992) Removal of toxic metals and nonmetals from contaminated water. J Toxicol Environ Health 35(4), 205-210

Belhaj, D., Jaabiri, I., Kallel, M., Zhou, J.L. (2014) Occurrence and removal of steroidal estrogens in Centre Eastern Tunisia municipal sewage treatment plant. Desalination Water Treatment 52(10-12):2330-2339

Benn, D., Doe, P.W. (1969) The disposal of sludge by the freezing and thawing process. Filtr Separat 6, 383-389

Bohm, N., Kulicke, W.M. (1997) Optimization of the use of polyelectrolytes for dewatering industrial sludge in various regions. Colloid Poly. Sci 275, 73-8

Bougrier, C., Albasi, C., Delgenès, J.P., Carrère, H. (2006) Effect of ultrasonic, thermal and ozone pre-treatments on waste activated sludge stabilization and anaerobic biodegradability. Chem Eng Process 45(8), 711-718

Bougrier, C., Carrère, H., Delgenès, J.P., (2005) Solubilisation of waste activated sludge by ultrasonic treatment. Chem Eng J 106(2), 163-169 
Bradford, M.M. (1976) A rapid and sensitive method for the quantification of microgram quantities of protein utilizing the principle of protein-dye binding. Analytical Biochemistry 72(1-2), 248-254

Bruce, A.M., Fisher, W.J. (1984) Sludge stabilisation - methods and measurement. Published in: Sewage Sludge Stabilisation and Disinfection, Bruce, A. (editor). Ellis Horwood, Chichester, England, pp. 23-47

Bruus, J.H., Nielson, P.H., Keiding, K., (1992) On the stability of activated sludge flocs with implications to dewatering. Water Res 26(12), 1597-1604

Cajthaml, T., Křesinová, Z., Svobodová, K., Sigler, K., Řezanka, T. (2009) Microbial transformation of synthetic estrogen 17 $\alpha$-ethinylestradiol. Environ Pollut 157(12):33253335

Canadian Council of Ministers of the Environment (CCME) (2010) A review of current Canadian legislative framework for wastewater biosolids. PN 1446 ISBN 978-1-89699795-7 PDF

Canfield, C., Sutphen, R.L. (1982) Mother nature as sludge thickener. Water Eng Manag $129(13), 22-24$

CEC (Council of the European Communities) (1986) Council Directive of 12 June 1986 on the protection of the environment, and in particular of soil, when sewage sludge is used in agriculture (86/278/EEC). Official J. European Communities L 181, 6-12

Chalmers, B. (1959) How water freezes. Sci Am 200, 114-122 
Chang, M.R., Chiang, L.I., Lee, D.J., Liu, J.C., Wu, N.M., Chen, W.C., Hsu, B.M. (2004)

Conditioning of wastewater sludge from science-based industrial park using freezing and thawing. J Environ Eng-ASCE 130(12), 1552-1555

Chang, I.L., Lee, D.J. (1998) Ternary expression stage in biological sludge dewatering. Wat Res 32(3), 905-914

Chen, Y.-C., Higgins, M.J., Beightol, S.M., Murthy, S.N., Toffey, W.E. (2011) Anaerobically digested biosolids odor generation and pathogen indicator regrowth after dewatering. Water Res 45(8):2616-2626

Chu, C.P., Lee, D.J., Peng, X.F. (2004) Structure of conditioned sludge flocs. Water Res 38(8), 2125-2134

Chu, C.P., Feng, W.C., Chang, B.-V., Chou, C.H., Lee, D.J. (1999) Reduction of microbial density level in wastewater activated sludge via freezing and thawing. Water Res 33(16), $3532-3535$

Chung, H.Y., Ju, S.P., Lee, D.J. (2003) Hydrodynamic drag force exerted on activated sludge floc at intermediate Reynolds number. J Colloid Interf Sci 263(2), 498-505 Clarke, B.O., Smith, S.R. (2011) Review of 'emerhing' orgainc contaminants in biosolids and assessment of international research priorities for the agricultural use of biosolids. Environ Int 37(1), 226-247

Clements, G.S., Stephenson, R.J., Regan, C.J. (1950) De-watering of sludge. Chem-Age 63(1636), 708-710 
Corte, A.E. (1962) The frost behavior of soils. Field and laboratory data for a new concept. Part1: Vertical sorting; Part II: Horizontal sorting. U.S. Army Cold Regions Research and Engineering Laboratory, Corps of Engineers, Research Report 85

Czajka, C.P., Londry, K.L. (2006) Anaerobic biotransformation of estrogens. Sci Total Environ 367:932-941

de Luca, S., Idle, C.N., Chao, A.C. (1996) Quality improvement of biosolids by ferrate(VI) oxidation of offensive odour compounds. Water Sci Technol 33(3), 119-130

Delaude, L., Laszlo, P. (1996) A novel oxidising agent based on potassium ferrate(VI). J Org Chem 61(18), 6360-6370

Desjardins, M.-A., Brière, F.G. (1996) Conditionnement et déshydration de boues d'étangs facultatifs à l'aide du gel - dégel naturel: résultats d'essais. Can J Civil Eng 23(2), 323-339

Devai, I., Delaune, R.D. (2002) Effectiveness of selected chemicals for controlling emission of malodorous sulfur gases in sewage sludge. Environ Techno/ 23(3), 319-329

Diak, J., Örmeci, B., Proux, C., (2011) Freeze-thaw treatment of RBC sludge from a remote mining exploration facility in subarctic Canada. Water Sci Technol 63(6), 13091313

Ding, L., Liang, H.C., Li, X.C. (2012) Oxidation of $\mathrm{CH}_{3} \mathrm{SH}$ by in situ generation of ferrate(VI) in aqueous alkaline solution for odour treatment. Sep Purif Technol 91, 117-124 
Djerassi, C., Miramontes, L., Rosenkranz, G., Sondheimer, F. (1954) Steroids. LIV. ${ }^{1}$ Synthesis of $19-N o v-17 \alpha$-ethynyltestosterone and 19-Nor-17 $\alpha$-methyltestosterone ${ }^{2} . J$ Am Chem Soc 76(16):4092-4094

El-Gohary, F., Tawfik, A., Mahmoud, U. (2010) Comparative study between chemical coagulation/precipitation (C/P) versus coagulation/dissolved air flotation (C/DAF) for pre-treatment of personal care products (PCPs) wastewater. Desalination 252(1-3), 106112

Environment Canada (2006) Atlantic Canada wastewater guidelines manual for Collection, Treatment, and Disposal. Online Document. Available: www.novascotia.ca/nse/water/docs/AtICanStdGuideSewage.pdfhttp://www.thermopile project.com/wp-content/uploads/2014/06/WERF EndocrineDisruptors.pdf, Accessed: Aug. 2015

Erdincler, A., Vesilind, P.A. (2003) Effect of sludge water distribution of the liquid-solid separation of a biological sludge. J Environ Sci Health A38(10), 2391-2003

Ezekwo, G., Tong, H.M., Gryte, C.C. (1980) On the mechanism of dewatering colloidal aqueous solutions by freeze-thaw processes. Water Res 14(8), 1079-1088

Faigle, J.W.; Schenkel, L. (1998) Pharmacokinetics of estrogens and progestogens. In: Fraser, I.S., Jansen, R.P.S., Lobo, R.A., Whitehead, M.I. (editors) Estrogens and Progestogens in Clinical Practice. London: Churchill Livingstone, pp 273-294.

Flint, O. (1972) The effect of freezing on precipitating solids. Acta Cryst A28, 548 
Furlong, E.T., Gray, J.L., Quanrud, D.M., Teske, S.S., Esposito, K., Marine, J., Ela, W.P., Stinson, B., Kolpin, D.W., Phillips, P.J. (2010) Fate of Estrogenic Compounds During Municipal Sludge Stabilization and Dewatering. Online Document. Available: www.thermopileproject.com/wpcontent/uploads/2014/06/WERF EndocrineDisruptors.pdf, Accessed: Mar.2015 Gabet-Giraud, V., Miège, C., Choubert, J.M., Martin Ruel, S., Coquery, M. (2010) Occurrence and removal of estrogens and beta blockers by various processes in wastewater treatment plants. Sci Total Environ 408(19), 4257-4269

Gao, W., Shao, Y., (2009) Freeze concentration for removal of pharmaceutically actives compounds in water. Desalination 249(1), 398-402

Gao, W., Leung, K., and Hawdon, N. (2009) Freezing inactivation of Escherichia coli and Enterococcus faecalis in water: Response to different strains. Water Environ Res 81(8), $824-830$

Gao, W., Smith, D.W., Li, Y. (2006) Natural freezing as a wastewater treatment method: E. Coli inactivation capacity. Water Res 40(12), 2321-2326

Gardner, J., Örmeci, B. (2010) Effect of aging, time, and temperature on fecal coliform counts during centrifugal dewatering and role of centrate in growth inhibition. Water Environ Res 82(2):51-61

Gendebien, A., Davis, B., Hobson, J., Palfrey, R., Pitchers, R., Rumsby, P., Carlton-Smith, C., Middleton, J. (2010) Environmental, economic and social impacts of the use of 
sewage sludge on land, Final Report, Part III: Project Interim Reports. Report prepared for: European Commission's Directorate-General Environment (DG Environment), Study Contract: DG ENV.G.4/ETU/2008/0076r, Feb. 2010

Godfree, A.F., Jones, F., Satchwell, M., Watson, D.C. (1984) The effectiveness of chemical disinfection on faecal bacteria in sludge. Published in: Sewage Sludge Stabilisation and Disinfection, Bruce, A. (editor). Ellis Horwood, Chichester, England, pp. 413-425

Gombos, E., Barkács, K., Felföldi, T., Vértes, C., Makó, M., Palkó, G., Záray, C. (2013) Removal of organic matters in wastewater treatment by ferrate(VI)-technology. Microchem J 107, 115-120

Gruber, F. (1970) Water removal from presedimentation and filtration sludges. German Patent $1,809,772$

Halde, R. (1980) Concentration of impurities by progressive freezing. Water Res 14, 575580

Hamid, H., Eskicioglu, C. (2013) Effect of microwave hydrolysis on transformation of steroidal hormones during anaerobic digestion of municipal sludge cake. Water Res 47(14), 4966-4977

Hamid, H., Eskicioglu, C. (2012) Fate of estrogenic hormones in wastewater and sludge treatment: A review of properties and analytical detection techniques in sludge matrix. Water Res 46(18), 5813-5833 
Harrison, E.Z., Oakes, S.R., Hysell, M., Hay, A. (2006) Organic chemicals in sewage sludges. Sci Total Environ 367(2-3), 481-497

Haug, R.T., Kuchenrither, R., Oerke, D., Prakasam, T., Soszynski, S., and Zenz, D. (1992) Sludge processing technology. Ch. 6 in Municipal Sewage Sludge Management, editors: C. Lue-Hing, D.R. Zenz, and R. Kuchenrither. Technomic Publishing, Lancaster PA, USA, pp. 223-298.

Havelaar, A.H. (1984) Sludge disinfection - an overview of methods and their effectiveness. Published in: Sewage Sludge Stabilisation and Disinfection, Bruce, A. (editor). Ellis Horwood, Chichester, England, pp.48-60

He, C., Li, X.-Z., Sharma, V.K., Li, S.-Y. (2009) Elimination of sludge door by oxidizing sulphur-containing compounds with ferrate(VI). Environ Sci Technol 43(15), 5890-5895

Hedström, A., Hanaeus, J. (1999) Natural freezing, drying, and composting for treatment of septic sludge. J Cold Reg Eng December 1999

Hellström, D., Kvarnström, E. (1997) Natural sludge dewatering. I: Combination of freezing, thawing, and drying as dewatering methods. J Cold Reg Eng March 1997 Henderson, J.P., Atwater, J.W. (1995) High ammonia landfill leachate treatment using anaerobic filter and rotating biological contactor. Can J Civil Eng 22(5), 992-1000

Higgins, M.J., Chen, Y., Murthy, S.N., Maas, N.A., Hendrickson, D. (2007) Reactivation and regrowth of non-culturable indicator bacteria in anaerobically digested biosolids after centrifuge dewatering. Water Res 41(3):665-673 
Hoekstra, P., Miller, R.D. (1967) On the mobility of water molecules in the transition layer between ice and a solid surface. J Colloid Interf Sci 25(2), 166-173

Hong, S.G., Young, Y.D., Chen, G.W., Chang, I.L., Hung, W.T., Lee, D.J. (1995)

Freeze/thaw treatment on waste activated sludge: an FTIR spectroscopic study. J Environ Sci Health A30(8):1717-1724

Hu, K., Jiang, J.-Q., Zhao, Q.-L., Lee, D.-J., Wang, K., Qiu, W. (2011) Conditioning of wastewater sludge using freezing and thawing: Role of curing. Water Res 45(18), 59695976

Huang, B., Li, X., Sun, W., Ren, D., Li., X., Li., X., Liu, Y., Li, Q., Pan, W. (2014) Occurrence, removal, and fate of preogestogens, androgens, estrogens, and phenols in six sewage treatment plants around Dianchi Lake in China. Environ Sci Pollut R 21(22):12898-12908

Hung, W.T., Feng, W.H., Tsai, I.H., Lee, D.J., Hong, S.G. (1997) Uni-directional freezing of waste activated sludges: vertical freezing versus radial freezing. Water Res 31(9), 22192228

Hung, W.T., Chang, I.L., Lin, W.W., Lee, D.J. (1996a) Unidirectional freezing of wasteactivated sludges: Effects of freezing speed. Environ Sci Technol 30(6), 2391-2396

Hung, W.T., Chang, I.L., Lee, D.J., Hong, S.G. (1996b) Sludge chemical composition changes under uni-directional freezing. Water Sci Technol 34(3-4), 525-531 Ivanov, A.N., Ivanov, N.A. (2010) Method for all-year decantation of municipal sewage sludge on sludge beds including winter conditions. Russian Patent RU2393122-C1 
Janex-Habibi, M.-L., Huyard, A., Esperanza, M., Bruchet, A. (2009) Reduction of endocrine disruptor emissions in the environment: The benefit of wastewater treatment. Water Res 43(6), 1565-1576

Jean, D.S., Lee, D.J., Chang, C.Y. (2001) Direct sludge freezing using dry ice. Adv Environ $\operatorname{Res}$ 5(2), 145-150

Jepsen, S.-E., Krause, M., Grüttner, H. (1997) Reduction of fecal streptococcus and Salmonella by selected treatment methods for sludge and organic waste. Water Sci Technol 36(11), 203-210

Jiang, J.-Q., Zhoo, Z. (2013) Removal of pharmaceutical residues by ferrate(VI). PLoS ONE 8(2): e55729. doi:10.1371/journal.pone.0055729

Jiang, J.Q., Stanford, C., Alsheyab, M. (2009) The online generation and application of ferrate for sewage treatment - A pilot scale trial. Sep Purif Technol 68(2), 227-231

Jiang, J.Q., Wang, S., Panagoulopoulos, A. (2007) The role of potassium ferrate(VI) in the inactivation of Escherichia coli and in the reduction of COD for water remediation. Desalination 210(1-3), 266-273

Jiang, J.Q. (2007) Research progress in the use of ferrate(VI) for the environmental remediation. J Hazard Mater 146(3), 617-623

Jiang, J.Q., Wang, S., Panagoulopoulos, A. (2006a) The exploration of potassium ferrate(VI) as a disinfectant/coagulant in water and wastewater treatment. Chemosphere 63(2), 212-219 
Jiang, J.Q., Panagoulopoulos, A., Bauer, M., Pearce, P. (2006b) The application of potassium ferrate for sewage treatment. J Environ Manage 79(2), 215-220

Jones-Lepp, T.L., Stevens, R. (2007) Pharmaceuticals and personal care products in biosolids/sewage sludge: the interface netween analytical chemistry and regulation. Anal Bioanal Chem 387(4), 1173-1183

Jiang, J.Q., Lloyd, B. (2002) Progress in the development and use of ferrate (vi) salt as an oxidant and coagulant for water and wastewater treatment. Water Res 36(6), 13971408

Kato, S., Jenkins, M.B., Fogarty, E.A., Bowman, D.D. (2002) Effects of freeze-thaw events on the viability of Cryptosporidium parvum oocysts in soil. J Parasitol 88(4), 718-722

Kimura, K., Hara, H., Watanabe, Y. (2010) Elimination of selected pharmaceuticals by biosolids from municipal wastewater treatment plants: importance of modest $\mathrm{pH}$ change and degree of mineralization. Water Sci Technol 62(5), 1084-1089 doi: 10.2166/wst.2010.356

Kinsley, C., Kennedy, K., Crolla, A. (2012) Modelling and application of an uncovered freezing bed technology for septage treatment. Can J Civil Eng 39(10), 1136-1144

Klaerschlamm, (2005) Waste from Municipal Waste Water Treatment Plants. Report for LANUV-NRW (Ministry of the Environment and Conservation, Agriculture and Consumer Protection of the German State of North Rhine-Westphalia), http://www.lanuv.nrw.de/abfall/pdf/Klaerschlamm 2005 EN.pdf, Accessed: Sept 2010 
Klee, A.J. (1993) A computer program for the determination of the most probable number and its confidence limits. J Microbiol Meth 18, 91-98.

Knocke, W.R., Trahern, P. (1989) Freeze-thaw conditioning of chemical and biological sludges. Water Res 23(1), 35-42

Kouloumbos, V.N., Shäffer, A., Corvini, P.F.-X. (2008) Impact of sewage sludge conditioning and dewatering on the fate of nonylphenol in sludge-amended soils. Water Res 42(14), 3941-3951

Layton, A.C., Gregory, B.W., Seward, J.R., Schultz, T.W., Sayler, G.S. (2000)

Mineralization of steroidal hormones by biosolids in wastewater treatment systems in Tennessee U.S.A.. Environ Sci Technol 34(18), 3925-3931

Lee, M.J., Kim, Y.S., Yoo, C.K., Song, J.H., Hwang, S.J. (2010) Sewage sludge reduction and system optimization in a catalytic ozonation process. Environ Technol B31(1), 7-14

Lee, D.J., Hsu, Y.H. (1994) Fast freeze/thaw treatment on excess activated sludges: Floc structure and sludge dewaterability. Environ Sci Technol 28(8), 1444-1449

Li, Y., Zhang, A. (2014) Removal of steroid estrogens from waste activated sludge using Fenton oxidation: Influencing factors and degradation intermediates. Chemosphere 105, 24-30

Li, Y.M., Zeng, Q.L., Yang, S.J. (2011) Removal and fate of estrogens in anaerobic-anoxicoxic activated sludge system. Water Sci Technol 63(1), 51-56 
Li, C., Li, X.Z., Graham, N., Gao, N.Y. (2008) The aqueous degradation of bisphenol A and steroid estrogens by ferrate. Water Res 42(1-2), 109-120

Li, C., Li, X.Z., Graham, N. (2005) A study of the preparation and reactivity of potassium ferrate. Chemosphere 61(4), 537-543

Lind, P.M., Öberg, D., Larsson, S., Kyle, C.E., Öberg, J., Rhind, S.M. (2010) Pregnant ewes exposed to multiple endocrine disrupting pollutants through sewage sludge-fertilized pasture show anti-estrogenic effect in their trabecular bone. Sci Total Environ 408(11), $2340-2346$

Liu, S.S., Ying, G.G., Liu, Y.S., Yang, Y.Y., He, L.Y., Chen, J., Liu, W.R., Zhao, J.L. (2015) Occurrence and removal of progestagens in two representative swine farms: Effectiveness of lagoon and digester treatment. Water Res 77, 146-154

Liu, S., Ying, G.G., Liu, Y.S., Peng, F.Q., He, L.Y. (2013) Degradation of norgestrel by bacteria from activated sludge: comparison to progesterone. Environ Sci Technol 47(18), 10266-10276

Liu, Z.H., Kanjo, Y., Mizutani, S. (2011) Removal of natural free estrogens and their conjugates in a municipal wastewater treatment plant. Clean - Soil, Air, Water 39(2):128-135

Liu, Z.H., Hashimoto, T., Okumura, Y., Kanjo, Y., Mizutani, S. (2010) Simultaneous analysis of natural free estrogens and their conjugates in wastewater by GC-MS. Clean 38(2):181-188 
Liu, Z.H., Kanjo, Y., Mizutani, S. (2009a) Urinary excretion rates of natural estrogens and adrogens from humans, and their occurrence and fate in the environment: A review. Sci Total Environ 407(18), 4975-4985

Liu, J.L., Nikolausz, M., Wang, X.C. (2009b) Biodegradation and detoxification of phenol using free and immobilized cells of Acinetobacter sp. XA05 and Shingomonas sp. FG03. J Environ Sci Heal A 44(2), 130-136

Liu, Z.H., Kanjo, Y., Mizutani, S. (2009c) Removal mechanisms for endocrine disrupting compounds (EDCs) in wastewater treatment - physical means, biodegradation, and chemical advanced oxidation: A review. Sci Total Environ 407:731-748

Logsdon, G.S., Edgerley, E. (1971) Sludge dewatering by freezing. J Am Water Works Ass 63(11) 740

Lumetta, G.L., Rapko, B.M. (1999) Removal of chromium from Hanford tank sludges. Separ Sci Technol 34(6-7), 1495-1506

Mácová, Z., Bouzek, K., Híveš, J., Sharma, V.K., Terryn, R.J., Baum, J.C. (2009) Research progress in the electrochemical synthesis of ferrate(VI). Electrochim Acta 54(10), 26732683

Mackay, K.M., Mackay, R.A. (1989) Introduction to Modern Inorganic Chemistry. Prentice Hall, Englewood Cliffs, NJ, pp. 228-229 
Madarász, J., Zbořil, R., Homonnay, Z., Sharma, V.K., Pokol, G. (2006) Thermal decomposition of iron(VI) oxides, $\mathrm{K}_{2} \mathrm{FeO}_{4}$ and $\mathrm{BaFeO}_{4}$, in an inert atmosphere. J Solid State Chem 179(5), 1426-1433

MAMROT (Ministère des Affaires municipales, des Régions et de l'Occupation du territoire) (2010) Évaluation de performance des ouvrages municipeaux d'assainissement des eaux (OMAE) pour l'année 2009. Online Document. Available: www.mamrot.gouv.qc.ca/pub/infrastructures/suivi ouvrages assainissement eaux/eva I perform rapport 2009.pdf, Accessed: Mar.2015

Martel, C.J. (2001) Design of freezing bed for sludge dewatering at McMurdo, Antarctica. US Army Corps of Engineers - Cold Regions Research \& Engineering Laboratory. Technical Report ERDC/CRREL TR, prepared for National Science Foundation Martel, C.J. (1993) Fundamentals of sludge dewatering in freezing beds. Water Sci Technol 28(1), 29-35

Martel, C.J., Diener, C.J. (1991a) A pilot-scale study of alum sludge dewatering in a freezing bed. J Am Water Works Ass, 83(12):51-55

Martel, C.J., Diener, C.J. (1991b) Pilot-scale studies of sludge dewatering in a freezing bed. Can J Civil Eng 18(4), 681-689

Martel, C.J. (1988a). Predicting freezing design depth of sludge freezing beds. J Cold Reg Eng 2(2), 145-156 
Martel, C.J. (1988b). Developing a thawing model for sludge freezing beds. Proc. $5^{\text {th }}$ Int. Conf. on Permafrost, International Permafrost Association, Aug 2-5

Mazur, P. (1986). Causes of injury in frozen and thawed cells. Fedn Proc Fedn Am Socs Exp Biol 24, Suppl. 15, S175-S182

McLean, D., Burns, N.J. (1997) Data Collection and Interpretation. Course Notes CHG 3337. University of Ottawa.

Metcalf and Eddy, Inc. (2003) Wastewater Engineering: Treatment and Reuse, $4^{\text {th }}$ ed., Tata-McGraw-Hill Pub. NY, pp. 930-935, 1499-1505, 1551-1559

MOE (Ministry of Environment) (2014) Nutrient Management Act, 2002 - Ontario Regulation 267/03 - last amendment: O. Reg. 284/13. Online Document. Available: http://www.e-laws.gov.on.ca/html/regs/english/elaws regs 030267 e.htm\#BK30, Accessed: Mar.2015

MOE (Ministry of Environment), Ministry of Agriculture, Food and Rural Affairs (1996) Guidelines for the utilization of biosolids and other wastes on agricultural land.

Montusiewicz, A., Lebiocka, M., Rozej, A., Zacharska, E., Pawlowski, L. (2010)

Freezing/thawing effects on anaerobic digestion of mixed sewage sludge. Bioresource Technol 101(10), 3466-3473

Morris, D.L. (1948) Quantitative determination of carbohydrates with Dreywood's anthrone reagent. Science 107(2775), 254-255 
Murmann, R.K., Robinson, P.R. (1974) Experiments utilising $\mathrm{FeO}_{4}{ }^{2-}$ for purifying water. Water Res 8(8), 543-547

Nagel, S.C., VomSaal, F.S., Thayer, K.A., Dhar, M.G., Boechler, M., Welshons, W.V. (1997) Relative binding affinity serum modified access (RBA-SMA) assay predicts the relative in vivo bioactivity of the xenoestrogens bisphenol A and octylphenol. Environ Health Persp 105(1), 70-76

Neyens, E., Baeyens, J.(2003) A review of classic Fenton's peroxidation as an advanced oxidation technique. J Hazard Mater B98(1-3), 33-50

Ning, B., Graham, N., Zhang, Y., Nakonechny, M., Gamal El-din, M. (2007) Degradation of endocrine disrupting compounds by ozone and AOPs. Ozone-Sci Eng 29(3), 153-176

Northcott, K.A., Snape, I., Scales, P.J., Stevens, G.W. (2005) Contaminated water treatment in cold region: an example of coagulation and dewatering modelling in Antarctica. Cold Reg Sci Technol 41(1):61-72

Nova Scotia Environment (2010) Guidelines for land application and storage of municipal biosolids in Nova Scotia, Revised March 2010. Online Document. Available: https://www.novascotia.ca/nse/water/docs/BiosolidGuidelines.pdf, Accessed: Mar.2015

OMAFRA (Ontario Ministry of Agriculture, Food and Rural Affairs) (2013) Information to Wastewater Treatment Plant Operators Relating To Non-Agricultural Source Materials 
(NASM). Online Document. Available:

http://www.omafra.gov.on.ca/english/nm/nasm/wastewater.htm, Accessed: Mar.2015

Örmeci, B., Vesilind, P.A. (2001) Effect of dissolved organic material and cations on freeze-thaw conditioning of activated and alum sludges. Water Res 35(18), 4299-4306

Parker, P.J., Collins, G. (1999) Dehydration of flocs by freezing. Environ Sci Technol 33(3), $482-488$

Parker, P.J., Collins, A.G., Dempsey, J.P. (1998) Effects of freezing rate, solids content, and curing time on freeze thaw conditioning of water treatment residuals. Environ Sci Technol 32(2), 383-387

Penman, A., Van Es, D.W. (1973) Winnipeg freezes sludge, slashes disposal costs 10 fold. CIV. ENGNG ASCE 43(11), 65-67

Pikal-Cleland, K.A., Rodriguez-Hornedo, N., Amidon, G.L., Carpenter, J.F. (2000) Protein denaturation during freezing and thawing in phosphate buffer systems: Monomeric and tetrameric $\beta$-galactosidase. Arch Biochem Biophys 384(2), 398-406

Pritchard, D.L., Penney, N., McLaughlin, M.J., Rigby, H., Scharz, K. (2010) Land application of sewage sludge (biosolids) in Australia: risks to the environment and food crops. Water Sci Technol 62(1), 48-57

Purdom, C.E., Hardiman, P.A., Bye, V.J., Eno, N.C., Tyler, C.R., Sumpter, J.P. (1994) Estrogenic effects of effluents from sewage treatment works. Chem Ecol 8(4), 275-285 
Read, J.F., Graves, C.R., Jackson, E. (2003) The kinetics and mechanism of the oxidation of the thiols 3-mercapto-1-propane sulfonic acid and 2-mercaptonicotinic acid by potassium ferrate. Inorg Chim Acta 348, 41-49

Read, J.F., Bewick, S.A., Graves, C.R., MacPherson, J.M., Salah, J.C., Theriault, A., Wyand, A.E.H. (2000) The kinetics and mechanism of the oxidation of S-methyl-L-cysteine, Lcystine and L-cysteine by potassium ferrate. Inorg Chim Acta 303, 244-255

Reed, S., Bouzoun, J., Medding, W. (1986) A rational method for sludge de-watering via freezing. J Water Pollut Con F 58(9), 911-916

Reinthaler, F.F., Feierl, G., Galler, H., Haas, D., Leitner, E., Mascher, F., Melkes, A., Posch, J., Winter, I., Zarfel, G., Marth, E. (2010) ESBL-producing E. coli in Austrian sewage sludge. Water Res 44(6), 1981-1985

Rhind, S.M., Kyle, C.E., Kerr, C., Osprey, M., Zhang, Z.I. (2011) Effect of duration of exposure to sewage sludge-treated pastures on liver tissue accumulation of persistent endocrine disrupting compounds (EDCs) in sheep. Sci Total Environ 409(19), 3850-3856 Saktaywin, W., Tsuno, H., Nagare, H., Soyama, T., Weerapakkaroon, J. (2005) Advanced sewage treatment process with excess sludge reduction and phosphorus recovery. Water Res 39(5), 902-910

Sanin, F.D., Vesilind, P.A., Martel, C.J. (1994) Pathogen reduction capabilities of freeze/thaw sludge conditioning. Water Res 28(11), 2393-2398 
Sawyer, C.N., McCarty, P.L. (1978) Chemistry for Environmental Engineering, $3^{\text {rd }}$ ed., McGraw-Hill Book Company, NY, 1978

Schink, T., Waite, T.D. (1980) Inactivation of f2 virus with ferrate (VI). Water Res 14(12), 1705-1717

Schleppenbach, F.X. (1984) Water filtration at Duluth. Report EPA 600/2-84-083, U.S. EPA, Cincinnati OH

Schuck, C.A., de Luca, S.J., Peralba, M., de Luca, M.A. (2006) Sodium ferrate(IV) and sodium hypochlorite in disinfection of biologically treated effluents. Ammonium nitrogen protection against THMs and HAAs. J Environ Sci Health A41(10), 2329-2343

Seol, Y., Zhang, H., Schwartz, F.W. (2003) A review of in situ chemical oxidation and heterogeneity. Environ Eng Geosci 9(1), 37-49

Sharpe, A.G. (1986) Inorganic Chemistry, $2^{\text {nd }}$ ed., Longman Scientific \& Technical, Burnt Mill, Harlow, England, pp. 601

Sharma, V.K., Yngard, R.A., Cabelli, D.E., Baum, J.C. (2008) Ferrate(VI) and ferrate(V) oxidation of cyanide, thiocyanide, and copper(II) cyanide. Radiat Phys Chem 77(6), 761767

Sharma, V.K., Mishra, S.K. (2006) Ferrate(VI) oxidation of Ibuprofen: A kinetic study. Environ Chem Lett 3(4), 182-185 
Sharma, V.K., Mishra, S.K., Ray, A.K. (2006) Kinetic assessment of the potassium ferrate(VI) oxidation of antibacterial drug sulfamethoxazole. Chemosphere 62(1), 128134

Silva, C.P., Otero, M., Esteves, V. (2012) Process for the elimination of estrogenic steroid homrmones from water: A review. Environ Pollut 165, 38-58

Silvares, O.M., Cravalho, E.G., Toscano, W.M., Higgins, C.E. (1975) The thermodynamics of water transport from biological cells during freezing. J Heat Transfer 97(4), 582-590

Stanford, C., Jiang, J.Q., Alsheyab, M. (2010) Electrochemical production of ferrate (iron $\mathrm{VI):} \mathrm{Application} \mathrm{to} \mathrm{the} \mathrm{wastewater} \mathrm{treatment} \mathrm{on} \mathrm{a} \mathrm{laboratory} \mathrm{scale} \mathrm{and} \mathrm{comparison} \mathrm{with}$ iron(III) coagulant. Water Air Soil Pollut 209(1), 483-488

Stumpf, M., Ternes, T.A., Wilken, R.-D., Vianna Rodrigues, S., Bauman, W. (1999) Polar drug residues in sewage and natural drug waters in the state of Rio de Janeiro, Brazil. Sci Total Environ 225(1-2), 135-141

Sumpter, J.P. (2007) The ecotoxicology of hormonally active micropollutants. Water Sci Technol 57(1), 125-130

Sylvester, P., Rutherford, L.A., Gonzalez-Martin, A., Kim, J., Rapko, B.M, Lumetta, G.J. (2001) Ferrate treatment for removing chromium from high-level radioactive tank waste. Environ Sci Technol 35(1), 216-221

Taber, S. (1930) The mechanics of frost heaving. J Geol 38(4), 303-317 
Tao, T., Peng, X.F., Lee, D.J. (2006a) Interaction between wastewater-sludge floc and moving ice front. Chem Eng Sci 61(10), 5369-5376

Tao, T., Peng, X.F., Lee, D.J., Hsu, J.P. (2006b) Micromechanics of wastewater sludge floc: Force-deformation relationship at cyclic freezing and thawing. J Colloid Interf Sci 298(2), 860-868

Ternes, T.A., Andersen, H., Gilberg, D., Bonerz, M. (2002) Determination of estrogens in sludge and sediments by liquid extraction and GC/MS/MS. Anal Chem 74(14), 3498-3504

Ternes, T.A., Stumpf, M., Mueller, J., Haberer, K., Wilken, R.D., Servos, M. (1999a) Behavior and occurrence of estrogens in municipal sewage treatment plants $-I$. Investigations in Germany, Canada and Brazil. Sci Total Environ 225(1-2), 81-90

Ternes, T.A., Kreckel, P., Mueller, J. (1999b) Behavior and occurrence of estrogens in municipal sewage treatment plants - II. Aerobic batch experiments with activated sludge. Sci Total Environ 225(1-2), 91-99

Thompson, G.W., Ockerman, G.W., Schreyer, J.M. (1951) Preparation and purification of potassium ferrate(VI). J Am Chem Soc 73(3), 1279-1281

Tremblay, L.A., Stewart, M., Peake, B.M., Gadd, J.B., Northcott, G. (2011) Review of the Risks of Emerging Organic Contaminants and Potential Impacts to Hawke's Bay. Prepared for: Hawke's Bay Regional Council. Cawthron Institute, Nelson, New Zealand Tyler, C.R., Filby, A.L., Bickley, L.K., Cumming, R.I., Gibson, R., Labadie, P., Katsu, Y., Liney, K.E., Shears, J.A., Silva-Castro, V., Urushitani, H., Lange, A., Winter, M.J., Iguchi, T., 
Hill, E.M. (2009) Environmental health impacts of equine estrogen derived from hormone replacement therapy. Environ Sci Technol 43(10), 3897-3904

U.S. EPA (United States Environmental Protection Agency) (2005a) Method 1681: Fecal Coliforms in Sewage Sludge (Biosolids) by Multiple Tube Fermentation using A-1 Medium. Washington DC, USA.

U.S. EPA (2005b) Method 1682: Salmonella in Sewage Sludge (Biosolids) by Modified Semisolid Rappaport-Vassiliadis (MSRV) Medium. Washington DC, USA.

U.S. EPA (2003) Environmental Regulations and Technology - Control of Pathogens and Vector Attraction in Sewage Sludge (Including Domestic Septage). Under 40 CFR Part 503, EPA/625/R-92/013, Revised July 2003

U.S. Geological Survey (2011) Emerging Contaminats in the Environment. Online Document. Available: http://toxics.usgs.gov/regional/emc/, Accessed: Mar.2015

Valo, A., Carrère, H., Delgenès, J.P. (2004) Thermal, chemical and thermo-chemical pretreatment of waste activated sludge for anaerobic digestion. J Chem Technol Biot 79(11), 1197-1203

Vesilind, P.A. and Örmeci, B. (2000) A modified capillary suction time apparatus for the measuring the filterability of super-flocculated sludges. Water Sci Technol 42(9), 135139

Veisilind, P.A. (1994) The role of water in sludge dewatering. Water Environ Res 66(1), 411 
Vesilind, P.A. and Martel, C.J. (1990) Freezing of water and wastewater sludges. J

Environ Eng ASCE, 116(5), 854-862

Waite, T.D. (2012) On-site production of ferrate for water and wastewater purification. American Laboratory. Online Document. Available:

http://www.americanlaboratory.com/914-Application-Notes/125788-On-Site-

Production-of-Ferrate-for-Water-and-Wastewater-Purification/, Accessed: Sept.2015

Waite, T.D. (1979) Feasibility of wastewater treatment with ferrate. J Environ Eng-ASCE

105(6), 1023-1034

Wang, Q., Fujisaki, K., Ohsumi, Y., Ogawa, H.I. (2001) Enhancement of dewaterability of thickened waste activated sludge by freezing and thawing treatment. J Environ Sci

Health A36(7), 1361-1371

Water UK (2006) Recycling of Biosolids on Land. Water UK, London SW1H 9Bt, UK, Online Document. Available: http://www.water.org.uk/publications/reports/recyclingbiosolids-land-briefing-pack, Accessed: Mar.2015

WEF (1995) Wastewater Residuals Stabilization, Manual of Practice no. FD-9, Water Environment Federation, Alexandria, VA

Wong, J.W.C., Selvam, A. (2009) Reduction of indicator and pathogenic microorganisms in pig manure through fly ash and lime addition during alkaline stabilization. J Hazard Mater 169(1-3), 882-889

Wood, C.W., Allanson, J.T. (1970) Dewatering of sludge. British Patent 1,812,019 
Wood, R.H. (1958) The heat, free energy and entropy of ferrate(VI) ion. J Am Chem Soc 80(9), 2038-2041

Wu, C., Zhang, G., Zhang, P., Chang, C.C. (2014) Disintegration of excess activated sludge with potassium permanganate: Feasibility, mechanisms and paramater optimisation. Chem Eng J 240, 420-425

Xu, J., Wu, L., Chen, W., Jiang, P., Chang, A.C.-S. (2009) Pharmaceuticals and Personal Care Products in Runoff from potato field irrigated with wastewater treated in Southern California. J Health Sci 55(2), 306-310

Yang, E.-L., Shi, J.-J., Liang, H.-C. (2012a) On-line electrochemical production of ferrate(VI) for odor control. Electrochim Acta 63, 369-374

Yang, B., Ying, G.-G., Zhao, J,-L, Liu, S., Zhou, L.-J., Chen, F. (2012b) Removal of selected endocrine disrupting chemicals (EDCs) and pharmaceuticals and personal care products (PPCPs) during ferrate(VI) treatment of secondary wastewater effluents. Water Res 46(7), 2194-2204

Ye, F.X., Peng, G., Li, Y. (2014) Fenton's oxidation to improve the filterability and dewaterability of excess activates sludge by affecting the extracellular polymeric substances. Asian J Chem 26(8), 2259-2263

Yin, X., Han, P., Lu X., Wang, Y. (2004) A review on the dewaterability of bio-sludge and ultrasound pretreatment. Ultrason Sonochem 11(6), 337-348 
Yoshimoto, T., Nagai, F., Fujimoto, J., Watanabe, K., Mizukoshi, H., Makino, T., Kimura, K., Saino, H., Sawada, H., Omura, H. Degradation of estrogens by Rhodococcus zopfii and Rhodococcus equi isolates from activated sludge in wastewater treatment plants. Appl Environ Microb 70(9):5283-5289

Zhang, A., Li, Y. (2014) Removal of phenolic endocrine disrupting compounds from waste activated sludge using UV, $\mathrm{H}_{2} \mathrm{O}_{2}$ and $\mathrm{UV} / \mathrm{H}_{2} \mathrm{O}_{2}$ oxidation processes: Effect of reaction conditions and sludge matrix. Sci Total Environ 493, 307-323

Zhu, J.-H., Yan, X.-L., Liu, Y., Zhang, B. (2006) Improving alachlor biodegradability by ferrate oxidation. J Hazard Mater 135(1-3), 94-99 


\section{Appendix A1}

Criteria for Selecting the Significant Dilutions Used to Determine the MPN Index and 95\% Confidence Limits (U.S. EPA, 2005a)

1. Choose the highest dilution (the most dilute, with the least amount of sample) giving positive results in all five tubes inoculated and the two succeeding higher (more dilute) dilutions. (For Example A from Table A1, $10^{-4}$ is higher/more dilute than $10^{-3}$.)

2. If the lowest dilution (least dilute) tested has less than five tubes with positive results, select it and the two next succeeding higher dilutions (Table A1, Examples B and C).

3. When a positive result occurs in a dilution higher (more dilute) than the three significant dilutions selected according to the rules above, change the selection to the lowest dilution (least dilute) that has less than five positive results and the next two higher dilutions (more dilute) (Table A1, Example D).

4. When the selection rules above have left unselected any higher dilutions (more dilute) with positive results, add those higher-dilution positive results to the results for the highest selected dilution (Table A1, Example E).

5. If there were not enough higher dilutions tested to select three dilutions, then select the next lower dilution (Table A1, Example F).

Table A1: Examples of significant dilutions and calculation of MPN/mL ${ }^{a}$ (U.S. EPA, 2005a).

\begin{tabular}{|c|c|c|c|c|c|c|}
\hline $\begin{array}{l}\text { Example } \\
\text { (liquid } \\
\text { or solid) }\end{array}$ & $\begin{array}{c}10^{-3} \\
\mathrm{~mL} \text { or } \mathrm{g}\end{array}$ & $\begin{array}{c}10^{4} \\
\mathrm{~mL} \text { or } \mathrm{g}\end{array}$ & $\begin{array}{c}10^{-5} \\
\mathrm{~mL} \text { or } \mathrm{g}\end{array}$ & $\begin{array}{c}10^{-6} \\
\mathrm{~mL} \text { or } \mathrm{g}\end{array}$ & $\begin{array}{l}\text { Step 1: } \\
\text { Significant } \\
\text { Dilutions }\end{array}$ & $\begin{array}{l}\text { Step 2: } \\
\text { (MPN from Table } 2 / \text { largest sig. dilution) } \\
=\text { MPN / } \mathrm{mL} \text { wet weight }\end{array}$ \\
\hline A & $5 / 5$ & $\underline{5 / 5}$ & $\underline{3 / 5}$ & $\underline{0 / 5}$ & $5-3-0$ & $\begin{array}{c}\left(7.92 / 10^{-4}\right)=79,200 \mathrm{MPN} / \mathrm{mL} \\
79,000 \mathrm{MPN} / \mathrm{mL}\end{array}$ \\
\hline B & $\underline{4 / 5}$ & $\underline{5 / 5}$ & $\underline{1 / 5}$ & $0 / 5$ & $4-5-1$ & $\begin{array}{c}\left(4.83 / 10^{-3}\right)=4830 \mathrm{MPN} / \mathrm{mL} \\
4800 \mathrm{MPN} / \mathrm{mL}\end{array}$ \\
\hline C & $\underline{0 / 5}$ & $\underline{1 / 5}$ & $\underline{0 / 5}$ & $0 / 5$ & $0-1-0$ & $\left(0.18 / 10^{-3}\right)=180 \mathrm{MPN} / \mathrm{mL}$ \\
\hline D & $5 / 5$ & $\underline{3 / 5}$ & $\underline{1 / 5}$ & $\underline{1 / 5}$ & $3-1-1$ & $\begin{array}{c}\left(1.37 / 10^{-4}\right)=13,700 \mathrm{MPN} / \mathrm{mL} \\
14,000 \mathrm{MPN} / \mathrm{mL}\end{array}$ \\
\hline$E$ & $\underline{4 / 5}$ & $\underline{4 / 5}$ & $\underline{0 / 5}$ & $1 / 5$ & $4-4-1$ & $\begin{array}{c}\left(3.98 / 10^{-3}\right)=3980 \mathrm{MPN} / \mathrm{mL} \\
4000 \mathrm{MPN} / \mathrm{mL}\end{array}$ \\
\hline $\mathrm{F}$ & $5 / 5$ & $\underline{5 / 5}$ & $\underline{5 / 5}$ & $\underline{2 / 5}$ & $5-5-2$ & $\begin{array}{c}\left(54.22 / 10^{-4}\right)=542,200 \mathrm{MPN} / \mathrm{mL} \\
540,000 \mathrm{MPN} / \mathrm{mL}\end{array}$ \\
\hline
\end{tabular}

a Significant dilutions are underlined and largest significant dilutions highlighted 


\section{Appendix A2}

Fecal Coliform MPN Index and 95\% Confidence Limits for Various Combinations of Positive Results When Fives Tubes are used per

Dilution (U.S. EPA, 2005 a; Klee, 1993)

\begin{tabular}{|c|c|c|c|c|c|c|c|}
\hline \multirow{2}{*}{$\begin{array}{c}\text { Combination of } \\
\text { Positives }\end{array}$} & \multirow{2}{*}{ MPN Index } & \multicolumn{2}{|c|}{ 95\% Confidence Limits } & \multirow{2}{*}{$\begin{array}{l}\text { Combination of } \\
\text { Positives }\end{array}$} & \multirow{2}{*}{ MPN Index } & \multicolumn{2}{|c|}{ 95\% Confidence Limits } \\
\hline & & Lower & Upper & & & Lower & Upper \\
\hline $0-0-0$ & $<0.1803$ & & & $1-3-0$ & 0.83 & 0.12 & 1.96 \\
\hline $0-0-1$ & 0.18 & 0.03 & 0.63 & $1-3-1$ & 1.04 & 0.20 & 2.43 \\
\hline $0-0-2$ & 0.36 & 0.03 & 1.01 & $1-3-2$ & 1.25 & 0.29 & 2.96 \\
\hline $0-0-3$ & 0.54 & 0.03 & 1.37 & $1-3-3$ & 1.47 & 0.38 & 3.64 \\
\hline $0-0-4$ & 0.72 & 0.08 & 1.74 & $1-3-4$ & 1.69 & 0.48 & 4.60 \\
\hline $0-0-5$ & 0.91 & 0.15 & 2.12 & $1-3-5$ & 1.91 & 0.57 & 5.66 \\
\hline $0-1-0$ & 0.18 & 0.03 & 0.63 & $1-4-0$ & 1.05 & 0.21 & 2.45 \\
\hline $0-1-1$ & 0.36 & 0.03 & 1.01 & $1-4-1$ & 1.27 & 0.30 & 3.00 \\
\hline $0-1-2$ & 0.55 & 0.03 & 1.38 & $1-4-2$ & 1.48 & 0.39 & 3.70 \\
\hline $0-1-3$ & 0.73 & 0.08 & 1.75 & $1-4-3$ & 1.70 & 0.48 & 4.68 \\
\hline $0-1-4$ & 0.91 & 0.15 & 2.14 & $1-4-4$ & 1.93 & 0.58 & 5.75 \\
\hline $0-1-5$ & 1.10 & 0.23 & 2.56 & $1-4-5$ & 2.15 & 0.67 & 6.57 \\
\hline $0-2-0$ & 0.37 & 0.03 & 1.02 & $1-5-0$ & 1.28 & 0.30 & 3.03 \\
\hline $0-2-1$ & 0.55 & 0.03 & 1.39 & $1-5-1$ & 1.50 & 0.40 & 3.75 \\
\hline $0-2-2$ & 0.74 & 0.08 & 1.76 & $1-5-2$ & 1.72 & 0.49 & 4.77 \\
\hline $0-2-3$ & 0.92 & 0.15 & 2.15 & $1-5-3$ & 1.95 & 0.58 & 5.83 \\
\hline $0-2-4$ & 1.11 & 0.23 & 2.58 & $1-5-4$ & 2.17 & 0.68 & 6.64 \\
\hline $0-2-5$ & 1.29 & 0.31 & 3.07 & $1-5-5$ & 2.40 & 0.77 & 7.31 \\
\hline $0-3-0$ & 0.56 & 0.03 & 1.40 & $2-0-0$ & 0.45 & 0.03 & 1.19 \\
\hline $0-3-1$ & 0.74 & 0.09 & 1.77 & $2-0-1$ & 0.68 & 0.06 & 1.64 \\
\hline $0-3-2$ & 0.93 & 0.16 & 2.17 & $2-0-2$ & 0.91 & 0.15 & 2.13 \\
\hline $0-3-3$ & 1.12 & 0.23 & 2.60 & $2-0-3$ & 1.15 & 0.25 & 2.69 \\
\hline $0-3-4$ & 1.30 & 0.31 & 3.10 & $2-0-4$ & 1.39 & 0.35 & 3.38 \\
\hline $0-3-5$ & 1.49 & 0.39 & 3.72 & $2-0-5$ & 1.64 & 0.46 & 4.37 \\
\hline $0-4-0$ & 0.75 & 0.09 & 1.79 & $2-1-0$ & 0.68 & 0.06 & 1.66 \\
\hline $0-4-1$ & 0.94 & 0.16 & 2.19 & $2-1-1$ & 0.92 & 0.15 & 2.16 \\
\hline $0-4-2$ & 1.12 & 0.24 & 2.63 & $2-1-2$ & 1.16 & 0.25 & 2.72 \\
\hline $0-4-3$ & 1.31 & 0.32 & 3.13 & $2-1-3$ & 1.41 & 0.36 & 3.43 \\
\hline $0-4-4$ & 1.50 & 0.40 & 3.77 & $2-1-4$ & 1.66 & 0.46 & 4.47 \\
\hline $0-4-5$ & 1.69 & 0.48 & 4.62 & $2-1-5$ & 1.92 & 0.57 & 5.71 \\
\hline $0-5-0$ & 0.94 & 0.16 & 2.21 & $2-2-0$ & 0.93 & 0.16 & 2.18 \\
\hline $0-5-1$ & 1.13 & 0.24 & 2.65 & $2-2-1$ & 1.18 & 0.26 & 2.76 \\
\hline $0-5-2$ & 1.33 & 0.32 & 3.17 & $2-2-2$ & 1.43 & 0.36 & 3.49 \\
\hline $0-5-3$ & 1.52 & 0.40 & 3.82 & $2-2-3$ & 1.68 & 0.47 & 4.56 \\
\hline $0-5-4$ & 1.71 & 0.48 & 4.70 & $2-2-4$ & 1.94 & 0.58 & 5.81 \\
\hline $0-5-5$ & 1.90 & 0.56 & 5.63 & $2-2-5$ & 2.21 & 0.69 & 6.75 \\
\hline $1-0-0$ & 0.20 & 0.03 & 0.68 & $2-3-0$ & 1.19 & 0.26 & 2.79 \\
\hline $1-0-1$ & 0.40 & 0.03 & 1.08 & $2-3-1$ & 1.44 & 0.37 & 3.55 \\
\hline $1-0-2$ & 0.60 & 0.03 & 1.49 & $2-3-2$ & 1.70 & 0.48 & 4.67 \\
\hline $1-0-3$ & 0.81 & 0.11 & 1.91 & $2-3-3$ & 1.97 & 0.59 & 5.91 \\
\hline $1-0-4$ & 1.01 & 0.19 & 2.36 & $2-3-4$ & 2.23 & 0.70 & 6.83 \\
\hline $1-0-5$ & 1.22 & 0.28 & 2.87 & $2-3-5$ & 2.51 & 0.82 & 7.59 \\
\hline $1-1-0$ & 0.40 & 0.03 & 1.09 & $2-4-0$ & 1.46 & 0.38 & 3.61 \\
\hline $1-1-1$ & 0.61 & 0.03 & 1.50 & $2-4-1$ & 1.72 & 0.49 & 4.77 \\
\hline $1-1-2$ & 0.81 & 0.11 & 1.92 & $2-4-2$ & 1.99 & 0.60 & 6.00 \\
\hline $1-1-3$ & 1.02 & 0.19 & 2.38 & $2-4-3$ & 2.26 & 0.72 & 6.92 \\
\hline $1-1-4$ & 1.23 & 0.28 & 2.90 & $2-4-4$ & 2.54 & 0.83 & 7.68 \\
\hline $1-1-5$ & 1.44 & 0.37 & 3.54 & $2-4-5$ & 2.82 & 0.94 & 8.36 \\
\hline $1-2-0$ & 0.61 & 0.03 & 1.51 & $2-5-0$ & 1.74 & 0.50 & 4.88 \\
\hline $1-2-1$ & 0.82 & 0.12 & 1.94 & $2-5-1$ & 2.01 & 0.61 & 6.10 \\
\hline $1-2-2$ & 1.03 & 0.20 & 2.40 & $2-5-2$ & 2.29 & 0.73 & 7.00 \\
\hline $1-2-3$ & 1.24 & 0.29 & 2.93 & $2-5-3$ & 2.57 & 0.84 & 7.76 \\
\hline $1-2-4$ & 1.46 & 0.38 & 3.59 & $2-5-4$ & 2.86 & 0.95 & 8.45 \\
\hline $1-2-5$ & 1.67 & 0.47 & 4.51 & $2-5-5$ & 3.15 & 1.07 & 9.10 \\
\hline
\end{tabular}




\section{Appendix A2 (cont.)}

Fecal Coliform MPN Index and 95\% Confidence Limits for Various Combinations of Positive Results When Fives Tubes Are Used per Dilution (U.S. EPA, 2005 a; Klee, 1993)

\begin{tabular}{|c|c|c|c|c|c|c|c|}
\hline \multirow{2}{*}{$\begin{array}{l}\text { Combination of } \\
\text { Positives }\end{array}$} & \multirow{2}{*}{ MPN Index } & \multicolumn{2}{|c|}{$95 \%$ Confidence Limits } & \multirow{2}{*}{$\begin{array}{l}\text { Combination of } \\
\text { Positives }\end{array}$} & \multirow{2}{*}{ MPN Index } & \multicolumn{2}{|c|}{ 95\% Confidence Limits } \\
\hline & & Lower & Upper & & & Lower & Upper \\
\hline $3-0-0$ & 0.79 & 0.10 & 1.88 & $4-3-0$ & 2.71 & 0.90 & 8.09 \\
\hline $3-0-1$ & 1.06 & 0.21 & 2.46 & $4-3-1$ & 3.26 & 1.11 & 9.34 \\
\hline $3-0-2$ & 1.35 & 0.33 & 3.23 & $4-3-2$ & 3.86 & 1.32 & 10.60 \\
\hline $3-0-3$ & 1.65 & 0.46 & 4.40 & $4-3-3$ & 4.51 & 1.54 & 11.92 \\
\hline $3-0-4$ & 1.96 & 0.59 & 5.89 & $4-3-4$ & 5.21 & 1.76 & 13.31 \\
\hline $3-0-5$ & 2.29 & 0.73 & 6.99 & $4-3-5$ & 5.93 & 1.96 & 14.77 \\
\hline $3-1-0$ & 1.07 & 0.22 & 2.50 & $4-4-0$ & 3.35 & 1.14 & 9.53 \\
\hline $3-1-1$ & 1.37 & 0.34 & 3.29 & $4-4-1$ & 3.98 & 1.37 & 10.84 \\
\hline $3-1-2$ & 1.67 & 0.47 & 4.52 & $4-4-2$ & 4.66 & 1.59 & 12.23 \\
\hline $3-1-3$ & 1.99 & 0.60 & 6.01 & $4-4-3$ & 5.39 & 1.81 & 13.68 \\
\hline $3-1-4$ & 2.32 & 0.74 & 7.10 & $4-4-4$ & 6.15 & 2.02 & 15.21 \\
\hline $3-1-5$ & 2.67 & 0.88 & 8.00 & $4-4-5$ & 6.93 & 2.23 & 16.81 \\
\hline $3-2-0$ & 1.38 & 0.35 & 3.35 & $4-5-0$ & 4.11 & 1.41 & 11.11 \\
\hline $3-2-1$ & 1.70 & 0.48 & 4.64 & $4-5-1$ & 4.83 & 1.64 & 12.56 \\
\hline $3-2-2$ & 2.02 & 0.62 & 6.13 & $4-5-2$ & 5.59 & 1.87 & 14.09 \\
\hline $3-2-3$ & 2.36 & 0.76 & 7.20 & $4-5-3$ & 6.39 & 2.09 & 15.70 \\
\hline $3-2-4$ & 2.71 & 0.90 & 8.10 & $4-5-4$ & 7.22 & 2.30 & 17.39 \\
\hline $3-2-5$ & 3.08 & 1.04 & 8.94 & $4-5-5$ & 8.06 & 2.50 & 19.16 \\
\hline $3-3-0$ & 1.72 & 0.49 & 4.77 & $5-0-0$ & 2.40 & 0.76 & 7.63 \\
\hline $3-3-1$ & 2.05 & 0.63 & 6.24 & $5-0-1$ & 3.14 & 1.06 & 9.08 \\
\hline $3-3-2$ & 2.40 & 0.77 & 7.31 & $5-0-2$ & 4.27 & 1.46 & 11.42 \\
\hline $3-3-3$ & 2.76 & 0.92 & 8.21 & $5-0-3$ & 5.78 & 1.92 & 14.46 \\
\hline $3-3-4$ & 3.13 & 1.06 & 9.06 & $5-0-4$ & 7.59 & 2.39 & 18.16 \\
\hline $3-3-5$ & 3.52 & 1.20 & 9.89 & $5-0-5$ & 9.53 & 1.65 & 22.34 \\
\hline $3-4-0$ & 2.09 & 0.64 & 6.36 & $5-1-0$ & 3.29 & 1.12 & 9.40 \\
\hline $3-4-1$ & 2.44 & 0.79 & 7.42 & $5-1-1$ & 4.56 & 1.56 & 12.02 \\
\hline $3-4-2$ & 2.81 & 0.93 & 8.33 & $5-1-2$ & 6.31 & 2.07 & 15.53 \\
\hline $3-4-3$ & 3.19 & 1.08 & 9.18 & $5-1-3$ & 8.39 & 2.57 & 19.85 \\
\hline $3-4-4$ & 3.58 & 1.23 & 10.02 & $5-1-4$ & 10.62 & 3.04 & 24.85 \\
\hline $3-4-5$ & 3.99 & 1.37 & 10.86 & $5-1-5$ & 12.93 & 3.04 & 30.90 \\
\hline $3-5-0$ & 2.48 & 0.80 & 7.53 & $5-2-0$ & 4.93 & 1.67 & 12.76 \\
\hline $3-5-1$ & 2.86 & 0.95 & 8.44 & $5-2-1$ & 7.00 & 2.24 & 16.94 \\
\hline $3-5-2$ & 3.25 & 1.10 & 9.31 & $5-2-2$ & 9.44 & 2.80 & 22.13 \\
\hline $3-5-3$ & 3.65 & 1.25 & 10.17 & $5-2-3$ & 12.05 & 3.31 & 28.43 \\
\hline $3-5-4$ & 4.07 & 1.40 & 11.03 & $5-2-4$ & 14.79 & 3.81 & 37.14 \\
\hline $3-5-5$ & 4.50 & 1.54 & 11.89 & $5-2-5$ & 17.67 & 5.03 & 52.30 \\
\hline $4-0-0$ & 1.30 & 0.31 & 3.11 & $5-3-0$ & 7.92 & 2.47 & 18.86 \\
\hline $4-0-1$ & 1.66 & 0.46 & 4.45 & $5-3-1$ & 10.86 & 3.08 & 25.44 \\
\hline $4-0-2$ & 2.07 & 0.64 & 6.31 & $5-3-2$ & 14.06 & 3.68 & 34.45 \\
\hline $4-0-3$ & 2.53 & 0.82 & 7.64 & $5-3-3$ & 17.50 & 4.34 & 51.31 \\
\hline $4-0-4$ & 3.02 & 1.02 & 8.81 & $5-3-4$ & 21.22 & 5.29 & 67.98 \\
\hline $4-0-5$ & 3.55 & 1.21 & 9.96 & $5-3-5$ & 25.27 & 8.14 & 79.71 \\
\hline $4-1-0$ & 1.69 & 0.48 & 4.60 & $5-4-0$ & 12.99 & 3.48 & 31.08 \\
\hline $4-1-1$ & 2.12 & 0.66 & 6.46 & $5-4-1$ & 17.24 & 4.29 & 49.75 \\
\hline $4-1-2$ & 2.58 & 0.85 & 7.79 & $5-4-2$ & 22.12 & 5.63 & 70.87 \\
\hline $4-1-3$ & 3.10 & 1.05 & 8.98 & $5-4-3$ & 27.81 & 8.82 & 86.00 \\
\hline $4-1-4$ & 3.65 & 1.25 & 10.16 & $5-4-4$ & 34.54 & 11.59 & 101.10 \\
\hline $4-1-5$ & 4.25 & 1.45 & 11.38 & $5-4-5$ & 42.56 & 14.37 & 118.00 \\
\hline $4-2-0$ & 2.16 & 0.67 & 6.61 & $5-5-0$ & 23.98 & 7.62 & 76.29 \\
\hline $4-2-1$ & 2.64 & 0.87 & 7.94 & $5-5-1$ & 34.77 & 11.72 & 101.60 \\
\hline $4-2-2$ & 3.17 & 1.08 & 9.15 & $5-5-2$ & 54.22 & 17.91 & 141.90 \\
\hline $4-2-3$ & 3.75 & 1.29 & 10.37 & $5-5-3$ & 91.78 & 26.72 & 220.10 \\
\hline $4-2-4$ & 4.38 & 1.50 & 11.64 & $5-5-4$ & 160.90 & 38.37 & 410.30 \\
\hline $4-2-5$ & 504 & 171 & 12.97 & $5-5-5$ & $\geq 160.90$ & & \\
\hline
\end{tabular}




\section{Appendix A3}

Salmonella MPN Index and 95\% Confidence Limits for Various

Combinations of Positive Results When Fives Tubes are used per 20.0, 10.0, and $1.0 \mathrm{~mL}$ Homogenised Sample Inoculation Volumes (U.S. EPA, 2005b; Klee, 1993)

\begin{tabular}{|c|c|c|c|c|c|c|c|}
\hline \multirow{2}{*}{$\begin{array}{l}\text { Combination } \\
\text { of Positives }\end{array}$} & \multirow[b]{2}{*}{ MPN Index } & \multicolumn{2}{|c|}{ 95\% Confidence Limits } & \multirow{2}{*}{$\begin{array}{c}\text { Combination of } \\
\text { Positives }\end{array}$} & \multirow[b]{2}{*}{ MPN Index } & \multicolumn{2}{|c|}{$95 \%$ Confidence Limits } \\
\hline & & Lower & Upper & & & Lower & Upper \\
\hline $0-0-0$ & $<0.006473$ & - & 0.0223 & $1-3-0$ & 0.0312 & 0.0055 & 0.0678 \\
\hline $0-0-1$ & 0.0065 & 0.0012 & 0.0223 & $1-3-1$ & 0.0393 & 0.0092 & 0.0821 \\
\hline $0-0-2$ & 0.0130 & 0.0012 & 0.0352 & $1-3-2$ & 0.0475 & 0.0132 & 0.0967 \\
\hline $0-0-3$ & 0.0195 & 0.0012 & 0.0472 & $1-3-3$ & 0.0559 & 0.0173 & 0.1119 \\
\hline $0-0-4$ & 0.0262 & 0.0033 & 0.0589 & $1-3-4$ & 0.0644 & 0.0216 & 0.1277 \\
\hline $0-0-5$ & 0.0328 & 0.0062 & 0.0706 & $1-3-5$ & 0.0730 & 0.0260 & 0.1444 \\
\hline $0-1-0$ & 0.0067 & 0.0012 & 0.0228 & $1-4-0$ & 0.0409 & 0.0099 & 0.0849 \\
\hline $0-1-1$ & 0.0134 & 0.0012 & 0.0360 & $1-4-1$ & 0.0495 & 0.0141 & 0.1002 \\
\hline $0-1-2$ & 0.0202 & 0.0012 & 0.0483 & $1-4-2$ & 0.0583 & 0.0185 & 0.1163 \\
\hline $0-1-3$ & 0.0270 & 0.0037 & 0.0604 & $1-4-3$ & 0.0672 & 0.0231 & 0.1331 \\
\hline $0-1-4$ & 0.0339 & 0.0067 & 0.0725 & $1-4-4$ & 0.0763 & 0.0277 & 0.1509 \\
\hline $0-1-5$ & 0.0408 & 0.0099 & 0.0847 & $1-4-5$ & 0.0855 & 0.0324 & 0.1700 \\
\hline $0-2-0$ & 0.0138 & 0.0012 & 0.0367 & $1-5-0$ & 0.0517 & 0.0152 & 0.1042 \\
\hline $0-2-1$ & 0.0208 & 0.0012 & 0.0495 & $1-5-1$ & 0.0609 & 0.0199 & 0.1212 \\
\hline $0-2-2$ & 0.0279 & 0.0040 & 0.0619 & $1-5-2$ & 0.0703 & 0.0247 & 0.1391 \\
\hline $0-2-3$ & 0.0350 & 0.0072 & 0.0745 & $1-5-3$ & 0.0799 & 0.0296 & 0.1583 \\
\hline $0-2-4$ & 0.0422 & 0.0106 & 0.0871 & $1-5-4$ & 0.0897 & 0.0346 & 0.1790 \\
\hline $0-2-5$ & 0.0494 & 0.0141 & 0.1001 & $1-5-5$ & 0.0998 & 0.0397 & 0.2015 \\
\hline $0-3-0$ & 0.0215 & 0.0012 & 0.0507 & $2-0-0$ & 0.0155 & 0.0012 & 0.0404 \\
\hline $0-3-1$ & 0.0288 & 0.0044 & 0.0636 & $2-0-1$ & 0.0226 & 0.0018 & 0.0526 \\
\hline $0-3-2$ & 0.0362 & 0.0077 & 0.0766 & $2-0-2$ & 0.0303 & 0.0051 & 0.0662 \\
\hline $0-3-3$ & 0.0437 & 0.0113 & 0.0898 & $2-0-3$ & 0.0382 & 0.0087 & 0.0801 \\
\hline $0-3-4$ & 0.0512 & 0.0051 & 0.1243 & $2-0-4$ & 0.0462 & 0.0125 & 0.0943 \\
\hline $0-3-5$ & 0.0588 & 0.0095 & 0.1428 & $2-0-5$ & 0.0543 & 0.0165 & 0.1090 \\
\hline $0-4-0$ & 0.0299 & 0.0049 & 0.0654 & $2-1-0$ & 0.0234 & 0.0022 & 0.0540 \\
\hline $0-4-1$ & 0.0375 & 0.0084 & 0.0789 & $2-1-1$ & 0.0315 & 0.0056 & 0.0683 \\
\hline $0-4-2$ & 0.0453 & 0.0121 & 0.0927 & $2-1-2$ & 0.0397 & 0.0094 & 0.0827 \\
\hline $0-4-3$ & 0.0531 & 0.0160 & 0.1069 & $2-1-3$ & 0.0480 & 0.0134 & 0.0976 \\
\hline $0-4-4$ & 0.0611 & 0.0200 & 0.1216 & $2-1-4$ & 0.0565 & 0.0177 & 0.1131 \\
\hline $0-4-5$ & 0.0691 & 0.0241 & 0.1369 & $2-1-5$ & 0.0652 & 0.0221 & 0.1293 \\
\hline $0-5-0$ & 0.0390 & 0.0090 & 0.0814 & $2-2-0$ & 0.0327 & 0.0062 & 0.0705 \\
\hline $0-5-1$ & 0.0470 & 0.0129 & 0.0958 & $2-2-1$ & 0.0413 & 0.0101 & 0.0856 \\
\hline $0-5-2$ & 0.0553 & 0.0170 & 0.1107 & $2-2-2$ & 0.0501 & 0.0144 & 0.1013 \\
\hline $0-5-3$ & 0.0636 & 0.0212 & 0.1262 & $2-2-3$ & 0.0590 & 0.0189 & 0.1176 \\
\hline $0-5-4$ & 0.0720 & 0.0255 & 0.1425 & $2-2-4$ & 0.0681 & 0.0236 & 0.1349 \\
\hline $0-5-5$ & 0.0806 & 0.0299 & 0.1596 & $2-2-5$ & 0.0774 & 0.0283 & 0.1533 \\
\hline $1-0-0$ & 0.0072 & 0.0012 & 0.0241 & $2-3-0$ & 0.0431 & 0.0110 & 0.0887 \\
\hline $1-0-1$ & 0.0139 & 0.0012 & 0.0369 & $2-3-1$ & 0.0523 & 0.0155 & 0.1053 \\
\hline $1-0-2$ & 0.0209 & 0.0012 & 0.0497 & $2-3-2$ & 0.0617 & 0.0203 & 0.1227 \\
\hline $1-0-3$ & 0.0281 & 0.0041 & 0.0623 & $2-3-3$ & 0.0714 & 0.0252 & 0.1412 \\
\hline $1-0-4$ & 0.0353 & 0.0073 & 0.0749 & $2-3-4$ & 0.0813 & 0.0303 & 0.1611 \\
\hline $1-0-5$ & 0.0425 & 0.0107 & 0.0878 & $2-3-5$ & 0.0914 & 0.0354 & 0.1826 \\
\hline $1-1-0$ & 0.0144 & 0.0012 & 0.0377 & $2-4-0$ & 0.0547 & 0.0168 & 0.1098 \\
\hline $1-1-1$ & 0.0217 & 0.0013 & 0.0509 & $2-4-1$ & 0.0647 & 0.0218 & 0.1284 \\
\hline $1-1-2$ & 0.0290 & 0.0045 & 0.0640 & $2-4-2$ & 0.0750 & 0.0271 & 0.1484 \\
\hline $1-1-3$ & 0.0365 & 0.0079 & 0.0771 & $2-4-3$ & 0.0855 & 0.0325 & 0.1700 \\
\hline $1-1-4$ & 0.0441 & 0.0115 & 0.0905 & $2-4-4$ & 0.0964 & 0.0380 & 0.1937 \\
\hline $1-1-5$ & 0.0517 & 0.0153 & 0.1043 & $2-4-5$ & 0.1076 & 0.0436 & 0.2201 \\
\hline $1-2-0$ & 0.0224 & 0.0017 & 0.0523 & $2-5-0$ & 0.0681 & 0.0235 & 0.1349 \\
\hline $1-2-1$ & 0.0301 & 0.0050 & 0.0658 & $2-5-1$ & 0.0791 & 0.0292 & 0.1566 \\
\hline $1-2-2$ & 0.0379 & 0.0085 & 0.0795 & $2-5-2$ & 0.0904 & 0.0349 & 0.1805 \\
\hline $1-2-3$ & 0.0457 & 0.0123 & 0.0935 & $2-5-3$ & 0.1021 & 0.0409 & 0.2070 \\
\hline $1-2-4$ & 0.0537 & 0.0162 & 0.1079 & $2-5-4$ & 0.1143 & 0.0469 & 0.2372 \\
\hline $1-2-5$ & 0.0618 & 0.0203 & 0.1229 & $2-5-5$ & 0.1268 & 0.0531 & 0.2725 \\
\hline
\end{tabular}




\section{Appendix A3 (cont.)}

Salmonella MPN Index and 95\% Confidence Limits for Various

Combinations of Positive Results When Fives Tubes are used per 20.0, 10.0 , and $1.0 \mathrm{~mL} \mathrm{Homogenised} \mathrm{Sample} \mathrm{Inoculation} \mathrm{Volumes} \mathrm{(U.S.} \mathrm{EPA,}$ 2005b; Klee, 1993)

\begin{tabular}{|c|c|c|c|c|c|c|c|}
\hline \multirow{2}{*}{$\begin{array}{l}\text { Combination of } \\
\text { Positives }\end{array}$} & \multirow[b]{2}{*}{ MPN Index } & \multicolumn{2}{|c|}{ 95\% Confidence Limits } & \multirow{2}{*}{$\begin{array}{c}\text { Combination of } \\
\text { Positives }\end{array}$} & \multirow[b]{2}{*}{ MPN Index } & \multicolumn{2}{|c|}{$95 \%$ Confidence Limits } \\
\hline & & Lower & Upper & & & Lower & Upper \\
\hline $3-0-0$ & 0.0255 & 0.0028 & 0.0585 & $4-3-0$ & 0.0797 & 0.0295 & 0.1579 \\
\hline $3-0-1$ & 0.0330 & 0.0063 & 0.0710 & $4-3-1$ & 0.0937 & 0.0366 & 0.1877 \\
\hline $3-0-2$ & 0.0417 & 0.0103 & 0.0863 & $4-3-2$ & 0.1086 & 0.0441 & 0.2228 \\
\hline $3-0-3$ & 0.0506 & 0.0147 & 0.1023 & $4-3-3$ & 0.1245 & 0.0520 & 0.2656 \\
\hline $3-0-4$ & 0.0598 & 0.0193 & 0.1191 & $4-3-4$ & 0.1414 & 0.0602 & 0.3218 \\
\hline $3-0-5$ & 0.0691 & 0.0241 & 0.1368 & $4-3-5$ & 0.1595 & 0.0686 & 0.4067 \\
\hline $3-1-0$ & 0.0344 & 0.0069 & 0.0734 & $4-4-0$ & 0.1012 & 0.0404 & 0.2049 \\
\hline $3-1-1$ & 0.0435 & 0.0112 & 0.0896 & $4-4-1$ & 0.1181 & 0.0489 & 0.2476 \\
\hline $3-1-2$ & 0.0529 & 0.0159 & 0.1065 & $4-4-2$ & 0.1364 & 0.0578 & 0.3038 \\
\hline $3-1-3$ & 0.0626 & 0.0207 & 0.1244 & $4-4-3$ & 0.1563 & 0.0672 & 0.3890 \\
\hline $3-1-4$ & 0.0725 & 0.0258 & 0.1434 & $4-4-4$ & 0.1780 & 0.0770 & 0.5273 \\
\hline $3-1-5$ & 0.0827 & 0.0310 & 0.1640 & $4-4-5$ & 0.2015 & 0.0873 & 0.6411 \\
\hline $3-2-0$ & 0.0456 & 0.0122 & 0.0932 & $4-5-0$ & 0.1304 & 0.0549 & 0.2836 \\
\hline $3-2-1$ & 0.0555 & 0.0171 & 0.1112 & $4-5-1$ & 0.1524 & 0.0653 & 0.3687 \\
\hline $3-2-2$ & 0.0657 & 0.0223 & 0.1303 & $4-5-2$ & 0.1769 & 0.0766 & 0.5210 \\
\hline $3-2-3$ & 0.0763 & 0.0277 & 0.1510 & $4-5-3$ & 0.2046 & 0.0886 & 0.6528 \\
\hline $3-2-4$ & 0.0872 & 0.0333 & 0.1735 & $4-5-4$ & 0.2357 & 0.1015 & 0.7516 \\
\hline $3-2-5$ & 0.0984 & 0.0390 & 0.1984 & $4-5-5$ & 0.2708 & 0.1150 & 0.8426 \\
\hline $3-3-0$ & 0.0583 & 0.0186 & 0.1164 & $5-0-0$ & 0.0549 & 0.0162 & 0.1116 \\
\hline $3-3-1$ & 0.0693 & 0.0241 & 0.1371 & $5-0-1$ & 0.0637 & 0.0213 & 0.1265 \\
\hline $3-3-2$ & 0.0806 & 0.0299 & 0.1597 & $5-0-2$ & 0.0763 & 0.0277 & 0.1510 \\
\hline $3-3-3$ & 0.0924 & 0.0359 & 0.1847 & $5-0-3$ & 0.0896 & 0.0345 & 0.1787 \\
\hline $3-3-4$ & 0.1046 & 0.0421 & 0.2128 & $5-0-4$ & 0.1037 & 0.0417 & 0.2107 \\
\hline $3-3-5$ & 0.1173 & 0.0484 & 0.2452 & $5-0-5$ & 0.0953 & 0.0165 & 0.2234 \\
\hline $3-4-0$ & 0.0733 & 0.0262 & 0.1450 & $5-1-0$ & 0.0678 & 0.0234 & 0.1344 \\
\hline $3-4-1$ & 0.0856 & 0.0325 & 0.1700 & $5-1-1$ & 0.0816 & 0.0304 & 0.1618 \\
\hline $3-4-2$ & 0.0984 & 0.0390 & 0.1982 & $5-1-2$ & 0.0963 & 0.0379 & 0.1936 \\
\hline $3-4-3$ & 0.1118 & 0.0457 & 0.2307 & $5-1-3$ & 0.1121 & 0.0459 & 0.2316 \\
\hline $3-4-4$ & 0.1258 & 0.0526 & 0.2695 & $5-1-4$ & 0.1291 & 0.0542 & 0.2796 \\
\hline $3-4-5$ & 0.1405 & 0.0597 & 0.3184 & $5-1-5$ & 0.1293 & 0.0304 & 0.3090 \\
\hline $3-5-0$ & 0.0913 & 0.0354 & 0.1825 & $5-2-0$ & 0.0879 & 0.0337 & 0.1751 \\
\hline $3-5-1$ & 0.1055 & 0.0426 & 0.2150 & $5-2-1$ & 0.1046 & 0.0421 & 0.2128 \\
\hline $3-5-2$ & 0.1204 & 0.0500 & 0.2538 & $5-2-2$ & 0.1227 & 0.0511 & 0.2605 \\
\hline $3-5-3$ & 0.1362 & 0.0577 & 0.3029 & $5-2-3$ & 0.1427 & 0.0608 & 0.3267 \\
\hline $3-5-4$ & 0.1529 & 0.0656 & 0.3715 & $5-2-4$ & 0.1646 & 0.0710 & 0.4385 \\
\hline $3-5-5$ & 0.1707 & 0.0738 & 0.4795 & $5-2-5$ & 0.1767 & 0.0503 & 0.5230 \\
\hline $4-0-0$ & 0.0381 & 0.0082 & 0.0809 & $5-3-0$ & 0.1151 & 0.0474 & 0.2394 \\
\hline $4-0-1$ & 0.0461 & 0.0125 & 0.0942 & $5-3-1$ & 0.1368 & 0.0580 & 0.3050 \\
\hline $4-0-2$ & 0.0563 & 0.0175 & 0.1126 & $5-3-2$ & 0.1614 & 0.0695 & 0.4183 \\
\hline $4-0-3$ & 0.0668 & 0.0229 & 0.1323 & $5-3-3$ & 0.1895 & 0.0821 & 0.5899 \\
\hline $4-0-4$ & 0.0777 & 0.0284 & 0.1537 & $5-3-4$ & 0.2216 & 0.0957 & 0.7101 \\
\hline $4-0-5$ & 0.0890 & 0.0342 & 0.1773 & $5-3-5$ & 0.2527 & 0.0814 & 0.7971 \\
\hline $4-1-0$ & 0.0484 & 0.0136 & 0.0983 & $5-4-0$ & 0.1571 & 0.0676 & 0.3935 \\
\hline $4-1-1$ & 0.0592 & 0.0190 & 0.1181 & $5-4-1$ & 0.1907 & 0.0826 & 0.5954 \\
\hline $4-1-2$ & 0.0705 & 0.0248 & 0.1395 & $5-4-2$ & 0.2319 & 0.0999 & 0.7409 \\
\hline $4-1-3$ & 0.0822 & 0.0308 & 0.1631 & $5-4-3$ & 0.2834 & 0.1196 & 0.8726 \\
\hline $4-1-4$ & 0.0945 & 0.0370 & 0.1894 & $5-4-4$ & 0.3475 & 0.1417 & 1.0160 \\
\hline $4-1-5$ & 0.1072 & 0.0434 & 0.2193 & $5-4-5$ & 0.4256 & 0.1437 & 1.1800 \\
\hline $4-2-0$ & 0.0626 & 0.0207 & 0.1244 & $5-5-0$ & 0.2398 & 0.0762 & 0.7629 \\
\hline $4-2-1$ & 0.0748 & 0.0269 & 0.1479 & $5-5-1$ & 0.3477 & 0.1172 & 1.0160 \\
\hline $4-2-2$ & 0.0875 & 0.0335 & 0.1742 & $5-5-2$ & 0.5422 & 0.1791 & 1.4190 \\
\hline $4-2-3$ & 0.1009 & 0.0403 & 0.2041 & $5-5-3$ & 0.9178 & 0.2672 & 2.2010 \\
\hline $4-2-4$ & 0.1150 & 0.0473 & 0.2392 & $5-5-4$ & 1.6090 & 0.3837 & 4.1030 \\
\hline $4-2-5$ & 0.1299 & 0.0546 & 0.2820 & $5-5-5$ & $\geq 1.609000$ & 0.3837 & $\ldots$ \\
\hline
\end{tabular}




\section{Appendix B1}

Temperature Logs for Freeze-Thaw Experiments with E. coli in PBS, using Freezer Temperatures of $-10,-20$ and $-30^{\circ} \mathrm{C}$

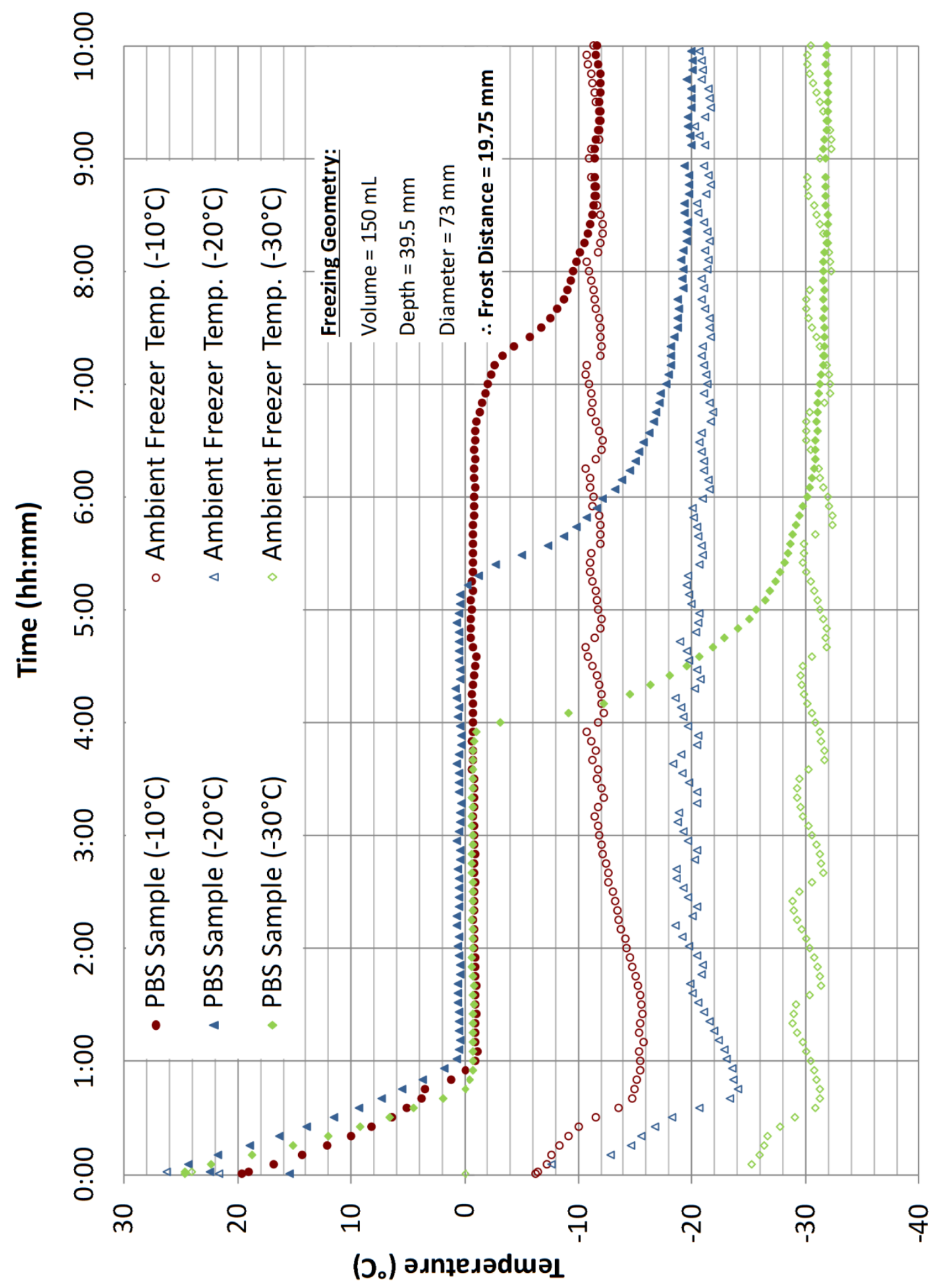




\section{Appendix B2}

Temperature Logs for Freeze-Thaw Experiments with Primary Sludge, using a Freezer Temperature of $-20^{\circ} \mathrm{C}$

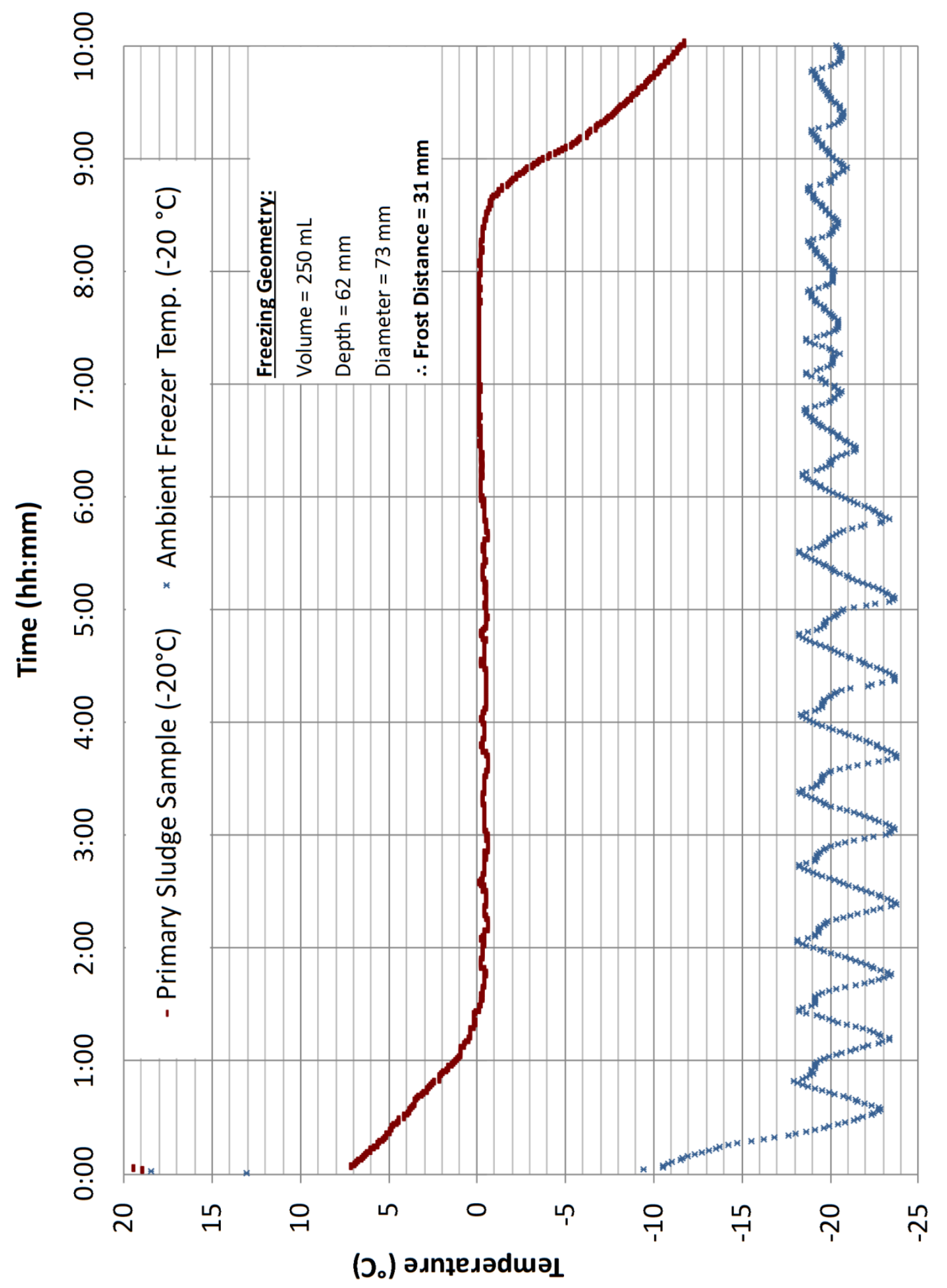




\section{Appendix B3}

Temperature Logs for Freeze-Thaw Experiments with Anaerobically Digested Sludge, using a Freezer Temperature of $-20^{\circ} \mathrm{C}$

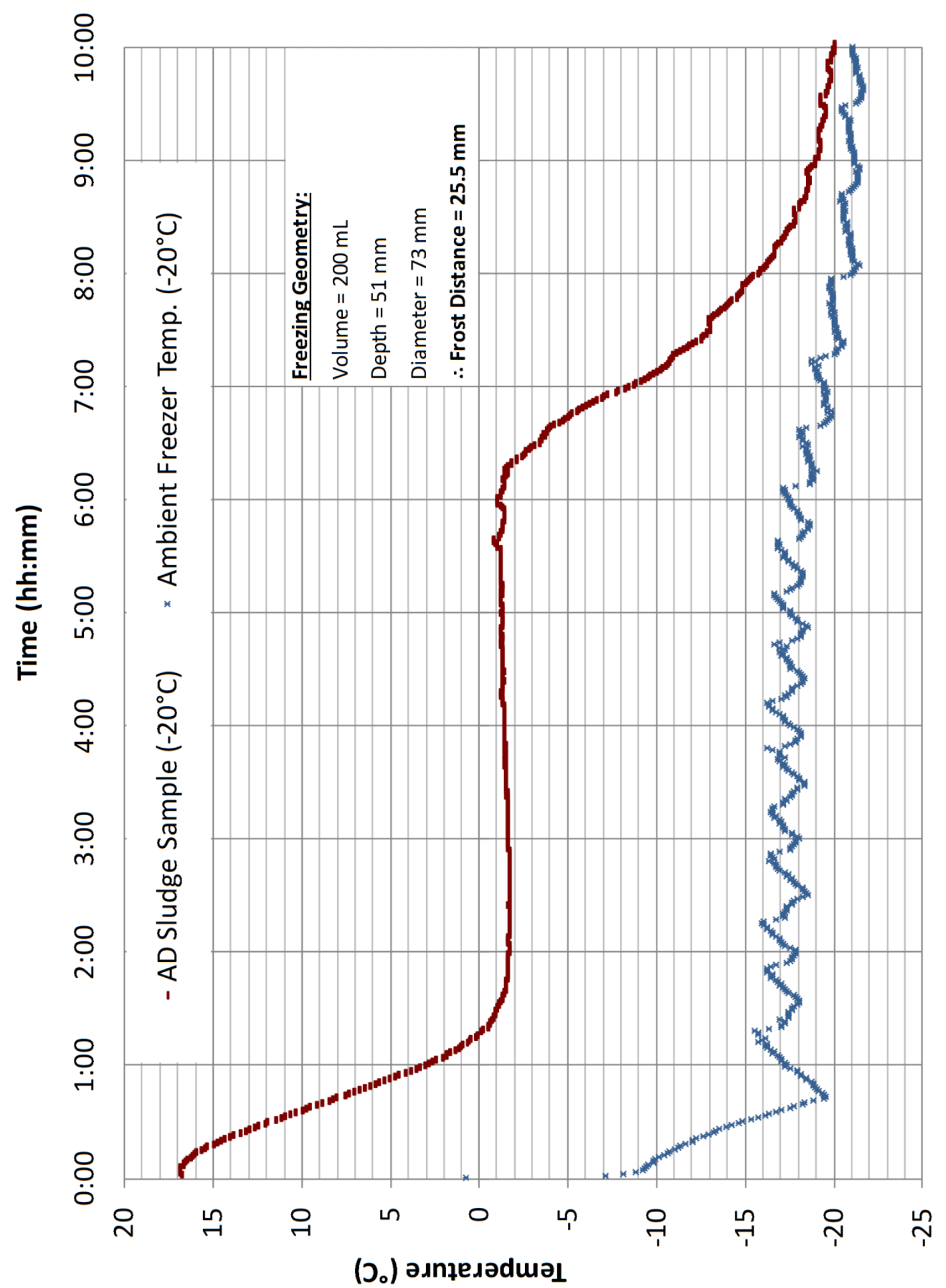




\section{Appendix B4}

Temperature Logs for Freeze-Thaw Experiments with Primary Sludge, using Freezer Temperatures of $-10,-20$ and $-30^{\circ} \mathrm{C}$

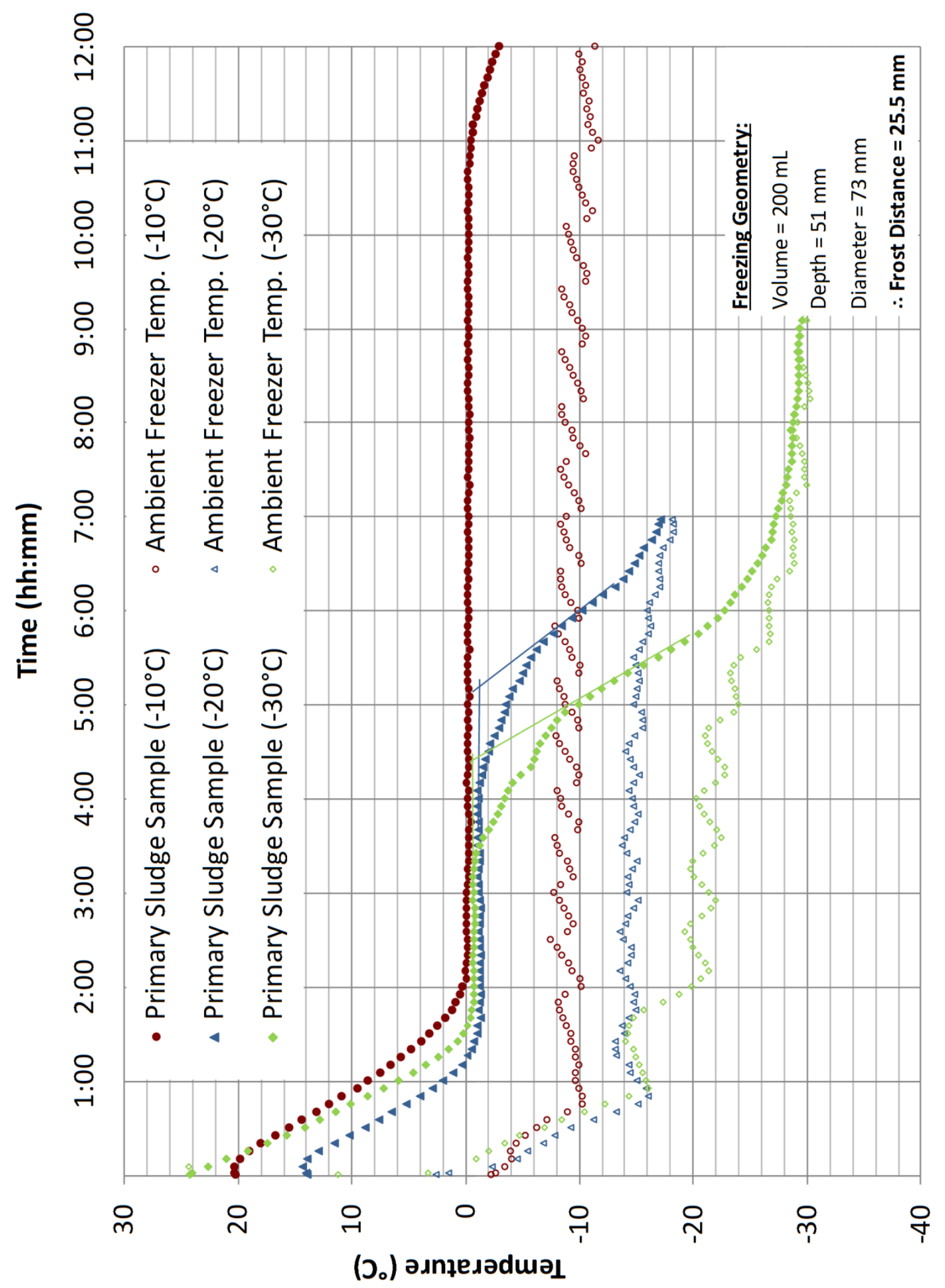




\section{Appendix B5}

Summary of Freezing Geometries, Temperature Log Data, Freezing Rates and Calculated Proportionality Coefficients

\begin{tabular}{|c|c|c|c|c|c|c|c|c|c|c|c|}
\hline 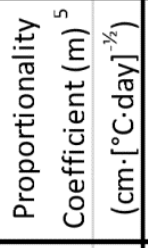 & $\stackrel{9}{-}$ & & & 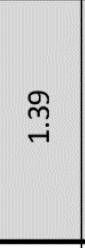 & $\stackrel{\text { mे }}{-}$ & & 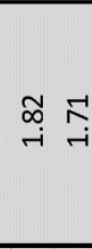 & 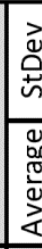 & & $\bar{c}$ & \\
\hline 告 & $\stackrel{-}{-}$ & 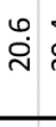 & ¿े. & $\stackrel{-1}{-1}$ & $\begin{array}{l}\sigma \\
\infty \\
\infty\end{array}$ & 웅 & $\stackrel{\substack{n \\
\ddot{g}}}{\stackrel{\infty}{\infty}}$ & : & 0 & & \\
\hline 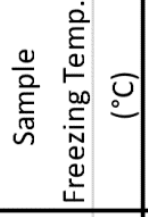 & 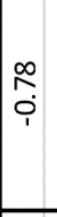 & भึ่ & & $\begin{array}{l}\tilde{m} \\
\stackrel{m}{i}\end{array}$ & 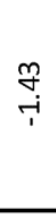 & 告 & 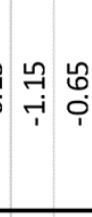 & & & & 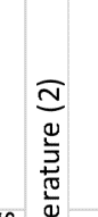 \\
\hline 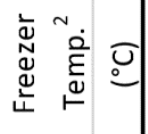 & 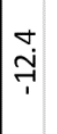 & 올 & - & ঙ. & ণั่ & क्. & 守 & $\frac{a}{0}$ & & & 崖 \\
\hline 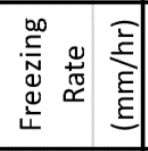 & $\stackrel{\sim}{m}$ & $\dot{f}$ & $\begin{array}{l}\varphi_{0} \\
\dot{0}\end{array}$ & $\stackrel{+}{+}$ & $\stackrel{\varphi}{\dot{n}}$ & $\stackrel{\circ}{\dot{m}}$ & $\stackrel{-}{\pi} \circ$ & 吾 & c & & 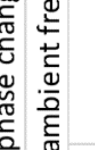 \\
\hline 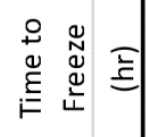 & $\begin{array}{l}\text { So } \\
\text { in }\end{array}$ & $\underset{+}{\vec{f}}$ & $\begin{array}{l}8 \\
\text { m. }\end{array}$ & $\stackrel{8}{r}$ & $\stackrel{\infty}{\stackrel{\infty}{\sim}} \underset{+}{+}$ & $\begin{array}{l}\text { h } \\
\infty \\
\infty\end{array}$ & 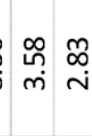 & $\frac{1}{2}$ & & & 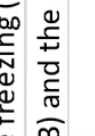 \\
\hline 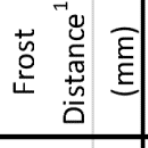 & $\begin{array}{l}\frac{n}{2} \\
\frac{9}{7}\end{array}$ & 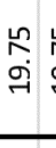 & & $\vec{m}$ & 呒 & 亗 & 赑 & 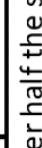 & & & 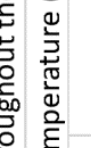 \\
\hline 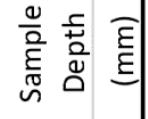 & ஸे & लें & 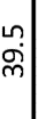 & $\widetilde{6}$ & ถี & กำ & ถู ถี & 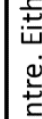 & & & 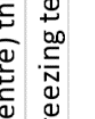 \\
\hline 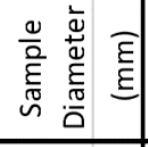 & $m$ & $\stackrel{m}{\sim}$ & $\stackrel{\Re}{\wedge}$ & $\stackrel{m}{\sim}$ & $\stackrel{m}{\wedge}$ & $\stackrel{m}{n}$ & $\stackrel{m}{N}$ & 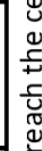 & & & 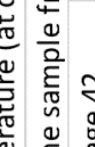 \\
\hline $\begin{array}{lll}\frac{0}{0} & \stackrel{0}{\xi} & \vec{E} \\
\stackrel{n}{n} & \frac{3}{\partial} & \vec{\xi}\end{array}$ & 옴 & 号 & ㅇํำ & 呙 & ষ্ণ & ষ্ণ & ৪্ণ & & & & 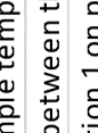 \\
\hline 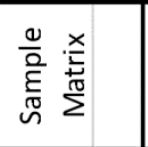 & & ณू & & 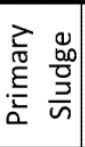 & $\begin{array}{l}0 \\
\frac{0}{00} \\
\frac{0}{n} \\
0 \\
0\end{array}$ & & 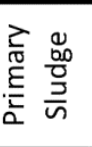 & & & & 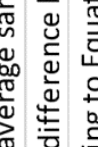 \\
\hline 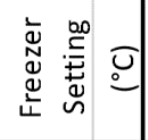 & 우 & શ્ર & 위 & Tิ & ণ্ণ & 구 & ণ্ণ & & & & 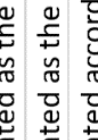 \\
\hline 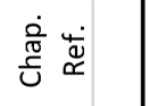 & 6 & 6 & 6 & 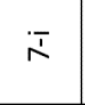 & 交 & $\infty$ & $\infty \infty$ & $\pi$ & & & 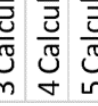 \\
\hline
\end{tabular}




\section{Appendix C1}

Sludge Generation in a CLEMENTINE $®$ Wastewater Treatment Unit with Combined Primary Settling and Biological Treatment using a RBC

Table C1: Assumptions, data and calculations used to determine the mass, volume and characteristics of the sludge generated in the CLEMENTINE ${ }^{\circledR}$ WWT Unit.

\section{Starting Assumptions}

\begin{tabular}{|lc|}
\hline Inlet TSS: & $500 \mathrm{mg} / \mathrm{L}$ \\
Outlet TSS: & $20 \mathrm{mg} / \mathrm{L}$ \\
Inlet $\mathrm{BOD}_{5}:$ & $500 \mathrm{mg} / \mathrm{L}$ \\
Outlet $\mathrm{BOD}_{5}:$ & $20 \mathrm{mg} / \mathrm{L}$ \\
Inlet $\mathrm{NH}_{3}-\mathrm{N}:$ & $50 \mathrm{mg} / \mathrm{L}$ \\
Inlet TN: & $85 \mathrm{mg} / \mathrm{L}$ \\
Inlet TP: & $20 \mathrm{mg} / \mathrm{L}$ \\
Average Daily Flow: & $14 \mathrm{~m}^{3} /$ day \\
\hline
\end{tabular}

Sludge Generation

Assumed

\begin{tabular}{|c|c|c|}
\hline & TSS Generation Rate: \\
\hline VSS Proportion and Generation Rate: & $90 \%$ & $6.05 \mathrm{~kg} / \mathrm{day}$ \\
\hline FSS Proportion and Generation Rate: & $10 \%$ & $0.67 \mathrm{~kg} / \mathrm{day}$ \\
\hline Auto-Digestion Efficiency: & $35 \%$ & $3.93 \mathrm{~kg} / \mathrm{day}$ \\
\hline $\mathrm{BOD}_{5}$ Removed in PST: & $25 \%$ & $1.75 \mathrm{~kg} \mathrm{BOD}_{5} /$ day \\
\hline \multicolumn{2}{|l|}{$\mathrm{BOD}_{5}$ Entering RBC: } & $5.25 \mathrm{~kg} \mathrm{BOD}_{5} /$ day \\
\hline \multicolumn{2}{|l|}{$\mathrm{BOD}_{5}$ Removed by RBC: } & $5.04 \mathrm{~kg} \mathrm{BOD}_{5} /$ day \\
\hline \multirow{2}{*}{$\begin{array}{l}\text { Biomass Yield and VSS Produced in RBC: } \\
\text { Auto-Digestion Efficiency: }\end{array}$} & $0.3 \mathrm{~kg} \mathrm{VSS} / \mathrm{kg} \mathrm{BOD}{ }_{5}$ & $1.51 \mathrm{~kg} \mathrm{VSS} /$ day \\
\hline & $35 \%$ & $0.98 \mathrm{~kg}$ VSS/day \\
\hline \multicolumn{2}{|l|}{ Total Dry Mass of Sludge } & $5.59 \mathrm{~kg} / \mathrm{day}$ \\
\hline \multirow{2}{*}{$\begin{array}{l}\text { TSS of Wet Sludge: } \\
\text { Density of Wet Sludge: }\end{array}$} & $2.6 \%$ & \\
\hline & $1000 \mathrm{~kg} / \mathrm{m}^{3}$ & \\
\hline \multirow[t]{3}{*}{ Volume of Wet Sludge: } & & $0.21 \mathrm{~m}^{3} /$ day \\
\hline & & $1.50 \mathrm{~m}^{3} / \mathrm{wk}$ \\
\hline & & $78.42 \mathrm{~m}^{3} / \mathrm{yr}$ \\
\hline Excess Sludge Storage Time: $\quad(0.25-0.5 \mathrm{yr})$ & $0.325 \mathrm{yr}$ & \\
\hline \multicolumn{2}{|l|}{ Additional Sludge Storage Volume Required: } & $25.49 \mathrm{~m}^{3}$ \\
\hline
\end{tabular}




\section{Appendix C2}

Annual Ferrate(VI) Production Costs for Electrochemically-Generated Ferrate(VI) Solutions and Ferrate(VI) Salts for Sludge Treatment

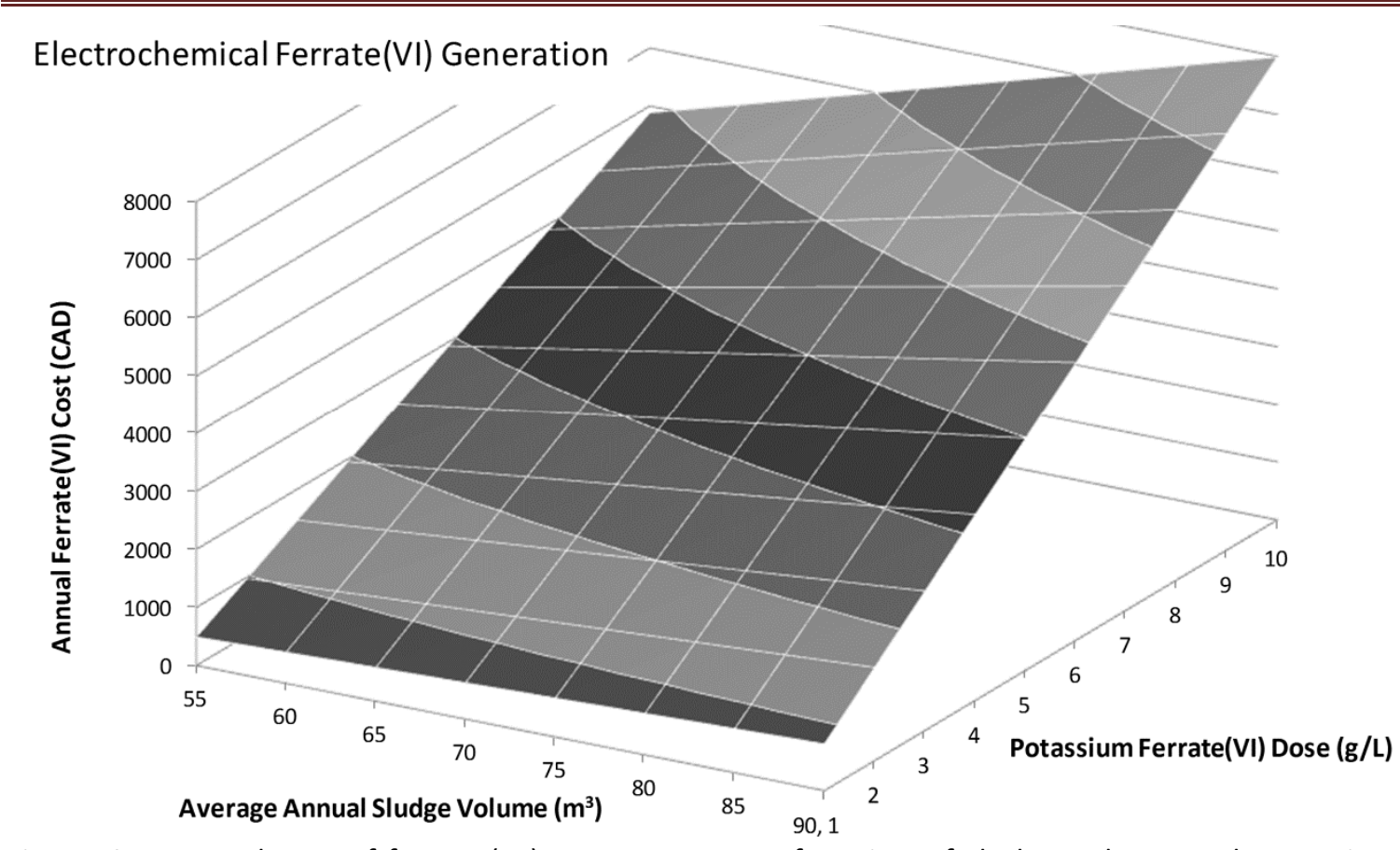

Figure C1: Annual cost of ferrate(VI) treatments as a function of sludge volume and potassium ferrate(VI) dose, using electrochemical ferrate(VI) production (after Waite, 2012).

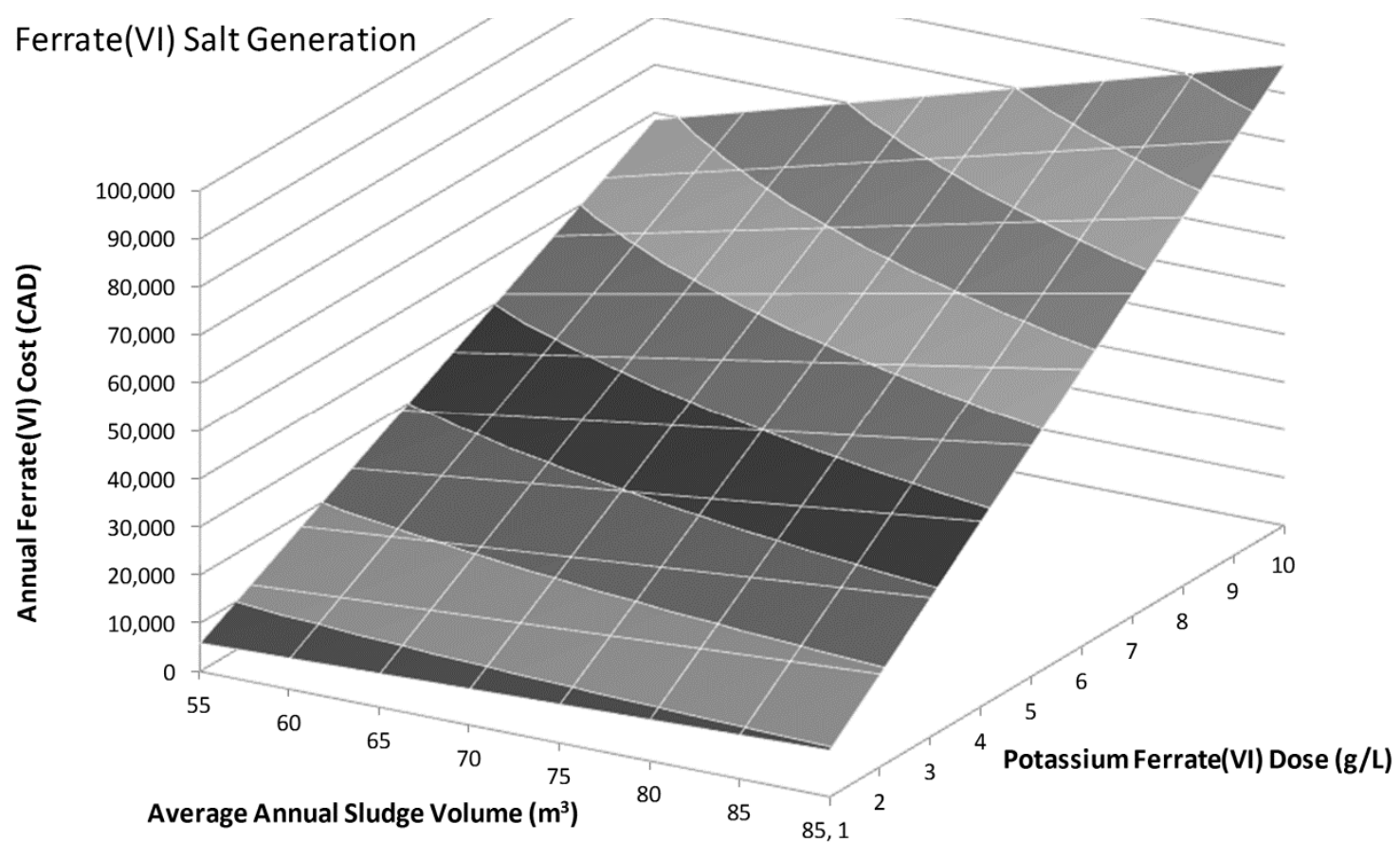

Figure C2: Annual cost of ferrate(VI) treatments as a function of sludge volume and potassium ferrate(VI) dose, using powdered ferrate(VI) salts (after Waite, 2012). 


\section{Appendix C3}

Operation of a Freeze-Thaw Sludge Dewatering Bed:

Estimated Sludge Freezing Time as a Function of Sludge Layer

Thickness and Average Daily Freezing Temperature

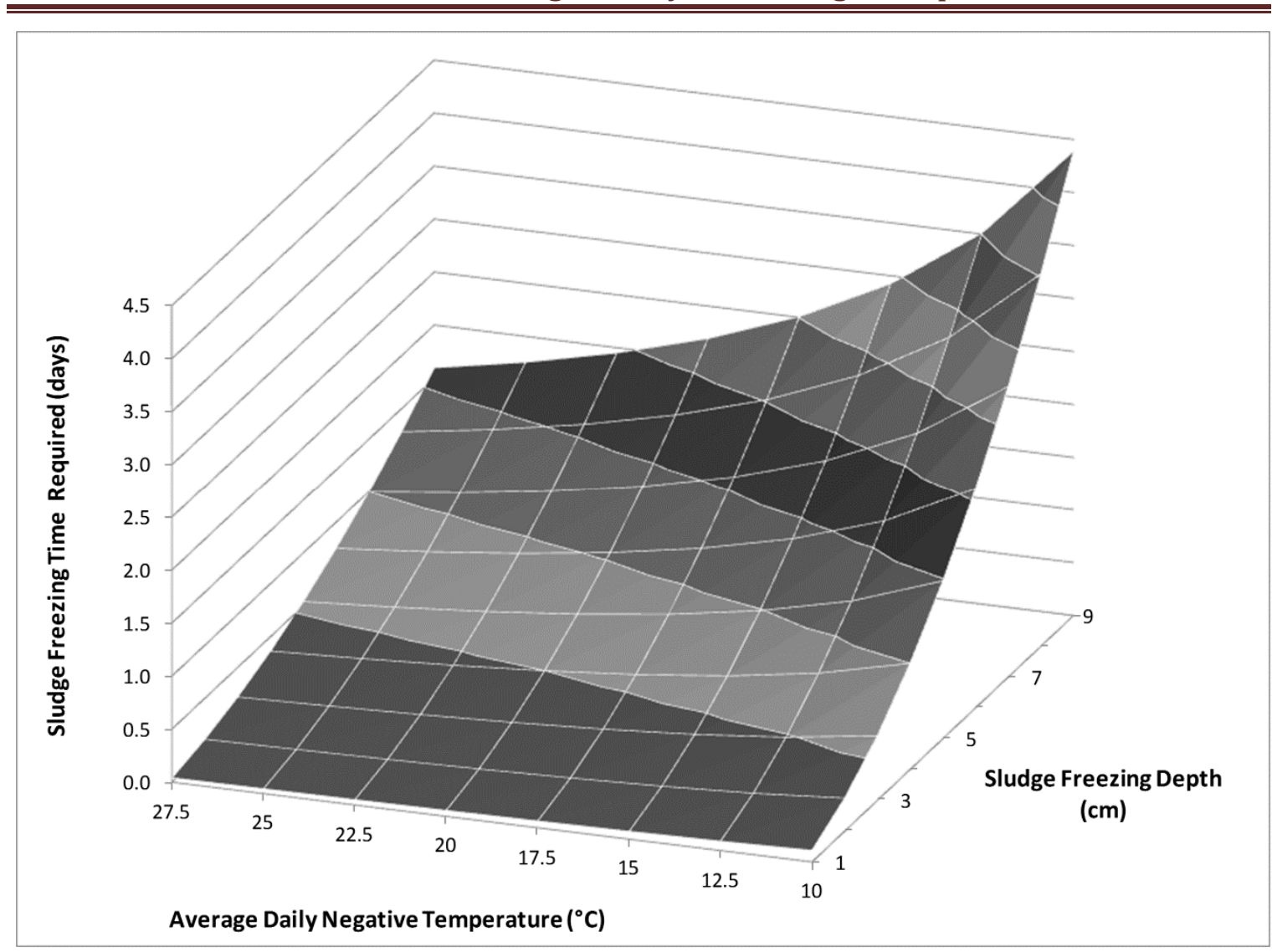

Figure C3: Time required to freeze sludge layers, with an initial temperature $=5^{\circ} \mathrm{C}$, as a function of sludge layer depth (thickness) and the average daily freezing temperature. Figure created using Equation 2 by Kinsley et al., 2012. 


\section{Appendix C4}

Meltwater Generation, Collection and Management

Table C1: Assumptions, data and calculations to determine the volume and characteristics of the meltwater generated during sludge thawing at an average daily temperature of $15{ }^{\circ} \mathrm{C}$.

Meltwater Collection

\begin{tabular}{|c|c|c|c|c|}
\hline Sludge Volume $\%$ and Total Meltwater: & $86.8 \%$ & \multicolumn{2}{|c|}{$68.07 \mathrm{~m}^{3}$} & \\
\hline Thaw Period: & 93 days & & & \\
\hline Meltwater Flow: & & \multicolumn{2}{|c|}{$0.73 \mathrm{~m}^{3} /$ day } & \\
\hline Peak Flow: & 7 days & \multicolumn{2}{|c|}{$0.97 \mathrm{~m}^{3} /$ day } & \\
\hline \multirow{2}{*}{\multicolumn{2}{|c|}{$\begin{array}{l}\text { Meltwater Characteristics } \\
\text { (Weighted Average) }\end{array}$}} & & & \\
\hline & & Avg & Peak & \\
\hline TSS & $51.1 \mathrm{mg} / \mathrm{L}$ & 0.037 & 0.050 & kg TSS/day \\
\hline VSS & $45.5 \mathrm{mg} / \mathrm{L}$ & 0.033 & 0.044 & kg VSS/day \\
\hline COD & $3077 \mathrm{mg} / \mathrm{L}$ & 2.252 & 2.992 & kg COD/day \\
\hline TN & $342.5 \mathrm{mg} / \mathrm{L}$ & 0.251 & 0.333 & kg N/day \\
\hline TP & $56.7 \mathrm{mg} / \mathrm{L}$ & 0.041 & 0.055 & $\mathrm{~kg} \mathrm{PO}_{4}^{-3} /$ day \\
\hline
\end{tabular}

Table C2: Calculated existing load on the WWT unit.

Existing Wastewater Loading

\begin{tabular}{|c|c|c|c|}
\hline $\begin{array}{l}\text { Total Surface Area of RBC: } \\
\text { Hydraulic loading: }\end{array}$ & $504 \mathrm{~m}^{2}$ & 0.028 & $\mathrm{~m}^{3} / \mathrm{m}^{2} \mathrm{~d}$ \\
\hline Influent TSS: & & 7.0 & kg TSS/day \\
\hline Influent VSS: & & 6.3 & kg VSS/day \\
\hline Influent $\mathrm{BOD}_{5}$ : & & 7.0 & $\mathrm{~kg} \mathrm{BOD}_{5} /$ day \\
\hline $\mathrm{BOD}_{5}$ loading: & & 13.89 & $\mathrm{~g} \mathrm{BOD}_{5} / \mathrm{m}^{2}$ day \\
\hline Influent $\mathrm{COD}\left(\mathrm{BOD}_{5} / \mathrm{COD}\right)$ & $75 \%$ & 9.33 & kg COD/day \\
\hline COD loading: & & 18.52 & $\mathrm{~g} \operatorname{COD} / \mathrm{m}^{2}$ day \\
\hline Influent $\mathrm{NH}_{3}-\mathrm{N}$ : & & 0.70 & $\mathrm{~kg} \mathrm{NH}_{3}-\mathrm{N} / \mathrm{day}$ \\
\hline $\mathrm{NH}_{3}-\mathrm{N}$ loading: & & 1.39 & $\mathrm{~g} \mathrm{NH}_{3}-\mathrm{N} / \mathrm{m}^{2}$ day \\
\hline Influent TN: & & 1.19 & kg N/day \\
\hline TN loading: & & 2.36 & $\mathrm{~g} \mathrm{~N} / \mathrm{m}^{2}$ day \\
\hline Influent TP: & & 0.20 & $\mathrm{~kg} \mathrm{PO}_{4}^{-3} /$ day \\
\hline TP loading: & & 0.39 & $\mathrm{gPO}_{4}^{-3} / \mathrm{m}^{2}$ day \\
\hline
\end{tabular}

Table C3: Calculated combined loading on the WWT unit, and corresponding percent increases in load during sludge thawing at an average daily temperature of $15^{\circ} \mathrm{C}$.

Combined Loading (Wastewater + Meltwater)

\begin{tabular}{|l|cc|c|cc|l|c|c|}
\cline { 2 - 8 } \multicolumn{1}{c|}{} & \multicolumn{2}{c|}{ Design Values } & $\begin{array}{c}\text { Existing } \\
\text { WW }\end{array}$ & Avg & Peak & Units & $\begin{array}{c}\% \text { Inc. } \\
\text { (Avg) }\end{array}$ & $\begin{array}{c}\% \text { Inc. } \\
\text { (Peak) }\end{array}$ \\
\hline Hydraulic loading: & 0.08 & 0.16 & 0.028 & 0.030 & 0.030 & $\mathrm{~m}^{3} / \mathrm{m}^{2} \cdot \mathrm{d}$ & $7 \%$ & $7 \%$ \\
BOD $_{5}$ loading: & 8 & 20 & 13.9 & 19.2 & 19.2 & $\mathrm{~g} \mathrm{BOD} / \mathrm{m}^{2}$ day & $38 \%$ & $38 \%$ \\
$\mathrm{NH}_{3}$-N loading: & 0.75 & 1.5 & 1.39 & 1.88 & 1.88 & $\mathrm{~g} \mathrm{NH}_{3}-\mathrm{N} / \mathrm{m}^{2}$ day & $36 \%$ & $36 \%$ \\
TN loading: & & 3.35 & 2.36 & 3.02 & 3.02 & $\mathrm{~g} \mathrm{NH}_{3}-\mathrm{N} / \mathrm{m}^{2}$ day & $28 \%$ & $28 \%$ \\
TP loading: & 0.24 & 0.75 & 0.39 & 0.50 & 0.50 & $\mathrm{~g} \mathrm{PO}_{4}^{-3} / \mathrm{m}^{2}$ day & $28 \%$ & $28 \%$ \\
\hline
\end{tabular}

